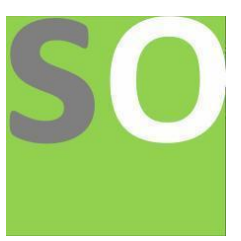

Article title: North Wakashan Comparative Root List (Updated)

Authors: John Rath[1]

Affiliations: Private researcher[1]

Orcid ids: 0000-0003-0515-5792[1]

Contact e-mail: john.c.rath@gmail.com

License information: This work has been published open access under Creative Commons Attribution License http://creativecommons.org/licenses/by/4.0/, which permits unrestricted use, distribution, and reproduction in any medium, provided the original work is properly cited. Conditions, terms of use and publishing policy can be found at https://www.scienceopen.com/.

Preprint statement: This article is a preprint and has not been peer-reviewed, under consideration and submitted to ScienceOpen Preprints for open peer review.

Funder: None

DOI: 10.14293/S2199-1006.1.SOR-.PPU1HYE.v1

Preprint first posted online: 19 October 2021

Keywords: Wakashan, Heiltsuk, Oowekyala, Haisla, Kwakwala, comparative linguistics 


\title{
North Wakashan Comparative Root List (Updated)
}

\author{
John C. Rath \\ Correspondence address: \\ 3375 Ponytrail Drive, Ste. 603 \\ Mississauga, Ontario, L4A 1V8 \\ Canada \\ John.C.Rath@Gmail.com
}

(905) 602-4446

October 19, 2021 


\begin{abstract}
This inventory of North Wakashan roots is a revised edition of a similar work published in 1980. Where applicable a root is cross-referenced to its occurrence in the earlier edition. For each root is shown the form(s) it takes in the four major varieties of North Wakashan and its known meanings. In selected cases editorial comments are added in footnotes. These comments may use phonemic and/or morphophonemic transcriptions whose interpretation requires experience with North Wakashan and for which the reader may wish to consult the literature referred to in the introductory paragraphs preceding the root list.
\end{abstract}

Keywords

Wakashan, Heiltsuk, Oowekyala, Haisla, Kwakwala, comparative linguistics 


\section{North Wakashan Comparative Root List (Updated)}

\section{Introduction}

1.1 North Wakashan divides into four main varieties hereafter called Heiltsuk, Oowekyala, Haisla and Kwakwala and referred to generically as isolects to avoid the language or dialect issue. Introductions to their phonemics and morphophonemics are provided in Rath (2020), a paper the reader is assumed to be familiar with. It can be viewed and downloaded at www.ScienceOpen.com (direct link: https://www.scienceopen.com/search\#author/ca9f5ab98d36-41b4-9bfc-49629f3c1cbb).

North Wakashan lexical words can consist of just a root with or without internal modifications but usually they begin in a root followed by one or more elements that for present purposes can all be called suffixes. This paper lists the forms and meanings of the roots identified to date, in selected cases with additional information in footnotes. It is a revised edition of Lincoln \& Rath (1980) and incorporates fresh data, particularly from Haisla. The paper's data is extracted from an MS Access-based all-North Wakashan lexicographic database maintained by this writer. Upon request the derivates of particular roots including their morphophonemic analysis can be made available in spreadsheet format. On-line accessibility of the database is a future project.

The Heiltsuk and Oowekyala in the database stems from this author's fieldwork since 1974. Most of the Haisla data was collected in the early 1980's in cooperation with Neville Lincoln of SFU. The Kwakwala data is from Franz Boas's Kwakiutl Grammar (1947) and unpublished Kwakiutl Dictionary (n.d.) but always checked against Neville Lincoln's unpublished Kwakwala dictionary of more than 22,000 lexical entries which he has kindly made available to this writer.

For this paper no financial support was received from funding agencies in the public, commercial, or not-for-profit sectors. 


\section{North Wakashan Comparative Root List (Updated)}

\section{Abbreviations and Other Notational Conventions}

\begin{tabular}{|l|l|l|}
\hline $\mathrm{HE}$ & & Heiltsuk \\
\hline $\mathrm{OO}$ & & Oowekyala \\
\hline $\mathrm{HA}$ & & Haisla \\
\hline $\mathrm{KW}$ & & Kwakwala \\
\hline sb. & & somebody \\
\hline sth. & & something \\
\hline
\end{tabular}

In phonemic and morphophonemic transcriptions this paper uses the notational conventions applied in the MS Access-based database. The following table shows how they differ from those applied in Rath (2020).

\begin{tabular}{|c|c|}
\hline Rath (2020) & This paper \\
\hline$\chi$ (a lambda with a stroke) & $£$ (pound sterling sign) \\
\hline ğ & г (a Cyrillic character) \\
\hline$\check{\mathrm{x}}$ & $\chi$ \\
\hline apostrophe above a glyph, as in $\hat{\mathrm{p}}$, ả & superscripted 3 after the glyph as in $\mathrm{p}^{3}, \mathrm{a}^{3}$ \\
\hline $\begin{array}{l}\mathrm{w} \text { (raised little } \mathrm{w} \text { signaling lip rounding as in } \\
\left.\check{\mathrm{g}}^{\mathrm{w}}, \mathrm{q}^{\mathrm{w}}, \mathrm{k}^{\mathrm{w}}\right)\end{array}$ & $\mathrm{v}\left(\right.$ as in $\left.\mathrm{rv}, \mathrm{qv}, \mathrm{k}^{3} \mathrm{v}\right)$ \\
\hline $\begin{array}{l}\text { ma, n, } 1 \text { (letter } m, n \text { or } 1 \text { with dot } \\
\text { underneath) }\end{array}$ & м, н, л (Cyrillic characters) \\
\hline
\end{tabular}

The elimination of diacritics allows print to stay legible even at small point sizes and avoids the problem that an apostrophe may not print properly or at all above a tall glyph. 


\section{North Wakashan Comparative Root List (Updated)}

Names of people and places are written with an initial capital letter. This concerns only obstruents and consonantal resonants as only these can occur word-initially. As capitals corresponding to $\lambda, £, \digamma, \chi$ are used $\Lambda, £$ (no special capital), $Г, X$.

No italics are used in this paper because they can be hard to read. In comments on roots phonemic transcriptions are surrounded by slants, as in OO /bipgvanm/, and morphophonemic ones by swung brackets, as in OO $\{[\mathrm{by}] \mathrm{bkv}-\% \mathrm{hnm}\}$. Submorphemic components of morphophonemic structures are however cited between verticals, as in $|\%|$.

When citing a series of cognate forms from different isolects, no commas separate the individual forms. Thus, $\mathrm{HE} / \mathrm{pkv}$ 'u/ OO /pkvu/ HA /bkvu/ "to borrow a boat" KW /bku/ "to borrow" and not $\mathrm{HE} / \mathrm{pkv}$ 'u/, OO /pkvu/, HA /bkvu/ "to borrow a boat," KW /bku/ "to borrow."

An asterisk after a phonemic or morphophonemic transcription means the item was found in literature but could not be re-elicited. An example is $\mathrm{KW}$ \{bns- $\}^{*}$ "bashful, embarrassed." The cases concerned are practically all from KW. An asterisk before a transcription means it is a hypothetical form. Thus, the policy regarding asterisk use differs from that stated in Lincoln \& Rath (1980:50-51). The warning given there bears repeating: one should be distrustful of KW forms that occur in materials collected by Franz Boas and George Hunt but could not be reelicited by Neville Lincoln, numerous as they regrettably are.

In this paper the phoneme $/ \mathrm{h}^{3} /$ is written $/ \mathrm{h}^{3} /$ when the result of a morphophonemic process and

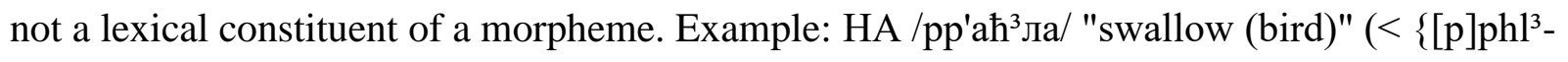
$\operatorname{lh}\}<\{[\mathrm{p}] \mathrm{ph}\}-+\mathrm{lh}\})$.

A root may have two or more variants that are not derivable from a common form in which case they are displayed one above the other, as in the following example: 
North Wakashan Comparative Root List (Updated)

\begin{tabular}{|l|l|l|l|l|}
\hline HE & OO & HA & \multicolumn{1}{|c|}{ KW } & \multicolumn{1}{|c|}{ Gloss } \\
\hline byx- & byx- & byx- & $\begin{array}{l}\text { byx- } \\
\text { bx-* } \\
\text { myx- }\end{array}$ & $\begin{array}{l}\text { to glow, sparkle, phosphoresce, } \\
\text { emit light }\end{array}$ \\
\hline
\end{tabular}

It may be undecidable which of several possible forms a root has. In these cases the alternatives are also listed one above the other but with a forward slash after the final hyphen, as in the following example:

\begin{tabular}{|c|c|c|c|c|}
\hline HE & OO & HA & KW & Gloss \\
\hline & $\begin{array}{l}\text { myk-I } \\
\text { myk }^{3}-\end{array}$ & $\begin{array}{ll}\text { myk-*/ } \\
\text { myk }^{3}-*\end{array}$ & $(?)$ \\
\hline
\end{tabular}

A gap in a set of cognate roots may be filled with a semantically similar but formally different root written between parentheses, see the following example from HA:

\begin{tabular}{|c|c|c|c|c|}
\hline HE & $\mathrm{OO}$ & HA & KW & Gloss \\
\hline $\mathrm{h}^{3} \mathrm{lkv}-$ & $\mathrm{h}^{3} \mathrm{lkv}-$ & (blk-) & $\begin{array}{l}\mathrm{h}^{3} \mathrm{lkv}- \\
\mathrm{h}^{3} \mathrm{l} \mathrm{xv}-\end{array}$ & $\begin{array}{l}\text { blood, to lose blood, to bleed; } \\
\text { (OO also:) precious }\end{array}$ \\
\hline
\end{tabular}




\section{North Wakashan Comparative Root List (Updated)}

\section{Root List}

Attention! The same root form can be listed under multiple root numbers. To find a particular root form in the below listing, first look up its number(s) in the alphabetic index of root forms at the end of this paper.

\begin{tabular}{|c|c|c|c|c|c|c|}
\hline Root \# & Old \# & $\mathrm{HE}$ & $\mathrm{OO}$ & HA & KW & Gloss \\
\hline 10 & 1 & & bns- & & bns-* & bashful, embarrassed \\
\hline 20 & 2 & $\left(\right.$ wnq $\left.^{3}-\right)$ & $\left(\mathrm{wnq}^{3}-\right)$ & $\left(\right.$ wnq $\left.^{3}-\right)$ & $\mathrm{bn}^{3}-$ & down, under, below, lower ${ }^{1}$ \\
\hline 30 & 3 & $b^{3}{ }^{3}$ & $b^{3}{ }^{3}$ & & & close, to fit closely \\
\hline 40 & 4 & & & & bs- & to bend metal \\
\hline 50 & 5 & bl- & bl- & bl- & bl- & to forbid, prevent \\
\hline 60 & 7 & $\left(\mathrm{~h}^{3} \mathrm{lkv}-\right)$ & $\left(\mathrm{h}^{3} \mathrm{lkV}-\right)$ & blk- & $\begin{array}{l}\left(\mathrm{h}^{3} \mathrm{lkv}-\right. \\
\left.\mathrm{h}^{3} 1 \mathrm{xv}-\right)\end{array}$ & $\begin{array}{l}\text { blood, to lose blood, to bleed; } \\
\text { (OO also:) precious }{ }^{2}\end{array}$ \\
\hline 70 & 8 & blxv- & blxv- & blxv- & blxv- & Bella Coola (place name) \\
\hline 80 & 54 & & & plq- & blq-* & $\begin{array}{l}\text { bud, excrescence, blossom; } \\
\text { abalone }^{3}\end{array}$ \\
\hline 90 & 9 & & & & $\mathrm{bl} \chi^{-*}$ & $\begin{array}{l}\text { scatter, spread (feathers, moss, } \\
\text { down, roots) }\end{array}$ \\
\hline 100 & 10 & by- & by- & by- & by-* & abandoned place \\
\hline 110 & & & & & bys- & $(?)^{4}$ \\
\hline 120 & 11 & & & był- & był- & $\begin{array}{l}\text { pull apart (moss, etc.), scatter, } \\
\text { spread }\end{array}$ \\
\hline 130 & 12 & byk- & byk- & byk- & byk- & to transfer fire \\
\hline
\end{tabular}

${ }^{1} \mathrm{KW}$ uses $\{$ wnq- $\}$ for "deep."

${ }^{2}$ Root occurs also in a few OO endearing terms for infants.

${ }^{3} \mathrm{KW}$ only in /bлrina/* "humpbacked whale"

${ }^{4}$ In $\mathrm{KW} / \mathrm{bisa} /$ "to pick-up trifles, small things needed for some purpose." Could be an alternant of root no. 120 . 
North Wakashan Comparative Root List (Updated)

\begin{tabular}{|c|c|c|c|c|c|c|}
\hline Root \# & Old \# & $\mathrm{HE}$ & $\mathrm{OO}$ & HA & KW & Gloss \\
\hline 140 & $\begin{array}{r}13 \\
120\end{array}$ & byx- & byx- & byx- & $\begin{array}{l}\text { byx- } \\
\text { bx-* } \\
\text { myx- }\end{array}$ & $\begin{array}{l}\text { to glow, sparkle, phosphoresce, } \\
\text { emit light }\end{array}$ \\
\hline 150 & 14 & pkv- & pkv- & bkv- & bkv- & $(?)^{5}$ \\
\hline 160 & $\begin{array}{r}15 \\
70 \\
317\end{array}$ & $\begin{array}{l}\text { dkv- } \\
\text { pkv- } \\
\text { bhkv- }\end{array}$ & $\begin{array}{l}\text { bkv- } \\
\text { pkv- } \\
\text { bhkv- }\end{array}$ & $\begin{array}{l}\text { bkv-, } \\
\text { bhkv- }\end{array}$ & $\begin{array}{l}\text { bkv- } \\
\text { bhkv- }\end{array}$ & $\begin{array}{l}\text { human, having personality (like } \\
\text { humans and supernatural beings); } \\
\text { male; (KW also:) testing, tough, } \\
\text { unbending character, macho }\end{array}$ \\
\hline 170 & 16 & bw- & bw- & bw- & bw- & leave, abandon, flee, escape \\
\hline 180 & 17 & & & bwt- & & (?) to swell, bulge, bud ${ }^{7}$ \\
\hline 190 & 18 & bws- & bws- & & bws- & to swell (esp. when pregnant) \\
\hline 200 & 19 & $\begin{array}{l}\left(\mathrm{m}^{3} \mathrm{w} £-\right. \\
\left.\mathrm{m}^{3} \mathrm{w} 1-\right)\end{array}$ & $\begin{array}{l}\left(\mathrm{m}^{3} \mathrm{w} £-\right. \\
\left.\mathrm{m}^{3} \mathrm{w} 1-\right)\end{array}$ & $\begin{array}{l}\left(\mathrm{m}^{3} \mathrm{w} £-\right. \\
\left.\mathrm{m}^{3} \mathrm{w} 1-\right)\end{array}$ & $\begin{array}{l}\text { bwf- } \\
\text { bwł- }\end{array}$ & $\begin{array}{l}\text { to swell, to bulge, to boil (tide), } \\
\text { to bud or flower }\end{array}$ \\
\hline 210 & 20 & bw1- & bw1- & bw1- & bw1- & $\begin{array}{l}\text { head or front going down, to } \\
\text { topple, to stumble }\end{array}$ \\
\hline 220 & 21 & bwXV- & bwXV- & bwXV- & bwxv- & pregnant (esp. illegitimately) \\
\hline 230 & $\begin{array}{l}22 \\
23 \\
24\end{array}$ & bwqv- & bwqv- & bwqv- & bwqv-* & $\begin{array}{l}\text { to swell (like bud, knob or when } \\
\text { pregnant), swelling or }\end{array}$ \\
\hline
\end{tabular}

\footnotetext{
${ }^{5}$ Only in HE /pkv'u/ OO /pkvu/ HA /bkvu/ "to borrow a boat" KW /bku/ "to borrow."

${ }^{6} \mathrm{HE}\{\mathrm{dkv}-\}$ OO $\{\mathrm{bkv}-\}$ are diagnosable only in $\mathrm{HE}\{[\mathrm{dy}] \mathrm{dkv}-\% \mathrm{hnm}\}>/ \mathrm{d}^{\prime} \mathrm{itgv}^{\prime} \mathrm{an}^{\prime} \mathrm{m} / \mathrm{OO}$ $\{[$ by]bkv-\%hnm $\}>$ /bipgvanm/ (plurals of HE /tgv'an'M/ OO /pgvanm/ "person, man," a term with the same ambiguity as French "homme").

${ }^{7}$ All examples refer to female genitals.

${ }^{8}$ \{bw£-\} only in KW /bu£kmala/ "heaping full." Some examples of $\{$ bwł- $\}$ refer to female reproductive power.
} 
North Wakashan Comparative Root List (Updated)

\begin{tabular}{|c|c|c|c|c|c|c|}
\hline Root \# & Old \# & $\mathrm{HE}$ & $\mathrm{OO}$ & $\mathrm{HA}$ & KW & Gloss \\
\hline & & & & & & $\begin{array}{l}\text { pressurized container discharging } \\
\text { its contents }{ }^{9}\end{array}$ \\
\hline 240 & 25 & pqv- & pqv- & bqv- & $\begin{array}{l}\text { bqv- } \\
\text { bhqv- }\end{array}$ & $(?)^{10}$ \\
\hline 250 & $\begin{array}{l}26 \\
27\end{array}$ & & & $\mathrm{~b} \chi-$ & $\mathrm{b} \chi-$ & $\begin{array}{l}\text { to slit, slash, lance cut; } \\
\text { (according to Boas (1948:116):) } \\
\text { to cut towards body with a } \\
\text { straight knife }\end{array}$ \\
\hline 260 & & $\begin{array}{l}\text { bh- } \\
\text { mh- } \\
\text { my- }\end{array}$ & $\begin{array}{l}\text { bh- } \\
\text { mh- } \\
\text { my- }\end{array}$ & $\begin{array}{l}\text { bh- } \\
\text { mh- } \\
\text { by- }\end{array}$ & bh- & (?) favoured, desired ${ }^{11}$ \\
\hline 270 & 28 & & & & bhs- & $\begin{array}{l}\text { wear through; chafe (rope, strap, } \\
\text { one's skin or tongue) }\end{array}$ \\
\hline 280 & $\begin{array}{r}6 \\
29\end{array}$ & bh£- & bhf- & bhf- & $\begin{array}{l}\text { bhf- } \\
\text { bhł-* }\end{array}$ & $\begin{array}{l}\text { to spread arms or fingers, to } \\
\text { measure using spread arms or } \\
\text { fingers; to spread wings or fins, } \\
\text { albatross }^{12}\end{array}$ \\
\hline
\end{tabular}

\footnotetext{
${ }^{9}$ Also in $\mathrm{KW}\left\{\mathrm{bw}[\mathrm{b}] \mathrm{qv}-\% \alpha \mathrm{y}^{3}\right\}>/ \mathrm{bubrv} \varepsilon^{3 / *}$ "gum of spruce or pine (believed to make one

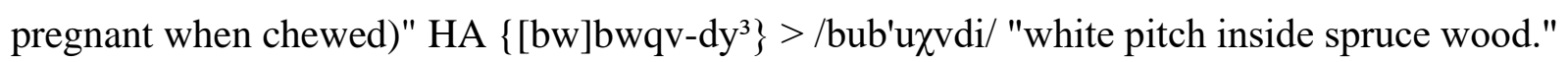
${ }^{10}\{$ pqv-, bqv- $\}$ occurs with presumed suffixes $\left\{-+\mathrm{t},-+\mathrm{c}^{3},-+\nmid\right\}$ in a stem meaning "sleepy, drowsy" and, in HE, "moth." \{bhqv-\} occurs in KW /baq³ vлаwi/ "bat."

${ }^{11}$ In feeding-related baby talk, endearing terms (e.g. for a grandfather) and possibly in the KW place name $/ \mathrm{Ba}^{3} \mathrm{a}^{3} \mathrm{~s} /$. What could be a metathesized form of this root, sc. the set $\{\mathrm{hm}-$, hp-, etc. $\}$ listed as root no. 22540, is also associated with desire for something. A meaning difference is associated with HA $\{$ bh- $\}$ and $\{$ mh- $\}$, the former being used for a male and the latter for a female.

12 \{bhł- $\}$ * may occur in $\mathrm{KW} /$ basbлi ${ }^{3} / *$ or /basbлє $\varepsilon^{3 / *}$ "side fins of fish or porpoise."
} 
North Wakashan Comparative Root List (Updated)

\begin{tabular}{|c|c|c|c|c|c|c|}
\hline Root \# & Old \# & $\mathrm{HE}$ & $\mathrm{OO}$ & HA & KW & Gloss \\
\hline 290 & 30 & bhkv- & bhkv- & bhkv- & bhkv- & $\begin{array}{l}\text { (?) wing, extending flaps or } \\
\text { wings from an axis }{ }^{13}\end{array}$ \\
\hline 300 & 31 & bhkv- & bhkv- & bhkv- & bhkv-* & $\begin{array}{l}(\mathrm{HE} / \mathrm{OO} / \mathrm{HA}) \text { to gather and } \\
\text { preserve food; }(\mathrm{KW}) \text { to fish for } \\
\text { halibut }\end{array}$ \\
\hline 310 & 32 & & bhkv- & & bhkv- & (?) to stay filled, replenish \\
\hline 311 & 33 & bhkv- & bhkv- & bhkv- & bhkv- & $(?)^{14}$ \\
\hline 320 & 34 & bhxv- & bhxv- & bhxv- & bhxv- & $\begin{array}{l}\text { to rise, swell, increase in size, } \\
\text { take shape; (KW also:) visible, } \\
\text { easily seen }\end{array}$ \\
\hline 330 & 35 & & & & bhxv-* & to leave, abandon ${ }^{15}$ \\
\hline 340 & 36 & & & & bhxv- & $\begin{array}{l}\text { human (as opposed to } \\
\text { supernatural), secular, profane, } \\
\text { uninitated }\end{array}$ \\
\hline 350 & 37 & bhqv- & bhqv- & bhqv- & & $\begin{array}{l}\text { to separate things that are joined } \\
\text { together (e.g. pull cap from pen, } \\
\text { toque from one's head) }\end{array}$ \\
\hline 360 & 38 & & & $\mathrm{bh} \chi \mathrm{v}^{-}$ & & to overload one's mouth \\
\hline 370 & 40 & & pt- & & pt- & to give or take medicine ${ }^{16}$ \\
\hline 380 & 41 & pn- & pn- & pn- & pn- & to fill sth., to pour in, to pour out \\
\hline 390 & 42 & pnc- & pnc- & pns- & pns- & bud, knob, swelling, blister \\
\hline
\end{tabular}

\footnotetext{
${ }^{13}$ In a stem referring to skate or ray fish.
}

${ }^{14}$ In the HE/OO/HA/KW stem $\{[$ bhkv]bhkv- $\}$ which refers to a mythological being associated in $\mathrm{HE} / \mathrm{OO} / \mathrm{KW}$ with cannibalism but in HA with always having food.

${ }^{15}$ Only in baxva* [2] 'to leave' which is from the Koskimo dialect.

${ }^{16}$ OO only in $/ \mathrm{pt}^{3} \mathrm{i} /$ and $/ \mathrm{pt}^{3} \mathrm{a}^{3} \mathrm{i} /$ "medicine." 
North Wakashan Comparative Root List (Updated)

\begin{tabular}{|c|c|c|c|c|c|c|}
\hline Root \# & Old \# & $\mathrm{HE}$ & $\mathrm{OO}$ & $\mathrm{HA}$ & $\mathrm{KW}$ & Gloss \\
\hline 400 & 43 & $\begin{array}{l}\text { pn£- } \\
p^{31-}\end{array}$ & $\begin{array}{l}\mathrm{pnf-} \\
\mathrm{pn}^{3} \mathrm{l}-\end{array}$ & $\begin{array}{l}\text { pnf- } \\
p^{3} 1-\end{array}$ & pnf- & $\begin{array}{l}\text { filled up, satiated, swollen, stout, } \\
\text { big with child }\end{array}$ \\
\hline 410 & 44 & $\begin{array}{l}\text { pnqv-, } \\
\text { pnq- }\end{array}$ & pnqv- & pnqv- & pnqv- & satiated, filled with food \\
\hline 420 & 45 & & & & pn $\chi \mathrm{v}-$ & swollen (like face after crying) \\
\hline 430 & 46 & & & & pnq-* & $\begin{array}{l}\text { to glare, to shine, to light (like a } \\
\text { match) }\end{array}$ \\
\hline 440 & $\begin{array}{l}39 \\
48 \\
49 \\
87 \\
88 \\
99\end{array}$ & $\begin{array}{l}\text { ps- } \\
\text { phs- }\end{array}$ & $\begin{array}{l}\text { ps- } \\
\text { pss- }^{3}{ }^{2} \\
\text { phs- }\end{array}$ & $\begin{array}{l}\text { ps- } \\
\text { phs- }\end{array}$ & $\begin{array}{l}\text { ps- } \\
\text { ps- } \\
\text { phs- } \\
\text { p3hs- }\end{array}$ & 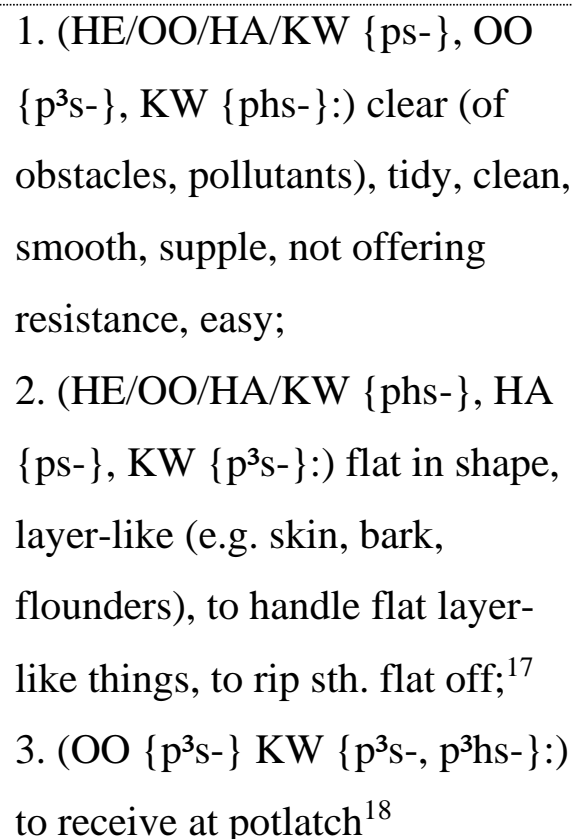 \\
\hline
\end{tabular}

${ }^{17} \mathrm{HA}$ \{ps-\} meaning "flat" is attested only in HA /pssi ${ }^{3} \mathrm{ud} /$ "to split dry ooligans in two" elicited from George Paul Wilson, a speaker of the Kitamaat dialect. The Kitlope consultant used HA /p'assi ${ }^{3} \mathrm{ud} /$ instead whose root $\{\mathrm{phs}-\}$ usually means "flat (etc.)" but also alternates freely with \{ps-\} "not offering resistance, easy, etc." as in KW \{phs-m-hlh $\}>$ /pasmala/ and KW \{ps-m-hlh $\}$ $>$ /psmala/ "to obtain easily."

${ }^{18} \mathrm{OO}\left\{\mathrm{p}^{3} \mathrm{~s}-\right\}$ is attested only in $\left\{\mathrm{p}^{3} \mathrm{~s}-\mathrm{m}-\mathrm{hlh}\right\}$ "obtain without exerting oneself" $\left\{\mathrm{p}^{3} \mathrm{~s}-\mathrm{lh}\right\}$ "a kind of potlatch" and the man's name $\left.\left\{\mathrm{p}^{3}[\mathrm{hh}] \mathrm{s}-1 \mathrm{~h}\right\}\right\}>/ \mathrm{P}^{3}$ aas The association of $\left\{\mathrm{p}^{3} \mathrm{~s}-, \mathrm{p}^{3} \mathrm{hs}\right\}$ with potlatching could be through "obtaining easily/freely" and the flat things (blankets) exchanged at potlatch. 
North Wakashan Comparative Root List (Updated)

\begin{tabular}{|c|c|c|c|c|c|c|}
\hline Root \# & Old \# & $\mathrm{HE}$ & $\mathrm{OO}$ & HA & KW & Gloss \\
\hline 450 & 50 & $\mathrm{p} £-$ & p£- & & $\begin{array}{l}\text { p£- } \\
\text { pł- } \\
\text { b1-* }\end{array}$ & $\begin{array}{l}\text { flat body part (e.g. palm of hand, } \\
\text { sole of foot, flap, fin, wing) }{ }^{19}\end{array}$ \\
\hline 460 & 51 & pl- & pl- & pl- & pl- & $\begin{array}{l}\text { thin and flat (said of sheets or } \\
\text { layers of sth.) }{ }^{20}\end{array}$ \\
\hline 470 & 52 & & plk- & & plk- & $\begin{array}{l}\text { flat thing is located somewhere, } \\
\text { moving or being handled }\end{array}$ \\
\hline 480 & 53 & plq- & plq- & & plq- & $\begin{array}{l}\text { flat thing is located somewhere, } \\
\text { is moving or being handled }\end{array}$ \\
\hline 500 & & & & & pk- & $\begin{array}{l}\text { to burst (like a balloon), blow off } \\
\text { (steam), to lose patience }{ }^{21}\end{array}$ \\
\hline 510 & $\begin{array}{l}56 \\
57 \\
58 \\
59\end{array}$ & px- & px- & $\begin{array}{l}\text { px- } \\
\text { tx- }\end{array}$ & px- & $\begin{array}{l}\text { warm, hot, to heat; (HA and } \\
\text { possibly KW also:) fierce, fiery, } \\
\text { red, dangerous, scary, off- } \\
\text { putting }^{22}\end{array}$ \\
\hline
\end{tabular}

${ }^{19}$ In KW alternates with $\{$ ph£- $\}$ "flat, to flatten." KW $\{$ bł- $\} *$ occurs possibly in /basbлє 3 /* 'side fins'. See also $\{£ \mathrm{p}-, \mathrm{bh} £-\}$.

${ }^{20} \mathrm{In} \mathrm{HE} / \mathrm{OO} / \mathrm{HA}$ root occurs also augmented to $\left\{\mathrm{pl}^{3}-\right\}$ and $\{\mathrm{pl}[\infty]-\}$.

${ }^{21} \mathrm{KW}$ consultants only gave glosses referring to losing patience. Boas's glosses however also refer to bursting and blowing off. This root may be an alternant of $\{\mathrm{px}-\}$.

${ }^{22}$ Lincoln (n.d.) indicates KW /pxa/* 'to bleed sb.' occurs in the Boas-Hunt materials but consultants rejected Boas's gloss and said it means 'to heat, warm up'. Likely Boas's gloss is in error and should have been a reference to heating and becoming red hot. This root $\{\mathrm{px}-\}$ may also have an association with redness in non-reelicitable $\mathrm{KW}$ words to do with kelpfish, e.g. $\mathrm{KW}$ /pxit/* "kelpfish." Kelpfish, a.k.a. giant kelpfish, live in kelp forests and according to http://www.sanroque.net/srstudents/foss/popeco/organismdatabase/orgpages/1122.html their dominant colour is red although they can alter their colour slightly to blend in with the brown or golden of the kelp they live in. 
North Wakashan Comparative Root List (Updated)

\begin{tabular}{|c|c|c|c|c|c|c|}
\hline Root \# & Old \# & $\mathrm{HE}$ & $\mathrm{OO}$ & $\mathrm{HA}$ & KW & Gloss \\
\hline 520 & 63 & $\begin{array}{l}\text { pys- } \\
\text { py }^{3} \text { s- }\end{array}$ & $\begin{array}{l}\text { pys- } \\
\text { py }^{3} \text { s- }\end{array}$ & pys- & pys- & $\begin{array}{l}\text { squeaking, whistling, pinched or } \\
\text { resonant sound }\end{array}$ \\
\hline 521 & $\begin{array}{r}60 \\
61 \\
62 \\
371\end{array}$ & $\begin{array}{l}\text { pys- } \\
\text { py }^{3} \mathrm{~s}^{-}\end{array}$ & $\begin{array}{l}\text { pys- } \\
\text { pyss- }\end{array}$ & py ${ }^{3} s-$ & $\begin{array}{l}\text { pys- } \\
\text { tys- }\end{array}$ & $\begin{array}{l}\text { unstable, unsteady, off and on, } \\
\text { back and forth, pliable, elastic, } \\
\text { yielding, wavering, fluttering, } \\
\text { fluctuating }\end{array}$ \\
\hline 530 & 64 & & & & pyf- & $\begin{array}{l}\text { unstable, unsteady, off and on, } \\
\text { etc. }\end{array}$ \\
\hline 540 & $\begin{array}{l}65 \\
68\end{array}$ & & pyk- & & $\begin{array}{l}\text { pyk- } \\
\text { pyq-* }\end{array}$ & $\begin{array}{l}\text { to whistle, to make a high pitched } \\
\text { sound; } \text { marmot }^{23}\end{array}$ \\
\hline 550 & $\begin{array}{l}66 \\
67\end{array}$ & py $\chi \mathrm{v}^{-}$ & $\begin{array}{l}\text { pyqv- } \\
\text { py } \chi v-\end{array}$ & pyqv- & pyqv- & $\begin{array}{l}\text { (HE/OO/HA) to soak, dampen, } \\
\text { sprinkle water; }(\mathrm{KW}) \text { agreeable, } \\
\text { pliable, soft, to soften (by } \\
\text { soaking) }\end{array}$ \\
\hline 580 & $\begin{array}{l}69 \\
71\end{array}$ & pkv- & $\begin{array}{l}\mathrm{pkv}- \\
\mathrm{p}^{3} \mathrm{kv}-\end{array}$ & & pkv- & $\begin{array}{l}\text { to congregate (like birds in flock, } \\
\text { fish in school), to compact, to } \\
\text { weave together }{ }^{24}\end{array}$ \\
\hline 590 & 72 & $\mathrm{p}^{3} \mathrm{xv}-$ & $p^{3} \mathrm{xv}-$ & $p^{3} \mathrm{xv}-$ & $\begin{array}{l}\text { pxv- } \\
\text { phxv- }\end{array}$ & $\begin{array}{l}\text { to float, to drift, to be buoyant; } \\
\text { (KW also:) to lie dead }\end{array}$ \\
\hline 600 & $\begin{array}{l}73 \\
74\end{array}$ & pw- & pw- & pw- & pw- & $\begin{array}{l}\text { capacious, lacking filling, deep, } \\
\text { hollow, starved for sth. }\end{array}$ \\
\hline 610 & 75 & pws- & pws- & & pws- & hollow, lacking filling, to starve \\
\hline 611 & 76 & pws- & pws- & pws- & pws- & $\begin{array}{l}\text { to expand, swell up (as through } \\
\text { soaking), rise (like dough); (HA }\end{array}$ \\
\hline
\end{tabular}

${ }^{23}\{\text { pyq- }\}^{*}$ only in KW /piradi/* "having marmots." Probably its $/ \mathrm{r} /$ is a spelling error and should have been $/ \mathrm{g} /$.

${ }^{24}$ The only HE example is possibly the place name $/ \mathrm{Pkvi}^{3}{ }^{\prime} \mathrm{a} /$ the meaning of which is however not known. Note the $|\mathrm{p}|-\left|\mathrm{p}^{3}\right|$ alternation in this root and no. 590. 
North Wakashan Comparative Root List (Updated)

\begin{tabular}{|c|c|c|c|c|c|c|}
\hline Root \# & Old \# & $\mathrm{HE}$ & $\mathrm{OO}$ & $\mathrm{HA}$ & $\mathrm{KW}$ & Gloss \\
\hline & & & & & & $\begin{array}{l}\text { also:) to rot (as fish after } \\
\text { spawning) }\end{array}$ \\
\hline 620 & 77 & & pw£- & pwf- & pwf- & $\begin{array}{l}\text { (OO/KW) satiated, full; (HA) } \\
\text { paunchy, stout }\end{array}$ \\
\hline 630 & 78 & pwxv- & pwxv- & pwXv- & pwxv- & $\begin{array}{l}\text { to blow (with the mouth, or like } \\
\text { whale), to inflate }\end{array}$ \\
\hline 640 & 79 & & & & pwxv-* & $(?)^{25}$ \\
\hline 650 & 80 & & & & pwqv- & (?) bladder, swelling, gas-filled ${ }^{26}$ \\
\hline 660 & 82 & & & & $\begin{array}{l}\text { pwqv-l } \\
\text { pw } \chi v-\end{array}$ & $(?)^{27}$ \\
\hline 670 & 81 & & & & pw $\chi v-$ & (?) to fill up on sth. ${ }^{28}$ \\
\hline 680 & 83 & $\mathrm{p} \chi \mathrm{v}-$ & $\mathrm{p} \chi \mathrm{v}^{-}$ & & & to wither (plant, etc.) \\
\hline 690 & $\begin{array}{l}84 \\
94\end{array}$ & phq- & phq- & phq- & $\begin{array}{l}\text { pq- } \\
\text { phq- }\end{array}$ & $\begin{array}{l}\text { flat in shape, to handle a flat } \\
\text { object, to lay down flat }\end{array}$ \\
\hline 700 & $\begin{array}{r}26 \\
400\end{array}$ & $\mathrm{p} \chi-$ & $\mathrm{p} \chi-$ & & $\begin{array}{l}\mathrm{p} \chi- \\
\mathrm{t} \chi-\end{array}$ & cedar strips, to split cedar ${ }^{29}$ \\
\hline
\end{tabular}

${ }^{25}$ Only in $\mathrm{KW} /$ puxvas. $\mathrm{m}^{3} \mathrm{~s} / *$ "willow tree"

${ }^{26}$ Occurs in two words referring to kelp. Because the root begins in $|\mathrm{pw}|$ there is a good chance it has something to do with floating or being swollen or bladder-like. Wikipedia in its article on kelp says gas-filled bladders (pneumatocysts) form at the base of blades of American kelp species such as Nereocystis lueteana (Mert. \& Post \& Rupr.), and keep the kelp blades close to the surface, holding up the blades by the gas they contain.

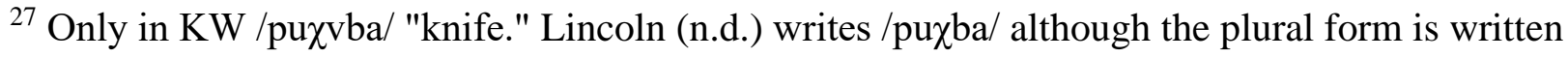
with $/ \chi v /$, cf. KW /pi ${ }^{3}$ pu $\chi v b a /$.

${ }^{28}$ In words referring to getting food.

${ }^{29}$ \{ $\mathrm{p} \chi$ - $\}$ only in $\mathrm{HE} / \mathrm{p} \chi^{\prime} \mathrm{a} / \mathrm{OO} / \mathrm{p} \chi \mathrm{a} /$ "cedar strip" KW /p $\chi \mathrm{a} /$ "to split cedar, cedar strip." 
North Wakashan Comparative Root List (Updated)

\begin{tabular}{|c|c|c|c|c|c|c|}
\hline Root \# & Old \# & $\mathrm{HE}$ & $\mathrm{OO}$ & HA & KW & Gloss \\
\hline 710 & 85 & $\mathrm{p} x-$ & $\mathrm{p} \chi-$ & & $\begin{array}{l}\mathrm{p} \chi- \\
\mathrm{ph} \chi-\end{array}$ & $\begin{array}{l}\text { to heal (said of shaman), to } \\
\text { exorcise a spirit }\end{array}$ \\
\hline 720 & 86 & ph- & ph- & ph- & ph- & $\begin{array}{l}\text { flat in shape, lay sth. down flat; } \\
\text { flat pieces splitting off (e.g. } \\
\text { pieces of cedar for basket } \\
\text { making); (KW also:) river } \\
\text { floods/overflows }\end{array}$ \\
\hline 740 & $\begin{array}{l}89 \\
92\end{array}$ & ph£- & ph£- & $\begin{array}{l}\text { ph£- } \\
\text { phł- }\end{array}$ & $\begin{array}{l}\text { ph£- } \\
\text { phł- } \\
\text { ph'3- } \\
\text { py }^{31-}\end{array}$ & flat, to flatten ${ }^{30}$ \\
\hline 750 & 90 & phł- & phł- & phł- & phł- & to flood, overflow (river) \\
\hline 760 & 91 & & & phł- & & $(?)^{31}$ \\
\hline 770 & & & & & phxv- & $(?)^{32}$ \\
\hline 780 & 93 & & & & phqv- & to put flat on a surface \\
\hline 790 & & $\operatorname{ph} \chi-$ & ph $\chi-$ & $\operatorname{ph} \chi-$ & & $\begin{array}{l}\text { peeling off, flaking off (like } \\
\text { dandruff, flakes from the skin), to } \\
\text { peel potatoes or fruit }\end{array}$ \\
\hline 800 & 95 & & $\mathrm{p}^{3} \mathrm{tk}-\mathrm{p}^{3} \mathrm{dk}-$ & & $\mathrm{p}^{3} \mathrm{dk}-$ & (?) dark ${ }^{33}$ \\
\hline 810 & 96 & & $\mathrm{p}^{3} \mathrm{t}-$ & & & dented \\
\hline
\end{tabular}

${ }^{30}\{$ ph£- $\}$ occurs in KW also with the meaning of $\{\mathrm{p} £-\}$ "flat body part." $\left\{\mathrm{ph}^{3} \mathrm{l}_{-}\right\}$and $\left\{\mathrm{py}^{3} \mathrm{l}_{-}\right\}$ occur only in $\mathrm{KW} / \mathrm{pa}^{3} ł ə g \mathrm{~g} /$ "flat dish" and its alternant /pi ${ }^{3} ł ə g a /$.

${ }^{31}$ Possibly in HA /pp'аћ'

${ }^{32}$ Only in $\mathrm{KW} /$ paxva/. Term recognized by informant but meaning not remembered. According to Boas it is "lungs, heart."

${ }^{33}$ In e.g. OO /ptkла/ KW /p³ dkla/ "dark night, darkness reigns." 
North Wakashan Comparative Root List (Updated)

\begin{tabular}{|c|c|c|c|c|c|c|}
\hline Root \# & Old \# & $\mathrm{HE}$ & $\mathrm{OO}$ & HA & KW & Gloss \\
\hline 820 & $\begin{array}{r}97 \\
547\end{array}$ & $n^{3} n q-$ & $\mathrm{n}^{3} \mathrm{nq}-$ & $\mathrm{h}^{3} \mathrm{nq}-$ & $p^{3} n q-$ & $\begin{array}{l}(\mathrm{HE} / \mathrm{OO} / \mathrm{HA}) \text { to moan, to groan; } \\
(\mathrm{KW}) \text { to gasp in annoyance }\end{array}$ \\
\hline 830 & $\begin{array}{r}98 \\
125\end{array}$ & $\begin{array}{l}\mathrm{p}^{3} \mathrm{~s}- \\
\mathrm{p}^{3} \mathrm{hs}-\end{array}$ & $\begin{array}{l}\mathrm{p}^{3} \mathrm{~s}- \\
\mathrm{p}^{3} \mathrm{hs}-\end{array}$ & $\mathrm{p}^{3} \mathrm{~s}-$ & $\begin{array}{l}\mathrm{p}^{3} \mathrm{~s}- \\
\mathrm{p}^{3} \mathrm{hs}-\end{array}$ & $\begin{array}{l}\text { bent or crooked in shape, warped; } \\
\text { to bend and give way; to force to } \\
\text { give way, to indent }\end{array}$ \\
\hline 850 & 98 & $p^{3} s-$ & $\mathrm{p}^{3} \mathrm{~s}-$ & & $p^{3} s-$ & (?) one's ears \\
\hline 860 & $\begin{array}{l}100 \\
101\end{array}$ & & & $\mathrm{p}^{3} \mathrm{f}_{-}$ & $\begin{array}{l}\mathrm{p}^{3} £- \\
\mathrm{p}^{3} \mathrm{~h} £-\end{array}$ & $\begin{array}{l}\text { (HA) to fling, to flap, to throw a } \\
\text { floppy object; (KW) to spread } \\
\text { wings, to fly }\end{array}$ \\
\hline 870 & 102 & $p^{3} 1-$ & $p^{3} 1_{-}$ & $\mathrm{p}^{3} \mathrm{l}^{-}$ & $p^{31-}$ & $\begin{array}{l}\text { (?) applying or removing a } \\
\text { covering layer }^{34}\end{array}$ \\
\hline 880 & 103 & $p^{3} 1-$ & $\mathrm{p}^{3} 1-$ & & $\mathrm{p}^{3} 1-$ & $\begin{array}{l}\text { to pinch and pull an outgrowth or } \\
\text { covering layer }\end{array}$ \\
\hline 890 & 106 & $\begin{array}{l}\mathrm{p}^{3} 1- \\
\mathrm{p}^{3} 1 \mathrm{xv-} / \\
\mathrm{p}^{3} 1 \mathrm{\chi v}^{-}\end{array}$ & $\begin{array}{l}p^{3} l x v-/ \\
p^{3} 1 \chi v\end{array}$ & $\mathrm{p}^{3} \mathrm{l}-$ & $\begin{array}{l}\mathrm{p}^{3} 1 \mathrm{xv}-/ \\
\mathrm{p}^{3} 1 \chi \mathrm{v}\end{array}$ & $(?)^{35}$ \\
\hline 900 & $\begin{array}{l}104 \\
105\end{array}$ & $\mathrm{p}^{3} \mathrm{lc}-$ & $p^{3} l c-$ & $p^{3} l c-$ & $p^{3} l c-$ & moss-covered ${ }^{36}$ \\
\hline 910 & 107 & $\mathrm{p}^{3} \mathrm{lq}-$ & $\mathrm{p}^{3} \mathrm{lq}-$ & $\mathrm{p}^{3} \mathrm{lqv}-$ & & blunt, dull ${ }^{37}$ \\
\hline 920 & $\begin{array}{l}108 \\
109\end{array}$ & & $p^{3} 1 \chi-$ & & $p^{3} 1 \chi-$ & $\begin{array}{l}\text { fluffy white layer (like fog } \\
\text { blanketing landscape, mountain } \\
\text { goat's fur, a goat wool blanket) }\end{array}$ \\
\hline
\end{tabular}

\footnotetext{
${ }^{34}$ Only in words to do with blinking or keeping the eyes closed.

${ }^{35}$ In a few words referring to a sister-in-law.

${ }^{36}$ The HA root $\left\{\mathrm{p}^{3} \mathrm{lc}-\right\}$ "to accompany" listed as no. 105 in the 1980 root list had only one example whose gloss "to accompany" is no doubt due to misunderstanding the informant.

${ }^{37}$ Note alternation of rounded and unrounded. Another example is HA /y $\chi \mathrm{su} / \mathrm{and} / \mathrm{y} \chi \mathrm{vsu} /$ "It's you who ..."
} 
North Wakashan Comparative Root List (Updated)

\begin{tabular}{|c|c|c|c|c|c|c|}
\hline Root \# & Old \# & $\mathrm{HE}$ & $\mathrm{OO}$ & HA & KW & Gloss \\
\hline 940 & $\begin{array}{l}110 \\
111 \\
112\end{array}$ & $\mathrm{p}^{3} \mathrm{x}-$ & $\begin{array}{l}\mathrm{p}^{3} \mathrm{k}- \\
\mathrm{p}^{3} \mathrm{x}-\end{array}$ & $\mathrm{p}^{3} \mathrm{x}-$ & $\mathrm{p}^{3} \mathrm{k}-$ & $\begin{array}{l}\text { to dent (hard material), to depress } \\
\text { (sth. resilient); dented, having } \\
\text { grooves or holes, hollow, } \\
\text { concave; a depression }\end{array}$ \\
\hline 960 & 113 & $\mathrm{p}^{3} \mathrm{ys}-$ & $\mathrm{p}^{3} \mathrm{ys}-$ & & $\mathrm{p}^{3} \mathrm{ys}-$ & hard, harsh \\
\hline 970 & 114 & $\mathrm{p}^{3} \mathrm{yxv}-$ & $\mathrm{p}^{3} \mathrm{yxv}-$ & $\mathrm{p}^{3} \mathrm{yxv}-$ & $\mathrm{p}^{3} \mathrm{yxv}-$ & $\begin{array}{l}\text { to grope, to touch, to feel with } \\
\text { the hand, to palpate }\end{array}$ \\
\hline 980 & & & & & $\mathrm{p}^{3} \mathrm{yq}^{3} \mathrm{v}-*$ & to press (crush) in hand \\
\hline 990 & 115 & $\mathrm{p}^{3} \mathrm{yq}-$ & $\mathrm{p}^{3} \mathrm{yq}-$ & $\mathrm{p}^{3} \mathrm{yq}-$ & $\mathrm{p}^{3} \mathrm{yq}-$ & rotten, moldy ${ }^{38}$ \\
\hline 1000 & & & & & $\mathrm{p}^{3} \mathrm{kv}-$ & (?) baggy, hollow \\
\hline 1010 & 116 & $\mathrm{p}^{3} \mathrm{w}-$ & $\mathrm{p}^{3} \mathrm{w}-$ & & $\mathrm{p}^{3} \mathrm{w}-$ & (?) halibut ${ }^{39}$ \\
\hline 1020 & 117 & & & $\mathrm{p}^{3} \mathrm{WS}-$ & & to bend sth. \\
\hline 1030 & 118 & & & $\left.\mathrm{p}^{3} \mathrm{w}\right\}-$ & & (?) spread fish open ${ }^{40}$ \\
\hline 1040 & 119 & $\mathrm{p}^{3} \mathrm{w}-$ & $\mathrm{p}^{3} \mathrm{w} 1-$ & & $\mathrm{p}^{3} \mathrm{w} 1-$ & $\begin{array}{l}\text { to bubble up, splash up (water, } \\
\text { fish), to boil (tide) }\end{array}$ \\
\hline 1050 & 120 & $\mathrm{p}^{3} \mathrm{wkV}-$ & $\mathrm{p}^{3} \mathrm{wkV}-$ & & $\mathrm{p}^{3} \mathrm{wkV}-$ & white, halibut \\
\hline 1060 & 121 & & & & $\mathrm{p}^{3} \mathrm{wqv}-$ & $\begin{array}{l}\text { to break off with the hands sth. } \\
\text { that is attached to a stem or } \\
\text { surface (e.g. leaves, dried fish) }\end{array}$ \\
\hline
\end{tabular}

\footnotetext{
${ }^{38}$ The glosses of the KW examples specify that reference is to a rope, mat or cloth.

${ }^{39}$ All examples are about halibut but the root could denote not the fish but a conspicuous property such as its flat shape or its white underside.

${ }^{40}$ Occurs in words to do with roasting or drying backbone of fish.
} 
North Wakashan Comparative Root List (Updated)

\begin{tabular}{|c|c|c|c|c|c|c|}
\hline Root \# & Old \# & $\mathrm{HE}$ & $\mathrm{OO}$ & HA & KW & Gloss \\
\hline 1070 & 122 & & & & $\mathrm{p}^{3} \mathrm{qv}-$ & to be flat on sth. ${ }^{41}$ \\
\hline 1080 & 123 & $\mathrm{p}^{3} \mathrm{q}-$ & $\mathrm{p}^{3} \mathrm{q}-$ & $\mathrm{p}^{3} \mathrm{q}-$ & $\mathrm{p}^{3} \mathrm{q}-$ & to taste \\
\hline 1090 & $\begin{array}{l}124 \\
130\end{array}$ & $\mathrm{p}^{3} \mathrm{~h}-$ & $\mathrm{p}^{3} \mathrm{~h}-$ & $\mathrm{p}^{3} \mathrm{~h}-$ & $\mathrm{p}^{3} \mathrm{~h}-$ & $\begin{array}{l}\text { to feel for sth., stretch out the } \\
\text { hand for sth., to use the hand; } \\
\text { (HE/OO/HA also:) to do work, } \\
\text { operate on sth. (physically or } \\
\text { magically) }{ }^{42}\end{array}$ \\
\hline 1100 & 132 & $\mathrm{p}^{3} \mathrm{~h}-$ & & & $\mathrm{p}^{3} \mathrm{~h}-$ & $\begin{array}{l}\text { (?) to wither, change colour } \\
\text { (plants) })^{43}\end{array}$ \\
\hline 1130 & $\begin{array}{l}126 \\
127\end{array}$ & $\mathrm{p}^{3} \mathrm{~h} £-$ & $p^{3} h £-$ & $\mathrm{p}^{3} \mathrm{~h} f-$ & & $\begin{array}{l}\text { (HE/OO) to string out, to spread } \\
\text { out from a centre, to extend from } \\
\text { a stem; (HA) to reflect }{ }^{44}\end{array}$ \\
\hline 1140 & 128 & $\mathrm{p}^{3} \mathrm{~h} t-$ & $p^{3} h 1-$ & $\mathrm{p}^{3} \mathrm{~h} t-$ & $\left.\mathrm{p}^{3} \mathrm{~h}\right\}-$ & woolly, woolly layer, moss \\
\hline 1150 & 129 & & & $\mathrm{p}^{3} \mathrm{hx}-$ & & (?) dark ${ }^{45}$ \\
\hline
\end{tabular}

${ }^{41} \mathrm{In} \mathrm{KW} / \mathrm{p}^{3} \mathrm{q}^{3} \mathrm{va} /$ "to be flat on sth. (e.g. stove)" and according to Boas also "to mold or press into shape."

${ }^{42} \mathrm{KW}$ uses $\left\{\left[\mathrm{h}^{3} \mathrm{y}\right] \mathrm{h}^{3} \chi-\right\}$ for "to work."

${ }^{43}$ For HE the root is attested only in $/ \mathrm{p}^{3} \mathrm{a} \chi \mathrm{v} \cdot \mathrm{h}^{3} \mathrm{it} /<\left\{\mathrm{p}^{3} \mathrm{~h}-=\mathrm{hqv}-\& \mathrm{~d}\right\}$ "to change colour (leaves in the fall)."

${ }^{44}$ The HE/OO examples all involve sprawling/stringing out (roots, line), spreading open (wings, arms), reaching/bending down (branches, shirt tail hanging out). The HA examples are about cognitive reflection. Cf. the polysemy of English "reflect" ("bend off/back" > "make manifest/show," "think" etc.)

${ }^{45}$ In two derivates of the stem $\left\{\mathrm{p}^{3} \mathrm{hny} \chi \chi_{\mathrm{v}-}\right\}$ "darkness." 
North Wakashan Comparative Root List (Updated)

\begin{tabular}{|c|c|c|c|c|c|c|}
\hline Root \# & Old \# & $\mathrm{HE}$ & $\mathrm{OO}$ & HA & $\mathrm{KW}$ & Gloss \\
\hline 1170 & $\begin{array}{l}131 \\
133\end{array}$ & & $\mathrm{p}^{3} h \mathrm{q}^{3} \mathrm{v}-$ & & $\begin{array}{l}\mathrm{p}^{3} h q^{3} \mathrm{v}_{-}^{*} \\
\mathrm{p}^{3} h \mathrm{q}^{3-}\end{array}$ & $\begin{array}{l}\text { spoiled, moldy, rotten (like } \\
\text { berries on bushes) }\end{array}$ \\
\hline 1190 & & $\begin{array}{l}\text { mmm- } \\
\text { hmm- }\end{array}$ & hmm- & hm- & $\begin{array}{l}\text { hwmo- } \\
\mathrm{hm}^{3} \mathrm{w}-\end{array}$ & (onomatopoeic) $^{47}$ \\
\hline 1200 & $\begin{array}{l}134 \\
135\end{array}$ & mmk- & $\begin{array}{l}\mathrm{mmk}- \\
\mathrm{mk}-\end{array}$ & hmk- & hmk- & $\begin{array}{l}\text { (onomatopoeic:) to hum, to buzz, } \\
\text { to mumble; (HE/OO also:) to } \\
\text { emit a drumming sound }{ }^{48}\end{array}$ \\
\hline 1210 & $\begin{array}{l}136 \\
137 \\
138 \\
139 \\
170\end{array}$ & $\begin{array}{l}\text { mt- } \\
\text { mht- }\end{array}$ & $\begin{array}{l}\text { mt- } \\
\text { mht- }\end{array}$ & $\begin{array}{l}\text { mt- } \\
\text { mht- }\end{array}$ & $\begin{array}{l}\text { mt- } \\
\text { mht- }\end{array}$ & $\begin{array}{l}\text { to quiver, twitch, tremble, } \\
\text { rumble; to flick, twitch, flutter, } \\
\text { flap (wings); to be agitated, } \\
\text { seething (like water bubbling or } \\
\text { boiling) }\end{array}$ \\
\hline
\end{tabular}

${ }^{46}\left\{\mathrm{p}^{3} \mathrm{hq}^{3} \mathrm{v}-\right\}$ occurs in $\mathrm{OO} / \mathrm{p}^{3} \mathrm{aq}^{3} \mathrm{vr} \mathrm{Mt} /$ "to make the face look moldy" and non-reelicitable KW $/ \mathrm{p}^{3} \mathrm{aq}^{3} \mathrm{VIMd} / *$ "to paint the face."

${ }^{47}$ In the word for "pigeon" and KW /hum:hum/* "white owl, myth name of blue grouse." The male blue grouse is known for its hooting in spring, cf.

https://nhpbs.org/natureworks/bluegrouse.htm. So is the male ruffed grouse, cf.

https://www.hww.ca/assets/pdfs/factsheets/ruffed-grouse-en.pdf.

${ }^{48}$ Occurs also in $\mathrm{HE} / \mathrm{m}^{\prime} \mathrm{mk}^{\prime} л \mathrm{~s} / \mathrm{OO} / \mathrm{mmkлs} /$ "ruffed grouse." In the spring the male ruffed grouse uses its wings to make a sound resembling a far-off motor boat or drumming, hence its English nickname "drummer." See Hinterland Who's Who at https://www.hww.ca/assets/pdfs/factsheets/ruffed-grouse-en.pdf. \{mk-\} occurs only in OO /mx:mka/ "bullroarer."

${ }^{49} \mathrm{In} \mathrm{HE} / \mathrm{OO}$ the form $\{$ mht- $\}$ usually refers specifically to flying by flapping wings and, by extension, to airplanes. Only two certain examples of HA $\{\mathrm{mht}-\}$ and according to the Kitlope consultant they could be dialect borrowings from HE. Perhaps HA $\left\{\right.$ mht- $\left.\% w^{3}{ }^{3} w\right\}>/$ mad'uy $^{3} u /$ "fox, bobcat" is another example of the root but it is unclear what its literal meaning, which is twitching in the middle, would have to do with the animals named. 
North Wakashan Comparative Root List (Updated)

\begin{tabular}{|c|c|c|c|c|c|c|}
\hline Root \# & Old \# & $\mathrm{HE}$ & $\mathrm{OO}$ & $\mathrm{HA}$ & KW & Gloss \\
\hline & 215 & & & & & \\
\hline & 216 & & & & & \\
\hline 1211 & 136 & & & & $\begin{array}{l}\text { mt- } \\
\text { mht- }\end{array}$ & (?) fat ${ }^{50}$ \\
\hline 1212 & & & & & $\mathrm{mn}-$ & (?) surplus, extra, surfeit ${ }^{51}$ \\
\hline 1220 & 140 & & & & mnz- & (?) to split kindling wood \\
\hline 1230 & 141 & mnk- & mnt- & mnk- & mnk- & $\begin{array}{l}\text { saturated, filled to capacity, filled } \\
\text { to excess; (KW also:) ooligan oil } \\
\text { (grease) feast }\end{array}$ \\
\hline 1240 & 142 & mnk- & mnk- & mnk- & mnk- & rust, excrement, manure \\
\hline 1250 & 143 & mlxv- & mlxv- & $\begin{array}{l}\text { mnxv- } \\
\text { mlxv- }\end{array}$ & mnxv- & to smile \\
\hline 1260 & 144 & & & & mnI-* & $(?)^{52}$ \\
\hline 1270 & 145 & & & & mnq- & to $\operatorname{light}^{53}$ \\
\hline 1280 & $\begin{array}{l}146 \\
149 \\
151\end{array}$ & $\mathrm{mc}-/ \mathrm{ms}-$ & $\mathrm{mc}-/ \mathrm{ms}-$ & & $\begin{array}{l}\text { mc- } \\
\text { ms- }\end{array}$ & $(?)^{54}$ \\
\hline
\end{tabular}

${ }^{50}$ In KW words referring to horse clams and the word for kidney fat. Is a cross-family stem, cf.

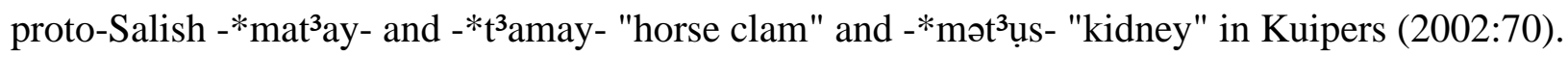

${ }^{51}$ In the KW stem $\{\mathrm{mn}-\%$ yqv- $\}$ "in a big way, more than necessary or expected" in which $\{-\%$ yqv- $\}$ probably refers to spreading around or showing off. Also in the KW stem $\{[\mathrm{my}] \mathrm{mn}-\}$ (with initial $|\mathrm{m}|$ becoming $|\mathrm{h}|$ ) "always, constantly."

${ }^{52}$ In KW (Koskimo dialect) /mnra/* "war canoe."

${ }^{53}$ Only in $\mathrm{KW} / \mathrm{mHqa} /$ 'to light'. Unclear what exactly is meant, illuminating or igniting.

${ }^{54}$ In names of living creatures. For HE/OO it is not diagnosable if the root form is $\{\mathrm{mc}-\}$ or $\{\mathrm{ms}-\}$ in the few available examples. KW diagnosably has $\{\mathrm{ms}-\}$ while the stem $\{\mathrm{mc}-\mathrm{h}\}$ specifically means "mink." 
North Wakashan Comparative Root List (Updated)

\begin{tabular}{|c|c|c|c|c|c|c|}
\hline Root \# & Old \# & $\mathrm{HE}$ & $\mathrm{OO}$ & HA & KW & Gloss \\
\hline 1290 & 147 & ms- & ms- & ms- & ms- & $\begin{array}{l}\text { to crave, covet, desire, long for, } \\
\text { lust after }\end{array}$ \\
\hline 1300 & $\begin{array}{l}148 \\
150 \\
152\end{array}$ & $\begin{array}{l}\text { ms- } \\
\mathrm{m}^{3} \mathrm{~s}- \\
\mathrm{mxs}-\end{array}$ & $\begin{array}{l}\text { ms- } \\
\mathrm{m}^{3} \mathrm{~s}- \\
\mathrm{mxs}-\end{array}$ & $\begin{array}{l}\mathrm{ms}- \\
\mathrm{m}^{3} \mathrm{~s}-\end{array}$ & ms- & $\begin{array}{l}\text { to tense, contract, throb, go back } \\
\text { and forth, agitate, recoil, } \\
\text { rebound }^{55}\end{array}$ \\
\hline 1320 & $\begin{array}{l}153 \\
154 \\
155 \\
224 \\
225\end{array}$ & $\begin{array}{l}\mathrm{mł}- \\
\mathrm{mł} \bullet- \\
\mathrm{mhł-}\end{array}$ & $\begin{array}{l}\mathrm{m}{ }^{-} \\
\mathrm{m} \bullet^{\bullet-} \\
\mathrm{mht}-\end{array}$ & $\begin{array}{l}\mathrm{mł}- \\
\mathrm{mł} \bullet- \\
\mathrm{młh-} \\
\mathrm{mht-}\end{array}$ & $\begin{array}{l}\mathrm{m} ł- \\
\mathrm{m} \nmid \bullet- \\
\mathrm{młh-} \\
\mathrm{mht-}\end{array}$ & $\begin{array}{l}\text { to whirl, rotate, stir, mix, churn, } \\
\text { interweave, swirl, swing; } \\
\text { sediment resulting from agitating } \\
\text { a liquid; ( \{mhł- }\} \text { in } \mathrm{HE} / \mathrm{OO} \text { and } \\
\text { rarely } \mathrm{KW} \text { also:) to swim }{ }^{56}\end{array}$ \\
\hline 1330 & 156 & & mt- & & $\begin{array}{l}\text { mł- } \\
\text { mht- }\end{array}$ & $(?)^{57}$ \\
\hline 1340 & 157 & ml- & ml- & & ml- & $\begin{array}{l}\text { to depart from straight course, to } \\
\text { slant, to go sideways, to turn } \\
\text { around, to twirl, to twist strands } \\
\text { together }\end{array}$ \\
\hline 1360 & 160 & mlc- & mlc- & mlc- & mls- & $\begin{array}{l}\text { to twist, turn, go or look } \\
\text { sideways }\end{array}$ \\
\hline 1370 & 161 & & & mlk- & & $(?)^{58}$ \\
\hline
\end{tabular}

${ }^{55}\left\{\mathrm{~m}^{3} \mathrm{~s}-\right\}$ is attested only in words to do with withering and shrinking. $\{$ mxs- $\}$ occurs only in the word for rainbow.

56 1. In one example KW uses $\{\mathrm{mt}-\}$ but HE/OO $\{\mathrm{mht}\}$, cf. KW /mлiqa/ HE /m'al'iqa/ OO /maliqa/ "to swing in a circle etc." The only HA example of $\{$ mhl- $\}$ is possibly /m'amał/ "soft underwool of mountain goat." In KW, \{mhł- $\}$ meaning "to swim" is in most cases replaced by $\{$ Ilq- $\}$. 2. The stems $\{\mathrm{m} \bullet-\}$ and $\{\mathrm{m} \nmid \mathrm{h}-\}$ refer more specifically to wind. For a similar alternation see $\left\{\mathrm{n}^{3} \mathrm{Xv} \mathrm{v}^{\bullet-}\right\}$ and $\left\{\mathrm{n}^{3} \mathrm{xvh}-\right\}$ "near." 3 . Perhaps this root occurs also in $\left.\{[\mathrm{mh}] \mathrm{mh}\}-\mathrm{yk}-+-\mathrm{h}\right\}>\mathrm{HE}$ /m'am'ałik³ a/ OO/KW /mamałikª/ "swallow (bird)."

${ }^{57}$ In words referring to sockeye salmon and halibut.

${ }^{58}$ In the HA stem $\{[\mathrm{mh}] \mathrm{mlk}-\}$ "berries." 
North Wakashan Comparative Root List (Updated)

\begin{tabular}{|c|c|c|c|c|c|c|}
\hline Root \# & Old \# & $\mathrm{HE}$ & $\mathrm{OO}$ & HA & KW & Gloss \\
\hline 1380 & $\begin{array}{l}162 \\
163\end{array}$ & & & mlk- & $\begin{array}{l}\text { mlk- } \\
\text { mlx- }\end{array}$ & $\begin{array}{l}\text { not following a straight course, } \\
\text { now here now there, lacking } \\
\text { order }\end{array}$ \\
\hline 1400 & $\begin{array}{l}164 \\
165 \\
166 \\
167 \\
168\end{array}$ & mlqv- & mlqv- & & $\begin{array}{l}\text { mlqv-, } \\
\text { ml } \chi v- \\
\text { mlq- }\end{array}$ & $\begin{array}{l}\text { to turn, twist, fold; to curl (like } \\
\text { wave breaking); (\{mlqv- }\} \text { usually } \\
\text { figuratively:) to mull, to turn over } \\
\text { (in thought), reminisce, } \\
\text { remember, cogitate }\end{array}$ \\
\hline 1401 & & & & & mlq- & to cover, to spread out over \\
\hline 1410 & $\begin{array}{r}159 \\
1245\end{array}$ & $\mathrm{ml}^{3}-$ & $1^{3} \mathrm{p}-$ & $\begin{array}{l}\mathrm{ml-} \\
\mathrm{ml}^{3}- \\
\mathrm{l}^{3} \mathrm{p}-\end{array}$ & $\begin{array}{l}\mathrm{ml}^{3-} \\
\mathrm{mhl}^{3-}\end{array}$ & to flash a light, beam a light ${ }^{59}$ \\
\hline 1420 & $\begin{array}{l}169 \\
171 \\
172\end{array}$ & & mk- & & mk- & $\begin{array}{l}\text { (?) sunken, down a hole, put in or } \\
\text { take out of pit or enclosure, to } \\
\text { bolt food }^{60}\end{array}$ \\
\hline 1440 & 173 & $\begin{array}{l}m x^{-} \\
m h x-\end{array}$ & $\begin{array}{l}\mathrm{mx}- \\
\mathrm{mhx}-\end{array}$ & $\begin{array}{l}m x^{-} \\
m h x-\end{array}$ & $\begin{array}{l}\mathrm{mx}- \\
\mathrm{mhx}-\end{array}$ & $\begin{array}{l}\text { to administer a sharp blow (as } \\
\text { with fist or bird's beak), to punch }\end{array}$ \\
\hline 1450 & $\begin{array}{l}174 \\
175 \\
176 \\
177 \\
178\end{array}$ & $\begin{array}{l}\text { my- } \\
\text { mhy³ }\end{array}$ & $\begin{array}{l}\text { my- } \\
\text { mhy³- }\end{array}$ & $\begin{array}{l}\text { my- } \\
\text { mhy³ }\end{array}$ & $\begin{array}{l}\text { my- } \\
\text { mhy }{ }^{3-} \\
m^{3} y_{-}^{-}\end{array}$ & $\begin{array}{l}\text { 1. pointed, pointing, wedge- } \\
\text { shaped, snouted, protruding, } \\
\text { sprouting, flaring up; } \\
\text { 2. (often but not always }\end{array}$ \\
\hline
\end{tabular}

${ }^{59} \mathrm{HA}\left\{1^{3} \mathrm{p}-\right\}$ is attested only in (Kitamaat dialect) $/ 1^{3} \mathrm{pa} /$ "to flash a light" instead of which the Kitlope consultant said $/ \mathrm{m}^{3} \mathrm{a} /$. HA $\{\mathrm{ml}-\}$ probably occurs in $\left\{\mathrm{ml}-=\mathrm{n}^{3} \mathrm{hkvlh}\right\}>\left\{\mathrm{ml} \infty-\mathrm{n}^{3} \mathrm{hkvlh}\right\}>$ /m'ллn³akvла/ "to shine a light on one thing after another," with $|=|$ becoming $|\infty|$ instead of glottalizing $|1|$.

${ }^{60} \mathrm{OO}$ only in /mka/ "to pick the stems off crabapples." 
North Wakashan Comparative Root List (Updated)

\begin{tabular}{|c|c|c|c|c|c|c|}
\hline Root \# & Old \# & $\mathrm{HE}$ & $\mathrm{OO}$ & $\mathrm{HA}$ & KW & Gloss \\
\hline & & & & & & $\begin{array}{l}\text { combined with }\{\text {-h }\} \text { :) fish (in } \\
\text { general), salmon }{ }^{61}\end{array}$ \\
\hline 1460 & 179 & & myt- & myt- & myt- & to twist strands together \\
\hline 1470 & 179 & & myt- & & myt- & $\begin{array}{l}\text { to point, poke, extend a long } \\
\text { thing }^{62}\end{array}$ \\
\hline 1480 & $\begin{array}{l}180 \\
257\end{array}$ & $\begin{array}{l}\text { mys- } \\
\text { mys- }^{3}\end{array}$ & mys- & & $\begin{array}{l}\text { mys- } \\
\text { m³ys- }^{3}\end{array}$ & (?) to point, poke, protrude ${ }^{63}$ \\
\hline 1490 & & mys- & mys- & & & meat, flesh ${ }^{64}$ \\
\hline
\end{tabular}

${ }^{61}$ The augmented form $\left\{\mathrm{mhy}^{3}-\right\}$ occurs only in the stem $\left\{\mathrm{mhy}^{3}-\mathrm{w} \mathcal{E}-\right\}$ "to be born, to point out of birth channel." $\left\{\mathrm{m}^{3} \mathrm{y}-\right\}$ occurs only in KW $\left\{\mathrm{m}^{3} \mathrm{y}-\mathrm{ynh}\right\}>/ \mathrm{m}^{3} \mathrm{ina} /$ "penis (baby talk)." See also comment on root no. 1530.

${ }^{62}$ Only OO example is possibly /Midawis/ (name of a creek).

${ }^{63}$ (1) \{mys-\} occurs in words referring to smelling and kissing, and in the non-reelicitable KW /mizłəхzayu/* "penis". The kissing may in the pre-contact period have been what is known by names like Eskimo kissing and sniff-kissing, which is not rubbing noses but pressing the nose and upper lip against somebody's skin and breathing in so that his/her skin or hair gets suctioned in. Neville Lincoln said in an e-mail dated October 16, 2008, that he believes Mabel Stanley told him that the Kwagiutl kissed traditionally using the nose. (2) \{mys-\} likely also occurs in HE /m'aisa/ "to spear cockles from canoe"; this term has an alternant in which the root is $\left\{\mathrm{m}^{3} \mathrm{ys}-\right\}$ instead of \{mys- $\}$. (3) $\left\{\mathrm{m}^{3} \mathrm{ys}-\right\}$ occurs also in $\mathrm{KW} / \mathrm{m}^{3} \mathrm{is}: \mathrm{m}^{3} \mathrm{c}^{3} \mathrm{a} /$ "chiton." According to Boas this means "penis on the rocks" which makes sense if the root means "to point etc." Wikipedia says of a chiton that "The majority of the body is a snail-like foot" and "A chiton creeps along slowly on a muscular foot."

${ }^{64}$ In only a few words. Root could be a foreign borrowing. 
North Wakashan Comparative Root List (Updated)

\begin{tabular}{|c|c|c|c|c|c|c|}
\hline Root \# & Old \# & $\mathrm{HE}$ & $\mathrm{OO}$ & HA & KW & Gloss \\
\hline 1510 & 1446 & $\begin{array}{l}\left(k^{3} y_{-}-\right. \\
\left.k^{3} y^{-}-\right)\end{array}$ & $\begin{array}{l}\left(k^{3} y_{-}-\right. \\
\left.k^{3} \mathrm{yk}^{-}\right)\end{array}$ & $\begin{array}{l}\left(k^{3} y_{-}-\right. \\
\left.k^{3} y^{-}\right)\end{array}$ & $\begin{array}{l}\text { myg- } \\
\text { mygh- }\end{array}$ & to caulk ${ }^{65}$ \\
\hline 1520 & & & & $\begin{array}{l}\text { myk-/ } \\
\text { myk }^{3}-\end{array}$ & $\begin{array}{l}\text { myk-*/ } \\
\text { myk }^{3}-*\end{array}$ & $(?)^{66}$ \\
\hline 1530 & 178 & & myx- & & myx- & pointed, pointing, snouted ${ }^{67}$ \\
\hline 1531 & 181 & & & & myx- & $\begin{array}{l}\text { to light a fire, set fire to sth., } \\
\text { strike a match }\end{array}$ \\
\hline 1540 & 182 & myx- & & myx- & & $(?)^{68}$ \\
\hline 1550 & 183 & & & & mykv- & $(?)^{69}$ \\
\hline 1560 & $\begin{array}{l}184 \\
502 \\
503\end{array}$ & $\begin{array}{l}\text { myqv- } \\
\text { nyqv- }\end{array}$ & $\begin{array}{l}\text { myqv- } \\
\text { nyqv- }\end{array}$ & & nyqv- & $\begin{array}{l}\text { murky, muddy, dirty (said of } \\
\text { water) }\end{array}$ \\
\hline
\end{tabular}

${ }^{65} \mathrm{KW}\{$ myg- $\}$ is present in $\left\{\right.$ myg-x. $\left.\hbar^{3} \mathrm{yd}\right\}>/$ migx. $\hbar^{3} \mathrm{id} /$ "to start caulking" and $\mathrm{KW}$ \{mygh- $\}$ in $\left\{\right.$ mygh- $\left.\mathrm{y}^{3} \mathrm{w}\right\}>$ migay $^{3} \mathrm{u}$ "caulking iron, caulking tool." In the other KW examples it is just not diagnosable if root is $\{$ myg- $\}$ or $\{$ mygh- $\}$ in which case $\{$ myg- $\}$ is assumed.

${ }^{66}$ 1. Perhaps in $\mathrm{HA} / \mathrm{mik}^{3} \mathrm{t} /$ "kind of hard stone used for arrows, probably obsidian" and KW $/ \mathrm{mik}^{3} \mathrm{a} / *$ "to throw up feathers, shoot arrow upwards." However, the semantic connection between the two words is tenuous. 2. HA / $\mathrm{mik}^{3} \mathrm{t} /$ could also be a loan from Bella Coola. It is glossed as "arrowhead" in Nater (n.d.:64, entry no. 769).

${ }^{67} \mathrm{In} \mathrm{OO} / \mathrm{mixt} / \mathrm{KW} / \mathrm{mix}$ ədi/ "porcupine" and note that HE uses $\{$ my- $\}$ instead of $\{$ myx- $\}$, cf. $\mathrm{HE} / \mathrm{m}$ 'it/ "porcupine."

${ }^{68}$ Only in HE /m'im'an³ uł/ HA /m'i:mhał/ "kid (of mountain goat)" (in HA also "lamb.")

${ }^{69}$ In KW /migvat/ "hair seal," term reminiscent of Halkomelem Salish /məkwólqəl/ "(have) coarse hair" (from a free root $\left\{\mathrm{m}^{\mathrm{w}^{\mathrm{w}}} \mathrm{\}}\right.$ "stout, thick, fat, coarse" and suffix referring to hair, fur, fleece, wool); cf. Galloway (2009:311). The KW root may have been borrowed (from Halkomelem or a third party) as $\{$ mygv- $\}$ but must have been reinterpreted as $\{$ mykv- $\}$ in $\left\{\right.$ mykv-=hc $\left.{ }^{3} \mathrm{y}\right\}>/ \operatorname{migvac}^{3} \mathrm{i} /$, synonym of $/$ migvadac $^{3} \mathrm{i} /$ "container for seal." 
North Wakashan Comparative Root List (Updated)

\begin{tabular}{|c|c|c|c|c|c|c|}
\hline Root \# & Old \# & $\mathrm{HE}$ & $\mathrm{OO}$ & $\mathrm{HA}$ & $\mathrm{KW}$ & Gloss \\
\hline 1570 & & & & & my $\chi v-*$ & $(?)^{70}$ \\
\hline 1580 & & & & & myq-* & $(?)^{71}$ \\
\hline 1590 & 185 & $\left(\mathrm{k}^{3} \mathrm{hl}-\right)$ & $\left(\mathrm{k}^{3} \mathrm{hl}-\right)$ & $\left(\mathrm{k}^{3} \mathrm{hl}-\right)$ & my $x-$ & to sleep, to dream \\
\hline 1600 & 186 & & & & $\mathrm{mkv}-$ & $(?)^{72}$ \\
\hline 1610 & & & & & $\begin{array}{l}\text { mkv-*/ } \\
\text { mxv-* }\end{array}$ & $(?)^{73}$ \\
\hline 1620 & 187 & & & $\mathrm{~m}^{3} \mathrm{xV}-$ & $\begin{array}{l}\text { mxv- } \\
\text { mhxv- }\end{array}$ & to envy, covet, desire, admire \\
\hline 1630 & $\begin{array}{l}188 \\
189\end{array}$ & mxv- & mxv- & & mxv- & fast, quick \\
\hline 1640 & 2441 & $\begin{array}{l}m w- \\
m h w^{3} y-\end{array}$ & mw- & & mw- & $(?)^{74}$ \\
\hline 1641 & & & & & mw-* & (onomatopoeic) $^{75}$ \\
\hline
\end{tabular}

${ }^{70} \mathrm{In} \mathrm{KW} / \mathrm{mi}_{\text {iquiwal }}^{3}$ is/* "bullhead." However, bullheads are not native to the western part of North America. Lincoln (n.d.) gives the taxonomic name of Myxocephalus polyacanthocephalus which belongs to the great sculpin. The great sculpin lives on sand and mud bottoms. It has a blotchy brown, black and/or grey coloration and relies on camouflage for survival. Perhaps the KW name refers to its conspicuous eyes high on the forehead rising up steeply above the mouth.

${ }^{71} \mathrm{In} \mathrm{KW} / \mathrm{miradi} / *$ (a place name), /miqmi/* "marriage mask."

${ }^{72}$ In KW /mkva/ "to kill fleas in the teeth" and KW /ma:mk ${ }^{3} \mathrm{va} /$ "bat."

${ }^{73} \mathrm{In} \mathrm{KW}$ (Koskimo dialect) /mxvstu/* "steam hole of oven"

${ }^{74}$ Root is probably an alternant of $\{1 \mathrm{w}-\}$ (no. 11310, q.v.), and like it may have a connection to the supernatural. It is assumed to occur in OO/KW /hmumu/ "butterfly" (HE cognate /hл'ulu/); $\mathrm{HE} / \mathrm{hm}^{\prime}$ uli/ OO /hmuli/ "limpet", HE /h'am'aw ${ }^{3}$ iala/ and /h'am'aw ${ }^{3}$ iali/ (a limpet) and, with the root $\left\{\mathrm{k}^{3} \mathrm{~h}-\right\}$ prefixed to it, in the compound word $\mathrm{HE} / \mathrm{k}^{3 \prime} \mathrm{am}$ 'amu/ OO $/ \mathrm{k}^{3} \mathrm{amumu} /$ (a kind of clams).

${ }^{75} \mathrm{In} \mathrm{KW} / \mathrm{mawawu} / *$ (crow's cry) $\mathrm{KW} / \mathrm{mo} / *$ (cry of raven). 
North Wakashan Comparative Root List (Updated)

\begin{tabular}{|c|c|c|c|c|c|c|}
\hline Root \# & Old \# & $\mathrm{HE}$ & $\mathrm{OO}$ & HA & KW & Gloss \\
\hline 1650 & 190 & mw- & mw- & mw- & mw- & four $^{76}$ \\
\hline 1660 & 191 & mwt- & mwt- & mwt- & mwt- & balsam fir \\
\hline 1670 & 192 & & & $\begin{array}{l}\text { mwc-/ } \\
\text { mws- }\end{array}$ & mws- & $(?)^{77}$ \\
\hline 1680 & 193 & & mWs- & & mws- & $\begin{array}{l}\text { to destroy, damage, hurt, abuse, } \\
\text { be careless or rough with }\end{array}$ \\
\hline 1690 & 194 & mwł- & mwł- & & mwł- & $\begin{array}{l}\text { to whirl, rotate, stir, mix, agitate, } \\
\text { seethe }\end{array}$ \\
\hline 1700 & 195 & & & & mwł-* & (?) chafed, chafing \\
\hline 1710 & 196 & & $\mathrm{mwl}^{3} \mathrm{~s}_{-}$ & & $\begin{array}{l}\text { mwl- } \\
\text { mwl }^{3}- \\
\text { mwl } \\
\text { mwl }\end{array}$ & grateful $^{79}$ \\
\hline 1720 & 197 & mwkv- & mwkv- & mwkv- & mwkv- & $\begin{array}{l}\text { to tie a rope to sth., tie a knot in a } \\
\text { rope, hang sb., dangle }\end{array}$ \\
\hline 1730 & 198 & & & & $\begin{array}{l}\text { mwkv-l } \\
\text { mwxv- }\end{array}$ & $(?)^{80}$ \\
\hline 1740 & 199 & mwXv- & & mwXv- & & hump, lump \\
\hline 1750 & 200 & & & & mwqv- & $(?)^{81}$ \\
\hline
\end{tabular}

76 This root always combines with an extender $\{-\mathrm{y}-\},\{- \pm \mathrm{y}-\}$ or $\{-\mathrm{w}\}$.

${ }^{77}$ In HA the root occurs in a stem meaning "chief's wife" and in KW in stems referring to gathering one's skirts and raising one's clothes.

${ }^{78}$ Occurs only in the stem $\{\mathrm{mw}[\mathrm{mh}] \mathrm{s}-\}$.

79 \{mwk-\} only in KW /muła/ "grateful."

${ }^{80}$ In some tree names.

${ }^{81}$ In KW /muqvała/ "to stand aside." 
North Wakashan Comparative Root List (Updated)

\begin{tabular}{|c|c|c|c|c|c|c|}
\hline Root \# & Old \# & $\mathrm{HE}$ & $\mathrm{OO}$ & HA & KW & Gloss \\
\hline 1760 & $\begin{array}{l}201 \\
202\end{array}$ & mwqv- & mwqv- & mwqv- & $\begin{array}{l}\text { mwqv-* } \\
m^{3} w q v-\end{array}$ & $\begin{array}{l}\text { to cover over, hide, keep under } \\
\text { wraps; (in some HE/OO/HA } \\
\text { examples:) to contain life, } \\
\text { animate }\end{array}$ \\
\hline 1770 & & & & & mwqv- & $(?)^{82}$ \\
\hline 1780 & 203 & & & & mwqv- & $(?)^{83}$ \\
\hline 1790 & 204 & & & & $\mathrm{mw} \chi \mathrm{v}^{-}$ & $(?)^{84}$ \\
\hline 1791 & & & & & mrv-* & (?) spread all over ${ }^{85}$ \\
\hline 1800 & 205 & mqv- & mqv- & mqv- & mqv- & $\begin{array}{l}\text { multiple layers building a } \\
\text { swollen bag-like structure, } \\
\text { bulbous }\end{array}$ \\
\hline 1810 & $\begin{array}{l}206 \\
235 \\
238\end{array}$ & $\begin{array}{l}\mathrm{m} \chi \mathrm{v}- \\
\mathrm{mh} \chi \mathrm{v}-\end{array}$ & $\begin{array}{l}m \chi v- \\
m h \chi v-\end{array}$ & $\begin{array}{l}m \chi v- \\
m h \chi v-\end{array}$ & $\mathrm{m} \chi \mathrm{v}-$ & $\begin{array}{l}\text { rhythmic movement up and down } \\
\text { or side to side, to undulate, } \\
\text { shaking movement, to shake } \\
\text { (clothes) in water, to rinse, to } \\
\text { launder; wet, damp }{ }^{86}\end{array}$ \\
\hline
\end{tabular}

${ }^{82}$ In words referring to fish stomach. Could be root no. 1760 and mean "covered, hidden inside." ${ }^{83} \mathrm{In} \mathrm{KW}\left\{[\mathrm{mh}] \mathrm{mwqv}-=\mathrm{yn}^{3}-\mathrm{h}\right\}>/$ mamurvin $^{3} \mathrm{a} /$ "quartzite." According to Wikipedia quartzite is white to gray if pure but can have other colours due to iron oxide or other mineral impurities. Possibly therefore the stem is a misrecording, its root being $\left\{\mathrm{m}^{3} \mathrm{wqv}-\right\}$ (root no. 2370). ${ }^{84}$ Only in KW /muұvла/ "slovenly, dirty."

${ }^{85}$ Of the examples in Boas (1948:133; 1947:353) only two are listed in Lincoln (n.d.) but as nonreelicitable.

${ }^{86}\{\mathrm{mh} \chi \mathrm{v}-\}$ occurs in words referring to bobbing. It is also assumed to occur in HE /m'a $\chi \mathrm{vada} /$ ОО /maұvadлі/ "a sandpiper-like bird" because sandpipers are said to bob up and down when they walk on the beach. It may also occur in $\mathrm{HE} / \mathrm{m}^{\prime} \mathrm{aw}^{\prime} \mathrm{ak}^{3} / \mathrm{OO} / \mathrm{mawak}^{3} \mathrm{a} /$ "sea lion" because sea 
North Wakashan Comparative Root List (Updated)

\begin{tabular}{|c|c|c|c|c|c|c|}
\hline Root \# & Old \# & $\mathrm{HE}$ & $\mathrm{OO}$ & HA & KW & Gloss \\
\hline 1830 & $\begin{array}{l}207 \\
273\end{array}$ & & mq- & $\mathrm{mq-}$ & & $(?)^{87}$ \\
\hline 1840 & 208 & & & & $\mathrm{~m} \chi-$ & group of hollow objects ${ }^{88}$ \\
\hline 1850 & & & $\mathrm{~m} \chi-$ & & & $(?)^{89}$ \\
\hline 1860 & $\begin{array}{l}158 \\
210 \\
212 \\
213\end{array}$ & mh- & mh- & mh- & mh- & $\begin{array}{l}\text { 1. flat, layer-like in shape, sheet; } \\
\text { to lie or put down flat; } \\
\text { 2. covered by layer-like } \\
\text { outgrowth, having hair, fur; } \\
\text { 3. level, lacking variation; } \\
\text { 4. on the bottom, on the belly; to } \\
\text { move on or close to an expanse } \\
\text { by crawling, squirming, } \\
\text { slithering, wriggling or stirring } \\
\text { fins (like fish) } 90\end{array}$ \\
\hline 1890 & 214 & mhq- & mhq- & mhq- & mhp-* & $\begin{array}{l}\text { to pluck, tweeze, snatch away, } \\
\text { snap apart (rope) }\end{array}$ \\
\hline
\end{tabular}

lions can move relatively well on land using all four legs but the hind legs have limited mobility and their back therefore curves sideways at every step.

${ }^{87} \mathrm{In} \mathrm{OO} / \mathrm{mq}^{3} \mathrm{al}^{3} \mathrm{ini} /$ "going across from point to point" $\mathrm{OO} / \mathrm{mqaw}^{3}$ is/ "way off when crossing from one point to another" HA /mr'ani/ "flying squirrel." Flying squirrels don't fly, they glide from tree to tree.

${ }^{88}$ Root is plural form of $\{\mathrm{hn}-\}$.

${ }^{89} \mathrm{In} \mathrm{OO} / \mathrm{m} \chi \mathrm{uc} /$ "long feather of eagle."

${ }^{90}$ The meaning "level" occurs in HA /m'aukvm/ "to always do the same thing." The root occurs possibly also in HA (Kitlope dialect) /mas:masdal'ai/ "to slide a flat object over the water" and

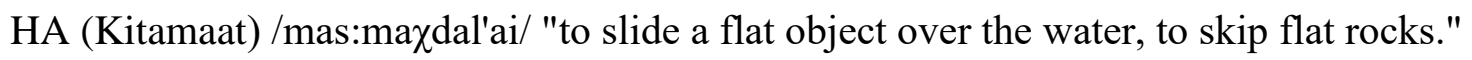


North Wakashan Comparative Root List (Updated)

\begin{tabular}{|c|c|c|c|c|c|c|}
\hline Root \# & Old \# & $\mathrm{HE}$ & $\mathrm{OO}$ & $\mathrm{HA}$ & KW & Gloss \\
\hline 1920 & 217 & & & & $\begin{array}{l}\text { mht- } \\
\text { mhdh- } \\
\text { mwdh-* }\end{array}$ & $(?)^{91}$ \\
\hline 1930 & 231 & mhnys- & $\mathrm{mhn}^{3} \mathrm{ys}-$ & mhn'hys- & & (?) flat, exposed, unshielded 92 \\
\hline 1940 & $\begin{array}{l}218 \\
219 \\
220\end{array}$ & $\begin{array}{l}\text { mhs- } \\
\text { mhzh- }\end{array}$ & $\begin{array}{l}\text { mhs- } \\
\text { mhz- }\end{array}$ & mhs- & $\begin{array}{l}\text { mhs- } \\
\text { mhsø- }\end{array}$ & $\begin{array}{l}\text { on the side, sideways, off course, } \\
\text { slanting, at an angle; to eat a side } \\
\text { dish }^{93}\end{array}$ \\
\hline 1950 & 221 & mhs- & mhs- & mhs- & mhs- & $\begin{array}{l}\text { stripe, striped, extending like a } \\
\text { strip, put down lengthwise }\end{array}$ \\
\hline 1960 & & $\mathrm{~m}^{3} \mathrm{hs}-$ & $\mathrm{m}^{3} \mathrm{hs}-$ & mhs- & & $(?)^{94}$ \\
\hline 1970 & 222 & mhf- & mh£- & mhf- & & $\begin{array}{l}\text { hand in hand, to shake hands, } \\
\text { take by the hand }\end{array}$ \\
\hline 1971 & & & & & mhk- & (?) spread out ${ }^{95}$ \\
\hline
\end{tabular}

${ }^{91}$ In some tribal and family names. Boas (1948:138) assumes the root is a borrowing from Nootka where he says it means "to fly." If the association with flying is correct, this root could be another instance of root no. 1210 .

${ }^{92}$ Stem possibly containing a root HE/HA $\{\mathrm{mhnh}-\}$ OO $\left\{\mathrm{mhn}^{3}-\right\}$ that is related to root no. 2200 . 93 \{mhzh-\} occurs only in HE /m'az'aiqvлª/ OO /maziqvлª/ "salmonberry blossom" and $\{$ mhs $\infty$ - $\}$ only in KW /masəkola/ "blossom, flowers." \{mhs- $\}$ may also be part of a HE/OO stem \{mhył- $\}$ "to lift and move the end of a long thing (e.g. a plank)." Perhaps \{mhs- $\}$ with the sense of eating a side dish is also present in OO /mazM/ "Indian cheese (made of salmon eggs)" and /masila/ "to make Indian cheese." Finally, it may be present in $\mathrm{HE} / \mathrm{m}^{\prime}$ ask'a/ the exact meaning of which is uncertain but involves one's lower back.

${ }^{94}$ In HA /M'as:masalanusiwa/ or / $\mathrm{M}^{3}$ as:m³asalanusiwa/ (uncertain which), HE

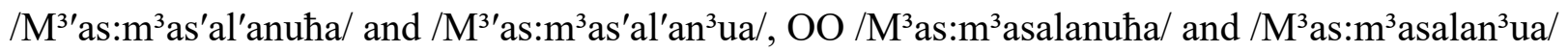
(name of the creator). For a text about the entity see Walkus (1982:157-162); however, Bella Bella elder Beatrice Brown explained in a low voice the name had to do with eating people). ${ }^{95}$ In the KW stem $\left.\{[\mathrm{mh}] \mathrm{mh}\}-\right\}>\{$ hhmhł- $\}$ "spread all over." 
North Wakashan Comparative Root List (Updated)

\begin{tabular}{|c|c|c|c|c|c|c|}
\hline Root \# & Old \# & $\mathrm{HE}$ & $\mathrm{OO}$ & $\mathrm{HA}$ & KW & Gloss \\
\hline 1980 & 223 & & & & mht- & $\begin{array}{l}\text { bitter, tart, tangy (like green } \\
\text { potatoes) }\end{array}$ \\
\hline 2000 & $\begin{array}{l}226 \\
228\end{array}$ & mhł- & mht- & & & (?) reddish, orange \\
\hline 2010 & 229 & & mhk- & & mhk- & close, near, next to \\
\hline 2020 & 230 & mhx- & mhx- & $\begin{array}{l}\text { mhx- } \\
\text { mht- }\end{array}$ & mhx- & (?) to take avoidance action ${ }^{96}$ \\
\hline 2021 & $\begin{array}{l}210 \\
211\end{array}$ & mhył- & mhył- & & $\mathrm{mh}^{3} \mathrm{yl}-*$ & (?) handle a long thing ${ }^{97}$ \\
\hline 2030 & & & & & $\begin{array}{l}\text { mhyh-* } \\
\text { mhyht- } \\
\text { mhyh }^{3} x-\end{array}$ & to value, spare, respect \\
\hline 2040 & 232 & mhkv- & mhkv- & mhkv- & mhkv- & to jerk, to jig \\
\hline 2050 & 234 & mhkv- & & & mhxv- & to tie a knot or bow ${ }^{98}$ \\
\hline 2060 & & mhw- & & & & meow! (onomatopoeia) \\
\hline 2070 & $\begin{array}{l}236 \\
282\end{array}$ & mhqv- & $\begin{array}{l}\text { mhqv- } \\
\text { mhqv- }\end{array}$ & mhqv- & mhqv- & $\begin{array}{l}\text { blocking one's view, opaque, } \\
\text { foggy, cover of darkness; (HA }\end{array}$ \\
\hline
\end{tabular}

96 The examples are about shame, shyness and being inconvenienced by the outside world (like blinded by glare). HA $\{\mathrm{mh}\}-\}$ is a free alternant of $\{\mathrm{mhx}-\}$ attested in only one example.

97 The HE/OO stem refers to moving the end of a plank by lifting and manipulating the other end. What the KW stem refers to is not clear but could be using the tip of a long thing to draw a marking line. Possibly the stem derives from a root $\{$ myk- $\}$ because several roots beginning in |my| are about extending a long thing.

$98\{$ mhkv- $\}$ only in HE $\{\mathrm{mh}[\mathrm{m}] \mathrm{kv}-=\mathrm{htw}-\mathrm{h}\}>$ /m'amgvatua/ "earrings, ear ornaments" (alternant of HE /m'umgvatua/). 
North Wakashan Comparative Root List (Updated)

\begin{tabular}{|c|c|c|c|c|c|c|}
\hline Root \# & Old \# & $\mathrm{HE}$ & $\mathrm{OO}$ & $\mathrm{HA}$ & KW & Gloss \\
\hline & & & & & & $\begin{array}{l}\text { also:) blocking one's way (like } \\
\text { uprooted tree) }\end{array}$ \\
\hline 2090 & 237 & & & & mhqv-* & $(?)^{99}$ \\
\hline 2100 & $\begin{array}{r}233 \\
283 \\
1254 \\
2582\end{array}$ & $\begin{array}{l}\mathrm{mh} \chi \mathrm{v}^{-} \\
\mathrm{m}^{3} \mathrm{~h} \chi \mathrm{v}-\end{array}$ & $\mathrm{m}^{3} \mathrm{~h} \chi \mathrm{v}-$ & $\mathrm{mh} \chi \mathrm{v}-$ & $\begin{array}{l}\mathrm{mh} \chi \mathrm{v}-* \\
\mathrm{~m}^{3} \mathrm{~h} \chi \mathrm{v}- \\
\mathrm{l}^{3} \mathrm{~h} \chi \mathrm{v}- \\
\mathrm{h}^{3} \mathrm{lqv}-\end{array}$ & $\begin{array}{l}\text { tongue hangs out, to wag the } \\
\text { tongue, covered with tongue-like } \\
\text { things or scales or plates }{ }^{100}\end{array}$ \\
\hline 2120 & 239 & & & & mhq-* & $(?)^{101}$ \\
\hline 2130 & 240 & $(\mathrm{hl} \chi-)$ & $\begin{array}{l}\operatorname{mh} \chi_{-}^{-} \\
\left(\mathrm{hl} \chi_{-}\right)\end{array}$ & $(\mathrm{hl} \chi-)$ & $\operatorname{mh} \chi-$ & killer whale ${ }^{102}$ \\
\hline 2140 & 241 & & & & $\operatorname{mh} \chi-*$ & (?) stripe ${ }^{103}$ \\
\hline 2150 & 227 & $\mathrm{mh}^{3} \mathrm{l}_{-}$ & $\mathrm{mh}^{3} \mathrm{t}-$ & $\mathrm{mh}^{3} \mathrm{l}^{-}$ & $\mathrm{mh}^{3} \mathrm{l}_{-}$ & two, both, forked ${ }^{104}$ \\
\hline 2160 & $\begin{array}{l}242 \\
243 \\
256\end{array}$ & $\begin{array}{l}m^{3} n- \\
m^{3} n y- \\
m^{3} h y h- \\
\left(w^{3} w-\right)\end{array}$ & $\begin{array}{l}m^{3} n- \\
m^{3} n y- \\
m^{3} h y h- \\
\left(w^{3} w-\right)\end{array}$ & $\begin{array}{l}\mathrm{m}^{3} \mathrm{n}- \\
\mathrm{m}^{3} \mathrm{y}- \\
\mathrm{m}^{3} \mathrm{hyh}-\end{array}$ & $\begin{array}{l}m^{3} n- \\
n^{3} m-\end{array}$ & $\begin{array}{l}\text { 1. (all } \mathrm{HE} / \mathrm{OO} / \mathrm{HA} \text { alternants and } \\
\mathrm{KW}\left\{\mathrm{n}^{3} \mathrm{~m}-\right\} \text { :) one, unit, item in a } \\
\text { set, individual, acting alone, in } \\
\text { unison with; to collect, } \\
\text { congregate, build a unit; }\end{array}$ \\
\hline
\end{tabular}

${ }^{99} \mathrm{In} \mathrm{KW} / \mathrm{maq}^{3} \mathrm{VHs} / *$, the story name of Heron.

$100\{\mathrm{mh} \chi \mathrm{v}-\}$ occurs possibly in HE /m'aw'alªs/ HA /m'awªs/ "Douglas-fir" because the bracts on the tree's cones look like tongues or like the tail and hind legs of a small animal sticking out from between the cone's scales.

${ }^{101}$ Only in KW /maqu/* "November" (a word from the Newettee dialect). Perhaps this is the root $\{$ mhq- $\}$ "to pluck etc." (root no. 1890) that occurs in HE/OO/HA.

$102 \mathrm{OO}$ only possibly in $/ \mathrm{Maxp}^{3} \mathrm{iq} /$ (name of a totem pole). For another case of $|\mathrm{m}|$ - $|1|$ correspondence see $\{\mathrm{mw}-\}$ and $\{1 \mathrm{w}-\}$ "flutter, whirl etc."

103 In KW /maxstola/* "raccoon."

${ }^{104}$ Also in HE /m'am'ałik³ a/ OO /mamałikª/ "swallow"; the root refers here to the forked tail. 
North Wakashan Comparative Root List (Updated)

\begin{tabular}{|c|c|c|c|c|c|c|}
\hline Root \# & Old \# & $\mathrm{HE}$ & $\mathrm{OO}$ & HA & KW & Gloss \\
\hline & & & & & & $\begin{array}{l}\text { 2. (KW }\left\{m^{3} n-\right) \text { and } \mathrm{HE} / \mathrm{OO} / \mathrm{HA} \\
\left.\left\{\mathrm{m}^{3} \mathrm{n}-\mathrm{hq}-\right\}:\right) \text { to single out, pick } \\
\text { out }^{105}\end{array}$ \\
\hline 2170 & $\begin{array}{l}244 \\
245 \\
246 \\
248\end{array}$ & $\mathrm{~m}^{3} \mathrm{nc}-$ & $m^{3} n c-$ & $\begin{array}{l}m^{3} n c- \\
m^{3} n^{3} c-\end{array}$ & $\mathrm{m}^{3} \mathrm{~ns}-$ & $\begin{array}{l}\text { to compare sth. with sth., to try to } \\
\text { match a standard, to measure, to } \\
\text { test, to try out, to attempt, to } \\
\text { rehearse }\end{array}$ \\
\hline 2180 & 247 & $\mathrm{~m}^{3} \mathrm{nc}-$ & $m^{3} n c-$ & $m^{3} n c-$ & $m^{3} n s-$ & to motion, to gesture \\
\hline 2190 & 249 & $\mathrm{~m}^{3} \mathrm{nq}-$ & & $\mathrm{m}^{3} \mathrm{nq}-$ & & stuck in a place, to stay behind \\
\hline 2200 & 250 & $\begin{array}{l}\text { mhnh- } \\
\text { mhnh- }\end{array}$ & $\begin{array}{l}\text { mhnh- }^{3} \text { m } \\
\text { mny- }\end{array}$ & $\begin{array}{l}\mathrm{m}^{3} \mathrm{nh}- \\
\mathrm{m}^{3} \mathrm{hnh}-\end{array}$ & $\begin{array}{l}\mathrm{n}^{3} \mathrm{mh}- \\
\mathrm{n}^{3} \mathrm{mhy}{ }^{3} \mathrm{~h}-\end{array}$ & $\begin{array}{l}\text { even, equal, similar, } \\
\text { simultaneous }^{106}\end{array}$ \\
\hline 2210 & 251 & $\begin{array}{l}\mathrm{m}^{3} \mathrm{c}-/ \\
\mathrm{m}^{3} \mathrm{~s}-\end{array}$ & & & & $(?)^{107}$ \\
\hline 2220 & 252 & $\mathrm{~m}^{3} \mathrm{stq}-$ & $\mathrm{m}^{3} \mathrm{stq}-$ & $\mathrm{m}^{3} \mathrm{stq}-$ & $\begin{array}{l}\mathrm{m}^{3} \mathrm{~s} \chi-^{*} \\
\mathrm{~m}^{3} \chi \mathrm{stq}-\end{array}$ & $\begin{array}{l}\text { to drop, release, discard, let go of } \\
\text { sth.; to release grip }{ }^{108}\end{array}$ \\
\hline
\end{tabular}

105 (1) For another example of KW using a metathesized form see HE/OO/HA \{nyk-\} versus $\mathrm{KW}$ \{ynk- $\}$ "to take revenge, retaliate, reciprocate." (2) HE/OO uses $\left\{\mathrm{W}^{3} \mathrm{~W}-\right\}$ "instance of" instead of $\left\{m^{3} n-\right\}$ before the suffix $\left\{-p^{3} n-\right\}$ "times." $\left\{m^{3} n-\right\}$ and $\left\{p^{3} n-\right\}$ are probably developments of one morpheme. (3) HA usually uses $\left\{n^{3} h x v-\right\}$ instead of $\left\{m^{3} n-\right\}$ for the meaning "one, single, alone etc."

${ }^{106}$ Root is an augmented form of $\left\{m^{3} n-, n^{3} m-\right\}$ "one, etc." (root no. 2160) but differs from it semantically in the same way Latin sem- and sim- (surviving in e.g. English "sim-ilar") differ semantically from Latin un- (surviving in e.g. English "un-ity"). HE \{mhnh- $\}$ occurs only in $\left\{\mathrm{mhnh}-=\mathrm{h}^{3} \mathrm{~s}\right\}>/ \mathrm{m}^{\prime}$ an'as/ "prairie, flat country" (cf. OO $/ \mathrm{m}^{3} \mathrm{anas} /$ ).

107 Only in $\mathrm{HE} / \mathrm{m}^{3} \mathrm{z}^{\prime} \mathrm{ay} \mathrm{y}^{\prime} \mathrm{u} /$ "stick for prying up roots." ${ }^{108} \mathrm{KW}\left\{\mathrm{m}^{3} \mathrm{~s} \chi-\right\} *$ only in $\left.\left\{\mathrm{m}^{3} \mathrm{~s} \chi-\mathrm{W}-\mathrm{hlh}\right\}>/ \mathrm{m}^{3} \mathrm{~s} \chi\right\lrcorner l \mathrm{a} / *$ "to squirt out." If this word is ignored, $\mathrm{KW}$ $\left\{\mathrm{m}^{3} \chi\right.$ stq- $\}$ could really be $\left\{\mathrm{m}^{3} \mathrm{q}-\mathrm{stq}-\mathrm{h}\right\}$, with root no. 2390 and suffix no. 1170 . In HE/OO/HA $\left\{m^{3} s t q-\right\}$ this same root $\left\{m^{3} q-\right\}$ may have lost its $|q|$. 
North Wakashan Comparative Root List (Updated)

\begin{tabular}{|c|c|c|c|c|c|c|}
\hline Root \# & Old \# & $\mathrm{HE}$ & $\mathrm{OO}$ & HA & KW & Gloss \\
\hline 2230 & 253 & $\mathrm{~m}^{3} 1-$ & $\mathrm{m}^{3} 1-$ & & $\mathrm{m}^{3} 1-$ & mountain goat colour ${ }^{109}$ \\
\hline 2240 & 254 & $\mathrm{~m}^{3} \mathrm{lqv}-$ & $\mathrm{m}^{3} \mathrm{lqv}-$ & $\mathrm{m}^{3} \mathrm{lqv}-$ & $\mathrm{m}^{3}$ lqv- & $\begin{array}{l}\text { to lick, spit, transfer moist } \\
\text { material from mouth, chew food } \\
\text { for baby, give food on tip of the } \\
\text { tongue; (KW also:) to flatter }\end{array}$ \\
\hline 2250 & 255 & $\mathrm{~m}^{3} \mathrm{lq}-$ & $\mathrm{m}^{3} \mathrm{lq}-$ & & $\mathrm{m}^{3} \mathrm{lq}-$ & mountain goat colour ${ }^{110}$ \\
\hline 2270 & $\begin{array}{l}258 \\
259 \\
260 \\
261\end{array}$ & $\mathrm{~m}^{3} \mathrm{y} £-$ & $\begin{array}{l}m^{3} y £- \\
m^{3} y^{3} £-\end{array}$ & $\begin{array}{l}m^{3} y £- \\
m^{3} y^{3} f-\end{array}$ & $\begin{array}{l}\mathrm{m}^{3} \mathrm{y} £- \\
\mathrm{m}^{3} \mathrm{y} 1-\end{array}$ & $\begin{array}{l}\text { to fail to hit target, deviate, go off } \\
\text { course, shift, move away; to } \\
\text { dodge, avoid accident; to make } \\
\text { mistake, be mistaken, be wrong; } \\
\text { to tease, provoke, do mischief; } \\
\text { (KW }\left\{\mathrm{m}^{3} \mathrm{y} \text { - }\right\} \text { only:) to spoil a } \\
\text { spell or magic, to make mistake } \\
\text { in ritual }{ }^{111}\end{array}$ \\
\hline 2300 & 262 & $\mathrm{~m}^{3} \mathrm{yk}-$ & $\mathrm{m}^{3} \mathrm{yk}-$ & $\mathrm{m}^{3} \mathrm{yk}-$ & $\mathrm{m}^{3} \mathrm{yk}-$ & (?) to move, movable ${ }^{112}$ \\
\hline 2310 & 263 & $\mathrm{~m}^{3} \mathrm{kv}-$ & $\mathrm{m}^{3} \mathrm{kv}-$ & $\mathrm{m}^{3} \mathrm{kv}-$ & $\begin{array}{l}\mathrm{m}^{3} \mathrm{kv}- \\
\mathrm{m}^{3} \mathrm{hkv}-\end{array}$ & $\begin{array}{l}\text { round and/or bulky object is } \\
\text { located somewhere, is being }\end{array}$ \\
\hline
\end{tabular}

109 The colour is called grey in the few HE/OO examples and one KW one. It is called white in the majority of KW examples.

${ }^{110} \mathrm{HE} / \mathrm{OO}$ only in $\mathrm{HE} / \mathrm{m}^{3 \prime л q і ' а / ~ O O ~ / m ³ л q q i a / ~ " g r e y ~ h a i r, ~ g r e y-h a i r e d . " ~}$

${ }^{111}$ In HA, $\left\{\mathrm{m}^{3} \mathrm{y} \mathfrak{f}-\right\}$ and $\left\{\mathrm{m}^{3} \mathrm{y}^{3} \mathfrak{f}-\right\}$ may seem to be in opposition in, respectively, $\left\{\mathrm{m}^{3} \mathrm{y} \mathfrak{f}-\mathrm{h}\right\}>$ $/ \mathrm{m}^{3} \mathrm{i} £ \mathrm{a} /$ "to miss a shot" and $\left\{\mathrm{m}^{3} \mathrm{y}^{3} £-\mathrm{h}\right\}>/ \mathrm{m}^{3} \mathrm{i} £^{\prime} \mathrm{a} /$ "to be (go) wrong, to make a mistake." 112 Possibly in $\mathrm{HE} / \mathrm{m}^{3} \mathrm{am}^{3} \mathrm{ikas} / \mathrm{HA} / \mathrm{m}^{3} \mathrm{am}^{3} \mathrm{ik}$ 'as/ OO/KW $/ \mathrm{m}^{3} \mathrm{am}^{3} \mathrm{ikas} /$ "possessions, household goods." 
North Wakashan Comparative Root List (Updated)

\begin{tabular}{|c|c|c|c|c|c|c|}
\hline Root \# & Old \# & $\mathrm{HE}$ & $\mathrm{OO}$ & HA & KW & Gloss \\
\hline & & & & & & $\begin{array}{l}\text { handled, thrown or dropped; } \\
\text { (HE/OO/HA also:) to hammer }{ }^{113}\end{array}$ \\
\hline 2320 & 264 & $\begin{array}{l}\mathrm{m}^{3} \mathrm{w}- \\
\mathrm{m}^{3} \mathrm{hw}^{3}- \\
\mathrm{w}^{3} \mathrm{hm}^{3}-\end{array}$ & $\begin{array}{l}\mathrm{m}^{3} \mathrm{~W}- \\
\mathrm{m}^{3} \mathrm{hw}^{3}- \\
\mathrm{w}^{3} \mathrm{hm}^{3-}\end{array}$ & $\begin{array}{l}\mathrm{m}^{3} \mathrm{w}- \\
\mathrm{w}^{3} \mathrm{hm}^{3}-\end{array}$ & $\begin{array}{l}\mathrm{m}^{3} \mathrm{w}- \\
\mathrm{m}^{3} \mathrm{hwh}- \\
\mathrm{m}^{3} \mathrm{hw}^{3-}\end{array}$ & $\begin{array}{l}\text { to transfer a load, to transport; } \\
\text { (KW also:) to pile, amass }\end{array}$ \\
\hline 2330 & 265 & $\mathrm{w}^{3} \mathrm{hmwt-}$ & $\mathrm{w}^{3} \mathrm{hmwt-}$ & $\left(h^{3} h m w y^{3}-\right)$ & $\mathrm{m}^{3} \mathrm{wt}-$ & take home leftovers from feast \\
\hline 2340 & 266 & $\mathrm{~m}^{3} \mathrm{wc}-$ & $\mathrm{m}^{3} \mathrm{wc}-$ & & & $\begin{array}{l}\text { to round the mouth, pucker the } \\
\text { lips }\end{array}$ \\
\hline 2350 & $\begin{array}{l}267 \\
268\end{array}$ & 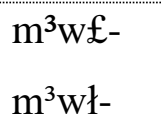 & $\begin{array}{l}\mathrm{m}^{3} \mathrm{w} £- \\
\mathrm{m}^{3} \mathrm{w}-\end{array}$ & $\begin{array}{l}\mathrm{m}^{3} \mathrm{w} £- \\
\mathrm{m}^{3} \mathrm{w} 1-\end{array}$ & $\begin{array}{l}\text { (bw£- } \\
\text { bwt-) }\end{array}$ & $\begin{array}{l}\text { to heap up, rise up, swell, } \\
\text { ferment }\end{array}$ \\
\hline 2360 & 269 & & & & $\mathrm{~m}^{3} \mathrm{wkV}-*$ & $(?)^{114}$ \\
\hline 2370 & $\begin{array}{r}270 \\
1913\end{array}$ & $\mathrm{~m}^{3} \mathrm{wqv}-$ & $\mathrm{m}^{3} \mathrm{wqv}-$ & $\mathrm{m}^{3} \mathrm{wqv}-$ & $\begin{array}{l}\mathrm{m}^{3} \mathrm{wqv-} * \\
\mathrm{~h}^{3} \mathrm{wqv}-\end{array}$ & $\begin{array}{l}\text { white, discoloured, grey, bland, } \\
\text { stale }\end{array}$ \\
\hline 2380 & 271 & $\mathrm{~m}^{3} \mathrm{w}^{3} \mathrm{qv}-$ & $\mathrm{m}^{3} \mathrm{w}^{3} \mathrm{qv}-$ & $\mathrm{m}^{3} \mathrm{w}^{3} \mathrm{qv}-$ & & $(?)^{115}$ \\
\hline 2390 & 272 & $\mathrm{~m}^{3} \mathrm{q}-$ & $m^{3} q^{-}$ & $\mathrm{m}^{3} \mathrm{q}-$ & $\mathrm{m}^{3} \mathrm{q}-$ & $\begin{array}{l}\text { to drop, release, discard, throw } \\
\text { away, to shoot power, transfer } \\
\text { magical power }\end{array}$ \\
\hline
\end{tabular}

113 The one certain example of $\mathrm{KW}\left\{\mathrm{m}^{3} \mathrm{hkv}-\right\}$ is $/ \mathrm{m}^{3} \mathrm{ak}^{3} \mathrm{vr} \varepsilon^{3} /$ "seed, pit, core" and it alternates with $/ \mathrm{m}^{3} \mathrm{k}^{3} \mathrm{VI} \varepsilon^{3} /$. In all other possible examples the presence of $|\mathrm{h}|$ in the root can also be explained as a suffix-triggered augment. HE/OO/HA often use $\left\{£^{3} \mathrm{k}-\right\}$ where $\mathrm{KW}$ uses $\left\{\mathrm{m}^{3} \mathrm{kv}-\right\}$. ${ }^{114} \mathrm{In} \mathrm{KW} / \mathrm{m}^{3} \mathrm{ukva} / *$ "young fur seal." Term is not in Boas's dictionary but may have been found in unspecified other Boas-Hunt materials. Could be a misrecording of $/ \mathrm{m}^{3} \mathrm{uqva} /{ }^{*}$ 'grey, whitish, blonde' which is a derivate of root no. 2370.

${ }^{115}$ Possibly in HE $/ \mathrm{m}^{3} \mathrm{u} \chi \mathrm{vd} \Omega^{\prime} \mathrm{i} / \mathrm{OO} / \mathrm{m}^{3} \mathrm{u}^{3} \chi \mathrm{vd} \mathrm{di} / \mathrm{HA} / \mathrm{m}^{3} \mathrm{u} \chi \mathrm{vd} \Omega^{\prime} \mathrm{i} /$ "scrotum; to get 'skunked' (= to have no catch)." However, the HE and HA items can also be pronounced $/ \mathrm{m}^{3} \chi \mathrm{vd} \mathrm{J}^{\prime} \mathrm{i} /$ and $/ \mathrm{m}^{3} \chi \mathrm{vd} \mathrm{i} /$, respectively, and it is not diagnosable if $/ \chi \mathrm{v} /$ before $/ \mathrm{d} /$ stems from $|\chi \mathrm{v}|$ or $|\mathrm{qv}|$. Root could therefore also be anyone of $\left\{\mathrm{m}^{3} \mathrm{w}^{3} \chi^{\mathrm{v}}, \mathrm{m}^{3} \mathrm{qv}^{-}, \mathrm{m}^{3} \chi \mathrm{v}^{-}\right\}$and even $\left\{\mathrm{m}^{3} \mathrm{wqv-}, \mathrm{m}^{3} \mathrm{w} \chi \mathrm{v}^{-}\right\}$. The word could be an unanalyzable loan. 
North Wakashan Comparative Root List (Updated)

\begin{tabular}{|c|c|c|c|c|c|c|}
\hline Root \# & Old \# & $\mathrm{HE}$ & $\mathrm{OO}$ & HA & KW & Gloss \\
\hline 2400 & 274 & $\mathrm{~m}^{3} \mathrm{~h}-$ & $\mathrm{m}^{3} \mathrm{~h}-$ & $\mathrm{m}^{3} \mathrm{~h}-$ & $\mathrm{m}^{3} \mathrm{~h}-$ & $(?)^{116}$ \\
\hline 2401 & & 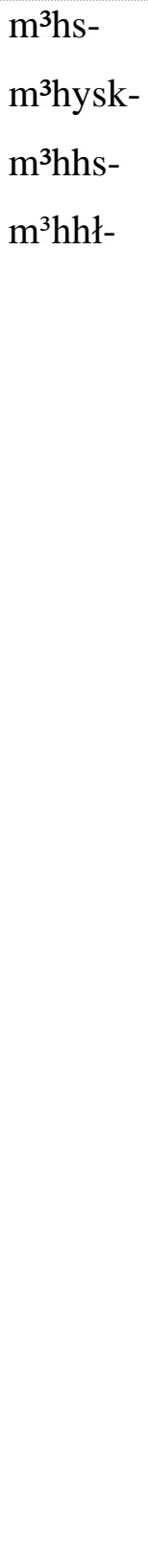 & $\begin{array}{l}\mathrm{m}^{3} \mathrm{hs}- \\
\mathrm{m}^{3} \mathrm{hy}- \\
\mathrm{m}^{3} \mathrm{hysk}- \\
\mathrm{m}^{3} \mathrm{hhs}- \\
\mathrm{m}^{3} \mathrm{hht-}\end{array}$ & $\begin{array}{l}\mathrm{m}^{3} \mathrm{hs}- \\
\mathrm{m}^{3} \mathrm{hy}- \\
\mathrm{m}^{3} \mathrm{hysk}- \\
\text { m³hs- }\end{array}$ & $\begin{array}{l}\mathrm{m}^{3} \mathrm{hs}- \\
\mathrm{m}^{3} \mathrm{hy}- \\
\mathrm{m}^{3} \mathrm{hsk}- \\
\mathrm{m}^{3} \mathrm{hy}{ }^{3} \mathrm{hf-}\end{array}$ & 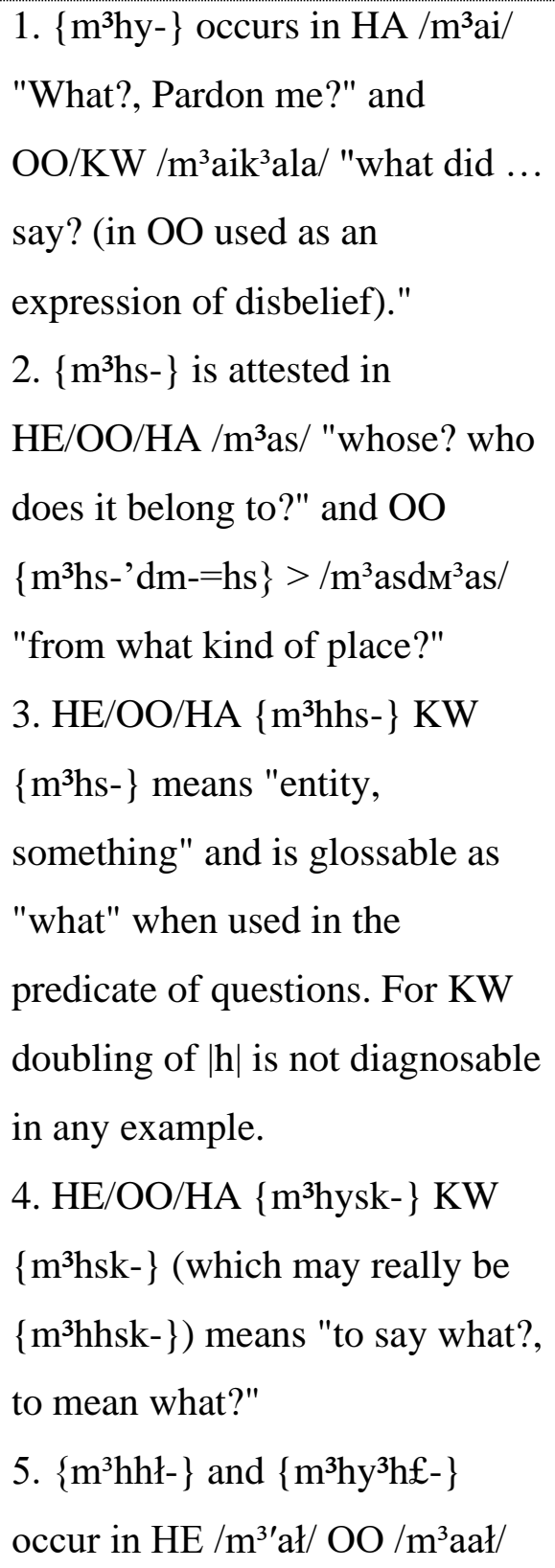 \\
\hline
\end{tabular}

${ }^{116}$ In the exclamation $\mathrm{HE} / \mathrm{m}^{3 /} \mathrm{a} / \mathrm{OO} / \mathrm{KW} / \mathrm{m}^{3} \mathrm{a} /$ "What?, Pardon me?" In $\mathrm{KW}$ also attested with some category B suffixes added. Is a component of the hard to analyze stems ending in $|\mathrm{s}|$ or $|\mathrm{H}|$ and listed as root no. 2401. 
North Wakashan Comparative Root List (Updated)

\begin{tabular}{|c|c|c|c|c|c|c|}
\hline Root \# & Old \# & $\mathrm{HE}$ & $\mathrm{OO}$ & HA & KW & Gloss \\
\hline & & & & & & $\begin{array}{l}\mathrm{KW} / \mathrm{m}^{3} \mathrm{ay}{ }^{3} \mathrm{a} f / \text { "where?, to which } \\
\text { place?" }\end{array}$ \\
\hline 2410 & $\begin{array}{l}275 \\
279\end{array}$ & & & & $\begin{array}{l}\mathrm{m}^{3} \mathrm{hl}- \\
\mathrm{m}^{3} \mathrm{l}-*\end{array}$ & $\begin{array}{l}\text { (?) rough, chafing, eating away } \\
\text { at }^{117}\end{array}$ \\
\hline 2420 & $\begin{array}{l}276 \\
277 \\
278\end{array}$ & $\mathrm{~m}^{3} \mathrm{~h} 1-$ & $\left.\mathrm{m}^{3} \mathrm{~h}\right\}-$ & $\mathrm{m}^{3} \mathrm{~h} 1-$ & $\mathrm{m}^{3} \mathrm{~h} 1-$ & $\begin{array}{l}\text { (?) to recognize, to } \\
\text { acknowledge }\end{array}$ \\
\hline 2430 & 280 & $\mathrm{~m}^{3} \mathrm{hkv}-$ & $\mathrm{m}^{3} \mathrm{hkv}-$ & & & $(?)^{119}$ \\
\hline 2440 & 281 & (yhx-) & $\mathrm{m}^{3} \mathrm{hxv}-$ & & $\mathrm{m}^{3} \mathrm{hxv}-$ & (?) potlatch ${ }^{120}$ \\
\hline 2450 & 284 & $\mathrm{dm}-$ & dm- & dm- & $\mathrm{dm}-$ & salt \\
\hline 2460 & $\begin{array}{l}285 \\
482\end{array}$ & $\mathrm{~nm}-$ & $\mathrm{nm}-$ & $\mathrm{nm}-$ & $\begin{array}{l}\text { dm- } \\
n m- \\
n^{3} m^{-}\end{array}$ & $\begin{array}{l}\text { lasting a certain time, having } \\
\text { duration }\end{array}$ \\
\hline 2470 & 286 & & & & dmł- & make a rumbling noise \\
\hline 2480 & 287 & dmkv- & dmkv- & dmkv- & dmkv- & $\begin{array}{l}\text { (HE/OO/HA) to hinge (like one's } \\
\text { limbs), to bend or straighten the } \\
\text { legs of V-shape; (HA also:) to } \\
\text { kick; (KW) tension in muscle, } \\
\text { cramps }\end{array}$ \\
\hline
\end{tabular}

$117\left\{\mathrm{~m}^{3} \mathrm{~h} 1-\right\}$ occurs perhaps in $\mathrm{KW}\left\{\mathrm{m}^{3} \mathrm{hlykv}\right\}$ "to chew" and $\mathrm{KW} / \mathrm{m}^{3}$ alizani/* "middle-sized clam with rough shell." $\left\{\mathrm{m}^{3} \mathrm{l}_{-}\right\}^{*}$ occurs perhaps in the Koskimo word $/ \mathrm{młxvid} / *$ or $/ \mathrm{m}^{3}$ łəxvid/* "to eat."

${ }^{118}$ In HA (Kitamaat dialect) $/ \mathrm{m}^{3} \mathrm{am}^{3} \mathrm{a} a /$ (Kitlope dialect) $/ \mathrm{m}^{3} \mathrm{am}^{3} \mathrm{a}$ ała "to pity sb." and the stem $\mathrm{HE} / \mathrm{OO} / \mathrm{HA} / \mathrm{KW}\left\{\mathrm{m}^{3} \mathrm{~h} \nmid t^{3} \mathrm{y}-\right\}$ "to recognize." See also the comment on $\left\{\mathrm{h}^{3} \mathrm{M}^{3}-, \mathrm{h}^{3} \mathrm{~m}^{3} \mathrm{~h}\right.$-, etc. $\}$ (root no. 23350).

${ }^{119}$ In HE /m'akvaradл'i/ OO /m³am³akvaradлi/ "dragonfly."

${ }^{120}$ The OO root occurs in proper names and is a dialect borrowing. 
North Wakashan Comparative Root List (Updated)

\begin{tabular}{|c|c|c|c|c|c|c|}
\hline Root \# & Old \# & $\mathrm{HE}$ & $\mathrm{OO}$ & HA & $\mathrm{KW}$ & Gloss \\
\hline 2490 & 288 & & & & dmxv-* & $(?)^{121}$ \\
\hline 2500 & 289 & dwqv- & & dwqv- & dmqv- & $(?)^{122}$ \\
\hline 2510 & 290 & & & & $\operatorname{dm} \chi-^{*}$ & white eelgrass $^{123}$ \\
\hline 2520 & 291 & & $\operatorname{dm} \chi-$ & & & to drone \\
\hline 2530 & 292 & dn- & dn- & dn- & dn- & $\begin{array}{l}\text { to pull with a rope, to haul, to } \\
\text { drag sth. }\end{array}$ \\
\hline 2550 & 293 & dnt- & dnt- & & dnt- & noisy discussion \\
\hline 2560 & 294 & dns- & dns- & dns- & dns- & $\begin{array}{l}\text { strips being ripped off (especially } \\
\text { red cedar bark strips) }\end{array}$ \\
\hline 2570 & & & & & dnkv-* & fat, grease \\
\hline 2580 & 295 & & dnxv- & & dnxv- & $\begin{array}{l}\text { in a row, one after the other (in } \\
\text { space or time), lined up; line of } \\
\text { rope }^{124}\end{array}$ \\
\hline 2590 & 296 & & & & $\operatorname{dn} \chi-$ & $\begin{array}{l}\text { to sing as an accompaniment to } \\
\text { the dancers }\end{array}$ \\
\hline 2600 & 297 & & $\operatorname{dn} \chi-$ & & & $(?)^{125}$ \\
\hline 2610 & 298 & & & & $\begin{array}{l}\mathrm{dn}^{3}-* \\
\mathrm{dn}^{3} \mathrm{~h}-\end{array}$ & (?) sandstone \\
\hline
\end{tabular}

${ }^{121}$ In KW /dmxva/* "to touch the genitals of a sleeping woman."

${ }^{122}$ In the word for "armpit." For another example of $|\mathrm{m}|-|\mathrm{w}|$ alternation see HE/OO $\left\{\mathrm{h}^{3} \mathrm{~m}\right.$ \%lhqv- $\} \mathrm{KW}\left\{\mathrm{h}^{3} \mathrm{~W}-\%\right.$ lhqv- $\}$ "quiet, faint (sound)."

${ }^{123}$ Eelgrass has ribbon-like leaves. According to Hill's Native Art's website they were bleached white in the sun and either as is or after dying used to imbricate baskets and hats.

124 OO only in /dHwac ${ }^{3} \mathrm{i} /$ "palette of painter"

125 In OO /dH $\chi \mathrm{a} /$ "to stop spawning (said of herring)." 
North Wakashan Comparative Root List (Updated)

\begin{tabular}{|c|c|c|c|c|c|c|}
\hline Root \# & Old \# & $\mathrm{HE}$ & $\mathrm{OO}$ & $\mathrm{HA}$ & KW & Gloss \\
\hline 2620 & & & & & dt- & to spread, open, unfold \\
\hline 2630 & 300 & & & & dls- & to $\operatorname{lisp}^{126}$ \\
\hline 2640 & 301 & dlk- & dlk- & dlk- & & $(?)^{127}$ \\
\hline 2650 & 302 & dlx- & dlx- & & dlx- & $\begin{array}{l}\text { damp, moist, white eruption on } \\
\text { skin due to dampness }\end{array}$ \\
\hline 2670 & 303 & & & & dk- & $\begin{array}{l}\text { to bury (in tree, cave, ground or } \\
\text { on rock) }\end{array}$ \\
\hline 2680 & $\begin{array}{l}304 \\
305 \\
306\end{array}$ & $\begin{array}{l}\mathrm{dx}- \\
\mathrm{tx}-\end{array}$ & $\mathrm{dx}-$ & $\mathrm{dx}-$ & dx- & $\begin{array}{l}\text { round, swollen, bulging; exposed, } \\
\text { unmistakable, harsh }{ }^{128}\end{array}$ \\
\hline 2690 & $\begin{array}{l}307 \\
308\end{array}$ & dy- & dy- & $\begin{array}{l}\text { dy- } \\
\text { dhyh- }\end{array}$ & $\begin{array}{l}\text { dy- } \\
\text { dhy- } \\
\text { dhyh- }\end{array}$ & to wipe, sweep ${ }^{129}$ \\
\hline 2700 & 309 & & & & dył- & $(?)^{130}$ \\
\hline 2710 & & & & & dyk- & $\begin{array}{l}\text { to dive with a flop (said of fish or } \\
\text { sea mammal) }\end{array}$ \\
\hline 2720 & 310 & dyx- & & dyx- & & $\begin{array}{l}\text { sexually excited (said of male), } \\
\text { having an erection }\end{array}$ \\
\hline
\end{tabular}

${ }^{126}$ Only in $\mathrm{KW} / \mathrm{d}$ лу ${ }^{3}$ ala/ "lisping, to lisp."

${ }^{127} \mathrm{In} \mathrm{HE} \mathrm{/d'лgм/} \mathrm{OO} \mathrm{/dлgм/} \mathrm{HA} \mathrm{/d'лgм/} \mathrm{"soft} \mathrm{roe} \mathrm{(milk)} \mathrm{of} \mathrm{fish."}$

${ }^{128} 1$. Occurs also in words referring to owls and hooting, probably because of the size and position of an owl's eyes. 2. HE/OO $\{\mathrm{dx}-\}$ is diagnosable only before a suffix that changes $|\mathrm{x}|$ into a non-obstruent.

${ }^{129}$ Root occurs probably also in HA /d'im ${ }^{3} a l^{3} a s /$ 'willow tree'. Brooms used to be made of willow in many parts of the world.

${ }^{130}$ In KW \{dylhk-\} "flopping about (fish out of water)." 
North Wakashan Comparative Root List (Updated)

\begin{tabular}{|c|c|c|c|c|c|c|}
\hline Root \# & Old \# & $\mathrm{HE}$ & $\mathrm{OO}$ & HA & KW & Gloss \\
\hline 2730 & 311 & dykv- & dykv- & dykv- & & (?) yellow ${ }^{131}$ \\
\hline 2740 & 312 & dyxv- & dyxv- & dyxv- & dyxv- & $\begin{array}{l}\text { yellow cedar bark, yellow cedar } \\
\text { wood }^{132}\end{array}$ \\
\hline 2750 & 313 & dyqv- & dyqv- & dyqv- & dyqv- & $\begin{array}{l}\text { to pound in piles, pegs, pins, } \\
\text { wedges }\end{array}$ \\
\hline 2760 & 314 & & dyqv- & dyqv- & & $(?)^{133}$ \\
\hline 2770 & 315 & & & & dy $x-$ & to defecate (said of an animal) \\
\hline 2780 & $\begin{array}{r}316 \\
2178 \\
2513 \\
2542 \\
2568 \\
2632\end{array}$ & $\begin{array}{l}\mathrm{h}^{3} \mathrm{~d}- \\
\mathrm{h}^{3} \mathrm{c}- \\
\mathrm{rh}- \\
\mathrm{rh}^{3}-\end{array}$ & $\begin{array}{l}\text { dy }{ }^{3}- \\
\text { dh- } \\
\text { dhc- } \\
h^{3} \mathrm{~d}^{-} \\
\mathrm{h}^{3} \mathrm{c}- \\
\mathrm{h}^{3} \mathrm{hc}- \\
\mathrm{rh}- \\
\mathrm{rh}^{3} \mathrm{q}-\end{array}$ & $\mathrm{h}^{3} \mathrm{~d}-, \mathrm{rh}-$ & $\begin{array}{l}\text { dy3- } \\
\text { dh- } \\
\text { dhc- } \\
\text { hhd- } \\
h^{3} d- \\
h^{3} h d- \\
h^{3} h d h c- \\
h^{3} h c- \\
\text { rh- } \\
\text { rh }\end{array}$ & $\begin{array}{l}\text { dear, valued, esteemed, } \\
\text { respected, deferred to }{ }^{134}\end{array}$ \\
\hline
\end{tabular}

${ }^{131}$ Only in the term for a young duck.

132 Also in HE /d'ixvalas/ OO /diixvalas/ HA /daixvalas/ "golden-crowned sparrow."

${ }^{133}$ Only in OO $/ \mathrm{diq}^{3} \mathrm{vmy}{ }^{3} \mathrm{a} /$ "cheek meat of fish" and HA /d'iqva/ "diseased fish with white spots in flesh."

${ }^{134}$ Entry is a family of roots and possibly polymorphemic but hard to analyze stems referring to one's relatives, often hypocoristically. Many of the examples are vocatives. The root $\{\mathrm{Ih}-\}$ is a special case. It is comparatively productive with most derivates referring non-hypocoristically to grandparents and ancestors. The following unusual plural forms are attested for it, $\mathrm{HE}\left\{\mathrm{Ih}^{3}-\right\} \mathrm{OO}$ $\left\{\mathrm{Ih}^{3} \mathrm{q}-\right\} \mathrm{KW}\left\{\mathrm{rh}^{3} \mathrm{~h}-\right\}$. It probably also occurs in KW/rarala/ and /rarax. $\hbar^{3} \mathrm{id} / *$ "to curry favour." The alternation of $|\mathrm{r}|$ and $\left|\mathrm{h}^{3}\right|$ has a parallel in that between $|\mathrm{r} v|$ and $\left|\mathrm{w}^{3}\right|$, see the alternants of root no. 17720 . 
North Wakashan Comparative Root List (Updated)

\begin{tabular}{|c|c|c|c|c|c|c|}
\hline Root \# & Old \# & $\mathrm{HE}$ & $\mathrm{OO}$ & HA & KW & Gloss \\
\hline & & & & & $\begin{array}{l}\text { rht- } \\
\text { rhc- } \\
\mathrm{q}^{3} \mathrm{hc}-\end{array}$ & \\
\hline 2790 & 457 & $\mathrm{t}^{3} \mathrm{kV}-$ & $\begin{array}{l}\mathrm{dkv-} \\
\mathrm{t}^{3} \mathrm{kv}-\end{array}$ & $\mathrm{t}^{3} \mathrm{kV}-$ & $\mathrm{t}^{3} \mathrm{kV}-$ & $(?)^{135}$ \\
\hline 2800 & 318 & $\begin{array}{l}\text { dxv- } \\
\text { txv- }\end{array}$ & $\begin{array}{l}\text { dxv- } \\
\text { txv- }\end{array}$ & dxv- & dxv- & $\begin{array}{l}\text { to jump, to bob, to move up and } \\
\text { down (like chest when breathing) }\end{array}$ \\
\hline 2810 & $\begin{array}{l}319 \\
321\end{array}$ & dw- & dw- & dw- & $\mathrm{dw}-*$ & $\begin{array}{l}\text { (?) tense, tenuous (hence: } 1 \text {. } \\
\text { dangerous; } 2 \text {. spanning a } \\
\text { distance, bridging, transmitting } \\
\text { (information), to communi- } \\
\text { cate) }{ }^{136}\end{array}$ \\
\hline 2820 & 320 & & & & $\mathrm{dw}-$ & $(?)^{137}$ \\
\hline 2830 & 319 & dwt- & dwt- & dwt- & dwt- & $\begin{array}{l}\text { spanning a distance, bridging, } \\
\text { transmitting (information), to } \\
\text { communicate }\end{array}$ \\
\hline 2840 & 322 & dws- & dws- & dws- & dws- & numb, slightly intoxicated \\
\hline 2850 & 323 & & & dw£- & & $\begin{array}{l}\text { to get out of the way, make sb. } \\
\text { get out of the way }\end{array}$ \\
\hline
\end{tabular}

${ }^{135} \mathrm{In} \mathrm{OO} / \mathrm{tk}^{3} \mathrm{vH} /\left(\right.$ plural $/ \mathrm{ditk}^{3} \mathrm{VH} /$ ) "death canoe, canoe carrying a corpse" and $\mathrm{HE} / \mathrm{t}^{3} \mathrm{gv}^{\prime} \mathrm{H} /$ $\mathrm{OO} / \mathrm{HA} / \mathrm{KW} / \mathrm{t}^{3} \mathrm{gvH} /$ (a kind of canoe).

${ }^{136} \mathrm{In}\{\mathrm{dw}-\%$ nt $\}>\mathrm{HE} / \mathrm{d}$ 'uHt/ OO/HA /duHt/ "name" and derivates. The sense of "dangerous" occurs in $\mathrm{HE} / \mathrm{d}$ 'udu/ OO /dudu/ "It's hot!" and HE /dud'u/ OO / $\mathrm{du}^{3} \mathrm{du} /$ (command to stay away from sth. hot). A morphophonemically problematic derivate of $\{\mathrm{dw}-\}, \mathrm{sc}\left\{\mathrm{dw}^{3}\right\}$, is used as a proclitic with a connective function and glossable as "and," "with" or "together with." KW uses $\left\{\lambda \mathrm{W}^{3}-\right\}$ deriving from $\left\{\lambda \mathrm{w}_{-}\right\}$(root no. 8650) for this same connective function.

${ }^{137} \mathrm{In} \mathrm{KW}\{\mathrm{dw}-\% \mathrm{yxv}\}>$ /dvixv/ "yellow cedar roots, rope made of branches." Boas has several more derivates but they could not be reelicited. 
North Wakashan Comparative Root List (Updated)

\begin{tabular}{|c|c|c|c|c|c|c|}
\hline Root \# & Old \# & $\mathrm{HE}$ & $\mathrm{OO}$ & $\mathrm{HA}$ & KW & Gloss \\
\hline 2860 & $\begin{array}{l}324 \\
461\end{array}$ & $\begin{array}{l}\text { dwt- } \\
\text { t }^{3} w 1-\end{array}$ & $\begin{array}{l}\text { dwt- } \\
\text { t }^{3} w 1-\end{array}$ & $\left.\mathrm{t}^{3} \mathrm{w}\right\}$ & dwk- & $\begin{array}{l}\text { to tickle, tingle; tight, cramped, } \\
\text { pins and needles, shocked (by } \\
\text { electricity); numb, dizzy, } \\
\text { delirious }^{138}\end{array}$ \\
\hline 2870 & 325 & & dwlw- & & dwlw- & to win (in a game or contest) $)^{139}$ \\
\hline 2880 & $\begin{array}{l}326 \\
327\end{array}$ & dwkv- & dwkv- & & dwkv- & $\begin{array}{l}\text { be dragged behind (as in } \\
\text { trolling), be dragged along; (KW } \\
\text { also:) to trail (like plant vine), } \\
\text { hang loose like end of rope or } \\
\text { fringe, straggle, dangle, stretch } \\
\text { out }\end{array}$ \\
\hline 2900 & 328 & dwxv- & dwxv- & dwxv- & dwxv-* & to sting (like nettles) \\
\hline 2910 & 329 & dwqv- & dwqv- & dwqv- & dwqv- & $\begin{array}{l}\text { to see, look at, look for sth., pay } \\
\text { attention, watch, examine, } \\
\text { consider }\end{array}$ \\
\hline 2920 & $\begin{array}{l}330 \\
394 \\
395 \\
396 \\
397 \\
398 \\
399 \\
468 \\
469 \\
2623 \\
2624\end{array}$ & $\begin{array}{l}\text { tq- } \\
t^{3} q_{-}^{-} \\
t \chi_{-}^{-} \\
h^{3} \chi^{-}\end{array}$ & $\begin{array}{l}\mathrm{tq}- \\
\mathrm{t}^{3} \mathrm{q}^{-} \\
\mathrm{t} \chi^{-}\end{array}$ & $\begin{array}{l}\text { dq- } \\
\text { tq- } \\
t^{3} q_{-}^{-} \\
t^{3} \chi^{-}\end{array}$ & $\begin{array}{l}\text { tq- } \\
\text { t } \chi- \\
t^{3} q_{-}^{-} \\
h^{3} \chi-\end{array}$ & $\begin{array}{l}\text { 1a. ( }\left\{\mathrm{dq}-, \text { tq-, } \mathrm{t}^{3} \mathrm{q}-\right\} \text { :) to take in } \\
\text { hand, to grasp (catch, hold, } \\
\text { squeeze, pin down) physically or } \\
\text { mentally, to control, to constrain, } \\
\text { to have power, to verify; } 1 \mathrm{~b} \text {. } \\
\text { (possibly in stem HE }\{\text { tq- }+1-\} \\
\text { HA }\{\text { dq-+1- }\} \text { meaning 'to itch':) } \\
\text { affected by, afflicted with; } \\
\text { 2. (HE/OO }\{\text { t } \chi-\} \text { HE/KW }\left\{\mathrm{h}^{3} \chi-\right. \\
\text { \}:) located somewhere, } \\
\text { moving/be moved somewhere, }\end{array}$ \\
\hline
\end{tabular}

$138\left\{t^{3} w 1-\right\}$ only in the stem $\left\{t^{3} w{ }^{3} \% y x-\right\}$ "intoxicated etc."

${ }^{139}$ From Chinook Jargon "tolu." 
North Wakashan Comparative Root List (Updated)

\begin{tabular}{|c|c|c|c|c|c|c|}
\hline Root \# & Old \# & $\mathrm{HE}$ & $\mathrm{OO}$ & HA & KW & Gloss \\
\hline & & & & & & $\begin{array}{l}\text { taking/be taken from. However, } \\
\mathrm{HE} / \mathrm{H}^{3} \chi \mathrm{a}^{3} \mathrm{i} / \text { ( name of Hakai } \\
\text { Passage) is the only non-KW } \\
\text { example of }\left\{\mathrm{h}^{3} \chi-\right\} \text {. It may be of } \\
\text { non-HE origin because derivates } \\
\text { have low tone where a high one } \\
\text { should occur if the name's } \\
\text { structure is }\left\{\mathrm{h}^{3} \chi-\alpha \mathrm{y}^{3}\right\} \text {; } \\
\text { 3. HE/OO }\{t \chi-\} \mathrm{HA}\left\{\mathrm{t}^{3} \chi-\right\} \mathrm{KW} \\
\left\{\mathrm{h}^{3} \chi-\right\} \text { are also used as substitutes } \\
\text { for any lexical word stem a } \\
\text { speaker cannot think of fast } \\
\text { enough: "thingamajig, } \\
\text { whatchamacallit"; } \\
4 . \mathrm{KW}\left\{\left[\mathrm{h}^{3} \mathrm{y}\right] \mathrm{h}^{3} \chi-\right\} \text { is equivalent } \\
\text { to HE/OO/HA }\left\{\mathrm{p}^{3} \mathrm{~h}-\right\} \text { in the } \\
\text { meaning of "to work"; } \\
5 .\left\{\mathrm{t}^{3} \mathrm{q}-\right\} \text { sometimes occurs as a } \\
\text { dummy attachment for category } \\
\text { A suffixes in the same manner as } \\
\left\{\mathrm{w}^{3} \mathrm{~W}-, \text { etc. }\right\} \text { (root no. } 17720 \text { ). }\end{array}$ \\
\hline 2930 & 331 & & & & $\mathrm{~d} \chi-$ & $\begin{array}{l}\text { to spread (legs), open (tongs, } \\
\text { clamshells), split (wood) }\end{array}$ \\
\hline 2940 & $\begin{array}{l}332 \\
333\end{array}$ & dh- & dh- & dh- & dhy ${ }^{3}-$ & $\begin{array}{l}\text { to take in hand, to grasp etc. } \\
\text { (same meanings as }\{\mathrm{dq}-\text {, tq-, } \\
\left.\mathrm{t}^{3} \mathrm{q}-\right\}^{140}\end{array}$ \\
\hline
\end{tabular}

${ }^{140}$ Occurs also in HE/OO/KW $\left\{\mathrm{dh}[\mathrm{dh}]-\mathrm{c}^{3} \mathrm{w}-\right\}$ HA $\left\{\mathrm{dh}[\mathrm{d}]-\mathrm{c}^{3} \mathrm{w}-\right\}$ "coat, coated." There are relatively few $\mathrm{HE} / \mathrm{OO}$ examples of this root compared to $\mathrm{HA} / \mathrm{KW}$ and they refer mostly to magical control; HE/OO usually use $\{\mathrm{sw}-\}$ for grasping, holding etc. In $\mathrm{KW}$ the root often 
North Wakashan Comparative Root List (Updated)

\begin{tabular}{|c|c|c|c|c|c|c|}
\hline Root \# & Old \# & $\mathrm{HE}$ & $\mathrm{OO}$ & $\mathrm{HA}$ & KW & Gloss \\
\hline 2950 & 334 & dhp- & dhp- & dhp- & dhp- & to tow \\
\hline 2960 & 335 & dhp- & dhp- & & & (?) at night, in darkness \\
\hline 2970 & $\begin{array}{l}336 \\
299\end{array}$ & dhs- & dhs- & dhs- & dhs- & $\begin{array}{l}\text { cover, covered, submerged, under } \\
\text { the surface of the water }\end{array}$ \\
\hline 2980 & & & dh1- & & dht- & to unfold ${ }^{141}$ \\
\hline 2981 & & & & $\begin{array}{l}\text { dhyd- } \\
\text { dlhyd- } \\
\text { dhyd- }\end{array}$ & & very, to a high degree ${ }^{142}$ \\
\hline 2982 & & & & & dhk-* & (?) \\
\hline 2990 & $\begin{array}{l}339 \\
340\end{array}$ & dhxv- & dhxv- & dhxv- & & $\begin{array}{l}\text { to skewer, pin together, interlock, } \\
\text { engage }\end{array}$ \\
\hline 3000 & 341 & dhq- & & dhq- & & $\begin{array}{l}\text { to get cedar bark (for roofing and } \\
\text { boxes) }\end{array}$ \\
\hline 3010 & 342 & dhq- & thq- & thq- & thq- & to catch ooligans \\
\hline 3020 & 338 & $\mathrm{dh}^{3} \mathrm{t}-$ & $\mathrm{dh}^{3} \mathrm{k}-$ & $\mathrm{dh}^{3} \mathrm{k}-$ & $\mathrm{dh}^{3} \mathrm{t}-$ & to laugh \\
\hline 3030 & 343 & tp- & tp- & tp- & tp- & $\begin{array}{l}\text { resilient object is subjected to or } \\
\text { gives way to pressure (hence: to }\end{array}$ \\
\hline
\end{tabular}

appears fully reduplicated as $\{\mathrm{dhdh}-\}$ and it can be difficult to tell which part is the reduplicand and which the reduplicate. Whenever in doubt, the first $|\mathrm{dh}|$ will be considered the reduplicate. A clear case of the first $|\mathrm{dh}|$ being the reduplicate is conative reduplication as in $\mathrm{KW}\{[\mathrm{dh}] \mathrm{dh}-+-\mathrm{h}\}$ $>/ \mathrm{dada}^{3} \mathrm{a} /$ "to try to grab." KW $\{[\mathrm{dy}] \mathrm{dh}-\}$ occurs in a few terms referring to property and credit. ${ }^{141}$ Only OO example is possibly the place name /Dalik/.

${ }^{142}$ These free stems are the counterparts to $\left\{\mathrm{w}^{3} \mathrm{w}-\mathrm{hlh}\right\}>\mathrm{HE} / \mathrm{w}^{3} \mathrm{u}^{\prime}$ ala/ OO $/ \mathrm{w}^{3} \mathrm{uala} / \mathrm{KW}\left\{\mathrm{h}^{3} \mathrm{w}-\right.$ hlh $\}>/ h^{3}$ ola/ "real(ly), true, truly, actual(ly), very"; they may be a foreign borrowing. \{dhyd-\} and $\{$ dlhyd- $\}$ altrnate freely, with $|\mathrm{d}| \mid$ being substituted by $|\lambda|$ in rapid speech. 
North Wakashan Comparative Root List (Updated)

\begin{tabular}{|c|c|c|c|c|c|c|}
\hline Root \# & Old \# & $\mathrm{HE}$ & $\mathrm{OO}$ & HA & KW & Gloss \\
\hline & & & & & & $\begin{array}{l}\text { squeeze, squash, shatter, crush; to } \\
\text { burst, explode) }\end{array}$ \\
\hline 3040 & 344 & & & & tm- & to vibrate, quiver ${ }^{143}$ \\
\hline 3050 & 345 & & & & $\begin{array}{l}\text { tms- } \\
\text { tmxse- }\end{array}$ & to vibrate, quiver, shake down \\
\hline 3060 & 346 & & tmt- & & tmi- & to shake, throb ${ }^{144}$ \\
\hline 3070 & 347 & $\operatorname{tmx}-$ & $\operatorname{tmx}-$ & & $\operatorname{tmx}-$ & to bud, sprout \\
\hline 3080 & $\begin{array}{l}348 \\
349\end{array}$ & tmkv- & tmkv- & tmkv- & tmkv- & $\begin{array}{l}\text { (HE/OO/HA possibly:) crunchy } \\
\text { (like cured salmon eggs); (KW) } \\
\text { to crunch, chop off, bite off }\end{array}$ \\
\hline 3090 & 350 & $\operatorname{tmxv-}$ & $\operatorname{tmxv-}$ & & $\mathrm{t}^{3} \mathrm{mxv}-$ & gooseberries \\
\hline 3100 & $\begin{array}{l}351 \\
403\end{array}$ & $\begin{array}{l}\text { tmqv- } \\
\text { thmhqv- }\end{array}$ & $\begin{array}{l}\text { tmqv- } \\
\text { thm }{ }^{3 h q v-}\end{array}$ & thm³ hqv- & tmqv-* & $\begin{array}{l}\text { to splash, to dip into water } \\
\text { violently to make it spatter (as } \\
\text { when kicking with the feet when } \\
\text { swimming or plunging into the } \\
\text { water to chase the fish back into } \\
\text { the net) }\end{array}$ \\
\hline
\end{tabular}

${ }^{143} \mathrm{In} \mathrm{KW}\{$ tm-ynhs $\}>/$ tminas/ "squirrel" and the name KW /Tmina ${ }^{3}$ ustolagil3akv/* which is said to mean "squirrel." Quivering is a characteristic of squirrels. They do it e.g. when an intruder moves into their territory. Body jerks and tail flicks are additional responses to an intruder. Cf. www.env.gov.yk.ca/wildlifebiodiversity/mammals/redsquirrel.php.

${ }^{144} \mathrm{OO}$ only in an ethnic name borrowed from KW.

145 The augmented root form $\left\{\right.$ thm $^{3}$ hqv- $\}$ means "to drip" in HE/OO but "to drift (like a seine net)" in HA. 
North Wakashan Comparative Root List (Updated)

\begin{tabular}{|c|c|c|c|c|c|c|}
\hline Root \# & Old \# & $\mathrm{HE}$ & $\mathrm{OO}$ & HA & KW & Gloss \\
\hline 3110 & 406 & & & $\begin{array}{l}\operatorname{tm} \chi v- \\
\operatorname{th} \chi v-\end{array}$ & th $\chi \mathrm{v}-$ & $\begin{array}{l}\text { to force one's way through, to } \\
\text { slog, proceed against } \\
\text { resistence }^{146}\end{array}$ \\
\hline 3120 & 352 & tmq- & tmq- & & & to snap, crack (wood or ice) \\
\hline 3130 & & & & $\begin{array}{l}\operatorname{tmq}-/ \\
\operatorname{tm} \chi-\end{array}$ & & (?) to hop ${ }^{147}$ \\
\hline 3140 & 353 & & tn- & & $\begin{array}{l}t^{3} n- \\
t^{3} h n h- \\
t^{3} h n y^{3}-\end{array}$ & $\begin{array}{l}\text { close to each other (in space or } \\
\text { the lineage) }{ }^{148}\end{array}$ \\
\hline 3150 & 354 & $\begin{array}{l}\text { tn- } \\
\text { tns- }\end{array}$ & $\begin{array}{l}\text { tn- } \\
\text { tns- }\end{array}$ & tn- & tn- & (?) to agitate ${ }^{149}$ \\
\hline 3160 & $\begin{array}{l}355 \\
356\end{array}$ & tnk- & tnk- & & tnk- & $\begin{array}{l}\text { mound, lump, heap, swelling; to } \\
\text { rise up, to boil }\end{array}$ \\
\hline 3170 & 357 & tnx- & tnx- & $\operatorname{tnx}-$ & & to vibrate, quake, rumble \\
\hline 3180 & 358 & & & $\operatorname{tn} \chi-$ & & $\begin{array}{l}\text { (?) close to each other, tightly } \\
\text { together }\end{array}$ \\
\hline 3190 & 360 & ts- & ts- & ts- & ts- & $\begin{array}{l}\text { to push, press against; (KW also:) } \\
\text { persistent action }\end{array}$ \\
\hline
\end{tabular}

146 All KW examples are about wading, with one example mentioning "in water up to knees."

${ }^{147}$ In HA /T'м $\chi . \mathrm{w}^{3}$ isла/ (chief's name referring to Eagle hopping up the shore on one leg) ${ }^{148} \mathrm{OO}\{\mathrm{tn}-\}$ is attested only in the expanded form $\left\{\operatorname{thhn}^{3} \mathrm{y}-\right\}$, cf. OO $\left\{\operatorname{thhn}^{3} \mathrm{y}-\mathrm{rW}\right\}>/ \operatorname{taan}^{3} \mathrm{iru} /$ "close to each other (as two people passing)." $149\{\mathrm{tn}-\}$ occurs only augmented to $\{$ thnh- $\}$ as in $\{\mathrm{t}[\mathrm{h}] \mathrm{n}[\mathrm{h}]-=\mathrm{ys}\}>\mathrm{HE} / \mathrm{t}^{\prime}$ an'is/ OO/KW $/$ tanis/ HA /t'anis/ (name of a dance and dancer). $\{$ tns- $\}$ is attested only in $\mathrm{HE} / \mathrm{t}^{\prime} \mathrm{Hzac}^{3} \mathrm{i} / \mathrm{OO} / \mathrm{tHzac}^{3} \mathrm{i} /$ "ceremonial dwelling of the HE/t'an'is/ OO /tanis/." 
North Wakashan Comparative Root List (Updated)

\begin{tabular}{|c|c|c|c|c|c|c|}
\hline Root \# & Old \# & $\mathrm{HE}$ & $\mathrm{OO}$ & $\mathrm{HA}$ & KW & Gloss \\
\hline 3200 & $\begin{array}{r}359 \\
47\end{array}$ & $\begin{array}{l}\mathrm{ttc}^{3}- \\
\mathrm{plc}^{3}-\end{array}$ & $\mathrm{ttc}^{3}-$ & $\mathrm{ttc}^{3}-$ & $\mathrm{ttc}^{3}-$ & (?) to warm oneself $\mathrm{f}^{150}$ \\
\hline 3210 & $\begin{array}{l}361 \\
372\end{array}$ & $\begin{array}{l}\operatorname{ty}^{3} s x- \\
t^{3} s x^{-}-\end{array}$ & $\begin{array}{l}\text { ty }{ }^{3} x s- \\
\text { ty }^{3} x s^{-}-\end{array}$ & tys- & $\begin{array}{l}\text { tłx } \bullet- \\
\text { tyłx }-\end{array}$ & to sprinkle, splash \\
\hline 3220 & 362 & & & & tlp- & $(?)^{151}$ \\
\hline 3230 & 363 & & & & tlkv- & Oregon grape \\
\hline 3240 & 364 & & & & tlkv-* & to run \\
\hline 3250 & 365 & & & & tlkv-* & to splash \\
\hline 3260 & 366 & tlqv- & tlqv- & tlqv- & tlqv- & soft, padding \\
\hline 3270 & 367 & & tlqv- & & thqv-* & $\begin{array}{l}\text { to eat beforehand (before a trip or } \\
\text { main course) }\end{array}$ \\
\hline 3280 & $\begin{array}{l}368 \\
369 \\
374 \\
433 \\
434\end{array}$ & $\begin{array}{l}\text { tk- } \\
t^{3} \mathrm{k}-\end{array}$ & $\begin{array}{l}\text { tk- } \\
t^{3} k- \\
t^{3} x-\end{array}$ & & $\begin{array}{l}\text { tk- } \\
\text { tyk- }\end{array}$ & $\begin{array}{l}\text { pressurized, tense, cushioned, } \\
\text { resilient, rebounding, spring- } \\
\text { loaded }\end{array}$ \\
\hline 3281 & 370 & & & & tk/ & $(?)^{152}$ \\
\hline 3290 & 373 & tył- & tył- & tył- & tył- & $\begin{array}{l}\text { bait, to bait, catch prey with a } \\
\text { baited hook }\end{array}$ \\
\hline
\end{tabular}

${ }^{150} \mathrm{HE} / \mathrm{OO} / \mathrm{HA}|£|$ and $|\mathrm{f}|$ are often elided before $\left|\mathrm{c}^{3}\right|$, as happens in derivates of this possibly polymorphemic stem and e.g. in HE/OO/HA $\left\{\mathrm{mh}^{3} \mathrm{l}-\mathrm{c}^{3} \mathrm{q}-\mathrm{lh}\right\}>\mathrm{HE} / \mathrm{mac}^{3} \mathrm{q} л^{\prime} \mathrm{a} / \mathrm{OO} / \mathrm{ma}^{3} \mathrm{c}^{3} \mathrm{q}$ ла/ НА

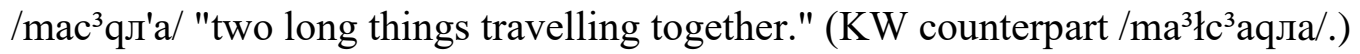
${ }^{151} \mathrm{In} \mathrm{KW} /$ tлра/ "heart beats in excitement, go meet a girl in the woods, one who follows a woman"

${ }^{152} \mathrm{In} \mathrm{KW}\left\{\mathrm{tx}-. \mathrm{m}^{3} \mathrm{hs}\right\}>/ \mathrm{tx} . \mathrm{m}^{3} \mathrm{as} /$ and $\left\{\mathrm{tx}-\mathrm{m}^{3} \mathrm{hs}\right\}>/ \mathrm{txM}^{3}$ as $/$ "gall, bile" and KW $\left\{\mathrm{tx}-\mathrm{p}^{3}-\mathrm{h}\right\}>$ /txpª/* bitter taste." 
North Wakashan Comparative Root List (Updated)

\begin{tabular}{|c|c|c|c|c|c|c|}
\hline Root \# & Old \# & $\mathrm{HE}$ & $\mathrm{OO}$ & HA & KW & Gloss \\
\hline 3310 & 375 & tyx- & tyx- & tyx- & & $(?)^{153}$ \\
\hline 3320 & $\begin{array}{l}376 \\
377 \\
378 \\
379\end{array}$ & tykv- & tykv- & tykv- & tykv- & $\begin{array}{l}\text { precarious, unstable, falling, } \\
\text { dangling, unsteady, unsupported; } \\
\text { (KW also:) not serious, in jest }\end{array}$ \\
\hline 3330 & $\begin{array}{l}380 \\
381\end{array}$ & tyq- & tyq- & tyq- & tyq- & $\begin{array}{l}\text { (HE/OO/HA and some } \mathrm{KW} \\
\text { examples:) to crack, shatter, } \\
\text { explode; (KW) released or } \\
\text { escaping from restraint, dropping } \\
\text { off, falling }\end{array}$ \\
\hline 3350 & 382 & $\operatorname{ty} \chi-$ & $\operatorname{ty} x-$ & $\operatorname{ty} \chi-$ & $\operatorname{ty} \chi-$ & $\begin{array}{l}\text { yellowish-green (like top of } \\
\text { hemlock needles, animal urine) }\end{array}$ \\
\hline 3360 & $\begin{array}{l}383 \\
384 \\
385\end{array}$ & & & & tkv- & $\begin{array}{l}\text { tight, fitting tightly (like deerskin } \\
\text { leather), tense, elastic, spanning a } \\
\text { distance, astringent (like } \\
\text { thimbleberry leaves and roots), to } \\
\text { contract, to throb (like boil), to } \\
\text { beat (heart) }\end{array}$ \\
\hline 3400 & 386 & tw- & tw- & tw- & $\begin{array}{l}\text { tw- } \\
\text { thw }{ }^{3-}\end{array}$ & $\begin{array}{l}\text { to walk, travel on land; (HA/KW } \\
\text { also:) to press forward, close in } \\
\text { on a target (as e.g. in an attack or } \\
\text { mountain goat hunting) }\end{array}$ \\
\hline 3410 & $\begin{array}{r}387 \\
459 \\
696\end{array}$ & $t^{3} w p-$ & $t^{3} w p-$ & $\begin{array}{l}\text { twp- } \\
t^{3} w p-\end{array}$ & $\begin{array}{l}\text { twp- } \\
t^{3} w p-\end{array}$ & $\begin{array}{l}\text { speck, dot, to be speckled, } \\
\left.\text { spotted; }\left\{t^{3} w p-\right\} \text { also: }\right) \text { fleabite }\end{array}$ \\
\hline 3420 & 388 & $(\chi \mathrm{vt}-)$ & $(\chi \mathrm{vt}-)$ & tws- & $\mathrm{t}^{3} \mathrm{WS}-$ & to cut with a knife ${ }^{154}$ \\
\hline
\end{tabular}

${ }^{153}$ Only in the stem $\{$ tyx-=yq- $\}>$ tynyq- $\}$ "to become covered with sweat or dew." ${ }^{154}$ A root $\left\{\mathrm{t}^{3} \mathrm{ws}-\right\}$ occurs in HE/OO/HA too but refers to cedar withes. 
North Wakashan Comparative Root List (Updated)

\begin{tabular}{|c|c|c|c|c|c|c|}
\hline Root \# & Old \# & $\mathrm{HE}$ & $\mathrm{OO}$ & HA & KW & Gloss \\
\hline 3430 & & & & & tw1-* & $(?)^{155}$ \\
\hline 3440 & 389 & & & twqv- & & Indian rhubarb ${ }^{156}$ \\
\hline 3441 & 390 & & & tw $\chi v-$ & tw $\chi \mathrm{v}-*$ & $\begin{array}{l}\text { to bunch up, concentrate, cluster } \\
\text { together }{ }^{157}\end{array}$ \\
\hline 3450 & $\begin{array}{l}391 \\
392 \\
393\end{array}$ & tqv- & tqv- & tqv- & $\begin{array}{l}\text { tqv- } \\
\text { tq-* }\end{array}$ & $\begin{array}{l}\text { soiled, dirty, dark; blackish ink } \\
\text { cloud ejected by octopus }\end{array}$ \\
\hline 3470 & 402 & th- & th- & th- & th- & to wade \\
\hline 3500 & 405 & thqv- & thqv- & thqv- & thqv-* & to cover (especially with a sheet) \\
\hline 3520 & $\begin{array}{l}407 \\
408\end{array}$ & $t^{3} p-$ & $t^{3} p-$ & $t^{3} p-$ & $t^{3} p-$ & $\begin{array}{l}\text { (KW) to rise and cover (like tide } \\
\text { over a rock), to envelop (like } \\
\text { water a swimmer); (HE/OO/HA) } \\
\text { to catch, hook, latch on, clasp (as } \\
\text { when fishing by trolling a baited } \\
\text { hook through the water and (in } \\
\mathrm{HE} / \mathrm{OO} \text { ) when gripping a pole or }\end{array}$ \\
\hline
\end{tabular}

${ }^{155}$ In KW $\{$ twlhs- $\}$ * probably meaning "to chop up or break up into many pieces" and occurring in three non-reelicitable derivates.

156 Perhaps this root is an alternant of $\left\{t_{w} \chi v^{-}\right\}$(no. 3441) because Indian rhubarb's many small flowers form a cluster atop a stalk that emerges from the ground in spring. The flower stalks are followed by leaves up to 24 inches across. The stalks grow in clusters so that "the foliage forms a vase-like clump, 4 feet tall and 3 feet wide," see http://www.finegardening.com/plantguide/darmera-peltata-indian-rhubarb.aspx. ${ }^{157}$ Only in $\{\mathrm{tw} \chi \mathrm{v}-\mathrm{h}\}>\mathrm{HA} / \mathrm{t}$ 'u $\chi \mathrm{va} /$ "to bunch up (said of nets when dragged by the tide)" KW /tu $\chi \mathrm{va} / *$ "to spin wool on thigh." 
North Wakashan Comparative Root List (Updated)

\begin{tabular}{|c|c|c|c|c|c|c|}
\hline Root \# & Old \# & $\mathrm{HE}$ & $\mathrm{OO}$ & HA & KW & Gloss \\
\hline & & & & & & $\begin{array}{l}\text { tree with the hands for } \\
\text { climbing } 158\end{array}$ \\
\hline 3540 & $\begin{array}{l}409 \\
410 \\
418\end{array}$ & $\begin{array}{l}\mathrm{t}^{3} \mathrm{~m}- \\
\mathrm{t}^{3} \mathrm{hm}^{3}-\end{array}$ & $t^{3} \mathrm{~m}-$ & $t^{3} \mathrm{~m}-$ & $\mathrm{t}^{3} \mathrm{~m}-$ & $\begin{array}{l}\text { to pin or sew together, to lace, to } \\
\text { lash; to lock, to use a bolt, to } \\
\text { insert rod in shaft, to drill in } \\
\text { wood or metal; to plunge } \\
\text { headlong into the water (like } \\
\text { kingfisher for prey) }\end{array}$ \\
\hline 3550 & $\begin{array}{l}411 \\
412 \\
413 \\
414 \\
415\end{array}$ & $\begin{array}{l}t^{3} m s- \\
t^{3} m^{3} s-\end{array}$ & $\begin{array}{l}t^{3} m s- \\
t^{3} m^{3} s-\end{array}$ & $\begin{array}{l}t^{3} m s- \\
t^{3} m^{3} s-\end{array}$ & $\begin{array}{l}t^{3} m s- \\
t^{3} m^{3} s-\end{array}$ & $\begin{array}{l}\text { tense, tight, taut; to tighten (one's } \\
\text { skin, drum skin, string), to pull at } \\
\text { a tight string; snare drum; } \\
\text { bunchberries } 160\end{array}$ \\
\hline 3560 & 416 & & $\mathrm{t}^{3} \mathrm{~m} ł-$ & & & tight, tense ${ }^{161}$ \\
\hline 3570 & & $\begin{array}{l}\mathrm{t}^{3} \mathrm{mkV}- \\
\mathrm{t}^{3} \mathrm{hm}^{3} \mathrm{hkv}-\end{array}$ & $\mathrm{t}^{3} \mathrm{mkV}-$ & $\mathrm{t}^{3} \mathrm{mhkV}-$ & & $(?)^{162}$ \\
\hline 3580 & 417 & $\mathrm{t}^{3} \mathrm{mkV}-$ & $\mathrm{t}^{3} \mathrm{mkV}-$ & $\mathrm{t}^{3} \mathrm{mkV}-$ & $\mathrm{t}^{3} \mathrm{mkv-}$ & to shrink, curl, shrivel, cramp \\
\hline 3590 & 419 & & $\mathrm{t}^{3} \mathrm{mq}-$ & $t^{3} m q-$ & $\mathrm{t}^{3} \mathrm{mq}-$ & $\begin{array}{l}\text { to pin or lace together, to close } \\
\text { opening }\end{array}$ \\
\hline 3600 & 420 & $\begin{array}{l}t^{3} n c- \\
t^{3} n s-\end{array}$ & $\begin{array}{l}t^{3} n c- \\
t^{3} n s-\end{array}$ & $\begin{array}{l}t^{3} n c- \\
t^{3} n s-\end{array}$ & & cold, cool, to chill \\
\hline
\end{tabular}

158 Root's core meaning could be "to overwhelm, to capture."

159 The form $\left\{\mathrm{t}^{3} \mathrm{hm}^{3}-\right\}$ occurs only in the Klemtu HE word for a kingfisher.

160 The connection between bunchberries and tenseness is in the backward flipping petals of their flowers, see the comment on the record for $\mathrm{KW} / \mathrm{t}^{3} \mathrm{Msa} / \mathrm{HE} / \mathrm{t}^{3} \mathrm{Ms}$ a $/ \mathrm{OO} / \mathrm{t}^{3} \mathrm{M}^{3} \mathrm{sa} / \mathrm{HA} / \mathrm{t}^{3} \mathrm{Ms} \mathrm{a} /$ "pick bunchberries (etc.)"

161 In OO / $\mathrm{t}^{3}$ мłла/ "to tighten one's muscles (as when having to stop suddenly)."

162 Perhaps in the word for kingfisher (bird). 
North Wakashan Comparative Root List (Updated)

\begin{tabular}{|c|c|c|c|c|c|c|}
\hline Root \# & Old \# & $\mathrm{HE}$ & $\mathrm{OO}$ & HA & KW & Gloss \\
\hline 3610 & 421 & $t^{3} n s-$ & & & $t^{3} n s-$ & $\begin{array}{l}\text { sheltered (from the elements, } \\
\text { from societal pressure); (KW } \\
\text { also:) within the clan }{ }^{163}\end{array}$ \\
\hline 3620 & 422 & $t^{3} n x-$ & $t^{3} n x-$ & $\left(£^{3} n x-\right)$ & $t^{3} n x-$ & $\begin{array}{l}\text { at an angle, branching off a shaft; } \\
\text { hard, dense (like a knot of wood) }\end{array}$ \\
\hline 3630 & 424 & & & & $t^{3} n q v-*$ & $(?)^{164}$ \\
\hline 3650 & 423 & $t^{3} n \chi-$ & & & $t^{3} n \chi-$ & $\begin{array}{l}\text { tense, rigid, stiff (like muscles); } \\
\text { elevated, erect, raised (like ridge } \\
\text { between grooves) }\end{array}$ \\
\hline 3660 & 425 & $t^{3} s-$ & $t^{3} s-$ & $t^{3} s-$ & $\begin{array}{l}\mathrm{t}^{3} \mathrm{~s}- \\
\mathrm{t}^{3} \mathrm{hs}-\end{array}$ & $\begin{array}{l}\text { to impact with heavy force } \\
\text { (hence: to crack (shells), bang } \\
\text { (head), pound with a rock, to } \\
\text { crash) }\end{array}$ \\
\hline 3670 & 472 & $\mathrm{t}^{3} \mathrm{~h}^{3} \mathrm{~s}-$ & $\mathrm{t}^{3} \mathrm{~h}^{3} \mathrm{~s}-$ & $\begin{array}{l}t^{3} s- \\
t^{3} h s-\end{array}$ & & to shiver, quiver, vibrate, shake \\
\hline 3680 & $\begin{array}{l}426 \\
427\end{array}$ & $t^{3} 1-$ & $t^{3} 1-$ & $t^{3} 1-$ & & $\begin{array}{l}\text { to uncover, open up, lay bare, } \\
\text { unveil; to show itself; a sheet } \\
\text { splits and the parts pull apart } \\
\text { (like stage curtain, fish tail) }\end{array}$ \\
\hline 3690 & 428 & $\begin{array}{l}\mathrm{t}^{3} \mathrm{lc}- \\
\mathrm{t}^{3} 1 \mathrm{~s}-\end{array}$ & $\begin{array}{l}\mathrm{t}^{3} \mathrm{lc}- \\
\mathrm{t}^{3} 1 \mathrm{~s}-\end{array}$ & $\begin{array}{l}\mathrm{t}^{3} \mathrm{lc}- \\
\mathrm{t}^{3} 1 \mathrm{~s}-\end{array}$ & $\mathrm{t}^{3} 1 \mathrm{~s}-$ & $(?)^{166}$ \\
\hline
\end{tabular}

${ }^{163} \mathrm{HE}$ only in $\left\{\mathrm{t}^{3} \mathrm{~ns}-\mathrm{lh}\right\}>/ \mathrm{t}^{3 \prime}{ }^{\prime}$ НАл'a/ "shelter against rain" and one derivate. Their matching OO structures however refer to a cool shaded place so contain the alternant $\left\{\mathrm{t}^{3} \mathrm{~ns}-\right\}$ of root no. 3600 . $164 \mathrm{In} \mathrm{KW} / \mathrm{t}^{3} \mathrm{Hqva} / *$ (name of a dance).

${ }^{165} \mathrm{HE}$ only in $/ \mathrm{t}^{3 \prime} \mathrm{н} \chi \mathrm{J}^{\prime} \mathrm{a} /$, a word whose meaning wasn't remembered except that it had to do with a cliff.

${ }^{166}$ In words referring to berries identified by informants as cranberries, highbush cranberries and chokecherries. According to https://en.wikipedia.org/wiki/Viburnum_trilobum highbush cranberries are not really cranberries but their red fruits look superficially like cranberries. Boas 
North Wakashan Comparative Root List (Updated)

\begin{tabular}{|c|c|c|c|c|c|c|}
\hline Root \# & Old \# & $\mathrm{HE}$ & $\mathrm{OO}$ & HA & KW & Gloss \\
\hline 3700 & 429 & $\left(t^{3} h t-\right)$ & $\left(t^{3} h t_{-}\right)$ & $\left(t^{3} h 1-\right)$ & $t^{3} 1 s-$ & to slice (fish or meat) \\
\hline 3710 & 430 & $t^{3} 1 x v-$ & $t^{3} 1 x v-$ & (ys-) & $t^{3} 1 \times v-$ & $\begin{array}{l}\text { to pound, to club, to soften by } \\
\text { pounding }\end{array}$ \\
\hline 3720 & 431 & & & & $t^{3} l q^{3}-$ & roe, spawn in fish ${ }^{167}$ \\
\hline 3730 & 432 & & & $t^{3} k-$ & $t^{3} k-$ & earth, dirt, soil, dirty \\
\hline 3740 & $\begin{array}{l}435 \\
437\end{array}$ & $t^{3} x-$ & $t^{3} x-$ & $t^{3} x-$ & $t^{3} x-$ & path, trail, door, way, custom ${ }^{168}$ \\
\hline 3750 & & & & & $t^{3} x-$ & $(?)^{169}$ \\
\hline 3760 & 436 & $t^{3} x-$ & $t^{3} x-$ & $t^{3} x-$ & & $(?)^{170}$ \\
\hline 3770 & 438 & $t^{3} y-$ & $t^{3} y-$ & $t^{3} y-$ & $t^{3} y-$ & $\begin{array}{l}\text { to weigh down, ballast, stabilize, } \\
\text { anchor; to handle stones and } \\
\text { heavy things }\end{array}$ \\
\hline 3780 & 439 & $t^{3} h y^{3}-$ & $\begin{array}{l}t^{3} y- \\
t^{3} h y^{3}-\end{array}$ & $\begin{array}{l}t^{3} y- \\
k^{3} h y^{3}-\end{array}$ & $\begin{array}{l}t^{3} y- \\
t^{3} h y^{3}-\end{array}$ & $\begin{array}{l}\text { out of reach, out of sight, } \\
\text { concealed, hidden; (KW also:) } \\
\text { sheltered, protected (from heat, } \\
\text { light) }{ }^{171}\end{array}$ \\
\hline
\end{tabular}

(Bella Bella Texts, p. 221) identifies the berries as Viburnum berries which is ambiguous because it could mean Viburnum trilobum (American highbush cranberry), the very similar Viburnum opulus (European highbush cranberry) or Viburnum edule (squashberry), cf. https://en.wikipedia.org/wiki/Highbush_cranberry.

167 The 1980 root list posits $\left\{\mathrm{t}^{3} \mathrm{lq}-\right\}$ but the two available derivates point to $\left\{\mathrm{t}^{3} 1 \mathrm{q}^{3}-\right\}$.

168 Possibly also in HA / $\mathrm{t}^{3} \mathrm{x}$ əłba/ "sewing needle."

${ }^{169} \mathrm{In} \mathrm{KW}\left\{\mathrm{t}^{3} \mathrm{x}-=\mathrm{hbyz}^{3} \mathrm{y}^{3}\right\}>/ \mathrm{t}^{3} \mathrm{Habiz} \varepsilon^{3 /}$ "pain in leg."

${ }^{170}$ Possibly in HA / $/ \mathrm{t}^{3} \mathrm{H}^{\prime} \mathrm{i} \chi \mathrm{v} \cdot \mathrm{l}^{3} \mathrm{as} /$ "maple" HE $/ \mathrm{t}^{3} \mathrm{H}^{\prime} \mathrm{i} \chi \mathrm{V} \cdot \mathrm{l}^{3} \mathrm{as} / \mathrm{OO} / \mathrm{t}^{3} \mathrm{Hi} \chi \mathrm{v} \cdot \mathrm{l}^{3} \mathrm{as} /$ (name of a tree, possibly a maple).

$171\left\{t^{3} y-\right\}$ only in HA $\left\{t^{3} y-h l h\right\}>/ t^{3} i^{\prime}$ ala/ "to wait for sth. or sb." and OO/KW $\left\{\left[t^{3} h\right] t^{3} y-h l h\right\}>$ OO $/ t^{3} a t^{3}$ iala/ "to meet sb. coming in" $\mathrm{KW} / \mathrm{t}^{3} a t^{3} \varepsilon l a /$ "to lie in wait, to try to conceal oneself." HA 
North Wakashan Comparative Root List (Updated)

\begin{tabular}{|c|c|c|c|c|c|c|}
\hline Root \# & Old \# & $\mathrm{HE}$ & $\mathrm{OO}$ & HA & KW & Gloss \\
\hline 3800 & $\begin{array}{l}440 \\
441\end{array}$ & $t^{3} y p-$ & $t^{3} y p-$ & $t^{3} y p-$ & $t^{3} y p-$ & $\begin{array}{l}\text { to step, tread on; (HE/OO/HA } \\
\text { also:) fern root (found by feeling } \\
\text { with the feet) }\end{array}$ \\
\hline 3810 & 442 & $t^{3} y s-$ & $\mathrm{t}^{3} \mathrm{ys}-$ & $t^{3} y s-$ & $t^{3} y s-$ & $\begin{array}{l}\text { stone, made of stone, stony; } \\
\text { (mostly in HE/OO/HA also:) to } \\
\text { weigh down, ballast, anchor }\end{array}$ \\
\hline 3820 & 443 & $\mathrm{t}^{3} \mathrm{y} \mathrm{l}^{-}$ & $t^{3} y 1-$ & $t^{3} y 1-$ & $\left.t^{3} y\right\}-$ & to soak (for softening) \\
\hline 3830 & 446 & & & & $t^{3} y k-$ & to sharpen (knife) \\
\hline 3840 & 445 & $t^{3} y x-$ & $t^{3} y x-$ & $t^{3} y x-$ & $\mathrm{t}^{3} \mathrm{yk}-$ & $\begin{array}{l}\text { to lean on back, to lie on back, } \\
\text { lean or lie back against }\end{array}$ \\
\hline 3850 & 448 & $\mathrm{t}^{3} \mathrm{yx}-$ & $t^{3} y x-$ & $t^{3} y x-$ & $t^{3} y x-$ & $\begin{array}{l}\text { to carry a round or bulky thing on } \\
\text { shoulder }\end{array}$ \\
\hline 3860 & 449 & & & & $t^{3} y x-*$ & $(?)^{172}$ \\
\hline 3870 & 450 & $t^{3} y k v-$ & $t^{3} y k v-$ & $t^{3} y_{k v-}$ & & $\begin{array}{l}\text { to hook (hence: to raise with } \\
\text { lever, pitch fork or gaff hook; to } \\
\text { fish with line and hook; to hang } \\
\text { on pin or nail, to dangle) }\end{array}$ \\
\hline 3880 & 451 & $t^{3} y x v-$ & $t^{3} y x v-$ & $t^{3} y x v-$ & $t^{3} y x v-$ & $\begin{array}{l}\text { bruised, darkened; (HA also:) } \\
\text { black bear }\end{array}$ \\
\hline 3890 & 452 & $t^{3} y q v-$ & $t^{3} y q v-$ & $t^{3} y q v-$ & $\mathrm{t}^{3} \mathrm{yqv}-*$ & $\begin{array}{l}\text { (HE/OO/HA) to smear, handle } \\
\text { syrupy or mushy substance; } \\
\text { (KW) to sip from spoon }\end{array}$ \\
\hline 3900 & 453 & & & & $t^{3} y q v-$ & $\begin{array}{l}\text { to use stones (in diving to sea } \\
\text { bottom or for cooking) }\end{array}$ \\
\hline
\end{tabular}

$\left\{\mathrm{khy}^{3}-\right\}$ is really an augmented form of $\left\{\mathrm{k}^{3} \mathrm{y}-\right\}$ (root no. 13200). To facilitate cross-dialect comparison derivates of $\left\{\mathrm{khy}^{3}-\right\}$ are listed under root no. 3780 .

${ }^{172} \mathrm{In} \mathrm{KW} / \mathrm{t}^{3} \mathrm{ixa} / *$ (name of a fish, perhaps the Pacific wolf eel). 
North Wakashan Comparative Root List (Updated)

\begin{tabular}{|c|c|c|c|c|c|c|}
\hline Root \# & Old \# & $\mathrm{HE}$ & $\mathrm{OO}$ & HA & KW & Gloss \\
\hline 3910 & 454 & $t^{3} y q-$ & $t^{3} y q-$ & $t^{3} y q-$ & $t^{3} y q-$ & bent backwards (head, body) \\
\hline 3920 & 455 & $t^{3} y x-$ & $t^{3} y x-$ & $t^{3} y x-$ & & $\begin{array}{l}\text { dead weight, ballast, to use as } \\
\text { ballast }\end{array}$ \\
\hline 3930 & 444 & $t^{3} y^{3} 1-$ & & $t^{3} y^{3} t-$ & & $(?)^{173}$ \\
\hline 3940 & 447 & $\mathrm{t}^{3} \mathrm{y}^{3} \mathrm{k}-$ & $t^{3} y^{3} k-$ & $t^{3} y^{3} k-$ & $t^{3} y^{3} k v-$ & $\begin{array}{l}\text { to set, thicken, curdle (said of a } \\
\text { liquid) }\end{array}$ \\
\hline 3950 & 456 & $t^{3} \mathrm{kv}-$ & $\mathrm{t}^{3} \mathrm{kv}-$ & $t^{3} \mathrm{kv}-$ & $\mathrm{t}^{3} \mathrm{kv}-$ & $\begin{array}{l}\text { to scratch, scrape, claw, grab or } \\
\text { pull with hooked fingers or claws }\end{array}$ \\
\hline 3960 & 458 & $t^{3} w-$ & $t^{3} \mathrm{~W}-$ & $t^{3} \mathrm{w}-$ & $t^{3} w-$ & $(?)^{174}$ \\
\hline 3970 & 460 & $t^{3} w s-$ & $t^{3} \mathrm{WS}^{-}$ & $t^{3} \mathrm{Ws}^{-}$ & & cedar withes \\
\hline 3980 & 462 & & & & $\mathrm{t}^{3} \mathrm{wxv}-*$ & $(?)^{175}$ \\
\hline 3990 & 463 & $\mathrm{t}^{3} \mathrm{WxV}-$ & $t^{3} w x v-$ & $t^{3} W x v-$ & $t^{3} \mathrm{WXV}-$ & waves, breakers, ocean swell \\
\hline 4000 & 464 & $t^{3} w q v-$ & $t^{3} w q v-$ & $t^{3} w q v-$ & $\mathrm{t}^{3} \mathrm{wqv}-$ & narrow, slim \\
\hline 4010 & & & & & $\begin{array}{l}\mathrm{t}^{3} \mathrm{wqv}-* / \\
\mathrm{t}^{3} \mathrm{w} \chi \mathrm{v}-*\end{array}$ & $(?)^{176}$ \\
\hline 4020 & 465 & & & & $\mathrm{t}^{3} \mathrm{w} \chi \mathrm{V}-*$ & $(?)^{177}$ \\
\hline 4030 & 466 & $t^{3} q v-$ & $t^{3} q v-$ & $t^{3} \mathrm{qv}^{-}$ & $t^{3} q v-$ & clover or cinquefoil root \\
\hline
\end{tabular}

${ }^{173}$ In words referring to saskatoon berries (Amelanchier alnifolia).

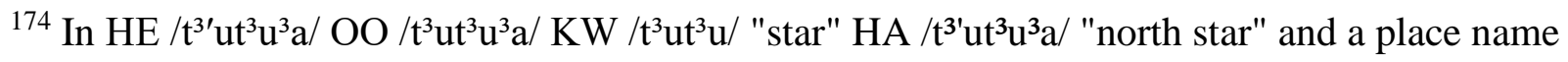
said to mean "star receptacle." Perhaps also in the non-reelicitable names of a speckled hawk and sea food that is unfit to eat.

175 Only in KW / $\mathrm{t}^{3} \mathrm{uxva} / *$ "to cut out intestines."

${ }^{176}$ In $\mathrm{KW} / \mathrm{t}^{3} \mathrm{u} \chi \mathrm{v} \cdot \mathrm{h}^{3} \mathrm{id} / *$ "to spin."

${ }^{177} \mathrm{In} \mathrm{KW} / \mathrm{t}^{3} \mathrm{u} \chi \mathrm{va} / *$ "to spear salmon guided by its wake" and "to mark trail in snow only." 
North Wakashan Comparative Root List (Updated)

\begin{tabular}{|c|c|c|c|c|c|c|}
\hline Root \# & Old \# & $\mathrm{HE}$ & $\mathrm{OO}$ & HA & KW & Gloss \\
\hline 4040 & 467 & $\left(\mathrm{q}^{3} \mathrm{vwt}-\right)$ & $\left(\mathrm{q}^{3} \mathrm{vwt}-\right)$ & $\left(\mathrm{q}^{3} \mathrm{wt}-\right)$ & $t^{3} q-$ & berry cake \\
\hline 4060 & 470 & $t^{3} \mathrm{~h}-$ & & $t^{3} h-$ & $t^{3} \mathrm{~h}-$ & $\begin{array}{l}\text { to lean, fall over (said of long } \\
\text { vertical object) }\end{array}$ \\
\hline 4070 & 471 & & $t^{3} h-$ & & $\mathrm{t}^{3} \mathrm{~h}-*$ & to cover with a layer ${ }^{179}$ \\
\hline 4071 & 473 & $t^{3} h t-$ & $t^{3} h 1-$ & $t^{3} h ł-$ & $\left(\mathrm{t}^{3} \mathrm{ls}-\right)$ & to slice fish \\
\hline 4080 & $\begin{array}{l}474 \\
840\end{array}$ & $\begin{array}{l}t^{3} h k- \\
t^{3} h x-\end{array}$ & $\begin{array}{l}t^{3} h k- \\
t^{3} h x-\end{array}$ & $t^{3} h k-$ & $\begin{array}{l}\mathrm{t}^{3} \mathrm{hk}- \\
\mathrm{c}^{3} \mathrm{hk}-\end{array}$ & $\begin{array}{l}\text { soft material, lining, padding, } \\
\text { bedding, to pad, to caulk a canoe; } \\
\text { (figuratively:) to lay foundation } \\
\text { for dinner courses to follow }{ }^{180}\end{array}$ \\
\hline 4100 & $\begin{array}{l}475 \\
476\end{array}$ & $\mathrm{t}^{3} \mathrm{hkv}-$ & $\mathrm{t}^{3} \mathrm{hkv}-$ & & $\mathrm{t}^{3} \mathrm{hkv}-$ & (?) to handle a stick ${ }^{181}$ \\
\hline 4110 & 477 & & & & $t^{3} h q v-$ & $(?)^{182}$ \\
\hline 4120 & $\begin{array}{l}404 \\
478\end{array}$ & & & & $\begin{array}{l}\mathrm{t}^{3} \mathrm{hqv}-* \\
\text { thqv-* }\end{array}$ & to spear salmon ${ }^{183}$ \\
\hline
\end{tabular}

${ }^{178} \mathrm{HE}$ only in $\left\{\left[\mathrm{t}^{3} \mathrm{~h}-\mathrm{k}\right] \mathrm{t}^{3} \mathrm{~h}-\mathrm{k}-\mathrm{h}\right\}>/ \mathrm{t}^{3}{ }^{\prime} \mathrm{axt} \mathrm{t}^{3} \mathrm{aka} /$ "to doze, be drowsy."

${ }^{179} \mathrm{In} \mathrm{OO} / \mathrm{t}^{3} \mathrm{a}^{3} \mathrm{kv} /$ "sth. covered with a layer of sth." and in KW non-reelicitable terms referring to gathering a blanket over one's shoulder.

$180\left\{\mathrm{t}^{3} \mathrm{hx}-\right\}$ only in $\left\{\mathrm{t}^{3} \mathrm{~h}[\infty] \mathrm{x}-\right\}>\mathrm{HE} / \mathrm{t}^{3} \mathrm{ax} / \mathrm{OO} / \mathrm{t}^{3} \mathrm{aax} /$ "gun powder." HA $/ \mathrm{t}^{3} \mathrm{aak} /$ "gun powder" is derivable from $\left.\left\{\mathrm{t}^{3} \mathrm{hk}-\right\}\right)$.

${ }^{181}$ Specific senses: to bend down sth. that sticks up (e.g. fingers when counting, a dog its ears), to smooth down (e.g. hair), to move a stick held vertically (as in bursting a blister, counting sticks lying side by side 182 In KW /tªqua/ "to belittle."

183 The form $\{$ thqv- $\} *$ occurs only in KW $\{$ thqv-h $\}>/$ taqva/* to spear salmon" which could be a misrecording of $/ \mathrm{t}^{3} \mathrm{aqva} / *$ [2] "to throw a spear in upward curve, to spear salmon.". 
North Wakashan Comparative Root List (Updated)

\begin{tabular}{|c|c|c|c|c|c|c|}
\hline Root \# & Old \# & $\mathrm{HE}$ & $\mathrm{OO}$ & HA & KW & Gloss \\
\hline 4130 & 479 & $\begin{array}{l}t^{3} h q- \\
t^{3} h \chi-\end{array}$ & $\begin{array}{l}t^{3} h q- \\
t^{3} h \chi-\end{array}$ & $\mathrm{t}^{3} \mathrm{hq}-$ & $\mathrm{t}^{3} \mathrm{hq}-*$ & $\begin{array}{l}\text { to distribute, spread, divide, } \\
\text { scatter; to detach particles from a } \\
\text { cluster }\end{array}$ \\
\hline 4150 & 480 & $t^{3} h^{3} \chi-$ & $\mathrm{t}^{3} \mathrm{~h}^{3} \chi^{-}$ & $t^{3} h^{3} \chi-$ & $t^{3} h^{3} \chi-$ & $\begin{array}{l}\text { to turn or hold right side up } \\
\text { (hollow thing) }\end{array}$ \\
\hline 4160 & $\begin{array}{r}481 \\
1202\end{array}$ & np- & np- & np- & $\begin{array}{l}\text { np- } \\
\text { nhp- } \\
\text { lp-* }\end{array}$ & $\begin{array}{l}\text { (HE/OO/HA) to knock down, } \\
\text { smash, hammer; to collapse (like } \\
\text { house); } \mathrm{KW} \text { ) to throw or fall } \\
\text { (round or bulky object) }\end{array}$ \\
\hline 4180 & $\begin{array}{l}483 \\
484\end{array}$ & & & & nms- & $\begin{array}{l}\text { (?) to move sideways, to cross } \\
\text { diagonally, to hit broadside }\end{array}$ \\
\hline 4190 & 485 & & & nt- & nt- & $(?)^{185}$ \\
\hline 4210 & 486 & nn- & nn- & hn- & & to sing \\
\hline 4211 & & & & & nnxw- & to walk alone ${ }^{186}$ \\
\hline 4220 & 509 & & nnkv- & & & $(?)^{187}$ \\
\hline 4230 & 508 & & & & nnkv- & $\begin{array}{l}\text { to order sth., to send for } s b \text {. or } \\
\text { sth. }\end{array}$ \\
\hline 4240 & 487 & & & & $\begin{array}{l}\text { ns-* } \\
\text { nhs-* }\end{array}$ & rank, putrid, decomposing \\
\hline 4250 & 488 & & & nł- & nł- & to wobble, to rock sth. \\
\hline
\end{tabular}

\footnotetext{
$184\{\mathrm{lp}-\}$ only in KW (Koskimo dialect) /lpa/* "to trow stones" and /lp 3 id/* "to strike with stones."

${ }^{185} \mathrm{In} \mathrm{KW} / \mathrm{nt}^{3} \mathrm{id} /$ "to pierce skin inflammation with needle" and HA words referring to decapitation with the guillotine trap.

${ }^{186}$ Unanalyzable stem occurring in KW $\{$ nnxw-hlh $\}>$ /nHxola/ "to walk alone."

${ }^{187}$ Possibly in OO $/$ naHk $^{3}$ va/ "to sing" $(<\{[n h] n n k v-+-h\})$.
} 
North Wakashan Comparative Root List (Updated)

\begin{tabular}{|c|c|c|c|c|c|c|}
\hline Root \# & Old \# & $\mathrm{HE}$ & $\mathrm{OO}$ & HA & KW & Gloss \\
\hline 4260 & $\begin{array}{l}489 \\
490\end{array}$ & & $\begin{array}{l}\text { n1- } \\
\text { nl- }\end{array}$ & & nl-* & to sing \\
\hline 4290 & 493 & & nk- & & nk- & mountain $^{188}$ \\
\hline 4300 & 494 & nx- & nx- & & nx- & $\begin{array}{l}\text { naughty, mischievous; (HE also:) } \\
\text { to beat sb. Up }\end{array}$ \\
\hline 4310 & 495 & ny- & ny- & ny- & ny- & $\begin{array}{l}\text { to shake, tremble, wobble (as in } \\
\text { earthquake), to lean over }\end{array}$ \\
\hline 4320 & 496 & nys- & nys- & nys- & nws- & belonging to 1 st person \\
\hline 4330 & 497 & nys- & & & nys- & $\begin{array}{l}\text { to pull (especially hair, roots, } \\
\text { grass; and into water for magic } \\
\text { revenge) }\end{array}$ \\
\hline 4350 & 499 & nył- & nył- & nył- & nył- & $\begin{array}{l}\text { to report, tell, reveal, expose, } \\
\text { disclose, show, show off }\end{array}$ \\
\hline 4360 & 500 & nyk- & nyk- & nyk- & ynk- & $\begin{array}{l}\text { to take revenge, retaliate, } \\
\text { reciprocate }\end{array}$ \\
\hline 4370 & $\begin{array}{l}501 \\
507\end{array}$ & nykv- & nykv- & nykv- & $\begin{array}{l}\text { nykv- } \\
n^{3} y k v- \\
\text { nkv- }\end{array}$ & nightly, to act at night ${ }^{190}$ \\
\hline 4380 & 504 & ny $\chi-$ & ny $\chi-$ & ny $\chi-$ & ny $\chi-$ & $\begin{array}{l}\text { (KW) to pull; (HE/OO) to pull } \\
\text { (esp. by the hair); (HA) to lift } \\
\text { fish by the gills }{ }^{191}\end{array}$ \\
\hline 4390 & 505 & ngv- & ngv- & ngv- & ngv- & in-law \\
\hline 4400 & 506 & nkv- & nkv- & nkv- & nkv- & salal berry \\
\hline
\end{tabular}

${ }^{188} \mathrm{OO}$ only in proper names of likely $\mathrm{KW}$ origin.

${ }^{189} \mathrm{HE}$ possibly in the place name $/ \mathrm{N}^{\prime} \mathrm{ic}^{3} \mathrm{a} /$.

190 \{n $\left.{ }^{3} \mathrm{ykv}-\right\}$ only in $\mathrm{KW} / \mathrm{n}^{3} \mathrm{ix}: \mathrm{n}^{3} \mathrm{k}^{3} \mathrm{vs} /$ "streetlights."

${ }^{191} \mathrm{KW}\{$ ny $\chi-\}$ has the productivity of HE/OO \{wht- $\}$. 
North Wakashan Comparative Root List (Updated)

\begin{tabular}{|c|c|c|c|c|c|c|}
\hline Root \# & Old \# & $\mathrm{HE}$ & $\mathrm{OO}$ & HA & KW & Gloss \\
\hline 4420 & 510 & $\begin{array}{l}\text { nw- } \\
\text { nwyh- } \\
\text { nynwyh- } \\
\text { nhwh- }\end{array}$ & $\begin{array}{l}\text { nw- } \\
\text { nwyh- } \\
\text { nhwh- }\end{array}$ & nw- & nw- & power, control, awareness ${ }^{192}$ \\
\hline 4421 & 518 & nwgvh- & nwgvh- & nwgvh- & $\begin{array}{l}\text { nw- } \\
\text { nwgvh- }\end{array}$ & $\begin{array}{l}\text { It is } \mathrm{I} / \text { we (1st. person } \\
\text { reference) }\end{array}$ \\
\hline 4430 & 511 & nwt- & nwt- & nwt- & nwt- & spoiled, polluted, poisoned, bitter \\
\hline 4440 & 512 & & & & nwn $\infty-$ & wolf (myth name) \\
\hline 4450 & $\begin{array}{l}513 \\
514\end{array}$ & nws- & nws- & nws- & nws- & $\begin{array}{l}\text { traces of past events, to reveal the } \\
\text { past, to tell story }\end{array}$ \\
\hline 4460 & 515 & $\begin{array}{l}\text { nw1- } \\
\text { nwł•- }\end{array}$ & $\begin{array}{l}\text { nwł- } \\
\text { nwł•- }\end{array}$ & $\begin{array}{l}\text { nw1- } \\
\text { nwł•- }\end{array}$ & $\begin{array}{l}\text { nwł- } \\
\text { nwł•- }\end{array}$ & $\begin{array}{l}\text { obsessed, possessed, disorderly, } \\
\text { crazy, not controlling self, } \\
\text { confused, uneasy, spooked, } \\
\text { scared }\end{array}$ \\
\hline 4470 & 516 & nhł- & nhł- & nwł- & nhł- & ling cod \\
\hline 4500 & $\begin{array}{l}519 \\
521\end{array}$ & nwkv- & nwkv- & nwkv- & nwkv- & worried, anxious, concerned \\
\hline 4510 & 520 & nwkv- & nwkv- & nwkv- & & fur seal \\
\hline 4520 & 522 & & & & $\mathrm{nw} \chi \mathrm{V}-$ & (?) fuzzy, confused \\
\hline 4530 & 523 & nqv- & nqv- & (h³hs-) & $\mathrm{n} \chi \mathrm{V}-$ & $\begin{array}{l}\text { soapberries, frothy (like whipped } \\
\text { soapberries) }\end{array}$ \\
\hline
\end{tabular}

192 Derivates are about controlling oneself (as one who is old, mature, wise, integrated in society); controlling the world (as when working magic); being possessed, controlled by the other world (as when singing); uncertainty, danger 193 \{nw- $\}$ as a 1 st person reference is attested in two KW examples. \{nwgvh- $\}$ is probably a combination of $\{$ nw- $\}$ and $\{\#$ gh $\}$ (suffix no. 3190). 
North Wakashan Comparative Root List (Updated)

\begin{tabular}{|c|c|c|c|c|c|c|}
\hline Root \# & Old \# & $\mathrm{HE}$ & $\mathrm{OO}$ & HA & KW & Gloss \\
\hline 4540 & 525 & & & nq- & & tired of sth. \\
\hline 4550 & $\begin{array}{r}526 \\
529 \\
531 \\
2460 \\
2565\end{array}$ & $\begin{array}{l}\text { nq- } \\
\text { hnq- } \\
h^{3} \text { nq- }^{-}\end{array}$ & $\begin{array}{l}\text { nq- } \\
\text { hnq- } \\
h^{3} \text { nq- }^{-}\end{array}$ & $\begin{array}{l}\text { nq- } \\
\text { nhq- } \\
\text { hnq- } \\
\text { hnh- } \\
\text { hnq- }\end{array}$ & $\mathrm{n} \chi-$ & $(?)^{194}$ \\
\hline 4560 & 528 & $n \chi-$ & $\mathrm{n} \chi-$ & $n \chi-$ & $\mathrm{n} \chi-$ & $\begin{array}{l}\text { flexible, elastic (like one's limbs), } \\
\text { adjusting to adverse force (as } \\
\text { when bucking the wind, paddling } \\
\text { against tide) }\end{array}$ \\
\hline 4580 & 531 & & nh- & & nh- & $(?)^{195}$ \\
\hline 4590 & 530 & nh- & nh- & & $\begin{array}{l}\text { nh- } \\
n^{3 h} h^{*}\end{array}$ & $(?)^{196}$ \\
\hline 4600 & $\begin{array}{l}532 \\
543\end{array}$ & nh- & nh- & nh- & nh- & $\begin{array}{l}\text { (?) soft ground, yielding } \\
\text { underfoot }^{197}\end{array}$ \\
\hline 4610 & $\begin{array}{l}534 \\
572\end{array}$ & & & nhp- & nhp- & hole, opening \\
\hline 4611 & 535 & nhn- & nhn- & $\begin{array}{l}\text { (shk- } \\
\text { shx-) }\end{array}$ & nhn- & grizzly bear ${ }^{198}$ \\
\hline
\end{tabular}

${ }^{194}$ In HA /nrc ${ }^{3}$ ai/ "fox" and names of ducks, geese and a monster identified by the OO consultant as a large fish that could swallow a whole canoe and by the HA (Kitlope) consultant as a monster that could swallow people.

$195 \mathrm{In} \mathrm{OO} /$ nask $^{3} \mathrm{a} \chi \mathrm{di} /$ "tail of bird" KW /na $\chi \mathrm{nsd}^{3} /$ "rear end of bird, 'parson's nose."'

${ }^{196}$ In words referring to daring and exclamations expressing affection, pain or fear. $\left\{\mathrm{n}^{3} \mathrm{~h}-\right\}^{*}$ only in $\mathrm{KW} / \mathrm{n}^{3} \mathrm{an}^{3} \mathrm{a} / *$ "shame on you!"

${ }^{197}$ In words for "snow" and possibly KW / a $^{3}$ хла/ "swampy, soggy underfoot, quicksand, soft (swampy, loose) ground."

198 The KW form is in many derivates regularized to $\{\mathrm{nn}-\}$. 
North Wakashan Comparative Root List (Updated)

\begin{tabular}{|c|c|c|c|c|c|c|}
\hline Root \# & Old \# & $\mathrm{HE}$ & $\mathrm{OO}$ & HA & $\mathrm{KW}$ & Gloss \\
\hline 4640 & 536 & nhs- & & & nhs- & overlay, covering layer \\
\hline 4650 & 537 & nhk- & nhk- & nhk- & & to wiggle (tail, hands) \\
\hline 4660 & 538 & & & nhx- & & (?) to do sth. to excess, to brag \\
\hline 4670 & 539 & $\begin{array}{l}\text { nhxv-l } \\
\text { nh } \chi v-\end{array}$ & $\begin{array}{l}\text { nhxv-/ } \\
\text { nh } \chi v-\end{array}$ & $\begin{array}{l}\text { nhxv-/ } \\
\text { nh } \chi v-\end{array}$ & & $(?)^{199}$ \\
\hline 4680 & 540 & nhxv- & nhxv- & nhxv- & nhxv- & vulva, shaped like a vulva \\
\hline 4690 & 541 & nhq- & nhq- & nhq- & nhq- & $\begin{array}{l}\text { to thirst, to ingest or handle } \\
\text { liquid, to quench thirst, to drain } \\
\text { off liquid }\end{array}$ \\
\hline 4700 & 542 & nhq- & & & & $(?)^{200}$ \\
\hline 4710 & 544 & $n^{3} n-$ & & & & sling, use the sling \\
\hline 4720 & 545 & $\begin{array}{l}n^{3} n k- \\
n^{3} k-\end{array}$ & $\begin{array}{l}\mathrm{n}^{3} \mathrm{nk}- \\
\mathrm{n}^{3} \mathrm{k}-\end{array}$ & & $\begin{array}{l}\text { ynk- } \\
\text { ynk }^{3-} \\
y^{3} n k-\end{array}$ & sling, use the sling 201 \\
\hline 4730 & 546 & $n^{3} n k-$ & $\mathrm{n}^{3} \mathrm{nk}-$ & & & $(?)^{202}$ \\
\hline 4740 & 548 & $n^{3} £-$ & $n^{3} £-$ & $\mathrm{n}^{3} \mathcal{L}_{-}$ & $n^{3} £-$ & $\begin{array}{l}\text { to lie on back, to put on back, } \\
\text { lean backwards }\end{array}$ \\
\hline 4741 & 491 & & & & $\mathrm{n}^{3} \mathrm{l}_{-} *$ & to fly \\
\hline
\end{tabular}

${ }^{199} \mathrm{In} \mathrm{HE} / \mathrm{n}^{\prime} \mathrm{aw}^{\prime} \mathrm{ac}^{3} \mathrm{i}$ / OO /nawac 3 i/ HA /nawac ${ }^{3}$ ai/ "butterball duck = bufflehead duck."

${ }^{200} \mathrm{In} \mathrm{HE} / \mathrm{n}^{\prime} \mathrm{aq}^{3} \mathrm{i}^{\prime} \mathrm{ukv} /$ "den of bear."

${ }^{201}\left\{\mathrm{n}^{3} \mathrm{k}-\right\}$ only possibly in $\left\{\left[\mathrm{n}^{3} \mathrm{~h}\right] \mathrm{n}^{3}[\mathrm{~h}] \mathrm{k}-\mathrm{+}-\mathrm{h}\right\}$ "to try to use the sling" which could however also be a euphonic adjustment of $\left\{\left[\mathrm{n}^{3} \mathrm{~h}\right] \mathrm{n}^{3} \mathrm{nk}-+-\mathrm{h}\right\}$. The form of the KW root is problematic because informants seem to be confused between using $\left\{\mathrm{ynk}^{3}-\right\}$ and $\left\{\mathrm{y}^{3} \mathrm{nk}-\right\}$.

${ }^{202} \mathrm{In} \mathrm{HE} / \mathrm{n}^{3 \prime} \mathrm{Hk}^{3} \mathrm{aut} / \mathrm{OO} / \mathrm{n}^{3} \mathrm{Hk}^{3} \mathrm{ut} /$ "to select a berry-picking spot" and perhaps the place name $\mathrm{HE} / \mathrm{N}^{3}{ }^{\prime} \mathrm{Hk}^{3} \mathrm{a} \chi \mathrm{V} /$. 
North Wakashan Comparative Root List (Updated)

\begin{tabular}{|c|c|c|c|c|c|c|}
\hline Root \# & Old \# & $\mathrm{HE}$ & $\mathrm{OO}$ & HA & KW & Gloss \\
\hline 4742 & 492 & & & & $\mathrm{n}^{3} l \mathrm{~s}-*$ & to whine \\
\hline 4750 & 550 & & & & $n^{3} 1 \chi-$ & $\begin{array}{l}\text { (?) damaged, bruised, sore, } \\
\text { chafed (said of body parts) }\end{array}$ \\
\hline 4760 & 549 & & & & $\mathrm{n}^{3} \mathrm{lh}-$ & $(?)^{203}$ \\
\hline 4770 & 552 & & $\mathrm{n}^{3} \mathrm{k}-$ & & $\mathrm{n}^{3} \mathrm{k}-$ & (?) steam, to use steam \\
\hline 4780 & $\begin{array}{r}553 \\
1612\end{array}$ & $\begin{array}{l}n^{3} y- \\
y^{3} y-\end{array}$ & $\begin{array}{l}n^{3} y- \\
y^{3} y-\end{array}$ & $\begin{array}{l}n^{3} y- \\
h^{3} y-\end{array}$ & $n^{3} y-$ & $\begin{array}{l}\text { to string up (fish, beads), thread } \\
\text { (needle), put rope through (hole) }\end{array}$ \\
\hline 4790 & 554 & & $\begin{array}{l}\mathrm{n}^{3} \mathrm{y}-/ \\
\mathrm{n}^{3} \mathrm{yk}-\end{array}$ & & & creaking sound $^{204}$ \\
\hline 4800 & 498 & $\mathrm{n}^{3} \mathrm{ys}-$ & $n^{3} y s-$ & & $\begin{array}{l}\mathrm{n}^{3} \mathrm{ys}-* / \\
\text { nys-* }\end{array}$ & $(?)^{205}$ \\
\hline 4810 & $\begin{array}{l}551 \\
555 \\
556 \\
557\end{array}$ & $\begin{array}{l}\mathrm{n}^{3} \mathrm{yk}- \\
\mathrm{n}^{3} \mathrm{k}-\end{array}$ & $\begin{array}{l}\mathrm{n}^{3} \mathrm{yk}- \\
\mathrm{n}^{3} \mathrm{ykv}-\end{array}$ & $\mathrm{n}^{3} \mathrm{yk}-$ & $\begin{array}{l}\mathrm{n}^{3} \mathrm{yk}- \\
\mathrm{n}^{3} \mathrm{yl}-\end{array}$ & $\begin{array}{l}\text { conduit, to let through, to let out } \\
\text { (hence } 1 \text {. spout, mouthpiece; } 2 \text {. to } \\
\text { say, tell, utter; to think (= say in } \\
\text { mind); 3. to permit to happen, } \\
\text { allow, obey; 4. to give } \\
\text { permission, tell to do, command, } \\
\text { instruct) }\end{array}$ \\
\hline
\end{tabular}

${ }^{203} \mathrm{In} \mathrm{KW} /$ пла/ "laughing goose."

${ }^{204} \mathrm{In} \mathrm{OO} / \mathrm{n}^{3} \mathrm{ik}^{3} \mathrm{ala} /$ "sound of creaking." Root could be onomatopoeic.

${ }^{205} \mathrm{In} \mathrm{HE} / \mathrm{n}^{3}$ isa/ OO $/ \mathrm{n}^{3} \mathrm{isa} /$ "to poke devilfish (underneath boulders, their hiding place, in order to get halibut bait)" and in non-reelicitable KW words referring to red cod, devilfish, octopus. Some of the latter derive from \{nys- $\}$ according to Boas and some from $\left\{n^{3} y s-\right\}$. ${ }^{206} 1$. KW $\left\{\mathrm{n}^{3} \mathrm{yl}-\right\}$ is attested only in two derivates of $\left.\left\{\left[\mathrm{n}^{3} \mathrm{~h}\right] \mathrm{n}^{3} \mathrm{y}\right\}-\right\}$ and could be a blend of $\left.\{\mathrm{ny}\}-\right\}$ "tell, reveal, etc." and $\left\{\mathrm{n}^{3} \mathrm{yk}-\right\}$. OO $\left\{\mathrm{n}^{3} \mathrm{ykv}-\right\}$ occurs only in $\left\{\mathrm{n}^{3} \mathrm{ykv}-\% \mathrm{~m}\right\}$ "beneficiary of permission." 2. At least in $\mathrm{HE} / \mathrm{OO} / \mathrm{HA},\left\{\mathrm{n}^{3} \mathrm{yk}-\right\}$ meaning "to say, utter" and derivates can be

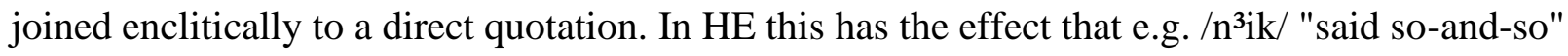


North Wakashan Comparative Root List (Updated)

\begin{tabular}{|c|c|c|c|c|c|c|}
\hline Root \# & Old \# & $\mathrm{HE}$ & $\mathrm{OO}$ & HA & KW & Gloss \\
\hline 4840 & 558 & $n^{3} y k v-$ & & $\mathrm{n}^{3} \mathrm{ykv}-$ & & to carry on the shoulder \\
\hline 4860 & 580 & & & & $\begin{array}{l}\mathrm{n}^{3} \mathrm{xv-} \\
\mathrm{n}^{3} \mathrm{~h} \times v_{-}\end{array}$ & $\begin{array}{l}\text { covered with a layer-like thing } \\
\text { (e.g. blanket, mat, lid), veiled, } \\
\text { shielded, skirted (like house } \\
\text { stilts) }\end{array}$ \\
\hline 4870 & 559 & $\begin{array}{l}n^{3} x v^{\bullet-} \\
n^{3} x v h-\end{array}$ & $\begin{array}{l}\mathrm{n}^{3} \mathrm{xv} \cdot- \\
\mathrm{n}^{3} \mathrm{xvh}-\end{array}$ & $\begin{array}{l}\mathrm{n}^{3} \mathrm{xv} \cdot- \\
\mathrm{n}^{3} \mathrm{xvh}-\end{array}$ & $n^{3} x v h-$ & $\begin{array}{l}\text { near, close, in the vicinity; low } \\
\text { (like building) }\end{array}$ \\
\hline 4880 & 560 & & & & $\mathrm{n}^{3} \mathrm{~W}-$ & $\begin{array}{l}\text { tense, cocked, on edge, ready to } \\
\text { go off; to aim; really or } \\
\text { pretendedly ready to strike, to } \\
\text { feign an action }\end{array}$ \\
\hline 4890 & $\begin{array}{r}561 \\
2546\end{array}$ & $\begin{array}{l}n^{3} w- \\
n^{3} w^{3} s- \\
h^{3} n^{3} w-\end{array}$ & $\begin{array}{l}n^{3} w- \\
n^{3} w^{3} s- \\
h^{3} n^{3} w-\end{array}$ & $\mathrm{n}^{3} \mathrm{~W}-$ & $\mathrm{n}^{3} \mathrm{w}-*$ & $\begin{array}{l}\text { light in the dark (from fire, torch, } \\
\text { moon), to carry fire (for lighting } \\
\text { the way or starting a fire } \\
\text { somewhere), to stoke the fire }{ }^{207}\end{array}$ \\
\hline 4900 & $\begin{array}{r}562 \\
2614\end{array}$ & $\mathrm{n}^{3} \mathrm{w}-$ & $\mathrm{n}^{3} \mathrm{wl}-$ & $\begin{array}{l}\mathrm{n}^{3} \mathrm{wl}- \\
\mathrm{h}^{3} \mathrm{w} 1-\end{array}$ & $\mathrm{h}^{3} \mathrm{wl}-$ & $\begin{array}{l}\text { (HE/OO/HA) to push or lean } \\
\text { with the shoulder or the side; } \\
(\mathrm{KW}) \text { to diverge from straight } \\
\text { course, to lean, to incline, to turn } \\
\text { a corner }^{208}\end{array}$ \\
\hline
\end{tabular}

is pronounced $/ \mathrm{n}^{3} \mathrm{k} /$. The latter has started behaving like a root different from $\left\{\mathrm{n}^{3} \mathrm{yk}-\right\}$ in HE $\left\{\left[\mathrm{n}^{3} \mathrm{~h}\right] \mathrm{n}^{3}[\mathrm{~h}] \mathrm{k}-\mathrm{+}-\mathrm{h}\right\}>/ \mathrm{n}^{3} \mathrm{an}^{3} \mathrm{ak} \mathrm{k}^{3} \mathrm{a} /$ "to try to say."

${ }^{207} \mathrm{HE} / \mathrm{OO}\left\{\mathrm{h}^{3} \mathrm{n}^{3} \mathrm{w}-\right\}$ corresponds to HA/KW $\left\{\left[\mathrm{n}^{3}\right] \mathrm{n}^{3} \mathrm{w}-\right\}$, compare HE/OO $\left\{\mathrm{h}^{3} \mathrm{n}^{3} \mathrm{w}-\mathrm{h}\right\}>\mathrm{HE}$ $/ h^{3} \mathrm{H}^{3} \mathrm{u}^{\prime} \mathrm{a} / \mathrm{OO} / \mathrm{h}^{3} \mathrm{H}^{3} \mathrm{ua} /$ and $\mathrm{HA} / \mathrm{KW}\left\{\left[\mathrm{n}^{3}\right] \mathrm{n}^{3} \mathrm{w}-\mathrm{h}\right\}>\left\{\mathrm{h}^{3} \mathrm{n}^{3} \mathrm{~W}-\mathrm{h}\right\}>\mathrm{HA} / \mathrm{h}^{3} \mathrm{H}^{3} \mathrm{ua} / \mathrm{KW} / \mathrm{h}^{3} \mathrm{H}^{3} \mathrm{~J} / *$ "to use torch to fetch fire from another house." $\mathrm{HE} / \mathrm{OO}\left\{\mathrm{h}^{3} \mathrm{n}^{3} \mathrm{w}-\right\}$ may ultimately stem from the same $\left\{\left[n^{3}\right] n^{3} W_{-}\right\}$as assumed for HA/KW but the dissimilation of $\left|n^{3} n^{3}\right|$ to $\left|h^{3} n^{3}\right|$ is not usual morphophonemically in HE/OO. Related to this root no. 4890 is $\left\{h^{3} n-\right\}$ (root no. 23410). ${ }^{208}$ Boas reports five supposedly HE examples of $\left.\left\{\mathrm{h}^{3} \mathrm{w}\right\}-\right\}$ on p. 187 of Bella Bella Texts. No text reference is given for any of them. 
North Wakashan Comparative Root List (Updated)

\begin{tabular}{|c|c|c|c|c|c|c|}
\hline Root \# & Old \# & $\mathrm{HE}$ & $\mathrm{OO}$ & HA & KW & Gloss \\
\hline 4910 & 563 & $\mathrm{n}^{3}$ wlh- & $\mathrm{n}^{3}$ wlh- & $\mathrm{n}^{3} \mathrm{wlh}-$ & $\mathrm{n}^{3} \mathrm{wlh}-$ & (?) sticking out, outstanding ${ }^{209}$ \\
\hline 4920 & 564 & & & & $\mathrm{n}^{3} \mathrm{WXV}-$ & $(?)^{210}$ \\
\hline 4930 & 565 & & & & $\mathrm{n}^{3} \mathrm{WXV}-$ & $(?)^{211}$ \\
\hline 4940 & $\begin{array}{l}1806 \\
1813\end{array}$ & wnyqv- & $\begin{array}{l}\text { wnyqv- } \\
\text { wn } \chi v-\end{array}$ & ywnwqv- & $\begin{array}{l}\mathrm{n}^{3} \mathrm{wqv}^{*} \\
\mathrm{n}^{3} \text { wyqv- } \\
\text { wnyqv- } \\
\text { wnhqv- } \\
\text { wnqv-* }\end{array}$ & $\begin{array}{l}\text { to scorch, to burn, to apply heat } \\
\text { (e.g. poultice), to get burned }{ }^{212}\end{array}$ \\
\hline
\end{tabular}

${ }^{209}$ In terms referring to an elder sibling, the middle finger and a flood (metaphor for potlatch) that rises higher than the last one.

${ }^{210}$ Only in KW /n³ uxva/ "to aim."

${ }^{211}$ In words referring to a small kind of blueberries.

212 1. The difference between $\mathrm{HE} / \mathrm{OO} / \mathrm{KW}$ \{wnyqv- $\}$ and $\mathrm{HA}$ \{ywnwqv- is reminiscent of that between HA $\left\{-p^{3}\right.$ nyxvls $\}$ and HE/OO $\left\{-p^{3}\right.$ ynyxvls $\}$. \{wnyqv- $\}$ and $\{$ wnhqv- $\}$ could be augmented forms of $\{w n q v-\}$ the one example of which is $\mathrm{KW} / \mathrm{wH} \chi \mathrm{v} \cdot \hbar^{3} \mathrm{H} / *$ "made of red pine" which could not be reelicited. \{wnhqv- $\}$ occurs only in the KW word for red pine. Red pine bark "is thick and gray-brown at the base of the tree, but thin, flaky and bright orange-red in the upper crown; the tree's [English] name derives from this distinctive character. Some red color may be seen in the fissures of the bark" (according to https://en.wikipedia.org/wiki/Pinus_resinosa). 2. $\{w n \chi v-\}$ occurs in OO $\{w n \chi v-! ! s y w h\}$ "to scorch through" and $\{w n \chi v-\& y d\}$ "to become scorched"; it could be a backformation from OO \{wnyqv-!!sywh $\}>$ /wHiðvsiwa/ "to scorch through." A root $\left\{w n \chi v^{-}\right\}$also occurs in one $\mathrm{KW}$ word referring to oil passing through one's body but its semantic relationship to scorching and burning is not clear and it is catalogued as a separate root. 
North Wakashan Comparative Root List (Updated)

\begin{tabular}{|c|c|c|c|c|c|c|}
\hline Root \# & Old \# & $\mathrm{HE}$ & $\mathrm{OO}$ & $\mathrm{HA}$ & KW & Gloss \\
\hline 4950 & 566 & $\mathrm{n}^{3} \mathrm{qv}-$ & & & $\mathrm{n}^{3} \mathrm{qv}-$ & $(?)^{213}$ \\
\hline & 567 & & & & & \\
\hline 4970 & 568 & $\begin{array}{l}\mathrm{n}^{3} \chi \mathrm{vh}- \\
\mathrm{h}^{3} \chi \mathrm{vh}- \\
\mathrm{h}^{3} \mathrm{nh} \chi \mathrm{vh}-\end{array}$ & $\mathrm{h}^{3} \mathrm{nhxvh}-$ & $\begin{array}{l}\mathrm{n}^{3} \chi v h- \\
h^{3} \chi v h- \\
h^{3} \chi v- \\
h^{3} y n h x v h- \\
h^{3} n h \chi v- \\
h^{3} \chi-\end{array}$ & & contingency, possibility ${ }^{214}$ \\
\hline 4980 & 569 & $n^{3} h-$ & $\mathrm{n}^{3} \mathrm{~h}-$ & $\mathrm{n}^{3} \mathrm{~h}-$ & & to pick berries \\
\hline 4990 & 570 & $\mathrm{n}^{3} \mathrm{~h}-$ & $n^{3} h-$ & & $\mathrm{n}^{3} \mathrm{~h}-$ & day, daylight, sky, weather \\
\hline 5000 & 571 & & $\mathrm{n}^{3} \mathrm{~h}-$ & $\mathrm{n}^{3} \mathrm{~h}-$ & & $(?)^{215}$ \\
\hline 5010 & 573 & $\mathrm{n}^{3} \mathrm{hs}-$ & $\mathrm{n}^{3} \mathrm{hs}-$ & & $\mathrm{n}^{3} \mathrm{hs}-$ & $(?)^{216}$ \\
\hline 5020 & 574 & $\mathrm{n}^{3} \mathrm{~h} £^{3}-$ & $\mathrm{n}^{3} \mathrm{~h} \AA^{3}-$ & & $\mathrm{n}^{3} \mathrm{~h}^{3}-$ & wolverine $\mathrm{e}^{217}$ \\
\hline 5030 & 576 & & & & $\mathrm{n}^{3} \mathrm{~h} 1-$ & $(?)^{218}$ \\
\hline
\end{tabular}

${ }^{213}$ In two KW words glossed as "to swallow" and possibly in $\mathrm{HE}\left\{\mathrm{n}^{3} \mathrm{qv}-=\mathrm{kv}-\& \mathrm{yd}\right\}>$ $/ \mathrm{n}^{3} \mathrm{qvxv} . \hbar^{3 \prime}$ it/ "to redeem a spouse or unborn child from clan membership."

214 Proclitics often glossable as "if," "when," "lest." HA $\left\{\mathrm{h}^{3} \chi-\right\}$ is only attested in combination with subject deictics and glossable as "it should be prevented from happening that ..." ${ }^{215} \mathrm{In} \mathrm{OO} / \mathrm{n}^{3} \mathrm{aas} /$ "an eldest son's eldest son" and HA $/ \mathrm{n}^{3} \mathrm{aka}^{3} \mathrm{u} /$ (endearing name for a grandchild).

${ }^{216} \mathrm{In} \mathrm{HE} / \mathrm{n}^{3} \mathrm{ask} / \mathrm{OO} / \mathrm{n}^{3} \mathrm{askv} / \mathrm{KW} / \mathrm{n}^{3} \mathrm{as} ə \mathrm{kv} /$. HE/OO elders said it refers to a kind of blueberries growing on high hills and tasting different from $\mathrm{HE} / \mathrm{si}^{\prime} \mathrm{ak}^{3} \mathrm{vHat} / \mathrm{OO} / \mathrm{siak}^{3} \mathrm{vHat} /$. The KW item can refer to blueberries that grow on high hills (mountains) but also to salal berries.

${ }^{217} \mathrm{In} \mathrm{HE} / \mathrm{n}^{3 \prime} \mathrm{a} £^{3 \prime} \mathrm{ay}$ 'a/ OO $/ \mathrm{n}^{3} \mathrm{a} £^{3} \mathrm{a} / \mathrm{KW} / \mathrm{n}^{3} \mathrm{a} £^{3} \varepsilon^{3 /}$ 'wolverine'.

${ }^{218} \mathrm{In} \mathrm{KW} / \mathrm{n}^{3} \mathrm{an}^{3} \mathrm{~J}^{3} \mathrm{imas} /$ "berries (of any kind)." 
North Wakashan Comparative Root List (Updated)

\begin{tabular}{|c|c|c|c|c|c|c|}
\hline Root \# & Old \# & $\mathrm{HE}$ & $\mathrm{OO}$ & HA & KW & Gloss \\
\hline 5040 & 575 & $\begin{array}{l}\mathrm{n}^{3} \mathrm{hlh}- \\
\mathrm{n}^{3} \mathrm{hl} \infty-\end{array}$ & $\begin{array}{l}\mathrm{n}^{3} \mathrm{hlh}- \\
\mathrm{n}^{3} \mathrm{hl} \infty \mathrm{-}\end{array}$ & $\begin{array}{l}\mathrm{n}^{3} \mathrm{hlh}- \\
\mathrm{n}^{3} \mathrm{hl} \infty-\end{array}$ & $\begin{array}{l}\mathrm{n}^{3} \mathrm{~h}- \\
\mathrm{n}^{3} \mathrm{hlh}- \\
\mathrm{n}^{3} \mathrm{hl} \infty-\end{array}$ & $\begin{array}{l}\text { 1. upriver; 2. (HE/OO/KW) } \\
\text { south; (HA) north }{ }^{219}\end{array}$ \\
\hline 5050 & $\begin{array}{r}577 \\
2548\end{array}$ & $\mathrm{n}^{3} \mathrm{hk}-$ & $\mathrm{n}^{3} \mathrm{hk}-$ & $\mathrm{n}^{3} \mathrm{hk}-$ & $\begin{array}{l}\mathrm{n}^{3} \mathrm{hk}- \\
\mathrm{n}^{3} \mathrm{hk}^{3}-\end{array}$ & $\begin{array}{l}\text { (HE/OO/HA) to keep an eye on } \\
\text { sth., to check, to watch out for } \\
\text { sth. (prey, trespassers); (HA } \\
\text { also:) 1. to look at, to look in the } \\
\text { mirror, to inspect; 2. to look for, } \\
\text { search for; (KW) expecting, } \\
\text { anticipating, apprehensive } 220\end{array}$ \\
\hline 5060 & 578 & & $\mathrm{n}^{3} \mathrm{hkv}-$ & & $\mathrm{n}^{3} \mathrm{hkV}-$ & $(?)^{221}$ \\
\hline 5070 & 579 & $\mathrm{n}^{3} \mathrm{hxv}-$ & $\mathrm{n}^{3} \mathrm{hxv}-$ & $\mathrm{n}^{3} \mathrm{hxv}-$ & $\begin{array}{l}\mathrm{n}^{3} \mathrm{hxv-} \\
\mathrm{n}^{3} \mathrm{hk}^{3} \mathrm{v}-*\end{array}$ & $\begin{array}{l}\text { collectivity, inclusiveness, all- } \\
\text { encompassing; (HA/KW also:) } \\
\text { one, alone }{ }^{222}\end{array}$ \\
\hline 5090 & 581 & $\mathrm{n}^{3} \mathrm{hqv-}$ & $\mathrm{n}^{3} \mathrm{hqv}-$ & $\mathrm{n}^{3} \mathrm{hqv-}$ & $\mathrm{n}^{3} \mathrm{hqv}-$ & $\begin{array}{l}\text { bright light, daylight, moonlight; } \\
\text { (HE/OO/HA also:) world, sky, } \\
\text { atmosphere, weather }\end{array}$ \\
\hline 5100 & & & & & $\mathrm{n}^{3} \mathrm{hqv-*}$ & $(?)^{223}$ \\
\hline
\end{tabular}

${ }^{219} \mathrm{KW}\left\{\mathrm{n}^{3} \mathrm{~h}-\right\}$ occurs possibly in $\left\{\mathrm{n}^{3} \mathrm{~h}-\mathrm{h} 1-\mathrm{h}\right\}>/ \mathrm{n}^{3}$ ała/ "facing south." In all KW examples of $\left\{\mathrm{n}^{3} \mathrm{hl} \infty-\right\},|\infty|$ becomes a replica of $|1|$ and merges with it.

${ }^{220} \mathrm{KW}\left\{\mathrm{n}^{3} \mathrm{hk}^{3}-\right\}$ attested only in $\left\{\mathrm{n}^{3} \mathrm{hk}^{3} \# \mathrm{q}^{3} \mathrm{hlh}\right\}>/ \mathrm{n}^{3} \mathrm{ak}^{3} \mathrm{q}^{3} \mathrm{ala} /$ "to really expect." $\mathrm{KW}\left\{\mathrm{n}^{3} \mathrm{hk}-\right\}$ occurs only in the free stem $\left\{\left[n^{3}\right] n^{3} h k-\right\}>\left\{h^{3} n^{3} h k-\right\}$ "to have learnt one's lesson (and not trying again), to desist."

${ }^{221}$ Only in names referring to the Seymour Inlet people.

${ }^{222} \mathrm{KW}$ sometimes uses $\left\{\mathrm{n}^{3} \mathrm{hxv}-\right\}$ also when HE/OO/HA use $\left\{\mathrm{h}^{3} h g-\right\}$. KW $\left\{\mathrm{n}^{3} \mathrm{hk}^{3} \mathrm{v}_{-}\right\}^{*}$ occurs only in $/ \mathrm{n}^{3} \mathrm{ak}^{3} \mathrm{vi}^{3} \mathrm{sta} / *$ likely meaning "integrated, encompassing every aspect."

${ }^{223}$ In KW $/ \mathrm{n}^{3} \mathrm{an}^{3}$ arvala/* "to help pack." 
North Wakashan Comparative Root List (Updated)

\begin{tabular}{|c|c|c|c|c|c|c|}
\hline Root \# & Old \# & $\mathrm{HE}$ & $\mathrm{OO}$ & HA & KW & Gloss \\
\hline 5110 & 582 & $\mathrm{n}^{3} \mathrm{~h} \chi-$ & $n^{3} h \chi-$ & & $\mathrm{n}^{3} \mathrm{~h} \chi-$ & $\begin{array}{l}\text { steep climb (as when after } \\
\text { mountain goat) }\end{array}$ \\
\hline 5120 & 583 & & & zblx- & & to fight ${ }^{224}$ \\
\hline 5130 & $\begin{array}{l}585 \\
587\end{array}$ & $\begin{array}{l}\text { zm- } \\
\text { zhmy- }\end{array}$ & $\begin{array}{l}\text { zm- } \\
\text { zhmy- }\end{array}$ & $\mathrm{zm}-$ & $\mathrm{zm}-$ & $\begin{array}{l}\text { to inter, cover with earth or sand; } \\
\text { (KW also:) to plant }{ }^{225}\end{array}$ \\
\hline 5140 & 636 & $\mathrm{zhm}^{3}-$ & $\mathrm{zhm}^{3}-$ & $\mathrm{zhm}^{3}-$ & $\begin{array}{l}\mathrm{zm}-* \\
\mathrm{zhm}^{3}-\end{array}$ & breast, to suckle, milk ${ }^{226}$ \\
\hline 5150 & 586 & & zmxv- & zmxv- & zmxv- & $\begin{array}{l}\text { (OO/HA) to suckle, give or draw } \\
\text { breast milk; (KW) milky, cloudy, } \\
\text { muddy; breast milk }{ }^{227}\end{array}$ \\
\hline 5170 & 588 & ct- & ct- & zt- & zt- & $\begin{array}{l}\text { to split, pull apart (roots, twigs), } \\
\text { to part company }\end{array}$ \\
\hline 5180 & $\begin{array}{l}635 \\
592\end{array}$ & & & $\mathrm{zn}-$ & & (?) to pierce, to sting 228 \\
\hline 5190 & 589 & & & & znk- & $\begin{array}{l}\text { stinging nettles, to sting (like } \\
\text { nettles or with words) }\end{array}$ \\
\hline 5200 & 590 & & & & znq- & to shore up with poles \\
\hline 5210 & 591 & & & & znq-* & $(?)^{229}$ \\
\hline
\end{tabular}

224 This stem is dimly reminiscent of "se battre" and may have been borrowed from French through some intermediary language(s).

${ }^{225}$ An expanded form of $\{\mathrm{zm}-\}$ occurs probably in $\mathrm{HE} / \mathrm{z}^{\prime} \mathrm{am}^{\prime} \mathrm{i} / \mathrm{OO} / \mathrm{zami} / \mathrm{HA} / \mathrm{zam}^{3}$ in'as/ "squirrel."

${ }^{226}$ The unaugmented root occurs possibly in $\mathrm{KW} / \mathrm{zM}^{3} \mathrm{isp}^{3} \mathrm{a} / *<\left\{\mathrm{zm}-=\mathrm{ys}-\mathrm{p}^{3}-\mathrm{h}\right\}$ "taste of milk"; in all other examples it is augmented to $\left\{\mathrm{zhm}^{3}-\right\}$.

227 OO only in /zMxvstu/ "milky."

${ }^{228}$ Possibly in HA /zH'awi/ "hummingbird" and HA/zaH/ "harpoon for catching seal."

${ }^{229}$ In KW /Zнднгłəхsла/* (name from the Newettee dialect meaning "smell of canoe" according to Boas; no indication what it is the name of). 
North Wakashan Comparative Root List (Updated)

\begin{tabular}{|c|c|c|c|c|c|c|}
\hline Root \# & Old \# & $\mathrm{HE}$ & $\mathrm{OO}$ & HA & KW & Gloss \\
\hline 5211 & 592 & $\mathrm{zn}^{3}-$ & $\mathrm{zn}^{3}-$ & $\mathrm{zn}^{3}-$ & $\mathrm{zn}^{3}-$ & emotionally attached, loyal to \\
\hline 5212 & & & & & zc-/zs- & $(?)^{230}$ \\
\hline 5220 & 593 & $(\chi \vee w s-)$ & $(\chi \vee w s-)$ & & zs- & $\begin{array}{l}\text { to foam, ferment, start to boil, } \\
\text { simmer, bubble }{ }^{231}\end{array}$ \\
\hline 5230 & 594 & & & & zł- & (?) to spread out, reach out ${ }^{232}$ \\
\hline 5240 & 595 & zlk- & zlk- & zlk- & zlk- & (?) white, mouldy 233 \\
\hline 5250 & $\begin{array}{l}596 \\
755\end{array}$ & zlx- & zlx- & $c^{3} 1 x-$ & $c^{3} 1 x-$ & $\begin{array}{l}\text { (HE, OO, HA) to stoop, lean } \\
\text { forward; }(\mathrm{KW}) \text { fish swimming } \\
\text { upriver to spawn }\end{array}$ \\
\hline 5260 & 597 & & & & zlxv- & to run on foot \\
\hline 5270 & $\begin{array}{l}598 \\
653 \\
656\end{array}$ & zl $\chi-$ & $\mathrm{zl} \chi-$ & zl $\chi-$ & $\begin{array}{l}\text { cnqv- } \\
\text { cnq- }\end{array}$ & $\begin{array}{l}\text { (HE/OO/HA) to bend over to } \\
\text { move forward, to stoop; (KW) to } \\
\text { hang the head, to stoop }\end{array}$ \\
\hline 5280 & 599 & ck- & ck- & zk- & zk- & to rub, to knead \\
\hline 5300 & 601 & & & & zk- & iron, silver \\
\hline 5310 & $\begin{array}{l}602 \\
603\end{array}$ & & & & $\mathrm{zX}-$ & (?) to repel, fight off ${ }^{234}$ \\
\hline
\end{tabular}

${ }^{230}$ Perhaps in KW /zs.h qv/ "young cedar."

${ }^{231}$ Probably onomatopoeic. See also root no. 5730.

232 Possibly in KW / $\mathrm{zJ}^{3} \mathrm{a}$ // "lake" and a small number of non-reelicitable words including KW /zлм/* "tentacles of squid" and $\mathrm{KW} / \mathrm{zła} / *$ to spread out (small pieces), to spread out property that is piled up."

${ }^{233}$ In words referring to old deteriorating salmon.

${ }^{234}$ In KW /zxila/* "to make war" and possibly KW /zxina/ "maidenhair fern" because maidenhair fern fronds repel water according to http://en.wikipedia.org/wiki/Adiantum. 
North Wakashan Comparative Root List (Updated)

\begin{tabular}{|c|c|c|c|c|c|c|}
\hline Root \# & Old \# & $\mathrm{HE}$ & $\mathrm{OO}$ & HA & KW & Gloss \\
\hline 5320 & 604 & zy- & & & & $(?)^{235}$ \\
\hline 5321 & & & & & zyp- & $(?)^{236}$ \\
\hline 5330 & 605 & & & & zyt-* & $(?)^{237}$ \\
\hline 5340 & 606 & & & & zyt- & $\begin{array}{l}\text { to spread (legs, fingers), to split } \\
\text { stick }\end{array}$ \\
\hline 5350 & 607 & & & & zys-* & $(?)^{238}$ \\
\hline 5360 & 608 & & & & zył- & fresh (meat or fish), new \\
\hline 5370 & $\begin{array}{l}600 \\
609 \\
611\end{array}$ & zyk- & zyk- & $\begin{array}{l}\text { zk- } \\
\text { zyk- }\end{array}$ & zyk- & $\begin{array}{l}\text { (HE/OO/HA) to push or poke } \\
\text { with a stick, to prod, to spur; } \\
\text { (KW also and mainly:) to lever } \\
\text { up (as in digging for clams, } \\
\text { prying up roots or using sprit to } \\
\text { hoist sail), to push with long } \\
\text { thing in cooking berries, to talk } \\
\text { in a pushy way } 239\end{array}$ \\
\hline 5380 & 610 & zyx- & zyx- & zyk- & & to give a feast ${ }^{240}$ \\
\hline
\end{tabular}

235 In HE / z'izizi'ala/ "chickadee"; probably onomatopoeic.

236 Possibly in KW /zibusa/ "irked, annoyed."

${ }^{237}$ In KW /zidaq/* "milky sea eggs."

${ }^{238}$ Possibly onomatopoeic. Occurs in words referring to a sea otter's whistling, a bird's cry and a kind of bird called a cape pigeon. (Cape pigeons are however from the Antarctic area.) ${ }^{239} \mathrm{HA}\{\mathrm{zk}-\}$ said to occur in the Kitamaat dialect only.

240 The HA root could be a semantic extension of root no. 5370. The HE/OO root occurs only in a proper name that is likely a dialect borrowing. 
North Wakashan Comparative Root List (Updated)

\begin{tabular}{|c|c|c|c|c|c|c|}
\hline Root \# & Old \# & $\mathrm{HE}$ & $\mathrm{OO}$ & HA & KW & Gloss \\
\hline 5400 & 612 & & & & zyx- & (?) spotted $^{241}$ \\
\hline 5410 & 615 & zykv- & zykv- & zykv- & & to move, function, operate \\
\hline 5420 & $\begin{array}{l}613 \\
614 \\
616\end{array}$ & zyqv- & zyqv- & zyqv- & $\begin{array}{l}\text { zykv- } \\
\text { zyqv-* }\end{array}$ & $\begin{array}{l}\text { leg or spine-like thing sticking } \\
\text { out, to stretch legs, to poke with } \\
\text { the leg }{ }^{242}\end{array}$ \\
\hline 5430 & 617 & zyq- & zyq- & zyq- & & $\begin{array}{l}\text { to stop vocal noise (speaking, } \\
\text { singing, crying) }\end{array}$ \\
\hline 5440 & 618 & & & & zyq- & (?) to smirk \\
\hline 5450 & 619 & ZXV- & ZXV- & ZXV- & ZXV- & silver (coho) salmon \\
\hline 5460 & 620 & $\mathrm{ZW}-$ & $\mathrm{ZW}-$ & ZW- & ZW- & (?) groove, rib $^{243}$ \\
\hline 5480 & $\begin{array}{l}584 \\
621 \\
645 \\
812\end{array}$ & zwp- & $\begin{array}{l}\text { zwp- } \\
\mathrm{cp-} \\
\mathrm{c}^{3} \mathrm{wp}-\end{array}$ & zwp- & $\begin{array}{l}\text { zp- } \\
\text { cp-* } \\
\text { zwp- } \\
c^{3} w p-\end{array}$ & $\begin{array}{l}\text { soft material (such as cloth) } \\
\text { stuffed into sth. or spread out } \\
\text { (like coat lying on floor); to plug } \\
\text { an opening (with soft material } \\
\text { but also e.g. cork) }\end{array}$ \\
\hline
\end{tabular}

${ }^{241}$ Possibly in KW /zinuma/ and /zinumara/ "(probably:) spotted ratfish" and its synonym /zinəł/ from the Nakoaktok (= Blunden Harbour) dialect.

$242\{$ zykv- $\}$ occurs also in $\mathrm{KW} / \mathrm{zik}^{3} \mathrm{vi}^{3} \mathrm{~S} /$ which refers to the oil of catfish, perch and seal. They are creatures with prominent barbels, a spiny fin and whiskers, respectively. $\mathrm{KW}\{\mathrm{zyqv}-\}^{*}$ means "prong, spear, point" in its examples.

${ }^{243}$ In the word for cockles. Almost all cockle shells have radial ribs. Root could also have the form of $\left\{\mathrm{zXv}_{-}\right\}$or $\left\{\mathrm{z} \chi \mathrm{v}^{-}\right\}$but $\{\mathrm{zw}-\}$ is more likely in view of HA $\{\mathrm{zws}-\}$ "to dig for cockles." ${ }^{244}$ Only three examples of $\{\mathrm{zp}-\} .\{\mathrm{cp}-\}$ only in $\mathrm{KW} / \mathrm{cbam}^{3} \mathrm{a} / *$ "old cedar bark blanket" and OO /cpa/ (meaning not remembered but had to do with cedar bark). $\left\{\mathrm{c}^{3} \mathrm{wp}-\right\}$ only in OO/KW $/ c^{3} u p^{3} \mathrm{ax} /$ "glove, mitten" and derivates. 
North Wakashan Comparative Root List (Updated)

\begin{tabular}{|c|c|c|c|c|c|c|}
\hline Root \# & Old \# & $\mathrm{HE}$ & $\mathrm{OO}$ & $\mathrm{HA}$ & KW & Gloss \\
\hline 5481 & & & & & $\begin{array}{l}\text { zwmyg-, } \\
\text { zwmyk- }\end{array}$ & $\begin{array}{l}\text { pole (for holding out sail or } \\
\text { punting) } \text { ) }^{245}\end{array}$ \\
\hline 5490 & 622 & & & & zwt-* & $(?)^{246}$ \\
\hline 5491 & 630 & zwnh- & zwnh- & & zwnh- & $(?)^{247}$ \\
\hline 5500 & $\begin{array}{l}623 \\
624 \\
625\end{array}$ & zws- & zws- & zws- & zws- & $\begin{array}{l}\text { (HE/OO/KW) to dig under the } \\
\text { surface, to hollow out; grooved } \\
\text { (like cockle shells); (HA) to dig } \\
\text { for cockles }{ }^{248}\end{array}$ \\
\hline 5510 & $\begin{array}{l}626 \\
631\end{array}$ & ZWXV- & zwxv- & zwXV- & ZWXV- & pole, post, log \\
\hline 5520 & 627 & & zwXv- & & zwXV- & $\begin{array}{l}\text { (?) to attract attention to a } \\
\text { desirable good, to advertise a } \\
\text { benefit }^{249}\end{array}$ \\
\hline 5540 & 628 & zwqv- & cwqv- & & & $(?)^{250}$ \\
\hline 5550 & 629 & & & & zwqv- & to bend (stiff, flat, long things) \\
\hline 5560 & 633 & & & & $\mathrm{zqv}-*$ & $(?)^{251}$ \\
\hline
\end{tabular}

${ }^{245}$ In just four words one of which is listed in Boas (1948:197) among derivates of $\{\mathrm{zwXV}-\}$ "pole, post, log."

${ }^{246} \mathrm{In} \mathrm{KW} / \mathrm{zud} \chi \mathrm{qa} / *$ "to have to let go, unable to hold on."

${ }^{247}$ Possibly in KW zwnh-wqv- $\}$ which refers to a sasquatch-like creature with puckered lips.

The only HEOO example is associated with a mask and magic power; it may be a dialect borrowing because the $\mathrm{OO}$ word for a sasquatch-like creature is $/ \mathrm{h}^{3} \mathrm{zi} /$ and the HE one $/ \mathrm{f}^{\prime} \mathrm{ała} /$. ${ }^{248}$ Also in $\mathrm{OO}$ and $\mathrm{KW}$ words referring to platforms in dug-out house.

${ }^{249} \mathrm{In} \mathrm{KW}$ words to do with raising a copper to show it off and promising a potlatch; in OO only in /zuxvzuxvzuxv/ (cry to call chickens, as when going to feed them).

${ }^{250}$ In HE /z'uq ${ }^{3}$ vqл'a/ OO /cuq ${ }^{3}$ vqла/ "rain and snow mixed."

${ }^{251}$ In KW /zrvat/* "dried sockeye salmon." 
North Wakashan Comparative Root List (Updated)

\begin{tabular}{|c|c|c|c|c|c|c|}
\hline Root \# & Old \# & $\mathrm{HE}$ & $\mathrm{OO}$ & HA & KW & Gloss \\
\hline 5570 & & & & zqv- & & opening, clearing \\
\hline 5580 & 632 & cq- & cq- & cqv- & zqv- & $\begin{array}{l}\text { soil, dirt, mud, earth, dust, dirty, } \\
\text { filthy }\end{array}$ \\
\hline 5590 & 634 & & $c \chi-$ & $z \chi-$ & $z \chi-$ & to split, crack (wood, bark) \\
\hline 5600 & & & $\begin{array}{l}\text { zhc-l } \\
\text { zhs- }\end{array}$ & & & $(?)^{252}$ \\
\hline 5610 & 637 & zhs- & zhs- & zhs- & zhs- & bruised, darkened, blue \\
\hline 5620 & 638 & zhkv- & $\begin{array}{l}\text { zhkv- } \\
\text { zhxv- }\end{array}$ & zhkv- & zhkv- & $\begin{array}{l}\text { V-shape widens (as elbow } \\
\text { straightening, blade of jackknife } \\
\text { opening), to spread apart, to } \\
\text { cock }^{253}\end{array}$ \\
\hline 5630 & 639 & & & & zhxv- & (?) to bend, bent ${ }^{254}$ \\
\hline 5640 & 640 & zhxv- & zhxv- & zhxv- & zhxv- & $\begin{array}{l}\text { ooligan (candlefish, Thaleichthys } \\
\text { pacificus) }\end{array}$ \\
\hline 5641 & & & & & zhwhdly- & robin call (unanalyzable) \\
\hline 5650 & 641 & & & & zhqv- & evening \\
\hline 5660 & 642 & $\mathrm{zhq}^{3} \mathrm{v}-$ & $\mathrm{zhq}^{3} \mathrm{v}-$ & & $\mathrm{zhq}^{3} \mathrm{v}-$ & wind off the sea \\
\hline 5670 & 643 & zhq- & zhq- & zhq- & & stained, darkened \\
\hline 5671 & & & & & $\mathrm{zh}^{3} \mathrm{n} \chi-$ & to nod head in pity ${ }^{255}$ \\
\hline
\end{tabular}

${ }^{252}$ In OO /zas.hiit/ [2] "to emerge before going deep (as a whale)."

${ }^{253} \mathrm{OO}\{$ zhxv- $\}$ only in $\{$ zhxv-'sm-d $\}>$ /zaxvsmt/ "to stretch the legs onto e.g. a box, table, stool."

${ }^{254} \mathrm{In} \mathrm{KW} /$ zaxvała/ "bent" KW /zaxvba/ "bent point" and perhaps KW /zaxvs.ћ $\mathrm{Hd} /$ "to break apart, tear to pieces."

${ }^{255} \mathrm{In} \mathrm{KW} / \mathrm{za}^{3} \mathrm{H} \chi \mathrm{a} /$ and $/ \mathrm{za}^{3} \mathrm{H} \chi \mathrm{h}^{3} \mathrm{id} /$ "to nod head in pity for sb." 
North Wakashan Comparative Root List (Updated)

\begin{tabular}{|c|c|c|c|c|c|c|}
\hline Root \# & Old \# & $\mathrm{HE}$ & $\mathrm{OO}$ & HA & KW & Gloss \\
\hline 5680 & 644 & & & & $\mathrm{zh}^{3} \mathrm{t}-$ & heavy swell ${ }^{256}$ \\
\hline 5700 & 646 & $\mathrm{~cm}-$ & $\mathrm{cm}-$ & $\mathrm{cm}-$ & & $(?)^{257}$ \\
\hline 5710 & $\begin{array}{l}647 \\
648\end{array}$ & & cmkv- & & cmkv-* & lump, hump \\
\hline 5720 & 649 & cmxv- & cmxv- & cmxv- & & $\begin{array}{l}\text { trickle, slow or controlled flow of } \\
\text { water }\end{array}$ \\
\hline 5721 & 646 & & & & $\mathrm{~cm}^{3} \mathrm{wt}-*$ & quiet, silent \\
\hline 5730 & 650 & $\begin{array}{l}\text { cyst- } \\
\text { sss- }\end{array}$ & ct- & cysd- & cy- & to hiss (onomatopoeic) (258 $^{258}$ \\
\hline 5740 & $\begin{array}{l}651 \\
717\end{array}$ & cnt- & cnt- & cnt- & & $\begin{array}{l}\text { to roll (ball, hoop, tire), to } \\
\text { revolve }\end{array}$ \\
\hline 5750 & 652 & cns- & cns- & & cns-* & $\begin{array}{l}\text { (?) club, stalk with a ball at the } \\
\text { end } \\
259\end{array}$ \\
\hline 5770 & $\begin{array}{l}654 \\
655\end{array}$ & cnq- & $\begin{array}{l}\operatorname{cn} \chi v^{-} \\
\text {cnq- }\end{array}$ & cnq- & $\operatorname{cn} \chi \mathrm{V}-$ & $\begin{array}{l}\text { (\{cn } \chi \mathrm{v}-\}:) \text { fat (of animal), } \\
\text { blubber, lard; fat as opposed to } \\
\text { lean; ( }\{\mathrm{cnq}-\}:) \text { to cook in fat or } \\
\text { oil, to fry } 260\end{array}$ \\
\hline
\end{tabular}

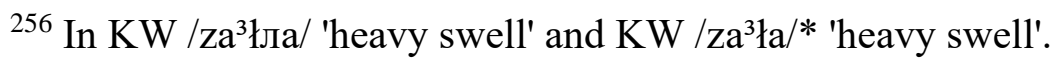

257 Possibly in $\mathrm{HE} / \mathrm{cm}^{3} \mathrm{ic}^{3} \mathrm{a} / \mathrm{OO} / \mathrm{cm}^{3} \mathrm{ic}^{3} / \mathrm{HA} / \mathrm{cmic}^{3} /$ "bored and fed up."

${ }^{258} \mathrm{In} \mathrm{HE} / \mathrm{c}^{\prime}$ ist $\chi \mathrm{a} / \mathrm{OO} / \mathrm{ct} \chi \mathrm{a} / \mathrm{HA} / \mathrm{c}^{\prime}$ isd $\chi \mathrm{a} /$ "to attract attention by hissing" HE $\{$ sss- $\chi-+\mathrm{xsyh}\}>$ /sss $\chi \cdot \hbar^{3} \mathrm{xsi}^{\prime} \mathrm{a} /$ "to say 'sss' through the teeth, to 'whistle' through the teeth" $\mathrm{KW} /$ cici/ "noise used to make children pee."

${ }^{259}$ In words referring to devil's club (Oplopanax horridum). The plant's small flowers form a cluster at the end of a stalk attached to a central flower stem.

${ }^{260} \mathrm{OO}\{\mathrm{cn} \chi \mathrm{V}-\}$ is likely a borrowing from $\mathrm{KW}$ since it occurs only in $\mathrm{OO} / \mathrm{c}^{3} \mathrm{HH} \chi \mathrm{v} /$ "greaves, cracklings (of fat)." 
North Wakashan Comparative Root List (Updated)

\begin{tabular}{|c|c|c|c|c|c|c|}
\hline Root \# & Old \# & $\mathrm{HE}$ & $\mathrm{OO}$ & HA & KW & Gloss \\
\hline 5800 & 657 & & cs- & cs- & cs- & $\begin{array}{l}\text { to swarm, flock together, act as a } \\
\text { group, form a school, gang up; to } \\
\text { press in on sth. }\end{array}$ \\
\hline 5810 & 658 & & cs- & & & $(?)^{261}$ \\
\hline 5820 & $\begin{array}{l}659 \\
743 \\
746\end{array}$ & $\mathrm{c}^{3} \mathrm{sk}-$ & $\mathrm{c}^{3} \mathrm{sk}-$ & $\mathrm{c}^{3} \mathrm{sk}-$ & $\begin{array}{l}\text { csqv- } \\
c^{3} \text { sqv- } \\
c^{3} \text { s- }^{*} \\
c^{3} \text { sk-* }^{2} \\
c^{3} \text { skv-* }^{2}\end{array}$ & $\begin{array}{l}\text { to chirp, twitter (perhaps } \\
\text { onomatopoeic) }^{262}\end{array}$ \\
\hline 5830 & $\begin{array}{l}660 \\
661\end{array}$ & cl- & ch- & & cl- & to make light of sth. ${ }^{263}$ \\
\hline 5840 & 662 & & cl- & & & $(?)^{264}$ \\
\hline 5850 & 663 & & & & clc- & $(?)^{265}$ \\
\hline 5860 & 664 & & clt- & & & to tear (cloth) \\
\hline 5870 & $\begin{array}{l}665 \\
666 \\
687\end{array}$ & cy $\chi^{\mathrm{v}-}$ & $\begin{array}{l}\text { clqv-/ } \\
\text { cl } \chi v- \\
\operatorname{cy} \chi v-\end{array}$ & cyzv- & $\begin{array}{l}\text { clqv- } \\
\text { cl } \chi v-\end{array}$ & sour, fermented; crabapples ${ }^{266}$ \\
\hline 5880 & & & & clq- & & shingle \\
\hline
\end{tabular}

${ }^{261} \mathrm{In} \mathrm{OO} / \mathrm{csa} /$ (meaning not remembered by consultant but had to do with splitting branches or roots).

${ }^{262}$ In words referring to various small birds.

${ }^{263} \mathrm{In} \mathrm{HE} /$ сл'іqа/ OO /сліqa/ "to deny, doubt, disbelieve" and KW words referring to women being good-natured, jolly or mischievous.

${ }^{264}$ In OО /сла/ "to cut through water."

${ }^{265} \mathrm{In} \mathrm{KW} /$ слслі/* (name of a scarlet-coloured berry)

${ }^{266} \mathrm{KW}$ \{clqv- $\}$ only in /слггvzu/ "place where crabapples grow." OO \{clqv-/cl $\left.\chi \mathrm{v}-\right\}$ (not diagnosable which) only in /слұ $\mathrm{vp}^{3} \mathrm{a} /$ "having a fermented taste" which is likely a loan from KW. 
North Wakashan Comparative Root List (Updated)

\begin{tabular}{|c|c|c|c|c|c|c|}
\hline Root \# & Old \# & $\mathrm{HE}$ & $\mathrm{OO}$ & HA & KW & Gloss \\
\hline 5890 & 667 & $\mathrm{cl} \chi-$ & $\mathrm{cl} \chi-$ & $\mathrm{cl} \chi-$ & $\mathrm{cl} \chi-$ & hail, spell of hail \\
\hline 5900 & $\begin{array}{l}668 \\
713\end{array}$ & & & & $\begin{array}{l}\text { ck- } \\
\text { chk- }\end{array}$ & $\begin{array}{l}\text { to slash (like with machete when } \\
\text { cutting a trail), to mow (like with } \\
\text { scythe), to chop, to cut straight } \\
\text { across; (\{chk-\} also specifically:) } \\
\text { to chop into and split sea urchins }\end{array}$ \\
\hline 5901 & 669 & & & & $\begin{array}{l}\text { ck-l } \\
\text { cx- }\end{array}$ & $(?)^{267}$ \\
\hline 5910 & 669 & & & cx- & & $\begin{array}{l}\text { to go up a difficult mountain for } \\
\text { goats }\end{array}$ \\
\hline 5920 & $\begin{array}{l}670 \\
671 \\
681 \\
768\end{array}$ & $\begin{array}{l}\text { cy- } \\
c^{3} y-\end{array}$ & cy- & $\begin{array}{l}c y- \\
c^{3} y-\end{array}$ & cy- & $\begin{array}{l}\text { to transfer liquid or particled } \\
\text { solid (berries, pellets, fish), to } \\
\text { draw water, to dip up, to use the } \\
\text { brailer (e.g. in transferring fish), } \\
\text { to serve at table }{ }^{268}\end{array}$ \\
\hline 5930 & 671 & & & & cyp- & (?) squeezed together ${ }^{269}$ \\
\hline 5940 & 672 & & & cyp- & & $(?)^{270}$ \\
\hline
\end{tabular}

${ }^{267}$ In KW /cxc 3 ola/ "shotgun." Root could be no. 5900 in view of the broad sweep of the pellets from a shotgun cartridge.

${ }^{268} \mathrm{HE}\left\{\mathrm{c}^{3} \mathrm{y}-\right\}$ only in $\left\{\mathrm{c}^{3} \mathrm{y}-\mathrm{xc} \mathrm{c}^{3} \mathrm{~h}^{3} \mathrm{~s}\right\}>/ \mathrm{c}^{3 \prime} \mathrm{ixc} \mathrm{c}^{3 \prime}$ as/ "open place with moss in the woods"; its root is associated with drawing water because its HA counterpart / $\mathrm{c}^{3} \mathrm{ixc} \mathrm{c}^{3} \mathrm{as} /$ "meadow" is said to be so called because water appears when stepping on it.

${ }^{269}$ Possibly in KW /cep/ "halibut or cod roe" and "apron." Perhaps the many buttons of a dancing apron have been associated with roe. Boas (1948) does not explain the association. KW /cєp/ could have a structure $\{\mathrm{c}[\mathrm{h}] \mathrm{yp}-\}$ or $\{\mathrm{c}[\mathrm{h}] \mathrm{y}[\mathrm{h}] \mathrm{p}-\} ;\{\mathrm{c}[\mathrm{h}] \mathrm{yp}-\}$ is assumed. The root $\{\mathrm{cyp}-\}$ could be a cross-family root occurring in proto-Salishan as *-c ip $^{3}$ - "to squeeze (shut), pinch" (Kuipers 2002:31).

${ }^{270}$ In two words referring to a person limping. 
North Wakashan Comparative Root List (Updated)

\begin{tabular}{|c|c|c|c|c|c|c|}
\hline Root \# & Old \# & $\mathrm{HE}$ & $\mathrm{OO}$ & HA & KW & Gloss \\
\hline 5950 & 673 & & & & cymh-* & $(?)^{271}$ \\
\hline 5960 & 674 & $\begin{array}{l}\text { cymh- } \\
\mathrm{cm}^{3} \mathrm{~s}-\end{array}$ & cymh- & cymh- & & $(?)^{272}$ \\
\hline 5970 & 675 & cyt- & cyt- & & cyt- & to tilt, lean, list, slope \\
\hline 5980 & & & & $\mathrm{cyn}^{3}-$ & & to cook shellfish \\
\hline 5990 & $\begin{array}{l}676 \\
680 \\
778\end{array}$ & cył- & cył- & cyk- & $\begin{array}{l}\text { cys- } \\
\text { cył- } \\
\text { c }^{3} y \text { - }^{*}\end{array}$ & $\begin{array}{l}\text { rough, frayed, unravelled, } \\
\text { ruffled, bushy; blunt (point) }\end{array}$ \\
\hline 6000 & 677 & & & & cys-* & $(?)^{274}$ \\
\hline 6010 & 678 & & cys- & & & $(?)^{275}$ \\
\hline 6020 & $\begin{array}{l}679 \\
683\end{array}$ & cyx- & cyx- & cyx- & $\begin{array}{l}\text { cył- } \\
\text { cyx-* }\end{array}$ & $\begin{array}{l}\text { water running (in stream, flood or } \\
\text { trickle) })^{276}\end{array}$ \\
\hline
\end{tabular}

${ }^{271}$ In KW /cimaxvḷ/** "to have a slight disagreement."

${ }^{272}$ In words referring to horse clams. Root may mean "to point," like $\left\{c^{3} m-\right\}$ (no. 6440), in reference to their conspicuous siphons.

273 1. All KW examples contain \{cys-\} except KW /ciłла/ "rough, frayed, unravelled, bushy" $\mathrm{KW}\{$ cył-\&yd $\}>/ \mathrm{cil}^{3} \mathrm{~h}^{3} \mathrm{id} /$ "to start fraying, become unravelled" and $\mathrm{KW} / \mathrm{c}^{3} \mathrm{iła} / *$ "to put loose things somewhere." The latter example's $/ \mathrm{c}^{3} /$ is likely a typing error by Boas. For an example where it is $\mathrm{HE} / \mathrm{OO} / \mathrm{HA}$ that have $|\mathrm{s}|$ but $\mathrm{KW}|\mathrm{H}|$ see suffix no. 4250. 2. This root parallels protoSalishan *-cix- in *cix-ups "fisher (animal)" (Kuipers 2002:26). Kuipers only says -ups means "tail" but *-cix- no doubt means "bushy," fishers having a bushy tail.

${ }^{274} \mathrm{In} \mathrm{KW} /$ cis/* "long round fish-basket (for salmon-trap)" and derivates.

275 In OO /сіsла/ "water bug."

276 The KW root comparable to HE/OO/HA \{cyx- $\}$ in terms of productivity and semantics is $\{\mathrm{cxv}\}$ (root no. 6140). Boas (1948) gives four examples of KW \{cyl-\} but they could not be reelicited. The only elicited example of KW $\{$ cył- $\}$ is KW $\{$ cył-h $\chi-\mathrm{h}\}>$ /ciła $\chi \mathrm{a} /$ "to sprinkle 
North Wakashan Comparative Root List (Updated)

\begin{tabular}{|c|c|c|c|c|c|c|}
\hline Root \# & Old \# & $\mathrm{HE}$ & $\mathrm{OO}$ & HA & KW & Gloss \\
\hline 6050 & 682 & & & & cyx- & $\begin{array}{l}\text { to burn sth. off, to singe, to } \\
\text { cauterize }\end{array}$ \\
\hline 6070 & 684 & & & & cyx- & (?) out of place, disarranged ${ }^{277}$ \\
\hline 6080 & 685 & & cykv- & & cykv- & $\begin{array}{l}\text { to shovel, to scoop, to lever up } \\
\text { sth. }\end{array}$ \\
\hline 6090 & 686 & & cyqv- & & & $(?)^{278}$ \\
\hline 6100 & 801 & & & $c^{3} \mathrm{yq}-$ & $\begin{array}{l}\text { cyq- } \\
\text { c }^{3} \mathrm{yq}^{-}\end{array}$ & $\begin{array}{l}\text { to scoop out of the water, to } \\
\text { skim }^{279}\end{array}$ \\
\hline 6110 & $\begin{array}{l}688 \\
689 \\
690\end{array}$ & $\operatorname{cy} \chi_{-}^{-}$ & $\begin{array}{l}\text { cyq- } \\
\text { cy } \chi-\end{array}$ & $\operatorname{cy} \chi_{-}^{-}$ & $\operatorname{cy} \chi-$ & $\begin{array}{l}\text { 1. to use heat to extract, preserve } \\
\text { or purify fat; to fry sth.; } 2 \text {. to } \\
\text { sting }^{280}\end{array}$ \\
\hline 6130 & $\begin{array}{l}691 \\
692\end{array}$ & ckv- & $\begin{array}{l}\text { ckv- } \\
\text { cxv- }\end{array}$ & ckv- & ckv-* & $\begin{array}{l}\text { rock fence, fish trap made of } \\
\text { stones }\end{array}$ \\
\hline 6140 & 693 & $(\operatorname{cyx}-)$ & $(\operatorname{cyx}-)$ & $(\operatorname{cyx}-)$ & cxv- & $\begin{array}{l}\text { running water (like tide, current, } \\
\text { waterfall, overflow) }\end{array}$ \\
\hline
\end{tabular}

down." KW \{cyx-\}* occurs in /сіхла/* mentioned in Lincoln \& Rath (1980:146) as a place name meaning "water flowing down."

${ }^{277}$ In KW /cixała/ "tilted" KW /cixcxsmala/ "having ruffled feathers."

${ }^{278}$ In words referring to curdled blood.

${ }^{279}$ In HA / c $^{3}$ iqa/ "to fish with a dipnet, to scoop (fish) out of the water" KW /ciruła/ "to skim off from surface with spoon" and possibly $\mathrm{KW} / \mathrm{c}^{3} \mathrm{iris} /$ "sea monster" $\mathrm{KW} / \mathrm{c}^{3} \mathrm{i} \mathrm{rac}^{3} \mathrm{i} / *$ "monster receptacle" (name of door of /Q ${ }^{3} u m u g v a /$ 's house)."

${ }^{280}$ The meaning "to sting" is preserved in two words from Boas (1948) that refer to killing wounded game but could not be reelicited. This root is a cross-family one. Its proto-Salishan correlate is $*_{-} \mathrm{c}^{3} \mathrm{i} \chi$ - "1. prickly, stinging; 2 . burn, fry; 3. cold" (Kuipers 2002:33). 
North Wakashan Comparative Root List (Updated)

\begin{tabular}{|c|c|c|c|c|c|c|}
\hline Root \# & Old \# & $\mathrm{HE}$ & $\mathrm{OO}$ & $\mathrm{HA}$ & KW & Gloss \\
\hline 6150 & $\begin{array}{l}694 \\
702\end{array}$ & cxv- & $\begin{array}{l}\text { cXv- } \\
\mathrm{cwXV}-\end{array}$ & & $\mathrm{c \chi v}-*$ & $\begin{array}{l}\text { quick chopping motions (as when } \\
\text { adzing off fine chips), quick } \\
\text { time-beating }\end{array}$ \\
\hline 6160 & 695 & & & & cxv-* & $\begin{array}{l}\text { (?) to gather mussels, to pry off } \\
\text { mussels }\end{array}$ \\
\hline 6170 & & & & & $\mathrm{cxV}^{*} *$ & $(?)^{283}$ \\
\hline 6180 & $\begin{array}{l}696 \\
697\end{array}$ & cwp- & cwp- & cwp- & $\begin{array}{l}\text { cwp- } \\
\text { swp- }\end{array}$ & $\begin{array}{l}\text { spotted, with black or dark spots } \\
\text { (like fledgling robins) }\end{array}$ \\
\hline 6181 & & & & & $\mathrm{cwm}^{3} \mathrm{ws}-*$ & to break or crush sth. brittle \\
\hline 6190 & 698 & & & cwt- & cwt-* & $(?)^{284}$ \\
\hline 6200 & 699 & cWs- & cWs- & cWs- & cWs- & dried-out, brittle, easily broken \\
\hline 6210 & 700 & & & & cw1- & to shatter, crumble 285 \\
\hline 6220 & 701 & cwkv- & cwkv- & & cwkv- & to shatter, crumble \\
\hline 6240 & 703 & cwqv- & cwqv- & cwqv- & & to beg, to go and ask for sth. \\
\hline 6250 & 704 & & & & $\begin{array}{l}\text { cwqv-*/ } \\
\mathrm{cw} \chi \mathrm{v}-*\end{array}$ & $(?)^{286}$ \\
\hline
\end{tabular}

${ }^{281}$ Lincoln \& Rath (1980:147) mistakenly identifies the KW root as $\{\mathrm{cxv}-\} *$ instead of $\left\{\mathrm{c} \chi \mathrm{v}^{-}\right\}$. Boas (1948:200) lists $\{\mathrm{c} \chi \mathrm{V}-\}^{*}$.

282 Only two examples. Root could be a misspelled alternant $\{$ cyxv- $\}$ of no. 6080.

${ }^{283}$ Perhaps in KW /cxva ${ }^{3} \mathrm{Mt} / *$ "heat (in poetry)" but the suffix would also be unknown.

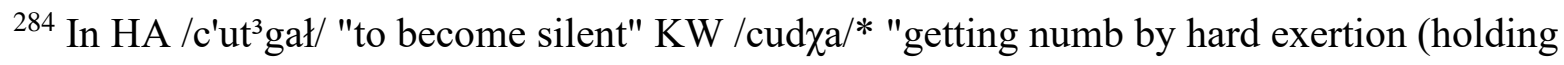
pen, etc.)." The KW item is suspiciously similar to /zud $\chi \mathrm{a} / *$ "to have to let go, unable to hold on" (only example of $\left\{\mathrm{zwt}_{-}\right\}^{*}$ ) and the HA example may not really be HA, said one of the consultants it was elicited from.

${ }^{285}$ In KW /culixa/ "to shatter on impact (canoes), to crush up into fine pieces."

${ }^{286} \mathrm{In} \mathrm{KW} / \mathrm{cu} \chi \mathrm{v} \cdot \mathrm{h}^{3} \mathrm{H} \varepsilon^{3 / *}$ "slime on body." 
North Wakashan Comparative Root List (Updated)

\begin{tabular}{|c|c|c|c|c|c|c|}
\hline Root \# & Old \# & $\mathrm{HE}$ & $\mathrm{OO}$ & $\mathrm{HA}$ & KW & Gloss \\
\hline 6260 & 705 & & & & $\mathrm{cw}^{3} \mathrm{kv}-$ & $\begin{array}{l}\text { flute- or trumpet-shaped, } \\
\text { puckered (lips) }\end{array}$ \\
\hline 6270 & $\begin{array}{l}706 \\
824\end{array}$ & cqv- & cqv- & & $\mathrm{c}^{3} \mathrm{qv}-$ & $(?)^{287}$ \\
\hline 6280 & 707 & $c \chi^{v-}$ & $c \chi v-$ & $c \chi v-$ & $c \chi \mathrm{v}-*$ & $\begin{array}{l}\text { (HE/OO/HA) to flap (like fish } \\
\text { when caught); (KW) stranded } \\
\text { (like whale or fish) } \\
288\end{array}$ \\
\hline 6290 & 708 & & & & cq- & $\begin{array}{l}\text { thimbleberry (Rubus parviflorus } \\
\text { Nutt.) }\end{array}$ \\
\hline 6300 & 709 & & & & $\begin{array}{l}\mathrm{c} \chi- \\
\operatorname{ch} \chi-\end{array}$ & $\begin{array}{l}\text { to walk with quick short steps (in } \\
\text { dancing), quick time-beating }\end{array}$ \\
\hline 6310 & 710 & & & & ch-* & $(?)^{289}$ \\
\hline 6330 & 711 & chs- & chs- & chs- & chs- & $\begin{array}{l}\text { to throw (pour, channel, move) a } \\
\text { liquid }^{290}\end{array}$ \\
\hline 6340 & 712 & chs- & & & & $(?)^{291}$ \\
\hline 6350 & 714 & & & & chk- & $(?)^{292}$ \\
\hline
\end{tabular}

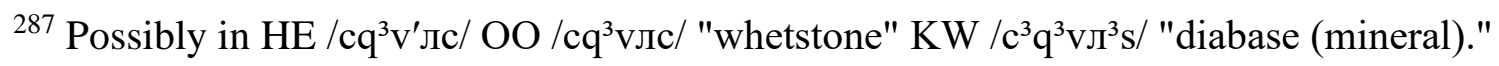
${ }^{288}$ Boas's examples of the KW root may all be typing errors, their root really being $\{\mathrm{cxv}-\}$, alternant of $\{\mathrm{ckv}-\}$ (root no. 6130).

${ }^{289} \mathrm{In} \mathrm{KW}\{\mathrm{ch}-=\mathrm{hs}\}>/ \mathrm{Ca}^{3} \mathrm{as} / *$ (place name glossed as "place of flowing") and unanalyzable $\mathrm{KW}$ $/ \mathrm{Ca}^{3} \mathrm{imi}$ ult/* (name of a place in Nimpkish country). See also the comment on root no. 6400. ${ }^{290}$ Probably occurs also in $\mathrm{KW} / \mathrm{cas} . \hbar^{3} \mathrm{H} \varepsilon^{3 /}$ "fish tail" where it refers to the water displaced when the tail fin moves to generate thrust.

${ }^{291}$ Possibly in HE /c'aixvsmkv/ (name of a small light-coloured bird with dark spots all over the body).

${ }^{292}$ In words referring to bracken fern and sword fern. Unclear what the root might refer to; could be the rhizomes or the fronds ("leaves"). 
North Wakashan Comparative Root List (Updated)

\begin{tabular}{|c|c|c|c|c|c|c|}
\hline Root \# & Old \# & $\mathrm{HE}$ & $\mathrm{OO}$ & HA & KW & Gloss \\
\hline 6360 & $\begin{array}{l}715 \\
716\end{array}$ & $\begin{array}{l}\text { chk- } \\
\text { khk- }\end{array}$ & chk- & & chk-* & $\begin{array}{l}\text { (HE/OO) to hop, jump on one } \\
\text { foot; (OO also:) to poke with one } \\
\text { leg (like dog scratching an itch); } \\
\text { (KW) (a kind of drum rhythm) }{ }^{293}\end{array}$ \\
\hline 6370 & $\begin{array}{l}718 \\
719 \\
807\end{array}$ & chxv- & chxv- & $\begin{array}{l}\text { chxv- } \\
c^{3} h \times v-\end{array}$ & $\begin{array}{l}\text { chxv- } \\
c^{3} h \times v-\end{array}$ & to leak, drip ${ }^{294}$ \\
\hline 6380 & 720 & & & & chq- & $\begin{array}{l}\text { rising up steeply (like wall, } \\
\text { partitioning board, roadblock) }\end{array}$ \\
\hline 6390 & 721 & chq- & chq- & chq- & & to chase away, to dismiss \\
\hline 6400 & 710 & & & & $\operatorname{ch} \chi-$ & $(?)^{295}$ \\
\hline 6410 & 722 & & & & $\operatorname{ch}^{3} x-$ & oily, slippery; wet ${ }^{296}$ \\
\hline 6411 & & & & $c^{3} b d y^{3}-$ & & (onomatopoeia, in bird names) \\
\hline
\end{tabular}

${ }^{293} \mathrm{HE}\{$ khk- $\}$ was used instead of $\{$ chk- $\}$ by younger speaker Doreen Robinson. KW in /cakла/* and /cagasil ${ }^{3} \varepsilon l a / *$ which refer to ways of time-beating Boas (1948:201) explains with musical notes.

${ }^{294} 1$. Occurs also in the HE/OO word for mother of pearl, a substance secreted continuously by molluscs' mantle tissue. 2. In Boas (1948:219) several derivates of KW $\left\{\mathrm{c}^{3} \mathrm{hxv}-\right\}$ are mistakenly listed with the rounded uvular fricative instead of the rounded velar one.

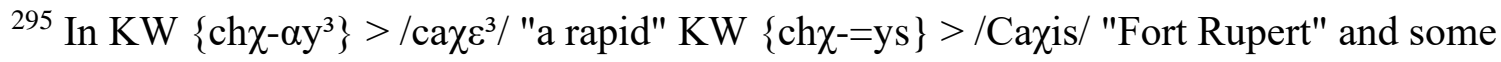
personal and place names deriving from $\{\operatorname{ch} \chi-=y s\}$. Not diagnosable if the stem $\{\operatorname{ch} \chi-\}$ is monomorphemic or analyzable as $\{\mathrm{ch}-\mathrm{h} \chi-\}$ (root no. 6310 plus suffix no. 6200). That the glosses for some $\{\operatorname{ch} \chi-\}$-derivates refer to rapids or a stream flowing down could be folk-etymology influenced by $\left\{\mathrm{c}^{3} \mathrm{~h}-\mathrm{h} \chi-\right\}$ "mass of water running down."

${ }^{296}$ The same connection between "oily/slippery" and "wet" occurs with proto-Salishan *-c3al-, *-c³als- and *-nis-, *-nos-, *-nas- (Kuipers 2002:30, 71-72). 
North Wakashan Comparative Root List (Updated)

\begin{tabular}{|c|c|c|c|c|c|c|}
\hline Root \# & Old \# & $\mathrm{HE}$ & $\mathrm{OO}$ & HA & KW & Gloss \\
\hline 6420 & $\begin{array}{l}723 \\
832\end{array}$ & $\begin{array}{l}\mathrm{c}^{3} \mathrm{p}- \\
\mathrm{c}^{3} \mathrm{hp}-\end{array}$ & $\begin{array}{l}\mathrm{c}^{3} \mathrm{p}- \\
\mathrm{c}^{3} \mathrm{hp}-\end{array}$ & $\begin{array}{l}\mathrm{c}^{3} \mathrm{p}- \\
\mathrm{c}^{3} \mathrm{hp}-\end{array}$ & $\begin{array}{l}\mathrm{c}^{3} \mathrm{p}- \\
\mathrm{c}^{3} \mathrm{hp}-\end{array}$ & 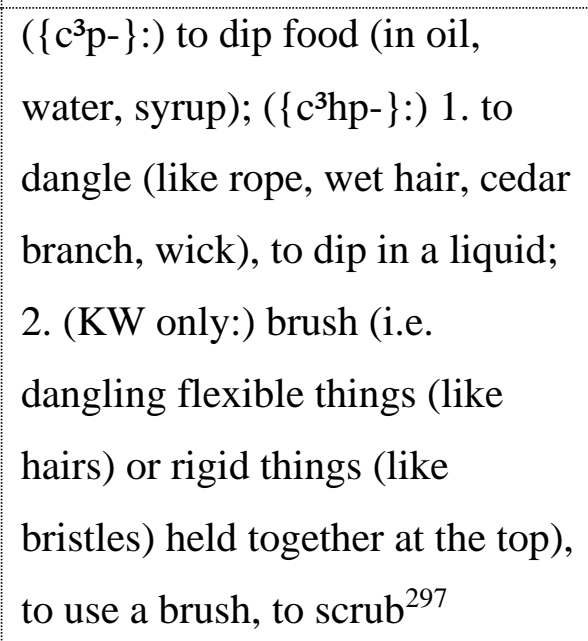 \\
\hline 6440 & 724 & $c^{3} \mathrm{~m}$ & $c^{3} \mathrm{~m}-$ & $c^{3} m-$ & $\begin{array}{l}c^{3} m- \\
c^{3} \mathrm{hm}^{3-}\end{array}$ & $\begin{array}{l}\text { to stick out, point, poke (like with } \\
\text { index-finger), to point out sth. }{ }^{298}\end{array}$ \\
\hline 6450 & 725 & $\mathrm{c}^{3} \mathrm{mt}-$ & $\mathrm{c}^{3} \mathrm{mt}-$ & $\begin{array}{l}c^{3} m t- \\
c^{3} n t-\end{array}$ & $\mathrm{c}^{3} \mathrm{mt}-$ & $\begin{array}{l}\text { to stretch out straight, standing } \\
\text { up straight, to stand on tiptoes }\end{array}$ \\
\hline 6470 & 727 & $\mathrm{c}^{3} \mathrm{mk}-$ & $c^{3} m k-$ & $c^{3} m^{3} k-$ & $\mathrm{c}^{3} \mathrm{mk}-$ & $\begin{array}{l}\text { to be careful with food, ration, } \\
\text { distribute sparingly }\end{array}$ \\
\hline 6480 & 728 & & & & $c^{3} m x^{-*}$ & $(?)^{299}$ \\
\hline 6490 & $\begin{array}{l}729 \\
730\end{array}$ & $\begin{array}{l}c^{3} m q v- \\
c^{3} m q-\end{array}$ & $\begin{array}{l}c^{3} m q v- \\
c^{3} m q-\end{array}$ & $\begin{array}{l}\mathrm{c}^{3} \mathrm{mqv}- \\
\mathrm{c}^{3} \mathrm{mq-}\end{array}$ & $c^{3} m q v-$ & $\begin{array}{l}\text { to force through a narrow } \\
\text { opening } \\
300\end{array}$ \\
\hline
\end{tabular}

${ }^{297}$ Proto-Salishan correlates: *-zəbł- "eyebrow, eyelash" (Kuipers 2002:24) and *-c ${ }^{3}$ aba 7 "cedar root" (ib.:31). The latter supposed root is near-identical in form to HE/OO/KW \{ $\mathrm{c}^{3} \mathrm{hp}$ $+\mathrm{h} \chi\}$ HA \{ $\left.\mathrm{c}^{3} \mathrm{hp}-+\mathrm{hq}\right\}$ (HE/OO) "leaves of red cedar" (HA) "branches of red cedar (used as diarrhea medicine)" (KW) "leaves or branches of red or yellow cedar (for dipping in water to collect herring eggs)."

${ }^{298}$ The augmented form $\left\{\mathrm{c}^{3} \mathrm{hm}^{3}-\right\}$ occurs in KW words referring to icicles and a kind of transparent stone that is used as ear ornament and probably icicle-like in shape. Proto-Salishan correlate: ${ }^{*}-\mathrm{c}^{3} 3 \mathrm{~m}$ - "sharp, pointed." (Kuipers 2002:30).

${ }^{299} \mathrm{In} \mathrm{KW} / \mathrm{c}^{3} \mathrm{Mxa} / *$ "to feel for sth. (e.g. lice) with the finger."

${ }^{300}$ In words referring to swallowing whole, crawling through a small hole and eel. 
North Wakashan Comparative Root List (Updated)

\begin{tabular}{|c|c|c|c|c|c|c|}
\hline Root \# & Old \# & $\mathrm{HE}$ & $\mathrm{OO}$ & HA & KW & Gloss \\
\hline 6510 & 853 & $c^{3} h \chi-$ & $\mathrm{c}^{3} \mathrm{~h} \chi-$ & $c^{3} \mathrm{~h} \chi-$ & $c^{3} m \chi-$ & fibre (from nettles or fireweed) ${ }^{301}$ \\
\hline & 854 & & & & & \\
\hline 6520 & 731 & & & & $\begin{array}{l}\mathrm{chm}^{3-*} \\
\mathrm{c}^{3} \mathrm{~m}^{3-} \\
\mathrm{c}^{3} \mathrm{hm}^{3-}\end{array}$ & $\begin{array}{l}\text { to melt, thaw, dissolve, wear } \\
\text { away; be consumptive }\end{array}$ \\
\hline 6521 & & & & & $\mathrm{c}^{3} \mathrm{dhq}-$ & woman $^{303}$ \\
\hline 6530 & 732 & $c^{3} t-$ & $c^{3} t-$ & $c^{3} t-$ & $c^{3} t-$ & $\begin{array}{l}\text { to crack, split, burst, fissure; to } \\
\text { open up (so inside shows), to } \\
\text { reveal }\end{array}$ \\
\hline 6540 & $\begin{array}{l}733 \\
745 \\
767 \\
773 \\
774\end{array}$ & $\begin{array}{l}c^{3} x^{-} \\
c^{3} x- \\
h c^{3} s- \\
c^{3} y t-\end{array}$ & $\begin{array}{l}\mathrm{c}^{3} \mathrm{kd}- \\
\mathrm{hc}^{3}- \\
\mathrm{c}^{3} \mathrm{yt}-\end{array}$ & $\begin{array}{l}\mathrm{c}^{3} \mathrm{t}- \\
\mathrm{h} \mathrm{c}^{3} \mathrm{t}- \\
\mathrm{c}^{3} \mathrm{yt}- \\
\mathrm{c}^{3} \mathrm{ht}-\end{array}$ & $\begin{array}{l}\mathrm{c}^{3} \mathrm{t}- \\
\mathrm{c}^{3} \mathrm{~s}- \\
\mathrm{h}^{3} \mathrm{~s}- \\
\mathrm{h}^{3} \mathrm{hs}- \\
\mathrm{c}^{3} \mathrm{yt}-\end{array}$ & $\begin{array}{l}\text { liquid or muck expelled under } \\
\text { pressure, to squirt, spout, sneeze, } \\
\text { diarrhoea }^{304}\end{array}$ \\
\hline
\end{tabular}

${ }^{301}$ Proto-Salishan correlate: $*_{-} c^{3} \mathrm{uy} \chi-{ }^{*}-\mathrm{c}^{3}$ ay $\chi$ - "fireweed" (Kuipers 2002:34).

$302\left\{\mathrm{chm}^{3}-\right\}$ occurs in the stems $\left\{\mathrm{chm}^{3} \mathrm{hqv}-\right\}$ and $\left\{\mathrm{chm}^{3} \mathrm{lqv}-\right\}$ "water dripping" of which altogether five examples occur in Boas (1948:201) but none of which could be reelicited. ProtoSalishan correlate: *-c ${ }^{3}$ əm $\chi$ "disappear, wear away" (Kuipers 2002:31).

${ }^{303}$ Boas reports (1948:208) that $\mathrm{KW} / \mathrm{c}^{3} \mathrm{daq} /$ is interpreted locally as meaning "split in crotch," in other words, that it analyzes as $\left\{c^{3} t-=h q\right\}$ with root no. 6530 and suffix no. 6100 . He indicates with a question mark that he is distrustful of this interpretation. Indeed the term could as well contain the alternant $\left\{\mathrm{c}^{3} \mathrm{t}-\right\}$ of root no. 6540 so that it means "squirting at crotch." Nor is the suffix necessarily no. 6100 , if there is a suffix in $\left\{\mathrm{c}^{3} \mathrm{dhq}-\right\}$ at all. It could be a borrowing. ${ }^{304}$ Alternants other than $\left\{c^{3} y t-\right\}$ and HE $\left\{c^{3} x_{-}\right\}$HA $\left\{c^{3} h t-\right\}$ are followed by an element $\{-x-\}$ or $\left\{-\mathrm{X}^{\bullet}\right\}$ of uncertain meaning but treated as a suffix because attested also after roots without formal or semantic connection to this one. The alternants beginning in $|\mathrm{h}|$ specifically refer to sneezing. $\left\{\mathrm{c}^{3} \mathrm{yt}-\right\}$ occurs mostly in words to do with cormorants, known for their runny droppings. 
North Wakashan Comparative Root List (Updated)

\begin{tabular}{|c|c|c|c|c|c|c|}
\hline Root \# & Old \# & $\mathrm{HE}$ & $\mathrm{OO}$ & HA & KW & Gloss \\
\hline & $\begin{array}{r}833 \\
2463\end{array}$ & & & & & \\
\hline 6550 & 734 & $c^{3} n-$ & $c^{3} n-$ & $c^{3} n-$ & $c^{3} n-$ & $\begin{array}{l}\text { to proceed in file (as in a } \\
\text { procession), to be part of a } \\
\text { sequence of events, to move in } \\
\text { mass rapidly (like tidal rapids, } \\
\text { clouds or wind) }\end{array}$ \\
\hline 6560 & $\begin{array}{l}736 \\
747 \\
751 \\
838\end{array}$ & $\begin{array}{l}c^{3} n ł d- \\
c^{3} l d-\end{array}$ & $\begin{array}{l}c^{3} n ł d- \\
c^{3} l d-\end{array}$ & $c^{3} v_{-}-$ & $\begin{array}{l}c^{3} n d- \\
c^{3} 1-\end{array}$ & $(?)^{305}$ \\
\hline 6570 & 735 & & $c^{3} n t-$ & & & $(?)^{306}$ \\
\hline 6580 & 737 & $c^{3} n c-$ & $c^{3} n c-$ & $c^{3} n c-$ & & $\begin{array}{l}\text { beads (of sweat), bubbles (as of } \\
\text { gas in a liquid), bumps on the } \\
\text { skin }\end{array}$ \\
\hline 6590 & 738 & & & & $c^{3} n x-$ & to singe $\mathrm{e}^{307}$ \\
\hline 6600 & $\begin{array}{l}739 \\
740\end{array}$ & $\mathrm{c}^{3} \mathrm{lkv}-$ & $\mathrm{c}^{3} \mathrm{kvv}-$ & $\begin{array}{l}c^{3} n k v- \\
c^{3} l k v-\end{array}$ & $c^{3} n k v-$ & (?) agitation, in turmoil ${ }^{308}$ \\
\hline
\end{tabular}

${ }^{305}$ In words referring to being surprised, startled, shocked, frightened.

${ }^{306}$ In OO $/ \mathrm{c}^{3} \mathrm{Hta} /$ "lean salmon." See comment on $\left\{\mathrm{c}^{3} \mathrm{ynh}-\right\}$ (root no. 6870).

${ }^{307} \mathrm{In} \mathrm{KW} / \mathrm{c}^{3} \mathrm{Hxa} /$ "to singe." A possible additional example is $\mathrm{KW} / \mathrm{c}^{3} \mathrm{Hkv} /$ [1] "furious, angry" if its structure is $\left\{\mathrm{c}^{3} \mathrm{nx}-=\mathrm{kv}\right\}$. It could however also be $\left\{\mathrm{c}^{3} \mathrm{x}-=\mathrm{kv}\right\}$, with root no. 6800 .

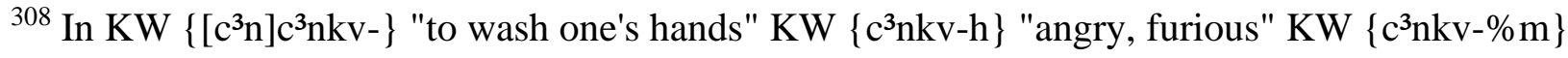
"cause of anger" and HE/OO $\left.\left\{\mathrm{c}^{3} \mathrm{lk}^{3} \mathrm{v}\right\}-\right\} \mathrm{HA} / \mathrm{KW}\left\{\mathrm{c}^{3} \mathrm{nk}^{3} \mathrm{v}-\right\}$ "to wriggle, squirm (like worm), to churn (like stomach content when nauseated). HA $\left\{\mathrm{c}^{3} \mathrm{lkv}-\right\}$ occurs possibly in $/ \mathrm{c}^{3} л \mathrm{c}^{3} л \mathrm{лv} \mathrm{p}^{31} \mathrm{ika} /(\mathrm{a}$ kind of woodpecker). 
North Wakashan Comparative Root List (Updated)

\begin{tabular}{|c|c|c|c|c|c|c|}
\hline Root \# & Old \# & $\mathrm{HE}$ & $\mathrm{OO}$ & $\mathrm{HA}$ & KW & Gloss \\
\hline 6610 & 741 & $\mathrm{c}^{3} \mathrm{lkv}-$ & $\mathrm{c}^{3} \mathrm{lkv}-$ & $\mathrm{c}^{3} \mathrm{lkv}-$ & $c^{3} n k v-$ & $\begin{array}{l}\text { to pay out line, stream out, } \\
\text { stream towards, wind off (rope, } \\
\text { net) }\end{array}$ \\
\hline 6620 & 767 & & & & $\begin{array}{l}c^{3} n^{3} y \chi v-* \\
c^{3} n^{3} y \chi^{*}\end{array}$ & $(?)^{309}$ \\
\hline 6630 & $\begin{array}{l}742 \\
744\end{array}$ & $c^{3} \mathrm{~S}^{-}$ & $c^{3} s-$ & $c^{3} S_{-}$ & $c^{3} S^{-}$ & $\begin{array}{l}\text { bowels, offensive in odour (like } \\
\text { bowel content), filthy, long and } \\
\text { tube-shaped (like bowels) }\end{array}$ \\
\hline 6640 & & & & & $\mathrm{c}^{3} \mathrm{sqv}-*$ & $(?)^{310}$ \\
\hline 6650 & 748 & $\begin{array}{l}c^{3} 1-/ \\
c^{3} 1-\end{array}$ & $\begin{array}{l}c^{3} 1-1 \\
c^{3} 1-\end{array}$ & $\begin{array}{l}c^{3} 1-1 \\
c^{3} 1-\end{array}$ & $\begin{array}{l}c^{3} k-1 \\
c^{3} l-\end{array}$ & $\begin{array}{l}\text { (?) to shine, reflect light; a flash } \\
\text { of light } \\
311\end{array}$ \\
\hline 6660 & 749 & $c^{3} 1-$ & $c^{3} 1-$ & $c^{3} 1-$ & $c^{3} 1-$ & to weave $\mathrm{e}^{312}$ \\
\hline 6670 & 751 & $c^{3} l t-$ & & $c^{3} 1 t-$ & $c^{3} l t-*$ & $\begin{array}{l}\text { to split, divide, move apart (e.g. } \\
\text { stack of papers, crowd, dried } \\
\text { ooligans on sticks), to split with } \\
\text { the grain (wood) } \\
313\end{array}$ \\
\hline 6680 & $\begin{array}{l}752 \\
750\end{array}$ & & & & $c^{3} 1 s-$ & $\begin{array}{l}\text { to ration, use or distribute } \\
\text { sparingly }\end{array}$ \\
\hline 6690 & $\begin{array}{l}753 \\
754\end{array}$ & $\mathrm{c}^{3} \mathrm{l} \mathrm{k}-$ & $c^{3} 1 k-$ & $c^{3} l_{k}-$ & $\begin{array}{l}\mathrm{c}^{3} \mathrm{lk}- \\
\mathrm{c}^{3} \mathrm{~lx}-\end{array}$ & $\begin{array}{l}\text { interwoven, intertwined, } \\
\text { interlocked (like strands in cloth, }\end{array}$ \\
\hline
\end{tabular}

${ }^{309}$ In non-reelicitable words from the Koskimo dialect referring to barnacles and intestines.

$310 \mathrm{In} \mathrm{KW} / \mathrm{c}^{3} \mathrm{ic}^{3} \mathrm{sqvanabalas} / *$ "sons of men who have given potlatch" and $/ \mathrm{c}^{3} \mathrm{~S} r \mathrm{r} H W \varepsilon^{3} / *$ "sth. small attached on sth., stomach of porpoise."

311 Perhaps in the stem $\left\{\mathrm{c}^{3} 1 \mathrm{yq}-\right\}$ "to shine, reflect light" and in HA only $\left\{\mathrm{c}^{3}\right.$ lhq- + my $\left.\mathrm{y}^{3}-\mathrm{h}\right\}>$ $/ \mathrm{c}^{3} \mathrm{л}^{\prime} \mathrm{aq}^{3} \mathrm{My}^{3} \mathrm{a} /$ "argillite (the black slate used by Haida carvers)." The HA stem $\left\{\mathrm{c}^{3}\right.$ lhq- $\}$ is reminiscent of OO $\left\{\mathrm{k}^{3} \mathrm{l}-+\mathrm{hq}\right\}>/ \mathrm{k}^{3} \mathrm{~J}^{3} \mathrm{aq} /$ "natural crystal."

312 In $\mathrm{HE} / \mathrm{c}^{3} \mathrm{~J}^{\prime} \mathrm{a} / \mathrm{OO} / \mathrm{HA} / \mathrm{c}^{3} л а / \mathrm{KW} / \mathrm{c}^{3} л \varepsilon^{3} / *$ "spruce root basket."

${ }^{313} \mathrm{HE}$ possibly in $/ \mathrm{c}^{3 \prime} \mathrm{It}^{3} \mathrm{a} /$ "heart of a tree." 
North Wakashan Comparative Root List (Updated)

\begin{tabular}{|c|c|c|c|c|c|c|}
\hline Root \# & Old \# & $\mathrm{HE}$ & $\mathrm{OO}$ & HA & KW & Gloss \\
\hline & & & & & & $\begin{array}{l}\text { osiers in basket, barbs in } \\
\text { feather) }^{314}\end{array}$ \\
\hline 6710 & 756 & & & & $\mathrm{c}^{3} 1 \mathrm{kv}-$ & to forbid 315 \\
\hline 6720 & 757 & (kvwXv-) & (kvwXv-) & $\begin{array}{l}\text { kwkv- } \\
\text { kwxv- }\end{array}$ & $c^{3} l q v-$ & hot, heat \\
\hline 6730 & 758 & & & & $c^{3} 1 \chi v-$ & $(?)^{316}$ \\
\hline 6740 & 759 & $c^{3} l q-$ & $c^{3} l q-$ & $c^{3} l q-$ & & $\begin{array}{l}\text { to crack, split (wood, china, } \\
\text { window) }\end{array}$ \\
\hline 6750 & 760 & $c^{3} l q-$ & $c^{3} l q-$ & $c^{3} l q-$ & $c^{3} l q-$ & to shine, reflect light \\
\hline 6760 & 761 & & & & $c^{3} 1 \chi-$ & to go head-first \\
\hline 6770 & 762 & $c^{3} k-$ & $\mathrm{c}^{3} \mathrm{k}-$ & $c^{3} k-$ & $\begin{array}{l}\mathrm{c}^{3} \mathrm{k}- \\
\mathrm{c}^{3} \mathrm{hk}-\end{array}$ & awake, attentive, watchful ${ }^{317}$ \\
\hline 6790 & 763 & & & & $\begin{array}{l}c^{3} \mathrm{x}- \\
c^{3} h x-\end{array}$ & sick, hurt \\
\hline 6800 & $\begin{array}{l}764 \\
765\end{array}$ & $c^{3} x-$ & $c^{3} \mathrm{x}-$ & $c^{3} \mathrm{x}-$ & $c^{3} x-$ & $\begin{array}{l}\text { to singe, roast on an open fire, } \\
\text { trim hair by singeing }\end{array}$ \\
\hline 6810 & 766 & & & & $\begin{array}{l}c^{3} x_{-*}^{*} \\
c^{3} h x-*\end{array}$ & $(?)^{318}$ \\
\hline
\end{tabular}

${ }^{314} \mathrm{KW}\left\{\mathrm{c}^{3} 1 \mathrm{x}-\right\}$ is attested only as the free form $/ \mathrm{c}^{3} \mathrm{Jx} /$ "feather."

${ }^{315}$ In a stem that needs reeliciting because it is uncertain if it is $\left.\left\{\mathrm{c}^{3} \mathrm{gvhl}-\right\}<\left\{\mathrm{c}^{3} \mathrm{lkv}-=\mathrm{h}\right\}-\right\}$ or $\left\{\mathrm{c}^{3} \operatorname{lgv} 1-\right\}<\left\{\mathrm{c}^{3} \mathrm{kv}-=1\right\}$.

${ }^{316} \mathrm{In} \mathrm{KW}\left\{\mathrm{c}^{3} \mid \chi \mathrm{v}-\%\right.$ hq- $\}$ "to praise, to pray" and two other derivates of $\left\{\mathrm{c}^{3} \chi \chi^{\mathrm{V}}-\right\}$ referring to speaking softly (like whispering)."

${ }^{317}$ Also probably in KW $\left\{\mathrm{c}^{3} \mathrm{k}-+\mathrm{hl}-\right\}$ and $\left\{\mathrm{c}^{3} \mathrm{hk}-+\mathrm{h} \mathrm{h}-\right\} \mathrm{HE} / \mathrm{OO} / \mathrm{HA}\left\{\left[\mathrm{c}^{3} \mathrm{y}\right] \mathrm{c}^{3} \mathrm{k}-\& \mathrm{hl}-\right\}$ "to tell news." The meaning of the suffix in these stems is problematic; it is assumed to be suffix no. 2790 . ${ }^{318} \mathrm{In} \mathrm{KW} / \mathrm{c}^{3} \mathrm{xas} /{ }^{*}$ "new (said of a mat or blanket)" $\mathrm{KW} / \mathrm{c}^{3} \mathrm{axay}^{3} \mathrm{M} /{ }^{*}$ and $/ \mathrm{c}^{3} \mathrm{axa}^{3} \mathrm{M} /{ }^{*}$ "new-made thing." 
North Wakashan Comparative Root List (Updated)

\begin{tabular}{|c|c|c|c|c|c|c|}
\hline Root \# & Old \# & $\mathrm{HE}$ & $\mathrm{OO}$ & HA & $\mathrm{KW}$ & Gloss \\
\hline 6830 & 769 & $c^{3} y-$ & $c^{3} y-$ & $c^{3} y-$ & & to push or throw with the horns \\
\hline 6840 & 770 & $c^{3} y p-$ & $c^{3} y p-$ & $c^{3} y p-$ & & wide, broad, moving sideways ${ }^{319}$ \\
\hline & 771 & & & $c^{3} y^{3} p-$ & & \\
\hline 6850 & 772 & & & $\mathrm{c}^{3} \mathrm{ymhws}-$ & & old driftlog 320 \\
\hline 6870 & 788 & & $c^{3}$ ynh- & & $c^{3}$ ynh- & $(?)^{321}$ \\
\hline 6880 & 775 & & & & $c^{3} y s-$ & $\begin{array}{l}\text { to roast at the side of the fire (sea } \\
\text { eggs, clams, shellfish), to use } \\
\text { roasting tongs }\end{array}$ \\
\hline 6890 & $\begin{array}{l}776 \\
777\end{array}$ & $\begin{array}{l}c^{3} y s- \\
c^{3} y^{3} s-\end{array}$ & $\begin{array}{l}c^{3} y s- \\
c^{3} y^{3} s-\end{array}$ & $\begin{array}{l}c^{3} y s- \\
c^{3} y^{3} s-\end{array}$ & $c^{3} y s-$ & $\begin{array}{l}\text { to aggregate, hoard, concentrate } \\
\text { (e.g. property); to retrieve, } \\
\text { collect, put away; to wind in, coil } \\
\text { or tangle up one's line }\end{array}$ \\
\hline 6910 & 779 & $\left.\mathrm{c}^{3} \mathrm{y}\right\} . \mathrm{w}^{3}-$ & $\begin{array}{l}c^{3} y ł . w^{3}- \\
c^{3} y ł \chi v-\end{array}$ & & $\mathrm{c}^{3} \mathrm{y}_{1} \mathrm{w}^{3}-$ & famous, talked about ${ }^{322}$ \\
\hline 6920 & $\begin{array}{l}780 \\
784\end{array}$ & $\begin{array}{l}c^{3} y k- \\
c^{3} y k v-\end{array}$ & $\begin{array}{l}c^{3} y k- \\
c^{3} y k v-\end{array}$ & $\begin{array}{l}c^{3} y k- \\
c^{3} y k v-\end{array}$ & $\begin{array}{l}c^{3} y k- \\
c^{3} y k v-\end{array}$ & $\begin{array}{l}\text { to squeak, screech, chirp, } \\
\text { cheep }^{323}\end{array}$ \\
\hline
\end{tabular}

${ }^{319}$ Also in HA / $\mathrm{c}^{3} \mathrm{ic}^{3} \mathrm{buy}^{3} \mathrm{u}$ / "shiner perch - Cymatogaster aggregata" (a fish whose sides are wide compared to its length).

${ }^{320}$ Unanalyzable stem. May contain a root $\left\{\mathrm{c}^{3} \mathrm{ymh}-\right\}$.

${ }^{321} \mathrm{In} \mathrm{OO} / \mathrm{c}^{3}$ ina/ OO/KW / $\mathrm{c}^{3}$ inas/, both of which mean "lean (meat)." Perhaps $\left\{\mathrm{c}^{3} \mathrm{ynh}-\right\}$ is an augmented form of $\left\{\mathrm{c}^{3} \mathrm{n}-\right\}$ and related to $\left\{\mathrm{c}^{3} \mathrm{nt}-\right\}$ in OO $/ \mathrm{c}^{3} \mathrm{Hta} /$ "lean salmon".

$322\left\{\mathrm{c}^{3} \mathrm{y} \nmid \chi \mathrm{v}-\right\}$ only in OO / $\mathrm{c}^{3}$ ił $\chi \mathrm{va} /$ "to cause rumours" which could be a backformation from

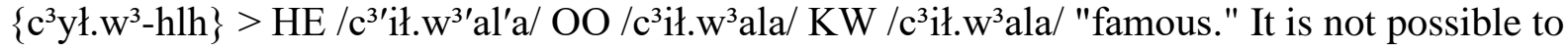

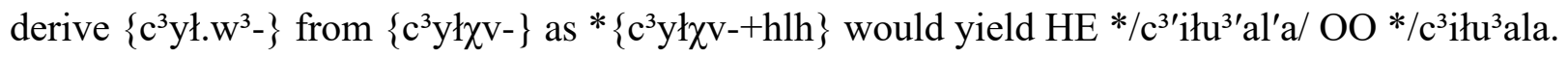
${ }^{323}$ Onomatopoeic root. $\left\{\mathrm{c}^{3} \mathrm{yk}-\right\}$ occurs in words for seagull and $\mathrm{KW} / \mathrm{c}^{3} \mathrm{ixc}^{3} \mathrm{ika} /$ "baby buggy."

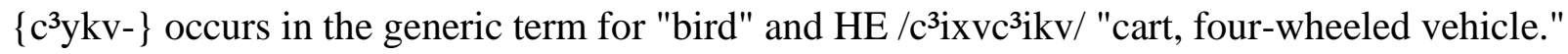
Proto-Salish correlate: *-c ${ }^{3}{ }^{1 x v{ }^{3}}{ }^{3} i x v-$ "osprey, fish hawk" (Kuipers 2002:33). 
North Wakashan Comparative Root List (Updated)

\begin{tabular}{|c|c|c|c|c|c|c|}
\hline Root \# & Old \# & $\mathrm{HE}$ & $\mathrm{OO}$ & HA & KW & Gloss \\
\hline & 794 & & & & & \\
\hline & 795 & & & & & \\
\hline 6930 & 781 & & & & $c^{3} \mathrm{yk}-*$ & $(?)^{324}$ \\
\hline 6940 & 782 & $c^{3} \mathrm{yk}-$ & $\mathrm{c}^{3} \mathrm{yk}-$ & $c^{3} \mathrm{yk}-$ & $c^{3} y_{k-*}$ & $\begin{array}{l}\text { to remove obstacles, clear a } \\
\text { surface }\end{array}$ \\
\hline 6950 & 783 & & & $c^{3} \mathrm{yk}-$ & & dirty \\
\hline 6960 & 785 & $\mathrm{c}^{3} \mathrm{yk}^{3} \mathrm{~h} 1-$ & $\mathrm{c}^{3} \mathrm{yk}^{3} \mathrm{~h} 1-$ & $\mathrm{c}^{3} \mathrm{yk}^{3} \mathrm{~h} 1-$ & & battle, fighting, rioting 325 \\
\hline 6970 & $\begin{array}{r}786 \\
1432\end{array}$ & $\mathrm{k}^{3} \mathrm{yp}-$ & $\mathrm{k}^{3} \mathrm{yp}-$ & $\mathrm{k}^{3} \mathrm{yp}-$ & $c^{3} \mathrm{yx}-$ & $\begin{array}{l}\text { elderberry (Sambucus } \\
\text { melanocarpa) }\end{array}$ \\
\hline 6980 & 787 & & & & $c^{3} y x-$ & to grill, roast ${ }^{327}$ \\
\hline 6990 & $\begin{array}{l}789 \\
790\end{array}$ & $c^{3} \mathrm{yx}^{-}$ & $\mathrm{c}^{3} \mathrm{yx}-$ & $c^{3} \mathrm{yx}-$ & $c^{3} \mathrm{yx}-$ & $\begin{array}{l}\text { (?) teasing, troubling, } \\
\text { preoccupying, irritating, boring }{ }^{328}\end{array}$ \\
\hline 7000 & 791 & & & & $c^{3} y x-$ & $(?)^{329}$ \\
\hline 7020 & 793 & $c^{3} \mathrm{ykv-}$ & $c^{3} y k v-$ & $c^{3} y k v-$ & $c^{3} \mathrm{ykv}-$ & shellfish (esp. clams) \\
\hline
\end{tabular}

${ }^{324}$ Perhaps in KW /c ${ }^{3}$ igulas/* "crosswise dimension."

${ }^{325}$ Could contain root no. 6940.

${ }^{326}$ Alternation of HE/OO/HA $\left|\mathrm{k}^{3}\right|$ and $\mathrm{KW}\left|\mathrm{c}^{3}\right|$ occurs also in the suffix for "hand and forearm." Alternation of $|\mathrm{p}|$ and $|\mathrm{x}|$ occurs in HE/OO when a root ending in $|\mathrm{p}|$ reduplicates completely, cf. OO /daxdapa/, frequentative of /dapa/ "to tow." Proto-Salish correlate: *-c 3ikv- "elderberry." ${ }^{327}$ Only in $\mathrm{KW} / \mathrm{c}^{3} \mathrm{ixa} /[2]$ "to grill, roast (dried fish)" and $\mathrm{KW} / \mathrm{c}^{3} \mathrm{ina}^{3} \mathrm{an}^{3} v \varepsilon^{3 /}$ "stick on which fat is roasted."

${ }^{328}$ Possibly in $\left\{\mathrm{c}^{3} \mathrm{ynhkv}-\right\}$ HE/OO/HA "cat's cradle" (a string game) KW "annoyed" and HE/OO/KW \{c $\left.\mathrm{c}^{3} \mathrm{ynyq}-\right\}$ (KW also $\left.\left\{\mathrm{c}^{3} \mathrm{yny} \chi-\right\}\right)$ "annoyed, irritated." ${ }^{329}$ In KW /c 3 ixa/ [3] "boiled blood." 
North Wakashan Comparative Root List (Updated)

\begin{tabular}{|c|c|c|c|c|c|c|}
\hline Root \# & Old \# & $\mathrm{HE}$ & $\mathrm{OO}$ & HA & KW & Gloss \\
\hline 7030 & $\begin{array}{l}798 \\
799 \\
800\end{array}$ & $\begin{array}{l}c^{3} y q v- \\
c^{3} y q-\end{array}$ & $\begin{array}{l}c^{3} y q v- \\
c^{3} y q-\end{array}$ & $\begin{array}{l}c^{3} y q v- \\
c^{3} y q-\end{array}$ & $\begin{array}{l}c^{3} \text { yqv- } \\
c^{3} y^{3}-\end{array}$ & $\begin{array}{l}\text { narrow (in width, in one's ways, } \\
\text { in comprehension), constricted, } \\
\text { astringent (like mountain ash } \\
\text { bark); narrowing, tapering, } \\
\text { narrow extension; shortcut; (KW } \\
\left.\text { \{c } \text { c }^{3} \text { yqv- }\right\} \text { also:) trail, traveling on } \\
\text { a trail }{ }^{330}\end{array}$ \\
\hline 7040 & $\begin{array}{l}792 \\
796 \\
797\end{array}$ & $c^{3} y \chi v-$ & & $c^{3} y \chi v-$ & $\begin{array}{l}c^{3} y x v-/ \\
c^{3} y \chi v-\end{array}$ & $\begin{array}{l}\text { at the end, exhausted; wastage, } \\
\text { offal }^{331}\end{array}$ \\
\hline 7070 & 802 & $\mathrm{c}^{3} \mathrm{y} \chi_{-}^{-}$ & $\mathrm{c}^{3} \mathrm{y} \chi_{-}^{-}$ & $c^{3} y \chi_{-}^{-}$ & $\mathrm{c}^{3} \mathrm{y} \chi-$ & precipitation stops \\
\hline 7080 & 803 & $\mathrm{c}^{3} \mathrm{kV}-$ & $\mathrm{c}^{3} \mathrm{kV}-$ & $\mathrm{c}^{3} \mathrm{kV}-$ & $c^{3} \mathrm{kv}-$ & short, compact, truncated \\
\hline 7090 & 805 & $\mathrm{c}^{3} \mathrm{kV}-$ & $\mathrm{c}^{3} \mathrm{kV}-$ & & & to invite ${ }^{332}$ \\
\hline 7100 & 804 & $c^{3} k v-$ & $\mathrm{c}^{3} \mathrm{kv}-$ & $\mathrm{c}^{3} \mathrm{kv}-$ & & (?) spiny $^{333}$ \\
\hline 7110 & 806 & $c^{3} x v-$ & $c^{3} x v-$ & $c^{3} x v-$ & $c^{3} x v-$ & $\begin{array}{l}\text { to stab, spear, push stick into; to } \\
\text { descend steeply (like bluff) }\end{array}$ \\
\hline
\end{tabular}

${ }^{330}$ Possibly also in HE / ${ }^{3} i^{\prime}$ aqva/ "person who does not eat very much."

${ }^{331}$ In HA words referring to fatigue, $\mathrm{HE} / \mathrm{c}^{3} \mathrm{ac}^{3} \mathrm{i} \chi \mathrm{vMq}^{3} \mathrm{a} /$ "to be tired of waiting" $\mathrm{KW} / \mathrm{c}^{3} \mathrm{uu}^{3} \chi \lambda \varepsilon^{3 /}$ "umbilical cord" and several non-reelicitable KW terms listed by Boas (1948) and referring to the anal fin and cutting out guts. In none of the KW examples is it diagnosable if root ends in $|\mathrm{xv}|$ or $|\chi \mathrm{v}|$. Boas (1948:223) lists one (non-reelicitable) word that could be taken as evidence for $|\mathrm{xv}|$ but it could well be a typing error; see the comment on lexical entry $\mathrm{KW} / \mathrm{c}^{3} \mathrm{i} \chi \mathrm{v}^{3} \mathrm{akv} / *^{*}$ "cut out (guts)."

${ }^{332} \mathrm{In} \mathrm{HE} / \mathrm{c}^{3} \mathrm{kv}$ 'i/ OO $/ \mathrm{c}^{3} \mathrm{kvi} /$ "to walk through the village inviting people."

${ }^{333}$ In words referring to sea urchins, sea eggs. Root could be an alternant of $\left\{c^{3} \mathrm{Xv}-\right\}$ (no. 7110$)$. $334\left\{\mathrm{c}^{3} \mathrm{WXV}-\right\}$ (root no. 7210) could be an alternant of this root. 
North Wakashan Comparative Root List (Updated)

\begin{tabular}{|c|c|c|c|c|c|c|}
\hline Root \# & Old \# & $\mathrm{HE}$ & $\mathrm{OO}$ & HA & KW & Gloss \\
\hline 7130 & $\begin{array}{l}808 \\
809\end{array}$ & $\begin{array}{l}c^{3} w- \\
c^{3} h w^{3}-\end{array}$ & $\begin{array}{l}c^{3} W_{-}^{-} \\
c^{3} h w^{3}-\end{array}$ & $\begin{array}{l}c^{3} W- \\
c^{3} h w^{3}-\end{array}$ & $c^{3} w-$ & $\begin{array}{l}\text { to bend, curve, circulate; to recur, } \\
\text { reciprocate; to give, put in } \\
\text { possession }^{335}\end{array}$ \\
\hline 7140 & $\begin{array}{l}726 \\
811 \\
816\end{array}$ & $\begin{array}{l}c^{3} W S^{-} \\
c^{3} W^{3} S_{-}^{-}\end{array}$ & $\begin{array}{l}c^{3} W- \\
c^{3} W S- \\
c^{3} W^{3} s-\end{array}$ & $\begin{array}{l}\mathrm{c}^{3} \mathrm{WS}- \\
\mathrm{c}^{3} \mathrm{WWS}-\end{array}$ & $\begin{array}{l}\mathrm{c}^{3} \mathrm{~W}- \\
\mathrm{c}^{3} \mathrm{~ms}- \\
\mathrm{c}^{3} \mathrm{w}^{3} \mathrm{~s}-\end{array}$ & $\begin{array}{l}\text { small outgrowth on a surface } \\
\text { (e.g. boil, pimple, barnacle) } \\
336\end{array}$ \\
\hline 7160 & 813 & $c^{3} w t-$ & $\mathrm{c}^{3} \mathrm{wt}-$ & $c^{3} w t-$ & & fresh (said of food) \\
\hline 7170 & 814 & & & & $\mathrm{c}^{3} \mathrm{Wnh}-$ & $(?)^{337}$ \\
\hline 7180 & 815 & & & & $c^{3} \mathrm{Ws}-$ & $\begin{array}{l}\text { to poke in the ground to dig up } \\
\text { roots }\end{array}$ \\
\hline 7190 & $\begin{array}{l}817 \\
818\end{array}$ & $c^{3} w 1-$ & $c^{3} w 1-$ & $c^{3} w 1-$ & $c^{3} w ł-$ & black \\
\hline 7200 & 810 & $c^{3} w k v-$ & $c^{3} w k v-$ & $c^{3} w k v-$ & $\mathrm{c}^{3} \mathrm{WkV}-$ & (?) to recur, reincarnate ${ }^{338}$ \\
\hline 7210 & 819 & & & & $\mathrm{c}^{3} \mathrm{WXV}-$ & (?) to insert, push in ${ }^{339}$ \\
\hline 7220 & 820 & $c^{3} w q v-$ & $c^{3} w q v-$ & $c^{3} w q v-$ & $c^{3} w q v-$ & $\begin{array}{l}\text { to rattle, to ring (like metal), to } \\
\text { jingle }\end{array}$ \\
\hline
\end{tabular}

$335\left\{\mathrm{c}^{3} \mathrm{hw}^{3}-\right\}$ occurs in OO / $\mathrm{c}^{3} \mathrm{aw}^{3} \mathrm{ikal} /$ "a ceremonial blanket" $\mathrm{HE} / \mathrm{c}^{3} \mathrm{aw}^{3} \mathrm{ikałba/}$ "nose of octopus" (i.e. bulbous front part of octopus swimming head first with arms trailing behind) HA $/ \mathrm{c}^{3} \mathrm{aw}^{3} \mathrm{ika} /$ "snowshoes."

$336\left\{\mathrm{c}^{3} \mathrm{w}-\right\}$ occurs likely in $\mathrm{OO} / \mathrm{KW} / \mathrm{c}^{3} \mathrm{uc}^{3} \mathrm{um}^{3} \mathrm{a} /$ "barnacles." It could be a cross-family stem since it resembles proto-Salish ${ }^{*} \mathrm{c}^{3} \mathrm{u} \mathrm{S}^{\mathrm{w}}$ "sore", cf. Kuipers (2002:35). $\left\{\mathrm{c}^{3} \mathrm{w}^{3} \mathrm{~s}-\right\}$ and $\left\{\mathrm{c}^{3} \mathrm{wws}-\right\}$ are augmented forms of $\left\{\mathrm{c}^{3} \mathrm{Ws}-\right\}$ both stemming from $\left\{\mathrm{c}^{3} \mathrm{w}[\infty] \mathrm{s}-\right\}$.

${ }^{337}$ In $\mathrm{KW} / \mathrm{c}^{3} \mathrm{una} /$ "thunderbird" and $\mathrm{KW} / \mathrm{c}^{3} \mathrm{una}^{3} \mathrm{as} / *$ "thunderbird place."

${ }^{338}$ In words referring to grandchildren.

${ }^{339}$ Could be an alternant of $\left\{c^{3} \mathrm{xv}-\right\}$ (root no. 7110 ) but only three examples and their glosses do not all support this interpretation. 
North Wakashan Comparative Root List (Updated)

\begin{tabular}{|c|c|c|c|c|c|c|}
\hline Root \# & Old \# & $\mathrm{HE}$ & $\mathrm{OO}$ & HA & KW & Gloss \\
\hline 7230 & $\begin{array}{l}807 \\
821 \\
822\end{array}$ & $\mathrm{c}^{3} \mathrm{w} \chi \mathrm{V}-$ & $\mathrm{c}^{3} \mathrm{w} \chi \mathrm{V}-$ & $\mathrm{c}^{3} \mathrm{w} \chi \mathrm{V}-$ & $\begin{array}{l}c^{3} w q v- \\
c^{3} w \chi v-\end{array}$ & $\begin{array}{l}\text { sloshing of water; to splash into } \\
\text { water, to wash, to launder }{ }^{340}\end{array}$ \\
\hline 7240 & 823 & $c^{3} \mathrm{qv}^{-}$ & & $\mathrm{c}^{3} \mathrm{qv}-$ & & $(?)^{341}$ \\
\hline 7250 & 825 & & & & $\mathrm{c}^{3} \mathrm{qv}-*$ & $(?)^{342}$ \\
\hline 7260 & 826 & $\begin{array}{l}c^{3} q- \\
c^{3} h q-\end{array}$ & $\left(\mathrm{m}^{3} \mathrm{q}-\right)$ & $\left(\mathrm{m}^{3} \mathrm{q}-\right)$ & $\begin{array}{l}c^{3} q^{-} \\
c^{3} h q-\end{array}$ & $\begin{array}{l}\text { to throw (e.g. a ball), to fall (said } \\
\text { of inanimate objects); (HE also:) } \\
\text { to propel violently, to smash; } \\
\text { (KW also:) to throw away, } \\
\text { discard }^{343}\end{array}$ \\
\hline 7270 & 827 & $c^{3} \chi-$ & & & & $(?)^{344}$ \\
\hline 7280 & 828 & $c^{3} \chi_{-}^{-}$ & $c^{3} \chi-$ & $c^{3} \chi-$ & $\mathrm{c}^{3} \chi^{-*}$ & $\begin{array}{l}\text { elastic, pliable, slack; taking it } \\
\text { easy; (KW also:) durable, tough, } \\
\text { resisting wear and tear }\end{array}$ \\
\hline 7290 & 829 & & & & $c^{3} \chi-$ & $(?)^{345}$ \\
\hline 7300 & 830 & $c^{3} h-$ & $c^{3} h-$ & $c^{3} h-$ & $c^{3} h-$ & $\begin{array}{l}\text { mass of water moving, water } \\
\text { flowing (as a stream, in a flood, } \\
\text { as a tide or as runoff (from e.g. } \\
\text { one's raincoat), current }\end{array}$ \\
\hline
\end{tabular}

340 \{ $\left.\mathrm{c}^{3} \mathrm{wqv}-\right\}$ occurs only in $\mathrm{KW} / \mathrm{c}^{3} \mathrm{urvayu} /$ "soap."

${ }^{341}$ Only in HE $/ \mathrm{c}^{3} \chi \mathrm{vc}^{3} \mathrm{rv}^{\prime} a t u^{\prime} \mathrm{a} / \mathrm{HA} / \mathrm{c}^{3} \mathrm{r} v^{\prime} a t u i /$ "wax in the ears."

$342 \mathrm{In} \mathrm{KW} / \mathrm{c}^{3} \mathrm{IVHW} \varepsilon^{3 / *}$ "stomach of porpoise."

${ }^{343} \mathrm{HE}\left\{\mathrm{c}^{3} \mathrm{hq}-\right\}$ only in $/ \mathrm{c}^{3 \prime} \mathrm{ar} \mathbf{r}^{\prime} \mathrm{i} \mathrm{a} /$, alternant of $/ \mathrm{c}^{3} \mathrm{r}^{\prime} \mathrm{i} £ \mathrm{a} /$ "to throw into a house."

344 In two words referring to low bush blueberries.

${ }^{345}$ In $\mathrm{KW} / \mathrm{c}^{3} \chi \mathrm{u}^{3} /$ and $/ \mathrm{c}^{3} \chi \mathrm{v}^{3} \mathrm{i} /$ "ling cod." 
North Wakashan Comparative Root List (Updated)

\begin{tabular}{|c|c|c|c|c|c|c|}
\hline Root \# & Old \# & $\mathrm{HE}$ & $\mathrm{OO}$ & HA & KW & Gloss \\
\hline 7310 & 831 & $c^{3} h-$ & & & & $\begin{array}{l}\text { to stagger, lurch, move to and } \\
\text { fro }^{346}\end{array}$ \\
\hline 7350 & $\begin{array}{l}834 \\
835\end{array}$ & $\mathrm{c}^{3} \mathrm{hs}-$ & $\mathrm{c}^{3} \mathrm{hs}-$ & & $\mathrm{c}^{3} \mathrm{hs}-$ & $\begin{array}{l}\text { elastic, stretchable; to stretch to } \\
\text { the limit, at the tail end; to strain, } \\
\text { to work hard; eelgrass (Zostera } \\
\text { marina) }{ }^{347}\end{array}$ \\
\hline 7370 & 836 & & & $c^{3}$ hs- & & to deceive, to disappoint \\
\hline 7380 & 837 & & & & $\mathrm{c}^{3} \mathrm{hs}-$ & curved, curving motion ${ }^{348}$ \\
\hline 7400 & 839 & $\mathrm{c}^{3} \mathrm{hk}-$ & $\mathrm{c}^{3} \mathrm{hk}-$ & $c^{3} h k-$ & $\mathrm{c}^{3} \mathrm{hk}-$ & (?) spearhead ${ }^{349}$ \\
\hline 7430 & 841 & $\mathrm{c}^{3} \mathrm{hk}-$ & & $\mathrm{c}^{3} \mathrm{hk}-$ & & to melt, thaw; to lose weight ${ }^{350}$ \\
\hline
\end{tabular}

${ }^{346} \mathrm{In} \mathrm{HE} / \mathrm{c}^{3} \mathrm{ac}^{3 \prime} \mathrm{an}^{3} \mathrm{akv}$ a/ "to stagger."

${ }^{347} \mathrm{HE} / \mathrm{OO}$ only in $\left\{\left[\mathrm{c}^{3} \mathrm{~h}\right] \mathrm{c}^{3} \mathrm{hs}-=\mathrm{m}\right\}>\mathrm{HE} / \mathrm{c}^{3 \prime} \mathrm{ac}^{3 \prime}$ aym/ OO $/ \mathrm{c}^{3} \mathrm{ac}^{3}$ aym/ "eelgrass, sea grass."

Eelgrass has long ribbon-like leaves that are still used industrially in some countries, among other things as mattress filling and in the production of mats, rugs and baskets, cf. (1) http://www.nrcs.usda.gov/Internet/FSE_PLANTMATERIALS/publications/mdpmcfs10921.pdf, (2) http://en.wikipedia.org/wiki/Seagrass and (3) p. 314 of The Chemical Composition and Technological Properties of Seagrasses a Basis for Their Use (A Review) by N. A. Milchakova, Benno Böer, L. I. Boyko, D. V. Mikulich, shown at http://link.springer.com/chapter/10.1007/978-94-007-7411-7_22\#page-1.

${ }^{348}$ In words referring to somersaulting and to a whale surfacing (as for blowing).

349 Possibly in $\mathrm{HE} / \mathrm{c}^{3} \mathrm{akH} / \mathrm{OO} / \mathrm{c}^{3} \mathrm{akH} /$ "point of seal or salmon spear" HA / $\mathrm{c}^{31} \mathrm{akH} /$ "harpoon for catching fish (made of baby goat horn)" KW (Koskimo dialect) / $\mathrm{c}^{3} \mathrm{akH} \chi / *$ "point of doubleheaded harpoon."

${ }^{350}$ The HE examples of $\left\{\mathrm{c}^{3} \mathrm{hk}-\right\}$ meaning "to melt etc." are from the Klemtu informant and not accepted by Bella Bella informant Angus Campbell. 
North Wakashan Comparative Root List (Updated)

\begin{tabular}{|c|c|c|c|c|c|c|}
\hline Root \# & Old \# & $\mathrm{HE}$ & $\mathrm{OO}$ & HA & $\mathrm{KW}$ & Gloss \\
\hline 7440 & 842 & & & & $\mathrm{c}^{3} \mathrm{hx}-$ & $(?)^{351}$ \\
\hline 7450 & 843 & & & & $\mathrm{c}^{3} \mathrm{hx}-*$ & $(?)^{352}$ \\
\hline 7460 & 844 & $c^{3} h x-$ & $c^{3} h x-$ & & & $\begin{array}{l}\text { (?) to veer off, to turn } \\
\text { sideways }^{353}\end{array}$ \\
\hline 7470 & 845 & & $c^{3} h x-$ & & $\mathrm{c}^{3} \mathrm{hx}-*$ & $(?)^{354}$ \\
\hline 7471 & & $c^{3}$ hyq- & $c^{3}$ hyq- & & $c^{3}$ hyq- & $\begin{array}{l}\text { 1. shaman, Indian doctor, } \\
\text { medicine man; } 2 \text {. Indian dancer; } \\
\text { 3. to sing; (KW also:) 4. winter } \\
\text { ceremonial }^{355}\end{array}$ \\
\hline
\end{tabular}

${ }^{351} \mathrm{In} \mathrm{KW} / \mathrm{c}^{3} \mathrm{axHs} /$ (probably:) "ironwood plant." According to

http://en.wikipedia.org/wiki/Holodiscus_discolor also known as ocean spray and creambush and noted for the strength of its wood that is often used for making spears, arrows, bows, harpoons and nails.

${ }^{352}$ Possibly in two derivates of $\left\{\mathrm{c}^{3} \mathrm{hnws}-\right\}^{*}$ "netting or snaring of deer, seal, ducks."

${ }^{353}$ Possibly in $\mathrm{HE} / \mathrm{c}^{3} \mathrm{an}^{3}{ }^{\prime} \mathrm{it} / \mathrm{OO} / \mathrm{c}^{3} \mathrm{an}{ }^{3} \mathrm{it} /$ "tail of fish" and $\mathrm{OO} / \mathrm{c}^{3} \mathrm{axsistayu} /$ "turned by the tide or back eddy."

${ }^{354}$ In four derivates of $\left\{\mathrm{c}^{3} \mathrm{hnwqv}-\right\}$ "to hush, soothe, comfort."

3551 . The large majority of KW derivates of this stem have a gloss referring to the winter ceremonial. 2. If the stem is monomorphemic its structure is likely $\left\{\mathrm{c}^{3}[\mathrm{~h}] \mathrm{yq}-\right\}$ because e.g. $*\left\{\mathrm{c}^{3}[\mathrm{~h}] \mathrm{y}[\mathrm{h}] \mathrm{q}-\% \mathrm{~m}\right\}$ would yield $\mathrm{HE} * / \mathrm{c}^{3}$ ay'arm/ OO $* / \mathrm{c}^{3}$ ayarm/ and not the attested HE $/ \mathrm{c}^{3}$ airm/ $\mathrm{OO} / \mathrm{c}^{3}$ airm/ " 1 . shaman's song; 2 . song text (of any kind)." If the stem is polymorphemic its root could have the form of $\left\{c^{3} h-\right\}$ or $\left\{c^{3} h s-\right\}$, with unclear meaning. Those taking a dim view of shamanic religion may want to identify the root as the $\left\{\mathrm{c}^{3} \mathrm{hs}-\right\}$ "to deceive, to disappoint" which occurs in HA (root no. 7370). There is however no evidence for this interpretation not counting one non-reelicitable term from Boas (1948:221) whose gloss could be made up by-Boas and not provided by an informant, $\mathrm{cf}$. KW $\left\{\mathrm{c}^{3} \mathrm{hyq}-=\mathrm{kv}\right\}>/ \mathrm{c}^{3}$ airkv $/ *$ "pretended." 3 . Most likely the stem is monomorphemic and has a structure $\left\{\mathrm{c}^{3}[\mathrm{~h}] \mathrm{yq}-\right\}$ because Sapir \& Swadesh (1939:306) list a South Wakashan (Nuuchahnulth) stem $\left\{\mathrm{c}^{3} \mathrm{i}: \mathrm{q}-\right\}$ "singing a secret chant with accompaniment of 
North Wakashan Comparative Root List (Updated)

\begin{tabular}{|c|c|c|c|c|c|c|}
\hline Root \# & Old \# & $\mathrm{HE}$ & $\mathrm{OO}$ & HA & KW & Gloss \\
\hline 7480 & 846 & $c^{3} h y^{3}-$ & $c^{3} h y^{3}-$ & $c^{3} h y^{3}-$ & $c^{3} h y^{3}-$ & younger sibling ${ }^{356}$ \\
\hline 7490 & & & $c^{3} h k v-$ & & $c^{3} h k v-$ & $(?)^{357}$ \\
\hline 7500 & 847 & & & & $c^{3} h w \infty-$ & (?) beaver 358 \\
\hline 7510 & 848 & & & & $\mathrm{c}^{3} \mathrm{hqv}-*$ & $(?)^{359}$ \\
\hline 7520 & 849 & & & & $\mathrm{c}^{3} \mathrm{hqv}-*$ & alive, (keeping) well ${ }^{360}$ \\
\hline 7530 & 850 & $c^{3} h q v-$ & $c^{3} h q v-$ & $\mathrm{c}^{3} \mathrm{hqv-}$ & $\mathrm{c}^{3} \mathrm{~h} \chi \mathrm{v}-$ & to stretch, make taut \\
\hline 7540 & 851 & $\mathrm{c}^{3} \mathrm{hr}-$ & $\mathrm{c}^{3} \mathrm{hr}-$ & $\mathrm{c}^{3} \mathrm{hr}-$ & $\left(\mathrm{m}^{3} \mathrm{l}-\right)$ & mountain goat \\
\hline 7550 & 852 & $\mathrm{c}^{3} \mathrm{hq}-$ & & $\mathrm{c}^{3} \mathrm{hq}-$ & $\mathrm{c}^{3} \mathrm{hq}-$ & outer bark of cedar \\
\hline 7551 & & & & & $\mathrm{c}^{3} \mathrm{hq}-$ & drifting with tide or current \\
\hline
\end{tabular}

rattle". Who borrowed from whom is unknown. Jacobsen (1968) lists /c 3 a yiq/ "doctoring ceremonial."

${ }^{356}$ Some derivates have confusing glosses due probably to the decline of the traditional kinship system.

${ }^{357}$ In OO/KW /C 3agvala/ (indigenous name of Chuckwallah River in the Rivers Inlet area)

358 1. All derivates of this root refer to beaver except that according to one informant $\mathrm{KW} / \mathrm{c}^{3} \mathrm{awi} /$ doesn't mean "beaver" but "fur seal." Possibly therefore the root has a more general meaning than "beaver." 2. That this root does not have the form of $\left\{c^{3} h x v-\right\}$ or $\left\{c^{3} h \chi v-\right\}$ is supported by the unfortunately non-reelicitable person's war name $/ \mathrm{C}^{3} \mathrm{\jmath gvлis} / *<\left\{\mathrm{c}^{3} \mathrm{hw} \infty\right.$-gł-=ys $\}$ which Boas glosses as "born to be beaver" (though with a question mark after it). If the root ended in $|\mathrm{xv}|$ or $|\chi v|$ the first suffix would have the form of $\{-\Delta 1-\}$ rather than $\{-g 1-\} .3$. This root could be crossfamily, cf. Proto-Salishan *-k3am- "beaver" (Kuipers 2002:140).

${ }^{359}$ In $\mathrm{KW} / \mathrm{c}^{3} \mathrm{aqva} / *$ "lean (said of salmon)."

${ }^{360}$ Derivates are archaic according to Boas. 
North Wakashan Comparative Root List (Updated)

\begin{tabular}{|c|c|c|c|c|c|c|}
\hline Root \# & Old \# & $\mathrm{HE}$ & $\mathrm{OO}$ & HA & KW & Gloss \\
\hline 7560 & 855 & $c^{3} h^{3} q-$ & $c^{3} h^{3} q-$ & & $c^{3} h^{3} \chi-$ & $\begin{array}{l}\text { (HE/OO) water dripping; (KW) } \\
(?)^{361}\end{array}$ \\
\hline 7570 & 856 & $\begin{array}{l}\text { sp- } \\
\text { sbh- }\end{array}$ & $\mathrm{sp}-$ & $\mathrm{sp}-$ & sp- & $\begin{array}{l}\text { to traverse a distance quickly and } \\
\text { suddenly (like flash of light or } \\
\text { burst of sound); to jut out (like } \\
\text { sharp edge), shoot forward (like } \\
\text { boat gaining headway) }\end{array}$ \\
\hline 7580 & 857 & $\mathrm{sp}^{3}-$ & & $\mathrm{sp}^{3}-$ & & $(?)^{363}$ \\
\hline 7590 & 858 & & & & smp- & to swear \\
\hline 7600 & 859 & sms- & sms- & sms- & sms- & mouth \\
\hline 7610 & 860 & smk- & smk- & smk- & smk- & to extract oil from ooligans \\
\hline 7620 & 861 & & & st- & & to eat meat \\
\hline 7630 & 862 & sn- & sn- & sn- & sn- & $\begin{array}{l}\text { to ponder, deliberate, think about } \\
\text { sth.; to plan, foresee, fret, to } \\
\text { commit, promise }\end{array}$ \\
\hline 7640 & 863 & sn- & sn- & sn- & sn- & $\begin{array}{l}\text { whole, complete, intact, } \\
\text { undamaged; spanning a whole } \\
\text { period (e.g. day) from beginning } \\
\text { to end } \\
364\end{array}$ \\
\hline
\end{tabular}

${ }^{361} \mathrm{KW}$ perhaps in $/ \mathrm{c}^{3} \mathrm{a}^{3} \chi \partial ł ə t u /$ or $/ \mathrm{c}^{3} \mathrm{a}^{3} \chi \partial ł t u /$ "to choke on liquid" (transcription in source document ambiguous).

362 The HE alternant $\{$ sbh- $\}$ occurs only in $\{[$ sp $]$ sbh- $\}>\{$ sxsbh- $\}$ "sharp" which likely is a metathesized form of $\{$ sk- $\}$ derivate $\{[s] \mathrm{sk}-\mathrm{b}-\mathrm{h}\}$ "sharp" that survives as such in HA.

${ }^{363} \mathrm{In} \mathrm{HE} / \mathrm{sp}^{3 \prime} \mathrm{ul}$ 'i/ "cherry-sized inedible red berries causing itchy anus when eaten" HA /sp ${ }^{3}$ auli/ "kind of small red berries growing close to the ground."

${ }^{364}$ Also possibly in some stems referring to asking back lent-out property. 
North Wakashan Comparative Root List (Updated)

\begin{tabular}{|c|c|c|c|c|c|c|}
\hline Root \# & Old \# & $\mathrm{HE}$ & $\mathrm{OO}$ & HA & KW & Gloss \\
\hline 7650 & 864 & & & sn- & & $\begin{array}{l}\text { to mention a name, to bestow a } \\
\text { name }^{365}\end{array}$ \\
\hline 7660 & 865 & snt- & snt- & snt- & snt- & to pick off nits \\
\hline 7670 & $\begin{array}{l}866 \\
879\end{array}$ & & & & $\begin{array}{l}\text { sny }{ }^{3}- \\
\text { sly }\end{array}$ & to pick out \\
\hline 7680 & & & & snkv- & & $\begin{array}{l}\text { to stick out of a tangled mass } \\
\text { (e.g. branches from thick brush, } \\
\text { hair from one's head) }\end{array}$ \\
\hline 7690 & 867 & snq- & snq- & snq- & snq- & to peel off cedar bark ${ }^{366}$ \\
\hline 7700 & 868 & & & & ss- & $\begin{array}{l}\text { washing is stiff (when frozen or } \\
\text { starched) }\end{array}$ \\
\hline 7710 & $\begin{array}{l}869 \\
870\end{array}$ & s1- & & s1- & s1-* & $\begin{array}{l}\text { (?) reluctant to commit, avoiding } \\
\text { work and responsibility }\end{array}$ \\
\hline 7720 & 872 & & & & st-* & $(?)^{367}$ \\
\hline 7730 & 871 & & & & słbxv- & to squirt \\
\hline 7740 & 874 & & & & sł-/sl- & $(?)^{368}$ \\
\hline 7750 & $\begin{array}{l}873 \\
874 \\
921\end{array}$ & sl- & $\begin{array}{l}\text { sl- } \\
\text { shlh- }\end{array}$ & sl- & $\begin{array}{l}\text { sl- } \\
\text { shlh- }\end{array}$ & $\begin{array}{l}\text { to drill (e.g. with sticks to make } \\
\text { fire), to turn a crank; wound up, } \\
\text { furled (like fiddleheads = young } \\
\text { fern's fronds) }\end{array}$ \\
\hline
\end{tabular}

${ }^{365}$ Could be root no. 7630 .

366 The HA examples refer specifically to red cedar but the other ones to young cedar or just cedar.

${ }^{367} \mathrm{In} \mathrm{KW} /$ słміqvла/* "to move a little (said of a person)."

${ }^{368}$ Only in KW /sлм/ [2] "berries of oval-leaf blueberry (Vaccinium ovalifolium)." Root may be related to $\{$ sls- $\}$ (root no. 7800) which refers to an (unidentified) kind of blueberry. 
North Wakashan Comparative Root List (Updated)

\begin{tabular}{|c|c|c|c|c|c|c|}
\hline Root \# & Old \# & $\mathrm{HE}$ & $\mathrm{OO}$ & HA & $\mathrm{KW}$ & Gloss \\
\hline 7760 & 874 & slp- & slp- & slp- & slp- & to twist, to turn (as in drilling) \\
\hline 7780 & 876 & & & & slt- & to drill ${ }^{369}$ \\
\hline 7790 & 877 & & & & slt- & still, quiet, inactive \\
\hline 7791 & 875 & & & & slt ${ }^{3} h-$ & little finger ${ }^{370}$ \\
\hline 7800 & 878 & sls- & sls- & & sls- & $\begin{array}{l}\text { (?) an (unidentified) type of } \\
\text { blueberry, colour of these berries }\end{array}$ \\
\hline 7810 & 880 & & & slq- & slqv- & twisted $^{371}$ \\
\hline 7820 & 881 & $\mathrm{sl} \chi \mathrm{v}-$ & & sl $\chi \mathrm{v}-$ & & $(?)^{372}$ \\
\hline 7840 & 882 & sk- & $\begin{array}{l}\text { sk- } \\
\text { shk- }\end{array}$ & sk- & sk- & $\begin{array}{l}\text { to spear, to harpoon, to poke with } \\
\text { sth. sharp }\end{array}$ \\
\hline 7850 & 883 & $\mathrm{sk}^{3} \mathrm{~h}-$ & $\mathrm{sk}^{3} \mathrm{~h}-$ & $\mathrm{sk}^{3} \mathrm{~h}-$ & $\mathrm{sk}^{3} \mathrm{~h}-$ & five \\
\hline 7860 & & & & & sx-* & $(?)^{373}$ \\
\hline 7880 & $\begin{array}{l}884 \\
885\end{array}$ & sy- & $\begin{array}{l}\text { sy- } \\
\text { shy- }\end{array}$ & sy- & sy- & $\begin{array}{l}\text { narrow long thing (pole, hair) } \\
\text { sticking out or into sth.; to push, } \\
\text { poke or propel with pole or stick; } \\
\text { (KW also:) vertical or horizontal }\end{array}$ \\
\hline
\end{tabular}

${ }^{369} \mathrm{In} \mathrm{KW} /$ sлsat.m ${ }^{3} \mathrm{ut} /$ "refuse from drilling."

${ }^{370}$ Unanalyzable free form.

${ }^{371}$ The two HA examples have $|\mathrm{q}|$, not $|\mathrm{qv}|$.

${ }^{372}$ In HA words referring to whistling through the teeth and in the name of the varied thrush, a bird with a whistle-like single pitch song. HE possibly in /s'лw'ilas/ "roof of the mouth."

${ }^{373} \mathrm{In} \mathrm{KW} /$ sхла/* "to want very much." 
North Wakashan Comparative Root List (Updated)

\begin{tabular}{|c|c|c|c|c|c|c|}
\hline Root \# & Old \# & $\mathrm{HE}$ & $\mathrm{OO}$ & HA & KW & Gloss \\
\hline & & & & & & $\begin{array}{l}\text { poles supporting a roof; putting } \\
\text { up a stanchion }^{374}\end{array}$ \\
\hline 7890 & $\begin{array}{l}886 \\
887\end{array}$ & syt- & syt- & & syt- & $\begin{array}{l}\text { to use a bar to pry, dislodge or } \\
\text { split; (hence these attested } \\
\text { meanings:) (HE/OO) to poke } \\
\text { through an opening (like } \\
\text { toothpick) or into a surface; } \\
\text { (KW) to split partially off a flat } \\
\text { surface or log (e.g. sliver still } \\
\text { attached to floor) }\end{array}$ \\
\hline 7900 & & & & synh- & synh- & (?) sideways, oblique 375 \\
\hline 7910 & 888 & sys- & sys- & sys- & sys- & $(?)^{376}$ \\
\hline 7920 & 889 & sy1- & sył- & & sył- & $\begin{array}{l}\text { to move in a winding manner } \\
\text { (like snake, like meandering } \\
\text { river), to slither, snake along }\end{array}$ \\
\hline 7930 & 890 & & & & sył- & $(?)^{377}$ \\
\hline 7940 & 891 & syx- & syx- & syx- & syx- & $\begin{array}{l}\text { to peel (fruit, sprouts), strip off, } \\
\text { clean off }\end{array}$ \\
\hline
\end{tabular}

${ }^{374}$ Also in $\left\{s y-k^{3} h q-\right\}$ "to walk with a cane" in which the suffix $\left\{-\mathrm{k}^{3} \mathrm{hq}\right\}$ may be an alternant of $\left\{-c^{3} q\right\}$ and $\left\{-c^{3} h q\right\}$ "long object."

${ }^{375}$ HA possibly in /sin'ai/ glossed by consultant as "spider crab" but probably referring to some other crab species because spider crabs are confined to Japanese waters. KW possibly in the stem \{synwqv-\} "oblique, slanting, bevelled, on a bias."

${ }^{376} \mathrm{In} \mathrm{HE} / \mathrm{s}^{\prime}$ isi'uł/ OO /sisiuł/ KW /sisul/, /sisuf/ and /sisiu 3 / / name of the mythical doubleheaded serpent) and HA /s'isiuł/ "prawns."

377 Possibly in KW $\{$ syl-=hnø $\infty$ > /silaH/ "one's grandchild's spouse." The term's /aH/ is an irregularity. Lincoln \& Rath (1980:170) uses the regularized form /silH/. 
North Wakashan Comparative Root List (Updated)

\begin{tabular}{|c|c|c|c|c|c|c|}
\hline Root \# & Old \# & $\mathrm{HE}$ & $\mathrm{OO}$ & HA & KW & Gloss \\
\hline 7950 & 892 & syx- & syx- & & syx- & $(?)^{378}$ \\
\hline 7960 & 893 & sykv- & sykv- & sykv- & & blueberry \\
\hline 7970 & 894 & syðv- & $\left(q^{3} w s-\right)$ & (£hk-) & $\operatorname{sy} \chi v$ & $\begin{array}{l}\text { to travel (in any vehicle), to } \\
\text { paddle }^{379}\end{array}$ \\
\hline 7980 & 895 & syq- & syq- & syq- & syq- & $\begin{array}{l}\text { (HE/OO/KW) to dry in the sun; } \\
\text { (HA) left high and dry by the tide }\end{array}$ \\
\hline 7990 & & & & & $\operatorname{sy}^{3} \mathrm{x}-$ & bowel or labour pains \\
\hline 8000 & 896 & & sy ${ }^{3} \mathrm{kv}-$ & $\mathrm{sy}^{3} \mathrm{kv}-$ & & crooked, to zigzag \\
\hline 8010 & 897 & & & & skv- & $\begin{array}{l}\text { leaning forward, bent over, to } \\
\text { bend to wishes, to comply }\end{array}$ \\
\hline 8020 & 898 & & & & skv- & $(?)^{380}$ \\
\hline 8040 & $\begin{array}{l}900 \\
905\end{array}$ & sw- & sw- & sw- & SW- & $\begin{array}{l}\text { to get, take, hold, carry in one's } \\
\text { hand; to control, to hold on to } \\
\text { sth.; one's cultural assets; to be } \\
\text { seized by sth. }\end{array}$ \\
\hline 8050 & 901 & SW- & SW- & & shwh- & $\begin{array}{l}\text { (?) to rumble; rattling noise, } \\
\text { clatter }^{382}\end{array}$ \\
\hline 8060 & 902 & $(q \# s w)$ & $(q \# s w)$ & $\begin{array}{l}(\mathrm{y} \chi \mathrm{v} \# \mathrm{sw} \\
\mathrm{y} \chi \# \mathrm{sw})\end{array}$ & SW- & it's you (who is/did ...) \\
\hline
\end{tabular}

${ }^{378}$ In OO $\left\{\right.$ syx-c $\left.{ }^{3} \mathrm{hs}\right\}$ "ambitious, having the will to achieve sth." and the HE/OO/KW free stem $\{$ syx $=$ ht $\}$ "purpose, reason, rationale, action plan."

${ }^{379} \mathrm{HE}$ possibly in the place name $/ \mathrm{S}^{\prime}{ }^{\prime} w^{\prime} \mathrm{ac}^{3} \mathrm{i} /$.

${ }^{380}$ In KW /skva/ "to eat dry food."

381 Productive root in HE/OO but only three possible HA examples. Only two of the possible KW examples found in Boas (1948) could be reelicited.

382 Possibly in $\mathrm{HE} / \mathrm{su}^{3} \mathrm{a} / \mathrm{OO} / \mathrm{su}^{3} \mathrm{a} /$ "thunder" and the KW stem \{shwlt- $\}$ "(to make) rattling or clattering noise." 
North Wakashan Comparative Root List (Updated)

\begin{tabular}{|c|c|c|c|c|c|c|}
\hline Root \# & Old \# & $\mathrm{HE}$ & $\mathrm{OO}$ & HA & KW & Gloss \\
\hline 8070 & 903 & & & & swp- & to chop with an axe \\
\hline 8080 & 904 & sw1- & & & swł-* & to twill \\
\hline 8090 & & & & & swkv- & (?) white w $^{383}$ \\
\hline 8110 & 906 & swqv- & & & & $(?)^{384}$ \\
\hline 8120 & $\begin{array}{l}907 \\
929\end{array}$ & & $\begin{array}{l}\text { sqv- } \\
\text { shqv- }\end{array}$ & shqv- & $\begin{array}{l}\mathrm{sqv}-* \\
\mathrm{~s} \chi \mathrm{v}-* \\
\mathrm{sq}^{3} \mathrm{v}- \\
\mathrm{shq}^{3} \mathrm{v}-\end{array}$ & $\begin{array}{l}\text { to peel, pry or tear off; to remove } \\
\text { covering layer (skin, bark, } \\
\text { clouds) }\end{array}$ \\
\hline 8130 & $\begin{array}{l}908 \\
909\end{array}$ & & & & sq- & $\begin{array}{l}\text { at an angle, leaning (like house, } \\
\text { chair, the back of a sitting frog or } \\
\text { toad) }\end{array}$ \\
\hline 8150 & 910 & $\mathrm{~s} \chi-$ & $\mathrm{s} \chi-$ & $s \chi-$ & & to crawl (like worm, insect) \\
\hline 8160 & 911 & $s \chi-$ & $\mathrm{s} \chi-$ & $s \chi-$ & $s \chi-$ & $(?)^{386}$ \\
\hline 8170 & $\begin{array}{l}912 \\
913 \\
914\end{array}$ & sh- & sh- & sh- & sh- & $\begin{array}{l}\text { level, even, lacking highs and } \\
\text { lows; (hence:) 1. levelheaded, } \\
\text { wise, sensible, temperate; } 2 \text {. flat }\end{array}$ \\
\hline
\end{tabular}

${ }^{383} \mathrm{In} \mathrm{KW} / \mathrm{st}^{3} \mathrm{ukv} /$ "widgeon duck" and its synonyms $/ \mathrm{si}^{3} \mathrm{uk}^{3} \mathrm{va} / *$, $/ \operatorname{sisuk}^{3} \mathrm{va} / *$. According to http://www.ducks.ca/learn-about-wetlands/wildlife/american-wigeon/, "The American wigeon was formerly known as Baldpate because the white stripe on the head resembled a bald man's head."

384 In HE /s'urvar'i/ "loin cloth."

${ }^{385}$ Could be same root as no. 8130 , with shared meaning "peel back, set at angle." Is also a cross-family stem, cf. proto-Salish *-səq ${ }^{3}$-glossed as "to split, crack" in Kuipers (2002:99) but should have included "opening up."

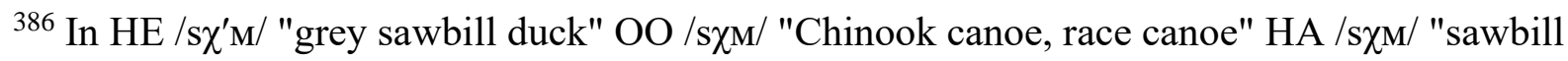
duck" KW /s $\chi \mathrm{M} /$ "figurehead of boat." 
North Wakashan Comparative Root List (Updated)

\begin{tabular}{|c|c|c|c|c|c|c|}
\hline Root \# & Old \# & $\mathrm{HE}$ & $\mathrm{OO}$ & $\mathrm{HA}$ & KW & Gloss \\
\hline & & & & & & $\begin{array}{l}\text { in shape, layer-like cover, } \\
\text { covered by a layer-like thing (e.g. } \\
\text { cloth, mat, board); } 3 \text {. (mostly in } \\
\mathrm{KW} \text { :) to stretch across or around } \\
\text { sth. (like lining, sheath), to } \\
\text { stretch out (e.g. one's arm), to } \\
\text { reach out, to reach a certain } \\
\text { point }^{387}\end{array}$ \\
\hline 8200 & 915 & shp- & shp- & shp- & shp- & $\begin{array}{l}\text { to remove a covering layer (skin, } \\
\text { veil, skirt, curtain); (KW also:) to } \\
\text { wear a covering layer }\end{array}$ \\
\hline 8210 & 916 & & sht- & & sht- & $(?)^{388}$ \\
\hline 8240 & 917 & $\begin{array}{l}\text { shc-/ } \\
\text { shs- }\end{array}$ & $\begin{array}{l}\text { shc-l } \\
\text { shs- }\end{array}$ & $\begin{array}{l}\text { shc-/ } \\
\text { shs- }\end{array}$ & shs- & spring salmon \\
\hline 8250 & 918 & shs- & shs- & shs- & shs- & offspring (of one couple) \\
\hline 8260 & 919 & shł- & sht- & shł- & sht- & $\begin{array}{l}\text { mental anguish, in love, in } \\
\text { mourning, jealous }\end{array}$ \\
\hline 8270 & 920 & sht- & sht- & & & $(?)^{390}$ \\
\hline
\end{tabular}

${ }^{387} \mathrm{In} \mathrm{HE} / \mathrm{OO} / \mathrm{HA}$ the root occurs also as alternant of $\{\mathrm{lh}-\}$ in interrogative perfective constructions, e.g. HE /sas l'axd'mn'ukv/ "Do you have a place to stay yet?" The root may occur also in HA /sa $\chi$ / "to want to."

${ }^{388}$ In a small number of words whose semantic relationship is problematic, cf. $\mathrm{KW} / \mathrm{sata} / *$ "desired as food" KW /sadqvла/* "to be greedy, to devour" KW /sadik³a/ "horsefly" OO /satzi/ "belly button."

${ }^{389}$ Also in HA /s'ała/ "jealous (and refusing to accept invitation to feast)."

${ }^{390} \mathrm{In} \mathrm{HE} / \mathrm{s}^{\prime}$ al'iqti/ and /s'al'iqvti/ OO /saliqti/ and /saliqvti/ "lips (of one's mouth)." 
North Wakashan Comparative Root List (Updated)

\begin{tabular}{|c|c|c|c|c|c|c|}
\hline Root \# & Old \# & $\mathrm{HE}$ & $\mathrm{OO}$ & HA & KW & Gloss \\
\hline 8290 & 922 & & & & $\begin{array}{l}\text { shlh-* } \\
\text { shyhł- }\end{array}$ & blind, visually impaired ${ }^{391}$ \\
\hline 8300 & 923 & $\mathrm{shl}^{3-}$ & $\operatorname{shl}^{3}-$ & $\operatorname{shl}^{3-}$ & & $(?)^{392}$ \\
\hline 8310 & 924 & shk- & shk- & $(\mathrm{gn}-)$ & shk-* & $\begin{array}{l}\text { (HE/OO) supplementary, } \\
\text { additional, extra; }(\mathrm{KW}) \text { low on } \\
\text { reserves }^{393}\end{array}$ \\
\hline 8320 & 925 & shk- & shk- & shk- & shk-* & $\begin{array}{l}\text { (HE/OO/HA) to travel in a } \\
\text { vehicle (e.g. boat, plane); (HA } \\
\text { also:) to move forward jerkily } \\
\text { (like baby moving in a sitting } \\
\text { position), to inch forward, to nod } \\
\text { the head forward (as when falling } \\
\text { asleep in a sitting position); } \\
\text { (KW) to use a canoe, to give } \\
\text { away a canoe }{ }^{394}\end{array}$ \\
\hline 8340 & & & & & shk- & (?) joint (of box) ${ }^{395}$ \\
\hline
\end{tabular}

391 \{ shlh- $\}$ is attested in KW /sala/* "blind (not totally)", a term from the Newettee dialect, and possibly in KW /salaxda3iliqała/* "half blind" which is a term reported by Boas (1948:187; 1947:328) and has a structure $\left\{\right.$ shlh-xdh $\left.{ }^{3} y l y q-h ł-h\right\}$. A stem $\{$ shyht- $\}$ rather than $\{$ shlh- $\}$ may be present in the synonyms KW $\{$ shyht-=yq-h1-h $\}>$ /sailiqała/ and KW $\{$ shyht-=yq-stw-h1-h $\}>$ /sailizstvała/ "half blindness of a drunk person." 392 Possibly in HE / $\mathrm{s}^{\prime} \mathrm{al}^{3 \prime} \mathrm{u} /$ and $/ \mathrm{s}^{\prime} \mathrm{al}^{3 \mathrm{a}} \mathrm{au} /$ and $/ \mathrm{s}^{\prime} \mathrm{al}^{3 \mathrm{a}} \mathrm{a} / \mathrm{OO} / \mathrm{sal}^{3} \mathrm{u} /$ and $/ \mathrm{sal}^{3} \mathrm{a} / \mathrm{HA} / \mathrm{s}^{\prime} \mathrm{al}{ }^{3} \mathrm{i} /$ "ring finger."

393 Possibly also in HE /s'ay'ak£uda/ "labour strike."

${ }^{394}$ Root may originally have referred to a sitting paddler bending forward before each stroke but subsequently became associated with canoe use, travelling in general and forward motion. ${ }^{395}$ In three words listed in Boas (1948:183) as derivates of $\{$ sk- $\}$ (no. 7840) although their glosses do not seem to relate to root no. 7840 . 
North Wakashan Comparative Root List (Updated)

\begin{tabular}{|c|c|c|c|c|c|c|}
\hline Root \# & Old \# & $\mathrm{HE}$ & $\mathrm{OO}$ & HA & KW & Gloss \\
\hline 8360 & 926 & $(\mathrm{nhn}-)$ & $(\mathrm{nhn}-)$ & $\begin{array}{l}\text { shk- } \\
\text { shx- }\end{array}$ & $(\mathrm{nhn}-)$ & grizzly bear ${ }^{396}$ \\
\hline 8370 & 927 & & $\begin{array}{l}\operatorname{sh} x- \\
\operatorname{sh}^{3} x^{-}\end{array}$ & & shx- & $\begin{array}{l}\text { to appropriate, to lay one's hands } \\
\text { on sth.; greed }\end{array}$ \\
\hline 8380 & 913 & & & shkv- & & $(?)^{397}$ \\
\hline 8390 & 928 & $\mathrm{sh}^{3} \mathrm{kv}-$ & $\mathrm{sh}^{3} \mathrm{kv}-$ & $\begin{array}{l}\text { shkv- } \\
\text { sh³ }^{3} \mathrm{kv}-\end{array}$ & $\mathrm{sh}^{3} \mathrm{kv}-$ & $\begin{array}{l}\text { animal, mammal (esp. seal), } \\
\text { animal products (e.g. skin, meat, } \\
\text { oil); (KW also:) to prepare } \\
\text { animal products (skinning, } \\
\text { butchering, carving) }\end{array}$ \\
\hline 8391 & 899 & & shkv- & & $\begin{array}{l}\text { shkv- } \\
\text { skv- }\end{array}$ & $\begin{array}{l}\text { bracken fern root (Pteridium } \\
\text { aquilinum) } \\
398\end{array}$ \\
\hline 8400 & & & & shxv- & & to use dip net for sea eggs \\
\hline 8410 & & & $\begin{array}{l}\operatorname{shxv-l} \\
\operatorname{sh} \chi v-\end{array}$ & & & $(?)^{399}$ \\
\hline 8430 & 930 & & & shqv- & & grass \\
\hline 8440 & 931 & $\operatorname{sh} \chi v-$ & $\operatorname{sh} \chi v^{-}$ & $\operatorname{sh} \chi v-$ & $\operatorname{sh} \chi v-$ & $\begin{array}{l}\text { isolated, by oneself, separated } \\
\text { from one's group, unique, one of } \\
\text { a kind }\end{array}$ \\
\hline
\end{tabular}

396 \{shk-\} only in HA (Kitamaat dialect) /saak/ and HA (Kitlope dialect) /sak/ "grizzly bear." The root form $\{\operatorname{shx}-\}$ is attested in HA (Kitlope) $\left\{\operatorname{shx}-+y^{3} s\right\}>/ s^{3}{ }^{3}$ is/ "grizzly fat." Possibly HA /saak/ and /sak/ are a loanword of unknown origin and because its /k/ is automatically fricativized word-finally the form $\{$ shx- $\}$ could result from backformation.

${ }^{397}$ Perhaps in HA $\{[\mathrm{sh}]$ shkv-+-h $\}>\left\{\right.$ shyhk $\left.^{3} \mathrm{v}-\mathrm{h}\right\}>/ \mathrm{saik}^{3} \mathrm{va} /$ "to want to get sth. (game, company or whatever)."

398 The only example of the form $\{\mathrm{skv}-\}$ is KW /skva/ attested in Lincoln \& Rath (1980:171) and could be an old misrecording or typing error.

${ }^{399}$ Possibly in OO /sawht/ "to suspect." 
North Wakashan Comparative Root List (Updated)

\begin{tabular}{|c|c|c|c|c|c|c|}
\hline Root \# & Old \# & $\mathrm{HE}$ & $\mathrm{OO}$ & $\mathrm{HA}$ & KW & Gloss \\
\hline 8450 & 932 & & & & shq- & to smart, to sting \\
\hline 8460 & 933 & & & shq- & & to shove, slide, skate \\
\hline 8470 & 934 & & & & $\operatorname{sh}^{3} \chi v-$ & oval, oblong, elongated, stretched \\
\hline 8480 & 935 & $\lambda \mathrm{m}-$ & $\lambda \mathrm{m}-$ & $\lambda \mathrm{m}-$ & $\lambda \mathrm{m}-$ & scab, sore, pock mark \\
\hline 8490 & 936 & & & & $\lambda \mathrm{ms}^{-}$ & $\begin{array}{l}\text { to pinch with the finger nails, to } \\
\text { pick a scab, to switch light on/off }\end{array}$ \\
\hline 8500 & $\begin{array}{l}937 \\
964 \\
977\end{array}$ & $\begin{array}{l}£ \mathrm{mxv}- \\
£ \text { hmw- }\end{array}$ & $£$ £mw- & & $\begin{array}{l}\lambda \mathrm{hmh}^{-} \\
\lambda \mathrm{hmhw}^{3}- \\
\lambda \mathrm{mxv}- \\
£ \mathrm{mxv}-\end{array}$ & (?) spotted ${ }^{400}$ \\
\hline 8510 & $\begin{array}{l}938 \\
940\end{array}$ & $\lambda \mathrm{n}-$ & $\lambda \mathrm{n}-$ & & $\lambda \mathrm{n}-$ & $\begin{array}{l}\text { to grip with the teeth (like fish } \\
\text { the bait, dog a stick), to take a } \\
\text { small bite, to nibble, to peck }\end{array}$ \\
\hline 8520 & 939 & $\lambda \mathrm{n}-$ & $\lambda \mathrm{n}-$ & & $\lambda n-*$ & $\begin{array}{l}\text { (?) across (like a barrier or a } \\
\text { crosspiece keeping things } \\
\text { together) })^{401}\end{array}$ \\
\hline
\end{tabular}

${ }^{400}$ In words referring to fish identified by informants as perch or shiner. The perch, a freshwater fish, and the shiner perch, a saltwater fish, belong to the order of the Perciformes and "perci-" means "spotted." A spotted appearance of perch and shiner perch would link this root's heterogenous forms to $\{\lambda \mathrm{m}-\}$ "scab etc." However, the identity of the fish in question is uncertain. It could also be Pacific ocean perch (Sebastes alutus) a.k.a Pacific rockfish, Rose fish, Red bream or Red perch and belong to a different order. Then again it could also be the Striped seaperch (Embiotica lateralis) which does belong to the Perciformes and whose stripes are series of spots.

${ }^{401}$ Uncertain root because the examples clearly pointing to the form $\{\lambda n-\}$ are from Boas but could not be reelicited. Among them is $\mathrm{KW} / \lambda_{\mathrm{Hy}}{ }^{3} \mathrm{u} / *$ "crossbar for barring door." The examples 
North Wakashan Comparative Root List (Updated)

\begin{tabular}{|c|c|c|c|c|c|c|}
\hline Root \# & Old \# & $\mathrm{HE}$ & $\mathrm{OO}$ & $\mathrm{HA}$ & KW & Gloss \\
\hline 8530 & 940 & & & & $\lambda$ ns- & (?) to nibble ${ }^{402}$ \\
\hline 8540 & 941 & & & & $\lambda \mathrm{n} £-$ & to peck \\
\hline 8550 & 942 & & £nk- & & $\lambda \mathrm{nk}^{*} *$ & to peck ${ }^{403}$ \\
\hline 8560 & 943 & & & & $\lambda \mathrm{nqv}-*$ & to peck $^{404}$ \\
\hline 8580 & 944 & $£ k-$ & $£ k-$ & $\lambda \mathrm{k}-$ & $\lambda \mathrm{k}-$ & cinquefoil (Potentilla pacifica) \\
\hline 8590 & $\begin{array}{l}945 \\
946 \\
995\end{array}$ & $\begin{array}{l}£ \mathrm{k}- \\
\lambda \mathrm{x}_{-} \\
£ \mathrm{x}- \\
£ \mathrm{x} \cdot-\end{array}$ & $\begin{array}{l}£ \mathrm{k}- \\
\lambda \mathrm{x}- \\
£ \mathrm{x}- \\
\mathfrak{£}^{3} \mathrm{yx} \cdot\end{array}$ & $\lambda x-$ & $\lambda x-$ & $\begin{array}{l}\text { to use a long thing as a barrier, } \\
\text { thwart or lever; to position } \\
\text { crosswise; to steer (boat with } \\
\text { tiller, car with steering wheel) }\end{array}$ \\
\hline 8600 & 947 & $\lambda y-$ & $\lambda y-$ & & & $\begin{array}{l}\text { to invite people from another } \\
\text { village to a potlatch }\end{array}$ \\
\hline 8601 & & & $\lambda y-$ & & & $(?)^{406}$ \\
\hline 8610 & 948 & & & & $\lambda y s^{-}$ & mosquito, mosquito bite \\
\hline 8620 & $\begin{array}{l}949 \\
967\end{array}$ & $\lambda y k v-$ & $\lambda y k v-$ & $\lambda \mathrm{y}^{3} \mathrm{kv}-$ & $\begin{array}{l}\lambda \mathrm{ykv}- \\
\lambda \mathrm{hkv-}\end{array}$ & to borrow ${ }^{407}$ \\
\hline
\end{tabular}

that could be elicited involve a stem \{ $\{$ nyk- $\}$ "to lock door inside with pin, bolt or bar" which may contain either $\{\lambda \mathrm{n}-\}$ or $\{\lambda \mathrm{x}-\}$ (root no. 8590).

${ }^{402} \mathrm{In} \mathrm{KW} / \lambda_{\mathrm{Hsa}} / *$ "to nibble" $\mathrm{KW} / \lambda_{\mathrm{Hs}} \hbar^{3} \mathrm{id} / * *$ "to take a nibble" and possibly $\mathrm{KW} / \lambda_{\text {Hs }}$ la/ "bow of boat is lower than the stern."

${ }^{403} \mathrm{In} \mathrm{OO} / \mathfrak{f H k a /}$ "to hold while pecking" KW (Newettee dialect) / $\lambda_{\mathrm{Hka}} / *$ "to peck."

${ }^{404} \mathrm{In} \mathrm{KW}$ (Newettee dialect.) / $\lambda$ Hqva/* "to peck."

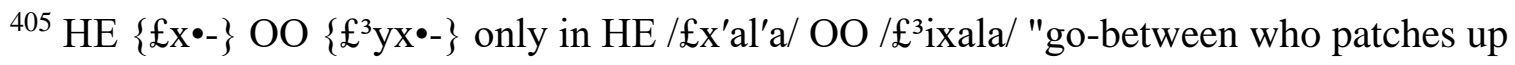
quarrels."

${ }^{406} \mathrm{In} \mathrm{OO} / \lambda$ iнcila/ "thicket" (synonym: $\left./ \mathfrak{\&}^{3} \mathrm{ic}^{3} \mathrm{~s} /\right)$.

$407\{\lambda \mathrm{hkv}-\}$ only in KW / $\lambda$ akubs/ "fond of borrowing." 
North Wakashan Comparative Root List (Updated)

\begin{tabular}{|c|c|c|c|c|c|c|}
\hline Root \# & Old \# & $\mathrm{HE}$ & $\mathrm{OO}$ & HA & KW & Gloss \\
\hline 8630 & 950 & $\lambda$ yq- & $\lambda$ yq- & & $\lambda y q-$ & $\begin{array}{l}\text { one's name; renown, scenario or } \\
\text { image one is associated with; } \\
\text { reputation; to give a name to sb., } \\
\text { to call sb.'s name, to call the next } \\
\text { line to be sung }\end{array}$ \\
\hline 8640 & 951 & & $£ \mathrm{kv}-$ & $\lambda \mathrm{kv}-$ & $\begin{array}{l}\lambda \mathrm{kv}- \\
£ \mathrm{kv}- \\
\lambda \mathrm{hkv-}\end{array}$ & thick in girth, voluminous, full ${ }^{408}$ \\
\hline 8650 & 952 & $\lambda w-$ & $\lambda w-$ & $\lambda w-$ & $\lambda w-$ & $\begin{array}{l}\text { proximity, contiguity, continuity, } \\
\text { recurrence, closeness, kinship }\end{array}$ \\
\hline 8670 & & $\lambda \mathrm{ws}^{-}$ & $\begin{array}{l}\lambda \mathrm{w}- \\
\lambda \mathrm{ws}-\end{array}$ & $\lambda \mathrm{ws}^{-}$ & & $\begin{array}{l}\text { luck, good fortune, supernatural } \\
\text { power }\end{array}$ \\
\hline 8680 & $\begin{array}{l}953 \\
955\end{array}$ & $\lambda w g v-$ & $\begin{array}{l}\lambda \mathrm{wgv-} \\
\lambda \mathrm{wkv-}\end{array}$ & $\lambda w g v-$ & $\begin{array}{l}\lambda \mathrm{wgv-} \\
\lambda w \mathrm{wV}--\end{array}$ & $\begin{array}{l}\text { touched by the supernatural, } \\
\text { lucky, spooked, alarmed }\end{array}$ \\
\hline 8690 & 954 & & & & $\lambda$ wgvm- & $\begin{array}{l}\text { using the paddle to steer the } \\
\text { canoe }\end{array}$ \\
\hline 8700 & 956 & $£ q v-$ & & & $\lambda q v-$ & defloration with the finger \\
\hline 8710 & 957 & & & & $\begin{array}{l}\lambda \mathrm{qv}^{-*} / \\
\lambda \mathrm{q}_{-}^{*}\end{array}$ & $(?)^{410}$ \\
\hline 8730 & 958 & $\begin{array}{l}\lambda \chi- \\
£ \chi-\end{array}$ & $\begin{array}{l}\lambda \chi- \\
£ \chi-\end{array}$ & $\lambda \chi-$ & $\lambda \chi-$ & $\begin{array}{l}\text { to slip, slide, shove; to gain } \\
\text { headway (said of canoe) }\end{array}$ \\
\hline
\end{tabular}

$408\{£ \mathrm{kv}-\}$ occurs in OO /£gvit/ "thick-bodied, thick in girth (like a tree)" KW /£kvM/ "brazen, arrogant" (= "thick-faced").

${ }^{409}$ Occurs also in the KW proclitic connective $/ \lambda \mathrm{u}^{3} /$ "and, and also, along with, together with, accompanied by." Instead of it HE/OO/HA use proclitic/du/.

${ }^{410} \mathrm{In} \mathrm{KW}\{\lambda \mathrm{qv} / \mathrm{q}-=\mathrm{kv}\}>/ \lambda \mathrm{rkv} / *$ "harpoon point." Could be a misspelling of $/ £ \mathrm{gkv} / *<\{£ \mathrm{k}-=\mathrm{kv}\}$ (with root no. 9060). The latter means "notched" and can refer to a harpoon point that is notched to create barbs. See Boas (1948:427). 
North Wakashan Comparative Root List (Updated)

\begin{tabular}{|c|c|c|c|c|c|c|}
\hline Root \# & Old \# & $\mathrm{HE}$ & $\mathrm{OO}$ & HA & KW & Gloss \\
\hline 8740 & 959 & & & & $\lambda \chi^{* *}$ & $(?)^{411}$ \\
\hline 8750 & $\begin{array}{l}960 \\
961 \\
962 \\
966\end{array}$ & $\lambda \mathrm{h}-$ & $\lambda \mathrm{h}-$ & $\lambda \mathrm{h}-$ & $\lambda \mathrm{h}-$ & $\begin{array}{l}\text { 1. erect, to stand up (like pole), to } \\
\text { stick out (like handle), to } \\
\text { protrude, to stand out (like } \\
\text { accomplishments); } 2 \text {. to raise by } \\
\text { wedging; to wedge apart, to drive } \\
\text { in wedge, to cut a wedge out of a } \\
\text { tree (as in logging); to chop down } \\
\text { a tree }{ }^{412}\end{array}$ \\
\hline 8760 & 963 & $\lambda \mathrm{hp}-$ & $\lambda \mathrm{hp}-$ & $\lambda \mathrm{hp}-$ & & to dig for clams or cockles \\
\hline 8761 & & $\lambda$ ht- & & & $\lambda \mathrm{ht}-*$ & $\begin{array}{l}\text { wedge, use a wedge, wedged } \\
\text { in }^{413}\end{array}$ \\
\hline 8770 & 965 & $\lambda \mathrm{hk}-$ & $\lambda \mathrm{hk}-$ & $\lambda \mathrm{hk}-$ & & $\begin{array}{l}\text { chopping motion (as of gillnet } \\
\text { being lowered vertically into the } \\
\text { water), ridge (as on rock cod's } \\
\text { head) }\end{array}$ \\
\hline 8800 & 968 & & & & $\lambda \mathrm{h} \chi_{\mathrm{v}}-$ & to go to get food \\
\hline 8810 & 969 & $\lambda \mathrm{h} \chi_{\mathrm{V}}-$ & $\lambda \mathrm{h} \chi \mathrm{v}-$ & $\lambda \mathrm{h} \chi \mathrm{v}-$ & $\lambda \mathrm{h} \chi_{\mathrm{v}}-$ & $\begin{array}{l}\text { to stand (said mostly of humans), } \\
\text { upright; sticking up, sticking up } \\
\text { for, to volunteer; to stand still, } \\
\text { barrier, to stand in the way; rank, } \\
\text { standing }\end{array}$ \\
\hline
\end{tabular}

${ }^{411} \mathrm{In} \mathrm{KW} / \lambda \times \mathrm{a} / *$ "to have a pungent smell."

${ }^{412}$ Root may occur also in HE / $\lambda$ 'ara/ "basking shark" and refer to the vertical gill slits that almost encircle the fish's head, and in HA / $\lambda$ 'a $\chi$ dala/ "grey cod" where it refers to the barbels hanging down from the lower jaw.

${ }^{413} \mathrm{HE}$ possibly in $/ \Lambda^{\prime}$ atul ${ }^{3}$ asbala/ (name of a point of land near Bella Bella). 
North Wakashan Comparative Root List (Updated)

\begin{tabular}{|c|c|c|c|c|c|c|}
\hline Root \# & Old \# & $\mathrm{HE}$ & $\mathrm{OO}$ & HA & $\mathrm{KW}$ & Gloss \\
\hline 8820 & 970 & & & & $\lambda \mathrm{h}^{3} \mathrm{p}-$ & to nail, to peg \\
\hline 8830 & 971 & $£ \mathrm{p}-$ & £p- & £p- & $\begin{array}{l}£ p- \\
£ \text { hp- }\end{array}$ & $\begin{array}{l}\text { to spread out (like cloth, sail, } \\
\text { wings), unfold, open up, split } \\
\text { apart, burst open; (HA also:) to } \\
\text { fly) }\end{array}$ \\
\hline 8840 & 972 & $£ \mathrm{~m}-$ & & & $£ \mathrm{~m}-*$ & $(?)^{414}$ \\
\hline 8850 & 973 & & & & $£ \mathrm{~ms}-*$ & to turn away in shame \\
\hline 8860 & 974 & & & & $£ \mathrm{mł-}$ & to burst, blast, blow up \\
\hline 8870 & 975 & & & & $£ \mathrm{mk}-$ & $\begin{array}{l}\text { wedge, to wedge, to split with } \\
\text { wedge }\end{array}$ \\
\hline 8880 & 976 & $£ \mathrm{mkv}-$ & $£ m k v-$ & $£ \mathrm{mkv}-$ & (qk-) & $\begin{array}{l}\text { bunchberries (Cornus } \\
\text { canadensis) })^{415}\end{array}$ \\
\hline 8900 & 979 & & & & $£ m q-$ & proud, haughty, arrogant \\
\hline 8910 & 980 & & & $£ m q-$ & $£ m q-$ & $(?)^{416}$ \\
\hline 8920 & 981 & & & & $£ t-$ & $\begin{array}{l}\text { floppy material covering sth. } \\
\text { (e.g. head or face) and } \\
\text { overhanging }\end{array}$ \\
\hline 8930 & 982 & & & & $£ \mathrm{t}-*$ & $(?)^{417}$ \\
\hline
\end{tabular}

${ }^{414}$ Perhaps in the man's name HE /£M'aba/ and in KW /£Mała/* "having the head backward." $415\{£ \mathrm{mkv}-\}$ may really refer to the springiness of filaments under a bunchberry flower's petals.

See the comment on $\{q k-\}$ (root no. 20620).

${ }^{416} \mathrm{In} \mathrm{KW} / £ \mathrm{Mqu} /$ "female sawbill duck" and perhaps HA /£мq ${ }^{3}$ 'auli/ (a kind of berries identified by the informant as bunchberries although the $\mathrm{HE} / \mathrm{OO} / \mathrm{HA}$ root referring to bunchberries is $\{£ m k v-\})$. The KW term may refer to the duck's spiky crest.

${ }^{417} \mathrm{In} \mathrm{KW} / £ t s \jmath / *$ "hole drilled in a slanting direction." 
North Wakashan Comparative Root List (Updated)

\begin{tabular}{|c|c|c|c|c|c|c|}
\hline Root \# & Old \# & $\mathrm{HE}$ & $\mathrm{OO}$ & HA & KW & Gloss \\
\hline 8940 & 983 & & & & $£ n-*$ & never ${ }^{418}$ \\
\hline 8950 & $\begin{array}{r}984 \\
1055\end{array}$ & & & & $\begin{array}{l}\text { Ens-* } \\
\text { Eny }{ }^{3} * *\end{array}$ & (?) to split wood ${ }^{419}$ \\
\hline 8960 & 985 & £nkv- & £nkv- & £nkv- & & $(?)^{420}$ \\
\hline 8970 & 986 & £nqv- & £nqv- & £nqv- & & $(?)^{421}$ \\
\hline 8980 & $\begin{array}{r}987 \\
988 \\
1161\end{array}$ & Enqv- & £nqv- & £nqv- & $\begin{array}{l}\text { £nqv- } \\
\text { łnq-* }\end{array}$ & lacking vigor, falling apart ${ }^{422}$ \\
\hline 8990 & 989 & & & & £nq-* & $(?)^{423}$ \\
\hline 9000 & $\begin{array}{r}990 \\
1042\end{array}$ & £s- & £s- & £s- & $\begin{array}{l}£ s- \\
£ h^{3} s-\end{array}$ & $\begin{array}{l}\text { to slip, slide, glide; to skim over } \\
\text { a surface }{ }^{424}\end{array}$ \\
\hline 9010 & 991 & & & & £- & $\begin{array}{l}\text { to chip off (paint, rock, bowl, } \\
\text { etc. })^{425}\end{array}$ \\
\hline
\end{tabular}

418 In KW (Newettee dialect) /£ $\mathrm{Ha} / *$ and /£Hala/* "never."

419 \{£ns- $\}$ only in $\mathrm{KW} / \mathfrak{f H S a} / *$ (possibly:) "to split with the grain." $\left\{£_{\left.n y^{3}-\right\}}\right.$ in $\mathrm{KW} / \mathfrak{H H}^{3} \mathrm{a} /$ "to split log so that the split misses heart of wood, to split wood across the grain through centre of tree" and KW /£нy ${ }^{3} \mathrm{a}^{3} \mathrm{akv} /$ "split across grain (wood)."

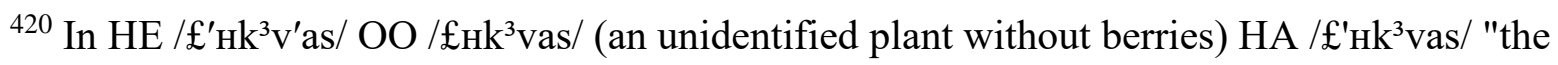
inside of one's nose (between nose and windpipe); species of berry (said to resemble dried snot)." ${ }^{421} \mathrm{In} \mathrm{HE} / £^{\prime} \mathrm{Hq}^{3} \mathrm{v}^{\prime} \mathrm{as} / \mathrm{OO} / \mathfrak{H q}^{3} \mathrm{vas} /$ (an unidentified plant with edible red berries) HA /£ $\mathrm{Hq}^{3} \mathrm{v}^{\prime} \mathrm{as} /$ and $/ \mathfrak{f}^{\prime} \mathrm{Hq}^{3} \mathrm{vas} /$ (a plant with purplish edible berries).

422 \{nq- $\}$ only in $\mathrm{KW} / \mathrm{HHq}^{3} \mathrm{a} / *$ "downcoast, uneasy." KW uses \{xvls- $\}$ for "unhappy, sad, withering."

${ }^{423}$ In KW /£Hqała/* "standing (said of inanimate object)."

$424\left\{\mathfrak{h}^{3} \mathrm{~s}-\right\}$ in $\mathrm{KW} / £ \mathrm{a}^{3} \mathrm{sa} /$ "slippery" and probably $\mathrm{KW} / £ \mathrm{a}^{3} \mathrm{~s} л \mathrm{a} /$ "weasel dance."

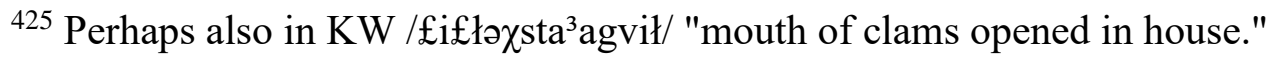


North Wakashan Comparative Root List (Updated)

\begin{tabular}{|c|c|c|c|c|c|c|}
\hline Root \# & Old \# & $\mathrm{HE}$ & $\mathrm{OO}$ & HA & KW & Gloss \\
\hline 9020 & 992 & $£^{3} 1-$ & $£^{3} 1-$ & $\mathfrak{f}^{3} 1-$ & $£ 1-$ & $\begin{array}{l}\text { to turn up the palms of the hands } \\
\text { (as in dancing); to raise on flat of } \\
\text { hands, scoop or shovel; (KW } \\
\text { also:) to lift or carry a canoe on } \\
\text { raised forearms }\end{array}$ \\
\hline 9040 & 1026 & & & & $\begin{array}{l}£ l p-* \\
£ h p-*\end{array}$ & to hesitate ${ }^{426}$ \\
\hline 9050 & 993 & & $£ k-$ & & $\begin{array}{l}£ k- \\
£ h k-\end{array}$ & $\begin{array}{l}\text { to take a break, to interrupt work, } \\
\text { to shirk }\end{array}$ \\
\hline 9060 & 994 & & & & $£ \mathrm{k}-*$ & to notch \\
\hline 9080 & 996 & $£ x-$ & $£ x-$ & & & to slip, slide \\
\hline 9090 & $\begin{array}{r}997 \\
1167\end{array}$ & $\begin{array}{l}\text { £xs- } \\
\text { łxs- }\end{array}$ & & $(\mathrm{xms})$ & & $(?)^{427}$ \\
\hline 9100 & $\begin{array}{r}998 \\
1010\end{array}$ & $\begin{array}{l}\text { £ys- } \\
£ y^{3} s-\end{array}$ & $£ y^{3} s-$ & & £ys- & $\begin{array}{l}\text { (HE/OO) to slap (a ball), to strike } \\
\text { at sth. with a flicking movement, } \\
\text { to flip; (KW) to put sth. up to dry } \\
\text { (in sun, in smokehouse). }{ }^{428}\end{array}$ \\
\hline 9110 & $\begin{array}{r}999 \\
1002 \\
1175\end{array}$ & $\begin{array}{l}\text { £yk- } \\
\text { £yx- }\end{array}$ & $\begin{array}{l}\text { £ył- } \\
\text { £yx- } \\
\text { łyx- }\end{array}$ & & £ył- & to invite to a feast ${ }^{429}$ \\
\hline
\end{tabular}

${ }^{426} \mathrm{In} \mathrm{KW} /$ Æлр$^{3}$ iqла/* "to think one is unable to do" and $\mathrm{KW} / £ a p a / *$ "to think sth. is insufficient, to hesitate on account of danger."

${ }^{427}$ In HE /ххsл'а/ "person who does everything in a big way (eating, working), cantankerous person" НЕ /ххsл'a/ "fierce person, someone who eats a lot."

${ }^{428} \mathrm{HE}\{£ y s-\}$ is attested only in the plural forms of some examples whose neutral form derives from $\left\{£ y^{3} s-\right\}$.

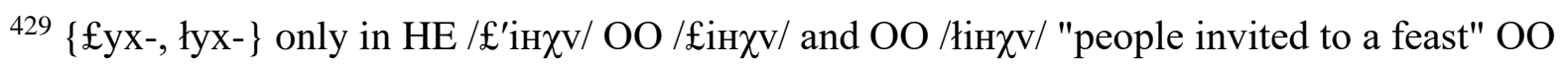
/lixa/ "to feast." 
North Wakashan Comparative Root List (Updated)

\begin{tabular}{|c|c|c|c|c|c|c|}
\hline Root \# & Old \# & $\mathrm{HE}$ & $\mathrm{OO}$ & HA & KW & Gloss \\
\hline 9120 & $\begin{array}{l}1000 \\
1001\end{array}$ & $\begin{array}{l}\text { £yt- } \\
£ y x- \\
\text { łyx- }\end{array}$ & $\begin{array}{l}\text { £ył- } \\
\text { tyx- }\end{array}$ & & łyx- & $\begin{array}{l}\text { (?) to appropriate, usurp, use } \\
\text { without authority }{ }^{430}\end{array}$ \\
\hline 9130 & 1037 & £ył- & £ył- & £ył- & & $\begin{array}{l}\text { to slap, to strike at sth. with a } \\
\text { flicking movement, to flap }{ }^{431}\end{array}$ \\
\hline 9150 & 1003 & $\left(q^{3} y k v-\right)$ & £ykv- & $\left(q^{3} y k v-\right)$ & £ykv- & $\begin{array}{l}\text { to tell a lie, cheat, deceive, lure, } \\
\text { decoy }^{432}\end{array}$ \\
\hline 9160 & $\begin{array}{l}1004 \\
1005\end{array}$ & & & & £yqv- & $\begin{array}{l}\text { to stray, to deviate, to change, to } \\
\text { move }^{433}\end{array}$ \\
\hline 9170 & 1006 & & & & £yqv-* & $(?)^{434}$ \\
\hline 9190 & $\begin{array}{l}1007 \\
1092\end{array}$ & £yq- & & $£^{3} \mathrm{yq}^{-}$ & £yq- & $\begin{array}{l}\text { (HA) to crack, split (as wood); } \\
(\mathrm{KW}) \text { to carve or hollow out } \\
\text { canoe }^{435}\end{array}$ \\
\hline 9200 & $\begin{array}{l}1008 \\
1009\end{array}$ & $£ y x-$ & $\begin{array}{l}\text { £yq- } \\
\text { £y }-\end{array}$ & $£ y x-$ & $\begin{array}{l}£ y q- \\
£ y x-\end{array}$ & $\begin{array}{l}\text { to beat time, rhythmic tapping, } \\
\text { woodpecker }\end{array}$ \\
\hline 9230 & 1012 & & & $£ w p-$ & & to burn, scald \\
\hline
\end{tabular}

$430\{£ y ł-\}$ occurs possibly in HE (Klemtu dialect) /£'il'mћa/ "to take for oneself what is intended for others" and $\mathrm{OO} / £$ ilakila/ "doing sth. different from what one is supposed to be doing." $\{£ y x-\}$ and $\{$ łyx- $\}$ occur possibly in HE /£'in'mћa/ OO /tinmћa/ "to take back lent out property" $\mathrm{KW} /$ hinmha/ "to grab, snatch, take by force, abduct."

${ }^{431}$ Possibly in HE/OO/HA \{£ylhq-\} "to flap like fish caught, to slap the tail (as a halibut caught)."

${ }^{432}$ Only one derivate of the OO form. It could be a KW borrowing because $\left\{\mathrm{q}^{3} \mathrm{ykv}-\right\}$ occurs and is productive in $\mathrm{OO}$ as in $\mathrm{HE} / \mathrm{HA}$.

${ }^{433}$ See also the polysemy of root no. 2270.

${ }^{434} \mathrm{In} \mathrm{KW} /$ fiqvaqa/* "split down (said of salmon prepared in a certain way for drying)."

${ }^{435} \mathrm{HE}$ possibly in the place name /E'iq'an'a/. 
North Wakashan Comparative Root List (Updated)

\begin{tabular}{|c|c|c|c|c|c|c|}
\hline Root \# & Old \# & $\mathrm{HE}$ & $\mathrm{OO}$ & HA & KW & Gloss \\
\hline 9240 & 1013 & & & & $\begin{array}{l}£ w m h- \\
£ w m \infty-\end{array}$ & $\begin{array}{l}\text { more than expected, in excess, } \\
\text { overdoing it }\end{array}$ \\
\hline 9250 & 1014 & & & & $£ w k-*$ & to be level \\
\hline 9260 & 1015 & $£ w y^{3}-$ & $£ w y^{3}-$ & & & to dress up, to decorate sth. \\
\hline 9270 & 1016 & & & & $£ w \chi v-$ & $\begin{array}{l}\text { small long things (like sticks, } \\
\text { bones, roots) being or moving } \\
\text { somewhere }\end{array}$ \\
\hline 9280 & 1017 & £qv- & £qv- & £qv- & £qv- & $\begin{array}{l}\text { to pet, caress; to stimulate with } \\
\text { finger or hand }\end{array}$ \\
\hline 9290 & 1018 & $£ \chi v-$ & $£ \chi v-$ & $£ \chi v-$ & $£ \chi v-$ & $\begin{array}{l}\text { to rub, stroke or press with flat of } \\
\text { hand }\end{array}$ \\
\hline 9300 & $\begin{array}{l}1019 \\
1037 \\
1039\end{array}$ & & & £hq- & $\begin{array}{l}£ q- \\
£ \text { hq- }\end{array}$ & $\begin{array}{l}\text { to slap, to clap, to hit with flat } \\
\text { object, to pat, to patch, to flatten } \\
\text { sth.; flat paw of bear or marten }{ }^{436}\end{array}$ \\
\hline 9310 & 1020 & & & $£^{3} q-$ & $£ q-*$ & $\begin{array}{l}\text { to drip, dripping wet, soaked; to } \\
\text { droop, dangle, hang limply }\end{array}$ \\
\hline 9320 & 1021 & & & £q- & $£ q-*$ & to catch (e.g. a ball) ${ }^{437}$ \\
\hline 9330 & 1022 & & £h- & & $£$ h- & shelf, stage, platform, scaffolding \\
\hline 9340 & 1024 & & & & £h-* & $\begin{array}{l}\text { (?) to move sth. or sb. } \\
\text { somewhere }{ }^{438}\end{array}$ \\
\hline
\end{tabular}

436 According to http://www.g-wow.org/en-us/pinemarten/default.aspx, "In winter, martens grow long hairs between the toe pads on their feet which work like insulated 'snowshoes' that keep their paws warm and help them travel over deep, fluffy snow."

${ }^{437} \mathrm{KW}$ only in words referring to fishing with a basket trap.

${ }^{438}$ Only three non-reelicitable derivates and Boas (1948) associates them with $\{£$ - $\}$ "shelf, etc." (root no. 9330). Root could be a synonym of $\{\mathrm{dh}-\}$ "to take in hand, to control etc." (root no. 2940). 
North Wakashan Comparative Root List (Updated)

\begin{tabular}{|c|c|c|c|c|c|c|}
\hline Root \# & Old \# & $\mathrm{HE}$ & $\mathrm{OO}$ & HA & $\mathrm{KW}$ & Gloss \\
\hline 9350 & 1025 & & & $£$ £hp- & & to growl, make threatening noise \\
\hline \multirow[t]{2}{*}{9360} & 1023 & \multirow[t]{2}{*}{ £ht- } & & \multirow[t]{2}{*}{ £ht- } & \multirow[t]{2}{*}{ £ht-* } & \multirow[t]{2}{*}{ backstop, to backstop ${ }^{439}$} \\
\hline & 1027 & & & & & \\
\hline 9380 & 1028 & fhs- & & & £hs- & $\begin{array}{l}\text { longish thing (e.g. sliver, pin, } \\
\text { bar) sticking out or being driven } \\
\text { in }{ }^{440}\end{array}$ \\
\hline 9400 & 1029 & £hs- & £hs- & fhs- & & to slap, strike with sth. flat ${ }^{441}$ \\
\hline & 1030 & $\mathrm{fh}^{3} \mathrm{~s}-$ & $£^{3} \mathrm{~s}-$ & & & \\
\hline 9410 & 1031 & £hk- & £hk- & fhk- & & $\begin{array}{l}\text { (HE/OO) to extend a long thing, } \\
\text { to steer with paddle; (HA) to } \\
\text { paddle }\end{array}$ \\
\hline 9420 & 1032 & £hx- & £hx- & £hx- & £hx- & to look for lice \\
\hline 9430 & 1033 & £hx- & £hx- & $£$ fhx- & & $(?)^{442}$ \\
\hline 9440 & $\begin{array}{l}1034 \\
1036\end{array}$ & £hqv- & £hqv- & £hqv- & £hqv- & $\begin{array}{l}\text { to pat; flat thing (like hand) } \\
\text { covering or pressing against sth.; } \\
\text { (KW also:) to push, press, shove } \\
\text { (like when kneading dough or } \\
\text { pushing sth. underwater) }\end{array}$ \\
\hline 9450 & 1035 & & & & $£$ £qv- & to blame, to accuse \\
\hline 9470 & 1038 & & & & £hq- & rancid \\
\hline
\end{tabular}

${ }^{439}$ Refers in HE/HA examples to a ball bouncing off or being passed to another player. In KW it refers to a structure for sth. to rest on or against.

${ }^{440} \mathrm{HE}$ only in $/ \mathfrak{E}^{\prime} \mathrm{ay} \mathbf{M}^{\prime} /$ "addition to sth.."

${ }^{441}$ Also in HA / $\mathfrak{E}^{\prime}$ alasayak/ "man-eating shark" (said to slap around with its tail). In the HE/OO examples of $\{£$ hs- $\}$ root-initial $|£|$ is reduplicated and next reduced to $|1|$ as in $\{£ h[£] s-\}>$ \{£hls-\}.

${ }^{442}$ Possibly in HE/OO $\left\{£^{3}{ }^{3} \mathrm{y} \chi-\right\}$ HA $\{£ h n y \chi-\}$ "vibrating movement." 
North Wakashan Comparative Root List (Updated)

\begin{tabular}{|c|c|c|c|c|c|c|}
\hline Root \# & Old \# & $\mathrm{HE}$ & $\mathrm{OO}$ & $\mathrm{HA}$ & KW & Gloss \\
\hline 9480 & 1040 & & & & $£$ hh -* $^{*}$ & $\begin{array}{l}\text { (?) small long string-like objects } \\
\text { (like cinquefoil roots) }\end{array}$ \\
\hline 9490 & 1041 & $£$ £h- & $£$ £h- & $£$ Łh $\chi-$ & fh $\chi-$ & $\begin{array}{l}\text { to make noise (to startle, flush } \\
\text { game, cheer) }\end{array}$ \\
\hline 9510 & 1043 & $£^{3} \mathrm{p}-$ & $£^{3} p-$ & $£^{3} p-$ & $£^{3} \mathrm{p}-$ & $\begin{array}{l}\text { opening narrows or gets plugged } \\
\text { up (as in closing or opening of } \\
\text { door, closing the gap between } \\
\text { legs of clothes pin or between } \\
\text { one's body and arms; to clutch to } \\
\text { one's body, to squat, to clamp, to } \\
\text { clasp, to climb) }\end{array}$ \\
\hline 9530 & 1044 & $£^{3} \mathrm{~m}^{3} \mathrm{~s}-$ & $£^{3} \mathrm{~m}^{3} \mathrm{~s}-$ & $£^{3} \mathrm{~ms}-$ & $£^{3} \mathrm{~ms}-$ & to emit sparks, to crackle \\
\hline 9550 & 1046 & $£^{3} \mathrm{~m} \mathbf{l}^{-}$ & $£^{3} \mathrm{mt}-$ & $£^{3} \mathrm{ml}-$ & $\left.£^{3} \mathrm{~m}\right\}^{-}$ & irritation (of eyes or throat) \\
\hline 9560 & 1047 & $£^{3} \mathrm{mkv}-$ & & $£^{3} \mathrm{mkv}-$ & $£^{3} \mathrm{mkv-*}$ & $\begin{array}{l}\text { (HE/OO/HA) to go up in the air, } \\
\text { to jump, to bounce, to walk with } \\
\text { a bounce; }(\mathrm{KW}) \text { ritual disposition } \\
\text { of roof boards (in potlatch) }\end{array}$ \\
\hline 9570 & 1048 & $£^{3} \mathrm{mkv}-$ & $£^{3} \mathrm{mkv}-$ & & $£^{3} \mathrm{mkv}-$ & $\begin{array}{l}\text { to tap, pound, poke with a stick; } \\
\text { (OO/KW also:) to play with } \\
\text { stick(s) in the air }\end{array}$ \\
\hline 9571 & 978 & $£^{3} \mathrm{~m} \chi \mathrm{v}-$ & $\mathfrak{f}^{3} \mathrm{~m} \chi \mathrm{v}-$ & $£^{3} \mathrm{~m} \chi \mathrm{v}^{-}$ & $\begin{array}{l}£^{3} \mathrm{~m} \chi \mathrm{v}- \\
£ \mathrm{~m} \chi \mathrm{v}-*\end{array}$ & $\begin{array}{l}\text { (?) inflexible, tough, hard, } \\
\text { hardy }{ }^{444}\end{array}$ \\
\hline
\end{tabular}

${ }^{443}$ Most KW examples refer to climbing.

${ }^{444} \mathrm{HE} / \mathrm{OO}$ only in a few words referring to wood being hard to split. HA in / $\mathfrak{f}^{31} \mathrm{~m} \chi \mathrm{va} /$ "hard,

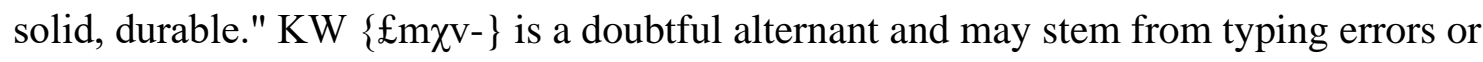
misrecording on the part of Boas or his staff; it occurs in $\mathrm{KW} / \mathfrak{\mathrm { M }}_{\mathrm{Mva}} /{ }^{*}$ "tough, hard on surface, a

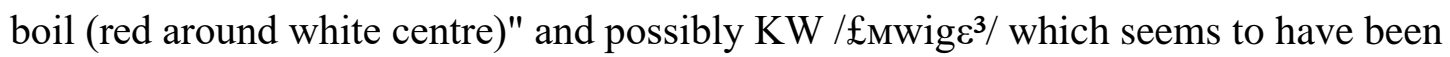
recognized by the informant but whose meaning is uncertain. $\mathrm{KW}\left\{\mathfrak{f}^{3} \mathrm{~m} \chi \mathrm{v}-\right\}$ occurs in 
North Wakashan Comparative Root List (Updated)

\begin{tabular}{|c|c|c|c|c|c|c|}
\hline Root \# & Old \# & $\mathrm{HE}$ & $\mathrm{OO}$ & HA & KW & Gloss \\
\hline 9580 & 1049 & & & $£^{3} \mathrm{mq}-$ & & to hop on one foot \\
\hline 9590 & 1050 & $£^{3} \mathrm{mq}-$ & $£^{3} \mathrm{mq}-$ & $£^{3} \mathrm{mq}-$ & $£^{3} \mathrm{mq}-$ & $\begin{array}{l}\text { 1. dirt, dirty; } 2 \text {. gelatinous, snotty } \\
\text { (like the substance surrounding } \\
\text { the seed cone inside a yew aril (= } \\
\text { "berry"); } 3 \text {. yew }{ }^{445}\end{array}$ \\
\hline 9600 & 1051 & & & & $£^{3} \mathrm{mh}-$ & $(?)^{446}$ \\
\hline 9601 & & & & & $£^{3} \mathrm{mhkv}-*$ & $(?)^{447}$ \\
\hline 9602 & & & & & $£^{3} \mathrm{mh} \chi \mathrm{v}-$ & $(?)^{448}$ \\
\hline 9603 & 1045 & $£^{3} \mathrm{~m}^{3} \mathrm{~s}-$ & $£^{3} \mathrm{~m}^{3} \mathrm{~s}-$ & $£^{3} \mathrm{~m}^{3} \mathrm{~s}-$ & & $(?)^{449}$ \\
\hline 9610 & 1052 & & & & $\begin{array}{l}\mathfrak{£}^{3} \mathrm{t}- \\
\mathfrak{£}^{3} \mathrm{ht}-\end{array}$ & $\begin{array}{l}\text { to desire a woman, to flirt, to } \\
\text { seduce }\end{array}$ \\
\hline 9620 & 1053 & $\left(£^{3} \mathrm{~h}-\right)$ & $\left(£^{3} h-\right)$ & $\left(t^{3} y x v-\right)$ & $\mathfrak{f}^{3} \mathrm{~ns}^{-}$ & black bear \\
\hline 9640 & 1057 & $£^{3} n k-$ & $£^{3} \mathrm{nk}-$ & $£^{3} n k-$ & $£^{3} \mathrm{nk}-*$ & hemlock bark \\
\hline
\end{tabular}

$/ \mathfrak{E}^{3} \mathrm{M} \chi \mathrm{v} \cdot \mathrm{h}^{3} \mathrm{id} /$ "to become stiff, hard" $\mathrm{KW} / \mathfrak{f}^{3} \mathrm{Mukv} /$ "made brittle" and possibly $\mathrm{KW} / \mathfrak{f}^{3} \mathrm{M} \chi \mathrm{vstalis} / *$ "to bathe in very cold weather."

4451 . A HA consultant said yew is a dirty tree when it dries out. It is unclear which phenomenon this refers to, however, dirtiness is mentioned in the glosses of many of the root's KW examples. Association with the mucus in yew "berries" may explain why the root has a HA derivate referring to boiling salmon eggs. 2 . The root may be the result of backformation from the tree name $\left(\mathrm{HE} / \mathfrak{f}^{3} \mathrm{Mq}^{3} / \mathrm{OO} / \mathrm{HA} / \mathrm{KW} / \mathfrak{E}^{3} \mathrm{Mq}^{3} /\right)$ which occurs also in Salish, cd. Kuipers (2002:143). ${ }^{446} \mathrm{In} \mathrm{KW} / \mathfrak{f}^{3} \mathrm{Ma \hbar}^{3}$ is/ "beach."

${ }^{447} \mathrm{In} \mathrm{KW} / \mathfrak{f}^{3} \mathrm{Mak}^{3} \mathrm{vbonu} / *$ and $/ \mathfrak{f}^{3} \mathrm{Mak}^{3} \mathrm{vbve}^{3 / *}$ "breast piece."

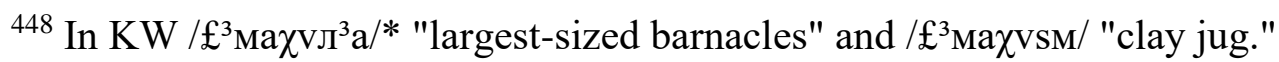

${ }^{449}$ In HE $/ \mathfrak{f}^{3} \mathrm{MSd}^{\prime} \mathrm{aic}^{3} / \mathrm{OO} / \mathfrak{£}^{3} \mathrm{M}^{3} \mathrm{Sdaic}^{3 /}$ HA $\mathfrak{£}^{3} \mathrm{MSd}^{\prime} \mathrm{aic}^{3 /}$ "bog cranberry." 
North Wakashan Comparative Root List (Updated)

\begin{tabular}{|c|c|c|c|c|c|c|}
\hline Root \# & Old \# & $\mathrm{HE}$ & $\mathrm{OO}$ & HA & KW & Gloss \\
\hline 9650 & $\begin{array}{l}1056 \\
1058\end{array}$ & $\left(t^{3} n x-\right)$ & $\left(t^{3} n x-\right)$ & $£^{3} n x-$ & $£^{3} n k-$ & $\begin{array}{l}\text { hard, tight, dense (like knot in } \\
\text { wood); (KW also:) stuffed, filled- } \\
\text { up (like belly) }\end{array}$ \\
\hline 9660 & 1054 & & & & $£^{3} n y^{3}-*$ & to covet 451 \\
\hline 9670 & & & & $£^{3} n q-$ & & to swell up (said of body parts) \\
\hline 9680 & & & & $\mathfrak{f}^{3} n^{3} q-$ & & $(?)^{452}$ \\
\hline 9681 & 1070 & & & & $\mathfrak{f}^{3} \mathrm{n}^{3} \mathrm{q}-$ & $\begin{array}{l}\text { to prick, to poke, to push or } \\
\text { shove long thing, to punch a hole }\end{array}$ \\
\hline 9690 & $\begin{array}{l}1059 \\
1060\end{array}$ & $£^{3} \mathrm{~s}-$ & $\mathfrak{f}^{3} \mathrm{~S}-$ & $£^{3} \mathrm{~s}-$ & $£^{3} \mathrm{~s}-$ & $\begin{array}{l}\text { deviating from main direction, } \\
\text { branching off, sideways (like } \\
\text { branches on tree, a sidelong } \\
\text { glance), to roll one's eyes; (KW } \\
\text { also:) alder tree }\end{array}$ \\
\hline 9710 & 1061 & & & & $£^{3} 1-$ & $(?)^{454}$ \\
\hline 9720 & 1062 & & & & $£^{3} 1-$ & $(?)^{455}$ \\
\hline
\end{tabular}

\footnotetext{
${ }^{450}$ Most examples of the full belly association are from the Koskimo dialect.

${ }^{451}$ Possibly in $\mathrm{KW} / \mathfrak{£}^{3} \mathrm{Hy}^{3} \mathrm{ała} / *$ "to covet."

452 In HA / $\mathfrak{£}^{3} \mathrm{H} \mathfrak{£}^{3} \mathrm{Hq}^{\prime} \mathrm{a} /$ "to go out alone in a boat."

453 The connection between alder trees and branching off is perhaps in the trees' female catkins which become woody cones that remain on the tree until the following spring (http://www.woodlands.co.uk/blog/tree-identification/alder/). According to http://oregonstate.edu/trees/broadleaf_genera/alder.htm, "Their peculiar woody cones (called strobiles) identify alders as surely as a flat tail identifies a beaver. They hang from the tree throughout winter like miniature lanterns."

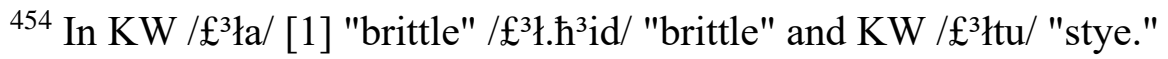

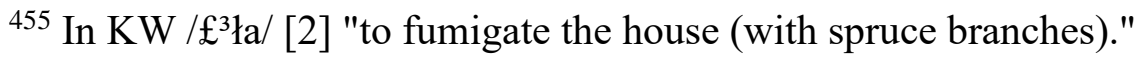


North Wakashan Comparative Root List (Updated)

\begin{tabular}{|c|c|c|c|c|c|c|}
\hline Root \# & Old \# & $\mathrm{HE}$ & $\mathrm{OO}$ & HA & KW & Gloss \\
\hline 9730 & 1063 & & & & $\mathfrak{£}^{3} 1-$ & $\begin{array}{l}\text { to push; (in some examples:) to } \\
\text { push up or hold up }\end{array}$ \\
\hline 9740 & 1064 & $£^{3} l-$ & $£^{3} 1-$ & $£^{3} l-$ & $£^{3} 1-$ & to forget \\
\hline 9750 & 1065 & $\mathfrak{f}^{3} 1^{3} \chi-$ & $\mathfrak{f}^{3} 1^{3} \chi-$ & & & $(?)^{456}$ \\
\hline 9760 & $\begin{array}{l}1066 \\
1067\end{array}$ & $£^{3} \mathrm{k}-$ & $£^{3} \mathrm{k}-$ & $£^{3} \mathrm{k}-$ & $\begin{array}{l}£^{3} \mathrm{k}-* / \\
£^{3} \mathrm{hk}-*\end{array}$ & $\begin{array}{l}\text { round, bulky, lump, lumpy, to } \\
\text { lump together, to pool resources, } \\
\text { to join together in a unit, } \\
\text { aggregate }^{457}\end{array}$ \\
\hline 9780 & 1068 & & & & $\mathfrak{£}^{3} \mathrm{k}-*$ & to break (said of waves) \\
\hline 9790 & 1069 & & & & $\mathfrak{f}^{3} \mathrm{k}-*$ & to be silent, to stop doing sth. \\
\hline 9800 & 1070 & & $\mathfrak{f}^{3} \mathrm{x}-$ & $£^{3} h x-$ & $£^{3} x-$ & $\begin{array}{l}\text { to poke, to prick, to flash (like } \\
\text { lightning), to mate }\end{array}$ \\
\hline 9810 & $\begin{array}{l}1071 \\
1077\end{array}$ & & $£^{3} y-$ & & & cluster, tangle, mass \\
\hline 9820 & 1072 & $\begin{array}{l}\mathfrak{f}^{3} \mathrm{y}- \\
\mathfrak{f}^{3} \mathrm{y}^{3}- \\
\mathfrak{£}^{3} \mathrm{hyh}- \\
\mathfrak{f}^{3} h y^{3}-\end{array}$ & 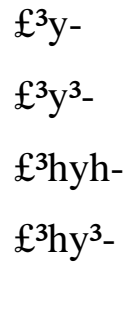 & 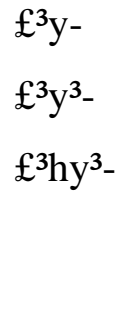 & $\mathfrak{£}^{3} \mathrm{hy}^{3}-$ & $\begin{array}{l}\text { to exchange, to trade (goods, } \\
\text { places), to switch, to substitute; } \\
\text { exchangeable (like twins), } \\
\text { convertible (like trade beads to } \\
\text { goods) }\end{array}$ \\
\hline
\end{tabular}

${ }^{456}$ In $\left\{£^{3} 1^{3} \chi\right.$ nw- $\}$ "to support with one's arm."

${ }^{457} \mathrm{KW}$ often uses $\left\{\mathrm{m}^{3} \mathrm{kv}-\right\}$ where HE/OO/HA use $\left\{£^{3} \mathrm{k}-\right\}$. The one possible KW example of root no. 9760 is $/ £^{3} \mathrm{ak}^{3} \mathrm{us} / *$ "crosspiece of halibut hook" which may contain a root $\left\{£^{3} \mathrm{k}-\right\}^{*}$ or $\left\{£^{3} \mathrm{hk}-\right\}^{*}$ meaning "aggregate." The example isn't reelicitable and may be in error.

${ }^{458} \mathrm{OO}$ only in $/ £^{3} \mathrm{xila} /$ "to mate (said of animals)." HA possibly in / $£^{31} \mathrm{a} £^{3}$ anuł/ "gooseberry"; reference may in this case be to a gooseberry plant's stem's sharp spines or the prickle on the berries. 
North Wakashan Comparative Root List (Updated)

\begin{tabular}{|c|c|c|c|c|c|c|}
\hline Root \# & Old \# & $\mathrm{HE}$ & $\mathrm{OO}$ & HA & KW & Gloss \\
\hline 9830 & 1073 & $£^{3}$ уур- & $£^{3}$ yp- & $£^{3}$ yp- & $£^{3}$ yp- & $\begin{array}{l}\text { to roll up (sleeves, trouser legs, } \\
\text { foreskin), to turn inside out }\end{array}$ \\
\hline 9840 & 1074 & $£^{3} \mathrm{yn}^{3} \mathrm{~h}-$ & $£^{3} \mathrm{yn}^{3} \mathrm{~h}-$ & $£^{3} \mathrm{yn}^{3} \mathrm{~h}-$ & $£^{3} \mathrm{yn}^{3} \mathrm{~h}-$ & ooligan oil (grease) $)^{459}$ \\
\hline 9850 & 1075 & $£^{3} \mathrm{yn}^{3} \mathrm{~h}-$ & $£^{3} \mathrm{yn}^{3} \mathrm{~h}-$ & & & jack pine $e^{460}$ \\
\hline 9860 & 1076 & $\begin{array}{l}\mathfrak{£}^{3} \mathrm{yc}-/ \\
\mathfrak{f}^{3} \mathrm{ys}-\end{array}$ & $\begin{array}{l}\mathfrak{£}^{3} \mathrm{yc}-/ \\
\mathfrak{£}^{3} \mathrm{ys}-\end{array}$ & $\begin{array}{l}\mathfrak{£}^{3} \mathrm{yc}-/ \\
\mathfrak{£}^{3} \mathrm{ys}-\end{array}$ & & cliff, steep rock \\
\hline 9880 & $\begin{array}{l}1078 \\
1079\end{array}$ & $£^{3} y s-$ & & $£^{3} y s-$ & $£^{3} y s-$ & $\begin{array}{l}\text { heat, hot; to shine, to hate, to } \\
\text { sting }^{461}\end{array}$ \\
\hline 9890 & 1080 & $£^{3} y s-$ & $£^{3} y s-$ & $£^{3} y s-$ & $£^{3} y s-$ & $\begin{array}{l}\text { skin (of fish, fruit, animal, } \\
\text { human), membrane, overlay }\end{array}$ \\
\hline 9891 & & & & & $£^{3} y s t-$ & $(?)^{462}$ \\
\hline 9900 & 1081 & & $£^{3} y £-$ & & & $(?)^{463}$ \\
\hline 9910 & 1082 & & $£^{3} y x-$ & & $£^{3} \mathrm{yx}^{-}$ & $\begin{array}{l}\text { to roll, tumble, move in a mass, } \\
\text { fall in a heap, disintegrate, scatter } \\
\text { about }\end{array}$ \\
\hline
\end{tabular}

${ }^{459}$ For general information on ooligan grease including a note on the English name's pronunciation and wildly varying spellings see "Ooligan Grease: a Nutritious Fat Used by Native People of Coastal British Columbia," Journal of Ethnobiology, 1982, Volume 2, Number 2: 154161.

${ }^{460}$ See also $\left\{£^{3} \mathrm{ykv}-\right\}$ (no. 9960).

${ }^{461} \mathrm{HA}$ only in $/ \mathfrak{f}^{3}$ isлa/ "capable of killing at a distance." HE probably in the stem $\left\{\mathfrak{f}^{3}[\mathrm{~h}] \mathrm{ys}-\right\}$ "to kill with bow and arrow."

${ }^{462} \mathrm{In} \mathrm{KW} / £^{3}$ isdaq/ "white goose, snow goose" (according to Boas); name of goose in legend according to consultants.

${ }^{463} \mathrm{In} \mathrm{OO} / \mathfrak{f}^{3} \mathrm{i} \mathfrak{f}^{3} \mathrm{M} /$ "whetstone." 
North Wakashan Comparative Root List (Updated)

\begin{tabular}{|c|c|c|c|c|c|c|}
\hline Root \# & Old \# & $\mathrm{HE}$ & $\mathrm{OO}$ & HA & KW & Gloss \\
\hline 9920 & $\begin{array}{l}1083 \\
1085\end{array}$ & $£^{3} \mathrm{ykv}-$ & $£^{3} \mathrm{ykv}-$ & & $£^{3} \mathrm{ykv}-$ & $(?)^{464}$ \\
\hline 9960 & $\begin{array}{l}1084 \\
1086 \\
1087 \\
1088 \\
1089\end{array}$ & $\begin{array}{l}£^{3} \mathrm{ykv}- \\
£^{3} \mathrm{yxv}-\end{array}$ & $£^{3} \mathrm{ykv}-$ & $£^{3} \mathrm{ykv}-$ & $£^{3} \mathrm{ykv}-*$ & $\begin{array}{l}\text { to pierce, to prick (as in } \\
\text { tattooing) }{ }^{465}\end{array}$ \\
\hline 9961 & & & & & $£^{3} y w h x-*$ & rattling, rumbling \\
\hline 9970 & $\begin{array}{l}1090 \\
1091 \\
1011\end{array}$ & $£^{3} \mathrm{yq}^{-}$ & $£^{3} \mathrm{yq}^{-}$ & $£^{3} y q-$ & $£^{3}$ yq- & $\begin{array}{l}\text { particled solid (e.g. sand), fine- } \\
\text { grained soil (e.g. clay), } \\
\text { suspended particles (e.g. mud in } \\
\text { water); (HE/OO/HA also:) } \\
\text { herring eggs, herring milt) }\end{array}$ \\
\hline 9980 & & & & & $£^{3} y q-*$ & $(?)^{466}$ \\
\hline
\end{tabular}

464 Perhaps in KW words referring to chokecherries (which taste sour and make lips pucker),

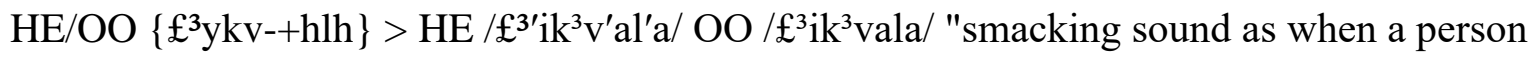
chews gum or tobacco" and KW $\{£ y k v-=\mathrm{kv}\}>/ £^{3}$ igkv/* (probably:) "roe in body, eaten raw." ${ }^{465}$ Most examples refer to tattooing and cauterizing, with Bella Bella HE using $\left\{£^{3} \mathrm{ykv}-\right\}$ but

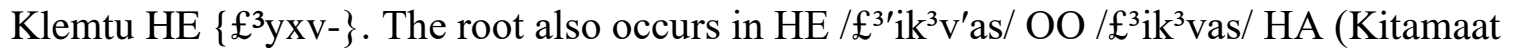
dialect) $/ \mathfrak{£}^{3} \mathrm{ik}^{3} \mathrm{v}^{\prime}$ as/ HA (Kitlope dialect) $/ \mathfrak{f}^{3} \mathrm{ik}^{3} \mathrm{vas} /$ "jack pine." According to HA elder Gordon Robertson tattooing and cauterizing was done with burnt jack pine twigs. For OO this tree name is the only attested instance of $\left\{£^{3} \mathrm{ykv}-\right\}$; the usual $\mathrm{OO}$ root for tattooing and cauterizing is $\{$ xvwp- $\}$ corresponding to KW $\{\mathrm{xwp}-\}$. The only KW examples of $\left\{£^{3} \mathrm{ykv}-\right\}$ are $/ £^{3} \mathrm{i} £^{3} \mathrm{kvM} / *(\mathrm{a}$ species of cod fish with a barbel sticking in its chin) and $/ £^{3} \mathrm{ik}^{3} \mathrm{vqa} / *$ "to make sure" (? = poke inside for probing).

${ }^{466}$ In KW /£3iqa/* "blunt." 
North Wakashan Comparative Root List (Updated)

\begin{tabular}{|c|c|c|c|c|c|c|}
\hline Root \# & Old \# & $\mathrm{HE}$ & $\mathrm{OO}$ & HA & KW & Gloss \\
\hline 10000 & 1093 & $\begin{array}{l}\mathfrak{f}^{3} \mathrm{y} x- \\
\mathfrak{E}^{3} \mathrm{y}^{3} \chi-\end{array}$ & $£^{3} y \chi-$ & $£^{3} y \chi-$ & $\mathfrak{f}^{3} \mathrm{y} \chi-$ & $\begin{array}{l}\text { rusty, red, or buff colour; } \\
\text { sealion }{ }^{467}\end{array}$ \\
\hline 10010 & 1094 & $£^{3} \mathrm{kv}-$ & $£^{3} \mathrm{kv}-$ & $£^{3} \mathrm{kv}-$ & $£^{3} \mathrm{kv}-$ & seaweed, kelp \\
\hline 10020 & 1095 & $£^{3} \mathrm{xv}-$ & $\mathfrak{f}^{3} \mathrm{xv}-$ & $£^{3} x v-$ & $\begin{array}{l}\mathfrak{f}^{3} \mathrm{Xv-} \\
\mathfrak{f}^{3} \mathrm{hxv-}\end{array}$ & $\begin{array}{l}\text { to prepare, distribute or eat food; } \\
\text { to eat a snack }\end{array}$ \\
\hline 10030 & $\begin{array}{l}1096 \\
1098\end{array}$ & $\begin{array}{l}£^{3} w p- \\
£^{3} \mathrm{wm}^{3-}\end{array}$ & $\begin{array}{l}\mathfrak{f}^{3} \mathrm{wp}- \\
\mathfrak{f}^{3} \mathrm{wm}^{3-}\end{array}$ & $£^{3} \mathrm{wp}-$ & $£^{3} \mathrm{wp}-$ & $\begin{array}{l}\text { ripe, cooked, roasted on open } \\
\text { fire; pus; cormorant (known for } \\
\text { runny droppings) }\end{array}$ \\
\hline 10040 & 1097 & $£^{3}$ wp-- & $\mathfrak{E}^{3} \mathrm{wp} \cdot-$ & $£^{3}$ wp-- & $\mathfrak{f}^{3} \mathrm{wp} \cdot-$ & $\begin{array}{l}\text { to slacken, to fall (said of the } \\
\text { tide) }\end{array}$ \\
\hline 10060 & 1099 & $\begin{array}{l}£^{3} \mathrm{wk}^{3} \mathrm{vp}- \\
£^{3} \mathrm{wk}^{3} \mathrm{vm}-\end{array}$ & $\begin{array}{l}\mathfrak{£}^{3} \mathrm{wk}^{3} \mathrm{vp}- \\
\mathfrak{f}^{3} \mathrm{wk}^{3} \mathrm{vm}-\end{array}$ & $£^{3} \mathrm{wp}^{3} \mathrm{kV}-$ & $£^{3} w p^{3} k-$ & root \\
\hline 10070 & 1100 & $\mathfrak{f}^{3} \mathrm{wt}$ & $\mathfrak{f}^{3} \mathrm{wt}$ & $£^{3} w t-$ & $\mathfrak{f}^{3} \mathrm{wt}$ & $\begin{array}{l}\text { to arch the back, to bend the } \\
\text { body, to make copulatory } \\
\text { movements }\end{array}$ \\
\hline 10071 & & & $\mathfrak{f}^{3} w c-$ & & & $(?)^{468}$ \\
\hline 10080 & 1103 & $\mathfrak{f}^{3} \mathrm{ws}-$ & $£^{3} w s-$ & & $\mathfrak{f}^{3} w s^{-}$ & $\begin{array}{l}\text { to chafe (also more specifically:) } \\
\text { to rub clitoris; in love, infatuated, } \\
\text { flirtatious }\end{array}$ \\
\hline 10090 & 1102 & & & & $£^{3}$ wsnhk- & $(?)^{469}$ \\
\hline 10100 & 1101 & & & & $£^{3}$ wskv- & bald $^{470}$ \\
\hline
\end{tabular}

${ }^{467} \mathrm{HE}\left\{£^{3} \chi-\right\}$ only in $/ \mathfrak{£}^{3} \mathrm{i} \chi \mathrm{J}^{\prime} \mathrm{i} /$ "unripe berry (of any kind)."

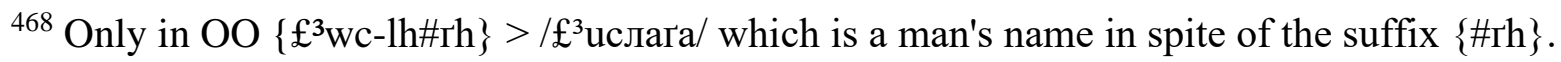

${ }^{469}$ Boas (1948:449) says it means turning up both ends but unclear what that refers to. Lincoln \& Rath (1980:200, item 1102) and Lincoln (n.d., item 23171) mention that the stem could also refer to the writhing of a snake.

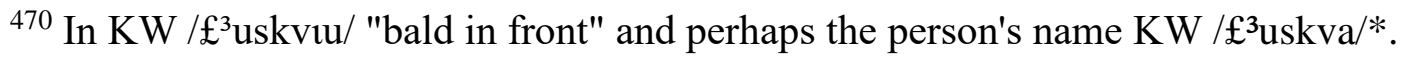


North Wakashan Comparative Root List (Updated)

\begin{tabular}{|c|c|c|c|c|c|c|}
\hline Root \# & Old \# & $\mathrm{HE}$ & $\mathrm{OO}$ & HA & KW & Gloss \\
\hline 10110 & 1104 & & & & $£^{3} w 1-$ & to scold \\
\hline 10120 & 1105 & $£^{3} w 1-$ & $\mathfrak{f}^{3} \mathrm{w}-$ & & $\mathfrak{f}^{3} \mathrm{w}-$ & $(?)^{471}$ \\
\hline 10130 & 1106 & & & & $£^{3} \mathrm{wk}^{3} \mathrm{v}-$ & $\begin{array}{l}\text { to peel, pluck or scrape sth. off } \\
\text { bark or } \operatorname{skin}^{472}\end{array}$ \\
\hline 10140 & 1107 & & & $\mathfrak{f}^{3} \mathrm{wqv}-$ & & to imitate \\
\hline 10150 & 1108 & $\mathfrak{f}^{3} \mathrm{wqv}-$ & $£^{3} w q v-$ & $£^{3} w q v-$ & $\mathfrak{f}^{3} w q v-$ & $\begin{array}{l}\text { bald (head), bare (island), } \\
\text { barkless (tree); to cut hair, to } \\
\text { make bare or bald }\end{array}$ \\
\hline 10151 & 1109 & $£^{3} w \chi v^{-}$ & $£^{3} w \chi v^{-}$ & $\mathfrak{f}^{3} w \chi v^{-}$ & $\mathfrak{f}^{3} w \chi v^{-}$ & ice, to freeze \\
\hline 10160 & 1110 & $£^{3} w \chi v^{-}$ & $£^{3} w \chi^{v-}$ & $\mathfrak{f}^{3} \mathrm{w} \chi \mathrm{v}^{-}$ & & $\begin{array}{l}\text { calcified (like limestone), rock- } \\
\text { hard (like pebble) }\end{array}$ \\
\hline 10161 & & fluxthx- & & & $f^{3} w \chi \mathrm{N}^{3} \mathrm{E}^{3} \mathrm{~N}^{-*}$ & oyster \\
\hline 10170 & 1111 & $\mathfrak{f}^{3} \mathrm{~W}^{3} \mathrm{xV} \cdot-$ & $\mathfrak{f}^{3} \mathrm{~W}^{3} \mathrm{xV} \bullet-$ & (łhxv-) & $\left(c^{3} x-\right)$ & pain, disease ${ }^{473}$ \\
\hline 10180 & 1113 & & & & $£^{3} q v-$ & $\begin{array}{l}\text { to break off branches (as for } \\
\text { sinking them to collect herring } \\
\text { spawn) }\end{array}$ \\
\hline 10190 & 1114 & $\mathfrak{E}^{3} \chi \mathrm{V}^{-}$ & $\mathfrak{E}^{3} \chi \mathrm{V}^{-}$ & $\mathfrak{E}^{3} \chi^{V-}$ & $\mathfrak{£}^{3} \chi \mathrm{v}-*$ & $\begin{array}{l}\text { to indicate one's guess in a game } \\
\text { (e.g. cards or lahel) by pointing }\end{array}$ \\
\hline
\end{tabular}

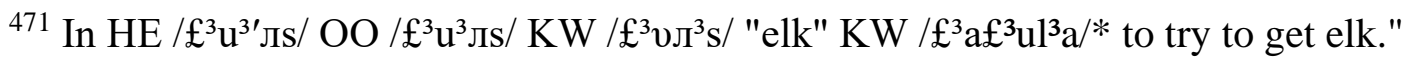

${ }^{472}$ Lincoln \& Rath (1980:200, item no. 1106) posits a root form with $|\mathrm{kv}|$ rather than $\left|\mathrm{k}^{3} \mathrm{v}\right|$ based on two non-reelicitable stems that are misspelled or copied wrong from the literature where they were found (references: C102.4 and R126.22).

${ }^{473}$ In several examples $|\bullet|$ is not merely preventing suffix-initial $|\%|,|=|$ or $|+|$ from coalescing with the root's $|\mathrm{xv}|$ but also causing them to behave irregularly. See the comments on the database records concerned. 
North Wakashan Comparative Root List (Updated)

\begin{tabular}{|c|c|c|c|c|c|c|}
\hline Root \# & Old \# & $\mathrm{HE}$ & $\mathrm{OO}$ & HA & KW & Gloss \\
\hline 10200 & 1115 & & & & $£^{3} \mathrm{q}-$ & $(?)^{474}$ \\
\hline & 1116 & & & & & \\
\hline 10210 & 1117 & & & & $\mathfrak{£}^{3} \chi-$ & silent, quiet, hushed, not crying \\
\hline & 1140 & & & & $\mathfrak{f}^{3} h \chi-$ & \\
\hline 10220 & 1118 & & & & $£^{3} \chi-$ & $\begin{array}{l}\text { (?) twisted, not straight, weaving } \\
\text { in and out }{ }^{475}\end{array}$ \\
\hline 10230 & 1139 & $£^{3} \mathrm{~h} \chi-$ & $\mathfrak{f}^{3} \mathrm{~h} \chi-$ & $\begin{array}{l}\mathfrak{f}^{3} \chi- \\
\mathfrak{f}^{3} \mathrm{~h} \chi-\end{array}$ & $£^{3} h \chi-$ & $\begin{array}{l}\text { stiff, rigid (like pole), retaining } \\
\text { shape under pressure }\end{array}$ \\
\hline 10240 & 1119 & $£^{3} h-$ & $£^{3} h-$ & $\left(t^{3} y x v-\right)$ & $£^{3} h-$ & black bear \\
\hline 10250 & 1120 & $£^{3} h-$ & $£^{3} h-$ & $£^{3} \mathrm{~h}-$ & & $\begin{array}{l}\text { (?) pointing downhill (like logs } \\
\text { on mountainside) }{ }^{477}\end{array}$ \\
\hline 10280 & 1122 & & & & $£^{3}$ hbht- & cedar bark basket ${ }^{478}$ \\
\hline 10290 & 1123 & & & & $£^{3} \mathrm{hp}-*$ & to $\operatorname{dip}^{479}$ \\
\hline 10300 & 1124 & $\mathfrak{f}^{3} \mathrm{ht}-$ & $£^{3} \mathrm{ht}-$ & $£^{3} \mathrm{ht}-$ & $\mathfrak{f}^{3} \mathrm{ht}-$ & $\begin{array}{l}\text { oil, grease; to apply oil or grease } \\
\text { (esp. to the hair) }\end{array}$ \\
\hline
\end{tabular}

${ }^{474}$ In words referring to scrofula, a form of tuberculosis causing glandular swellings and skin inflammation, especially in the neck. Possibly also in $\mathrm{KW} / £^{3} \mathrm{q}^{3} \chi u l i /$ "fruit (i.e. berries) of the black twinberry (Lonicera involucrata)." These berries had very many medicinal uses including to treat swelling, see http://plants.usda.gov/factsheet/pdf/fs_loin5.pdf

${ }^{475}$ In two words referring to weaving and knitting and perhaps in $\mathrm{KW} / £^{3} \chi \mathrm{um}^{3} \partial \mathrm{s} / *^{*}$ "twisted stalk." Twisted stalk owes its name to the $90^{\circ}$ twist in the flower stalks, cf.

http://www.fs.fed.us/wildflowers/plant-of-the-week/streptopus_amplexifolius.shtml.

${ }^{476}$ HA $\left\{\mathfrak{£}^{3} \mathrm{~h} \chi-\right\}$ is attested only in HA (Kitamaat dialect) $/ \mathfrak{£}^{3} \mathrm{a} \chi /$ "stiff, rigid."

${ }^{477}$ In one HE and several OO words referring to logs moving down lengthwise and in the stem HE/OO $\left\{£^{3} h^{3} n \chi-\right\}$ HA $\left\{£^{3} h n^{3} \chi-\right\}$ possibly meaning "mountain slope."

${ }^{478}$ Cross-family stem. See Kuipers (2002:63).

${ }^{479}$ In KW (Newettee dialect) /£3apsta/* "to dip into water." 
North Wakashan Comparative Root List (Updated)

\begin{tabular}{|c|c|c|c|c|c|c|}
\hline Root \# & Old \# & $\mathrm{HE}$ & $\mathrm{OO}$ & HA & KW & Gloss \\
\hline 10301 & & & & & $\begin{array}{l}£^{3} \text { hnys-* } \\
£^{3} h^{3} \text { hnys-* }\end{array}$ & seagull \\
\hline 10310 & 1125 & & & $£^{3} \mathrm{hs}-$ & & $(?)^{480}$ \\
\hline 10320 & 1126 & $£^{3} \mathrm{hs}-$ & $£^{3} \mathrm{hs}-$ & $£^{3} \mathrm{hs}^{-}$ & $£^{3} \mathrm{hs}^{-}$ & oil, fat, grease, blubber \\
\hline 10350 & 1127 & $\AA^{3} \mathrm{hs}^{\bullet-}$ & $\mathfrak{f}^{3} \mathrm{hs}^{\bullet-}$ & $\begin{array}{l}£^{3} \mathrm{hs}^{\bullet-} \\
£^{3} \mathrm{hs}-\end{array}$ & $\begin{array}{l}£^{3} \mathrm{hs} \bullet- \\
£^{3} \mathrm{hsh}-\end{array}$ & $\begin{array}{l}\text { seawards, outwards, towards the } \\
\text { central fire in the longhouse, off } \\
\text { the wall }\end{array}$ \\
\hline 10360 & 1128 & $£^{3} \mathrm{hl}-$ & $\mathfrak{f}^{3} \mathrm{ht}-$ & $£^{3} \mathrm{hl}-$ & $£^{3} \mathrm{~h} k-$ & $\begin{array}{l}\text { to surface (said of whale, } \\
\text { porpoise, fish) }\end{array}$ \\
\hline 10361 & & $\left(£ w y^{3}-\right)$ & $\left(£ w y^{3}-\right)$ & $f^{3} \mathrm{hk}-$ & & to dress up, to decorate sth. \\
\hline 10370 & 1129 & $£^{3} h x-$ & & & $£^{3} h x-$ & $(?)^{481}$ \\
\hline 10371 & 1120 & & & & $£^{3} \mathrm{hykm}-$ & piling up, heaping up \\
\hline 10372 & & & & & $f^{3}$ hyqu- & mourn, wail \\
\hline 10380 & 1130 & & & & $£^{3}$ hyhqv-* & $(?)^{482}$ \\
\hline 10390 & & & & & $£^{3} h y^{3}-$ & $(?)^{483}$ \\
\hline 10400 & 1131 & & & & $£^{3} h y^{3}-$ & thin, skinny, lean \\
\hline 10410 & 1132 & $£^{3} \mathrm{hkv}-$ & $£^{3} h \mathrm{hv}-$ & $£^{3} \mathrm{hkv}-$ & $£^{3} h k v-$ & $\begin{array}{l}\text { hinged V-shape (like elbow or } \\
\text { knee) opens, closes, leans on or } \\
\text { pushes against sth. }\end{array}$ \\
\hline
\end{tabular}

480 Perhaps in HA (Kitamaat dialect) / $\mathfrak{£}^{3}$ 'asiagvmik/ "wolf."

${ }^{481} \mathrm{In} \mathrm{KW} / \mathfrak{f}^{3} \mathrm{axm} /$ "wooden spoon" and $\mathrm{KW} / \mathfrak{f}^{3} \mathrm{axaw}^{3}$ inira/* (Crow's name:) "spoon-bringing

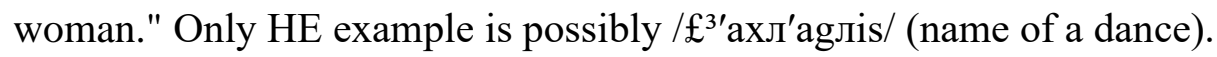

${ }^{482}$ In two words referring to equipment for a trap.

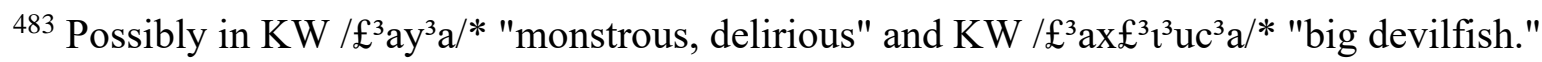


North Wakashan Comparative Root List (Updated)

\begin{tabular}{|c|c|c|c|c|c|c|}
\hline Root \# & Old \# & $\mathrm{HE}$ & $\mathrm{OO}$ & HA & KW & Gloss \\
\hline 10420 & 1133 & $£^{3} h x v-$ & $£^{3} h x v-$ & $£^{\mathfrak{3} h x v-}$ & $£^{3} h x v-$ & $\begin{array}{l}\text { to mark (with paint, by notching } \\
\text { or by rubbing) }\end{array}$ \\
\hline 10421 & & $£^{3} h w q^{3} v-$ & $£^{3} h w q^{3} v-$ & & $\mathfrak{£}^{3} \mathrm{hwq}^{3} \mathrm{v}-$ & Indian tobacco \\
\hline 10430 & $\begin{array}{l}1134 \\
1135\end{array}$ & $£^{3} h q v-$ & $\mathfrak{f}^{3}$ hqv- & $£^{3}$ hqv- & $£^{3} h q v-$ & $\begin{array}{l}\text { red, metal; (usually but not } \\
\text { exclusively when combined with } \\
\{-\mathrm{h}\}: \text { ) copper }\end{array}$ \\
\hline 10440 & 1136 & $£^{3} h \chi^{v-}$ & & $£^{3} \mathrm{~h} \chi \mathrm{v}-$ & $\mathfrak{f}^{3} h \chi \mathrm{v}^{-*}$ & $(?)^{485}$ \\
\hline 10450 & $\begin{array}{l}1137 \\
1138\end{array}$ & & & $£^{3} h q-$ & $f^{3} \mathrm{hq}-$ & $\begin{array}{l}\text { angle, hook; (hence:) arranged } \\
\text { crosswise (like logs in pyre), } \\
\text { hook-shaped (like gaff), barbed; } \\
\text { to fish with one hooked line or } \\
\text { multiple parallel hooked lines } \\
\text { reaching down from one floating } \\
\text { line }\end{array}$ \\
\hline 10460 & 1138 & $\mathfrak{£}^{3} \mathrm{hq}-$ & $£^{3} \mathrm{hq}-$ & $£^{3} \mathrm{hq}-$ & $\mathfrak{f}^{3} \mathrm{hq}-$ & $\begin{array}{l}\text { to stretch out (legs, a line), pay } \\
\text { out line }\end{array}$ \\
\hline 10480 & 1141 & & & & $\mathfrak{f}^{3} \mathrm{~h} \chi-$ & the least thing \\
\hline 10481 & & & $£^{3} h^{3} w x V-$ & & & $(?)^{486}$ \\
\hline 10482 & 1121 & & & & $£^{3} h^{3} h £^{3} y-$ & bufflehead duck \\
\hline 10490 & 1142 & & & & tp- & $(?)^{487}$ \\
\hline
\end{tabular}

${ }^{484} \mathrm{KW}$ only in $/ \mathfrak{£}^{3} \mathrm{axv} \cdot \mathrm{m}^{3} \mathrm{~s} /$ "alder tree." Alder bark was used in dying and tanning.

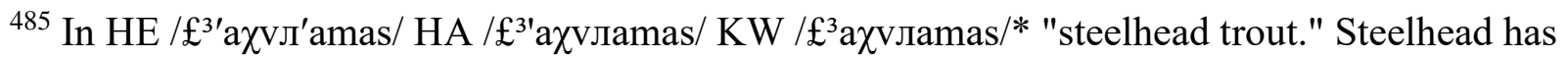
pinkish fin tips when coming up the river and steelhead flesh can be bright red. The root may really be an alternant of $\left\{£^{3} \mathrm{hqv}-\right\}$ "red."

${ }^{486} \mathrm{In} \mathrm{OO} / \mathfrak{f}^{3} \mathrm{a}^{3} \mathrm{uxvла/} \mathrm{"to} \mathrm{take} \mathrm{care} \mathrm{of} \mathrm{a} \mathrm{cry-baby"}$

${ }^{487}$ In KW /łpa/ "to drip with grease (as food after dipping)." 
North Wakashan Comparative Root List (Updated)

\begin{tabular}{|c|c|c|c|c|c|c|}
\hline Root \# & Old \# & $\mathrm{HE}$ & $\mathrm{OO}$ & HA & KW & Gloss \\
\hline 10500 & $\begin{array}{l}1143 \\
1144\end{array}$ & łm- & tm- & łm- & $\begin{array}{l}\text { tm- } \\
\text { thm }\end{array}$ & $\begin{array}{l}\text { curtained off, concealed; (KW } \\
\text { also:) difficult to identify with } \\
\text { the senses (sight, hearing, taste), } \\
\text { dimly perceptible, indefinable, } \\
\text { uncertain, going out of } \\
\text { existence }^{488}\end{array}$ \\
\hline 10510 & $\begin{array}{l}1145 \\
1146\end{array}$ & tm- & $\mathrm{lm}-$ & łm- & tm- & $\begin{array}{l}\text { secured by line strung to other } \\
\text { object (like boat anchored, } \\
\text { moored, tied up), to string a line } \\
\text { to sth., to mend boards }\end{array}$ \\
\hline 10530 & 1147 & & & $\begin{array}{l}\text { łmp- } \\
\text { łwp- }\end{array}$ & łmp- & $\begin{array}{l}\text { soft, soggy, baggy, sagging, } \\
\text { loose, wrinkled }{ }^{489}\end{array}$ \\
\hline 10540 & 1148 & & łms- & & łms- & to sip \\
\hline 10550 & 1149 & & & & łmqv- & (?) to suck out sickness ${ }^{490}$ \\
\hline 10560 & 1150 & tmq- & tmq- & łmq- & & $\begin{array}{l}\text { to tie sth. to sth. else with rope; } \\
\text { (HA also:) to sew together }\end{array}$ \\
\hline 10570 & 1151 & $\operatorname{tm} \chi-$ & $\operatorname{lm} \chi-$ & $\operatorname{lm} x-$ & $\ln \chi-$ & to inhale smoke \\
\hline 10580 & 1152 & & & & $\mathrm{tt}-$ & $\begin{array}{l}\text { (?) 1. to reach out and pull; } 2 \text {. } \\
\text { stream, small waterway }\end{array}$ \\
\hline 10590 & 1153 & ln- & th- & ln- & th- & $\begin{array}{l}\text { lost, forgotten, to be missing } \\
\text { sth. }^{491}\end{array}$ \\
\hline
\end{tabular}

${ }^{488}$ Most KW examples of the meaning "difficult to identify, etc." use the augmented root form $\left\{\mathrm{hhm}^{3}-\right\}$. Boas lists several non-reelicitable examples of a stem $\left\{\mathrm{hhm}^{3} \mathrm{hq}-\right\}$ "embroidery, tying" as derivates of this root no. 10500. The examples' glosses leave it uncertain but possible that reference is to covering sth. The stem's structure could be $\left\{\mathrm{hhm}^{3}-\% \mathrm{hq}-\right\}$. ${ }^{489}$ HA \{łmp-\} only in /f'mbis/ "soft beach" elicited from the Kitamaat informant. ${ }^{490}$ Only in KW /hmqva/ "to suck out sickness (said of a shaman)." ${ }^{491}$ HA examples of $\{\mathrm{ln}-\}$ could only be obtained from the Kitamaat consultant. 
North Wakashan Comparative Root List (Updated)

\begin{tabular}{|c|c|c|c|c|c|c|}
\hline Root \# & Old \# & $\mathrm{HE}$ & $\mathrm{OO}$ & HA & KW & Gloss \\
\hline 10600 & $\begin{array}{l}1155 \\
1165\end{array}$ & $\begin{array}{l}\text { ln- } \\
11-\end{array}$ & th- & & tn- & (?) salal ${ }^{492}$ \\
\hline 10610 & 1156 & lnp- & lnp- & lnp- & lnp- & $\begin{array}{l}\text { saggy, loose, soft, pliable, } \\
\text { wrinkled }\end{array}$ \\
\hline 10620 & 1158 & & & & lnt- & $\begin{array}{l}\text { pliable, soft (like cloth), weak } \\
\text { (person) }\end{array}$ \\
\hline 10630 & 1157 & lnt- & lnt- & lnt- & lnt- & $\begin{array}{l}\text { to blow the nose; snot, snotty, } \\
\text { slimy }\end{array}$ \\
\hline 10650 & 1160 & & & & lnxv- & $\begin{array}{l}\text { (?) rustling sound (of leaves in } \\
\text { the wind or as when passing } \\
\text { through bushes) }\end{array}$ \\
\hline 10660 & 1162 & lnq- & lnq- & & & $(?)^{494}$ \\
\hline 10670 & 1163 & $\ln \chi-$ & $\ln \chi-$ & & $\ln \chi-$ & $\begin{array}{l}\text { crabapples, crabapple colours } \\
\text { (red, green, yellow, golden) }{ }^{495}\end{array}$ \\
\hline 10680 & 1164 & & & & ts-* & (?) to pull 196 \\
\hline 10690 & 1166 & $\begin{array}{l}\text { tl- } \\
\qquad 1^{3}- \\
\text { thl }\end{array}$ & $\begin{array}{l}\mathrm{Hl}- \\
\mathrm{Hl}^{3}-\end{array}$ & $\begin{array}{l}\text { tl- } \\
\qquad 1^{3}- \\
t h l^{3}-\end{array}$ & $\begin{array}{l}\mathrm{Hl}^{3}- \\
\text { thl }\end{array}$ & $\begin{array}{l}\text { dead, inactive, paralyzed, worn } \\
\text { out }\end{array}$ \\
\hline 10700 & 1154 & & & & $\mathrm{tx}-*$ & $(?)^{497}$ \\
\hline
\end{tabular}

492 Only in words for salal bush. Root may really refer to some property of salal, perhaps the leatheriness of the leaves.

493 In words referring to salal, bird cherry and paper birch.

494 Only in HE /ł' $\mathrm{Hqa/OO} / \mathrm{Hqq} /$ "to peel bark from a tree."

495 The root's HE/OO derivates are mostly about crabapples and actions concerning them whereas the KW derivates are mostly about the colours.

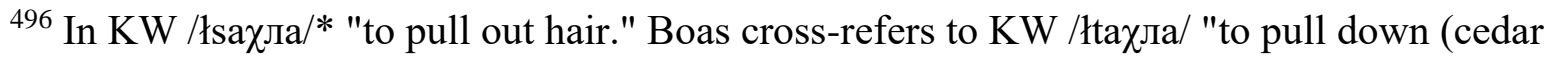
withes, hair in mourning)" of which this item could be a misrecording or misspelling.

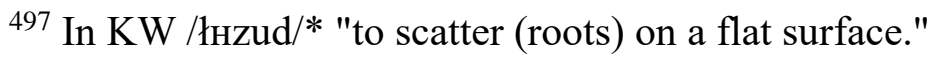


North Wakashan Comparative Root List (Updated)

\begin{tabular}{|c|c|c|c|c|c|c|}
\hline Root \# & Old \# & $\mathrm{HE}$ & $\mathrm{OO}$ & HA & KW & Gloss \\
\hline 10710 & 1168 & & ty- & ły- & & $\begin{array}{l}\text { to lie (said of a plurality of } \\
\text { people) }\end{array}$ \\
\hline 10720 & 1169 & & & & $\mathrm{lym}^{3}-*$ & $(?)^{498}$ \\
\hline 10730 & 1170 & łyt- & łyt- & & tyt- & tattered, ragged, split \\
\hline 10740 & $\begin{array}{l}1171 \\
1173\end{array}$ & $\begin{array}{l}\text { łyc-l } \\
\text { łys- }\end{array}$ & $\begin{array}{l}\text { łyc-/ } \\
\text { łys- }\end{array}$ & & łys- & pale, blonde ${ }^{499}$ \\
\hline 10760 & 1174 & łys- & tys- & łys- & łys- & $\begin{array}{l}\text { (HE/OO/HA) to find sth. } \\
\text { accidently, }(\mathrm{KW}) \text { to find dead } \\
\text { game accidently }\end{array}$ \\
\hline 10770 & $\begin{array}{l}1177 \\
1000\end{array}$ & ty $x-$ & ty $x-$ & łyq- & łyx- & $\begin{array}{l}\text { dangling loose end or edge; to } \\
\text { unravel, unlace, rip off, } \\
\text { dismantle, take apart }\end{array}$ \\
\hline 10780 & 1176 & łyxv- & Hyxv- & łyxv- & łyxv- & $\begin{array}{l}\text { ribbon or strip torn or cut from } \\
\text { flat material such as cedar bark, } \\
\text { cedar bark mat }\end{array}$ \\
\hline 10800 & 1178 & tkv- & $\mathrm{kkv}-$ & $\mathrm{kkv}-$ & tkv- & $\begin{array}{l}\text { tense, tight, to tighten; (hence:) to } \\
\text { draw in or out (line, breath, } \\
\text { drawer), to jerk, to yank, to jig, to } \\
\text { pull line lengthwise (as when } \\
\text { jigging for fish) or perpendicular } \\
\text { to length (as when pulling one's } \\
\text { bowstring) }\end{array}$ \\
\hline 10810 & 1179 & łkv- & tkv- & łkv- & łkv- & old woman 501 \\
\hline
\end{tabular}

${ }^{498} \mathrm{In} \mathrm{KW} /$ tim³ ${ }^{3}$ a $/$ /* "landslide."

${ }^{499} \mathrm{HE} / \mathrm{OO}$ only in HE / $/$ 'isk $^{3} \mathrm{vs} / \mathrm{OO} /$ fisk $^{3} \mathrm{vs} /$ "young yellow cedar."

${ }^{500}$ The core meaning "tight, to tighten" is preserved in the KW stem $\{\nmid \mathrm{kv}-+\mathrm{t}-\}$.

${ }^{501}$ Root may really refer to some physical or social affliction of old women. It could be exhaustion, cf. HA $\{$ kkv-=1-\%hlysm $\}>$ /gvvi'alism/ "woman who has grown too old and is about 
North Wakashan Comparative Root List (Updated)

\begin{tabular}{|c|c|c|c|c|c|c|}
\hline Root \# & Old \# & $\mathrm{HE}$ & $\mathrm{OO}$ & $\mathrm{HA}$ & KW & Gloss \\
\hline 10820 & 1180 & & & & tkv- & $\begin{array}{l}\text { licorice fern (Polypodium } \\
\text { glycyrrhiza) }\end{array}$ \\
\hline 10830 & 1181 & lxv- & & łxv- & txv- & $(?)^{503}$ \\
\hline 10840 & 1182 & & & & txv- & to eat sea eggs or sea urchins \\
\hline 10850 & 1183 & & & & lwmo-* & $(?)^{504}$ \\
\hline 10870 & $\begin{array}{l}1184 \\
1185\end{array}$ & $7 w^{3} t-$ & & twt- & twt- & $\begin{array}{l}\text { (HE/KW) to make copulatory } \\
\text { movements; (HA) moving like a } \\
\text { crippled or lame person, to move } \\
\text { over a surface while sitting and } \\
\text { pulling oneself along by the } \\
\text { legs } 505\end{array}$ \\
\hline
\end{tabular}

to die." The root could even be the same as no. 10800 if old age is associated with osteoporosiscaused dowager's hump and the image of a tense shooting bow.

${ }^{502}$ Possibly the root really is no. 10800 and refers in this case to the curving of fern fronds and/or the coiling of young fronds (fiddleheads). This is suggested by KW $\left\{\nmid \mathrm{kv}-+\mathrm{y}^{3} \mathrm{~s}\right\}>/ \mathrm{kk}^{3} \mathrm{vi}^{3} \mathrm{~s} /$ meaning both "bow for shooting arrows" and "small fern (likely licorice fern)."

${ }^{503}$ Only in HE $\{[\mathrm{lx}] \mathrm{txv}-+-\mathrm{h}\}>/ \mathrm{x}_{\mathrm{x}} \mathrm{u}^{3} \mathrm{a} /$ " "broad-leaved seaweed, shallow water kelp" HA

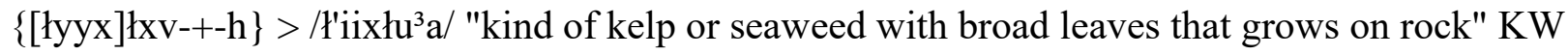
$\{[$ łyx $] \mathrm{xxv}=\mathrm{ys}\}>/$ /ix:łvis/ "sea lettuce."

${ }^{504} \mathrm{In} \mathrm{KW} / \mathrm{hum} / *$ and /tom/* "to belch, to have an aftertaste of grease." The form /hum/ fits the more frequently occurring KW pattern for two consecutive vocalic resonants. The word could be a cross-family stem, cf. proto-Salish *-łum³- "soup, to eat soup" and particularly Bella Coola łum, łuma "to sip," cf. Kuipers (2002:58).

505 The only HE example is possibly the proper name /Łut'ał/ which is said to mean "lover." The name needs to be re-elicited; the low tone of /u/ may be wrong. 
North Wakashan Comparative Root List (Updated)

\begin{tabular}{|c|c|c|c|c|c|c|}
\hline Root \# & Old \# & $\mathrm{HE}$ & $\mathrm{OO}$ & HA & KW & Gloss \\
\hline 10880 & 1186 & łwxv- & łwxv- & & łwxv- & $\begin{array}{l}\text { avoidance, dodging, shunning, } \\
\text { spurning } 506\end{array}$ \\
\hline 10890 & 1187 & łwqv- & łwqv- & łwqv- & łwqv- & $\begin{array}{l}\text { shallow container (i.e. dish, bowl, } \\
\text { basin); contents of shallow } \\
\text { container }\end{array}$ \\
\hline 10900 & 1188 & & & & łwqv- & to slip, slide, scoot along \\
\hline 10910 & 1112 & $\begin{array}{l}\text { łqv- } \\
\text { fhqv- } \\
\text { thqv- }\end{array}$ & łqv- & $\begin{array}{l}\text { łqv- } \\
£ q v- \\
£^{3} q v-\end{array}$ & łqv- & $\begin{array}{l}\text { (?) contents or core inside a hard } \\
\text { or soft shell (e.g. seeds in fruit, } \\
\text { brains in skull) })^{507}\end{array}$ \\
\hline 10920 & 1189 & łq- & łq- & $\begin{array}{l}\text { tq- } \\
\text { thq- }\end{array}$ & łq- & $\begin{array}{l}\text { red laver seaweed (Porphyra } \\
\text { abbotiae) }\end{array}$ \\
\hline 10930 & & & & & łq-* & $(?)^{508}$ \\
\hline 10940 & 1190 & & & & $1 x-$ & $(?)^{509}$ \\
\hline 10970 & $\begin{array}{l}1191 \\
1195\end{array}$ & th- & & & th- & $\begin{array}{l}\text { unclear, mysterious, puzzling, } \\
\text { unexpected, surprising, } \\
\text { dangerous }^{510}\end{array}$ \\
\hline 10980 & $\begin{array}{l}1192 \\
1193\end{array}$ & & & & $\begin{array}{l}\text { thp- } \\
\text { tht- }\end{array}$ & $\begin{array}{l}\text { to glance off, to strike lightly (as } \\
\text { when striking a match), to brush } \\
\text { past }^{511}\end{array}$ \\
\hline
\end{tabular}

${ }^{506} \mathrm{HE} / \mathrm{OO}$ only in $\mathrm{HE} / \mathrm{f}^{\prime} \mathrm{uxva} / \mathrm{OO} /$ huxva/ "almost." Its gloss is likely wrong, cf. KW /łuxva/ "to turn head away."

507 \{£hqv- $\}$ and $\{$ hhqv- $\}$ are attested only in $\mathrm{HE} / \mathfrak{f}^{\prime} \mathrm{a} \chi \mathrm{vc}^{3} \mathrm{ua} /$ and $/ \mathrm{f}^{\prime} \mathrm{a} \chi \mathrm{vvc}^{3} \mathrm{ua} /$ "seed or pit of any fruit." HA $\{£ q v-\}$ was used by the Kitlope informant, $\left\{£^{3} \mathrm{qv}^{-}\right\}$by the Kitamaat one.

${ }^{508} \mathrm{In} \mathrm{KW} / \mathrm{l \chi :}: \mathrm{lq}^{3} \mathrm{tH}^{\prime *}$ which Boas says may refer to a small sea animal.

${ }^{509}$ In words referring to loose or stringlike things such as grass, roots, or hair being somewhere and to throwing a cloth or net. Also perhaps in $\mathrm{KW} / \mathrm{t \chi} . \mathrm{h}^{3} \mathrm{a} /$ "wrecked."

${ }^{510} \mathrm{HE}$ only in / $/$ 'ała/ "child-snatching monster with the basket on the back."

511 \{tht-\} only in KW /łatabala/, synonym of KW /łapabala/ "to glance off, to brush past." 
North Wakashan Comparative Root List (Updated)

\begin{tabular}{|c|c|c|c|c|c|c|}
\hline Root \# & Old \# & $\mathrm{HE}$ & $\mathrm{OO}$ & HA & KW & Gloss \\
\hline 11010 & 1194 & & & & $\begin{array}{l}\text { tht- } \\
\text { tht }\end{array}$ & $\begin{array}{l}\text { to split shakes or boards off a } \\
\text { block, to send an advance on a } \\
\text { gift of blankets }\end{array}$ \\
\hline 11011 & $\begin{array}{l}1159 \\
1172\end{array}$ & $\begin{array}{l}\text { łhnc- } \\
\text { łync- }\end{array}$ & łhnc- & thnc- & łns- & one day from today ${ }^{512}$ \\
\hline 11012 & & & & & $\begin{array}{l}\text { ths- } \\
\text { thws-/ } \\
\text { thwhs- }\end{array}$ & $(?)^{513}$ \\
\hline 11013 & 1179 & & & & tht- & $(?)^{514}$ \\
\hline 11020 & 1196 & thk- & thk- & & $\begin{array}{l}\text { thk- } \\
\text { tyk- }\end{array}$ & $\begin{array}{l}\text { (?) unknown, unexpected, } \\
\text { surprising, dangerous }\end{array}$ \\
\hline 11030 & 1197 & thx- & thx- & thx- & & (?) difficulty, trouble $\mathrm{e}^{516}$ \\
\hline
\end{tabular}

${ }^{512} \mathrm{KW}$ form could also be $\{$ thns- $\}$ but $|\mathrm{h}|$ not diagnosable. Stem can be followed by few suffixes of the kind that can follow a bound stem but is in all HE/OO/HA and most $\mathrm{KW}$ examples followed by suffixes of the kind that can only follow a complete word stem and/or a deictic with grammatical function, e.g. \{łhnc\#h\}> HE / /'анса/ OO/łанса/ "yesterday" (with grammatical deictic $\{\#$ h $\}$ "near neither you nor me") and $\{$ hnc\#£•\#h\#ts\} $>$ HE /ł'aHs£ats/ OO /łahs£ats/ "tomorrow" (with this same $\{\# \mathrm{~h}\}$ and also $\{\# £ \bullet\}$ "in future" and $\{\#$ ts $\}$ "invisible").

${ }^{513}$ Perhaps in KW /łay ${ }^{3} \mathrm{H} \chi /<\{$ ths- $+\mathrm{H} \chi\}$ "autumn, fall" and its synonym KW /łəу ${ }^{3} \mathrm{H} \chi /$ (from either $\{$ thws- $+\mathrm{n} \chi\}$ or $\{$ thwhs- $+\mathrm{n} \chi\}$ ).

${ }^{514}$ Possibly in KW /łałani/* "teal duck," /łalanm/ "little mallard duck" and /aałk³/ "mallard duck."

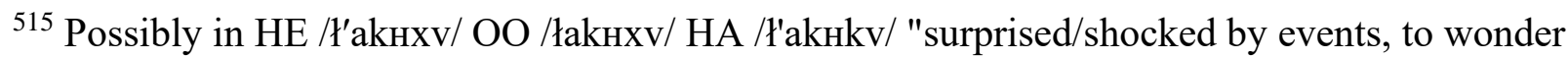
what could be the case" and the KW stems \{thyk- $\}$ and \{łyk- $\}$ "damaged, disabled (said of a canoe)." \{łyk-\} is directly observable only in the synonym /tika/ of KW /teka/ "canoe gets wrecked."

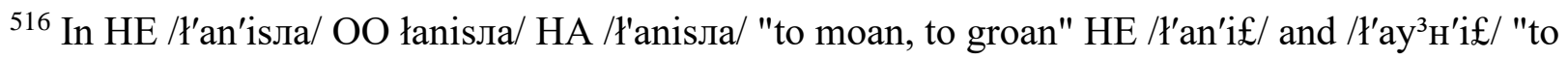
enter the wrong house." 
North Wakashan Comparative Root List (Updated)

\begin{tabular}{|c|c|c|c|c|c|c|}
\hline Root \# & Old \# & $\mathrm{HE}$ & $\mathrm{OO}$ & HA & KW & Gloss \\
\hline 11050 & 1198 & thxv- & thxv- & thxv- & thxv- & $\begin{array}{l}\text { tight, strong, intense, difficult, } \\
\text { painful, valuable, dear }{ }^{517}\end{array}$ \\
\hline 11060 & $\begin{array}{l}1191 \\
1199\end{array}$ & thq- & thq- & thq- & $\begin{array}{l}\text { thq-* } \\
\text { th'3 hq- }\end{array}$ & $\begin{array}{l}\text { overhang, overhanging (in } \\
\mathrm{HE} / \mathrm{OO} / \mathrm{HA} \text { with the connotation } \\
\text { of offering shelter (like a tent or a } \\
\text { lean-to), in the } \mathrm{KW} \text { examples } \\
\text { referring to an overhanging rock } \\
\text { or lifting the edge of a cover, as } \\
\text { for shaking particles off a mat or } \\
\text { blanket) }\end{array}$ \\
\hline 11070 & 1200 & & & & th $\chi-$ & $(?)^{518}$ \\
\hline 11080 & 1201 & th $\chi-$ & th $\chi-$ & & th $\chi-$ & to itch, irritated \\
\hline 11090 & 1203 & & $\operatorname{lmxv-}$ & & $\operatorname{lmxv-}$ & $\begin{array}{l}\text { dry, thirsty; high and dry, } \\
\text { marooned }^{519}\end{array}$ \\
\hline 11100 & 1204 & & $\operatorname{lm} \chi v-$ & & & $(?)^{520}$ \\
\hline 11110 & 1205 & & & & $\operatorname{lm} \chi-$ & to beat or clap on a surface \\
\hline 11120 & 1206 & & & & ls- & to pound with a stone \\
\hline 11130 & 1207 & lk- & lk- & lk- & lk- & $\begin{array}{l}\text { (HE/OO/HA) to play games with } \\
\text { stones; }(\mathrm{KW}) \text { to crush, pound or } \\
\text { hammer with a stone; to throw }\end{array}$ \\
\hline
\end{tabular}

${ }^{517}$ HA uses $\{\mathrm{xmt}-\}$ for "strong" said of the wind.

${ }^{518}$ In KW /łахла/ [1] "to defend (person)."

${ }^{519} \mathrm{OO}$ only in /lmxva/ "dry enough (to be ironed)."

${ }^{520} \mathrm{In} \mathrm{OO} / \mathrm{l} \chi \mathrm{va} /$ (precise meaning not remembered but had to do with limping or infirmity). 
North Wakashan Comparative Root List (Updated)

\begin{tabular}{|c|c|c|c|c|c|c|}
\hline Root \# & Old \# & $\mathrm{HE}$ & $\mathrm{OO}$ & $\mathrm{HA}$ & KW & Gloss \\
\hline & & & & & & $\begin{array}{l}\text { rocks (as in stone-throwing } \\
\text { game) }\end{array}$ \\
\hline 11140 & 1208 & & & & lk-* & $(?)^{522}$ \\
\hline 11150 & 1209 & lyp- & lyp- & lyp- & lyp- & to roll sth., to wrap sth. up \\
\hline 11160 & 1210 & lyt- & lyt- & lyt- & lyt- & $\begin{array}{l}\text { to rummage in search of sth., to } \\
\text { uncover; (HA also:) to shovel } \\
\text { snow }\end{array}$ \\
\hline 11170 & 1211 & $\begin{array}{l}\text { lyno-/ } \\
\text { lyx- }\end{array}$ & & $\begin{array}{l}\text { lyno-/ } \\
\text { lyx- }\end{array}$ & & $(?)^{523}$ \\
\hline 11180 & 1212 & lys- & & lys- & & boiled fish, fish soup \\
\hline 11190 & 1213 & & & & lyk-* & $(?)^{524}$ \\
\hline 11200 & 1214 & & & & lyx- & $(?)^{525}$ \\
\hline 11210 & 1215 & lyx- & lyx- & lyx- & lyx- & $\begin{array}{l}\text { to roll, turn over (cylindrical } \\
\text { object) }\end{array}$ \\
\hline 11220 & 1216 & & & & lyxv- & $\begin{array}{l}\text { to pull out a long thing (e.g. nail. } \\
\text { tooth) }\end{array}$ \\
\hline
\end{tabular}

${ }^{521}$ It means "rocky" in two non-reelicitable examples from the Koskimo dialect and "to gamble" in one non-reelicitable word from the dialect of the $/ \mathrm{Gva}^{3} \mathrm{~s} л \mathrm{a} /$.

522 In KW $\{$ lk-hlhs $\}>/$ kalas/* "thimbleberries when not quite ripe." Possibly this root is no. 11130 and refers here to pounding unripe thimbleberries.

523 In HA /liн/ "love song" HE (Klemtu dialect) /1'iH/ (an unidentified kind of song, to sing this kind of song). Not diagnosable if this is a free root $\{1 y n \infty-\}$ or $\{1 y x-=n\}$. Could be a foreign loan. ${ }^{524} \mathrm{In} \mathrm{KW} /$ likusa/* "wild cabbage leaves (used in making berry cake)" KW /lix:lk³/* "(kind of plant, possibly a lichen).

${ }^{525}$ In KW /lixa/ [1] "only." 
North Wakashan Comparative Root List (Updated)

\begin{tabular}{|c|c|c|c|c|c|c|}
\hline Root \# & Old \# & $\mathrm{HE}$ & $\mathrm{OO}$ & HA & KW & Gloss \\
\hline 11230 & 1217 & lyqv- & lyqv- & lyqv- & lyqv- & $\begin{array}{l}\text { flat thing folds onto itself, to } \\
\text { hinge; to fold in a flat thing, to } \\
\text { roll up a flat thing }\end{array}$ \\
\hline 11231 & & lyqv- & & lyqv- & lyqv- & $(?)^{526}$ \\
\hline 11240 & 1218 & lyq- & lyq- & & & reef, rocky ridge \\
\hline 11250 & & & & lyq- & & (?) lacking, missing ${ }^{527}$ \\
\hline 11260 & 1219 & lyx- & lyx- & lyx- & ly $\chi^{* *}$ & red cedar ${ }^{528}$ \\
\hline 11270 & $\begin{array}{l}1220 \\
1221 \\
1222 \\
1223 \\
1224\end{array}$ & $\begin{array}{l}\text { lkv- } \\
\text { lxv- }\end{array}$ & lkv- & lkv- & lkv- & $\begin{array}{l}\text { inconstant, inclined to change, } \\
\text { unreliable (hence: } 1 \text {. different, } \\
\text { strange, foreign, wrong, evil; } 2 . \\
\text { giddy, fainting, weak, tired; } 3 . \\
\text { limping, unsteady motion, } \\
\text { fluttering) } \\
529\end{array}$ \\
\hline 11300 & 1225 & lw- & lw- & & lw-* & (?) white, light, bright ${ }^{530}$ \\
\hline 11310 & $\begin{array}{l}1225 \\
2468\end{array}$ & lw- & & lw- & lw- & $\begin{array}{l}\text { (?) instability, unrest (of motion, } \\
\text { light or sound), fluttering, }\end{array}$ \\
\hline
\end{tabular}

${ }^{526}$ In the name of the Lekwiltok division of Cape Mudge and Campbell River on Vancouver Island. Also likely in HA /l'i:lqvalis/ "different villages" and НЕ /1'ілqvла£i/ KW /liлqvла£ $\varepsilon^{3} /$ "tribe, tribespeople."

${ }^{527}$ In HA /l'i:l $\chi s k^{3} \mathrm{ana} /$ "coat without sleeves, vest, waistcoat" and HA /l'iq ${ }^{3} \mathrm{ksi}^{3} \mathrm{a} /$ "toothless." 528 The HE/OO examples refer to red cedar but the HA ones to barkless red cedar. The only KW example is /Lixsm/* "November."

529 \{lxv-\} only in HE /l'al'axvmas/ "person who always does things the wrong way." The root likely refers to fluttering in HA /lalagvd'ai/ "butterfly."

${ }^{530}$ In terms referring to the sky, the milky way, northern lights and water with a milky colour. 
North Wakashan Comparative Root List (Updated)

\begin{tabular}{|c|c|c|c|c|c|c|}
\hline Root \# & Old \# & $\mathrm{HE}$ & $\mathrm{OO}$ & HA & KW & Gloss \\
\hline & & & & & & $\begin{array}{l}\text { flapping, flickering, whirling, } \\
\text { twirling, spiralling } 531\end{array}$ \\
\hline 11320 & 1231 & lw- & lw- & lw- & lw- & $(?)^{532}$ \\
\hline 11330 & 1226 & lwp- & lwp- & lwp- & lwp- & empty, hollow inside; to wither \\
\hline 11340 & 1226 & lwm³- & lwm³- & lwm³- & & $(?)^{533}$ \\
\hline 11350 & 1227 & & & & lwt- & to uncover, bare, reveal \\
\hline 11360 & 1228 & & & & lws- & to uncover, bare, reveal \\
\hline 11370 & 1229 & lwł- & lwł- & & lw1- & haunted, possessed \\
\hline 11380 & 1230 & lwqv- & lwqv- & & lwqv- & $\begin{array}{l}\text { longlining (for halibut, black } \\
\text { cod) }\end{array}$ \\
\hline 11390 & 1231 & lwqv- & lwqv- & lwqv- & lwqv-* & to go after hemlock bark \\
\hline 11400 & 1232 & $\operatorname{lw} \chi \mathrm{v}^{-}$ & $\operatorname{lw} \chi \mathrm{v}^{-}$ & $\operatorname{lw} \chi \mathrm{v}^{-}$ & $\operatorname{lw} \chi \mathrm{v}^{-}$ & $\begin{array}{l}\text { round, rolling; (HA and } \mathrm{HE} \mathrm{(Kl)} \\
\text { also:) rolling like thunder, } \\
\text { rumbling, trouble; (HE/KW } \\
\text { also:) patch, batch, aggregation, } \\
\text { grouping }\end{array}$ \\
\hline
\end{tabular}

${ }^{531}$ In KW /lumutла/ "to make a hollow rumbling noise, to cause turmoil" HE /hл'ulu/ "butterfly (cf. ОО /hмumu/) KW hлиlu/* (a reddish bird) HA /lul'u/ "sole." Could be the same root as no. 11300. In the case of HA /lul'u/ the root is perhaps associated with flatfish having a pale side that faces the sea bottom and a pigmented side that faces upward and sometimes serves as camouflage.

532 Possibly in HE /lu'aq/ OO /luaq/ HA /lu³aq/ KW /loq/ "hemlock sap." Reference is probably not to juice but western hemlock cambium which is edible and scraped off the harvested bark. It is believed to ward off evil. It is believed to ward off evil. It is also possible that the four items are augmented forms of a root $*\{l w q-\}$, older form of $\{$ lwqv- $\}$ (root no. 11390).

${ }^{533}$ In $\left\{1 \mathrm{wm}^{3} \mathrm{hs}-\right\}$ "to scorch (clothing); (HA also:) brown, orangy (like leaves in fall)." 
North Wakashan Comparative Root List (Updated)

\begin{tabular}{|c|c|c|c|c|c|c|}
\hline Root \# & Old \# & $\mathrm{HE}$ & $\mathrm{OO}$ & HA & KW & Gloss \\
\hline 11410 & 1233 & $1 \mathrm{w}^{3} \chi \mathrm{v}-$ & $1 w^{3} \chi v-$ & $\begin{array}{l}\mathrm{l} \chi \mathrm{v}^{-} \\
\mathrm{n} \chi \mathrm{v}^{-}\end{array}$ & $1 w^{3} \chi v-$ & $(?)^{534}$ \\
\hline 11420 & $\begin{array}{l}1234 \\
1235\end{array}$ & lqv- & lqv- & lqv- & lqv- & $\begin{array}{l}\text { tended fire, campfire, camp; } \\
\text { village }^{535}\end{array}$ \\
\hline 11421 & & & & & lrw- & strawberries 536 \\
\hline 11430 & 1237 & & & & $1 \chi-$ & open-work basket \\
\hline 11440 & 1238 & $1 \chi-$ & & $1 \chi-$ & $1 \chi-$ & $\begin{array}{l}\text { to pound with stone (or other } \\
\text { round thing) }\end{array}$ \\
\hline 11450 & $\begin{array}{r}533 \\
1239 \\
1244\end{array}$ & lh- & $\begin{array}{l}\text { lh- } \\
\text { nh- } \\
\text { hh- }\end{array}$ & $\begin{array}{l}\text { lh- } \\
\text { nh- }\end{array}$ & $\begin{array}{l}\text { lh- } \\
\text { nh- } \\
\text { hh- }\end{array}$ & $\begin{array}{l}\text { to go (away from origin, towards } \\
\text { target, about business); ongoing, } \\
\text { applicable; to reach, attain }{ }^{537}\end{array}$ \\
\hline
\end{tabular}

$534\left\{1 \mathrm{w}^{3} \chi \mathrm{v}-\right\}$ occurs only in $\left\{1 \mathrm{w}^{3} \chi \mathrm{v}-\infty \mathrm{kv}-\right\}$ "rocks, boulders." HA $\{1 \chi \mathrm{v}-\}$ occurs in $\{1 \chi \mathrm{v}-\infty \mathrm{kv}-\}$ "rocks, boulders" and $\{1 \chi \mathrm{v}-\infty \mathrm{t}-\}$ "personal belongings." $\{\mathrm{n} \chi \mathrm{V}-\}$ is attested only in the alternant /n $\chi \operatorname{vad}^{\prime} \mathrm{ac}^{3} \mathrm{i} /$ of HA /l $\chi \mathrm{vad}^{\prime} \mathrm{ac}^{3} \mathrm{i} /$ and $/ \mathrm{l} \chi \mathrm{ud}^{\prime} \mathrm{ac}^{3} \mathrm{i} /$ "place where one puts one's valuables."

535 \{lqv-h\} has the idiomatic meaning "firewood."

${ }^{536}$ Wild strawberries (Fragaria vesca) can survive mild fires or regrow after fire (cf.

https://en.wikipedia.org/wiki/Wild_strawberries). Possibly therefore this stem derives from $\{$ lqv- $\}$ (root no. 11420).

$537\{\mathrm{lh}-\}$ is the most frequently occurring alternant by far. $\{\mathrm{nh}-\}$ and $\{\mathrm{hh}-\}$ occur mostly in exclamations, e.g. HA /n³ $\mathrm{Ha} / \mathrm{OO} / \mathrm{hah}^{3} \mathrm{a} /$ "yes"; $\mathrm{HE} / \mathrm{hi}$ 'a/ OO /hia/ (mock encouragement and anxious encouragement); HE $/ h^{3} \mathrm{Han}^{\prime} \mathrm{a} / \mathrm{OO} / \mathrm{h}^{3}$ anana/ $\mathrm{KW} / \mathrm{h}^{3} \mathrm{Hana} /$ "ouch!" The form $\{\mathrm{ly}-\}$ occurs only as a proclitic signalling resumption of a thread of discourse, cf. English "well!"; in HE it alternates with $\{\mathrm{lh}-\}$. KW $\{\mathrm{hlh}-\}$, like $\mathrm{HA} / \mathrm{KW}\{\mathrm{hh}-\}$, occurs in a number of expressions broadly meaning "go!" and "farewell." In one example $\{$ hlh- $\}$ is replaced by $\{$ hlhk- $\}$, cf. KW $\{$ hlhk- $\Delta \mathrm{xs} \bullet-\mathrm{hlh}\}>$ /hлagxsala/ "go and take your chance!" The presence of $|\mathrm{k}|$ in $\{\mathrm{hlhk}-\}$ may be influenced by KW $\left\{\mathrm{hhl}^{3 \mathrm{~h}} \mathrm{~h}\right\}$ "to rush" having an extended form $\left\{\mathrm{hhl}{ }^{3} \mathrm{hk}-\right\}$. This stem $\left\{\mathrm{hhl} \mathrm{l}^{3 \mathrm{~h}}-\right\}$ and its alternants are listed as root no. 23142 but could be augmented forms of $\{\mathrm{hh}-\}$ or $\{\mathrm{lh}-, \mathrm{nh}-\}$. The form $\{\mathrm{hh}-\}$ of the root occurs possibly also in HA /ha风g/ "poor, destitute" in which case this 
North Wakashan Comparative Root List (Updated)

\begin{tabular}{|c|c|c|c|c|c|c|}
\hline Root \# & Old \# & $\mathrm{HE}$ & $\mathrm{OO}$ & HA & KW & Gloss \\
\hline & 2469 & hh- & & hh- & hlh- & \\
\hline & 2498 & $1 \mathrm{y}-$ & & ly- & hlhk- & \\
\hline & 2510 & & & & & \\
\hline 11460 & 1240 & lhxv- & lhxv- & lhxv- & lhxv- & to wrap sth. around \\
\hline 11461 & & & & & $\mathrm{lhq}^{3} \mathrm{vt}-$ & bundle, bundling ${ }^{538}$ \\
\hline 11470 & 1241 & & lh $\chi \mathrm{v}-$ & & $\operatorname{lh} \chi \mathrm{v}-$ & (?) cover, concealment ${ }^{539}$ \\
\hline & 1242 & & & & & \\
\hline 11490 & 1243 & $\operatorname{lh} \chi-$ & & $\operatorname{lh} \chi-$ & $\operatorname{lh} \chi-$ & $\begin{array}{l}\text { clear, unclouded (water, sky), } \\
\text { watery (liquid), runny (sore) }\end{array}$ \\
\hline 11500 & 1246 & & & & $1^{3} y x-$ & wide open, broad \\
\hline 11510 & 1247 & $1^{3} \chi \mathrm{VW}-$ & $1^{3} \chi \mathrm{vW}-$ & $1^{3} \chi \mathrm{VW}-$ & $1^{3} \chi \mathrm{w}-$ & to cough ${ }^{541}$ \\
\hline 11520 & 1248 & $1^{3} \mathrm{q}-$ & $1^{3} \mathrm{q}-$ & $1^{3} \mathrm{q}-$ & $1^{3} \mathrm{q}-$ & shapeless soft moist mass (e.g. \\
\hline & 1249 & & & & & putty) \\
\hline & 1236 & & & & & \\
\hline 11530 & 1252 & & & & $l^{3} \mathrm{~h}-$ & $(?)^{542}$ \\
\hline
\end{tabular}

stem contains a semantically opaque suffix $\{-\chi g\}$ whose only other occurrence is in HA /sa $\chi g /$ "to want to."

${ }^{538}$ Only two examples, KW /laq ${ }^{3} \mathrm{vta} /$ "to bundle up, bale, make bundle (of skins, blankets)" and KW /laq ${ }^{3} \mathrm{vdkv} /$ "bundle." Stem $\left\{\mathrm{lhq}^{3} \mathrm{vt}-\right\}$ may contain a root $\{\mathrm{lhqv}-\}$ and a suffix $\{-+\mathrm{t}\}$ but no clues to their meaning. Stem could also be a foreign loan.

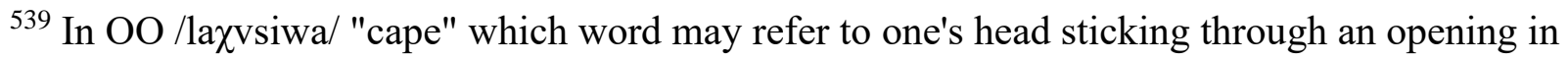

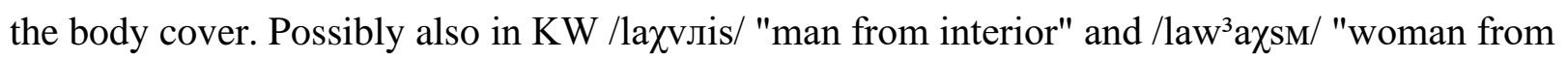
interior."

540 Only HE example is possibly the place name /L'aл $\chi \mathrm{sk}^{3} \mathrm{vs} /$.

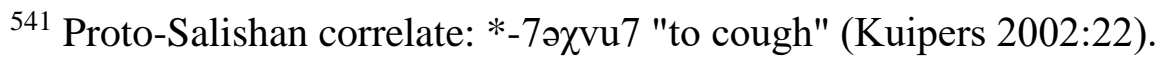

${ }^{542} \mathrm{In} \mathrm{KW}\left\{1^{3} \mathrm{~h}-=\mathrm{ys}\right\}>/ 1^{3} \mathrm{a}^{3} \mathrm{is} /$ "small mussel" and KW $\left\{1^{3} \mathrm{~h}-\% y d y\right\}>/ \mathrm{L}^{3} \varepsilon d i /$ (a place name: "where you go for small mussels"). 
North Wakashan Comparative Root List (Updated)

\begin{tabular}{|c|c|c|c|c|c|c|}
\hline Root \# & Old \# & $\mathrm{HE}$ & $\mathrm{OO}$ & HA & KW & Gloss \\
\hline 11540 & 1250 & $1^{3} \mathrm{hp}-$ & $1^{3} \mathrm{hp}-$ & $1^{3} \mathrm{hp}-$ & $1^{3} \mathrm{hp}-$ & $\begin{array}{l}\text { to spread apart (lips, legs, edges } \\
\text { of slit); (OO/KW also:) to dig a } \\
\text { pit, to excavate; (HA also:) to } \\
\text { expose what's under the cover }\end{array}$ \\
\hline 11550 & 1251 & & $1^{3} \mathrm{hc}-$ & & & $(?)^{543}$ \\
\hline 11560 & 1253 & & & & $\begin{array}{l}\text { l3hqv- }^{2} \\
1^{3} \mathrm{~h} \chi \mathrm{v}-*\end{array}$ & to shout, holler ${ }^{544}$ \\
\hline 11570 & 1255 & g- & g- & g- & $\mathrm{g}$-* & $\begin{array}{l}\text { and further, and then; (in } \\
\text { subordinate clauses:) when, } \\
\text { whenever }^{545}\end{array}$ \\
\hline 11580 & 1256 & kp- & kp- & gp- & gp- & $\begin{array}{l}\text { to plug (hole), button up, tuck in, } \\
\text { jam in, insert (lever); (HA also:) } \\
\text { to tuck away, to store for later } \\
\text { use, to stock up }\end{array}$ \\
\hline 11590 & 1257 & gm- & gm- & & gm- & $(?)^{546}$ \\
\hline 11600 & 1258 & (ghmwl3-) & $\left(\right.$ ghmwl $\left.^{3}-\right)$ & (ghmwl3-) & gmt- & fish hook \\
\hline 11610 & 1259 & & & & gmt- & $(?)^{547}$ \\
\hline
\end{tabular}

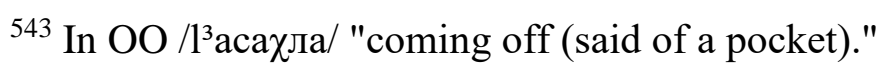

$544\left\{1^{3} \mathrm{~h} \chi \mathrm{v}-\right\}^{*}$ only in $\mathrm{KW} / 1^{3} \mathrm{a} \chi \mathrm{v}: 1^{3} \mathrm{a} \chi \mathrm{v} / *$ "yellow-beaked cormorant" (so called because of its cry, says Boas).

545 The only available KW example is possibly $/ \mathrm{gm}^{3} \mathrm{H} / *$ which Boas $(1947: 358)$ uses in $/ \mathrm{gm}^{3} \mathrm{H}$ $\mathrm{h}^{3} \mathrm{a}^{3} \mathrm{llak}^{3} \varepsilon^{3} /$ "this is my witness."

${ }^{546} \mathrm{In} \mathrm{HE} / \mathrm{gm}^{3}$ 'in'u $\mathrm{uv} / \mathrm{OO} / \mathrm{KW} / \mathrm{gm}^{3} \mathrm{inu} \chi \mathrm{v} / \mathrm{HE} / \mathrm{OO}$ "clan" KW "fellow clansmen." Root could mean "offshoot, barb, branch in network" and be related to $\{$ ghmh- $\}$ (no. 12100) and \{gm\}-\} (no. 11600).

${ }^{547}$ In KW /gmła/ [2] "to get tired of eating sth." 
North Wakashan Comparative Root List (Updated)

\begin{tabular}{|c|c|c|c|c|c|c|}
\hline Root \# & Old \# & $\mathrm{HE}$ & $\mathrm{OO}$ & HA & KW & Gloss \\
\hline 11620 & 1260 & & & & gmxv- & $\begin{array}{l}\text { lumpy object (like one's head) } \\
\text { hangs down, to hang heads }\end{array}$ \\
\hline 11630 & 1262 & & & & $\operatorname{gd} \chi v-$ & $(?)^{548}$ \\
\hline 11640 & 1261 & & & & gt- & to make, create, construct sth. \\
\hline 11650 & 1263 & & & & gt- & $(?)^{549}$ \\
\hline 11660 & $\begin{array}{l}1264 \\
1269\end{array}$ & gn- & $\begin{array}{l}\text { gn- } \\
\text { gnth- }\end{array}$ & gn- & gn- & $\begin{array}{l}\text { extension, supplement, addition, } \\
\text { topping up } \\
550\end{array}$ \\
\hline 11670 & 1268 & & & & $\begin{array}{l}\text { gn- } \\
\text { gnt- }\end{array}$ & child, person of tender age ${ }^{551}$ \\
\hline 11680 & 1266 & gnc- & $\begin{array}{l}\text { gnc- } \\
\text { gn }^{3} \mathrm{c}-\end{array}$ & gnc- & & $\begin{array}{l}\text { to own, ownership, possession, } \\
\text { responsibility for sth. }\end{array}$ \\
\hline 11690 & $\begin{array}{l}1265 \\
1267\end{array}$ & $\begin{array}{l}\text { gnc•- } \\
\text { gnch- }\end{array}$ & $\begin{array}{l}\text { gnc•- } \\
\text { gnch- }\end{array}$ & gnc $\bullet-$ & $\begin{array}{l}\text { gn- } \\
\text { gns- }\end{array}$ & how many \\
\hline 11710 & 1270 & & & & $\begin{array}{l}\text { gnw }^{3}- \\
\text { gnw }^{3} \text { h- } \\
\text { gwn } \infty-\end{array}$ & $\begin{array}{l}\text { to add, give more; to lend a } \\
\text { helping hand } \\
552\end{array}$ \\
\hline 11720 & 1271 & & & & gn $\chi v^{-}-$ & $(?)^{553}$ \\
\hline
\end{tabular}

${ }^{548} \mathrm{In} \mathrm{KW} / \mathrm{gd} \chi \mathrm{va} /$ "to tickle."

${ }^{549} \mathrm{In} \mathrm{KW} / \mathrm{gt}^{3} \mathrm{ala} /[2]$ "to tell sb. not to do sth., to forbid" and possibly KW /gt ${ }^{3} \mathrm{a}$ ллud/ "to fit in."Q ${ }^{550} \mathrm{OO}$ \{gnłh-\} only in /ghłut/ "to add money to an account" which has a structure \{gnłh-wd (with suffix no. 830) that parallels that of the synonym $\left\{\mathrm{q}^{3} \mathrm{n} ł \mathrm{~h}-\mathrm{wd}\right\}>\mathrm{HE} / \mathrm{q}^{3}{ }^{\prime} \mathrm{H}^{\prime}$ aut $/ \mathrm{OO} / \mathrm{q}^{3} \mathrm{H}$ hut $/$. ${ }^{551}$ The form $\{\mathrm{gn}-\}$ occurs only before $\{-\% \mathrm{hnm}\}$ (suffix no. 1290) and is probably the result of this process, $\{$ gnt-\%hnm $\}>\{$ gnlo-hnm $\}>\{$ gnl-hnm $\}>\{$ gnØ-hnm $\}>/$ gнапм $/$ "child." ${ }^{552}$ Direct evidence of the alternant $\left\{\mathrm{gnw}^{3} \mathrm{~h}-\right\}$ is $\mathrm{KW}\left\{[\mathrm{g}] \mathrm{gnw}^{3} \mathrm{~h}-\mathrm{rw}-\mathrm{d}\right\}>/ \mathrm{ggHw}^{3}$ arud/ "to add in between."

${ }^{553} \mathrm{In} \mathrm{KW} / \mathrm{gH} \chi \mathrm{va} /$ [1] "to fart (especially after eating fresh ooligan oil)" 
North Wakashan Comparative Root List (Updated)

\begin{tabular}{|c|c|c|c|c|c|c|}
\hline Root \# & Old \# & $\mathrm{HE}$ & $\mathrm{OO}$ & HA & KW & Gloss \\
\hline 11730 & $\begin{array}{l}1272 \\
1280 \\
1281\end{array}$ & & gl $\chi \mathrm{v}-$ & $\begin{array}{l}\mathrm{gl} \chi- \\
\mathrm{gl}^{3} \chi-\end{array}$ & $\begin{array}{l}\text { gn } \chi \mathrm{v}- \\
\text { gl } \chi-*\end{array}$ & to rub, rub in ${ }^{554}$ \\
\hline 11740 & 1273 & gł- & gł- & g1- & g1- & $\begin{array}{l}\text { to raise the head in fear of } \\
\text { danger }^{555}\end{array}$ \\
\hline 11750 & 1274 & gl- & gl- & gl- & gl- & $\begin{array}{l}\text { to go on all fours, to crawl, to } \\
\text { creep, to move stealthily, to stay } \\
\text { low to the ground }\end{array}$ \\
\hline 11760 & 1275 & & & & gl-* & $(?)^{556}$ \\
\hline 11780 & 1277 & glt- & glt- & glt- & glt- & long, tall \\
\hline 11790 & 1278 & klx- & klx- & klx- & glx- & $\begin{array}{l}\text { to leak (said of a canoe, kettle, } \\
\text { hollow container), leak, leaky }\end{array}$ \\
\hline 11800 & 1279 & $\mathrm{glw}^{3-}$ & glw $^{3}-$ & $\mathrm{glw}^{3}-$ & $\begin{array}{l}\text { glw }^{3}- \\
\text { glxv-* }\end{array}$ & canoe $^{557}$ \\
\hline 11820 & 1276 & & & & glh- & grizzly bear \\
\hline 11830 & 1282 & gy- & $\begin{array}{l}\text { gy- } \\
\mathrm{gy}^{3}-\end{array}$ & $\begin{array}{l}\text { gy- } \\
\text { gh- }\end{array}$ & gy- & located, situated somewhere \\
\hline
\end{tabular}

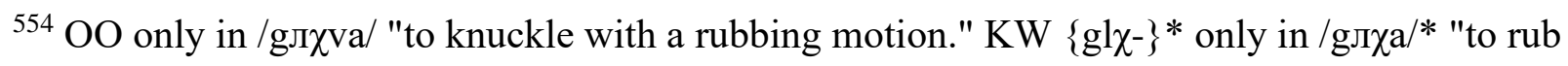
with a wet finger" and $\mathrm{KW} / \mathrm{g}$ лаs/* "clitoris."

${ }^{555} \mathrm{In}$ words referring to the weasel and $\mathrm{KW} / \mathrm{g}^{3} \mathrm{ala} / *$ "birds raise heads in expectation of danger." Weasels have long necks and raise their heads when disturbed. Evidence that the root is $\{\mathrm{gl}-\}$ rather than $\{\mathrm{gl}-\}$ is the insertion of $|\mathrm{h}|$ in the root in HA $\{[\mathrm{gh}] \mathrm{g}[\mathrm{h}] \mathrm{l}-\mathrm{+m}-\mathrm{h}\}>/ \mathrm{gagal}^{3} \mathrm{~m}^{\prime} \mathrm{a} /$ "to go after weasels." This root may be related to $\left\{\mathrm{gwl}^{3}-\right\}$ and $\left\{\mathrm{ghl}^{3}-\right\}$ "apprehensive, uneasy, alarmed" (root no. 11991).

${ }^{556} \mathrm{In} \mathrm{KW} \mathrm{/gла/*} \mathrm{[2]} \mathrm{"salmon} \mathrm{when} \mathrm{dried} \mathrm{put} \mathrm{lengthwise"} \mathrm{and} \mathrm{KW} \mathrm{/gліз3stnd/*} \mathrm{"to} \mathrm{turn} \mathrm{over}$ lengthwise (salmon, small animal)."

557 \{glxv-\}* only in $\mathrm{KW} / \mathrm{g}$ лхvila/* "to make a canoe." 
North Wakashan Comparative Root List (Updated)

\begin{tabular}{|c|c|c|c|c|c|c|}
\hline Root \# & Old \# & $\mathrm{HE}$ & $\mathrm{OO}$ & HA & KW & Gloss \\
\hline 11850 & $\begin{array}{l}1284 \\
1294\end{array}$ & $\begin{array}{l}\text { gy- } \\
\text { gys- } \\
\text { gy }^{3}-\end{array}$ & $\begin{array}{l}\text { gy- } \\
\text { gys- } \\
\text { gy³- }\end{array}$ & $\begin{array}{l}\text { gy- } \\
\text { gyx- } \\
\text { gy }{ }^{3} \text { s- }\end{array}$ & $\begin{array}{l}\text { gy- } \\
\text { gys- } \\
\text { gyx- } \\
\text { gyy3- }^{3} \text { - } \\
\text { gy³ }^{3} \\
\text { gy³- }^{3} \text { - } \\
\text { ghy³- }\end{array}$ & beloved, respected, revered 558 \\
\hline 11860 & 1285 & & & & gys- & to shake head in dissent \\
\hline 11870 & $\begin{array}{l}1283 \\
1286\end{array}$ & $\begin{array}{l}\text { gyc- } \\
\text { kyc- }\end{array}$ & kyc- & & $\begin{array}{l}\text { gys- } \\
\text { kys- }\end{array}$ & $\begin{array}{l}\text { material with pale shiny surface } \\
\text { (like enamel of teeth or the pearly } \\
\text { material used for buttons and } \\
\text { decorating) } \text { s }^{55}\end{array}$ \\
\hline 11880 & 1287 & gy£- & gy£- & & & dear, beloved \\
\hline 11890 & 1288 & $\begin{array}{l}\text { gyg- } \\
\text { gyk- }\end{array}$ & $\begin{array}{l}\text { gyg- } \\
\text { gyk- }\end{array}$ & gyg- & $\begin{array}{l}\text { gyg- } \\
\text { gyk- }\end{array}$ & tooth, teeth \\
\hline 11891 & 1298 & gyw³hlh- & gwy³hlh- & & & be careful, take care ${ }^{560}$ \\
\hline 11900 & 1289 & gyw³hlh- & & & & $(?)^{561}$ \\
\hline
\end{tabular}

${ }^{558}$ Derivates refer to kinship and marital relations.

${ }^{559} \mathrm{KW}$ \{gys- $\}$ only in /gis $\chi$ stala/ "ceremonial box cover with sea otter teeth at the opening." The examples of KW \{kys-\} probably include KW/kiziłbai $3 / *$ "nose ornament" and KW /kiziłbala/* "to wear nose ornament" (which latter according to Boas (1948:22) is a synonym of $\mathrm{KW} / \mathrm{h}^{3}$ iziłbala/* "abalone nose ornament."

${ }^{560} \mathrm{In} \mathrm{HE} / \mathrm{g}^{\prime} \mathrm{iw}^{3} \mathrm{al}^{\prime} \mathrm{a} /$ [1] "to take care" OO /guy ${ }^{3} \mathrm{ala} /$ "to be careful." The HE item is listed in Boas (1928:240).

${ }^{561}$ In HE /g'iw ${ }^{3 \prime} \mathrm{al}^{\prime} \mathrm{a} /$ [2] "to make love to one's sister-in-law." 
North Wakashan Comparative Root List (Updated)

\begin{tabular}{|c|c|c|c|c|c|c|}
\hline Root \# & Old \# & $\mathrm{HE}$ & $\mathrm{OO}$ & HA & KW & Gloss \\
\hline 11910 & 1290 & & & & gy $\chi v-*$ & (?) foolish f62 $^{56}$ \\
\hline 11911 & $\begin{array}{l}1291 \\
1305\end{array}$ & & & & $\begin{array}{l}\text { gy } \chi v- \\
g \chi v-\end{array}$ & steelhead salmon \\
\hline 11920 & 1292 & & & & gyq- & chief, lord, authority ${ }^{563}$ \\
\hline 11930 & 1293 & gy $\chi-$ & gy $\chi-$ & gy $\chi-$ & gy $\chi-$ & $\begin{array}{l}\text { to grind, rub, file (as for } \\
\text { sharpening) }\end{array}$ \\
\hline 11931 & & & & gvy- & $\begin{array}{l}\text { gw- } \\
\text { gvy- }\end{array}$ & (onomatopoeic) $^{564}$ \\
\hline 11940 & 1296 & gvwmh- & gvwmh- & gwmh- & gwmh- & (?) large eyes ${ }^{565}$ \\
\hline 11950 & 1297 & & & & gwt- & head is underwater \\
\hline 11980 & 1299 & gvws- & gvws- & gws- & gwys- & frost, to freeze ${ }^{566}$ \\
\hline 11990 & 1301 & gvwł- & gvwł- & gwł- & gwt- & $\begin{array}{l}\text { (HE/OO/HA) to gather and } \\
\text { preserve food staples (meat, } \\
\text { berries, salmon), make travel } \\
\text { provisions, to will sth. to sb. } \\
\text { (KW) travelling provisions, } \\
\text { snack, lunch }\end{array}$ \\
\hline
\end{tabular}

${ }^{562} \mathrm{In} \mathrm{KW} /$ gi $\chi \mathrm{va} / *$ "foolish" KW /giw ${ }^{3} \mathrm{ala} / *$ "to speak foolishly" (both from the $/ \mathrm{N}^{3} \mathrm{ak}^{3} \mathrm{vaxda}^{3} \chi \mathrm{v} /$ dialect)

563 This very productive root could be a backformation from KW $\left\{\right.$ gy-rm- $\left.\alpha y^{3}\right\}>/ g i r M \varepsilon^{3} /$ "chief" (literally "the one in front").

${ }^{564}$ In KW /gugugu/ "screech owl" HA /gvigvigv'i/ KW /gvigvigvi/ "oystercatcher (bird)."

${ }^{565}$ In words referring to ratfish and the grey or snowy owl. Ratfish like owls have large eyes.

${ }^{566} \mathrm{KW}$ \{gwys- $\}$ could have the structure $\{\mathrm{gw}[\mathrm{y}] \mathrm{s}-\}<\{\mathrm{gw}[\infty] \mathrm{s}-\}$. 
North Wakashan Comparative Root List (Updated)

\begin{tabular}{|c|c|c|c|c|c|c|}
\hline Root \# & Old \# & $\mathrm{HE}$ & $\mathrm{OO}$ & HA & $\mathrm{KW}$ & Gloss \\
\hline 11991 & & & & & $\begin{array}{l}\mathrm{gwl}^{3-} \\
\mathrm{ghl}^{3} \_*\end{array}$ & (?) to agitate, be agitated ${ }^{567}$ \\
\hline 12000 & 1295 & gvwgvy3- & gvwgvy3- & gwgvy3- & gwgvy $^{3} w-$ & foot, leg 568 \\
\hline 12010 & $\begin{array}{l}1302 \\
1303\end{array}$ & gvwkv- & gvwkv- & gwkv- & $\begin{array}{l}\text { gwkv- } \\
\text { gwxv- }\end{array}$ & residence, home, settlement ${ }^{569}$ \\
\hline 12030 & 1295 & $\mathrm{gvw}^{3}-$ & $\mathrm{gvw}^{3}-$ & $\mathrm{gw}^{3-}$ & $\mathrm{gw}^{3}-$ & $(?)^{570}$ \\
\hline 12040 & 1300 & & & $\mathrm{gw}^{3} \mathcal{E}-$ & & to borrow \\
\hline 12050 & 1304 & & & IVXV- & $\mathrm{g} \chi \mathrm{V}-$ & to drift (like canoe, log) \\
\hline 12070 & 1306 & & & & $\mathrm{~g} \chi-$ & seat in canoe \\
\hline 12080 & 1307 & gh- & gh- & gh- & gh- & this (near speaker) ${ }^{571}$ \\
\hline 12090 & 1310 & gh- & gh- & gh- & $\begin{array}{l}\text { gh- } \\
\text { zh-* }\end{array}$ & $\begin{array}{l}\text { (?) reference point, starting } \\
\text { point }^{572}\end{array}$ \\
\hline
\end{tabular}

${ }^{567}$ In KW /gטл ${ }^{3} л^{3} \mathrm{qa} /$ "to stir about among things." Another example may be KW /gul3ala/ and /gal3ala/* "to expect unseen danger, uneasy for fear, on the alert against danger."

568 This free stem could derive from root no. 12010 if the latter means "foundation."

${ }^{569} \mathrm{KW}\{$ gwxv- $\} *$ only in /guxva/* "to dwell" (from the Gwasla dialect).

${ }^{570}$ Perhaps in HE /gvu'a a a/ OO /gvu³ a£a/ HA /gu' ${ }^{31} \mathrm{a} a / \mathrm{KW} / \mathrm{gv}^{3} \mathrm{ala} /$ "to help, to assist."

${ }^{571}$ Demonstrative root occurring as free form and proclitic. Only a small number of suffixes can be attached; which ones is different in $\mathrm{HE} / \mathrm{OO}, \mathrm{HA}$ and $\mathrm{KW}$.

5721 . $\{$ gh- $\}$ is assumed to be a subcomponent of the stem $\left\{\operatorname{gh} \chi^{\bullet-}, \operatorname{gh} \chi-\right\}$ "hither, towards the speaker, etc." listed as entry no. 12093 in this root list because in HA the pluralizing suffix $\{-\mathrm{m}-$, -mळ-, etc. $\}$ (suffix no. 430) can separate $|\mathrm{gh}|$ and $|\chi \bullet, \chi|$, cf. HA $\{\mathrm{gh} \chi-\mathrm{m}-=\mathrm{y} £\}>/ \mathrm{g}^{\prime} \mathrm{a} \chi \mathrm{M}^{3} \mathrm{i} £ /$ and

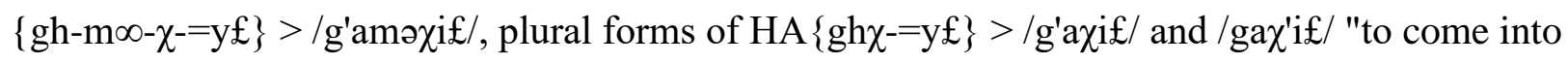
sth." 2. The following stems are also listed as separate entries (nos. 12091 and 12092) but are probably augmented forms of \{gh-\}, sc. (a) \{ghhlh-, ghloo-, ghlh-\} "before, first, etc."; (b) \{ghy-, ghyh- $\}$ "starting from, etc." 3. $\{$ zh- $\} *$ occurs in a non-reelicited word Boas (1948:335) mentions 
North Wakashan Comparative Root List (Updated)

\begin{tabular}{|c|c|c|c|c|c|c|}
\hline Root \# & Old \# & $\mathrm{HE}$ & $\mathrm{OO}$ & HA & KW & Gloss \\
\hline 12091 & 1311 & $\begin{array}{l}\text { ghlh- } \\
\text { ghlœ- } \\
\text { ghhlh- }\end{array}$ & $\begin{array}{l}\text { ghlh- } \\
\text { ghlœ- } \\
\text { ghhlh- }\end{array}$ & $\begin{array}{l}\text { ghlh- } \\
\text { ghlœ- } \\
\text { ghhlh- }\end{array}$ & $\begin{array}{l}\text { ghlh- } \\
\text { ghlœ- }\end{array}$ & $\begin{array}{l}\text { before, first, ahead, earlier on, } \\
\text { ancient; (in certain syntactic } \\
\text { constructions:) when, as soon } \\
\text { as }^{573}\end{array}$ \\
\hline 12092 & 1313 & $\begin{array}{l}\text { ghy- } \\
\text { ghyh- }\end{array}$ & $\begin{array}{l}\text { ghy- } \\
\text { ghyh- }\end{array}$ & $\begin{array}{l}\text { ghy- } \\
\text { ghyh- }\end{array}$ & $\begin{array}{l}\text { ghy- } \\
\text { ghyh- }\end{array}$ & $\begin{array}{l}\text { starting point, origin, reason, } \\
\text { cause }\end{array}$ \\
\hline 12093 & 1315 & $\begin{array}{l}\operatorname{gh} \chi- \\
\operatorname{gh} \chi^{\bullet-}\end{array}$ & $\begin{array}{l}\operatorname{gh} \chi- \\
\operatorname{gh} \chi^{\bullet-}\end{array}$ & $\begin{array}{l}\operatorname{gh} \chi- \\
\operatorname{gh} \chi^{\bullet-}\end{array}$ & $\begin{array}{l}\operatorname{gh} \chi- \\
\operatorname{gh} \chi \bullet-\end{array}$ & $\begin{array}{l}\text { hither, to come towards the } \\
\text { speaker(s), to move this way, to } \\
\text { come back home }\end{array}$ \\
\hline 12100 & 1308 & & & & ghmh- & $\begin{array}{l}\text { branching off, off the beaten } \\
\text { track, strange, different }{ }^{574}\end{array}$ \\
\hline 12101 & 1258 & ghmwl $^{3}-$ & ghmwl $^{3-}$ & ghmwl $\mathbf{3}_{-}$ & ghmwl³_* & fish hook ${ }^{575}$ \\
\hline 12110 & 1309 & & & & ghd- & far, to a great extent ${ }^{576}$ \\
\hline 12130 & 1312 & & & $\mathrm{ghl}^{3}-$ & $\mathrm{ghl}^{3}-$ & $\begin{array}{l}\text { (?) to hold a suspicion, verify an } \\
\text { assumption }\end{array}$ \\
\hline 12140 & 1314 & ghwyq- & ghwyq- & ghwyq- & $\begin{array}{l}\text { ghwyq- } \\
\text { ghwhq- }\end{array}$ & $\begin{array}{l}\text { (HE/OO/HA) seafood; (KW) } \\
\text { clams }^{577}\end{array}$ \\
\hline 12150 & 1316 & & & & kp- & to embrace, to carry in one's arms \\
\hline
\end{tabular}

as an example of the $/ \mathrm{DH}^{3} \mathrm{axda}^{3} \chi \mathrm{v} /$ (Knight Inlet people) using a dental-alveolar plosive where KW has a palatal-velar one.

${ }^{573}$ Possibly $\{$ gh- $\}$ (no. 12090) expanded into $\{\mathrm{g}[\mathrm{h}] \mathrm{h}[\mathrm{h}]-\}$ and $\{\mathrm{g}[\mathrm{h}] \mathrm{h}[\infty]-$, with the middle $|\mathrm{h}|$ dissimilating into $|1|$.

${ }^{574}$ Could be an augmented form of $\{$ gm- $\}$ (no. 11590).

575 Only in HE /g'am'ul³a/ OO /gamulª/ "black cod hook made out of yew wood" HA /g'amulay ${ }^{3} \mathrm{u} /$ "black cod hook" KW (Newettee dialect) /gamul³a/* "halibut hook."

${ }^{576}$ In KW /gadala/ "far ahead, far away" and KW /gagada3isla/* "to take sth. seriously."

${ }^{577}$ Could contain a root $\{\mathrm{ghxv}-\},\left\{\mathrm{gh} \chi \mathrm{v}^{-}\right\}$or $\{\mathrm{ghwh}-\}$. If the root is $\{\mathrm{ghwh}-\}$ it might be related to $\left\{\mathrm{gw}^{3}-, \mathrm{gvw}^{3}-\right\}$ and mean "sustenance, support." 
North Wakashan Comparative Root List (Updated)

\begin{tabular}{|c|c|c|c|c|c|c|}
\hline Root \# & Old \# & $\mathrm{HE}$ & $\mathrm{OO}$ & HA & KW & Gloss \\
\hline 12151 & 1319 & & & & $\begin{array}{l}\mathrm{km}-* / \\
\mathrm{kmk}-* / \\
\mathrm{kmx}-*\end{array}$ & $(?)^{578}$ \\
\hline 12160 & 1317 & & kmt- & kmt- & kmt- & $\begin{array}{l}\text { (OO) to gnaw with the upper } \\
\text { teeth, }(\mathrm{HA} / \mathrm{KW}) \text { to remove } \\
\text { protrusions (e.g. stems from } \\
\text { berries or berries from plant) }\end{array}$ \\
\hline 12170 & 1318 & & & & $\begin{array}{l}\text { kms-* } \\
\text { kmy }^{3}-\end{array}$ & $\begin{array}{l}\text { captured, immobilized by } \\
\text { surroundings (like saw that } \\
\text { "binds" when moved too fast or } \\
\text { forcefully), corralled, surrounded } \\
\text { (like fish or the novices who had } \\
\text { disappeared during the initiation } \\
\text { ceremony) }\end{array}$ \\
\hline 12190 & 1320 & & & & kmq- & $(?)^{581}$ \\
\hline 12200 & 1321 & kmq- & kmq- & kmq- & kmq- & $\begin{array}{l}\text { gap between objects closing } \\
\text { forcefully; to collide with sth., to } \\
\text { bump or collapse into sth., to jam } \\
\text { together, to get shut in }\end{array}$ \\
\hline
\end{tabular}

${ }^{578} \mathrm{In} \mathrm{KW} / \mathrm{kmx} . \hbar^{3} \mathrm{id} / *$ "to give a pull, to start to pull." No telling if root is $\{\mathrm{km}-\},\{\mathrm{kmk}-\}$ or $\{\mathrm{kmx}-\}$.

${ }^{579}$ Only one OO example of this root but relatively many HA/KW ones. The HA/KW ones suggest that $\{\mathrm{kmt}-\}$ refers to gripping or pinching the object to be removed. Blocking escape seems to be a recurring theme among roots beginning in $|\mathrm{k}|$ plus $|\mathrm{p}|$ or $|\mathrm{m}|$. $580\{\mathrm{kms}\}^{*}$ only in $\mathrm{KW} / \mathrm{kmsa} / *$ and $/ \mathrm{kms} . \mathrm{h}^{3} \mathrm{id} / *$ "to close up, to bind (saw)." ${ }^{581} \mathrm{In} \mathrm{KW} / \mathrm{kmqa} /$ "to use adze." Not listed in Boas's dictionary but mentioned in the 1980 root list (p. 230). 
North Wakashan Comparative Root List (Updated)

\begin{tabular}{|c|c|c|c|c|c|c|}
\hline Root \# & Old \# & $\mathrm{HE}$ & $\mathrm{OO}$ & HA & KW & Gloss \\
\hline 12220 & 1322 & & kdh- & & & $(?)^{582}$ \\
\hline 12230 & 1323 & & & kt- & & to shoot with a gun \\
\hline 12240 & $\begin{array}{l}1324 \\
1325 \\
1367\end{array}$ & khnh- & & $\mathrm{khn}^{3}-$ & $\begin{array}{l}\text { kn- } \\
\mathrm{kn}^{3}- \\
\text { khnh- }\end{array}$ & $\begin{array}{l}\text { circle, circular, back and forth; to } \\
\text { encircle, embrace; to surround } \\
\text { (isolate) a portion of a mass (e.g. } \\
\text { small fish) with a scoop or net }{ }^{583}\end{array}$ \\
\hline 12250 & 1326 & knc- & knc- & knc- & kns-* & $\begin{array}{l}\text { (HE/OO/HA) to loathe, be } \\
\text { disgusted with sth.; }(\mathrm{KW}) \text { to fear, } \\
\text { to be ashamed }\end{array}$ \\
\hline 12260 & $\begin{array}{l}1327 \\
1329 \\
1330 \\
1333\end{array}$ & knk- & knk- & knk- & knk- & $\begin{array}{l}\text { in a circle, wrapping around, } \\
\text { back and forth (as when shaking } \\
\text { the head), in a loop } \\
584\end{array}$ \\
\hline 12290 & $\begin{array}{l}1328 \\
1331\end{array}$ & & & & knx- & $\begin{array}{l}\text { to roll (like ring or hoop), to } \\
\text { wrap, to surround, to capture, to } \\
\text { scoop }\end{array}$ \\
\hline 12310 & 1332 & knq- & knq- & knq- & knq- & $\begin{array}{l}\text { to bend one' head, to put one's } \\
\text { head close to sth. }\end{array}$ \\
\hline
\end{tabular}

${ }^{582} \mathrm{In} \mathrm{OO} / \mathrm{kdau} /$ (a vocative to address one's daughter) and OO $/ \mathrm{Kdaka}^{3} \mathrm{u} /$ (a woman's name). $583\{\mathrm{khnh}-\}$ and $\left\{\mathrm{khn}^{3}-\right\}$ only in $\mathrm{HE} / \mathrm{k}^{\prime} \mathrm{an}^{\prime} \mathrm{an}^{3} \mathrm{a} / \mathrm{HA} / \mathrm{kak}^{\prime} \mathrm{an}^{3} \mathrm{i} / \mathrm{KW} / \mathrm{kan} \varepsilon^{3} /$ "1. hoop game; 2. punk (a white pancake-like growth on old trees that was used to preserve fire)."

584 1. Also in terms referring to a fungus, most likely because it is a hemicircular shelf fungus that sort of wraps itself around a stem or branch. 2. Also in HA $\{$ knk-m $\}>/ \mathrm{k}^{\prime} \mathrm{kkm} /$ "marten." The root refers here to the throat and chest "bib" hanging from the animal's neck. The suffix is no. 490 of the suffix catalogue and means "in front." 3. The 1980 root list has a KW root $\left\{\mathrm{kn} \chi^{-}\right\}$"to wrap around." One of its three examples is in error and the other two are really examples of $\{\mathrm{knk}-\}$ but with the root allomorph's $/ \mathrm{x} /$ misspelled $/ \chi /$. 
North Wakashan Comparative Root List (Updated)

\begin{tabular}{|c|c|c|c|c|c|c|}
\hline Root \# & Old \# & $\mathrm{HE}$ & $\mathrm{OO}$ & HA & KW & Gloss \\
\hline 12330 & $\begin{array}{l}1335 \\
1342\end{array}$ & & kys- & ks- & $\begin{array}{l}\text { ks- } \\
\text { kys- }\end{array}$ & $\begin{array}{l}\text { sweeping motion with hand, arm, } \\
\text { tail or tool; to rake, to motion } \\
\text { sideways, to move to one side }\end{array}$ \\
\hline 12340 & 1334 & & & & $\mathrm{ksp}^{3-}$ & (?) musky ${ }^{585}$ \\
\hline 12350 & 1336 & & & & $\mathrm{kf-}$ & $\begin{array}{l}\text { to use arm (to beckon, carry } \\
\text { under arm, hold sb.'s arm) }\end{array}$ \\
\hline 12360 & 1337 & & & & $\mathrm{k£-*}$ & to notch \\
\hline 12370 & 1338 & kł- & & kł- & $\begin{array}{l}\text { k1- } \\
\text { kykhł- }\end{array}$ & $\begin{array}{l}\text { (HE) amazed, impressed, in awe; } \\
\text { (HA) apprehensive, unhappy } \\
\text { with what's going on; (KW) } \\
\text { afraid, fearful }\end{array}$ \\
\hline 12380 & 1339 & & klx- & & klx- & $\begin{array}{l}\text { circular, round; (KW also:) to go } \\
\text { around, to operate a vehicle, run } \\
\text { an engine }\end{array}$ \\
\hline 12390 & 1340 & & & & klxv- & to buy, purchase ${ }^{586}$ \\
\hline 12400 & 1341 & & & & $\mathrm{kl} \chi-*$ & $(?)^{587}$ \\
\hline 12410 & & & & & klhkv- & muskrat $^{588}$ \\
\hline 12420 & 1343 & & & & kyf- & $\begin{array}{l}\text { to fish with a net; to fish sth. out } \\
\text { of water (by any means) }\end{array}$ \\
\hline
\end{tabular}

${ }^{585}$ In KW / $\mathrm{ksp}^{3} \mathrm{uli} /$ "trailing black currant" KW /kakasp3 $\mathrm{a}^{3} \mathrm{a} /$ "to gather trailing black currants." Boas (1948:263) wonders if /ksp33uli/ could mean "musky smell berry" and it probably does because according to http://nativeplantspnw.com/trailing-black-currant-ribes-laxiflorum/ the plant's leaves give off a strong odor when bruised.

${ }^{586}$ All examples are about buying goods but the root could more generally refer to the circulation of goods through trading and thus be related to $\{\mathrm{klx}-\}$ (root no. 12380).

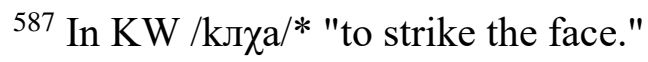

${ }^{588}$ Cross-family stem, cf. proto-Salish *-kəlaxv- "muskrat" (Kuipers 2002:36). 
North Wakashan Comparative Root List (Updated)

\begin{tabular}{|c|c|c|c|c|c|c|}
\hline Root \# & Old \# & $\mathrm{HE}$ & $\mathrm{OO}$ & HA & KW & Gloss \\
\hline 12430 & 1344 & kyxv- & kyxv- & kyxv- & kyxv- & $\begin{array}{l}\text { (HE/OO/KW) fair or light in } \\
\text { colour (e.g. hair); (HA) grey, } \\
\text { whitish }\end{array}$ \\
\hline 12440 & 1345 & ky $\chi-$ & ky $\chi-$ & $\mathrm{k}^{3} \mathrm{y} \chi-$ & ky $x-$ & $\begin{array}{l}\text { to scrape, to scrub, to rub } \\
\text { together; (HE/OO also:) to use a } \\
\text { saw }\end{array}$ \\
\hline 12450 & 1347 & kvXv- & kvXv- & kvXv- & kxv- & $\begin{array}{l}\text { to rise or blow out forcefully (hot } \\
\text { air, steam, breath, dust, smoke); } \\
\text { (KW also:) to give off a smell }\end{array}$ \\
\hline 12460 & 1348 & kvwp- & kvwp- & kwp- & kwqv- & $\begin{array}{l}\text { to snap, to break (said of a stick } \\
\text { or long stick-like thing) }\end{array}$ \\
\hline 12470 & 1349 & xvwt- & kvwt- & kwt- & kwt- & to suspect, guess \\
\hline 12480 & 1350 & kws- & kws- & kws- & kws- & $\begin{array}{l}\text { to shave, to scrape off with a } \\
\text { knife (fur, fish scales) }\end{array}$ \\
\hline 12490 & 1351 & & & & kws-* & $(?)^{589}$ \\
\hline 12500 & 1352 & kw1- & & & kw1- & dried up, dried out $t^{590}$ \\
\hline 12510 & 1353 & kvwkv- & kvwkv- & & kwkv- & pearly, mother-of-pearl \\
\hline 12520 & 1354 & kvwXv- & kvwXv- & $\begin{array}{l}\mathrm{kwXv-} \\
\mathrm{kW}^{3} \mathrm{kv}-\end{array}$ & kwXv- & $\begin{array}{l}\text { (HE/OO/HA) warm, hot; }(\mathrm{KW}) \\
\text { at or towards the right } \\
\text { temperature, weather is } \\
\text { improving } 591\end{array}$ \\
\hline 12530 & 1355 & & & & kw $\chi v^{-}$ & $\begin{array}{l}\text { to scrape (for inner hemlock } \\
\text { bark, for cockles) }\end{array}$ \\
\hline
\end{tabular}

\footnotetext{
${ }^{589}$ In KW (Koskimo dialect) /kus.h ${ }^{3} \mathrm{id} / *$ "breath stops."

${ }^{590} \mathrm{HE}$ only in $\{\mathrm{kw}[\mathrm{k}] \mathrm{k}-=\mathrm{hly}\}>/ \mathrm{k}^{\prime} \mathrm{ukv} \Omega^{\prime} \mathrm{ali} /$ "dried clams."

$591\left\{\mathrm{kw}^{3} \mathrm{kv}-\right\}$ only in HA /kukvл'a/ "warm clothes"; the glottalization of $\left|\mathrm{w}^{3}\right|$ accounts for the accent on the suffix, $\{-1 \mathrm{~h}\}$.
} 
North Wakashan Comparative Root List (Updated)

\begin{tabular}{|c|c|c|c|c|c|c|}
\hline Root \# & Old \# & $\mathrm{HE}$ & $\mathrm{OO}$ & HA & KW & Gloss \\
\hline 12540 & 1356 & & & & $k w \chi v-$ & (?) to give off stench 592 \\
\hline 12550 & 1357 & kv $\chi v-$ & kv $\chi v-$ & & $\mathrm{k} \chi \mathrm{V}-$ & to fart, give off stench ${ }^{593}$ \\
\hline 12560 & $\begin{array}{l}1358 \\
1375 \\
1376\end{array}$ & $\begin{array}{l}\text { kq- } \\
\text { khq- }\end{array}$ & $\begin{array}{l}\text { kq- } \\
\text { khq- }\end{array}$ & $\begin{array}{l}\text { khq- } \\
k^{3} h^{\prime}-\end{array}$ & $\begin{array}{l}\text { kq- } \\
\text { khq- }\end{array}$ & $\begin{array}{l}\text { one's head or the end of a moving } \\
\text { object (e.g. log, canoe) strikes or } \\
\text { touches sth., to collide with, to } \\
\text { bump into sth; to head towards } \\
\text { sth., to aim; (HA also:) to nod, to } \\
\text { bend the head forward }{ }^{594}\end{array}$ \\
\hline 12570 & $\begin{array}{l}1359 \\
1377 \\
1952 \\
1961 \\
2190\end{array}$ & $\begin{array}{l}\text { Iv } \chi v^{-} \\
\text {rvh } \chi v^{-} \\
\text {rh } \chi-\end{array}$ & $\begin{array}{l}\text { IV } \chi v- \\
\text { rvh } \chi v- \\
\text { rvhqv- } \\
\text { rh } \chi-\end{array}$ & $\begin{array}{l}\mathrm{k}^{3} \mathrm{~h} \chi- \\
\text { rvhkv- } \\
\text { rvh } \chi \mathrm{v-} \\
\text { rhq- } \\
\text { rh } \chi-\end{array}$ & $\begin{array}{l}\mathrm{k} \chi- \\
\mathrm{kh} \chi- \\
\text { rvh } \chi \mathrm{v}^{-} \\
\mathrm{rh} \chi-\end{array}$ & $\begin{array}{l}\text { (onomatopoeic:) to croak (crow } \\
\text { or raven), cry of crow or raven }\end{array}$ \\
\hline 12580 & 1360 & kh- & kh- & kh- & kh- & $\begin{array}{l}\text { dish-shaped object is being held } \\
\text { up (e.g. waiter-style on the palms } \\
\text { of one's hands) or put } \\
\text { somewhere; to use plate, pot or } \\
\text { pan }\end{array}$ \\
\hline 12590 & 1361 & kh- & & kh- & kh- & $(?)^{596}$ \\
\hline
\end{tabular}

${ }^{592} \mathrm{In} \mathrm{KW} / \mathrm{ku} \mathrm{vac}^{3} \mathrm{i} /$ "musk bag of an animal."

${ }^{593}$ All HE/OO examples refer to farting with the specification "noiselessly."

${ }^{594} \mathrm{HE} / \mathrm{OO}\{\mathrm{kq}-\}$ only in $\{\mathrm{k}[\mathrm{hh}] \mathrm{q}-\mathrm{w}\}>\mathrm{HE} / \mathrm{k}$ 'aqu/ OO /kaaqu/ "to collide." Possibly HA $\{\mathrm{khq}-\}$ once referred to bending the head and bumping the head while $\left\{\mathrm{k}^{3} \mathrm{hq}-\right\}$ referred to steering and collision but both forms can now have either meaning.

${ }^{595} \mathrm{In} \mathrm{HA}$ reference is to a frog's croaking but in $\mathrm{HE} / \mathrm{OO} / \mathrm{KW}$ to that of a raven. $\{\mathrm{r} \mathrm{V} \chi \mathrm{V}-\}$ only in $\mathrm{HE} / \mathrm{rvu}^{3} \mathrm{i} / \mathrm{OO} / \mathrm{rvu}^{3} \mathrm{i} /$ "raven" and derivates.

${ }^{596}$ Possibly in HE $/ \mathrm{kay}^{3 / \mathrm{i}}$ / (expression of scorn) HA $/ \mathrm{ka} /$ (proclitic said to be used in Kitamaat for "I don't know") HA /ka3a/ (form of phatic communion when sb. is talking: "yes, yes"; "sure, 
North Wakashan Comparative Root List (Updated)

\begin{tabular}{|c|c|c|c|c|c|c|}
\hline Root \# & Old \# & $\mathrm{HE}$ & $\mathrm{OO}$ & HA & KW & Gloss \\
\hline 12591 & 1373 & $(\chi £-)$ & $(\chi £-)$ & & kh- & $(?)^{597}$ \\
\hline 12600 & $\begin{array}{l}1362 \\
1363\end{array}$ & khp- & khp- & khp- & & $\begin{array}{l}\text { (HE/OO) to apply, change or } \\
\text { remove a covering layer; to } \\
\text { separate layers; (HA) frozen } \\
\text { over, frost-covered }{ }^{598}\end{array}$ \\
\hline 12610 & 1364 & kht- & kht- & kht- & kht- & $\begin{array}{l}\text { long object (e.g. log) is or moves } \\
\text { somewhere }\end{array}$ \\
\hline 12620 & $\begin{array}{l}1365 \\
1371\end{array}$ & kht- & kht- & kht- & $\begin{array}{l}\text { kht-* } \\
\text { khs- }\end{array}$ & $\begin{array}{l}\text { head or heavy hammer-like } \\
\text { object (like deadfall) coming } \\
\text { down, to nod the head, to bob up } \\
\text { and down (like head, canoe) }\end{array}$ \\
\hline 12630 & 1366 & kht- & kht- & & & $(?)^{599}$ \\
\hline 12640 & 1372 & & & & $\mathrm{khc}^{3} \mathrm{~s}-$ & cone of coniferous tree 600 \\
\hline 12650 & 1368 & khs- & khs- & khs- & khs- & crooked, bent \\
\hline 12660 & 1369 & & & & khs- & to shred cedar bark \\
\hline
\end{tabular}

sure"). Also perhaps in $\mathrm{KW} / \mathrm{kat}^{3} \mathrm{u} /$ and $/ \mathrm{kat}^{3} \mathrm{\jmath} /$ "not enough, insufficient, mediocre" which could however also be singular developments of $\mathrm{KW} / \mathrm{k}^{3} \mathrm{uta} /[2]$ "to judge insufficient, etc." (from $\left\{\mathrm{k}^{3} \mathrm{wt}-\right\}$, root no. 13440).

${ }^{597}$ In KW $\{[\mathrm{kh}] \mathrm{kh}-+\chi \mathrm{w}-\mathrm{hlh}\}>/ \mathrm{kaka}^{3} \chi$ ola/ "to wear a necktie" and $\left\{\mathrm{kh}-\mathrm{hlh}-+\chi \mathrm{hhw} \alpha \mathrm{y}^{3}\right\}>$ /kala $\chi a w \varepsilon^{3} /$ "necktie." Root may be a borrowing from Nuuchahnulth ("Nootka") which has a root $\{\mathrm{ka}-\}$ "stick-like object protruding" (Sapir \& Swadesh 1939:283). The HE/OO equivalents of the KW terms derive from $\{\chi £-\}$ "long object points or pokes out, etc." 598 Probably also in OO / $\mathrm{kapi}^{3} \mathrm{ala} /$ "living together to give a child a recognized father." 599 Only in HE/OO \{kht-w $\}$ "to meet sb." Form and gloss of this term are reminiscent of a HA/KW derivate of root no. 12610 , sc. $\{\mathrm{kh}[\mathrm{k}] \mathrm{t}-\mathrm{w}\}>\mathrm{HA} / \mathrm{kakt} \mathrm{u} / \mathrm{KW} / \mathrm{kaktu} /$ "to meet, to fall to the same spot (logs), to lie together (logs)."

${ }^{600}$ Free stem. Could be loanword. 
North Wakashan Comparative Root List (Updated)

\begin{tabular}{|c|c|c|c|c|c|c|}
\hline Root \# & Old \# & $\mathrm{HE}$ & $\mathrm{OO}$ & HA & KW & Gloss \\
\hline 12690 & 1374 & khx- & khx- & khx- & & $\begin{array}{l}\text { to lift, hold up or carry on the } \\
\text { hands and/or forearms (e.g. } \\
\text { canoe); (for HE/OO figurative } \\
\text { use is also attested:) to uphold } \\
\text { law and traditions }\end{array}$ \\
\hline 12700 & 1370 & & & & khy ${ }^{3}-$ & to chase off, to drive away ${ }^{601}$ \\
\hline 12710 & & & & & $\begin{array}{l}\text { khwt-/ } \\
\text { khwht- }\end{array}$ & $\begin{array}{l}\text { (?) non-serious, for fun (e.g. cat's } \\
\text { cradle) }{ }^{602}\end{array}$ \\
\hline 12740 & $\begin{array}{l}1378 \\
1379\end{array}$ & $\mathrm{k}^{3} \mathrm{p}-$ & $\mathrm{k}^{3} \mathrm{p}-$ & $\mathrm{k}^{3} \mathrm{p}-$ & $\mathrm{k}^{3} \mathrm{p}-$ & $\begin{array}{l}\text { to hold by squeezing (with tongs, } \\
\text { a clip, under the arm); (HA/KW } \\
\text { also:) to clip, to cut with } \\
\text { scissors }^{603}\end{array}$ \\
\hline 12750 & $\begin{array}{l}1380 \\
1433 \\
1472\end{array}$ & & & $\begin{array}{l}\mathrm{k}^{3} \mathrm{~m}- \\
\mathrm{k}^{3} \mathrm{ymh}-\end{array}$ & $\begin{array}{l}\mathrm{k}^{3} \mathrm{~m}-* \\
\mathrm{k}^{3} \mathrm{hmh}-\end{array}$ & $(?)^{604}$ \\
\hline 12760 & $\begin{array}{l}1381 \\
1384\end{array}$ & $\mathrm{k}^{3} \mathrm{mt}-$ & $\mathrm{k}^{3} \mathrm{mt}-$ & $\mathrm{k}^{3} \mathrm{mt}-$ & $k^{3} m y^{3}-$ & to shut out, seal up, lock in \\
\hline 12770 & 1382 & & & & $\mathrm{k}^{3} \mathrm{mt}-$ & $\begin{array}{l}\text { to make a notch; to cut off or cut } \\
\text { up with knife or adze }\end{array}$ \\
\hline
\end{tabular}

${ }^{601}$ Could be a cross-family stem; there is partial semantic and formal similarity to Proto-Salish *-kal- "go after, follow, chase" considering that Proto-Salish *1 > y in e.g. Comox and Sliammon spoken in areas adjacent to KW territory (Kuipers 2002:3, 36).

602 Stem could contain a root $\{\mathrm{khxv}-\}$ or $\{\mathrm{kh} \chi \mathrm{v}\}$ reminiscent of Proto-Salish *-caxv- "glad, happy, enjoy" (Kuipers 2002:26). The suffix would be $\{-=t\}$ or $\{-=h t\}$ (suffix no. 1060). ${ }^{603}$ Probably also in $\mathrm{HE} / \mathrm{k}^{3} \mathrm{Mst} \mathrm{u}^{\mathrm{u}} \mathrm{OO} / \mathrm{k}^{3} \mathrm{M}^{3} \mathrm{stu} /$ "to show discontent by closing both eyes and turning the face away." HE/OO uses $\{\mathrm{ql} \chi-\}$ (root no. 20590) for cutting with scissors. ${ }^{604}$ Possibly in the names of a young herring, salmon fry and a young land otter. 
North Wakashan Comparative Root List (Updated)

\begin{tabular}{|c|c|c|c|c|c|c|}
\hline Root \# & Old \# & $\mathrm{HE}$ & $\mathrm{OO}$ & HA & KW & Gloss \\
\hline 12780 & 1383 & & & & $\mathrm{k}^{3} \mathrm{~ms}-$ & $(?)^{605}$ \\
\hline 12790 & $\begin{array}{l}1385 \\
1472\end{array}$ & $\mathrm{k}^{3} \mathrm{hmhs}-$ & $\mathrm{k}^{3} \mathrm{hmhs}-$ & & $\begin{array}{l}\mathrm{k}^{3} \mathrm{~ms}-* \\
\mathrm{k}^{3} \mathrm{hmhs}^{*}\end{array}$ & cascade, rapids ${ }^{606}$ \\
\hline 12800 & 1386 & & & & $\begin{array}{l}\mathrm{k}^{3} \mathrm{mf-} \\
\mathrm{c}^{3} \mathrm{mf-}\end{array}$ & to adze $^{607}$ \\
\hline 12810 & 1387 & & & & $\mathrm{k}^{3} \mathrm{mxv}-$ & hemlock needles ${ }^{608}$ \\
\hline 12820 & 1388 & $\mathrm{k}^{3} \mathrm{mq}-$ & $\mathrm{k}^{3} \mathrm{mq}-$ & & $\begin{array}{l}\mathrm{k}^{3} \mathrm{mq}-^{*} \\
\mathrm{k}^{3} \mathrm{mhq} \mathbf{-}^{*} \\
\mathrm{k}^{3} \mathrm{mh} \chi_{-}\end{array}$ & calm (weather, sea) \\
\hline 12830 & & $k^{3} m \chi-$ & $k^{3} m \chi-$ & $k^{3} m \chi-$ & & canyon $^{609}$ \\
\hline 12840 & $\begin{array}{l}1389 \\
1390 \\
1392\end{array}$ & $\mathrm{k}^{3} \mathrm{t}-$ & $\mathrm{k}^{3} \mathrm{t}-$ & $\begin{array}{l}k^{3} t- \\
k^{3} h t-\end{array}$ & $\begin{array}{l}\mathrm{k}^{3} \mathrm{t}- \\
\mathrm{k}^{3} \mathrm{ht}-\end{array}$ & $\begin{array}{l}\text { unstable, dizzy; tippy, cranky } \\
\text { (like boat), in and out (as when } \\
\text { weaving), up and down, back and } \\
\text { forth (as when sweeping, } \\
\text { brushing or shaking off) }\end{array}$ \\
\hline 12860 & 1391 & & & & $k^{3} t-$ & to extend, protrude, stick out \\
\hline
\end{tabular}

${ }^{605} \mathrm{In} \mathrm{KW} / \mathrm{k}^{3} \mathrm{MSa} /$ "to rough out canoe" and $/ \mathrm{k}^{3} \mathrm{MSM} \varepsilon^{3} / *$ "tough butt of $\log . "$

${ }^{606} \mathrm{HE} / \mathrm{OO}$ only in $\mathrm{HE} / \mathrm{k}^{3} \mathrm{am}$ 'azh/ OO / $\mathrm{k}^{3} \mathrm{amazH} /$ "small waterfall."

${ }^{607}\left\{\mathrm{c}^{3} \mathrm{~m} £-\right\}^{*}$ occurs in one never reelicited word Boas (1948:335) mentions as an example of the $/ \mathrm{DH}^{3} \operatorname{axda}^{3} \chi \mathrm{v} /$ (Knight Inlet people) using $/ \mathrm{c}^{3} /$ where $\mathrm{KW}$ has $/ \mathrm{k}^{3} /$.

${ }^{608}$ Could be a backformation from $\mathrm{KW} / \mathrm{k}^{3}$ amuma/ "needles of coniferous trees" which is a cross-family stem, cf. PS *-k3amu/a(ma7)- in Kuipers (2002:41). See also the comment on $\left\{\mathrm{k}^{3} \mathrm{~h}-\right\}$ (root no. 13590).

${ }^{609}$ Only HE/OO example is a place name.

${ }^{610}$ Insufficiently clear glosses make it hard to tell if it is this root or no. 12860 that is present in

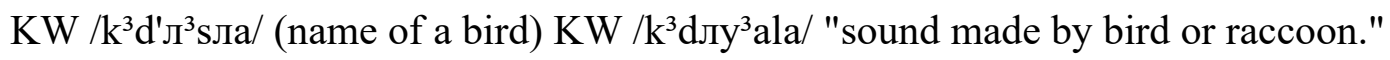


North Wakashan Comparative Root List (Updated)

\begin{tabular}{|c|c|c|c|c|c|c|}
\hline Root \# & Old \# & $\mathrm{HE}$ & $\mathrm{OO}$ & HA & KW & Gloss \\
\hline 12880 & 1393 & & & $k^{3} n-$ & $k^{3} n-$ & $\begin{array}{l}\text { hanging loose (like hair, snot); } \\
\text { (KW also:) unstable, unsteady; } \\
\text { detached, separated, disconnected } \\
\text { (from origin) }{ }^{611}\end{array}$ \\
\hline 12890 & $\begin{array}{l}1394 \\
1395\end{array}$ & $k^{3} n-$ & $k^{3} n-$ & $\mathrm{k}^{3} \mathrm{nh}-$ & $\begin{array}{l}\mathrm{k}^{3} \mathrm{n}- \\
\mathrm{k}^{3} \mathrm{nh}-\end{array}$ & feeling cold \\
\hline 12900 & $\begin{array}{l}1396 \\
1397 \\
1398 \\
1402\end{array}$ & & & & $\begin{array}{l}\mathrm{k}^{3} \mathrm{nt}-* \\
\mathrm{k}^{3} \mathrm{nt}-* \\
\mathrm{k}^{3} \mathrm{ny1}-* \\
\mathrm{k}^{3} \mathrm{nxv}-* \\
\mathrm{k}^{3} \mathrm{nwt}\end{array}$ & (?) chiton ${ }^{612}$ \\
\hline 12930 & 1399 & $\mathrm{k}^{3} \mathrm{nk}-$ & $\mathrm{k}^{3} \mathrm{nk}-$ & $\mathrm{k}^{3} \mathrm{nk}-$ & $(\chi q-)$ & to comb, to rake \\
\hline 12940 & $\begin{array}{l}1400 \\
1401\end{array}$ & $\mathrm{k}^{3} \mathrm{nxv}-$ & $\mathrm{k}^{3} \mathrm{nxv}-$ & $\begin{array}{l}k^{3} n k v- \\
k^{3} n x v-\end{array}$ & $\mathrm{k}^{3} \mathrm{nxv}-$ & $\begin{array}{l}\text { tangled, matted together, a } \\
\text { twisted mass, curled up; } \\
\text { grappling and grabbing } \\
\text { movement (as of crabs) }\end{array}$ \\
\hline 12960 & 1403 & $k^{3} n q-$ & $k^{3} n q-$ & & & $\begin{array}{l}\text { grappling and grabbing } \\
\text { movement (of crabs or as in } \\
\text { wrestling) }\end{array}$ \\
\hline 12970 & $\begin{array}{l}1404 \\
1405\end{array}$ & $\begin{array}{l}k^{3} S^{-} \\
k^{3} s^{\bullet}-\end{array}$ & $\begin{array}{l}\mathrm{k}^{3} \mathrm{~S}- \\
\mathrm{k}^{3} \mathrm{~s} \bullet- \\
\mathrm{sk}^{3} \mathrm{~s}^{\bullet-}\end{array}$ & $\mathrm{k}^{3} \mathrm{~s}-$ & $\begin{array}{l}\mathrm{k}^{3} \mathrm{~s}- \\
\mathrm{sk}^{3} \mathrm{~s}^{\bullet}-\end{array}$ & $\begin{array}{l}\text { (negative and privative } \\
\text { meanings:) } \\
\text { 1. not the case, not as before (or } \\
\text { could or should be), not any }\end{array}$ \\
\hline
\end{tabular}

${ }^{611}$ Also in HA (Kitamaat dialect) $/ \mathrm{k}^{3} \mathrm{ak}^{3} \mathrm{H}^{31} \mathrm{a} /$ "hummingbird" (said to mean "trying to get hair"). ${ }^{612}$ In altogether ten words reported by Boas (1948) but eight of which could not be reelicited. According to Boas they are about Cryptochiton stelleri which, according to https://en.wikipedia.org/wiki/Gumboot_chiton, is commonly known as gumboot chiton or Giant Western Firey chiton. Which property of this large kind of chiton the roots may refer to is unclear. 
North Wakashan Comparative Root List (Updated)

\begin{tabular}{|c|c|c|c|c|c|c|}
\hline Root \# & Old \# & $\mathrm{HE}$ & $\mathrm{OO}$ & HA & KW & Gloss \\
\hline & & & & & & $\begin{array}{l}\text { more, deprived of; to undo, back } \\
\text { out of; (HE/OO also:) one-sided; } \\
\text { 2. lacking control or vigour, lax, } \\
\text { slack, loose, lazy }{ }^{613}\end{array}$ \\
\hline 12990 & 1406 & & $\mathrm{k}^{3} \mathfrak{f}-$ & & & $(?)^{614}$ \\
\hline 12991 & 1407 & & & $\mathrm{k}^{3} \mathcal{f}-$ & & $(?)^{615}$ \\
\hline 13000 & 1408 & $\mathrm{k}^{3} \mathrm{k}-$ & $\mathrm{k}^{3} \mathrm{k}-$ & $\mathrm{k}^{3} \mathrm{k}-$ & $\mathrm{k}^{3} \mathrm{l}^{-}$ & $\begin{array}{l}\text { particles (e.g. dust, berries) are } \\
\text { moving; to brush, sweep or shake } \\
\text { off particles; (KW also:) to shake } \\
\text { huckleberries off the bush }\end{array}$ \\
\hline 13010 & 1410 & $\mathrm{k}^{3} \mathrm{l}-$ & $\mathrm{k}^{3} \mathrm{l}-$ & $\mathrm{k}^{3} \mathrm{l}^{-}$ & $\begin{array}{l}\mathrm{k}^{3} 1- \\
\mathrm{k}^{3} 1-\end{array}$ & $\begin{array}{l}\text { movement of bodily organ } \\
\text { (tongue, clitoris, heart), to lick } \\
\text { with tongue }^{616}\end{array}$ \\
\hline 13020 & 1409 & $\mathrm{k}^{3} \mathrm{l}-$ & $\mathrm{k}^{3} 1-$ & $k^{3} 1-$ & $\mathrm{k}^{3} 1-*$ & $(?)^{617}$ \\
\hline 13021 & & & & $\mathrm{k}^{3} \mathrm{lhm}-$ & & $(?)^{618}$ \\
\hline 13030 & 1411 & $k^{3} l p-$ & $\mathrm{k}^{3} \mathrm{lp}-$ & $k^{3} l p-$ & $\mathrm{k}^{3} \mathrm{lp}-$ & to twist, wring \\
\hline
\end{tabular}

${ }^{613}\left\{\mathrm{sk}^{3} \mathrm{~s}^{\bullet}\right\}$ is attested only in $\mathrm{OO} / \mathrm{sk}^{3}$ sirvił/ "(single) side of the longhouse (seen from the entrance)" and perhaps the non-reelicitable $\mathrm{KW} / \mathrm{sk}^{3} \mathrm{sala} / *$ "to be seen but not to see" [sic]. It can be undiagnosable if $\mathrm{HE} / \mathrm{OO}$ is using $\left\{\mathrm{k}^{3} \mathrm{~s}-\right\}$ or $\left\{\mathrm{k}^{3} \mathrm{~s} \bullet\right\}$ in which case $\left\{\mathrm{k}^{3} \mathrm{~s}-\right\}$ is assumed.

${ }^{614} \mathrm{In} \mathrm{OO} / \mathrm{k}^{3} \mathfrak{f}^{3} \mathrm{a}^{3} \mathrm{f} /$ "long flat chief's dish with flat carved ends."

${ }^{615}$ In HA words referring to a bullhead.

${ }^{616}$ The only example of $\{\mathrm{kl}-\}$ is $\mathrm{KW} / \mathrm{k}^{3}$ ллатаs/ "to cause the heart to beat fast (as when running)."

${ }^{617}$ Perhaps in HA $/ \mathrm{k}^{3} л \mathrm{лм} /$ [2] "red hot stone or iron for heating grease" OO $/ \mathrm{k} \pi^{3} \mathrm{aq} / \mathrm{HE}$ $/ \mathrm{k}^{3} \mathrm{ik}^{3} \mathrm{~J}^{3} \mathrm{aq} / \mathrm{HA} / \mathrm{k}^{3} \mathrm{\Omega k}^{3} \mathrm{~J}^{3} \mathrm{aq} /$ "natural crystal" KW $/ \mathrm{k}^{3} \Omega \mathrm{p}^{3} \mathrm{ala} / *$ "copper smell." ${ }^{618}$ In HA $/ \mathrm{k}^{3} \mathrm{I}^{\prime}$ амр ${ }^{3} \mathrm{a} /$ "sour, fermented" and derivates. 
North Wakashan Comparative Root List (Updated)

\begin{tabular}{|c|c|c|c|c|c|c|}
\hline Root \# & Old \# & $\mathrm{HE}$ & $\mathrm{OO}$ & HA & KW & Gloss \\
\hline 13040 & 1412 & $\mathrm{k}^{3} \mathrm{lt}-$ & $\mathrm{k}^{3} \mathrm{l}^{\mathrm{s}}-$ & $\mathrm{k}^{3} \mathrm{lt}-$ & $\mathrm{k}^{3} \mathrm{lt}-$ & reluctant to act, put off by sth. \\
\hline 13050 & 1413 & & & & $\mathrm{k}^{3} 1 \mathrm{~s}-$ & easily kinked or twisted \\
\hline 13060 & 1414 & & & & $k^{3} 1 s-$ & $(?)^{619}$ \\
\hline 13070 & 1415 & $\mathrm{k}^{3} \mathrm{lk}-$ & $\mathrm{k}^{3} l \mathrm{k}-$ & $\mathrm{k}^{3} l \mathrm{k}-$ & $\mathrm{k}^{3} \mathrm{lk}-$ & $\begin{array}{l}\text { to bind, bandage, tie with a string } \\
\text { or ribbon, wrap, wind around, } \\
\text { strangle (in trap), interweave }\end{array}$ \\
\hline 13080 & $\begin{array}{l}1416 \\
1423\end{array}$ & $\begin{array}{l}k^{3} 1 x- \\
k^{3} 1^{3} x-\end{array}$ & $\begin{array}{l}k^{3} 1 x^{-} \\
k^{3} l^{3} x^{-}\end{array}$ & $\begin{array}{l}\mathrm{k}^{3} \mathrm{~lx}- \\
\mathrm{k}^{3} \mathrm{l}^{3} \mathrm{x}-\end{array}$ & $\mathrm{k}^{3} 1^{3} \mathrm{x}-$ & $\begin{array}{l}\text { raw, untreated (e.g. food, } \\
\text { metal) }{ }^{620}\end{array}$ \\
\hline 13090 & 1417 & $\mathrm{k}^{3} \mathrm{lkv}-$ & $\mathrm{k}^{3} \mathrm{kvV}-$ & $\mathrm{k}^{3} \mathrm{lkv}-$ & $\mathrm{k}^{3} \mathrm{lkv}-$ & to prick ${ }^{621}$ \\
\hline 13100 & 1418 & $k^{3} l q v-$ & k3lqv- & $\mathrm{k}^{3} \mathrm{lqv}-$ & $\mathrm{k}^{3} \mathrm{lqv}-$ & $\begin{array}{l}\text { to urinate (said of a male), to } \\
\text { spout, to gush out }\end{array}$ \\
\hline 13110 & 1419 & $\mathrm{k}^{3} \mathrm{lq}-$ & $\mathrm{k}^{3} \mathrm{lq}-$ & $\mathrm{k}^{3} \mathrm{lq}-$ & $\mathrm{k}^{3} \mathrm{lq}-$ & to lick \\
\hline 13120 & 1420 & $\mathrm{k}^{3} \mathrm{l} \chi-$ & $\mathrm{k}^{3} \mid \chi-$ & & & to wrap \\
\hline 13130 & 1421 & $\mathrm{k}^{3} 1 \chi-$ & $\mathrm{k}^{3} 1 \chi-$ & $k^{3} 1 \chi-$ & $\mathrm{k}^{3} 1 \chi-$ & $\begin{array}{l}\text { to extinguish, go out, put out } \\
\text { (light, fire) }\end{array}$ \\
\hline 13140 & 1422 & & & & $\mathrm{k}^{3} \mathrm{l} \chi^{*} *$ & $(?)^{622}$ \\
\hline 13150 & 1424 & $\left(\mathrm{y}^{3} \mathrm{yt}-\right)$ & $\left(y^{3} y t-\right)$ & $\left(\mathrm{h}^{3} \mathrm{yt}-\right)$ & $\mathrm{k}^{3} \mathrm{k}-$ & $\begin{array}{l}\text { going backwards, to row a boat, } \\
\text { to back up, to defecate }\end{array}$ \\
\hline 13160 & 1425 & $\mathrm{k}^{3} \mathrm{k}-$ & $\mathrm{k}^{3} \mathrm{k}-$ & $\mathrm{k}^{3} \mathrm{k}-$ & $\mathrm{k}^{3} \mathrm{k}-$ & to stretch skins \\
\hline
\end{tabular}

\footnotetext{
${ }^{619} \mathrm{In} \mathrm{KW} / \mathrm{k}^{3}$ Лsu/ "to beg for sth." KW / $\mathbf{k}^{3}$ лsэnм/ "obtained by begging."

${ }^{620} \mathrm{HE} / \mathrm{OO} / \mathrm{HA}\left\{\mathrm{k}^{3} 1 \mathrm{x}-\right\}$ occurs possibly in $\mathrm{HE} / \mathrm{k}^{3 \prime} л \mathrm{xk}^{3 \prime} \mathrm{axa} / \mathrm{OO} / \mathrm{k}^{3} л \mathrm{xk}^{3} \mathrm{axa} / \mathrm{HA} / \mathrm{k}^{3} л x \mathrm{k}^{3 \prime} \mathrm{axa} /$ and $/ \mathrm{k}^{3} \mathrm{xxk}^{3} \mathrm{ax} \mathrm{a} /$ "to eat sth. raw."

${ }^{621}$ In three words referring to a digging stick and the thorn-like core of a corn or callus.

${ }^{622}$ In words referring to a thistle-like plant with milky juice.
} 
North Wakashan Comparative Root List (Updated)

\begin{tabular}{|c|c|c|c|c|c|c|}
\hline Root \# & Old \# & $\mathrm{HE}$ & $\mathrm{OO}$ & HA & KW & Gloss \\
\hline 13170 & 1426 & $\mathrm{k}^{3} \mathrm{k}-$ & $\mathrm{k}^{3} \mathrm{k}-$ & $\mathrm{k}^{3} \mathrm{k}-$ & $\begin{array}{l}\mathrm{k}^{3} \mathrm{vk}- \\
\mathrm{k}^{3} \mathrm{vhk}-\end{array}$ & $\begin{array}{l}\text { to squeak, to creak (like sth. } \\
\text { bursting, new shoes) }\end{array}$ \\
\hline 13180 & 1427 & & $\mathrm{k}^{3} \mathrm{x}-$ & & $\mathrm{k}^{3} \mathrm{X}-$ & to bifurcate, to split in two \\
\hline 13190 & 1428 & & & & $k^{3} x^{-}$ & mouldy \\
\hline 13200 & $\begin{array}{l}1429 \\
1430 \\
1442\end{array}$ & $\begin{array}{l}k^{3} y \\
k^{3} y^{3}-\end{array}$ & $\begin{array}{l}k^{3} y \\
k^{3} y^{3}-\end{array}$ & $\begin{array}{l}k^{3} y- \\
k^{3} h y h- \\
k^{3} h y^{3}-\end{array}$ & $\begin{array}{l}\mathrm{k}^{3} \mathrm{y}- \\
\mathrm{k}^{3} \mathrm{hyh}-\end{array}$ & $\begin{array}{l}\text { 1. (privative meanings:) } \\
\text { removed, gone, missing, } \\
\text { deceased, in memory only; not as } \\
\text { it could, used to or should be; } \\
\text { lacking sth., deprived of, poor; } \\
\text { 2. (when combined with }\left\{-\mathrm{s.} \mathrm{h}^{3} \mathrm{w}\right\} \\
\text { (suffix no. } 2610 \text { ) as in the free } \\
\text { stem }\left\{\mathrm{k}^{3} \mathrm{y}-\mathrm{s} . \mathrm{h}^{3} \mathrm{w}\right\} \text { and derivates:) } \\
\text { memorabilia (e.g. rights, } \\
\text { privileges, songs, masks); } \\
\text { 3. (HE/OO also:) to carve (wood, } \\
\text { meat), to dress or fillet fish; (KW } \\
\text { also:) to carve (wood, pole, } \\
\text { blubber); (HA also:) to cut } \\
\text { anything with a knife; } \\
\text { 4. (HA }\left\{\mathrm{k}^{3} h y^{3}-\right\} \text { :) out of sight, } \\
\text { disappeared. }{ }^{623}\end{array}$ \\
\hline 13210 & 1431 & $\mathrm{k}^{3} \mathrm{y}-$ & $\mathrm{k}^{3} \mathrm{y}-$ & $\mathrm{k}^{3} \mathrm{y}-$ & $\mathrm{k}^{3} \mathrm{hyh}-$ & $(?)^{624}$ \\
\hline 13230 & $\begin{array}{l}1434 \\
1437\end{array}$ & & & & $\mathrm{k}^{3} \mathrm{yt}-$ & upright, prominent, notable ${ }^{625}$ \\
\hline
\end{tabular}

${ }^{623} \mathrm{HA}\left\{\mathrm{k}^{3} \mathrm{~h} \mathrm{y}^{3}-\right\}$ corresponds to HE/OO/KW $\left\{\mathrm{t}^{3} h y^{3}-\right\}$, augmented form of $\left\{\mathrm{t}^{3} \mathrm{y}-\right\}$ (root no. 3780).

To facilitate cross-dialect comparison the derivates of $\left\{\mathrm{k}^{3} \mathrm{~h} \mathrm{y}^{3}-\right\}$ are listed under root no. 3780 .

${ }^{624}$ Possibly in $\mathrm{HE} / \mathrm{k}^{3} \mathrm{id}$ 'a/ OO $/ \mathrm{k}^{3} \mathrm{ida} / \mathrm{HA} / \mathrm{k}^{3} \mathrm{id}$ 'a/ "little finger" $\mathrm{KW} / \mathrm{k}^{3} \varepsilon \mathrm{da} /$ "ring finger."

${ }^{625}$ Several derivates refer to a princess (chief's eldest daughter). 
North Wakashan Comparative Root List (Updated)

\begin{tabular}{|c|c|c|c|c|c|c|}
\hline Root \# & Old \# & $\mathrm{HE}$ & $\mathrm{OO}$ & $\mathrm{HA}$ & KW & Gloss \\
\hline 13240 & 1435 & $\mathrm{k}^{3} \mathrm{yt}-$ & $k^{3} y t-$ & $\mathrm{k}^{3} \mathrm{yt}-$ & & to rake in (herrings) \\
\hline 13250 & 1436 & $k^{3} y t-$ & $k^{3} y t-$ & & $k^{3} y t-$ & grass, grassy, to get grass \\
\hline 13260 & $\begin{array}{l}1438 \\
1439 \\
1440 \\
2594\end{array}$ & $\begin{array}{l}\mathrm{k}^{3} \mathrm{ys}- \\
\mathrm{k}^{3} \mathrm{Wws}-\end{array}$ & $\begin{array}{l}k^{3} y s- \\
k^{3} y w s-\end{array}$ & $\begin{array}{l}k^{3} y s- \\
k^{3} \text { wws- }\end{array}$ & $\begin{array}{l}k^{3} y s- \\
h^{3} y s- \\
h^{3} y^{3} s- \\
k^{3} y^{3} s- \\
k^{3} y^{3} s \bullet- \\
c^{3} y^{3} s-* \\
k^{3} y w s- \\
k^{3} y h w s- \\
k^{3} h y w s-\end{array}$ & $\begin{array}{l}\text { 1. absent, gone, etc. (see the } \\
\text { privative meanings of no. } \\
\text { 13200); } \\
\text { 2. immaterial, spiritual; } \\
\text { 3. not the case, to the contrary, } \\
\text { non-existent; } \\
\text { 4. (HE/OO/HA }\left\{\mathrm{k}^{3} \mathrm{ys}-\right\} \text { also:) } \\
\text { water is drained off, stream goes } \\
\text { dry } 626\end{array}$ \\
\hline 13290 & 1441 & & & $\mathrm{k}^{3} \mathrm{ys}-$ & $\mathrm{k}^{3} \mathrm{ys}^{-*}$ & (?) layer of clothing \\
\hline 13300 & 1443 & & & & $\mathrm{k}^{3} \mathrm{y} £-$ & $\begin{array}{l}\text { to clip off, to cut down (e.g. } \\
\text { grass), to cut open (a can) }\end{array}$ \\
\hline 13310 & 1444 & & & & $\mathrm{k}^{3} \mathrm{y} \mathrm{l}^{-}$ & $(?)^{627}$ \\
\hline 13320 & 1445 & & & & $\begin{array}{l}k^{3} y^{\prime-} \\
k^{3} y^{3}- \\
k^{3} y l^{3} h-\end{array}$ & slime \\
\hline 13330 & & & & $\mathrm{k}^{3} \mathrm{y} \mathbf{1}^{-}$ & & $(?)^{628}$ \\
\hline 13340 & 1446 & $\begin{array}{l}\mathrm{k}^{3} \mathrm{ygh}- \\
\mathrm{k}^{3} \mathrm{yk}-\end{array}$ & $\begin{array}{l}k^{3} y g h- \\
k^{3} y k-\end{array}$ & $\begin{array}{l}\mathrm{k}^{3} \mathrm{ygh}- \\
\mathrm{k}^{3} \mathrm{yk}-\end{array}$ & $\begin{array}{l}\text { (myg- } \\
\text { mygh-) }\end{array}$ & to caulk a boat \\
\hline
\end{tabular}

${ }^{626}$ The $|\mathrm{s}|$ component in the alternants could be the extender listed as suffix no. 2140. There is only one example of $\left\{\mathrm{k}^{3} \mathrm{y}^{3} \mathrm{~s}^{\bullet}\right\}$, cf. KW $\left\{\mathrm{k}^{3} \mathrm{y}^{3} \mathrm{~s}^{\bullet-h l h}\right\}>/ \mathrm{k}^{3} \mathrm{i}^{3} \mathrm{sala} /$ "not being." The form $\left\{\mathrm{c}^{3} \mathrm{y}^{3} \mathrm{~s}^{-}\right\}^{*}$ is mentioned by Boas (1948:335) as an example of KW using a palatal-velar obstruent but the $/ \mathrm{DH}^{3} \mathrm{axda}^{3} \chi \mathrm{v} /$ (Knight Inlet people) a dental-alveolar one.

${ }^{627}$ Possibly in the KW stem $\left\{\mathrm{k}^{3} \mathrm{y}\right.$ - $\left.=\mathrm{hkv}-\right\}$ "to kill, to beat up, to club"

${ }^{628}$ Possibly in HA (Kitamaat dialect) / ${ }^{3}{ }^{3} 1^{3}{ }^{3}$ / "rat." The word could however be a borrowing. 
North Wakashan Comparative Root List (Updated)

\begin{tabular}{|c|c|c|c|c|c|c|}
\hline Root \# & Old \# & $\mathrm{HE}$ & $\mathrm{OO}$ & HA & KW & Gloss \\
\hline 13341 & 1447 & $\begin{array}{l}\mathrm{k}^{3} \mathrm{ww}- \\
\mathrm{k}^{3} \mathrm{hwh}-\end{array}$ & $\mathrm{k}^{3} \mathrm{yw}-$ & $\begin{array}{l}\mathrm{k}^{3} \mathrm{ww}- \\
\mathrm{k}^{3} \mathrm{hwh}-\end{array}$ & $\begin{array}{l}k^{3} \mathrm{yw}- \\
k^{3} \mathrm{yhw} \\
k^{3} h y w-\end{array}$ & $\begin{array}{l}\text { not the case, to the contrary, non- } \\
\text { existent }{ }^{629}\end{array}$ \\
\hline 13350 & 1448 & $\mathrm{k}^{3} \mathrm{y} \chi \mathrm{v}^{-}$ & $\mathrm{k}^{3} \mathrm{y} \chi \mathrm{v}-$ & $\mathrm{k}^{3} \mathrm{y} \chi \mathrm{v}-$ & $\mathrm{k}^{3} \mathrm{y} \chi \mathrm{v}-$ & $\begin{array}{l}\text { (HE/OO/HA) to run on foot; } \\
(\mathrm{KW}) \text { to run away, escape, flee } \\
\text { from }\end{array}$ \\
\hline 13360 & 1449 & $k^{3} y_{q-}$ & $\mathrm{k}^{3} \mathrm{yq}-$ & $\mathrm{k}^{3} \mathrm{yq}-$ & $\mathrm{k}^{3} \mathrm{yq}-*$ & to poke one's head forward \\
\hline 13370 & 1450 & & & & $\mathrm{k}^{3} \mathrm{y} \chi^{*}$ & (?) bloodied skin, scraped skin ${ }^{630}$ \\
\hline 13371 & & & & & $\mathrm{k}^{3} \mathrm{y}^{3}-$ & virgin \\
\hline 13380 & 1451 & $\mathrm{k}^{3} \mathrm{vxv}-$ & $\mathrm{k}^{3} \mathrm{vxv}-$ & $\mathrm{k}^{3} \mathrm{vxv}-$ & $\mathrm{k}^{3} \mathrm{xv}-$ & to suck \\
\hline 13390 & $\begin{array}{l}1452 \\
1748\end{array}$ & & $\mathrm{k}^{3} \mathrm{vw}-$ & $\mathrm{k}^{3} \mathrm{~W}-$ & & $\begin{array}{l}\text { (?) to forge social ties, to link up } \\
\text { with others }\end{array}$ \\
\hline 13400 & $\begin{array}{l}1453 \\
1749\end{array}$ & $\mathrm{k}^{3} \mathrm{vw}-$ & $\mathrm{k}^{3} \mathrm{vw}-$ & $\mathrm{k}^{3} \mathrm{~W}-$ & $\mathrm{k}^{3} \mathrm{~W}-$ & $\begin{array}{l}\text { to split open, spread apart (like } \\
\text { clam shells, wood, jaw's legs); to } \\
\text { dry clams }\end{array}$ \\
\hline 13410 & 1454 & $\mathrm{k}^{3} \mathrm{vwp}-$ & $\mathrm{k}^{3} \mathrm{vwp}-$ & $\mathrm{k}^{3} \mathrm{wp}-$ & $\mathrm{k}^{3} \mathrm{wp}-$ & $\begin{array}{l}\text { flake or strip peeling off or being } \\
\text { torn off; to handle a flat object; } \\
\text { (HA/KW also:) to break or tear } \\
\text { flat brittle object }\end{array}$ \\
\hline 13420 & 1455 & & & & $\mathrm{k}^{3} \mathrm{wt}-$ & $\begin{array}{l}\text { flat narrow object stands or lies } \\
\text { on edge, to put sth. on edge }\end{array}$ \\
\hline 13430 & 1456 & & & & $\mathrm{k}^{3} \mathrm{wt}-$ & salmon \\
\hline
\end{tabular}

${ }^{629} \mathrm{HE} / \mathrm{HA}\left\{\mathrm{k}^{3} \mathrm{hwh}-\right\}$ only in HE $/ \mathrm{k}^{3} \mathrm{aw}^{\prime} \mathrm{aq}^{3} \mathrm{a} /$ (name of a monster) HA $/ \mathrm{k}^{3} \mathrm{awaq}^{3} \mathrm{a} /$ "wild caveman." It may mean "to be avoided."

${ }^{630} \mathrm{In} \mathrm{KW} / \mathrm{k}^{3} \mathrm{i} \chi \mathrm{k}^{3} \chi \mathrm{is} / *$ "red-throated loon" $\mathrm{KW} / \mathrm{k}^{3} \mathrm{i} \chi \mathrm{a} / *$ "to scrape skin (drawing towards body)" $\mathrm{KW} / \mathrm{k}^{3}$ ixayu/* "skin scraper." These words' $/ \mathrm{k}^{3} /$ may be in error because there is a $\mathrm{KW}$ root $\{\mathrm{ky} \chi-\}$ "to scrape, to scrub, to rub together" (root no. 12440). 
North Wakashan Comparative Root List (Updated)

\begin{tabular}{|c|c|c|c|c|c|c|}
\hline Root \# & Old \# & $\mathrm{HE}$ & $\mathrm{OO}$ & HA & KW & Gloss \\
\hline 13440 & 1457 & & & & $\mathrm{k}^{3} \mathrm{wt}-$ & $\begin{array}{l}\text { to judge insufficient, to criticize } \\
\text { what is given, to look down upon }\end{array}$ \\
\hline 13450 & 1458 & $\mathrm{k}^{3} \mathrm{vwt}-$ & $\mathrm{k}^{3} \mathrm{vwt}-$ & $\mathrm{k}^{3} \mathrm{wt}-$ & & $\begin{array}{l}\text { long slender pointed object (like } \\
\text { quill, nail) sticking in or driven } \\
\text { into sth. }\end{array}$ \\
\hline 13460 & 1459 & & & $\mathrm{k}^{3} \mathrm{WS}-$ & $\mathrm{k}^{3} \mathrm{WS}-$ & (?) to handle clams \\
\hline 13470 & 1460 & $\begin{array}{l}\mathrm{k}^{3} \text { WS- } \\
\mathrm{k}^{3} \text { vws- }\end{array}$ & $\begin{array}{l}\mathrm{k}^{3} \mathrm{wc}-/ \\
\mathrm{k}^{3} \mathrm{w}^{3} \mathrm{~s}- \\
\mathrm{k}^{3} \mathrm{WS}- \\
\mathrm{k}^{3} \mathrm{VWs}-\end{array}$ & $\mathrm{k}^{3} \mathrm{WS}-$ & $\mathrm{k}^{3} \mathrm{WS}-$ & $\begin{array}{l}\text { 1. surface is bent or folded at a } \\
\text { sharp angle (as at a ridge, edge, } \\
\text { backbone, keel); } \\
\text { 2. (OO/HA/KW also:) bent or } \\
\text { curled up (like finger, one's body, } \\
\text { box made by bending single } \\
\text { cedar board) } 631\end{array}$ \\
\hline 13480 & 1461 & & & & $\mathrm{k}^{3} \mathrm{w} \mathcal{-}-$ & rough (to sight or touch) \\
\hline 13490 & $\begin{array}{l}1462 \\
1464\end{array}$ & $\mathrm{k}^{3} \mathrm{vw} 1-$ & $\mathrm{k}^{3} \mathrm{vw1-}$ & $\mathrm{k}^{3} \mathrm{w} 1-$ & $\mathrm{k}^{3} \mathrm{w} 1-$ & $\begin{array}{l}\text { to form a sharp angle (as at a } \\
\text { ridge, edge, backbone, keel), V- } \\
\text { shaped, triangular, square, to } \\
\text { square off }\end{array}$ \\
\hline 13500 & 1463 & & & & $\mathrm{k}^{3} \mathrm{w} 1-$ & $(?)^{632}$ \\
\hline 13510 & 1465 & $\mathrm{k}^{3} \mathrm{vwkV}-$ & $\mathrm{k}^{3} \mathrm{vwkV}-$ & $\mathrm{k}^{3} \mathrm{wkV}-$ & $\mathrm{k}^{3} \mathrm{WkV}-$ & $\begin{array}{l}\text { to stand on edge, to stand } \\
\text { sideways or on the side (said of } \\
\text { thin, flat object), to use an }\end{array}$ \\
\hline
\end{tabular}

${ }^{631} \mathrm{OO}\left\{\mathrm{k}^{3} \mathrm{wc}-\right\}$ or $\left\{\mathrm{k}^{3} \mathrm{w}^{3} \mathrm{~s}-\right\}$ (not diagnosable which) occurs only in $/ \mathrm{k}^{3} \mathrm{u}^{3} \mathrm{ssmala} /$ "curled up, having a hoop-like shape (as a person curled up)."

${ }^{632} \mathrm{In} \mathrm{KW} / \mathrm{k}^{3} \mathrm{ulisa} / *$ "noise made by whale when making long dive" and two reelicited $\mathrm{KW}$ words referring to a porpoise and seemingly deriving from a stem $\left\{\mathrm{k}^{3} \mathrm{wlwt}-\right\}<\left\{\mathrm{k}^{3} \mathrm{w} t-=\mathrm{wt}-\right\}$. This stem $\left\{\mathrm{k}^{3} \mathrm{wlwt}\right\}$ could however be a backformation from $/ \mathrm{k}^{3} \mathrm{ulut}^{3} / *$ "porpoise" which is a crossfamily stem, "porpoise" being k ${ }^{3} v^{\prime} u n u t^{3}$ in Squamish, cf. Kuipers (1967:346). 
North Wakashan Comparative Root List (Updated)

\begin{tabular}{|c|c|c|c|c|c|c|}
\hline Root \# & Old \# & $\mathrm{HE}$ & $\mathrm{OO}$ & $\mathrm{HA}$ & KW & Gloss \\
\hline & & & & & & $\begin{array}{l}\text { object's edge (as in chopping), } \\
\text { layer splits off a stem and falls } \\
\text { down flat (like banana peel, } \\
\text { skunk cabbage leaves) }\end{array}$ \\
\hline 13520 & 1750 & $\mathrm{k}^{3} \mathrm{vwXv}-$ & $\mathrm{k}^{3} \mathrm{vwXV-}$ & $\mathrm{k}^{3} \mathrm{wXV}-$ & & $(?)^{634}$ \\
\hline 13530 & $\begin{array}{l}1466 \\
2175\end{array}$ & $q^{3}$ vwqv- & $q^{3} v_{w q v-}$ & $\mathrm{k}^{3} \mathrm{wqv}-$ & $\begin{array}{l}\mathrm{k}^{3} \mathrm{wqv-} \\
\text { rwqv- }\end{array}$ & $\begin{array}{l}\text { to carry by handle or loop; to } \\
\text { pull, tug, hoist }{ }^{635}\end{array}$ \\
\hline 13540 & 1467 & & $\mathrm{k}^{3} \mathrm{w} \chi \mathrm{V}-$ & & $\mathrm{k}^{3} \mathrm{w} \chi \mathrm{v}^{-}$ & to bend, to fold \\
\hline 13550 & $\begin{array}{l}1468 \\
1476 \\
1488 \\
2060\end{array}$ & $q^{3} v q v-$ & $q^{3} v q v-$ & $q^{3} v q v-$ & $\begin{array}{l}\mathrm{k}^{3} \mathrm{qv}- \\
\mathrm{k}^{3} \mathrm{~h}^{3} \mathrm{qv}- \\
\mathrm{k}^{3} \mathrm{vh}^{3} \mathrm{qv}-\end{array}$ & $\begin{array}{l}\text { to disengage things that fit } \\
\text { together, to pull sth. out of what } \\
\text { it fits in; (KW also:) long thing } \\
\text { pokes out of sth., to poke into } \\
\text { sth. }{ }^{636}\end{array}$ \\
\hline 13560 & $\begin{array}{l}1469 \\
1470\end{array}$ & $k^{3} q^{-}$ & $\mathrm{k}^{3} \mathrm{q}-$ & $\mathrm{k}^{3} \mathrm{q}-$ & $\mathrm{k}^{3} \mathrm{q}-$ & $\begin{array}{l}\text { to move, deliver or put down a } \\
\text { pile, load or cargo of things (said } \\
\text { of humans handling food staples, } \\
\text { mats or blankets, but in HA also } \\
\text { said of beaver) }\end{array}$ \\
\hline
\end{tabular}

${ }^{633}$ Uncertain if this root occurs in OO $/ \mathrm{k}^{3} \mathrm{vuuk}^{3} \mathrm{v} / \mathrm{HE} / \mathrm{k}^{3} \mathrm{vk}^{3} \mathrm{v}^{\prime} \mathrm{uk}^{3} \mathrm{v} / \mathrm{HA} / \mathrm{k}^{3} \mathrm{k}^{3} \mathrm{uk}^{3} \mathrm{v} / \mathrm{KW}$ $/ \mathrm{k}^{3} \mathrm{k}^{3} \mathrm{a}^{3} \mathrm{ukv} /$ "skunk cabbage." Term could be a cross-linguistic stem, cf. ${ }^{*}-\mathrm{k}^{3} \mathrm{vuk}^{3} \mathrm{v}$ - in protoSalish (Kuipers 2002:141).

${ }^{634}$ Only in HE $/ \mathrm{k}^{3} \mathrm{v}^{\prime} \mathrm{uxv}^{\prime}$ an'i/ OO $/ \mathrm{k}^{3} \mathrm{v} u x v a n i / \mathrm{HA} / \mathrm{k}^{3} \mathrm{uxvani} /$ "butter clams" which however is a cross-family stem, cf. Proto-Salish * $\mathrm{k}^{3} \mathrm{vuxvani} 7$ "clam" (Kuipers 2002:142).

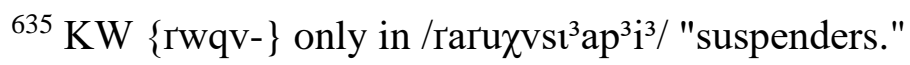

${ }^{636} \mathrm{KW}\left\{\mathrm{k}^{3} \mathrm{qv}-\right\}$ likely also in three slightly different names of fireweed, $/ \mathrm{k}^{3} \mathrm{ak}$ aqvama/,

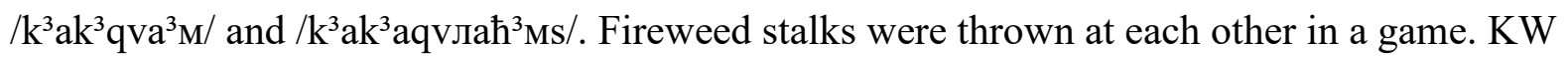
$\left\{\mathrm{k}^{3} \mathrm{~h}^{3} \mathrm{qv}-\right\}$ only in $/ \mathrm{k}^{3} \mathrm{a}^{3} \mathrm{q}^{3} \mathrm{vs} /$ "young tree, pole or stick in the ground."

${ }^{637}$ Probably also in HA / $\mathrm{k}^{3} \mathrm{q}$ 'ita/ "to undress." 
North Wakashan Comparative Root List (Updated)

\begin{tabular}{|c|c|c|c|c|c|c|}
\hline Root \# & Old \# & $\mathrm{HE}$ & $\mathrm{OO}$ & HA & $\mathrm{KW}$ & Gloss \\
\hline 13570 & 1471 & $\mathrm{k}^{3} \chi-$ & $\mathrm{k}^{3} \chi-$ & $\mathrm{k}^{3} \chi-$ & $\mathrm{k}^{3} \chi^{-*}$ & $\begin{array}{l}\text { to close one's eyes, shut-eye, } \\
\text { sleep }^{638}\end{array}$ \\
\hline 13580 & $\begin{array}{l}1472 \\
1487\end{array}$ & $\mathrm{k}^{3} \mathrm{~h}-$ & $\mathrm{k}^{3} \mathrm{~h}-$ & $\mathrm{k}^{3} \mathrm{~h}-$ & $\mathrm{k}^{3} \mathrm{~h}-$ & $\begin{array}{l}\text { small loose things (e.g. ooligans; } \\
\text { particled solids like sand, ashes, } \\
\text { gravel) are located somewhere or } \\
\text { being handled (spread, poured, } \\
\text { scattered) }\end{array}$ \\
\hline 13590 & $\begin{array}{l}1472 \\
1474 \\
1475\end{array}$ & $k^{3} h-$ & $k^{3} h-$ & $k^{3} h-$ & $\mathrm{k}^{3} \mathrm{~h}-*$ & $(?)^{639}$ \\
\hline 13600 & 1473 & & & & $\mathrm{k}^{3} \mathrm{~h}-$ & (?) to handle a flat thing ${ }^{640}$ \\
\hline 13610 & 1477 & $\mathrm{k}^{3} \mathrm{hp}-$ & $\mathrm{k}^{3} \mathrm{hp}-$ & $\mathrm{k}^{3} \mathrm{hp}-$ & $\mathrm{k}^{3} \mathrm{hp}-$ & to gnaw \\
\hline 13620 & 1478 & $\mathrm{k}^{3} \mathrm{hp}-$ & $\mathrm{k}^{3} \mathrm{hp}-$ & $\mathrm{k}^{3} \mathrm{hp}-$ & (hnxv-) & humpback salmon \\
\hline 13630 & 1479 & $\mathrm{k}^{3} \mathrm{ht}-$ & $\mathrm{k}^{3} \mathrm{ht}-$ & $\mathrm{k}^{3} \mathrm{ht}-$ & $\mathrm{k}^{3} \mathrm{ht}-$ & to write, to paint, to draw pictures \\
\hline 13640 & 1758 & $\mathrm{k}^{3} \mathrm{vht}-$ & $\mathrm{k}^{3} \mathrm{vht}-$ & $\begin{array}{l}\mathrm{k}^{3} \mathrm{ht}- \\
\mathrm{k}^{3} \mathrm{vht}-\end{array}$ & & $\begin{array}{l}\text { to rake in, to pull towards oneself } \\
\text { with a stick, to strike a match, to } \\
\text { reclaim goods }\end{array}$ \\
\hline
\end{tabular}

${ }^{638}$ The KW examples are all from the Koskimo dialect.

${ }^{639}\left|\mathrm{k}^{3} \mathrm{~h}\right|$ is the shared initial component of the following hard to analyze terms and their derivates.

It is a root in example no. 3 but may or may not be that in the other ones. $1 . \mathrm{HE} / \mathrm{k}^{3 /} \mathrm{am} / \mathrm{amu} /$ and $/ \mathrm{k}^{3} \mathrm{a} \mathrm{l}^{\prime} \mathrm{ulu} / \mathrm{OO} / \mathrm{k}^{3} \mathrm{amumu}$ / (a small kind of clams) KW $/ \mathrm{k}^{3} \mathrm{amuma} /$ "needles of coniferous trees"; 2. $\mathrm{HE} / \mathrm{k}^{3} \mathrm{an}^{3} \mathrm{a} / \mathrm{OO} / \mathrm{k}^{3} \mathrm{an}^{3} \mathrm{a} /$ "saltwater bullhead"; 3 . HE $/ \mathrm{k}^{3} \mathrm{au} / \mathrm{HA} / \mathrm{k}^{3} \mathrm{a} . \mathrm{u} /$ (exclamation, meaning unknown); 4. HA $/ \mathrm{k}^{3}$ arqas/ OO $/ \mathrm{k}^{3}$ aaqa/ "crow" $\mathrm{HE} / \mathrm{k}^{3} \mathrm{aqa} /$ "crow" and "black rock cod" OO /K $\mathrm{K}^{3}$ aanawira/ (name of Raven's sister); 5. KW / $\mathrm{k}^{3} \mathrm{ada} /$ "starry flounder."

${ }^{640}$ In KW $\left\{\mathrm{k}^{3} \mathrm{~h}-\mathrm{h} \nmid-\mathrm{h}\right\}>/ \mathrm{k}^{3} \mathrm{ała} / *$ [2] "to throw flat thing overhand" and perhaps also in the stem $\left\{\mathrm{k}^{3} \mathrm{~h}^{3} \mathrm{ht}-\right\}$ "hold salmon flat in tongs." 
North Wakashan Comparative Root List (Updated)

\begin{tabular}{|c|c|c|c|c|c|c|}
\hline Root \# & Old \# & $\mathrm{HE}$ & $\mathrm{OO}$ & HA & KW & Gloss \\
\hline 13650 & 1480 & & & & $\mathrm{k}^{3} \mathrm{hs}-$ & $\begin{array}{l}\text { to send away sb. unwelcome, to } \\
\text { separate, divorce (like couple), to } \\
\text { come apart (like bundle) }{ }^{641}\end{array}$ \\
\hline 13660 & 1481 & $\begin{array}{l}\mathrm{k}^{3} \mathrm{hs}- \\
\mathrm{k}^{3} \mathrm{~h}^{3} \mathrm{~s}-\end{array}$ & $\mathrm{k}^{3} \mathrm{~h}^{3} \mathrm{~s}-$ & $\mathrm{k}^{3} \mathrm{hs}-$ & $\mathrm{k}^{3} \mathrm{~h} \mathrm{~s}-$ & to grip, pinch, claw ${ }^{642}$ \\
\hline 13670 & 1482 & & & & $\mathrm{k}^{3} \mathrm{~h} £-*$ & flat thing sails through the air \\
\hline 13680 & 1483 & & & & $\mathrm{k}^{3} \mathrm{~h} \mathrm{-}^{-*}$ & $(?)^{643}$ \\
\hline 13690 & 1484 & $\mathrm{k}^{3} \mathrm{~h} 1-$ & $\left.\mathrm{k}^{3} \mathrm{~h}\right\}-$ & $\mathrm{k}^{3} \mathrm{~h} 1-$ & $(\mathrm{my} \chi-)$ & to sleep, to dream \\
\hline 13700 & 1485 & $\mathrm{k}^{3} \mathrm{~h} t-$ & $\mathrm{k}^{3} \mathrm{~h} t-$ & $\left.\mathrm{k}^{3} \mathrm{~h}\right\}-$ & & $(?)^{644}$ \\
\hline 13710 & 1486 & $\mathrm{k}^{3} \mathrm{hk}-$ & k³k- & $\mathrm{k}^{3} \mathrm{hk}-$ & $\mathrm{k}^{3} \mathrm{hk}-$ & to raise the eyebrows \\
\hline 13720 & 1489 & $(\chi \mathrm{yx}-)$ & $(\chi \mathrm{yx}-)$ & $(\chi y x-)$ & $\begin{array}{l}\mathrm{k}^{3} \mathrm{~h} \chi \mathrm{v}^{-} \\
\mathrm{k}^{3} \mathrm{hwh} \chi \mathrm{v}^{-}\end{array}$ & $\begin{array}{l}\text { to whittle, shave, slice thinly, } \\
\text { peel }\end{array}$ \\
\hline 13730 & & & & & $\mathrm{k}^{3} \mathrm{~h} y^{3} \mathrm{~h} \chi \mathrm{V}-*$ & half dry \\
\hline 13750 & 1490 & & & & $\mathrm{k}^{3} \mathrm{hq}-$ & $\begin{array}{l}\text { positioned crosswise (logs, } \\
\text { boards) }\end{array}$ \\
\hline
\end{tabular}

${ }^{641}$ Root could be a backformation from KW $/ \mathrm{k}^{3} \mathrm{asu} /<\left\{\mathrm{k}^{3}[\mathrm{~h}] \mathrm{s}-\mathrm{w}\right\}$ (with $\left\{\mathrm{k}^{3} \mathrm{~s}-\right\}$ "slack, etc." (root no. 12970) and suffix no. 4290) which Boas (1948:275) glosses as "to come loose from each other, bundle comes apart, married couple separates." Alternately, $\left\{\mathrm{k}^{3} \mathrm{hs}-\right\}$ may refer first of all to "throwing lower arm horizontally sideways [with] palm inward" which gesture serves to chase sb. away, cf. Boas (1948:282).

${ }^{642}$ Could be a backformation from $\mathrm{HE} / \mathrm{k}^{3{ }^{\prime}} \mathrm{ask}^{3 \prime} \mathrm{as} /$ "talons, claws" $\mathrm{HE} / \mathrm{k}^{3 \prime} \mathrm{az}^{\prime} \mathrm{al}^{\prime} \mathrm{ac}^{3} \mathrm{i} /$ and $/ \mathrm{k}^{3} \mathrm{az}^{\prime} \mathrm{al}^{\prime} \mathrm{ic}^{3} \mathrm{i} / \mathrm{HA} / \mathrm{k}^{3} \mathrm{azan}^{\prime} \mathrm{ic}^{3} / \mathrm{KW} / \mathrm{k}^{3}$ azalac $^{3} \mathrm{i} /$ "ant." Note the occurrence in South Wakashan (Nuuchahnulth) of kªc- "to pinch." ${ }^{643}$ In KW (Koskimo dialect) $/ \mathrm{k}^{3} \mathrm{a} ł a / *$ "to menstruate." ${ }^{644}$ Possibly in HE $/ \mathrm{k}^{3 \prime} \mathrm{al}$ 'ax/ OO $/ \mathrm{k}^{3}$ alax/ "a species of fern" HA $/ \mathrm{k}^{3}$ alak/ "long green fern species." 
North Wakashan Comparative Root List (Updated)

\begin{tabular}{|c|c|c|c|c|c|c|}
\hline Root \# & Old \# & $\mathrm{HE}$ & $\mathrm{OO}$ & HA & KW & Gloss \\
\hline 13760 & 1491 & & & & $\mathrm{k}^{3} \mathrm{~h}^{3} \mathrm{l}-$ & to emit steam, to menstruate \\
\hline 13770 & 1492 & $\mathrm{k}^{3} \mathrm{~h}^{3} \mathrm{x}-$ & & & & to slip, slide, scoot \\
\hline 13780 & 1493 & & & & $k^{3} h^{3} x-$ & to go backward \\
\hline 13790 & & & & & $\mathrm{k}^{3} \mathrm{~h}^{3} \mathrm{qw}-$ & engine idles ${ }^{645}$ \\
\hline 13810 & 1494 & & & xmt- & & strong wind \\
\hline 13820 & 1495 & & & & $\mathrm{xms}-$ & to skim off oil ${ }^{646}$ \\
\hline 13830 & 1496 & & $\mathrm{xms}-$ & & $\mathrm{xms}-*$ & (?) silly, foolish ${ }^{647}$ \\
\hline 13840 & 1497 & $\begin{array}{l}(£ X s- \\
ł x s-)\end{array}$ & & $\mathrm{xms}-$ & & to eat too much ${ }^{648}$ \\
\hline 13841 & 1498 & $\mathrm{xm}^{3}-$ & & & $\mathrm{xm}^{3}-$ & to lasso, snare, set a snare \\
\hline 13850 & 1499 & & & & $\mathrm{xt}-$ & $\begin{array}{l}\text { to suck, siphon, pump, inhale, } \\
\text { breathe } \\
649\end{array}$ \\
\hline 13860 & $\begin{array}{l}1500 \\
1501\end{array}$ & & xnt- & & xnt- & $\begin{array}{l}\text { to spin around while held in a } \\
\text { sling or at the end of a rope, to } \\
\text { swing overhead, to use a } \\
\text { bullroarer } 650\end{array}$ \\
\hline
\end{tabular}

${ }^{645}$ Stem could be the form $\left\{\mathrm{k}^{3} \mathrm{~h}^{3} \mathrm{qv}-\right\}$ of root no. 13550 followed by $\{-\mathrm{w}\}$ (suffix no. 4290).

${ }^{646} \mathrm{In} \mathrm{KW} / \mathrm{xMsa} /[1]$ "to skim off oil" KW / $\mathrm{xMzac}^{3} \mathrm{i}$ / "oil vat."

${ }^{647} \mathrm{In} \mathrm{OO} / \mathrm{xmsa} /$ "to act in a silly or foolish way" KW /xMsa/* [2] "to feel happy" KW /xмyasнukv/* "having laughed with happiness."

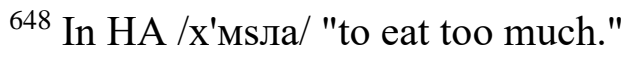

${ }^{649}$ Perhaps also in KW /xi:xtba/* "puffin." Its unreduplicated root could be $\left\{\mathrm{xt}^{-}\right\}$or $\{\mathrm{xyt}-\}$ as far as form is concerned. Boas (1948:374) lists the name as a derivate of $\{\mathrm{xt}-\}$.

${ }^{650}$ Possibly also in words to do with giant fawn lily (Erythronium giganteum) because the plant has tall thin naked stalks capped with usually only one pendant flower (cf. 
North Wakashan Comparative Root List (Updated)

\begin{tabular}{|c|c|c|c|c|c|c|}
\hline Root \# & Old \# & $\mathrm{HE}$ & $\mathrm{OO}$ & $\mathrm{HA}$ & KW & Gloss \\
\hline 13880 & 1502 & xnt- & $(\mathrm{hn} \chi-)$ & xnt- & $\left(\mathrm{hn} \chi^{-}\right)$ & $\begin{array}{l}\text { (HE) to look at one's reflection } \\
\text { (in water, in mirror); (HA) to } \\
\text { look, to peek }\end{array}$ \\
\hline 13890 & 1503 & xnc- & xnc- & & xns- & to sniff \\
\hline 13900 & 1504 & & & & $\mathrm{xnk}^{3}-*$ & $(?)^{651}$ \\
\hline 13910 & 1505 & & & & xnqv- & to snort $^{652}$ \\
\hline 13920 & $\begin{array}{l}1506 \\
1507\end{array}$ & & & xnq- & xnq- & $\begin{array}{l}\text { (?) to stick out (like fingers, toes, } \\
\text { nose), to stick up (like plant } \\
\text { stalk) }{ }^{653}\end{array}$ \\
\hline 13940 & 1509 & & & & XzX- & mould, mouldy \\
\hline 13950 & $\begin{array}{l}1508 \\
1543\end{array}$ & $\begin{array}{l}\text { Xs- } \\
\text { xhs- }\end{array}$ & Xs- & Xs- & $\begin{array}{l}\text { XS- } \\
x h c^{3-}\end{array}$ & $\begin{array}{l}(\{\mathrm{xs}-, \mathrm{xhs}-\}:) \text { to disappear, } \\
\text { vanish; lost from view, hidden; to } \\
\text { dissolve (e.g. in water); } \\
\left(\left\{\mathrm{xhc}^{3}-\right\}:\right) \text { liquid drains off, tide } \\
\text { goes down }^{654}\end{array}$ \\
\hline 13970 & 1510 & & & xs- & Xs- & $\begin{array}{l}\text { (?) to spray, to sprinkle (from } \\
\text { mouth or like rain) }\end{array}$ \\
\hline 13980 & 1511 & $x £-$ & $\mathrm{x} £-$ & $x £-$ & & to move to another place ${ }^{655}$ \\
\hline
\end{tabular}

http://science.halleyhosting.com/nature/gorge/3petal/lily/erythronium/fawn.htm). The shape of stalk pus flower could be associated with holding up an object to be swung around.

${ }^{651} \mathrm{In} \mathrm{KW} / \mathrm{xHk}^{3} \mathrm{a} / *$ "to repent" $\mathrm{KW} / \mathrm{xixHk}^{3} \mathrm{u} / *$ "to repent and do better next time." ${ }^{652}$ The 1980 root list incorrectly indicates this root is also attested for OO.

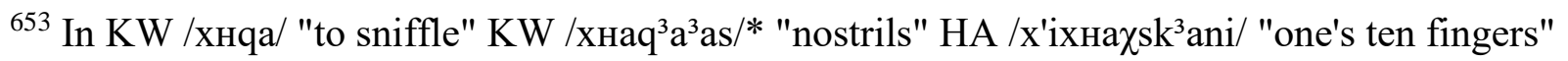
HA /x'ixнахsiza/ "one's ten toes."

${ }^{654}$ \{xhs-\} occurs only in HE \{xhs-=hp-\} "twilight" (= "disappear to bottom," with reference to setting sun). $\left\{\mathrm{xhc}^{3}-\right\}$ may have developed from $*\left\{\mathrm{xh}^{3} \mathrm{~s}-\right\}$.

${ }^{655}$ HA only in /x£uy'a/ "to leave one's spouse behind." 
North Wakashan Comparative Root List (Updated)

\begin{tabular}{|c|c|c|c|c|c|c|}
\hline Root \# & Old \# & $\mathrm{HE}$ & $\mathrm{OO}$ & $\mathrm{HA}$ & KW & Gloss \\
\hline 13990 & 1512 & & & & xł- & $\begin{array}{l}\text { to hang up (to dry in wind, sun or } \\
\text { smoke) }\end{array}$ \\
\hline 14000 & 1513 & & & & $\mathrm{xl}-*$ & to fish for dentalia \\
\hline 14020 & 1515 & & & & $\mathrm{xl}-*$ & $(?)^{656}$ \\
\hline 14030 & 1516 & xlp- & & & & to lay bare ${ }^{657}$ \\
\hline 14040 & 1517 & & & xlp- & xlp- & $\begin{array}{l}\text { (HA) to twist strands of fibre into } \\
\text { rope; }(\mathrm{KW}) \text { to revolve, to spin } \\
\text { around (like flywheel or rotors of } \\
\text { helicopter) }\end{array}$ \\
\hline 14050 & 1518 & & & & xlt- & to saw \\
\hline 14060 & 1519 & & & & xls-* & $(?)^{658}$ \\
\hline 14070 & 1520 & & & & xlkv- & $(?)^{659}$ \\
\hline 14080 & 1521 & xlq- & xlq- & & xlq- & $\begin{array}{l}\text { limp object (e.g. rope, clothing, } \\
\text { bedding), to fling a limp object }\end{array}$ \\
\hline 14090 & 1522 & & & & $\mathrm{xk}^{3}-$ & $(?)^{660}$ \\
\hline 14100 & 1523 & xyt- & xyt- & xyt- & xyt- & $\begin{array}{l}\text { to raise the end of sth. long (e.g. } \\
\text { pole, paddle, head of a person } \\
\text { lying down); (HE/OO/HA also:) }\end{array}$ \\
\hline
\end{tabular}

${ }^{656} \mathrm{In} \mathrm{KW}$ (Newettee dilaect) /хла/* "ribs of halibut."

${ }^{657}$ In HE / $x^{\prime}$ лра/ and / $x^{\prime} л m^{3}$ it/ "to lift one's dress."

${ }^{658} \mathrm{In} \mathrm{KW} /$ хлsа/* and /хльла/* "top of waves blown by wind."

${ }^{659}$ Possibly in the stem $\left\{\mathrm{xlk}^{3} \mathrm{vt}-\right\}$ which refers to twisting a line and making a single coil in basket-making. Only two examples.

${ }^{660} \mathrm{In} \mathrm{KW} / \mathrm{xk}^{3} \mathrm{a} / *$ and $/ \mathrm{xk}^{3} \mathrm{x} . \mathrm{h}^{3} \mathrm{id} /{ }^{*}$ "to take sth. out of the tongs" $\mathrm{KW} / \mathrm{xk}^{3} \mathrm{ala} /$ "Coleman stove." 
North Wakashan Comparative Root List (Updated)

\begin{tabular}{|c|c|c|c|c|c|c|}
\hline Root \# & Old \# & $\mathrm{HE}$ & $\mathrm{OO}$ & HA & KW & Gloss \\
\hline & & & & & & $\begin{array}{l}\text { to use a long thing to lever up } \\
\text { sth. to a vertical position }\end{array}$ \\
\hline 14110 & 1524 & xyc- & $\mathrm{xy}^{3} \mathrm{~s}-$ & xyq- & xys- & to show the teeth; to grin \\
\hline 14111 & & & & & xyc ${ }^{3} h x-$ & $\begin{array}{l}\text { to witness, observe, be a } \\
\text { spectator }\end{array}$ \\
\hline 14120 & 1526 & xył- & xył- & & & to squirt, to spray \\
\hline 14130 & $\begin{array}{l}1525 \\
1544\end{array}$ & xhł- & xhł- & xh1- & $\mathrm{xyl}^{3}{ }_{-}$ & to respect, admire, hold dear \\
\hline 14140 & 1527 & & & & xyk-* & $\begin{array}{l}\text { to strip off (berries), to squeeze } \\
\text { out (from a tube) }\end{array}$ \\
\hline 14150 & 1528 & xyq- & xyq- & xyq- & xyq- & $\begin{array}{l}\text { (HE/OO/HA) red hot, sparkling, } \\
\text { fiery (colour); (KW) to burn, on } \\
\text { fire, red hot }{ }^{662}\end{array}$ \\
\hline 14160 & 1529 & & & & $\mathrm{xkv}-*$ & $\begin{array}{l}\text { in parallel, side by side (as clams } \\
\text { for cooking) }\end{array}$ \\
\hline 14170 & 1530 & xvwp- & xvwp- & & xwp- & $(?)^{663}$ \\
\hline 14180 & 1531 & $\left(£^{3} \mathrm{ykv}-\right)$ & xvwp- & xwp- & xwp- & $\begin{array}{l}\text { burning end of a stick; (hence:) } \\
\text { (OO) to tattoo, to cauterize; (HA) } \\
\text { to cauterize, to transfer fire; } \\
\text { (KW) to light a fire }\end{array}$ \\
\hline
\end{tabular}

${ }^{661}$ Uncertain if raising to a vertical position is a KW meaning too. Boas (1948:376) glosses KW /xita/ and / $\mathrm{xit}^{3} \mathrm{id} /$ as "to lift up head" and comments that "to raise a pole" is an OO meaning. ${ }^{662}$ For HA attested only in words referring to stars. For "to burn, be on fire" HE/OO/HA use $\{$ xvlt- $\}$.

${ }^{663}$ In the names of butterball duck, goldeneye duck and the Western screech owl (which happens to have yellow eyes, like the goldeneye). The root occurs across families, cf. Proto-Salish *-xvup- "a night bird" (Kuipers 2002:121). 
North Wakashan Comparative Root List (Updated)

\begin{tabular}{|c|c|c|c|c|c|c|}
\hline Root \# & Old \# & $\mathrm{HE}$ & $\mathrm{OO}$ & HA & KW & Gloss \\
\hline 14190 & 1532 & & & & $\mathrm{XWm} \mathrm{S}^{3} \mathrm{-}$ & head of land animal ${ }^{664}$ \\
\hline 14200 & 1533 & & & & xwt-* & severed head of seal or porpoise \\
\hline 14220 & 1535 & & & & XWs- & (?) head of person or animal ${ }^{665}$ \\
\hline 14230 & 1534 & XVWS- & XVWS- & XWS- & & ball, airbag, lungs \\
\hline 14240 & 1534 & & XVWS- & & XWS- & $\begin{array}{l}(\mathrm{OO}) \text { tired, weary; }(\mathrm{KW}) \text { rest, } \\
\text { breather; to take a rest, go on } \\
\text { holiday }\end{array}$ \\
\hline 14250 & & & & XWs- & & $(?)^{666}$ \\
\hline 14260 & 1536 & & & & $\mathrm{xw} £-*$ & $(?)^{667}$ \\
\hline 14270 & 1537 & xVwł- & xVwł- & xw1- & Xwł-* & fresh boiled salmon eggs \\
\hline 14280 & $\begin{array}{l}1538 \\
1539\end{array}$ & XVwkV- & XVwkV- & xwkv- & xwkv- & swollen, bulbous 668 \\
\hline 14281 & & & & & xwkv- & (probably onomatopoeic) $^{669}$ \\
\hline 14290 & & & & & $\begin{array}{l}\text { xwqv-*/ } \\
\text { xwq-* }\end{array}$ & $(?)^{670}$ \\
\hline
\end{tabular}

664 Only in $\mathrm{KW} / \mathrm{xuM}^{3} \mathrm{~s} /$ "head of land animal."

${ }^{665} \mathrm{In} \mathrm{KW} / \mathrm{xusut} /<$ xws-wt $\}$ "to cut off head of person or animal" and perhaps two nonreelicitable terms whose transcription and meaning are problematic.

666 In HA /x'usalał/ "twilight; northern lights."

${ }^{667} \mathrm{In} \mathrm{KW} / \mathrm{xu£a/*} \mathrm{"to} \mathrm{beat} \mathrm{fast} \mathrm{time,} \mathrm{make} \mathrm{a} \mathrm{noise."}$

${ }^{668}$ One HA example refers to thick fat on goat legs. All other ones refer to Indian rice which is a kind of lily with edible bulbs known as chocolate lily.

${ }^{669}$ In KW /xukv xukv/ "cry of raven signifying bad news."

${ }^{670}$ In KW /xurkv/* "mock suns." 
North Wakashan Comparative Root List (Updated)

\begin{tabular}{|c|c|c|c|c|c|c|}
\hline Root \# & Old \# & $\mathrm{HE}$ & $\mathrm{OO}$ & HA & KW & Gloss \\
\hline 14300 & 1540 & & & xqv- & xqv- & $\begin{array}{l}\text { to stretch head out, to peek, to } \\
\text { crane neck }^{671}\end{array}$ \\
\hline 14310 & 1541 & & & & $\mathrm{xhm}^{3}-*$ & scarcity of food, famine \\
\hline 14320 & 1542 & xht- & xht- & xht- & $(\mathrm{xqv}-)$ & $\begin{array}{l}\text { to peek, to peer, make effort to } \\
\text { look (like stretching head out, } \\
\text { standing on tip of one's toes) }\end{array}$ \\
\hline 14340 & 1545 & xhlh- & & & & $(?)^{672}$ \\
\hline 14350 & 1546 & xhk- & xhk- & xhk- & & rib, rafter, supporting beam \\
\hline 14360 & 1547 & & & & xhxv-* & $\begin{array}{l}\text { to hear insufficiently well to be } \\
\text { able to understand }\end{array}$ \\
\hline 14370 & 1548 & & & & $\mathrm{xhw}^{3}-$ & to take sth. out of sth. \\
\hline 14380 & $\begin{array}{l}1549 \\
1551 \\
1559 \\
2587\end{array}$ & $\begin{array}{l}\text { y- } \\
\text { yy- } \\
\text { yh- } \\
\text { hy- } \\
\text { h}^{3} y- \\
h^{3} h y h-\end{array}$ & $\begin{array}{l}\text { yy- } \\
\text { yh- } \\
\text { hy- } \\
\text { h}^{3} y- \\
\text { h'hyh- }\end{array}$ & $\begin{array}{l}\text { y- } \\
\text { h- } \\
\text { yy- } \\
\text { yh- } \\
\text { hy- } \\
\text { yh- }\end{array}$ & $\begin{array}{l}\text { y- } \\
\text { yh- } \\
\text { hy- } \\
y^{3 h}- \\
h^{3} y-\end{array}$ & $\begin{array}{l}\text { 1. the one in point, the one under } \\
\text { discussion; } \\
\text { 2. instance of, satisfying a } \\
\text { description; real, actual, true; } \\
\text { straight away, undelayed, direct, } \\
\text { abrupt }^{673}\end{array}$ \\
\hline
\end{tabular}

${ }^{671}$ Only HA example is /xaxaquy'a/ $<\{[\mathrm{xh}] \mathrm{x}[\mathrm{h}] \mathrm{qv}-$ wyh-+-h $\}$ "to stretch out the head in order to look at a person as he goes by."

${ }^{672}$ In HE / $x^{\prime} a l^{\prime} a /$ "come here!"

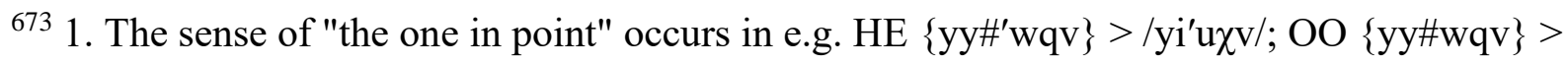

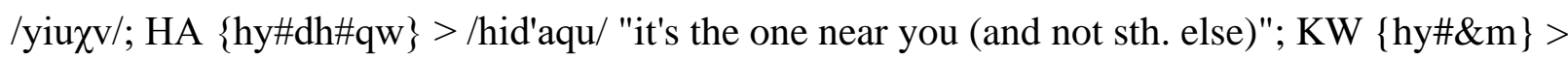
$/$ hi ${ }^{3} \mathrm{M} /$ "that's it!" 2. The examples of the "instance of, etc." sense have varying degrees of semantic transparency. In some the root is merely an attachment point to turn suffixes into words, just like $\left\{\mathrm{w}^{3} \mathrm{w}-, \mathrm{h}^{3} \mathrm{w}-\right.$, etc. $\}$ (root no. 17720). Alternation with forms of root no. 17720 occurs, cf. HE / $\mathrm{y}^{\prime} \mathrm{im}^{3} \mathrm{as} / \mathrm{OO} / \mathrm{yim}^{3}$ as/ HA / $\mathrm{h}^{\prime} \mathrm{im}^{3}$ as/ "chief" and $\mathrm{HA} / \mathrm{h}^{3}{ }^{\prime} \mathrm{um}^{3}$ as/ "big" which latter contains the same suffix as the word for "chief." Whereas $\{y y-\}$ is used in the HE/OO word for 
North Wakashan Comparative Root List (Updated)

\begin{tabular}{|c|c|c|c|c|c|c|}
\hline Root \# & Old \# & HE & $\mathrm{OO}$ & HA & KW & Gloss \\
\hline 14390 & 1550 & yp- & yp- & yp- & yp- & $\begin{array}{l}\text { to weave (e.g. net, mat, cedar } \\
\text { strip fence), to intertwine, to } \\
\text { arrange and secure long things in } \\
\text { parallel, to be abreast }{ }^{674}\end{array}$ \\
\hline 14400 & 1552 & & & & yn- & (?) to drag along ${ }^{675}$ \\
\hline 14410 & 1553 & & & & ynt-* & $\begin{array}{l}\text { to gnaw (said of human or large } \\
\text { animal) }\end{array}$ \\
\hline 14420 & $\begin{array}{l}1555 \\
1556\end{array}$ & ys- & ys- & ys- & & $\begin{array}{l}\text { to soften inner cedar bark by } \\
\text { pounding; (HA also:) to hit (any } \\
\text { object) with a stick }\end{array}$ \\
\hline 14421 & 1554 & & & & ys-* & $(?)^{676}$ \\
\hline 14430 & 1557 & & $y \mathcal{f}-$ & & 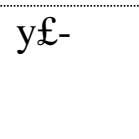 & $\begin{array}{l}\text { to wrap or tie around (rope, } \\
\text { bandage, strap), to bandage }\end{array}$ \\
\hline 14440 & 1558 & & & & yls- & $\begin{array}{l}\text { to twirl a fire drill, to rub with } \\
\text { palm of hand } \\
677\end{array}$ \\
\hline
\end{tabular}

"chief," $\left\{\mathrm{w}^{3} \mathrm{w}-\right\}$ is used in $\mathrm{HE} / \mathrm{w}^{3} \mathrm{um}^{3} \mathrm{aqs} / \mathrm{OO} / \mathrm{w}^{3} \mathrm{uum}^{3} \mathrm{aqs} /$ "chieftainness." 3. This root is likely also the base of various hard to analyze exclamations including e.g. HA /y'aiyiyiyi/ used to encourage a speaker or to accompany the raising of a pole. The HE equivalent of this expression (recorded in Klemtu from William Freeman) is /yilil'ai/. Another exclamation, HE /улаl'a/ $\mathrm{OO} / \mathrm{KW} / \mathrm{y}$ лаla/ "serving sb. right, etc." is assumed to contain a root $\{\mathrm{h}-\}$.

4. The alternant $\left\{\mathrm{h}^{3} \mathrm{y}-\right\}$ occurs in $\mathrm{HE} / \mathrm{h}^{3} \mathrm{i}^{3} \mathrm{us} / \mathrm{OO} / \mathrm{h}^{3} \mathrm{i}^{3} \mathrm{u}^{3} \mathrm{~s} / \mathrm{KW} / \mathrm{h}^{3} \mathrm{l}^{3} \mathrm{u}^{3} \mathrm{~s} /$ "to understand (language, meaning)."

${ }^{674}$ One HE speaker of the /W3'uyalit $\chi \mathrm{v} /$ dialect consistently pronounced /yp/ as /yip/). Kitamaat informant George Paul Wilson for HA pronounced /yp/ as /yup/.

${ }^{675}$ Only in KW /yнgtt ${ }^{3} a l a /$ "dragging along on water."

${ }^{676}$ Only in KW /ysała/* "open (said of a cut, crack, boil)."

${ }^{677}$ Many derivates found in the Boas-Hunt materials could not be re-elicited. 
North Wakashan Comparative Root List (Updated)

\begin{tabular}{|c|c|c|c|c|c|c|}
\hline Root \# & Old \# & $\mathrm{HE}$ & $\mathrm{OO}$ & HA & KW & Gloss \\
\hline 14460 & 1560 & & & & yl $\chi v-*$ & $\begin{array}{l}\text { to hang heads or skulls of } \\
\text { enemies on poles }\end{array}$ \\
\hline 14480 & $\begin{array}{l}1561 \\
1562\end{array}$ & yl $\chi-$ & ylx- & & ylq-* & to rub, to smear \\
\hline 14490 & 1563 & & & & yk- & to limp \\
\hline 14500 & 1564 & & yk- & yk- & yk- & to catch halibut \\
\hline 14510 & 1565 & $\begin{array}{l}\mathrm{yx}- \\
\mathrm{yx} \cdot-\end{array}$ & $\begin{array}{l}y x- \\
y x \cdot-\end{array}$ & $\begin{array}{l}\mathrm{yx}- \\
\mathrm{yx} \cdot-\end{array}$ & $\begin{array}{l}\text { yx- } \\
\text { yxh- } \\
\text { yhx- }\end{array}$ & $\begin{array}{l}\text { swift, fast, speedy; to ride the } \\
\text { current, shoot the rapids }\end{array}$ \\
\hline 14520 & & yx- & & & & to heap up ${ }^{678}$ \\
\hline 14530 & 1566 & & & & ykv- & $(?)^{679}$ \\
\hline 14550 & $\begin{array}{l}1567 \\
1568\end{array}$ & yxv- & yxv- & yxv- & $\begin{array}{l}\text { yxv- } \\
\text { ykv-* }\end{array}$ & $\begin{array}{l}\text { covering layer, flat object (e.g. a } \\
\text { card) }\end{array}$ \\
\hline 14560 & 1569 & & yxv- & & yxv- & to fan the fire \\
\hline 14570 & & $\begin{array}{l}\text { yxv-l } \\
\text { yhxv- }\end{array}$ & & & & $(?)^{680}$ \\
\hline 14580 & 1571 & yw- & yw- & yw- & yw- & $\begin{array}{l}\text { wind, draft; catching wind (like } \\
\text { sail, billowing curtain or canvas) } \\
\text { to sail }\end{array}$ \\
\hline 14581 & 1572 & & & & yw- & this one (near addressee) $)^{681}$ \\
\hline
\end{tabular}

${ }^{678}$ In HE $\{\mathrm{yx}-\mathrm{yw}-\mathrm{t}\}$ "ocean swell" and its derivate $\{\mathrm{yx}-\mathrm{yw}-\mathrm{t}-=\mathrm{y}\}$ "sth. heaped up or piled up on the floor of the house."

${ }^{679}$ In the stem KW \{ykvył- $\}$ "twins, having twins"

${ }^{680} \mathrm{In} \mathrm{HE} / \mathrm{y}^{\prime} a y^{\prime} a x v a n i /<\{[\mathrm{yh}] \mathrm{yhxv}-\mathrm{hny}\}$ or $\{[\mathrm{yh}] \mathrm{y}[\mathrm{h}] \mathrm{xv}-\mathrm{hny}\}$ (a kind of clams).

${ }^{681}$ Free form but syntactic status uncertain. Could contain $\{y-\}$ (root no. 14380) and $\{\# w\}$

(suffix no. 4690). 
North Wakashan Comparative Root List (Updated)

\begin{tabular}{|c|c|c|c|c|c|c|}
\hline Root \# & Old \# & $\mathrm{HE}$ & $\mathrm{OO}$ & HA & $\mathrm{KW}$ & Gloss \\
\hline 14590 & 1573 & ywd- & ywd- & ywd- & ywd- & four $^{682}$ \\
\hline 14600 & 1549 & ywt- & $\begin{array}{l}\text { ywt- } \\
\text { ywł- }\end{array}$ & ywt- & $\begin{array}{l}\text { ywt- } \\
\text { ywt- }\end{array}$ & $\begin{array}{l}\text { settled in a routine or framework, } \\
\text { trained, accustomed to sth., } \\
\text { tamed }\end{array}$ \\
\hline 14610 & 1574 & yws- & yws- & yws- & yws- & $\begin{array}{l}\text { (HE/OO/KW) to spoon out, eat } \\
\text { with a spoon; (HE/OO/HA) to } \\
\text { sip; (HA) to drink }\end{array}$ \\
\hline 14620 & 1575 & & & & yw£-* & $(?)^{683}$ \\
\hline 14630 & 1576 & & & & $\begin{array}{l}\text { ywlh- } \\
\text { ywloo-* }\end{array}$ & to drift downriver \\
\hline 14640 & 1577 & & & ywkv- & ywkv-* & $(?)^{684}$ \\
\hline 14660 & 1578 & & & yqv- & & cedar bark for baskets \\
\hline 14661 & $\begin{array}{l}2193 \\
2366 \\
2495\end{array}$ & $\begin{array}{l}\mathrm{q}- \\
\chi-\end{array}$ & $\begin{array}{l}\mathrm{q}^{-} \\
\chi^{-}\end{array}$ & $\begin{array}{l}\text { yqv- } \\
\text { yq- } \\
\text { I- } \\
\text { q- }\end{array}$ & $\begin{array}{l}\mathrm{q}- \\
\chi- \\
\mathrm{h}-\end{array}$ & $\begin{array}{l}\text { in regard to, for the benefit of, for } \\
\text { the purpose of }{ }^{685}\end{array}$ \\
\hline
\end{tabular}

${ }^{682}$ In most derivates followed by $\{-\mathrm{w}\},\{-\mathrm{xv}\}$ or, in $\mathrm{KW},\{-\mathrm{kv}\}$.

${ }^{683} \mathrm{In} \mathrm{KW} / \mathrm{Yuf}^{3} \varepsilon^{3} / *$ (a place name, probably that of Triangle Island) $\mathrm{KW} / \mathrm{Yu} \mathfrak{f}^{3}$ inu $\chi \mathrm{V} / *$ "Triangle Island people." Perhaps the root means "three."

${ }^{684} \mathrm{HA}$ in /y'ukva/ "soft, brittle (said of rock)"; KW in /yuk ${ }^{3} \mathrm{va} / *$ "slate, throw flat thing overhand."

${ }^{685} \mathrm{In}$ a number of full words, proclitics and enclitic-like elements referring to the 1st, 2nd and 3rd person and having various syntactic functions. The forms $\{\mathrm{yqv}-\}$ and $\{\mathrm{yq}-\}$ are attested only in HA (Kitamaat) $\{\mathrm{yqv \# sw}\}>/ \mathrm{y} \chi \mathrm{vsu} / \mathrm{HA}$ (Kitlope) $\{\mathrm{yq} \# \mathrm{sw}\}>/ \mathrm{y} \chi \mathrm{su} /$ "It is you" (and note that in HA before /s/ there is no opposition between /qv/ and / $\chi \mathrm{v} /, / \mathrm{q} /$ and $/ \chi /$ ). The HE/OO counterparts have $\{\mathrm{q}-\}, \mathrm{cf}$. HE $\{\mathrm{q} \# \mathrm{sw}\}>/ \mathrm{qs} \mathbf{u}^{\prime} \mathrm{u} ;$ OO $\{\mathrm{q} \# \mathrm{sw}\}>/ \mathrm{qsu} /$ "It is you." 
North Wakashan Comparative Root List (Updated)

\begin{tabular}{|c|c|c|c|c|c|c|}
\hline Root \# & Old \# & $\mathrm{HE}$ & $\mathrm{OO}$ & HA & KW & Gloss \\
\hline & & & & $\begin{array}{l}\chi- \\
h^{3}-\end{array}$ & & \\
\hline 14670 & $\begin{array}{l}1579 \\
1570\end{array}$ & $y \chi^{\mathrm{V}-}$ & $y \chi v^{-}$ & $\mathrm{y} \chi \mathrm{V}-$ & $y \chi^{\mathrm{V}-}$ & $\begin{array}{l}\text { to shake, tremble, twirl, spin, } \\
\text { Indiandance }\end{array}$ \\
\hline 14680 & 1580 & yq- & yq- & yq- & yq- & to knit, to make net or web \\
\hline 14690 & 1581 & $\begin{array}{l}\text { yq- } \\
\text { yhq- }\end{array}$ & $\begin{array}{l}\text { yq- } \\
\text { yhq- }\end{array}$ & & $\begin{array}{l}\text { yhq- } / \\
\text { yh } \chi-\end{array}$ & $(?)^{686}$ \\
\hline 14700 & 1582 & & & & yh- & to hang down, trail \\
\hline 14701 & 1549 & & & & yh- & $\begin{array}{l}\text { to quit, give up trying, } \\
\text { discontinue; to deviate from a } \\
\text { course }\end{array}$ \\
\hline 14702 & & & & yhmh- & & $(?)^{687}$ \\
\hline 14710 & 1583 & yhp- & yhp- & yhp- & & $\begin{array}{l}\text { to send on an errand, to order sth. } \\
\text { done }\end{array}$ \\
\hline 14720 & 1584 & yht- & yht- & yht- & yht- & $\begin{array}{l}\text { to rattle (with a rattle, gambling } \\
\text { bones) }\end{array}$ \\
\hline 14730 & 1585 & yht- & yht- & yht- & & backwards, in reverse ${ }^{688}$ \\
\hline 14740 & 1586 & yht- & & yht- & & $(?)^{689}$ \\
\hline
\end{tabular}

${ }^{686} \mathrm{In} \mathrm{HE} / \mathrm{y} \chi £^{\prime} \mathrm{H} / \mathrm{OO} / \mathrm{y} \chi £_{\mathrm{H}} /$ "property to be given away at potlatch" and possibly in in a number of proper names among which $\mathrm{OO} / \mathrm{KW} / \mathrm{Ya \chi £}_{\mathrm{H}} /$ (person's name, meaning unknown). ${ }^{687}$ In HA /y'ama/ "small (compared to what one expected)" and possibly the place name /Yam'acisa/ said to mean "fine beach (all gravel and little stones)."

${ }^{688}$ In HE /y'ay'atªla/ OO / yayat ${ }^{3}$ ala/ "remorseful" HA /y'aita/ "to regret doing sth." ${ }^{689}$ In HE /y'ay'atu£i/ (an unidentified bird) HE /y'adHsr'm/ HA(Kitlope) /y'adhsr'm/ HA (Kitamaat) /y'aidhsז́/ "harlequin duck." Boas (1928:184) adds "(= rattle face)" to the gloss "harlequin duck" and must believe this root \{yht- $\}$ is identical to that for "rattle." 
North Wakashan Comparative Root List (Updated)

\begin{tabular}{|c|c|c|c|c|c|c|}
\hline Root \# & Old \# & $\mathrm{HE}$ & $\mathrm{OO}$ & $\mathrm{HA}$ & KW & Gloss \\
\hline 14750 & 1587 & yhzh- & & & & to complain, whimper ${ }^{690}$ \\
\hline 14760 & 1588 & & & & yhs $\infty$ kv- & suet, tallow ${ }^{691}$ \\
\hline 14780 & 1625 & $\mathrm{y}^{3} \mathrm{~h} £-$ & $\mathrm{y}^{3} \mathrm{~h} £-$ & $\begin{array}{l}\text { yh£- } \\
y^{3} h £-\end{array}$ & $\begin{array}{l}\text { yh£- } \\
\mathrm{y}^{3} \mathrm{~h} £-\end{array}$ & bad $^{692}$ \\
\hline 14790 & 1589 & & & & yht- & to rock the cradle \\
\hline 14800 & 1590 & & & yht- & & $\begin{array}{l}\text { floppy object (e.g. towel, } \\
\text { blanket) is somewhere }\end{array}$ \\
\hline 14810 & 1591 & & & & yht- & to dig clams \\
\hline 14820 & $\begin{array}{l}1592 \\
1594\end{array}$ & & & & $\begin{array}{l}\text { yhtk }{ }^{3-*} \\
\text { yhxk }^{3-}\end{array}$ & to hop on one foot ${ }^{693}$ \\
\hline 14830 & 1593 & yhx- & yhx- & yhx- & yhx- & $\begin{array}{l}(\mathrm{HE} / \mathrm{OO} / \mathrm{HA}) \text { to potlatch; }(\mathrm{KW}) \\
\text { to give dowry }\end{array}$ \\
\hline 14840 & 1595 & & yhwhs- & & yhwhs- & to stay a little while \\
\hline 14850 & 1595 & & & & $\mathrm{yhw}^{3} \mathrm{yx}-$ & $\begin{array}{l}\text { 1. in motion, moving about, } \\
\text { moving things about, to } \\
\text { rummage; } 2 \text {. to give a winter } \\
\text { ceremonial }\end{array}$ \\
\hline
\end{tabular}

${ }^{690}$ Possibly in HE /y'aizakªla/ "to ask for a favour" HE /y'ay'azasila/ "to take care of a cry-baby or any spoiled person."

${ }^{691}$ Possibly a polymorphemic stem but form and meaning of root and suffix(es) problematic. See also the similar case of $\mathrm{HE} / \mathrm{OO} / \mathrm{HA} / \mathrm{KW}$ \{hysookv-\} "married."

${ }^{692} \mathrm{HA}\{\mathrm{yh} £-\}$ only in $\left\{\mathrm{yh}^{3} \mathrm{wp}-\right\}$ "gassy feeling' in throat or stomach (sign of arrival of visitors)." KW \{yhf-\} only in $\left\{\mathrm{hhy}^{3} \mathrm{~h}^{\left.\mathfrak{3}^{3} \mathrm{w}-\right\}}\right.$ "to warn." KW $\left\{\mathrm{y}^{3} \mathrm{~h} f-\right\}$ only in $\left\{\mathrm{y}^{3} \mathrm{~h} \mathfrak{f}^{3} \mathrm{wp}-\right\}$ (same meaning as HA $\left.\left\{y h £^{3} w p-\right\}\right)$ and $\left\{y^{3} h £^{3} w-\right\}$ "wary, treating with caution." ${ }^{693}\left\{\mathrm{yhłk}^{3}-\right\}^{*}$ is Koskimo dialect according to Boas-Hunt. 
North Wakashan Comparative Root List (Updated)

\begin{tabular}{|c|c|c|c|c|c|c|}
\hline Root \# & Old \# & $\mathrm{HE}$ & $\mathrm{OO}$ & HA & KW & Gloss \\
\hline 14860 & 1596 & yhqv- & yhqv- & yhqv- & yhqv- & to distribute gifts ${ }^{694}$ \\
\hline 14870 & 1597 & yhqv- & yhqv- & & yhqv- & $\begin{array}{l}\text { (HE) long thing (e.g. log) lies } \\
\text { somewhere; }(\mathrm{OO}) \text { flat thing (e.g. } \\
\text { board) lies somewhere; }(\mathrm{KW}) \text { to } \\
\text { lie still, lie dead }\end{array}$ \\
\hline 14880 & 1598 & yhqv- & yhqv- & & yhqv- & $(?)^{695}$ \\
\hline 14890 & 1599 & yhqv- & yhqv- & yhqv- & & to wager, bet, put at stake \\
\hline 14900 & 1600 & & & & yh $\chi \mathrm{v}-*$ & $(?)^{696}$ \\
\hline 14910 & 1601 & yhrht- & yhrht- & yhrht- & & $(?)^{697}$ \\
\hline 14920 & 1602 & yhq- & yhq- & yhq- & yhq- & $\begin{array}{l}\text { (?) to talk, engage in } \\
\text { conversation }^{698}\end{array}$ \\
\hline 14930 & 1603 & yh $\chi-$ & yh $\chi-$ & yh $\chi-$ & yh $\chi-$ & $\begin{array}{l}\text { thin (said of liquid), molten, of } \\
\text { low viscosity, liquefied (as lead, } \\
\text { butter, ice) }\end{array}$ \\
\hline 14940 & $\begin{array}{l}1604 \\
1619\end{array}$ & & & & $\begin{array}{l}\mathrm{y}^{3} \mathrm{mt}- \\
\mathrm{y}^{3} \mathrm{w} 1-*\end{array}$ & $\begin{array}{l}\text { to burst, split open (berries, } \\
\text { frozen pipe, clams), to split sth. } \\
\text { open (e.g. game) }{ }^{699}\end{array}$ \\
\hline
\end{tabular}

${ }^{694}$ Some KW examples link the giving to a potlatch.

${ }^{695}$ In words referring to woodworms and dentalia (i.e. tooth- or tusk-like shells as used in Indian jewellery).

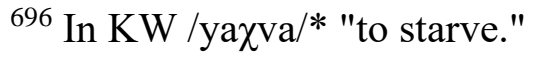

${ }^{697}$ In HE /y'arał'a/ OO /yarała/ HA /y'arała/ "a man's brother-in-law."

${ }^{698}$ Only in the stems HE/OO/HA/KW \{yhq-+gh3ł $\}$ "to utter sth." and KW \{yhq-+nt- $\}$ "to talk." $\left.699\left\{\mathrm{y}^{3} \mathrm{w}\right\}-\right\}^{*}$ only in $\mathrm{KW} / \mathrm{y}^{3} \mathrm{u}$ a/* "to burst (e.g. clams and potatoes when being boiled)." 
North Wakashan Comparative Root List (Updated)

\begin{tabular}{|c|c|c|c|c|c|c|}
\hline Root \# & Old \# & $\mathrm{HE}$ & $\mathrm{OO}$ & $\mathrm{HA}$ & KW & Gloss \\
\hline 14941 & 1623 & & & & $\begin{array}{l}\mathrm{y}^{3} \mathrm{mk}-l \\
\mathrm{y}^{3} \mathrm{mx}- \\
\mathrm{y}^{3} \mathrm{hp}-\end{array}$ & $(?)^{700}$ \\
\hline 14950 & 1605 & & & & $\mathrm{y}^{3} \mathrm{mqv}-*$ & to sit flat \\
\hline 14960 & 1606 & & & & $\mathrm{y}^{3} \mathrm{mq}_{-}^{-*}$ & to spread apart, to burst apart \\
\hline 14970 & 1607 & $y^{3} 1-$ & $y^{3} 1-$ & $y^{3} 1-$ & $y^{3} 1-$ & $\begin{array}{l}\text { to spread out (e.g. things for } \\
\text { drying), to spread apart (canoe, } \\
\text { jaws of spring trap, one's legs) }\end{array}$ \\
\hline 14980 & 1608 & $\mathrm{y}^{3} 1 \mathrm{x}-$ & $\mathrm{y}^{3} 1 \mathrm{x}-$ & & & to spread or pour out over an area \\
\hline 14990 & 1609 & & & & $\mathrm{y}^{3} \mathrm{lkv}-*$ & $\begin{array}{l}\text { to carry a long, stiff object on the } \\
\text { shoulder }\end{array}$ \\
\hline 15000 & & & & $y^{3} x-$ & & to pour liquid into a container \\
\hline 15010 & 1610 & $y^{3} y-$ & $y^{3} y-$ & $h^{3} y-$ & & $\begin{array}{l}\text { to keep sth. raised (like a net- } \\
\text { buoy), to put the hand under sth. } \\
\text { to raise it, to raise the hand, to } \\
\text { wave }\end{array}$ \\
\hline 15020 & 1611 & $y^{3} y-$ & $y^{3} y-$ & $h^{3} y-$ & $\mathrm{h}^{3} \mathrm{y}-$ & $\begin{array}{l}\text { (exclamation expressing a } \\
\text { negative experience) }\end{array}$ \\
\hline 15030 & $\begin{array}{l}1613 \\
2590\end{array}$ & $\mathrm{y}^{3} \mathrm{yt}-$ & $\mathrm{y}^{3} \mathrm{y} t-$ & $h^{3} y t-$ & $h^{3} y t-$ & $\begin{array}{l}\text { back, backwards, back again, in } \\
\text { reverse; (HE/OO/HA also:) to } \\
\text { row (facing backwards); (HA } \\
\text { also:) to defecate; (KW also:) re- } \\
\text { occurring }{ }^{701}\end{array}$ \\
\hline
\end{tabular}

\footnotetext{
${ }^{700}$ Only example of $\left\{\mathrm{y}^{3} \mathrm{mk}-/ \mathrm{y}^{3} \mathrm{mx}-\right\}$ (not diagnosable if $|\mathrm{k}|$ or $|\mathrm{x}|$ ) is $\mathrm{KW} / \mathrm{h}^{3} \mathrm{l}^{3} \mathrm{Mxq}^{3} \mathrm{~s} /$ "enjoying oneself." Only examples of $\left\{\mathrm{y}^{3} \mathrm{hp}-\right\}$ are $\mathrm{KW} / \mathrm{h}^{3} \mathrm{l}^{3} \mathrm{apq}^{3} \mathrm{~s} /\left(\right.$ synonym of $/ \mathrm{h}^{3} \mathrm{l}^{3} \mathrm{Mxq}^{3} \mathrm{~s} /$ ) and $\mathrm{KW}$ $/ h^{3} \mathrm{ay}^{3} \mathrm{apq}^{3} \mathrm{~s}$ sla/ "noise of people enjoying something."

${ }^{701}$ Possibly also in a word from the Koskimo dialect, /h3idayu/* "gaff hook."
} 
North Wakashan Comparative Root List (Updated)

\begin{tabular}{|c|c|c|c|c|c|c|}
\hline Root \# & Old \# & $\mathrm{HE}$ & $\mathrm{OO}$ & HA & KW & Gloss \\
\hline 15040 & 1614 & $\mathrm{y}^{3} \mathrm{ys}-$ & $\mathrm{y}^{3} \mathrm{ys}-$ & $\mathrm{h}^{3} \mathrm{ys}-$ & $\mathrm{h}^{3} \mathrm{ys}-$ & $\begin{array}{l}(\mathrm{HE} / \mathrm{OO} / \mathrm{KW}) \text { to stay, to remain; } \\
\text { (KW also:) to wait; (HA) to } \\
\text { restore, renew }\end{array}$ \\
\hline 15050 & 1615 & $\begin{array}{l}\mathrm{y}^{3} \mathrm{yx}^{\bullet-} \\
\mathrm{y}^{3} \mathrm{yxh}-\end{array}$ & $\begin{array}{l}\mathrm{y}^{3} \mathrm{yx} \cdot- \\
\mathrm{y}^{3} \mathrm{yxh}-\end{array}$ & $\begin{array}{l}h^{3} y x \cdot- \\
h^{3} y x h-\end{array}$ & $\begin{array}{l}\mathrm{h}^{3} \mathrm{yx} \bullet- \\
\mathrm{h}^{3} \mathrm{yxh}-\end{array}$ & $\begin{array}{l}\text { target or expected event getting } \\
\text { closer, about to happen, almost }\end{array}$ \\
\hline 15060 & 1616 & $\mathrm{y}^{3} \mathrm{yq}-$ & $\mathrm{y}^{3} \mathrm{yq}-$ & $\mathrm{h}^{3} \mathrm{yq}-$ & $\mathrm{h}^{3} \mathrm{yq}^{-}$ & $\begin{array}{l}\text { witchcraft; (HE/OO/HA also:) } \\
\text { modern medicine }\end{array}$ \\
\hline 15070 & 1617 & $\mathrm{y}^{3} \mathrm{y} \chi-$ & $\mathrm{y}^{3} \mathrm{y} x-$ & $\mathrm{h}^{3} \mathrm{y} \chi-$ & $\mathrm{h}^{3} \mathrm{y} \chi-$ & $(?)^{702}$ \\
\hline 15080 & 1618 & $\mathrm{y}^{3} \mathrm{wmh}-$ & $\mathrm{y}^{3} \mathrm{wmh}-$ & & & $\begin{array}{l}\text { to visit for the purpose of getting } \\
\text { a free drink or meal }\end{array}$ \\
\hline 15100 & $\begin{array}{l}1620 \\
1621\end{array}$ & $\begin{array}{l}y^{3} w g v- \\
y^{3} w g v h-\end{array}$ & $\begin{array}{l}y^{3} w g v- \\
y^{3} w g v h-\end{array}$ & $\begin{array}{l}y^{3} w g v- \\
y^{3} w g v h-\end{array}$ & $\begin{array}{l}\mathrm{y}^{3} \mathrm{wgv-} \\
\mathrm{y}^{3} \mathrm{wgvh}-\end{array}$ & rain $^{703}$ \\
\hline 15110 & 1622 & $\mathrm{y}^{3} \chi^{\mathrm{v}-}$ & $\mathrm{y}^{3} \chi^{\mathrm{v}-}$ & $\mathrm{y}^{3} \chi^{\mathrm{v}-}$ & $\mathrm{y}^{3} \chi^{\mathrm{V}-}$ & to rise (tide), to flood \\
\hline 15130 & 1624 & & & & $\mathrm{y}^{3} \mathrm{hs}-*$ & $\begin{array}{l}\text { to spread sth. open, to crack open } \\
\text { (like cut, boil) }\end{array}$ \\
\hline 15150 & 1626 & & & & $\mathrm{y}^{3} \mathrm{hlhq-}$ & to send sb. ${ }^{704}$ \\
\hline 15160 & $\begin{array}{l}1627 \\
1628\end{array}$ & $\begin{array}{l}\mathrm{y}^{3} \mathrm{hk}- \\
\mathrm{y}^{3} \mathrm{hxv}-\end{array}$ & $\begin{array}{l}\mathrm{y}^{3} \mathrm{hk}- \\
\mathrm{y}^{3} \mathrm{hxv}-\end{array}$ & $\begin{array}{l}\mathrm{y}^{3} \mathrm{hk}- \\
\mathrm{y}^{3} \mathrm{hxv}-\end{array}$ & $\begin{array}{l}\mathrm{y}^{3} \mathrm{hk}- \\
\mathrm{y}^{3} \mathrm{hxv}-\end{array}$ & $\mathrm{bad}^{705}$ \\
\hline
\end{tabular}

702 Possibly in HE/OO $\left\{\mathrm{y}^{3} \mathrm{y} \chi \mathrm{nt}-\right\} \mathrm{HA} / \mathrm{KW}\left\{\mathrm{h}^{3} \mathrm{y} \chi \mathrm{nt}-\right\}$ "to menstruate."

${ }^{703}$ In many examples it is not diagnosable which of the two forms the root has. In these cases $\left\{y^{3}\right.$ wgvh- $\}$ is assumed because there is one example in which a locative suffix does diagnosably follow $\left\{\mathrm{y}^{3} \mathrm{wgvh}-\right\}$, cf. HA $\left\{\mathrm{y}^{3} \mathrm{wgvh}-\mathrm{yst} \bullet-\mathrm{kv}\right\}>/ \mathrm{y}^{3} \mathrm{ugv}$ 'aisdakv/ "caught in the rain (without a coat)."

${ }^{704}$ May contain a root $\left\{\mathrm{y}^{3} \mathrm{hlh}-\right\}$ or $\left\{\mathrm{y}^{3} \mathrm{~h} 1-\right\}$.

${ }^{705}$ In all examples of $\left\{\mathrm{y}^{3} \mathrm{hxv}-\right\}$ except one it is followed by a suffix beginning in $\left|\mathrm{p}^{3}\right|$ so that it is not diagnosable if the root ends in $|\mathrm{kv}|$ or $|\mathrm{xv}|$. The exception pointing to $|\mathrm{xv}|$ is $\mathrm{KW} / \mathrm{y}^{3} \mathrm{ol}^{3} \mathrm{\jmath} \mathrm{l} /$ "ugly old (thing or person)." In HA and KW $\left\{\mathrm{y}^{3} \mathrm{hk}-\right\}$ can be substituted for $\left\{\mathrm{y}^{3} \mathrm{hxv}-\right\}$ (not vice versa). Perhaps this is a normalization because $\left\{y^{3} h k-\right\}$ occurs in very many more words than 
North Wakashan Comparative Root List (Updated)

\begin{tabular}{|c|c|c|c|c|c|c|}
\hline Root \# & Old \# & $\mathrm{HE}$ & $\mathrm{OO}$ & HA & KW & Gloss \\
\hline 15170 & $\begin{array}{l}1629 \\
2647\end{array}$ & & $\mathrm{~h}^{3} \mathrm{hr}-$ & & $\begin{array}{l}\mathrm{y}^{3} \mathrm{hr}- \\
\mathrm{h}^{3} \mathrm{hr}-\end{array}$ & $(?)^{706}$ \\
\hline 15200 & 1630 & & & gvd- & gvd-* & $\begin{array}{l}\text { to become separated, } \\
\text { disconnected, untied (like rope), } \\
\text { to become disentangled }\end{array}$ \\
\hline 15201 & 1632 & & & & gvd- & $(?)^{707}$ \\
\hline 15210 & 1631 & kvt- & kvt- & gvt- & $\begin{array}{l}\text { gvt- } \\
\text { kvt-* }\end{array}$ & (?) goldeneye duck 708 \\
\hline 15230 & 1633 & & & & gvt-* & $\begin{array}{l}\text { to scream (from fear, love, } \\
\text { excitement) }\end{array}$ \\
\hline 15240 & $\begin{array}{l}1634 \\
1652\end{array}$ & & & & $\begin{array}{l}\text { gvn-* } \\
\text { gvhn }^{3-}\end{array}$ & 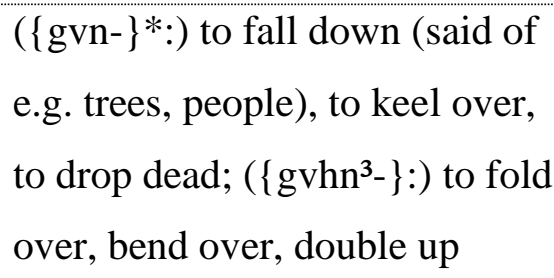 \\
\hline
\end{tabular}

$\left\{\mathrm{y}^{3} \mathrm{hxv}-\right\}$. For HA the examples of $\left\{\mathrm{y}^{3} \mathrm{hxv}-\right\}$ are either from the Kitamaat informant or provided by the Kitlope one with the warning they are from Kitamaat.

${ }^{706}$ In all examples combined with $\{$-hs $\}$ which is suffix no. 2150 and occurs in kinship terms. The combination means "a woman's female friend."

${ }^{707}$ In KW /Gvagvada ${ }^{3} \chi$ ла/ "always potlatching" (proper name).

${ }^{708} \mathrm{KW}\{\mathrm{kvt}-\}^{*}$ is from the Koskimo dialect. Root may really refer to some physical characteristic of goldeneyes and possibly other ducks. The HA consultant referred to some kind of black ducks in the root's examples. Goldeneyes could be confused with black ducks because the back, wings, and tail of the male common goldeneye are black (cf.

http://www.allaboutbirds.org/guide/Common_Goldeneye/id). Male scoters are also black, see http://www.allaboutbirds.org/guide/Black_Scoter/id. HE/OO informants however positively identified goldeneyes, as does Boas (1928:248). 
North Wakashan Comparative Root List (Updated)

\begin{tabular}{|c|c|c|c|c|c|c|}
\hline Root \# & Old \# & $\mathrm{HE}$ & $\mathrm{OO}$ & HA & KW & Gloss \\
\hline 15250 & 1643 & & & & $\begin{array}{l}\text { gvn-/ } \\
\text { gvx- }\end{array}$ & $(?)^{709}$ \\
\hline 15260 & 1643 & & gvnyp- & $\operatorname{gvyn}^{3} \mathrm{p}$ & $\begin{array}{l}\text { gvnp- } \\
\text { gvnyp- }\end{array}$ & (?) to disintegrate, perish ${ }^{710}$ \\
\hline 15270 & 1635 & & & & gvn $\chi-*$ & dentalia $^{711}$ \\
\hline 15280 & $\begin{array}{l}1636 \\
1654\end{array}$ & & gvhs- & & $\begin{array}{l}\text { gvs- } \\
\text { gvhs- }\end{array}$ & $\begin{array}{l}\{\text { gvs- }\}:) \text { to rub, to scrub; to wring } \\
\text { out (washing); }\{\text { gvhs- }\} \text { :) to fray, } \\
\text { chafe, } \text { rub }^{712}\end{array}$ \\
\hline 15290 & 1637 & & & gvf- & gvf- & $\begin{array}{l}\text { (HA) to appropriate, arrogate, } \\
\text { take more than one's share; }(\mathrm{KW}) \\
\text { to keep in reserve, to leave } \\
\text { standing for future use }\end{array}$ \\
\hline
\end{tabular}

${ }^{709}$ Possibly in KW /gviqu/ "embers of a fire" and a proper name with unknown meaning, KW /GvH3alalis/. A HA consultant the name was read to associated it with a Tsimshian term he pronounced as $/ \mathrm{gvH}^{3} \mathrm{a} /$ and which he said means "here!, take it!" ${ }^{710}$ Only examples are KW /gvнpa/ "to go after dry wood" and KW /gvнip/ OO /gvнiћ' M/ HA /gvin $\mathrm{p} /$ "dry, powdery alder wood." The $\mathrm{OO}$ term is a rare example of morphophonemically unpredictable $\mathrm{OO}$ accent. It shows change from $|\mathrm{p}|$ to $\left|\mathrm{m}^{3}\right|$ (as is usual in $\mathrm{HE} / \mathrm{OO}$ word-finally and before an obstruent, cf. $\left\{\mathrm{w}^{3} \mathrm{hp}-\right\}>\left\{\mathrm{w}^{3} \mathrm{hm}^{3}\right\}>/ \mathrm{w}^{3} \mathrm{a}^{3} \mathrm{M} /$ "water") while the subsequent split of $\left|\mathrm{m}^{3}\right|$ into $\left|\hbar^{3} \mathrm{M}\right|$ is precedented in $\mathrm{OO}\{$ mhył- $\chi \mathrm{d}-\mathrm{hlh}\}>/$ mai $^{3} л \chi \mathrm{dala} /$ "lean body sideways (so that one can take away what one is sitting on)." Possibly OO $/ \mathrm{gvHi \hbar}^{31} \mathrm{M} /$ is an adaptation of borrowed $\mathrm{KW} / \mathrm{gvnip} /$. There is however some uncertainty about the KW term. It is listed in Boas (1948) and could be re-elicited except that one consultant pronounced it $/ \mathrm{gvH}^{3} \mathrm{ip} /$ rather than /gvHip/.

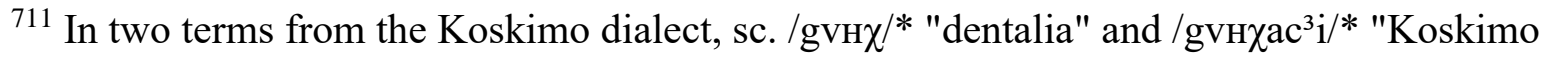
basket for dentalia."

${ }^{712}$ Boas (1948) gives examples of \{gvs- $\}$ also meaning "to put a patch on" but they could not be reelicited. 
North Wakashan Comparative Root List (Updated)

\begin{tabular}{|c|c|c|c|c|c|c|}
\hline Root \# & Old \# & $\mathrm{HE}$ & $\mathrm{OO}$ & HA & $\mathrm{KW}$ & Gloss \\
\hline 15300 & 1638 & & & & gvt- & $(?)^{713}$ \\
\hline 15301 & 1640 & & & & $\begin{array}{l}\text { gvl- } \\
\text { gvls- }\end{array}$ & $(?)^{714}$ \\
\hline 15310 & $\begin{array}{l}1639 \\
1769\end{array}$ & xvlt- & xvlt- & xvlt- & $\begin{array}{l}\text { gvlt- } \\
\text { xvmt- }\end{array}$ & fire, on fire \\
\hline 15330 & 1641 & gvlkv- & gvlkv- & gvlkv- & gvlkv- & $(?)^{715}$ \\
\hline 15340 & 1642 & & & & gvlkv- & $(?)^{716}$ \\
\hline 15350 & 1644 & gvy- & gvy- & gvy- & gvy- & to awaken, wake up \\
\hline 15360 & 1645 & gvyt- & gvyt- & gvyt- & gvyt- & $\begin{array}{l}\text { (HA/HE) Tsimshian; }(\mathrm{OO}) \\
\text { belonging to more than one clan; } \\
(\mathrm{KW}) \text { belonging to other Indian } \\
\text { tribes (esp. Salishan) }\end{array}$ \\
\hline 15370 & & & & & $\begin{array}{l}\text { gvyc-*/ } \\
\text { gvys*- }\end{array}$ & $(?)^{718}$ \\
\hline
\end{tabular}

${ }^{713} \mathrm{In} \mathrm{KW} / \mathrm{gvła} /$ "to lower the price of sth" KW /gvлisbrd/ "to put harpoon point on its shaft." ${ }^{714}$ \{gvl-\} possibly in $\mathrm{KW} / \mathrm{gval}^{3} \mathrm{ac}^{3} \mathrm{i} /$ and $/ \mathrm{gval}^{3} \mathrm{ic}^{3} \mathrm{a} /$ "abalone." $\{$ gvls- $\}$ in $\mathrm{KW} / \mathrm{gvлyadi} /$ "having abalone."

${ }^{715}$ In HA \{[gvh]gvlkv-bhlh\} > /gvagvлxvbal'a/ "hard of hearing" and the HE/OO/HA/KW free stem $\{$ gvlkvm- $\}$ "deaf, hard of hearing." If stem's root \{gvlkv- $\}$ means "deaf" it's unclear what the suffix $\{-\mathrm{m}\}$ means. If the suffix is the one referring to one's head, the root could mean "unyielding, hard to get through."

${ }^{716}$ In words referring to rubbing with sth. rough (e.g. with hemlock branches) or against sth. hard (e.g. with a flint to make fire, on a stone to sharpen sth.). It could be the same root as no. 15330. ${ }^{717}$ One KW consultant indicated the derivate /gvitлa/ does not just refer to foreign Indians tribes but can be derogatory: "slow to understand, stupid."

${ }^{718}$ In KW /gvissmut/* and /gvis.w w $^{3}$ /* both meaning "head sparrow fellow." 
North Wakashan Comparative Root List (Updated)

\begin{tabular}{|c|c|c|c|c|c|c|}
\hline Root \# & Old \# & $\mathrm{HE}$ & $\mathrm{OO}$ & HA & KW & Gloss \\
\hline 15380 & 1646 & gvył- & gvył- & & gvył- & $\begin{array}{l}\text { to dismantle, tear down, pull } \\
\text { apart; (KW also:) to scatter }{ }^{719}\end{array}$ \\
\hline 15390 & 1647 & gvwt- & gvwt- & & & to stack, pile things up \\
\hline 15400 & 1648 & & & & gvq- & to pour out, to throw out a liquid \\
\hline 15410 & 1649 & gvh- & gvh- & gvh- & gvh- & $\begin{array}{l}\text { low, down; (KW also:) } \\
\text { downriver, north }\end{array}$ \\
\hline 15420 & 1650 & $\begin{array}{l}\text { gvh-/ } \\
\text { gvhk-/ } \\
\text { gvhx- }\end{array}$ & & & & $(?)^{720}$ \\
\hline 15430 & 1651 & gvhn ${ }^{3}-$ & gvhn $^{3}-$ & gvhn ${ }^{3}-$ & $\begin{array}{l}\text { gvhnh- } \\
\text { gvhn³- }\end{array}$ & $\begin{array}{l}\text { to relate to a standard of quantity } \\
\text { or quality: to assess, test, judge, } \\
\text { weigh etc.; (HA also:) to restrict, } \\
\text { to control, to ration }\end{array}$ \\
\hline 15460 & 1649 & gvhs- & gvhs- & gvhs- & gvhs- & tears, to weep \\
\hline 15470 & 1653 & $\begin{array}{l}\text { gvh- } \\
\text { gvhs•- }\end{array}$ & gvhs•- & $\begin{array}{l}\text { gvhs•- } \\
\text { gvhsh- } \\
\text { gvhs- }\end{array}$ & $\begin{array}{l}\text { Ivhs•- } \\
\text { rvhsh- } \\
\text { rvhs- }\end{array}$ & (on) this side ${ }^{721}$ \\
\hline 15490 & 1655 & & & & gvhkv- & $\begin{array}{l}\text { discoloured by smoke (like a } \\
\text { pulp mill's cellulose sheets that } \\
\text { look like off-white paper) }\end{array}$ \\
\hline 15500 & 1656 & & & & gvhxv- & sea parsley \\
\hline
\end{tabular}

\footnotetext{
${ }^{719}$ Most KW examples point to the "scatter" sense only.

${ }^{720}$ In HE /gv'ax. $\hbar^{3}$ it/ "to move back suddenly (as a fish when startled)."

721 gvh- $\}$ only in HE /gv'aki ${ }^{3 \prime}$ a/ "on this side of the top of sth. bulky (e.g. a cabin)" and derivates. The KW root may have $|\mathrm{r} v|$ instead of $|\mathrm{gv}|$ due to contamination with the uvular of its antonym KW \{qvys $\bullet\}\left(\mathrm{HE} / \mathrm{OO} / \mathrm{HA}\left\{\chi_{\mathrm{vys}}^{\bullet}-\right\}\right)$ "over there, on that side."
} 
North Wakashan Comparative Root List (Updated)

\begin{tabular}{|c|c|c|c|c|c|c|}
\hline Root \# & Old \# & $\mathrm{HE}$ & $\mathrm{OO}$ & HA & KW & Gloss \\
\hline 15510 & 1657 & & & & gvhq $^{3}$ l- $^{-}$ & $\begin{array}{l}\text { to prevent sth. undesirable (e.g. } \\
\text { fire from going out) }\end{array}$ \\
\hline 15520 & 1658 & & & & kvm- & $\begin{array}{l}\text { to stoop (whole body), to bend } \\
\text { the body down }\end{array}$ \\
\hline 15530 & 1659 & & & kvmt- & kvmt- & $\begin{array}{l}\text { to stoop, to have or move one's } \\
\text { head down }\end{array}$ \\
\hline 15540 & 1660 & kvmc- & & & kvmc- & to stoop, to lean forward \\
\hline 15550 & 1661 & & & & $\begin{array}{l}\text { kvmł- } \\
\text { kvmx- }\end{array}$ & $(?)^{723}$ \\
\hline 15560 & 1662 & & & & $\begin{array}{l}\text { kvdkv- } \\
\text { kvhdkv- }\end{array}$ & continuously, always ${ }^{724}$ \\
\hline 15570 & 1663 & & kvt- & & kvt- & $\begin{array}{l}\text { (KW:) rising low (like hill, } \\
\text { island, floating log), sinking a } \\
\text { small distance, shallow (like } \\
\text { depression, low-sided basket); } \\
\text { (OO possibly:) concave or } \\
\text { convex object }^{725}\end{array}$ \\
\hline
\end{tabular}

722 Only two examples. Stem may be analyzable as $\{$ gvhq- $+1-\}$ but meaning of root and suffix are unknown.

${ }^{723}$ Root may mean "to stoop, move head downward" because grouse spend most of their time on the ground and bob when foraging.

${ }^{724}$ In KW /kvdkva/ "continuously" and two synonymous proper names, KW /Kvdgvisila ${ }^{3} \mathrm{urva} /$ and /Kvadgvisila ${ }^{3} u r v a /$ "always (potlatching) woman."

${ }^{725} \mathrm{OO}$ only in $/ \mathrm{kvti}^{3} \mathrm{a} /$ and $/ \mathrm{kvtqi}^{3} \mathrm{a} /$ "cover, lid," words that are synonymous with $\mathrm{OO} / \mathrm{h}^{3} \mathrm{Mki}^{3} \mathrm{a} /$ and like it contain suffix no. 4070. 
North Wakashan Comparative Root List (Updated)

\begin{tabular}{|c|c|c|c|c|c|c|}
\hline Root \# & Old \# & $\mathrm{HE}$ & $\mathrm{OO}$ & HA & KW & Gloss \\
\hline 15580 & $\begin{array}{l}1664 \\
1669 \\
1676\end{array}$ & $\begin{array}{l}\text { kvn- } \\
\text { kvl- }\end{array}$ & kvn- & $\begin{array}{l}\text { kvn- } \\
k^{3}{ }^{3}\end{array}$ & kvn-* & pungent ${ }^{726}$ \\
\hline 15590 & $\begin{array}{l}1665 \\
1666 \\
1684\end{array}$ & $\begin{array}{l}\text { kvnc- } \\
\text { kvns- }\end{array}$ & $\begin{array}{l}\text { kvnc- } \\
\text { kvns- }\end{array}$ & $\begin{array}{l}\text { kvnc- } \\
\text { kvns- } \\
\text { kvyx- }\end{array}$ & kvns- & $\begin{array}{l}\text { to use steam (e.g. to poultice, } \\
\text { bend wood, take a steam bath, } \\
\text { bake); (KW also:) to steam up, } \\
\text { fog up, cloud over; (HA }\{\mathrm{kvyx}-\} \\
\text { also:) to get steamed up about } \\
\text { sth. }{ }^{727}\end{array}$ \\
\hline
\end{tabular}

${ }^{726}$ Only in (1) the names for mink, (2) $\mathrm{OO} / \mathrm{kvH}^{3} \chi \mathrm{di} / \mathrm{KW} / \mathrm{kvH}^{3} \chi \mathrm{sd} \varepsilon^{3} / *$ "musk bag of animals (e.g. mink)," (3) the HA name for a frog (more likely toad), (4) HA terms deriving from $\left\{\mathrm{kvhn}^{3}-\right\}$ and having to do with yellow cedar. According to http://www.for.gov.bc.ca/hfd/library/documents/treebook/yellowcedar.htm "the inside of yellow cedar bark smells like potatoe skins." A commentator at http://forum.norwoodindustries.com/board/viewtopic.php?f=2\&t=1084 wrote that "the smell of yellow cedar is strong and unique. Pungent. When we would get in the crummy at the end of the shift, you could smell who had cut yellow cedar that day. Very strong odour." The frog connection is problematic because there is a frog that smells like mink and is therefore called mink frog, however, it lives no further west than Manitoba. The mountain yellow-legged frog emits a pungent odor when disturbed but belongs to the Sierra Nevada and southern California (cf. http://earthjustice.org/irreplaceable/mountain-yellow-legged-frog\#). Perhaps the frog the HA term refers to is really a toad. At http://www.kidzone.ws/lw/frogs/facts8.htm one reads "Toad's skin lets out a bitter taste and smell that burns the eyes and nostrils of its predators, much like a skunk does." The form $\{k v l-\}$ instead of $\{k v n-\}$ occurs in HE $/ k v л \chi d ' i /$ which is the counterpart to $\mathrm{OO} / \mathrm{kvH}^{3} \chi \mathrm{di} /$ "musk bag of animals."

${ }^{727} \mathrm{HE} / \mathrm{OO}$ \{kvns- $\}$ only in the word for a steam box and old-fashioned box stove. HA \{kvyx- $\}$ may originate by backformation from examples where $\{\mathrm{kvns}-\}$ is followed by a suffix beginning in $|=|$ and metathesis occurs between $|\mathrm{n}|$ and $|\mathrm{y}|$ (from $|\mathrm{s}-=|$ ), as in $\left\{\mathrm{kvns}-=\mathrm{hc}^{3} \mathrm{y}\right\}>\left\{\mathrm{kvny} \propto-\mathrm{hc} \mathrm{c}^{3} \mathrm{y}\right\}$ $>\left\{\right.$ kvny-hc $\left.^{3} \mathrm{y}\right\}>\{$ kvyn-hc $3 \mathrm{y}\}>/ \mathrm{kvin}^{\prime} \mathrm{ac}^{3} \mathrm{i} /$ "steam box" and $\{\mathrm{kvns}-=\mathrm{kv}\}>\{\mathrm{kvny} \infty-\mathrm{kv}\}>$ 
North Wakashan Comparative Root List (Updated)

\begin{tabular}{|c|c|c|c|c|c|c|}
\hline Root \# & Old \# & $\mathrm{HE}$ & $\mathrm{OO}$ & HA & KW & Gloss \\
\hline 15600 & 1667 & kvnxv- & kvnxv- & kvnxv- & kvnxv- & loud noise, rumbling, thunder \\
\hline 15610 & $\begin{array}{l}1668 \\
1680\end{array}$ & kvnq- & kvnq- & kvnq- & $\begin{array}{l}\mathrm{kvn}^{3} \mathrm{q}- \\
\mathrm{kvn}^{3} \chi-\end{array}$ & $\begin{array}{l}\text { pungent, musky; repugnant, } \\
\text { repulsive; (in most KW } \\
\text { examples:) spoiled, rotten (said } \\
\text { of clams) }\end{array}$ \\
\hline 15630 & $\begin{array}{l}1670 \\
1672\end{array}$ & kvs- & kvs- & kvs- & $\begin{array}{l}\text { kvs- } \\
\text { kvsx॰- } \\
\text { kvsx- } \\
\text { kvsxh- }\end{array}$ & $\begin{array}{l}\text { to splash, spray, wash, bathe } \\
\text { (with water or urine) }\end{array}$ \\
\hline 15650 & 1671 & kvs- & kvs- & kvs- & $\begin{array}{l}\text { kvs- } \\
\mathrm{k}^{3} \mathrm{Vs}- \\
\mathrm{q}^{3} \mathrm{Vs}-\end{array}$ & dark colour (grey, blue, black) ${ }^{729}$ \\
\hline
\end{tabular}

$\{\mathrm{kvyn} \infty-\mathrm{kv}\}>/ \mathrm{kvin} ə \mathrm{kv} /$ "meat steamed in an underground oven." Both these examples are from the Kitamaat consultant; the Kitlope one used /kviniy'ac ${ }^{3} \mathrm{i} /<\left\{\mathrm{kvyx}-=\mathrm{ys}=\mathrm{hc} \mathrm{c}^{3} \mathrm{y}\right\}$ and $/ \mathrm{kviniy}$ 'ukv $/<$ $\{\mathrm{kvyx}-=\mathrm{ys}-=\mathrm{kv}\}$, respectively.

${ }^{728}$ Some examples refer to what informants call frogs but are probably toads. On toads and bad smell see the comment on root no. 15580. Toads "are associated with sickness and disease," cf. p. 145 of Kwakiutl String Figures by Julia P. Averkieva, UBC Press, 1992. Boas's listing of $\left\{\mathrm{kvn}^{3} \chi_{-}\right\}$derivates (1948:299) suggests the root is primarily about a kind of colour but this is likely a misinterpretation.

${ }^{729}$ 1. \{kvs-\} occurs in KW /kvskvs/ "Steller's jay" KW /kvskvssm/ "blue blanket" HE /kvi'uqv/ $\mathrm{HA} / \mathrm{kvi}^{3}$ auqv/ (an unidentified small grey diving sea bird). The Stellar's jay is often mislabelled "bluejay" in the Pacific Northwest; its lower body is blue like a bluejay but its upper body and head are black. The base form $\{\mathrm{kvs}-\}$ and various augmented forms occur across families, see first of all Proto-Salish *-kvas- represented by Sechelt kvəsim "blue" Comox kv'əsim "colour of evening or morning sky" Shushwap kvəses "getting dark" etc., see Kuipers (2002:47). In augmented form and with $|\mathrm{s}|$ replaced by $|\mathrm{s}|$ the root also occurs in South Wakashan, cf. Makah kviškviši Ditidaht xviišxviiš "bluejay" (= Stellar's jay) cited in Fortescue (2007). 2. It is uncertain 
North Wakashan Comparative Root List (Updated)

\begin{tabular}{|c|c|c|c|c|c|c|}
\hline Root \# & Old \# & $\mathrm{HE}$ & $\mathrm{OO}$ & HA & KW & Gloss \\
\hline 15660 & $\begin{array}{l}1673 \\
1970 \\
1971\end{array}$ & kv£m- & $\mathrm{kv£} \bullet-$ & $\begin{array}{l}\mathrm{kv£m-} \\
\mathrm{qv£m-}\end{array}$ & $\begin{array}{l}\mathrm{kv£-*} \\
\mathrm{qv} £-\end{array}$ & $\begin{array}{l}\text { sticking out or hanging down } \\
\text { from a point of attachment (like } \\
\text { roots still attached to a stump or } \\
\text { log, or strips in a tassel); (HA } \\
\text { possibly also:) to stick together } \\
\text { (like group of people or } \\
\text { animals) }\end{array}$ \\
\hline 15670 & 1674 & kvł- & kv1- & & & $\begin{array}{l}\text { pile of things collapses or } \\
\text { disintegrates }\end{array}$ \\
\hline 15680 & 1675 & kvl- & kvl- & kvl- & kvl- & $\begin{array}{l}\text { to lie somewhere, to lie down } \\
\text { (said of animate beings) }\end{array}$ \\
\hline 15690 & 1677 & & & & kvlxv- & greyish, blond, faded \\
\hline 15700 & 1678 & & & & kvk- & $\begin{array}{l}\text { to burst (like boil), to pop (like } \\
\text { berries in mouth) }\end{array}$ \\
\hline 15710 & 1679 & & & & kvk- & $\begin{array}{l}\text { to trip, stumble, fall down } \\
\text { (persons/animals) }\end{array}$ \\
\hline 15720 & $\begin{array}{l}1681 \\
1682\end{array}$ & kvyt- & kvyt- & kvyt- & & $\begin{array}{l}\text { (HE/OO) to break (said of } \\
\text { waves); (HA) to rip, to tear }\end{array}$ \\
\hline 15730 & 1683 & kvys- & kvys- & kvys- & kvys- & to spit \\
\hline 15750 & $\begin{array}{l}1685 \\
1844\end{array}$ & $\begin{array}{l}\text { kvykv-, } \\
\text { wykv- }\end{array}$ & $\begin{array}{l}\text { kvykv-, } \\
\text { wykv- }\end{array}$ & kvykv- & kvykv- & $\begin{array}{l}(\mathrm{kvykv}-\}:) \text { to whistle (with } \\
\text { fingers or like eagle or marmot); } \\
\{\text { wykv-\}:) eagle }\end{array}$ \\
\hline 15760 & 1686 & kvyq- & kvyq- & kvyq- & kvyq-* & $\begin{array}{l}\text { to sting (like wound, pepper), to } \\
\text { scold, to act angrily }\end{array}$ \\
\hline
\end{tabular}

if $\{\mathrm{kvs}-\}$ occurs also in $\mathrm{HE} / \mathrm{kv}^{\prime} \mathrm{ay}^{3} \mathrm{alaqs} / \mathrm{HA} / \mathrm{kvay}^{3} \mathrm{al}$ 'axs/ OO /kvaira/ "Steller's jay." The name could be a foreign borrowing with native suffixes added.

${ }^{730} \mathrm{If}|\mathrm{m}|$ in the forms $\{\mathrm{kv£m}-\}$ and $\{\mathrm{qvfm}-\}$ is suffix no. 490 and means "protruding," it would in all examples but one be followed by the form $\{-\mathrm{rm}\}$ of this same suffix no. 490 . 
North Wakashan Comparative Root List (Updated)

\begin{tabular}{|c|c|c|c|c|c|c|}
\hline Root \# & Old \# & $\mathrm{HE}$ & $\mathrm{OO}$ & $\mathrm{HA}$ & KW & Gloss \\
\hline 15770 & 1687 & $\operatorname{kvy} \chi-$ & kvy $\chi-$ & & kvy $\chi-$ & $\begin{array}{l}\text { (KW) to strike with stick (as in } \\
\text { an attack or to crack open nuts or } \\
\text { clamshells), to murder, to bang } \\
\text { on floor with stick, to beat time; } \\
\text { (HE/OO) to crack open (nuts, } \\
\text { clam shells) }{ }^{731}\end{array}$ \\
\hline 15771 & 1688 & xvwmyt- & kvwmyt- & & & $\begin{array}{l}\text { to move up and down, to move to } \\
\text { and fro, to seesaw, to teeter }\end{array}$ \\
\hline 15780 & 1689 & kvw£- & kvw£- & & & $\begin{array}{l}\text { (HE) to break a branch; }(\mathrm{OO}) \text { to } \\
\text { bend sth. long (e.g. a piece of } \\
\text { wire) }\end{array}$ \\
\hline 15781 & & kvw1- & & & & $(?)^{732}$ \\
\hline 15800 & $\begin{array}{l}1690 \\
1699\end{array}$ & kvhq- & & kvhq- & kvq- & $\begin{array}{l}\text { to split (wood), to part (bushes), } \\
\text { to open up (crack) }\end{array}$ \\
\hline 15810 & 1691 & kvhs- & kvhs- & & kvhs- & $\begin{array}{l}\text { to trample, stamp the feet, push } \\
\text { with the feet; (KW also:) to scorn } \\
\text { sb., to kick sb. out }\end{array}$ \\
\hline 15820 & 1693 & & & & kvh£- & $\begin{array}{l}\text { to decrease (in size or volume, } \\
\text { like berries settling), to become } \\
\text { indistinct (to sight) }\end{array}$ \\
\hline 15830 & 1694 & & & & kvhx- & smoke (from fire or chimney) \\
\hline 15840 & 1695 & kvhkv- & kvhkv- & kvhkv- & kvhkv- & $(?)^{733}$ \\
\hline
\end{tabular}

${ }^{731} \mathrm{HE} / \mathrm{OO} / \mathrm{HA}$ use $\left\{\mathrm{h}^{3} \mathrm{l} \chi-\right\}$ to refer to murdering and beating up.

732 Only in HE $\left.\{[\mathrm{kvh}] \mathrm{kvw}\}-+\chi \lambda-\alpha \mathrm{y}^{3}\right\}>/ \mathrm{kv}^{\prime} \mathrm{akvu}^{3} л \chi \lambda \lambda^{\prime} \mathrm{ai} /$ "one's rear end."

${ }^{733}$ In names of tribal divisions on northern Vancouver Island and the adjacent mainland, and in words referring to the tongue of one subdivision, the Kwakiutl. Boas tried but failed to get to this root's meaning and complains for example on p.112 of The Social Organization of the Kwakiutl 
North Wakashan Comparative Root List (Updated)

\begin{tabular}{|c|c|c|c|c|c|c|}
\hline Root \# & Old \# & $\mathrm{HE}$ & $\mathrm{OO}$ & $\mathrm{HA}$ & KW & Gloss \\
\hline 15850 & $\begin{array}{l}1696 \\
1697 \\
1698 \\
2001 \\
2003 \\
2006\end{array}$ & $\begin{array}{l}\text { kvhxv- } \\
k^{3} v h x v- \\
\text { qvhqv- }\end{array}$ & $\begin{array}{l}\text { kvhxv- } \\
k^{3} v h x v- \\
\text { qvhqv- }\end{array}$ & $\begin{array}{l}\text { kvhxv- } \\
k^{3} v h x v- \\
\text { qvhqv- }\end{array}$ & kvhxv- & $\begin{array}{l}\text { hole, hollow, pit; wind-filled, } \\
\text { lungs, airbag, swelling, pustule }{ }^{734}\end{array}$ \\
\hline 15860 & 1692 & $\begin{array}{l}\mathrm{kvh}^{3} \mathrm{c}-/ \\
\mathrm{kvh}^{3} \mathrm{~s}-\end{array}$ & $\begin{array}{l}\mathrm{kvh}^{3} \mathrm{c}-/ \\
\mathrm{kvh}^{3} \mathrm{~s}-\end{array}$ & & & $(?)^{735}$ \\
\hline 15870 & $\begin{array}{l}1700 \\
1701 \\
1755 \\
1756 \\
1997\end{array}$ & $\begin{array}{l}k^{3} \mathrm{vp}- \\
k^{3} \mathrm{vhp}-\end{array}$ & $\begin{array}{l}\mathrm{k}^{3} \mathrm{vp}- \\
\mathrm{k}^{3} \mathrm{vhp}-\end{array}$ & & $\begin{array}{l}\mathrm{k}^{3} \mathrm{vp}- \\
\mathrm{k}^{3} \mathrm{vhp}-* \\
\text { qvhp- }\end{array}$ & $\begin{array}{l}\text { to make a slit in a layer and rip } \\
\text { one side off, to tear off along a } \\
\text { line (e.g. sheet off a writing } \\
\text { tablet) }{ }^{736}\end{array}$ \\
\hline 15890 & 1702 & $\mathrm{k}^{3} \mathrm{vp}-$ & $\mathrm{k}^{3} \mathrm{vp}-$ & & & (?)muddy 737 \\
\hline
\end{tabular}

(in American Anthropologist - New Series, Vol. 22 No 2 of April - June 1920) that informants derive /Kvagu³ ${ }^{3} /$ "Kwakiutl" folk-etymologically from $\{\mathrm{kvhx}-\}$ "smoke" (root no. 15830). However, a connection with smoke is possible if $\{\mathrm{kvhkv}-\}$ is related historically to $\mathrm{HE} / \mathrm{OO} / \mathrm{HA}$ $\{\mathrm{kvxv}-\} \mathrm{KW}\{\mathrm{kxv}\}$ "to rise or blow out forcefully (hot air, steam, breath, dust, smoke); (KW also:) to give off a smell" (root no. 12450).

${ }^{734} \mathrm{HE} / \mathrm{OO}\{\mathrm{kvhxv}-\}$ only in HE/OO $\left\{\mathrm{kvhw}^{3} \mathrm{t}-\right\}$ "musical instrument."

${ }^{735}$ In HE /kv'ikv'aziłba/ OO /kvikvaziłba/ "ocean bird with a parrot-like beak - probably a puffin."

${ }^{736} \mathrm{OO}\left\{\mathrm{k}^{3} \mathrm{vhp}-\right\}$ only in $/ \mathrm{k}^{3} \mathrm{vapa} /$ "to part because of arguments (as husband and wife)." KW $\left\{\mathrm{k}^{3} \mathrm{vhp}-\right\}^{*}$ in $/ \mathrm{k}^{3}$ vabir $\chi \mathrm{st}^{3} / *$ "lips."

${ }^{737}$ In HE/OO $\left\{\mathrm{k}^{3} \mathrm{vp}^{3} \mathrm{~s}-\right\}$ "mud." 
North Wakashan Comparative Root List (Updated)

\begin{tabular}{|c|c|c|c|c|c|c|}
\hline Root \# & Old \# & $\mathrm{HE}$ & $\mathrm{OO}$ & HA & KW & Gloss \\
\hline 15900 & 1703 & & & $\mathrm{k}^{3} \mathrm{vhm}^{3}-$ & $\begin{array}{l}\mathrm{k}^{3} \mathrm{vm}^{-*} \\
\mathrm{k}^{3} \mathrm{vm}^{3-} \\
\mathrm{k}^{3} \mathrm{wm}^{3-}\end{array}$ & $(?)^{738}$ \\
\hline 15920 & 1705 & $\mathrm{q}^{3} \mathrm{vm}-$ & & $\mathrm{k}^{3} \mathrm{vm}-$ & $\mathrm{k}^{3} \mathrm{vm}-$ & $\begin{array}{l}\text { to become detached, break away } \\
\text { (like in landslide) }{ }^{739}\end{array}$ \\
\hline 15930 & 1706 & $\mathrm{k}^{3} \mathrm{vmt}-$ & $\mathrm{k}^{3} \mathrm{vmt}-$ & $\mathrm{k}^{3} \mathrm{vmt}-$ & $\mathrm{k}^{3} \mathrm{vmt}-$ & $\begin{array}{l}\text { to suck; (HA/KW also:) to inhale } \\
\text { tobacco smoke }\end{array}$ \\
\hline 15940 & $\begin{array}{l}1704 \\
1707\end{array}$ & & & $\mathrm{k}^{3} \mathrm{vms}-$ & $\begin{array}{l}\mathrm{k}^{3} \mathrm{vms}- \\
\mathrm{k}^{3} \mathrm{vmws}-\end{array}$ & $\begin{array}{l}\text { to tighten the opening of a bag or } \\
\text { conduit, to gather material (as in } \\
\text { sewing), to pucker the lips }{ }^{740}\end{array}$ \\
\hline 15950 & $\begin{array}{l}1708 \\
1711\end{array}$ & $\mathrm{k}^{3} \mathrm{vms}-$ & $\mathrm{k}^{3} \mathrm{vms}-$ & $\mathrm{k}^{3} \mathrm{vms}-$ & $\begin{array}{l}\mathrm{k}^{3} \mathrm{vml}- \\
\mathrm{k}^{3} \mathrm{vml}^{3-}\end{array}$ & to char, burn, scorch, go dry \\
\hline 15960 & 1709 & & & & $\mathrm{k}^{3} \mathrm{vm} £-*$ & to shrink back, to draw back \\
\hline 15970 & 1710 & & & $\mathrm{k}^{3} \mathrm{vm} £-$ & & to grow up (child, plant) \\
\hline 15980 & 1712 & & & & $\mathrm{k}^{3} \mathrm{vml}-$ & battledore and shuttlecock game \\
\hline 15990 & 1713 & $\mathrm{k}^{3} \mathrm{vt}-$ & $\mathrm{k}^{3} \mathrm{vt}-$ & $\mathrm{k}^{3} \mathrm{vt}-$ & $\begin{array}{l}\mathrm{k}^{3} \mathrm{vt}- \\
\mathrm{k}^{3} \mathrm{vht}-\end{array}$ & $\begin{array}{l}\text { to stick on, sticky, to stick } \\
\text { together; (KW also:) to infect }{ }^{741}\end{array}$ \\
\hline
\end{tabular}

${ }^{738} \mathrm{In} \mathrm{KW} / \mathrm{k}^{3} \mathrm{VM}^{3} \mathrm{a} /, / \mathrm{k}^{3} \mathrm{um}^{3} \mathrm{a} /$ and $/ \mathrm{k}^{3} \mathrm{VMS}^{3} \mathrm{a} / *$ "bullhead" and in $\mathrm{HA} / \mathrm{k}^{3} \mathrm{vam}^{3} \chi \mathrm{S} /$ "baby bullhead." Uncertain which fish is meant by "bullhead" but it is likely a sculpin. The root could refer to the wide, heavy head of sculpins (cf. http://www.britannica.com/animal/sculpin). The root's various forms could derive from a cross-family stem * $\left\{\mathrm{k}^{3} \mathrm{vwm} \infty-\right\}$, cf. Proto-Salish ${ }^{*}$-q $\mathrm{q}^{3} \mathrm{vum}-[1]$ "skull, head, hair on head" and *-q3vum- [2] "lump, pile, top, high" in Kuipers (2002:97).

${ }^{739} \mathrm{HA}$ only in $/ \mathrm{k}^{3} \mathrm{Vm}$ 'axa/ "to come off, become detached (e.g. boards from a wall)." KW only in $/ \mathrm{k}^{3} \mathrm{vmx} . \hbar^{3} \mathrm{id} /$ "to rush away, to slide (mountain)."

${ }^{740} \mathrm{KW}\left\{\mathrm{k}^{3} \mathrm{vmws}-\right\}$ may have developed via $\left\{\mathrm{k}^{3} \mathrm{vm}[\mathrm{m}] \mathrm{s}-\right\}$ from $\left\{\mathrm{k}^{3} \mathrm{vm}[\infty] \mathrm{s}-\right\}$.

${ }^{741}\left\{\mathrm{k}^{3} \mathrm{vht}-\right\}$ only in $\mathrm{KW} / \mathrm{k}^{3}$ vatлa/ "burrs." 
North Wakashan Comparative Root List (Updated)

\begin{tabular}{|c|c|c|c|c|c|c|}
\hline Root \# & Old \# & $\mathrm{HE}$ & $\mathrm{OO}$ & HA & KW & Gloss \\
\hline 16000 & 1714 & $\mathrm{k}^{3} \mathrm{vt}-$ & $\mathrm{k}^{3} \mathrm{vt}-$ & $\mathrm{k}^{3} \mathrm{vt}-$ & $\mathrm{k}^{3} \mathrm{vt}-$ & $(?)^{742}$ \\
\hline 16010 & 1715 & & & $\mathrm{k}^{3} \mathrm{vt}-$ & $\mathrm{k}^{3} \mathrm{vt}-$ & $(?)^{743}$ \\
\hline 16020 & 1716 & $\mathrm{k}^{3} \mathrm{vn}-$ & $\mathrm{k}^{3} \mathrm{vn}-$ & $\mathrm{k}^{3} \mathrm{vn}-$ & $\mathrm{k}^{3} \mathrm{vn}-*$ & $(?)^{744}$ \\
\hline 16030 & 1717 & $\mathrm{k}^{3} \mathrm{vnc}-$ & $\mathrm{k}^{3} \mathrm{vnc}-$ & $\mathrm{k}^{3} \mathrm{vnc}-$ & & wrinkled \\
\hline 16040 & 1718 & $\mathrm{k}^{3} \mathrm{vnf}-$ & $\mathrm{k}^{3} \mathrm{vnf}-$ & $\mathrm{k}^{3} \mathrm{vnf}-$ & & intestines $^{745}$ \\
\hline 16050 & 1719 & & & & $\mathrm{k}^{3} \mathrm{vnXv-}$ & sunburnt \\
\hline 16060 & $\begin{array}{l}1720 \\
1765\end{array}$ & $\mathrm{k}^{3} \mathrm{vnq}-$ & $\mathrm{k}^{3} \mathrm{vnq}-$ & $\mathrm{k}^{3} \mathrm{vnq-}$ & $\mathrm{k}^{3} \mathrm{vnq}-$ & wet, damp \\
\hline 16070 & 1721 & $\begin{array}{l}\mathrm{k}^{3} \mathrm{vc}-/ \\
\mathrm{k}^{3} \mathrm{vs}-\end{array}$ & $\begin{array}{l}\mathrm{k}^{3} \mathrm{vc}-/ \\
\mathrm{k}^{3} \mathrm{vs}-\end{array}$ & $\begin{array}{l}\mathrm{k}^{3} \mathrm{vc}-/ \\
\mathrm{k}^{3} \mathrm{vs}-\end{array}$ & $\begin{array}{l}\mathrm{k}^{3} \mathrm{vc}-1 \\
\mathrm{k}^{3} \mathrm{VS}-\end{array}$ & leather, hide \\
\hline 16080 & $\begin{array}{l}1722 \\
1723\end{array}$ & $\mathrm{k}^{3} \mathrm{VS}-$ & $\mathrm{k}^{3} \mathrm{vS}-$ & $\mathrm{k}^{3} \mathrm{vS}-$ & $\mathrm{k}^{3} \mathrm{vS}-$ & light (in weight) $)^{76}$ \\
\hline 16090 & 1724 & $\mathrm{k}^{3} \mathrm{vy}-$ & $\mathrm{k}^{3} \mathrm{vy}-$ & & $\mathrm{k}^{3} \mathrm{vS}-$ & $\begin{array}{l}\text { to sit (optionally used instead of } \\
\left\{\mathrm{k}^{3} \mathrm{vh}-\right\} \text { when talking about a } \\
\text { plurality) }\end{array}$ \\
\hline 16091 & & & & & $\mathrm{k}^{3} \mathrm{VS}-$ & $(?)^{747}$ \\
\hline 16100 & 1760 & $\begin{array}{l}\mathrm{k}^{3} \mathrm{vhs}- \\
\mathrm{k}^{3} \mathrm{vhxs}-\end{array}$ & $\begin{array}{l}\mathrm{k}^{3} \text { vhs- } \\
\mathrm{k}^{3} \text { vhxs- }\end{array}$ & $\mathrm{k}^{3} \mathrm{vhs}-$ & $\begin{array}{l}\mathrm{k}^{3} \mathrm{vs}-* \\
\mathrm{k}^{3} \mathrm{vhs}^{*}\end{array}$ & $\begin{array}{l}\text { dish-shaped things piled up, } \\
\text { mussels }\end{array}$ \\
\hline
\end{tabular}

${ }^{742}$ In the name of an unidentified plant called Indian tobacco.

${ }^{743}$ In words referring to a blanket. Boas (1948:304) interprets them as derivates of $\left\{\mathrm{k}^{3} \mathrm{vt}-\right\}$ "to stick on etc." (root no. 15990) but its semantic basis is unclear.

${ }^{744}$ Possibly in $\left\{\mathrm{k}^{3} \mathrm{vnhxv-}\right\}$ "to split off boards."

745 The HA informant specified reference is to the guts of deer or mountain goat, not fish.

${ }^{746}$ Also in $\mathrm{HE} / \mathrm{k}^{3} \mathrm{vs} \Omega \mathrm{S} / \mathrm{OO} / \mathrm{k}^{3} \mathrm{vs}^{3} \mathrm{~s} /$ "wolf." Wolves are said to move across snow as if having little weight. This must be thanks to their large feet with slight webbing between the toes.

${ }^{747}$ Perhaps in KW /k³ vuqv/ [2] "mischievous, proud" 
North Wakashan Comparative Root List (Updated)

\begin{tabular}{|c|c|c|c|c|c|c|}
\hline Root \# & Old \# & $\mathrm{HE}$ & $\mathrm{OO}$ & HA & KW & Gloss \\
\hline 16110 & 1725 & & & & $\mathrm{k}^{3} \mathrm{vl}-$ & $\begin{array}{l}\text { to pluck out (hair, beard, } \\
\text { eyebrows, roots, seaweed) }\end{array}$ \\
\hline 16120 & $\begin{array}{l}1726 \\
1727 \\
1728\end{array}$ & $\mathrm{k}^{3} \mathrm{vl}-$ & $\mathrm{k}^{3} \mathrm{vl}-$ & $\mathrm{k}^{3} \mathrm{vl}-$ & & $\begin{array}{l}\text { to stir, squirm, move slightly; } \\
\text { stop-and-go when swimming }{ }^{748}\end{array}$ \\
\hline 16150 & 1730 & & & $\mathrm{k}^{3} \mathrm{vlp}-$ & & (?) to squirm ${ }^{749}$ \\
\hline 16160 & 1731 & & & & $\mathrm{k}^{3} \mathrm{vlp}-$ & $\begin{array}{l}\text { to pluck off (berries), to tear } \\
\text { apart (meat) }\end{array}$ \\
\hline 16170 & 1732 & & & $\mathrm{k}^{3} \mathrm{vlt}-$ & & $(?)^{750}$ \\
\hline 16180 & 1733 & & & & $\mathrm{k}^{3} \mathrm{vls}-$ & $\begin{array}{l}\text { to take fish off the hook or out of } \\
\text { the net; to take game out of the } \\
\text { trap }\end{array}$ \\
\hline 16190 & 1729 & $\begin{array}{l}k^{3} v^{3} x v-/ \\
k^{3} v^{3} \chi v-\end{array}$ & $\begin{array}{l}\mathrm{k}^{3} \mathrm{vl}^{3} \mathrm{xv}-/ \\
\mathrm{k}^{3} \mathrm{vl}^{3} \chi \mathrm{v}-\end{array}$ & $\begin{array}{l}\mathrm{k}^{3} \mathrm{vlxv}-1 \\
\mathrm{k}^{3} \mathrm{vl} \chi \mathrm{v}-\end{array}$ & & $(?)^{751}$ \\
\hline 16200 & & & & $\mathrm{k}^{3}$ vlq- & & (?) fur-bearing aquatic animal ${ }^{752}$ \\
\hline
\end{tabular}

${ }^{748}$ In words referring to the movements of fish, seal and also probably in the word for land otter because according to the HA (Kitlope) informant a land otter owes its name to being fast when swimming in any direction.

${ }^{749}$ In HA (Kitamaat dialect) /k³ль'м/ "worm."

750 Only in HA $/ \mathrm{k}^{3} \mathrm{v}^{\prime} л \mathrm{k}^{3} \mathrm{v} л \mathrm{t} /$ "wild raspberry."

${ }^{751} \mathrm{In} \mathrm{HE} / \mathrm{k}^{3} \mathrm{vл}^{3 \prime} \mathrm{uн} / \mathrm{OO} / \mathrm{k}^{3} \mathrm{v}{ }^{3} \mathrm{uH} / \mathrm{HA} / \mathrm{k}^{3} \mathrm{v} л \mathrm{unH} /$ "black bud of cottonwood" and derivates; HE $/ \mathrm{k}^{3} \mathrm{vлш}^{3} \mathrm{as} /$ (with two low tones) OО $/ \mathrm{k}^{3} \mathrm{vлш}^{3} \mathrm{as} /$ (with accent on /л/) "northern black cottonwood, balsam poplar." According to http://www.fs.fed.us/wildflowers/plant-of-theweek/populus_balsamifera.shtml "The bark is rough and dark-colored, thus 'black cottonwood.'" The root could be a cross-family one, cf. Proto-Salish *-q33al-/-q3 vay- "to scorch, black, (burn to) ashes; roast, ripen; berry" in Kuipers (2002:95).

${ }^{752}$ In НA $/ \mathrm{k}^{3} \mathrm{v}^{\prime} л \mathrm{q}^{3} \chi \lambda \mathrm{a} /$ "fur seal" and HA $/ \mathrm{k}^{3} \mathrm{v}^{\prime} \mathrm{ak}^{3} \mathrm{vлдq}^{3} \mathrm{a} /$ "to hunt for land otter." 
North Wakashan Comparative Root List (Updated)

\begin{tabular}{|c|c|c|c|c|c|c|}
\hline Root \# & Old \# & $\mathrm{HE}$ & $\mathrm{OO}$ & $\mathrm{HA}$ & KW & Gloss \\
\hline 16210 & 1735 & & & & $\mathrm{k}^{3} \mathrm{vl} \chi-$ & to sight sth. \\
\hline 16211 & 1734 & & & & $\mathrm{k}^{3} \mathrm{v}^{3} \mathrm{k}-$ & (?) to sight, to recognize $e^{753}$ \\
\hline 16220 & $\begin{array}{l}1736 \\
1761\end{array}$ & $\mathrm{k}^{3} \mathrm{vhx}-$ & $\begin{array}{l}\mathrm{k}^{3} \mathrm{vhl-} \\
\mathrm{k}^{3} \mathrm{vhx}-\end{array}$ & $\mathrm{k}^{3} \mathrm{vhx}-$ & $\mathrm{k}^{3} \mathrm{vX}-$ & scum, slime, saliva, mucous \\
\hline 16230 & 1738 & $k^{3}$ vyt- & $\mathrm{k}^{3} \mathrm{vyt}-$ & $\mathrm{k}^{3} \mathrm{vyt}-$ & $k^{3} \mathrm{vyt}-$ & $\begin{array}{l}\text { to pry loose, to pry apart, to use } \\
\text { leverage; (KW also:) barnacles } \\
\text { (by back-formation) }\end{array}$ \\
\hline 16240 & 1739 & $\mathrm{k}^{3}$ vys- & $\mathrm{k}^{3}$ vys- & $\mathrm{k}^{3}$ vys- & $\mathrm{k}^{3} \mathrm{vys}-$ & snow \\
\hline 16250 & 1740 & & & & $\mathrm{k}^{3} \mathrm{vyl-}$ & to feast \\
\hline 16260 & 1741 & $\mathrm{k}^{3} \mathrm{vykv-}$ & $k^{3} v y k v-$ & $\mathrm{k}^{3} \mathrm{vykv-}$ & $\mathrm{k}^{3} \mathrm{vykv-}$ & $\begin{array}{l}\text { to squirm, wriggle, twist, distort, } \\
\text { warp, skew; misaligned (in HA } \\
\text { also:) to sidestep, miss out on sth. }\end{array}$ \\
\hline 16270 & 1742 & $\mathrm{k}^{3} \mathrm{vyxv}-$ & & & $\mathrm{k}^{3} \mathrm{vyxv}-*$ & $(?)^{754}$ \\
\hline 16280 & 1743 & & & $\mathrm{k}^{3} \mathrm{vy} \chi \mathrm{v}-$ & & to smile \\
\hline 16290 & $\begin{array}{l}1744 \\
1752\end{array}$ & $\mathrm{k}^{3} \mathrm{v} \chi-$ & $\mathrm{k}^{3} \mathrm{v} \chi-$ & $\mathrm{k}^{3} \mathrm{vyq}-$ & $\mathrm{k}^{3} \mathrm{v} \chi-$ & $\begin{array}{l}\text { to wink, blink, flash, keep one } \\
\text { eye closed }\end{array}$ \\
\hline 16300 & 1745 & $\mathrm{k}^{3} \mathrm{vyq}-$ & $\mathrm{k}^{3} \mathrm{vyq}-$ & $\mathrm{k}^{3} \mathrm{vyq}-$ & & $\begin{array}{l}\text { to choose, select (as from a list), } \\
\text { elect, set a target, determine } \\
\text { which is best }\end{array}$ \\
\hline 16310 & $\begin{array}{l}1746 \\
1747\end{array}$ & $\mathrm{k}^{3} \mathrm{vy} \chi-$ & $\mathrm{k}^{3} \mathrm{vy} \chi-$ & $\mathrm{k}^{3} \mathrm{vy} \chi-$ & $\mathrm{k}^{3} \mathrm{vy} \chi-$ & $\begin{array}{l}\text { to make arrangements, to } \\
\text { negotiate (deal, marriage), to } \\
\text { agree to sth., to plan; (KW also:) }\end{array}$ \\
\hline
\end{tabular}

\footnotetext{
${ }^{753}$ Possibly in the unanalyzable $\mathrm{KW}$ proper name $/ \mathrm{K}^{3} \mathrm{VI}^{3} \mathrm{k}^{3} \varepsilon \mathrm{S} /$ said to mean "always recognized, prominent."

${ }^{754} \mathrm{In} \mathrm{KW}$ (Koskimo dialect) / $\mathrm{k}^{3} \mathrm{vixva/*}$ "to twist" HE $/ \mathrm{k}^{3} \mathrm{v}^{\prime} \mathrm{ixv} \mathrm{an}^{\prime} \mathrm{i} / \mathrm{KW} / \mathrm{k}^{3} \mathrm{vixvan} \varepsilon^{3 / *}$ "geoduck clam." Root could be an alternant of $\left\{\mathrm{k}^{3} \mathrm{vykv}-\right\}$ (root no. 16260).
} 
North Wakashan Comparative Root List (Updated)

\begin{tabular}{|c|c|c|c|c|c|c|}
\hline Root \# & Old \# & $\mathrm{HE}$ & $\mathrm{OO}$ & $\mathrm{HA}$ & KW & Gloss \\
\hline & & & & & & $\begin{array}{l}\text { to advise, to council; (HE/OO } \\
\text { also:) to request sth., to ask a } \\
\text { favour, to persuade sb. to do } \\
\text { sth. }\end{array}$ \\
\hline 16320 & 1751 & $\mathrm{k}^{3} \mathrm{vq}-$ & $\mathrm{k}^{3} \mathrm{vq}-$ & $\mathrm{k}^{3} \mathrm{vq}-$ & $\mathrm{k}^{3} \mathrm{vq}-$ & $\begin{array}{l}\text { light, bright, daylight; to flash } \\
\text { (with a light or like a bird with } \\
\text { colours) }\end{array}$ \\
\hline 16330 & 1753 & & & & $\mathrm{k}^{3} \mathrm{v} \chi-$ & square \\
\hline 16340 & 1754 & $\mathrm{k}^{3} \mathrm{vh}-$ & $\mathrm{k}^{3} \mathrm{vh}-$ & $\mathrm{k}^{3} \mathrm{vh}-$ & $\mathrm{k}^{3} \mathrm{vh}-$ & $\begin{array}{l}\text { to sit, to be in a fixed position } \\
\text { (said mainly of a single animate } \\
\text { being) }\end{array}$ \\
\hline 16350 & & & & $\mathrm{k}^{3} \mathrm{vh}-$ & & $(?)^{756}$ \\
\hline 16380 & 1757 & & & & $\mathrm{k}^{3} \mathrm{vht}-*$ & to $\mathrm{bet}^{757}$ \\
\hline 16400 & 1759 & $\mathrm{k}^{3} \mathrm{vhs}-$ & & & & $(?)^{758}$ \\
\hline 16410 & & & & & k³hywkv- & $(?)^{759}$ \\
\hline 16420 & 1762 & & & & $\mathrm{k}^{3} \mathrm{vhy}^{3-}$ & rather, quite \\
\hline 16430 & 1763 & & & & $\mathrm{k}^{3} \mathrm{vhxv}-$ & tallow $^{760}$ \\
\hline
\end{tabular}

${ }^{755}$ Boas lists HE and/or OO derivates of $\left\{\mathrm{k}^{3} \mathrm{vy} \chi^{-}\right\}$on p. 252 of Bella Bella Texts that could not be reelicited but point to the root also having the sense "to advise, to counsel" as in KW.

${ }^{756} \mathrm{In} \mathrm{HA} / \mathrm{k}^{3} \mathrm{vaa} /$ "to pay ransom for an enslaved relative."

${ }^{757} \mathrm{In} \mathrm{KW} / \mathrm{k}^{3} \mathrm{vata} / *$ to stake in a bet" and $/ \mathrm{k}^{3} \mathrm{vato} \mathrm{l}^{3} \mathrm{a} /{ }^{*}$ "to bet against each other" (both from the Newettee dialect).

${ }^{758}$ In HE / $\mathrm{k}^{3} \mathrm{v}^{\prime}$ ask ${ }^{3}$ vasa/ "to limp."

${ }^{759} \mathrm{In} \mathrm{KW} / \mathrm{k}^{3}$ vayukvла/ "to shake one's body up and down (dancing)."

${ }^{760} \mathrm{In}$ the place name $\mathrm{KW} / \mathrm{K}^{3} \mathrm{vaxvm} /$ "tallow on face" and two non-reelicitable $\mathrm{KW}$ words, $/ \mathrm{k}^{3} \mathrm{vaxva} / *$ "to rub tallow on" and the place name $/ \mathrm{K}^{3} \mathrm{vaxvлаwad} / *$ (meaning unknown). 
North Wakashan Comparative Root List (Updated)

\begin{tabular}{|c|c|c|c|c|c|c|}
\hline Root \# & Old \# & $\mathrm{HE}$ & $\mathrm{OO}$ & HA & KW & Gloss \\
\hline 16431 & & & & & $\mathrm{k}^{3} \mathrm{vhwh}-*$ & (?) to sever, split off ${ }^{761}$ \\
\hline 16440 & 1764 & & & $\mathrm{k}^{3} \mathrm{vhq}-$ & $\begin{array}{l}k^{3} v^{-} q^{-} \\
k^{3} \mathrm{vh}^{3} q^{-}\end{array}$ & wood (especially cedar) \\
\hline 16450 & 1766 & $\mathrm{k}^{3} \mathrm{vh}^{3} \mathrm{qv}-$ & & & & $(?)^{762}$ \\
\hline 16460 & $\begin{array}{l}1767 \\
2085\end{array}$ & & & & xvp- & hole, cave, hollow \\
\hline 16461 & $\begin{array}{l}1768 \\
1793\end{array}$ & $\begin{array}{l}\mathrm{xvm}- \\
\mathrm{xvhm^{3 } -}\end{array}$ & $\begin{array}{l}\text { xvm- } \\
\mathrm{xvhm}^{3-}\end{array}$ & & & $\begin{array}{l}\text { long pointed thing shoots out } \\
\text { (like bee's stinger, a flame) }\end{array}$ \\
\hline 16470 & 1770 & xvmt- & xvmt- & xvmt- & & $\begin{array}{l}\text { to suck in, siphon, pump, draw in } \\
\text { breath }\end{array}$ \\
\hline 16480 & 1771 & & & $\begin{array}{l}\text { xvmc- } / \\
\text { xvms- }\end{array}$ & xvms- & to sniffle \\
\hline 16490 & $\begin{array}{l}1772 \\
1773\end{array}$ & xvmq- & $\begin{array}{l}\text { xvmqv- } \\
\text { xvmq- }\end{array}$ & xvmq- & & $\begin{array}{l}\text { sharp point penetrating a surface } \\
\text { (as a bee stinging), pointed thing } \\
\text { touching a surface (as in sniff- } \\
\text { kissing); nose; to smell sth. }\end{array}$ \\
\hline 16500 & 1774 & & & & xvt- & $\begin{array}{l}\text { to decrease in size or volume } \\
\text { (like water level in well or berries } \\
\text { settling in basket) }\end{array}$ \\
\hline 16501 & & & & $x v t^{3} q-$ & & $(?)^{763}$ \\
\hline 16502 & & & & & xvn-* & $(?)^{764}$ \\
\hline 16503 & 1776 & & & & xvn£-* & to get faint \\
\hline
\end{tabular}

\footnotetext{
${ }^{761}$ In five words listed in Boas (1948:307) but never yet reelicited.

762 Possibly in HE / $\mathrm{k}^{3} \mathrm{v}^{\prime} \mathrm{ik}^{3} \mathrm{v}^{\prime} \mathrm{aqv} \chi £$ £awa/ "lichen."

763 Only in HA / $\mathrm{xvt}^{3} \mathrm{qa} /$ "tidal wave."

${ }^{764}$ Only in KW /xvHa/* "mud with air in it."
} 
North Wakashan Comparative Root List (Updated)

\begin{tabular}{|c|c|c|c|c|c|c|}
\hline Root \# & Old \# & $\mathrm{HE}$ & $\mathrm{OO}$ & HA & KW & Gloss \\
\hline 16510 & 1775 & xvnys- & xvnys- & xvnys- & & cold spring ${ }^{765}$ \\
\hline 16520 & $\begin{array}{l}1777 \\
1779 \\
1784\end{array}$ & $\begin{array}{l}\text { xvs- } \\
\text { xvhs- } \\
\text { xvh }^{3} \text { s- }^{-}\end{array}$ & $\begin{array}{l}\text { xvs- } \\
\text { xvhs- } \\
\text { xvh }^{3} \text { s- }^{-}\end{array}$ & $\begin{array}{l}\text { xvs- } \\
\text { xvhs- }\end{array}$ & Xvs- & $\begin{array}{l}\text { long thing spans a distance, long } \\
\text { thing sweeps or sways across } \\
\text { (e.g. thread, tree branch), to lean } \\
\text { over; (KW also:) to whip, to beat } \\
\text { with stick; (HA also:) to bob (like } \\
\text { end of sth. that leans over at } \\
\text { intervals, e.g. a seesaw) }\end{array}$ \\
\hline 16540 & 1778 & & & & Xvs- & fort $^{767}$ \\
\hline 16560 & $\begin{array}{l}1780 \\
1781\end{array}$ & xvlp- & xvlp- & xvlp- & xvlp- & $\begin{array}{l}\text { open, gaping, hollow inside, to } \\
\text { cave in }\end{array}$ \\
\hline 16570 & 1782 & & & & xvls- & $\begin{array}{l}\text { to wither, shrivel up; lonely, } \\
\text { longing for sth. }\end{array}$ \\
\hline 16580 & 1783 & xvyqv- & xvyqv- & xvykv- & xvyp- & to whistle (with the lips) ${ }^{768}$ \\
\hline 16590 & 1785 & xvyt- & xvyt- & xvyt- & xvyt- & $\begin{array}{l}\text { (HE/OO/KW) to stick out, } \\
\text { exceed; to handle sth. sticking } \\
\text { out (e.g. by tipping it up), to lever } \\
\text { up; (HA) long thing (e.g. tree) } \\
\text { leans over, to sway, to swing }\end{array}$ \\
\hline 16600 & 1787 & $x \vee y^{3} s-$ & $x v y^{3} s-$ & xvys- & & to whip, whipping movement \\
\hline
\end{tabular}

765 May contain a root $\{x v n-\}$ or $\{$ xvns- $\}$.

${ }^{766}$ Related to this root is HE/OO $\left\{\mathrm{xvy}^{3} \mathrm{~s}-\right\}$ HA $\{\mathrm{xvys}-\}$ (root no. 16600) which refers specifically to whipping.

${ }^{767}$ Boas (1948:379) considers this root a semantic extension of root no. 16520, perhaps because a fort dominates (holds sway over) an area.

768 A cross-family stem, cf. Proto-Salish *-xviw- "to whistle" and the alternation of labial, velar and uvular among its descendants in Kuipers (2002:121). 
North Wakashan Comparative Root List (Updated)

\begin{tabular}{|c|c|c|c|c|c|c|}
\hline Root \# & Old \# & $\mathrm{HE}$ & $\mathrm{OO}$ & HA & KW & Gloss \\
\hline 16610 & 1786 & & & & xvys-* & $(?)^{769}$ \\
\hline 16620 & 1788 & xvył- & xvył- & xvył- & xvył- & $\begin{array}{l}\text { movement in alternating } \\
\text { directions, to pitch (hence: at an } \\
\text { angle; to turn a corner sharply, to } \\
\text { zigzag, to stagger, to lurch, to } \\
\text { wrestle, to reverse directions, to } \\
\text { return, to back out; (HE/OO/HA } \\
\text { also:) to throw to a target, to } \\
\text { drop, to fall) }\end{array}$ \\
\hline 16630 & 1789 & & & & xvyq- & $\begin{array}{l}\text { potlatch gift that is to be } \\
\text { returned }^{771}\end{array}$ \\
\hline 16640 & 1790 & & & & xvyq-* & $\begin{array}{l}\text { streak, marking (with coal or } \\
\text { paint) }\end{array}$ \\
\hline 16650 & 1791 & xvyq- & xvyq- & xvyq- & xvyq-* & $\begin{array}{l}\text { to string a line, to swing a line or } \\
\text { long thing, to throw a rope }\end{array}$ \\
\hline 16651 & & & & & XVW-* & $(\text { onomatopoeic) })^{772}$ \\
\hline 16660 & 1792 & & xvh- & & $\mathrm{xvh}-*$ & $(?)^{773}$ \\
\hline
\end{tabular}

${ }^{769}$ Only in KW /xvisa/* "marriage present (to bride's father)."

${ }^{770}$ On the relationship between movement in alternating directions and just falling see the description of the polysemy of "to pitch" in Webster's Collegiate Dictionary.

${ }^{771} \mathrm{In} \mathrm{KW} / \mathrm{xviqa} / *$ "potlatch gift that is to be returned" $\mathrm{KW}$ /xvaxvirala/ "to return gift due in the potlatch."

${ }^{772} \mathrm{In} \mathrm{KW} / \mathrm{xvuxvuxvu/*} \mathrm{"Raven's} \mathrm{cry} \mathrm{(presaging} \mathrm{poor} \mathrm{salmon} \mathrm{year)."}$

${ }^{773}$ In OO /xvaan ${ }^{3} \mathrm{ia} /$ "a game in which people run around sb." OO /xvaan 3 iazuis/ (an unidentified stellar configuration) KW /xvan ${ }^{3}$ ala/* "tree sways in wind." 
North Wakashan Comparative Root List (Updated)

\begin{tabular}{|c|c|c|c|c|c|c|}
\hline Root \# & Old \# & $\mathrm{HE}$ & $\mathrm{OO}$ & HA & KW & Gloss \\
\hline 16670 & 1794 & Xvht- & Xvht- & Xvht- & Xvht- & $\begin{array}{l}\text { (HE/OO/HA) to rake, to pull sth. } \\
\text { towards oneself with a stick; } \\
(\mathrm{KW}) \text { to } \text { scratch }^{774}\end{array}$ \\
\hline 16680 & $\begin{array}{l}1795 \\
1796 \\
1797\end{array}$ & xvhs- & xvhs- & xvhs- & xvhs- & $\begin{array}{l}\text { poised for moving or acting, on } \\
\text { edge, aroused, excited, agitated, } \\
\text { coming into sight }\end{array}$ \\
\hline 16690 & 1798 & & & xvhł- & xvhł- & crosswise, in crossed directions \\
\hline 16700 & 1799 & & xvhkv- & & xvhkv- & canoe $^{775}$ \\
\hline 16710 & 1800 & & & & xvhkv- & croaking sound \\
\hline 16720 & 1801 & Xvhkv- & xvhkv- & xvhkv- & xvhkv- & to disintegrate, to separate \\
\hline 16730 & 1802 & xvhq- & xvhq- & xvhq- & xvhq- & to scratch, to mark by scratching \\
\hline 16740 & & & & wp- & & $\begin{array}{l}\text { water rising and rippling over } \\
\text { objects, water washes over sth. }\end{array}$ \\
\hline 16760 & 1806 & wn- & wn- & wn- & $\begin{array}{l}\text { wn- } \\
\text { wnk-/ } \\
\text { wnx- }\end{array}$ & $\begin{array}{l}\text { to enter, penetrate, go inside; } \\
\text { (hence:) (HE/OO/HA) to insert a } \\
\text { plug, fill an opening; (KW) to } \\
\text { drill (with auger) }{ }^{776}\end{array}$ \\
\hline 16780 & 1808 & $\begin{array}{l}\text { wny- } \\
\text { wly- }\end{array}$ & $\begin{array}{l}\text { wn- } \\
\text { wny- }\end{array}$ & wny- & wly-* & $\begin{array}{l}\text { to go on, to proceed, to go } \\
\text { ahead }^{777}\end{array}$ \\
\hline
\end{tabular}

774 The only KW example is /xvata/ "to scratch swelling to relieve pressure."

${ }^{775} \mathrm{OO}$ only in / $\mathrm{xvak}^{3} \mathrm{vHa} /$ "a type of canoe fit for one or two persons." Could be a dialect borrowing from $\mathrm{KW}$.

$776\{w n k / x-\}$ only in $\mathrm{KW}\left\{\mathrm{wn}[\mathrm{wh}] \mathrm{k} / \mathrm{x}-. \mathrm{m}^{3} \mathrm{wt}\right\}>/ \mathrm{wHwax} \cdot \mathrm{m}^{3} \mathrm{ut} /$ "leftovers from drilling" (alternant of $\left.\mathrm{KW} / \mathrm{wHWam}^{3} \mathrm{ut} /\right)$.

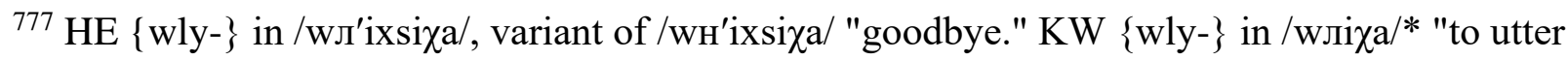
war-cry." 
North Wakashan Comparative Root List (Updated)

\begin{tabular}{|c|c|c|c|c|c|c|}
\hline Root \# & Old \# & $\mathrm{HE}$ & $\mathrm{OO}$ & HA & KW & Gloss \\
\hline 16790 & 1806 & & & & $\begin{array}{l}\text { wnmt- } \\
\text { whnm- }\end{array}$ & immobile, dead \\
\hline 16800 & $\begin{array}{l}1805 \\
1867\end{array}$ & $\begin{array}{l}\text { wn- } \\
\text { whn'3- }\end{array}$ & $\begin{array}{l}\text { wn- } \\
\text { whn }^{3-}\end{array}$ & $\begin{array}{l}\text { wn- } \\
\text { whn }^{3-}\end{array}$ & $\begin{array}{l}\text { wn- } \\
\text { whn'- }\end{array}$ & $\begin{array}{l}\text { white, milky (like the sea when } \\
\text { the herring release their milt), } \\
\text { herring }\end{array}$ \\
\hline 16810 & 1809 & wnc- & wnc- & wnc- & wns- & $\begin{array}{l}\text { sunken, submerged, floating } \\
\text { deep, soaked; (HA also:) at low } \\
\text { level (as one's mood) }\end{array}$ \\
\hline 16820 & $\begin{array}{l}1810 \\
1812\end{array}$ & & & & $\begin{array}{l}\text { wnt- } \\
\text { wnx- }\end{array}$ & $\begin{array}{l}\text { (?) impressive, incredible, } \\
\text { excessive }^{778}\end{array}$ \\
\hline 16830 & 1811 & & & & wnł-* & $(?)^{779}$ \\
\hline 16860 & 1814 & & & & wn $\chi v^{-}$ & $(?)^{780}$ \\
\hline 16870 & $\begin{array}{l}1815 \\
1832\end{array}$ & wnq- & wnq- & wnq- & $\begin{array}{l}\text { wnq- } \\
\text { wlq-* }\end{array}$ & $\begin{array}{l}\text { (KW) deep (eyes, water etc.), } \\
\text { deep inside sth.; (HE/OO/HA) } \\
\text { deep in the water, low in the } \\
\text { water (like boat) }\end{array}$ \\
\hline 16871 & 1816 & wnq $^{3}-$ & wnq $^{3}-$ & wnq $^{3}-$ & $\left(b^{3}-\right)$ & $\begin{array}{l}\text { down, under, below, lower, at } \\
\text { bottom, deep down }\end{array}$ \\
\hline 16872 & 1807 & $\mathrm{w}^{3} \mathrm{n}^{3} \mathrm{~h} 1-$ & $\mathrm{w}^{3} \mathrm{n}^{3} \mathrm{~h} 1-$ & $\begin{array}{l}\mathrm{w}^{3} \mathrm{nh} 1- \\
\mathrm{w}^{3} \mathrm{n}^{3} \mathrm{~h} 1-\end{array}$ & wn ${ }^{3} \mathrm{~h} 1-$ & $\begin{array}{l}\text { physical or mental disturbance } \\
\text { (as when feeling one is being }\end{array}$ \\
\hline
\end{tabular}

778 In KW /whwhłxəs/ "wonderful, incredible" (also name of Cannibal society) KW /whwhxəs/ "grand, great, huge, big, wonderful (superlative)" KW /wHwhxəsa/ "to overdo, do sth. to extremes." The root forms $\{$ wnt- $\}$ and $\{w n x-\}$ are both uncertain.

779 In KW /whła/* "damp ground."

${ }^{780} \mathrm{In} \mathrm{KW} / \mathrm{wH} \chi \mathrm{va} /$ which refers to oil passing through one's body. Unclear if root is related to that referring to scorching and burning (no. 4940).

${ }^{781}$ Few HE/OO/HA examples. Some KW derivates of \{wnq- $\}$ correspond to HE/OO/HA derivates of $\left\{\mathrm{wnq}^{3}-\right\}$. 
North Wakashan Comparative Root List (Updated)

\begin{tabular}{|c|c|c|c|c|c|c|}
\hline Root \# & Old \# & $\mathrm{HE}$ & $\mathrm{OO}$ & HA & KW & Gloss \\
\hline & & & & & & $\begin{array}{l}\text { touched, having a presentiment } \\
\text { or being drunk, dazed) }{ }^{782}\end{array}$ \\
\hline 16880 & 1893 & $\begin{array}{l}\text { wc-l } \\
\text { ws- }\end{array}$ & & $\begin{array}{l}\text { wc-l } \\
\text { ws- }\end{array}$ & & $(?)^{783}$ \\
\hline 16881 & 1820 & & & & $\begin{array}{l}\mathrm{wc}-* / \\
\mathrm{ws}-*\end{array}$ & $(?)^{784}$ \\
\hline 16890 & 1817 & & & & ws-* & $(?)^{785}$ \\
\hline 16900 & 1819 & & & ws- & ws- & $\begin{array}{l}\text { to roll, to twist (for spinning or } \\
\text { removing debris) }\end{array}$ \\
\hline 16920 & 1803 & & & & wsdys- & up landwards from the beach ${ }^{786}$ \\
\hline 16930 & 1821 & & & & $\begin{array}{l}\text { w£- } \\
\text { wh£- }\end{array}$ & $\begin{array}{l}\text { to question, to ask; to hear } \\
\text { (speech or any sound) }\end{array}$ \\
\hline 16940 & 1822 & $\mathrm{w} £-$ & w£- & $\mathrm{w} £-$ & $\mathrm{w} £-$ & to have antlers or horns \\
\hline 16941 & & & & & $\begin{array}{l}\mathrm{w} £-* / \\
\mathrm{w}\}-*\end{array}$ & $(?)^{787}$ \\
\hline 16950 & 1823 & w1- & w1- & & w1- & $\begin{array}{l}\text { without help, unspported, without } \\
\text { or as if without cause, }\end{array}$ \\
\hline
\end{tabular}

${ }^{782}$ Stem may contain a root HE/OO/HA $\left\{w^{3} n-\right\} \mathrm{KW}\{w n-\}$ or HE/OO/HA $\left.\left\{w^{3} n\right\}-\right\}$ KW $\{$ wnt- $\}$. HA derivates with both initial $/ \mathrm{w}^{3} \mathrm{H} /$ and $/ \mathrm{w}^{3} \mathrm{H}^{3} /$ have been recorded.

${ }^{783}$ Possibly in HE (Klemtu dialect) /wz'ik/ HA /wzik/ "moose." The HA informant said the term is onomatopoeic. The 1980 root list mistakenly writes the term with / $\mathrm{w}^{3} /$ instead of $/ \mathrm{w} /$.

${ }^{784}$ In KW /wZHris/* "mud."

785 In KW /wsali/* "small barnacle."

${ }^{786} \mathrm{In} \mathrm{KW}\left(\mathrm{N}^{3} \mathrm{ak}^{3} \mathrm{vaxda}^{3} \chi \mathrm{v}\right.$ dialect) /wsdisлах. $\hbar^{3 \mathrm{id}} /$ "to carry up from beach." This is suffix no. 4430 used as a root.

${ }^{787}$ In KW /włra/* glossed by Boas or his editor as "a diving sea bird, loon" and "kind of spoon of Haida." 
North Wakashan Comparative Root List (Updated)

\begin{tabular}{|c|c|c|c|c|c|c|}
\hline Root \# & Old \# & $\mathrm{HE}$ & $\mathrm{OO}$ & HA & KW & Gloss \\
\hline & & & & & & $\begin{array}{l}\text { effortlessly; to try one's luck, to } \\
\text { venture; in vain }\end{array}$ \\
\hline 16960 & $\begin{array}{l}1826 \\
1875\end{array}$ & wl- & wl- & wl- & $\begin{array}{l}\text { wl- } \\
\text { whl }^{3}- \\
\mathrm{w}^{3} \mathrm{hl}^{3}-\end{array}$ & $\begin{array}{l}\text { stopped, stalling, arrested, } \\
\text { confined, imprisoned }^{788}\end{array}$ \\
\hline 16970 & 1827 & & & & wlt-* & bear grease \\
\hline 16980 & $\begin{array}{l}1828 \\
1829\end{array}$ & & wlk- & & $\begin{array}{l}\text { wlk- } \\
\text { wlx- }\end{array}$ & $\begin{array}{l}\text { to encircle, constrain, contain, } \\
\text { bind, tie up }\end{array}$ \\
\hline 16990 & 1824 & wlyqv- & wlyqv- & wlyqv- & wly ${ }^{3} \chi v-*$ & baby seal ${ }^{789}$ \\
\hline 17000 & 1830 & wlxv- & wlxv- & wlxv- & wlxv-* & $\begin{array}{l}\text { before other things, with priority, } \\
\text { at short notice }\end{array}$ \\
\hline 17010 & 1831 & wl $\chi v-$ & wl $\chi \mathrm{v}-$ & & wl $\chi v-$ & to use a love charm \\
\hline 17020 & 1833 & wlq- & wlq- & wlq- & & afraid, scared \\
\hline 17030 & 1834 & wlq- & wlq- & & $\mathrm{wl} \chi^{-*}$ & $\begin{array}{l}\text { to encircle, constrain, contain, } \\
\text { bind, tie up }\end{array}$ \\
\hline 17040 & 1835 & & & wk- & wk- & $\begin{array}{l}\text { to break off (said of crumbly or } \\
\text { friable things) }\end{array}$ \\
\hline 17041 & $\begin{array}{l}1862 \\
1907 \\
2502 \\
2619\end{array}$ & $\begin{array}{l}\text { wh- } \\
\text { why- } \\
\text { hhy- } \\
\text { h}^{3} \text { wh- } \\
\text { w }^{3} \text { w- }\end{array}$ & $\begin{array}{l}\text { ww- } \\
\text { wh- } \\
\text { why- } \\
\text { hhy- }\end{array}$ & $\begin{array}{l}\text { wy- } \\
\text { wh- } \\
h^{3} w w-\end{array}$ & $\begin{array}{l}\text { wy- } \\
\text { wh- } \\
\text { why- }\end{array}$ & $\begin{array}{l}\text { 1. proceed!, go ahead!, to affirm, } \\
\text { to say Yes; 2. (interjection } \\
\text { marking the beginning or } \\
\text { termination of an act or a train of } \\
\text { thought, and to capture or }\end{array}$ \\
\hline
\end{tabular}

788 The alternant $\left\{\mathrm{w}^{3} \mathrm{hl}^{3}-\right\}$ may be the result of confusing the augmented form $\left\{\mathrm{whl}^{3}-\right\}$ of $\{\mathrm{wl}-\}$ with the stem $\left\{\mathrm{w}^{3} \mathrm{~h}-\mathrm{l}^{3}-\right\}$ "distance (e.g. length, height, depth, radius, scope), limit of a course." Boas (1948) only lists examples of $\left\{\mathrm{w}^{3} \mathrm{hl}^{3}-\right\}$, not $\left\{\mathrm{whl}^{3}-\right\}$.

${ }^{789} \mathrm{In} \mathrm{HE} /$ wл'iqv/ OO/НА /wліqv/ KW /wлі $\chi{ }^{3}$ /* "baby seal." It could contain a root \{w1- \}, $\{w l-\}$ or $\{w l s-\}$, and it could be monomorphemic $\left\{\right.$ wlqv $\left./ \chi v^{-}\right\}$with a $|y|$ or $\left|y^{3}\right|$ augment. 
North Wakashan Comparative Root List (Updated)

\begin{tabular}{|c|c|c|c|c|c|c|}
\hline Root \# & Old \# & $\mathrm{HE}$ & $\mathrm{OO}$ & HA & KW & Gloss \\
\hline & & & $\begin{array}{l}\text { hhw- } \\
h^{3} \text { wh- }\end{array}$ & & & $\begin{array}{l}\text { maintain the listener's attention:) } \\
\text { well!, well now!, behold! }{ }^{790}\end{array}$ \\
\hline 17050 & 1836 & wy- & wy- & wy- & wy- & $\begin{array}{l}\text { to reach for the limit, to fail to } \\
\text { reach the limit, to come up short, } \\
\text { to lack }\end{array}$ \\
\hline 17060 & 1836 & wy- & wy- & wy- & wy- & to lift off, to rise \\
\hline 17070 & 1837 & & & & wyp-* & $(?)^{791}$ \\
\hline 17080 & 1838 & $\begin{array}{l}\text { wynh- } \\
\text { wyn } \infty-\end{array}$ & $\begin{array}{l}\text { wynh- } \\
\text { wynœ- }\end{array}$ & $\begin{array}{l}\text { wynh- } \\
\text { wyn } \infty-\end{array}$ & $\begin{array}{l}\text { wynh- } \\
\text { wyn } \infty-\end{array}$ & $\begin{array}{l}\text { to raid, attack, make war; to } \\
\text { challenge; in turmoil }\end{array}$ \\
\hline 17090 & 1839 & wys- & wys- & wys- & wys- & male, forceful \\
\hline 17100 & 1840 & wy£- & wy£- & wy£- & wy£- & $\begin{array}{l}\text { weak, feeble; to get the better of } \\
\text { sb., to overpower; to overcome } \\
\text { difficulty }\end{array}$ \\
\hline 17110 & 1841 & & & & wy£- & $(?)^{792}$ \\
\hline 17120 & 1842 & & & & wy1- & cedar \\
\hline 17121 & & & & & whylyq- & $\begin{array}{l}\text { to zigzag (around the village to } \\
\text { announce termination of the } \mathrm{KW} \\
\left./ \mathrm{c}^{3} \varepsilon q a /\right)^{793}\end{array}$ \\
\hline
\end{tabular}

${ }^{790} \mathrm{HE}\left\{\mathrm{w}^{3} \mathrm{w}-\right\}$ only in $\left\{\mathrm{w}^{3} \mathrm{w}-\chi-\mathrm{h}\right\}>/ \mathrm{w}^{3} \mathrm{u} \chi \mathrm{va} /$ "to set free etc." and HA $\left\{\mathrm{h}^{3} \mathrm{ww}-\right\}$ only in $\left\{\mathrm{h}^{3} \mathrm{ww}-\right.$ $\chi-h\}>/ h^{3}$ uu $\chi v a /$ "free (of debt, obligations)." HA may have $\left\{\mathrm{h}^{3} \mathrm{ww}-\right\}$ in this latter instead of just $\left\{\mathrm{h}^{3} \mathrm{w}-\right\}$ to distinguish $/ \mathrm{h}^{3} \mathrm{uu} \chi \mathrm{va} /$ from HA $/ \mathrm{h}^{3} \mathrm{u} \chi \mathrm{va} /$ "also, likewise, too" which presumably contains the root $\left\{\mathrm{h}^{3} \mathrm{w}-\right\}$ (no. 17720) followed by $\{\# \chi \mathrm{h}\}$ (suffix no. 5450).

791 Only in KW /wipa/* "to redden (skin, by heat of fire)"

792 Only in KW /wi£a/ "to pack on the back."

${ }^{793}$ Stem may have structure $\{w[h] y k-\% y q-\}$ or $\{[w h] w y k-\% y q-\}$ but unclear what the root \{wyk- could mean. The suffix would be no. 4020 of the suffix catalogue. Boas (1948:68) lists the stem without attempting to analyze. 
North Wakashan Comparative Root List (Updated)

\begin{tabular}{|c|c|c|c|c|c|c|}
\hline Root \# & Old \# & $\mathrm{HE}$ & $\mathrm{OO}$ & HA & KW & Gloss \\
\hline 17130 & 1843 & $\mathrm{wyl}^{3}-$ & & $\mathrm{wyl}^{3}-$ & & beach \\
\hline 17131 & & & & & wyk- & weak, moribund \\
\hline 17140 & $\begin{array}{l}1836 \\
1905\end{array}$ & $w^{3} y x-$ & wyx- & & wyx-* & $(?)^{794}$ \\
\hline 17160 & 1845 & & wyqv- & & wyqv- & $\begin{array}{l}\text { (OO) to slip, slide, skate; (KW) } \\
\text { to shove a long thing; to praise, } \\
\text { ennoble (i.e. push forward), to } \\
\text { talk about one's deeds }\end{array}$ \\
\hline 17180 & 1847 & wy $\chi-$ & wy $\chi-$ & & & to lie on one's back, to lean back \\
\hline 17181 & 1885 & & & & $\begin{array}{l}\mathrm{wy}^{3} \chi^{-} \\
\mathrm{wh}^{3} \chi^{-}\end{array}$ & to lift \\
\hline 17190 & $\begin{array}{l}1818 \\
1848\end{array}$ & Wws- & WWs- & & $\begin{array}{l}\text { WS- } \\
\text { Wws- }\end{array}$ & $\begin{array}{l}\text { (HE/OO) scared, frightened; } \\
(\mathrm{KW}) \text { pity, sympathy }{ }^{795}\end{array}$ \\
\hline 17200 & 1849 & ww£- & & & & $(?)^{796}$ \\
\hline 17210 & 1850 & ww1- & Wwł- & $\left(h^{3} w p-\right)$ & & to heap up, rise, uprising, riot \\
\hline 17220 & $\begin{array}{l}1851 \\
2496\end{array}$ & WWW- & WwW- & $\begin{array}{l}\text { ww- } \\
\text { hw- }\end{array}$ & $\begin{array}{l}\text { ww- } \\
\text { hw- } \\
\text { hww-* }\end{array}$ & $\begin{array}{l}\text { Ooh! (onomatopoeia expressing } \\
\text { fear, discomfort or awe) } \text { ) }^{797}\end{array}$ \\
\hline
\end{tabular}

$794\left\{w^{3} \mathrm{yx}-\right\}$ in $\mathrm{HE} / \mathrm{w}^{3}$ ay'ax/ "skirt, woman's garment in general." \{wyx- $\}$ may occur in unanalyzable $\mathrm{OO} /$ wawixiaqs/ "woman with power" and \{wyk-\} in non-reelicitable KW /wawekila/* "skirt."

795 KW \{ws- $\}$ only in $\{[w y] w s-y l h\}>$ /wi:wsila/ "to desire sympathy."

796 Possibly in HE /w'u£ª M/ "club."

${ }^{797} \mathrm{KW}\{$ hww- \} would be diagnosable only in KW (Newettee dialect) /huhagila/* "warn against danger, notify" (from $\{$ hww-h-gylh $\}$, with telescoping of $|w w|)$. 
North Wakashan Comparative Root List (Updated)

\begin{tabular}{|c|c|c|c|c|c|c|}
\hline Root \# & Old \# & $\mathrm{HE}$ & $\mathrm{OO}$ & HA & KW & Gloss \\
\hline 17230 & $\begin{array}{l}1852 \\
1853\end{array}$ & wwqv- & wwqv- & hwqv- & hwqv- & $\begin{array}{l}\text { to spill out of a place (hence 1. to } \\
\text { vomit; } 2 \text {. to move in a group (like } \\
\text { people) }\end{array}$ \\
\hline 17250 & 1854 & & wq- & wq- & wq- & frog, toad; to croak \\
\hline 17260 & 1855 & & & & wq-* & $(?)^{798}$ \\
\hline 17270 & 1857 & & & wq- & wq-* & $\begin{array}{l}\text { to move in one direction, to go in } \\
\text { a school }\end{array}$ \\
\hline 17290 & $\begin{array}{l}1858 \\
2501\end{array}$ & $\mathrm{w} \chi-$ & $\mathrm{w} \chi-$ & $\mathrm{w} \chi-$ & $\begin{array}{l}\mathrm{w} \chi- \\
\mathrm{hw} \chi \mathrm{v}^{-}\end{array}$ & to split, crack, shatter, break off \\
\hline 17300 & 1859 & & $\mathrm{w} \chi-$ & & & $(?)^{799}$ \\
\hline 17310 & 1860 & $\mathrm{w} \chi-$ & & & & $(?)^{800}$ \\
\hline 17330 & $\begin{array}{l}1861 \\
1863\end{array}$ & wh- & wh- & wh- & wh- & river, stream, flowing water \\
\hline 17340 & 1864 & wht- & wht- & wht- & wht- & $\begin{array}{l}\text { to pull or drag using hands; (KW } \\
\text { also:) to lead by the hand }\end{array}$ \\
\hline 17350 & 1865 & wht- & wht- & wht- & wht- & $\begin{array}{l}\text { kelp (probably Nereocystis } \\
\text { luetkeana, known by many } \\
\text { everyday names including e.g. } \\
\text { giant kelp and bladder wrack } \\
\text { kelp) }\end{array}$ \\
\hline
\end{tabular}

${ }^{798} \mathrm{In} \mathrm{KW} / \mathrm{wa}: \mathrm{wq}^{3} \mathrm{a}^{3} \mathrm{ayu} / *$ "to eat what is left over after feast, to beg for remains of feast, to eat with wife."

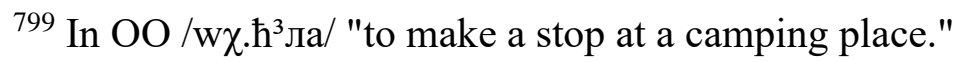

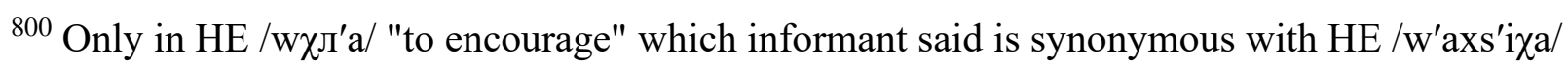
$\left(<\left\{w h \# x s^{\prime} y-\chi-h\right\}\right)$.

${ }^{801}$ Uncertain if this is the root referring to pulling (no. 17340). Kelp was used for making fishing nets and harvested by pulling it into the canoe. 
North Wakashan Comparative Root List (Updated)

\begin{tabular}{|c|c|c|c|c|c|c|}
\hline Root \# & Old \# & $\mathrm{HE}$ & $\mathrm{OO}$ & HA & KW & Gloss \\
\hline 17360 & $\begin{array}{l}1868 \\
1869\end{array}$ & whs- & & whs- & whs-* & $\begin{array}{l}\text { (?) muscular tension, force, } \\
\text { agitation; forceful, unbending } 802\end{array}$ \\
\hline 17370 & 1871 & whs- & whs- & whs- & whs- & fish eggs, to spawn ${ }^{803}$ \\
\hline 17380 & 1870 & & & & $\begin{array}{l}\text { whz-* } \\
\text { whs-* } \\
\text { whłqy- } \\
\text { whłqys-* }\end{array}$ & $(?)^{804}$ \\
\hline 17390 & 1872 & wh1- & wht- & wht- & wht- & $\begin{array}{l}\text { thought, deliberation, discourse, } \\
\text { content of one's words }\end{array}$ \\
\hline 17400 & $\begin{array}{l}1804 \\
1866 \\
1872 \\
1873\end{array}$ & & $\begin{array}{l}\text { w1- } \\
\text { wht- }\end{array}$ & wht- & wht- & $\begin{array}{l}\text { directionless, uncoordinated, } \\
\text { undecided, misdirected; } \\
\text { unserious, unreal, imagined only, } \\
\text { deceitful, wishful, wished for }{ }^{805}\end{array}$ \\
\hline 17410 & 1874 & & & whł- & wh1-* & (?) smelly ${ }^{806}$ \\
\hline 17420 & 1876 & whk- & whk- & whk- & whk- & $\begin{array}{l}\text { to bend sth., to be bent or ring- } \\
\text { shaped, to be around sth. }\end{array}$ \\
\hline 17430 & 1877 & whk- & whk- & whk- & $\begin{array}{l}\text { wwkv- } \\
w^{3} w^{3} k-\end{array}$ & to bark (dog, wolf), to woof ${ }^{808}$ \\
\hline
\end{tabular}

${ }^{802}$ Probably also in KW \{whyhd- \} "ruthlessness, disregard to danger or consorts."

${ }^{803}$ Most examples are about herring spawn in particular, especially in HE/OO/KW.

${ }^{804}$ In a small number of words referring to hunger, food and marriage presents that are mentioned by Boas but can be neither analyzed nor re-elicited, with the partial exception of KW $\{[$ wh]whłqy-hlh $\}>$ /wawałqela/ "to give presents (man to son-in-law in marriage feast)." ${ }^{805} \mathrm{OO}$ only in /włkin ${ }^{3} \mathrm{i} /$, /włcin $3 \mathrm{i}$ /, /wałkin ${ }^{3} \mathrm{i} /$ "child" (endearing term) which matches KW /wawałkin $\varepsilon^{3 /}$ "child" (endearing term meaning "one wished for"). ${ }^{806}$ In KW words referring to the land otter or its musk bag; HA possibly in /wałp"as/ "flavour." ${ }^{807}$ Possibly also in KW /wagiga/ "fisher (mammal)." ${ }^{808}$ Also in HE/OO $\left\{w_{h k}^{3} p-\right\}$ "to hiccup." 
North Wakashan Comparative Root List (Updated)

\begin{tabular}{|c|c|c|c|c|c|c|}
\hline Root \# & Old \# & $\mathrm{HE}$ & $\mathrm{OO}$ & HA & KW & Gloss \\
\hline 17440 & 1878 & whx- & whx- & whx- & whx- & $\begin{array}{l}\text { to attempt (without knowing the } \\
\text { outcome), to try one's luck, in } \\
\text { vain, for nothing }\end{array}$ \\
\hline 17450 & 1879 & whkv- & & & & to try; nevertheless, although \\
\hline 17460 & 1880 & & & whxv- & whxv-* & water hemlock \\
\hline 17470 & 1881 & whxv- & whxv- & & whxv-* & to fart noisily \\
\hline 17480 & $\begin{array}{l}1856 \\
1882 \\
1883\end{array}$ & whqv- & whqv- & $\begin{array}{l}\text { whqv- } \\
\text { wq- }\end{array}$ & $\begin{array}{l}\text { whqv- } \\
\text { whq- }\end{array}$ & $\begin{array}{l}\text { ringed in, encircled (as when } \\
\text { wrapped in a blanket, wearing a } \\
\text { cape); to go in circle from one } \\
\text { point to another (like rainbow); } \\
\text { to bridge a gap } \\
\text { 809 }\end{array}$ \\
\hline 17490 & 1883 & & & & whqv- & $\begin{array}{l}\text { to run (said of a colour); washing } \\
\text { is soiled or discoloured }\end{array}$ \\
\hline 17500 & & & & & wh $\chi v-$ & (?) (perhaps onomatopoeic) $)^{810}$ \\
\hline 17510 & & wh $\chi-$ & wh $\chi-$ & wh $\chi-$ & wh $\chi-$ & gully, trough, trench, grave \\
\hline 17520 & 1884 & & & & $w^{3}{ }^{3}-$ & $\begin{array}{l}\text { to help, to do a favour, to be so } \\
\text { good as to, please }\end{array}$ \\
\hline 17540 & 1887 & & & & $w^{3} p^{-}$ & to fall into a hole 811 \\
\hline 17550 & 1888 & & & & $\mathrm{w}^{3} \mathrm{~d}-$ & cold, cool \\
\hline
\end{tabular}

${ }^{809} \mathrm{HA}\{\mathrm{wq}-\}$ possibly in /wr'ari/ "ring tied to an ooligan net and put on a stick so as to keep the net in place." Perhaps the ring is the wooden hoop around the bag net. The hoop is anchored to poles driven into the river bed and thus helps keep the net stable in the river mouth.

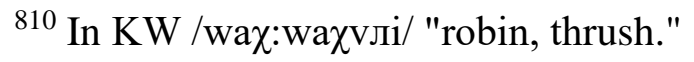

${ }^{811} \mathrm{In} \mathrm{KW} / \mathrm{w}^{3} \mathrm{pa} /$ "to fall into a hole" and $\mathrm{KW} / \mathrm{w}^{3} \mathrm{aw}^{3} \mathrm{ap}^{3} \mathrm{ay}{ }^{3} \mathrm{u} /$ "hole dug and covered so that game falls in." 
North Wakashan Comparative Root List (Updated)

\begin{tabular}{|c|c|c|c|c|c|c|}
\hline Root \# & Old \# & $\mathrm{HE}$ & OO & HA & KW & Gloss \\
\hline 17560 & 1889 & $w^{3} n-$ & $w^{3} n-$ & $\begin{array}{l}\mathrm{w}^{3} \mathrm{n}- \\
\mathrm{w}^{3} \mathrm{ynh}- \\
\mathrm{w}^{3} \mathrm{yn} \infty- \\
\mathrm{w}^{3} \mathrm{hnh}- \\
\mathrm{w}^{3} \mathrm{hn} \infty-\end{array}$ & $\begin{array}{l}w^{3} n- \\
w^{3} h^{3}-\end{array}$ & $\begin{array}{l}\text { to act inconspicuously or } \\
\text { stealthily, to hide oneself, to } \\
\text { sneak about, to stalk }\end{array}$ \\
\hline 17570 & $\begin{array}{l}1890 \\
1926\end{array}$ & $\mathrm{w}^{3} \mathrm{nk}-$ & $\mathrm{w}^{3} \mathrm{nk}-$ & $w^{3}$ nk- & $\mathrm{w}^{3} \mathrm{~h} £-$ & lover, sweetheart \\
\hline 17580 & 1891 & & & & $\mathrm{w}^{3} \mathrm{nxv}-*$ & $(?)^{812}$ \\
\hline 17590 & 1892 & $\begin{array}{l}w^{3} n x v- \\
w^{3} w n w- \\
w^{3} w_{n} w^{3}-\end{array}$ & & & & $(?)^{813}$ \\
\hline 17600 & 1894 & $\mathrm{w}^{3} \mathrm{~s}-$ & $\mathrm{w}^{3} \mathrm{~S}-$ & $\mathrm{w}^{3} \mathrm{~S}-$ & $\begin{array}{l}\mathrm{w}^{3} \mathrm{~s}- \\
\mathrm{w}^{3} \mathrm{xS}-\end{array}$ & fur, hair; hide; belt \\
\hline 17610 & 1895 & & & & $\mathrm{w}^{3} \mathrm{~s}-*$ & to store away for future use \\
\hline 17620 & 1896 & $\mathrm{w}^{3} l-$ & $\mathrm{w}^{3} 1-$ & $w^{3} l-$ & & $\begin{array}{l}\text { to use branches (e.g. for } \\
\text { camouflaging or to gather herring } \\
\text { eggs) }\end{array}$ \\
\hline 17630 & 1897 & & & & $\mathrm{w}^{3} 1-*$ & $\begin{array}{l}\text { to bend wood into a one-piece } \\
\text { box }\end{array}$ \\
\hline 17631 & & & & & w3lwp-* & to eat fresh roasted ooligan \\
\hline 17640 & 1898 & & & & $w^{3} \mathrm{x}-$ & to beg for food \\
\hline
\end{tabular}

\footnotetext{
${ }^{812} \mathrm{In} \mathrm{KW} / \mathrm{w}^{3} \mathrm{nxva} / *$ "to hide."
}

$813\left\{\mathrm{w}^{3} \mathrm{nXv}-\right\}$ occurs only in $\mathrm{HE} / \mathrm{w}^{3 \prime} \mathrm{HXv}^{\prime} \mathrm{il}^{\prime} \mathrm{akv} /$ "cured mountain goat meat." This term has a synonym $/ \mathrm{w}^{3 \prime} \mathrm{un}^{\prime} \mathrm{u} / \mathrm{which}$ is difficult to analyze; the available diagnostic derivates point to its stem being $\left\{w^{3} w n w-\right\}$ and $\left\{w^{3} w_{n} w^{3}-\right\}$. The relationship between $\left\{w^{3} n x v-\right\}$ and $\left\{w^{3} w n w-\right.$, $\left.\mathrm{W}^{3} \mathrm{Wnw}^{3}-\right\}$ is reminiscent of that between $\{£ \mathrm{mxv}-\}^{*}$ and $\{£ \mathrm{hmw}-\}$ "perch (fish)." 
North Wakashan Comparative Root List (Updated)

\begin{tabular}{|c|c|c|c|c|c|c|}
\hline Root \# & Old \# & $\mathrm{HE}$ & $\mathrm{OO}$ & HA & KW & Gloss \\
\hline 17650 & 1899 & $\begin{array}{l}\mathrm{w}^{3} \mathrm{y}- \\
\mathrm{w}^{3} \mathrm{ys}-\end{array}$ & $w^{3} y-$ & $\begin{array}{l}w^{3} y_{-}^{-} \\
w^{3} y s^{-}-\end{array}$ & $w^{3} y-$ & $\begin{array}{l}\text { where, when, how, to what } \\
\text { degree, which one } \\
814\end{array}$ \\
\hline 17660 & & & & & $\mathrm{w}^{3} \mathrm{yp}-$ & $(?)^{815}$ \\
\hline 17670 & 1901 & $\left.\mathrm{w}^{3} \mathrm{y}\right\}_{-}$ & $\mathrm{w}^{3} \mathrm{y} 1-$ & $\left.\mathrm{w}^{3} \mathrm{y}\right\}_{-}$ & $\mathrm{w}^{3} \mathrm{y} 1-$ & $\begin{array}{l}\text { thin (said of sth. tall such as tree } \\
\text { or person) }\end{array}$ \\
\hline 17680 & $\begin{array}{l}1902 \\
2641\end{array}$ & $\left(h^{3} h g-\right)$ & $\left(h^{3} h g-\right)$ & $\left(h^{3} h g-\right)$ & $\begin{array}{l}\mathrm{w}^{3} \mathrm{yl}^{3}- \\
\mathrm{wyl}{ }^{3} \mathrm{~h}-\end{array}$ & all, everything, everybody ${ }^{816}$ \\
\hline 17690 & 1903 & $\mathrm{w}^{3} \mathrm{yk}-$ & $\mathrm{w}^{3} \mathrm{yk}-$ & $\mathrm{w}^{3} \mathrm{yk}-$ & $\mathrm{w}^{3} \mathrm{yk}-$ & $\begin{array}{l}\text { to lift a load onto the shoulder } \\
\text { and carry it }{ }^{817}\end{array}$ \\
\hline 17700 & 1904 & $w^{3} y^{\bullet} \bullet-$ & $\mathrm{w}^{3} \mathrm{yx} \bullet-$ & $\begin{array}{l}w^{3} y_{x-}^{-} \\
w^{3} y^{\bullet-}\end{array}$ & & slow, delayed, delaying \\
\hline 17710 & 1846 & $\mathrm{w}^{3} \mathrm{yq}-$ & $\mathrm{w}^{3} \mathrm{yq}-$ & $\mathrm{h}^{3} \mathrm{wyq}-$ & $\begin{array}{l}h^{3} y^{3} q v-/ \\
h^{3} y^{3} \chi v-\end{array}$ & $\begin{array}{l}\text { devil's club (Oplopanax horridus, } \\
\text { a.k.a. Fatsia horrida) })^{818}\end{array}$ \\
\hline 17711 & 1900 & $\mathrm{w}^{3} \mathrm{yw} £-$ & $\mathrm{w}^{3} \mathrm{yw} £-$ & & & early morning \\
\hline 17720 & $\begin{array}{l}1886 \\
1906 \\
1914 \\
1929\end{array}$ & $\begin{array}{l}\mathrm{W}^{3} \mathrm{~W}- \\
\mathrm{W}^{3} \mathrm{hwh}- \\
\text { rVw- }\end{array}$ & $\begin{array}{l}\mathrm{W}^{3} \mathrm{~W}- \\
\mathrm{W}^{3} \mathrm{hwh}- \\
\text { rVw- }\end{array}$ & $\begin{array}{l}h^{3} \mathrm{w}- \\
\mathrm{h}^{3} \mathrm{hwh}- \\
\mathrm{w}^{3} \mathrm{hwh}-\end{array}$ & $\begin{array}{l}h^{3} \mathrm{w}- \\
\mathrm{h}^{3} \mathrm{hwh}- \\
\mathrm{h}^{3} \mathrm{hw}^{3}-\end{array}$ & $\begin{array}{l}\text { instance of, satisfying a } \\
\text { description; real, actual, true; } \\
\text { straight away, undelayed, direct, } \\
\text { abrupt }^{819}\end{array}$ \\
\hline
\end{tabular}

${ }^{814} \mathrm{HE}\left\{\mathrm{w}^{3} \mathrm{ys}-\right\}$ only in $/ \mathrm{w}^{3}$ 'istu'ala/ "to concern, be the business of."

815 Only in $\mathrm{KW} / \mathrm{w}^{3} \mathrm{ip} /$ "cry of the KW /hMs:hmc ${ }^{3} \mathrm{~s} / *$ (= a member of one of the lower-ranked Cannibal societies)."

${ }^{816}$ If it is not diagnosable if a suffix is attached to the bound root $\left\{\mathrm{wyl}^{3^{3}}\right\}$ or the free stem $\left\{\mathrm{w}^{3} \mathrm{yl}^{3} \mathrm{~h}-\right\}$, attachment to $\left\{\mathrm{w}^{3} \mathrm{yl}^{3}-\right\}$ is assumed.

${ }^{817}$ In some of Boas's KW examples the load is more particularly a long stiff thing.

${ }^{818} \mathrm{HE} / \mathrm{OO}$ root misrecorded as $\{$ wyq- $\}$ in 1980 root list.

819 1. In many examples this root is followed by a locative or somatic suffix and serves as a prop to make a word with the meaning of the suffix. This is also the case in the only example of

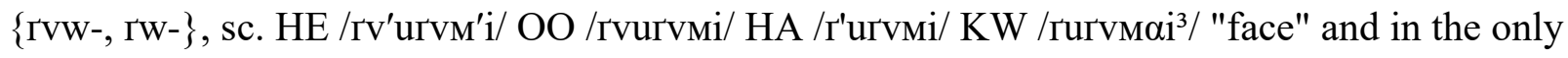


North Wakashan Comparative Root List (Updated)

\begin{tabular}{|c|c|c|c|c|c|c|}
\hline Root \# & Old \# & $\mathrm{HE}$ & $\mathrm{OO}$ & HA & KW & Gloss \\
\hline & $\begin{array}{l}1947 \\
2052 \\
2606 \\
2645\end{array}$ & $\begin{array}{l}q^{3} \mathrm{VW}- \\
\mathrm{h}^{3} \mathrm{~W}-\end{array}$ & $\begin{array}{l}q^{3} v w- \\
h^{3} w-\end{array}$ & $\begin{array}{l}\mathrm{rW}- \\
\mathrm{q}^{3} \mathrm{~W}-\end{array}$ & $\begin{array}{l}\mathrm{rW}- \\
\mathrm{q}^{3} \mathrm{~W}-\end{array}$ & \\
\hline 17730 & 1908 & $\mathrm{w}^{3} \mathrm{wt}-$ & $\mathrm{w}^{3} \mathrm{wt}-$ & $\mathrm{h}^{3} \mathrm{wt}-$ & $\mathrm{h}^{3} \mathrm{wt}-$ & $\begin{array}{l}\text { to prick, pierce, pin, perforate; } \\
\text { (KW also:) to vaccinate }\end{array}$ \\
\hline 17740 & 1909 & $\begin{array}{l}\mathrm{w}^{3} \mathrm{wz}- \\
\mathrm{w}^{3} \mathrm{wzh}- \\
\mathrm{w}^{3} \mathrm{w}^{3} \mathrm{zh}- \\
\mathrm{h}^{3} \mathrm{wc} \cdot-\end{array}$ & $\begin{array}{l}w^{3} w z- \\
w^{3} w z h- \\
h^{3} w z h-\end{array}$ & $\begin{array}{l}h^{3} w Z- \\
h^{3} w z h- \\
h^{3} w Z w- \\
h^{3} w^{3} z_{-}^{-}\end{array}$ & $\mathrm{h}^{3} \mathrm{wzh}-$ & $\begin{array}{l}\text { to go wrong, suffer misfortune, } \\
\text { have trouble, give trouble }{ }^{820}\end{array}$ \\
\hline 17750 & 1910 & $\mathrm{~W}^{3} \mathrm{wlh}-$ & $\mathrm{w}^{3}$ wlh- & $\mathrm{w}^{3} \mathrm{wlh}-$ & $\mathrm{h}^{3} \mathrm{wlh}-$ & $(?)^{821}$ \\
\hline
\end{tabular}

certain example of $\left\{q^{3} v-, q^{3} w-\right\}$, sc. $H E / q^{3} v^{\prime} u^{3} v^{\prime} u^{3} i / O O / q^{3} v q^{3} v^{3} i / H A / q^{3} u^{3} v^{3} H^{3} / K W$ $/ q^{3} \mathrm{uq}^{3} \mathrm{un}^{3} \mathrm{i} /$ "neck, throat." 2. The senses of this root overlap with those of the set $\{y-$, h-, yy- yh-, hy-, etc. $\}$ (root no. 14380). 3. HE/OO $\left\{\mathrm{h}^{3} \mathrm{w}-\right\}$ occurs in $\mathrm{HE} / \mathrm{h}^{3 \prime} \mathrm{um} / \mathrm{OO} / \mathrm{h}^{3} \mathrm{um} /$ "father" and related words, and in $\left\{\mathrm{h}^{3} \mathrm{~W}- \pm \chi £ \bullet-\right\}$ "to carry on the back." 4. HE/OO $\left\{\mathrm{w}^{3} \mathrm{w}-\right\}$ is used instead of $\left\{h^{3} m-\right\}$ "one, unit" (root no. 23230) before $\left\{-p^{3} n-\right\}$ "times" or one of the many derivatives of $\left\{-p^{3} n-\right\}$, see suffix no. 220 ff. 5. Root no. $17820\left(\left\{w^{3} h-\right.\right.$, etc. $\left.\}\right)$ is probably just an alternant of this one.

${ }^{820} \mathrm{HE}\left\{\mathrm{h}^{3} \mathrm{wc} \bullet-\right\}$ OO $\left\{\mathrm{h}^{3} \mathrm{wzh}-\right\}$ occur in a proper name that is likely a $\mathrm{KW}$ borrowing. Note that in all HE/OO examples where $|\mathrm{z}|$ of $\left\{\mathrm{w}^{3} \mathrm{wz}-\right\}$ becomes /c/ due to being followed by an obstruent, the root could also have the form of $\left\{\mathrm{w}^{3} \mathrm{Wc} \bullet-\right\}$ and that the only actual evidence for $\left\{\mathrm{w}^{3} \mathrm{WZ}-\right\}$ as opposed to $\left\{\mathrm{w}^{3} \mathrm{wzh}-\right\}$ are the two high tones of $/{ }^{\prime} \mathrm{al}$ 'a $/$ in $\left\{\mathrm{w}^{3} \mathrm{wz}-\mathrm{hlh}\right\}>\mathrm{HE} / \mathrm{w}^{3} \mathrm{uz}$ 'al'a/ "to have bad luck, etc." HA $\left\{h^{3} w Z w-\right\}$ is assumed to occur in $\left\{h^{3} w Z w-\Delta ł-k^{3} h n h\right]$ and $\left\{h^{3} w z w-\Delta ł-k^{3} h n y^{3}\right\}$ "(to have a) hand or arm that hurts/is sprained" and $\left\{\mathrm{h}^{3} \mathrm{WZW}-\Delta \mathfrak{l}-\mathrm{sys}-\mathrm{h}\right\}$ "foot or leg that hurts." ${ }^{821} \mathrm{HE} / \mathrm{OO}$ in $\mathrm{HE} / \mathrm{w}^{3 \prime} \mathrm{ul}^{\prime} \mathrm{a} / \mathrm{OO} / \mathrm{w}^{3} \mathrm{ula} /$ "to visit a sick person." $\mathrm{KW}$ in words referring to expecting and waiting for sth. Root may also occur in $\mathrm{HE} / \mathrm{w}^{3 /} \mathrm{ul}$ 'ala/ OO / $\mathrm{w}^{3} \mathrm{ulala}$ / (name of a dance) HA / $w^{3} \mathrm{ul}$ 'ala/ "hemlock branch dance (second dance in the HA /h'ilikла/ series)" KW (Newettee dialect) $/ h^{3}$ ulala/* (a ceremonial). 
North Wakashan Comparative Root List (Updated)

\begin{tabular}{|c|c|c|c|c|c|c|}
\hline Root \# & Old \# & $\mathrm{HE}$ & $\mathrm{OO}$ & HA & KW & Gloss \\
\hline 17760 & 1911 & $\mathrm{w}^{3} \mathrm{wgv-}$ & $\mathrm{w}^{3} \mathrm{wgV-}$ & $\mathrm{h}^{3} \mathrm{wgV}-$ & $\mathrm{h}^{3} \mathrm{wgV}-$ & different, other, separate, private \\
\hline 17761 & & $\mathrm{w}^{3} \mathrm{wkV}-$ & $\mathrm{w}^{3} \mathrm{wkV}-$ & $\left(h^{3} y g h-\right)$ & & to store away, to save ${ }^{822}$ \\
\hline 17770 & & $\mathrm{~W}^{3} \mathrm{WW}-$ & $\mathrm{W}^{3} \mathrm{WW}-$ & & $\mathrm{h}^{3} \mathrm{~W}-$ & (onomatopoeic) \\
\hline 17780 & 1912 & & $\mathrm{w}^{3} \mathrm{wqV}-$ & & & $(?)^{823}$ \\
\hline 17790 & $\begin{array}{l}2618 \\
2646\end{array}$ & $\begin{array}{l}\mathrm{w}^{3} \mathrm{wqv}- \\
\mathrm{h}^{3} \mathrm{wqv}-\end{array}$ & $\begin{array}{l}\mathrm{w}^{3} \mathrm{wqv-} \\
\mathrm{h}^{3} \mathrm{wqv}-\end{array}$ & $\mathrm{h}^{3} \mathrm{wqV}-$ & $\begin{array}{l}\mathrm{h}^{3} \mathrm{wqv}-* \\
\mathrm{~h}^{3} \mathrm{~h} \chi \mathrm{v}-\end{array}$ & $(?)^{824}$ \\
\hline 17810 & 1915 & $\mathrm{w}^{3} \mathrm{~h}^{3} \mathrm{q}^{3} \mathrm{vh}-$ & $w^{3} h^{3} q^{3} v h-$ & $w^{3} h^{3} q^{3} v-$ & $\begin{array}{l}w q^{3} v- \\
w^{3} q v- \\
w^{3} q v h-\end{array}$ & sibling of opposite sex ${ }^{825}$ \\
\hline
\end{tabular}

${ }^{822} \mathrm{HE} / \mathrm{OO}$ only in $\left.\left\{\mathrm{w}^{3} \mathrm{wkv}-=\mathrm{y}\right\}-\mathrm{h}\right\}>\mathrm{HE} / \mathrm{w}^{3}$ ugviła/ OO / $\mathrm{w}^{3} \mathrm{ugviła} /$ "to put sth. away indoors." ${ }^{823} \mathrm{In} \mathrm{OO} / \mathrm{w}^{3}$ uqvла/ "to throw away property at one's daughter's wedding."

${ }^{824}$ In the names of flying squirrel (a kind of bat), hellebore and jellyfish. It is not diagnosable if $\mathrm{HE} / \mathrm{OO}$ has $\left\{\mathrm{h}^{3} \mathrm{wqv}-\right\}$ or $\left\{\mathrm{h}^{3} \mathrm{w} \chi \mathrm{v}^{-}\right\}$; the former is assumed because the HA root diagnosably has |qv|. The hellebore terms probably refer to white hellebore (Veratrum viride), also known as false or Indian hellebore. It is toxic and was used for medicinal and ritual purposes, cf. Compton (1993: 80, 123, 184, 201-203 and passim). What links a flying squirrel to jellyfish and hellebore is unclear. It could be a parachute-like appearance in the case of flying squirrel and jellyfish but judging from pictures the bowl- or saucer-shape of hellebore flowers is not reminiscent of a parachute. The connection may concern the supernatural.

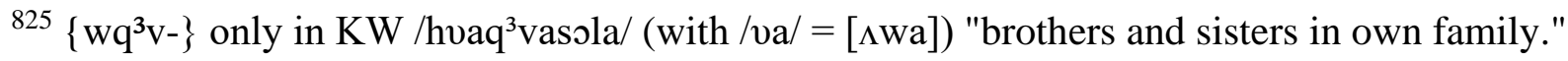
$\left\{\mathrm{w}^{3} \mathrm{qv}-\right\}^{*}$ only in $/ \mathrm{w}^{3} \mathrm{rvad} / *$ "having a sibling of opposite sex" which term Boas says is from the dialect of the /Zawada ${ }^{3}$ inu $\chi \mathrm{v} / .\left\{\mathrm{w}^{3} \mathrm{qvh}-\right\}$ only in $\mathrm{KW} / \mathrm{w}^{3} \mathrm{qvad} /$ "having a sibling of opposite sex." The KW example $/ \mathrm{w}^{3} \mathrm{q}^{3} \mathrm{vu}^{3} \mathrm{t} /$ "former sibling of opposite sex"/ is the only available evidence for KW $\left\{\mathrm{w}^{3} \mathrm{q}^{3} \mathrm{v}-\right\}$ beside $\left\{\mathrm{w}^{3} \mathrm{q}^{3} \mathrm{vh}-\right\}$. Contamination with $\left\{\mathrm{w}^{3} \mathrm{~h}^{3} \chi \mathrm{v}-\right\}$ "group of people" (as e.g. in HE $/ w^{3} a \chi v^{\prime} u y^{\prime} a / O O / w^{3} a^{3} \chi v u y a /$ "male's brothers") may have changed an original HE/OO/HA root $\left\{w^{3} q^{3} v h-\right\}$ into $\left\{w^{3} h^{3} q^{3} v-\right\}$. 
North Wakashan Comparative Root List (Updated)

\begin{tabular}{|c|c|c|c|c|c|c|}
\hline Root \# & Old \# & $\mathrm{HE}$ & $\mathrm{OO}$ & HA & KW & Gloss \\
\hline & & & & & $\begin{array}{l}w^{3} q^{3} v- \\
w^{3} q^{3} v h-\end{array}$ & \\
\hline 17820 & 1917 & $\begin{array}{l}w^{3} h- \\
w^{3} h w^{3}-\end{array}$ & $\begin{array}{l}w^{3} h- \\
w^{3} w h w^{3} \\
-\end{array}$ & $\begin{array}{l}\mathrm{w}^{3} \mathrm{~h}- \\
\mathrm{h}^{3} \mathrm{ywh}- \\
\mathrm{h}^{3} \mathrm{hwh}- \\
\mathrm{w}-\end{array}$ & $\begin{array}{l}\mathrm{w}^{3} \mathrm{~h}- \\
\mathrm{wh}^{3}- \\
\mathrm{w}^{3}- \\
\mathrm{h}^{3} \mathrm{w}- \\
\mathrm{h}^{3} \mathrm{hwh-}\end{array}$ & $\begin{array}{l}\text { 1. (all forms except HE } \\
\left\{w^{3} h\left[w^{3}\right]-\right\} \text { HA }\left\{h^{3} y w h-\right\} \text { OO } \\
\left.\left\{w^{3} w_{h} w^{3}-\right\}:\right) \text { quantity, size, } \\
\text { measure, dimension; } \\
\text { 2. (HE }\left\{w^{3} h w^{3}-\right\} \text { HA }\left\{h^{3} y w h-\right\} \\
\left.\text { OO }\left\{w^{3} w h w^{3}-\right\}:\right) \text { equal in size, } \\
\text { measure, dimensions, direction }{ }^{826}\end{array}$ \\
\hline 17840 & $\begin{array}{l}1916 \\
1928\end{array}$ & $\begin{array}{l}\mathrm{w}^{3} \mathrm{~h}- \\
\mathrm{w}^{3} \mathrm{~h}^{3} \mathrm{xS} \bullet- \\
\mathrm{w}^{3} \mathrm{hx} \mathrm{S}^{\bullet-}\end{array}$ & $\begin{array}{l}\mathrm{w}^{3} \mathrm{~h}- \\
\mathrm{w}^{3} \mathrm{~h}^{3} \mathrm{xS} \bullet- \\
\mathrm{w}^{3} \mathrm{hxs} \bullet-\end{array}$ & $h^{3} y^{3} s \bullet-$ & $\begin{array}{l}\mathrm{w}^{3} \mathrm{hxs}- \\
\mathrm{w}^{3} \mathrm{hxs} \mathrm{s}^{-}\end{array}$ & duality 827 \\
\hline
\end{tabular}

8261 . HE $\left\{w^{3} h w^{3}-\right\}$ is $\left\{w^{3} h-\right\}$ with reduplication of the initial element: $\left\{w^{3} h\left[w^{3}\right]-\right\} .2$. In some examples HE $\left\{\mathrm{w}^{3} h \mathrm{w}^{3}-\right\}$ alternates with $\left\{\mathrm{w}^{3} \mathrm{~h}-\mathrm{hw} \mathrm{w}^{3}\right\}$ in which $\{-\mathrm{hw}\}$ is suffix no. 4230. For "equal in size etc." OO only uses $\left\{\mathrm{w}^{3} \mathrm{w}-\right\}$ plus this suffix no. 4230; to prevent a useless proliferation of records the combination is conveniently written $\left\{w^{3} w h w^{3}-\right\}$ when HE has $\left\{w^{3} h w^{3}\right\}$ but $\left\{w^{3} w-h w^{3}-\right\}$ when HE has $\left\{w^{3} h-h w^{3}-\right\}$. It is unclear how HA $\left\{h^{3} y w h-\right\}$ and $\left\{h^{3} h w h-\right\}$ derive from $\left\{w^{3} h-\right\}$. No KW $\left\{w^{3} h-\right\}$-related equivalent has been recorded meaning "equal in ..."; however, $\mathrm{KW}\left\{\mathrm{w}^{3} \mathrm{~h}-\mathrm{hw}^{3}-\right\}$ does occur but with the meaning "big, large, great." 3. $\mathrm{KW}\left\{\mathrm{w}^{3}-\right\}$ occurs only in the stem $\left\{\mathrm{w}^{3}-\mathrm{wkv}-\right\}$, alternant of $\left\{\mathrm{w}^{3} \mathrm{~h}-\mathrm{wkv}\right\}$ "thick layer." $\mathrm{KW}\left\{\mathrm{h}^{3} \mathrm{w}-\right\}$ occurs in the stem $\left\{\mathrm{h}^{3} \mathrm{w}-\mathrm{hw}^{3}-\right\}$ "big, large." 4. This root occurs only with a suffix signifying quantity, number, size or dimension (e.g. length, width) and is just like root no. 17720 only a device to turn a suffix into a word. It could be identical historically to no. 17720.

${ }^{827} 1$. $\left\{\mathrm{w}^{3} \mathrm{~h}-\right\}$ by itself refers to duality only in the HE/OO stem $\left\{\mathrm{w}^{3} \mathrm{~h}-\%\right.$ lhqv- $\}$ "talking together." Compare this stem with HE/OO $\left\{\mathrm{w}^{3} \mathrm{w}-\%\right.$ lhqv- $\}$ "to admit to sth." and its HA/KW cognate $\left\{\mathrm{h}^{3} \mathrm{w}-\right.$ \%lhqv- $\}$ HA "to deny an accusation" KW "to fall silent." This root $\left\{\mathrm{w}^{3} \mathrm{~h}-\right\}$ is likely related historically to suffix no. 4220 with the sense of "across." The stems $\left\{\mathrm{w}^{3} \mathrm{hxs} \mathrm{s}^{\bullet-}\right\}$ and $\left\{\mathrm{w}^{3} \mathrm{~h}^{3} \mathrm{Xs}^{\bullet-}\right\}$ consist of $\left\{w^{3} h-\right\}$ plus suffix no. 2140. 2. In HE the stem $\left\{w^{3} h x s^{\bullet-}\right\}$ is rare and in OO it is not 
North Wakashan Comparative Root List (Updated)

\begin{tabular}{|c|c|c|c|c|c|c|}
\hline Root \# & Old \# & $\mathrm{HE}$ & $\mathrm{OO}$ & HA & KW & Gloss \\
\hline 17850 & 1918 & $\begin{array}{l}\mathrm{w}^{3} \mathrm{hp}- \\
\mathrm{w}^{3} \mathrm{hm}^{3}- \\
\mathrm{w}^{3} \mathrm{hm}^{3} \mathrm{p}-\end{array}$ & $\begin{array}{l}\mathrm{w}^{3} \mathrm{hp}- \\
\mathrm{w}^{3} \mathrm{hm}^{3-} \\
\mathrm{w}^{3} \mathrm{hm}^{3} \mathrm{p}-\end{array}$ & $\mathrm{w}^{3} \mathrm{hp}-$ & $\mathrm{w}^{3} \mathrm{hp}-$ & water 828 \\
\hline 17870 & 1922 & $\mathrm{w}^{3} \mathrm{hnh}-$ & $\mathrm{w}^{3} \mathrm{hnh}-$ & $\begin{array}{l}\mathrm{w}^{3} \mathrm{hnh}- \\
\mathrm{w}^{3} \mathrm{hn} \infty-\end{array}$ & $\begin{array}{l}\mathrm{w}^{3} \mathrm{n}-* \\
\mathrm{w}^{3} \mathrm{hnh}-*\end{array}$ & to exchange, take turns ${ }^{829}$ \\
\hline 17880 & 1921 & & & & $\begin{array}{l}\mathrm{w}^{3} \mathrm{n}-* \\
\mathrm{w}^{3} \mathrm{hnh}-\end{array}$ & poor 830 \\
\hline 17881 & & & & & $\begin{array}{l}\text { w³nwf- } \\
\text { w }^{3} \text { hnwh-* }\end{array}$ & $(?)^{831}$ \\
\hline 17890 & $\begin{array}{l}1923 \\
1924 \\
1925\end{array}$ & $\mathrm{w}^{3} \mathrm{hs}-$ & $\mathrm{w}^{3} \mathrm{~h} \mathrm{~s}-$ & $\mathrm{w}^{3} \mathrm{hs}-$ & $\mathrm{w}^{3} \mathrm{~h} \mathrm{~s}-$ & $\begin{array}{l}\text { dog, dog-like; (KW also:) dear, } \\
\text { loved }\end{array}$ \\
\hline 17891 & 1926 & $\left(w^{3} n k-\right)$ & $\left(w^{3} n k-\right)$ & $\left(w^{3} n k-\right)$ & $\mathrm{w}^{3} \mathrm{~h} £-$ & lover, sweetheart \\
\hline 17900 & 1927 & & & $\mathrm{w}^{3} \mathrm{~h} 1-$ & & (?) in a tight spot ${ }^{832}$ \\
\hline
\end{tabular}

even diagnosable directly but assumed when the HE cognate has it. 3. HA $\left\{\mathrm{h}^{3} \mathrm{y}^{3} \mathrm{~s}^{\bullet-}\right\}$ may stem from $\left\{\left[h^{3} y\right] h^{3} s^{\bullet-}\right\}$ or $\left\{\left[h^{3}\right] h^{3} s^{\bullet-}\right\}$.

${ }^{828} \mathrm{HE} / \mathrm{OO}\left\{\mathrm{w}^{3} \mathrm{hm}^{3}-, \mathrm{w}^{3} \mathrm{hm}^{3} \mathrm{p}-\right\}$ are the free form versions of $\left\{\mathrm{w}^{3} \mathrm{hp}-\right\}$, with $\left\{\mathrm{w}^{3} \mathrm{hm}^{3}-\right\}$ occurring before category B and C suffixes beginning in an obstruent and if no suffix follows, and $\left\{w^{3} h m^{3} p-\right\}$ before vocalic elements. A HE free form $\left\{w^{3} h p-\right\}$ is rumoured to have existed. Note that in $\mathrm{HE} / \mathrm{w}^{3} \mathrm{a}^{3 \prime} \mathrm{Mx} . \hbar^{3} \mathrm{it} /$ "to turn into fresh water" the suffix form $\left\{-\mathrm{x} . \hbar^{3} \mathrm{yd}\right\}$ has been joined to $\left\{\mathrm{w}^{3} \mathrm{hm}^{3}-\right\}$ instead of the suffix's alternant $\{-\& y d\}$ to $\left\{\mathrm{w}^{3} \mathrm{hp}-\right\}$ (resulting in $* / \mathrm{w}^{3} \mathrm{am}^{3} \mathrm{it} /$ ).

${ }^{829} \mathrm{HA}\left\{\mathrm{w}^{3} \mathrm{hn} \infty-\right\}$ is diagnosable in $\left\{\mathrm{w}^{3} \mathrm{hn} \infty-\mathrm{w}-\mathrm{d}\right\}>/ \mathrm{w}^{3} \mathrm{an}$ 'ud/ "to trade in, exchange."

${ }^{830}$ Only reelicitable example is $\mathrm{KW} / \mathrm{w}^{3}$ anała/ "poor."

$831\left\{\mathrm{w}^{3} \mathrm{hnw} \mathfrak{-}\right\}$ is a free KW stem meaning "not up to a task, not really capable" according to Lincoln (n.d.). It doesn't seem to occur in Boas's 1948 dictionary. What Boas does list (1948:75) is $\left\{\mathrm{w}^{3} \mathrm{hnw} \mathrm{f}^{*}\right.$, a bound stem referring to helpful medicine. Boas's examples could however not be reelicited.

${ }^{832}$ In HA (Kitamaat dialect) / $w^{3}$ 'ała/ "to narrowly escape an accident." 
North Wakashan Comparative Root List (Updated)

\begin{tabular}{|c|c|c|c|c|c|c|}
\hline Root \# & Old \# & $\mathrm{HE}$ & $\mathrm{OO}$ & $\mathrm{HA}$ & KW & Gloss \\
\hline 17920 & 1930 & $\begin{array}{l}\mathrm{w}^{3} \mathrm{~h} \chi \mathrm{v}- \\
\mathrm{w}^{3} \mathrm{~h}^{3} \chi \mathrm{v}^{-} \\
\mathrm{w}^{3} \mathrm{~h}^{3} \chi-\end{array}$ & $\begin{array}{l}\mathrm{w}^{3} \mathrm{~h} \chi \mathrm{v}- \\
\mathrm{w}^{3} \mathrm{~h}^{3} \chi \mathrm{v}^{-} \\
\mathrm{w}^{3} \mathrm{y}^{3} \chi \mathrm{v}^{-}\end{array}$ & $\begin{array}{l}\mathrm{w}^{3} \mathrm{~h} \chi \mathrm{v}- \\
\mathrm{w}^{3} \mathrm{~h}^{3} \chi \mathrm{v}-\end{array}$ & $\mathrm{w}^{3} \mathrm{~h}^{3} \chi \mathrm{v}^{-}$ & group of people ${ }^{833}$ \\
\hline 17930 & 1931 & $\mathrm{w}^{3} \mathrm{hq}-$ & $\mathrm{w}^{3} \mathrm{hq}-$ & $\mathrm{w}^{3} \mathrm{hq}-$ & & $\begin{array}{l}\text { to feed a visitor, to welcome, to } \\
\text { host sb. }\end{array}$ \\
\hline 17940 & 1932 & $\mathrm{w}^{3} \mathrm{~h} \chi-$ & $\mathrm{w}^{3} \mathrm{~h} \chi-$ & $\mathrm{w}^{3} \mathrm{~h} \chi-$ & $\mathrm{w}^{3} \mathrm{~h} \chi-$ & $\begin{array}{l}\text { plume (of smoke in } \mathrm{HE} / \mathrm{OO} / \mathrm{KW} \text {, } \\
\text { of feathers in HA) }\end{array}$ \\
\hline 17960 & 1953 & $\begin{array}{l}\text { IVms- } \\
\text { rvh- }\end{array}$ & $\begin{array}{l}\text { IVms- } \\
\text { rvh- }\end{array}$ & $\begin{array}{l}\text { IVms- } \\
\text { IVnc- }\end{array}$ & IVms- & ochre $^{835}$ \\
\hline 17970 & 1933 & IVn- & IVn- & IVn- & IVn- & to pay a debt, to repay \\
\hline 17980 & $\begin{array}{l}1934 \\
1956\end{array}$ & IVn- & & IVn- & IVhn ${ }^{3}-$ & (?) bending, curved 836 \\
\hline 17990 & $\begin{array}{l}1935 \\
1936\end{array}$ & rVn- & IVn- & rVn- & IVn- & to burn ${ }^{837}$ \\
\hline 18000 & 1937 & & & & $\begin{array}{l}\text { rVnt- } \\
\text { rVnt }^{3}-\end{array}$ & heavy \\
\hline 18010 & 1938 & & IVnc- & IVnc- & IVns- & excrescence, to bud out ${ }^{838}$ \\
\hline
\end{tabular}

${ }^{833} \mathrm{KW}$ only in $/ \mathrm{w}^{3} \mathrm{a}^{3} \mathrm{ukv} /$ "some, others, the rest (of the group)" and derivates. $\left\{\mathrm{w}^{3} \mathrm{y}^{3} \chi \mathrm{v}-\right\}$ occurs only in $\mathrm{OO} / \mathrm{w}^{3} \mathrm{i}^{3} \chi$ vuyaqs/, plural form of $/ \mathrm{w}^{3} \mathrm{a}^{3} \chi$ vuyaqs/ "sisters (term used by females)." ${ }^{834}$ In most examples $\left\{\mathrm{w}^{3} \mathrm{hq}-\right\}$ is followed by $\{-+-\}$ the significance of which is unclear.

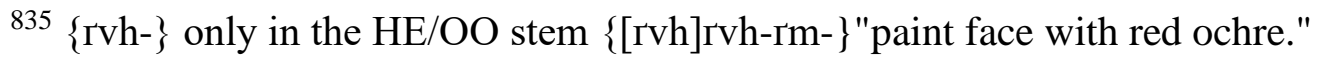

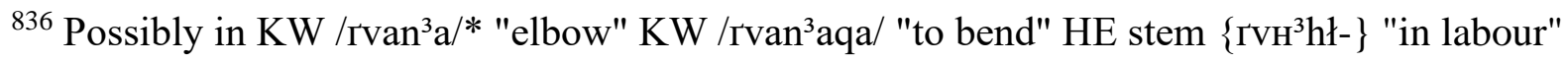
and HE/HA $\{\mathrm{rv}[\mathrm{h}] \mathrm{n}[\mathrm{y}]-\}>\mathrm{HE} / \mathrm{rv}^{\prime}$ an'i/ "ladle made of mountain goat horn" HA /rvan'i/ "horseclam shells (used as dishes at potlatches)."

${ }^{837}$ In HE/OO only in the word for ashes of a fire. Cf. English "ash" which is akin to Latin "aridus" ("dry"), "ardor" and "ardēre" ("to burn").

${ }^{838}$ In terms for a bud of cottonwood, a horseclam and a carbuncle. 
North Wakashan Comparative Root List (Updated)

\begin{tabular}{|c|c|c|c|c|c|c|}
\hline Root \# & Old \# & $\mathrm{HE}$ & $\mathrm{OO}$ & HA & KW & Gloss \\
\hline 18020 & $\begin{array}{l}1939 \\
2136\end{array}$ & $\begin{array}{l}\text { rVn- } \\
\text { rvl-, } \\
\text { rl- }\end{array}$ & $\begin{array}{l}\text { IVn- } \\
\text { IVl- }\end{array}$ & IVl- & IVl- & $\begin{array}{l}\text { pitch, resin, gum, sap from } \\
\text { plant }^{839}\end{array}$ \\
\hline 18030 & 1940 & Ivlx- & Ivlx- & Ivlx- & rvlx- & pitch, resin, gum; sticky \\
\hline 18050 & 1941 & rvy- & rvy- & rvy- & IVy- & $\begin{array}{l}\text { thus, in such a way, of such a } \\
\text { kind }\end{array}$ \\
\hline 18060 & 1942 & rvy- & IVy- & rvy- & $\begin{array}{l}\text { IVy- } \\
\text { rvhy³- }\end{array}$ & heavy in weight, whale \\
\hline 18070 & 1943 & $\begin{array}{l}\text { IVyc-/ } \\
\text { IVys- }\end{array}$ & $\begin{array}{l}\text { IVyc-/ } \\
\text { IVys- }\end{array}$ & $\begin{array}{l}\text { IVyc-/ } \\
\text { IVys- }\end{array}$ & IVys-* & sparrow $^{840}$ \\
\hline 18080 & 1944 & IVys- & IVys- & & & unsoaked dried salmon \\
\hline 18090 & 1945 & IVyk- & IVyk- & IVyk- & IVyk- & whale \\
\hline 18100 & 1946 & rvy $\chi-$ & rvy $\chi-$ & rvy $\chi-$ & & flour, bread \\
\hline 18110 & 1948 & IVwy ${ }^{3}-$ & $\begin{array}{l}\mathrm{IVW}^{3}- \\
\mathrm{IVy}^{3}-\end{array}$ & rwy ${ }^{3} \mathrm{~h}-$ & & $\begin{array}{l}\text { (?) sameness of shape, action or } \\
\text { timing; occurrence at regular } \\
\text { intervals }^{841}\end{array}$ \\
\hline 18120 & 1949 & $\begin{array}{l}\text { IVwł-/ } \\
\text { IVwlh- }\end{array}$ & $\begin{array}{l}\text { IVwł-/ } \\
\text { IVwlh- }\end{array}$ & $\begin{array}{l}\text { rwł-/ } \\
\text { rwlh-/ } \\
\text { rwlœ- }\end{array}$ & rwlh- & trout \\
\hline
\end{tabular}

839 \{rvn- $\}$ only in HE/OO \{[rvh]rvn-+-h $\}$ "to go after pitch."

${ }^{840}$ In several non-reelicitable KW words referring to a sparrow and the Sparrow Society. Perhaps also in HA /rv'isrvi/ and /rvisrv'i/ "sparrow" and HE /rv'isqv/ OO /rvist $\chi \mathrm{v} /$ which informants said refers to a dark brownish kind of robin but which Boas-Hunt gloss as "sparrow" (see Bella Bella Texts, p. 258).

${ }^{841}$ The HE/OO forms are attested only in HE /rv'uy ${ }^{3} \mathrm{al}^{\prime} \mathrm{a} / \mathrm{OO} / \mathrm{rvu}^{3} л \mathrm{~s} /$ and OO /rvi ${ }^{3} л а /$ "regular(ly)." 
North Wakashan Comparative Root List (Updated)

\begin{tabular}{|c|c|c|c|c|c|c|}
\hline Root \# & Old \# & $\mathrm{HE}$ & $\mathrm{OO}$ & HA & KW & Gloss \\
\hline 18130 & 1950 & IVWł- & IVWł- & IW1- & rW1-* & $\begin{array}{l}\text { fat, tallow, marrow; (KW } \\
\text { possibly also:) core (e.g. of fruit), } \\
\text { innards }{ }^{842}\end{array}$ \\
\hline 18140 & 1951 & IVWXV- & 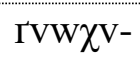 & IW $\chi \mathrm{V}-$ & & skinny, lean, slender \\
\hline 18150 & $\begin{array}{l}1994 \\
2004 \\
2061 \\
2245 \\
2246\end{array}$ & $\begin{array}{l}\text { qvqv- } \\
\text { qq- } \\
\text { qhq- }\end{array}$ & $\begin{array}{l}\text { qvqv- } \\
\text { qq- } \\
\text { qhq- }\end{array}$ & $\begin{array}{l}\text { rqv- } \\
\text { rwqv- } \\
\text { qq- } \\
\text { qhq- }\end{array}$ & $\begin{array}{l}\text { rvqv- } \\
\text { rqv- } \\
\text { qvhqv- } \\
\text { rwqv-, } \\
\text { rq- }\end{array}$ & (possibly onomatopoeic) $)^{843}$ \\
\hline 18160 & 1954 & & & & $\begin{array}{l}\text { rvh- } \\
\text { rvhlh- }\end{array}$ & $\begin{array}{l}\text { to stop doing sth., to refrain from } \\
\text { doing sth. }\end{array}$ \\
\hline 18170 & 1955 & & & Ivh- & & $(?)^{844}$ \\
\hline 18180 & 1957 & rvht- & rvht- & rvht- & rvht- & red huckleberry \\
\hline 18190 & 1958 & & & & IVh£- & $\begin{array}{l}\text { to moan, groan, holler (when } \\
\text { startled) }\end{array}$ \\
\hline 18200 & 1959 & rvhł- & rvhł- & rvhł- & rvhł- & $\begin{array}{l}\text { ready, finished, completed; to } \\
\text { make ready, arrange, appoint, } \\
\text { order }\end{array}$ \\
\hline 18210 & 1960 & Ivhlhs- & Ivhlhs- & Ivhlhs- & Ivhlhs- & lizard \\
\hline
\end{tabular}

${ }^{842}$ None of the KW examples in the Boas-Hunt materials could be reelicited.

${ }^{843}$ \{qq- $\}$ occurs in words referring to dogs giving the hunting cry when chasing animals, and in $\mathrm{HE} / \mathrm{qr}^{\prime} \mathrm{ig}^{\prime} \mathrm{a} / \mathrm{OO} / \mathrm{qriga/}$ "a species of white diving bird that says $\mathrm{HE} / \mathrm{q}^{3} \mathrm{q}^{3} \mathrm{q}^{3} \mathrm{q}^{3} \mathrm{a} /$. " The other forms occur in the names of water birds including long-necked ones such as heron, crane, swan and the long-billed sawbill duck, but also KW /qvarviłbe $3 / *$ "lance" (possibly literally "heronnosed"). The heron's call is a harsh croak resembling /qa qaa qa qa/.

${ }^{844}$ In HA /rvar'auy ${ }^{3}$ uala/ "bumble bee" (according to the Kitlope informant), "horsefly" (according to the Kitamaat one). 
North Wakashan Comparative Root List (Updated)

\begin{tabular}{|c|c|c|c|c|c|c|}
\hline Root \# & Old \# & $\mathrm{HE}$ & $\mathrm{OO}$ & HA & $\mathrm{KW}$ & Gloss \\
\hline 18220 & & & & & rvhk- & $(?)^{845}$ \\
\hline 18230 & $\begin{array}{l}1962 \\
1963\end{array}$ & rvh $\chi-$ & $\operatorname{rvh} \chi-$ & rvh $\chi-$ & rvh $\chi-$ & $\begin{array}{l}\text { striped, streak-marked (like dog } \\
\text { salmon near spawning time); } \\
\text { dyed (with alder bark) } \\
846\end{array}$ \\
\hline 18240 & 1964 & qvp- & qvp- & qvp- & qvp- & $\begin{array}{l}\text { powder, powdery (like dry snow, } \\
\text { rotten wood) }{ }^{847}\end{array}$ \\
\hline 18250 & $\begin{array}{l}1965 \\
2063\end{array}$ & $\begin{array}{l}\text { qvmc- } \\
\text { qvms- }\end{array}$ & $\begin{array}{l}\text { qvmc- } \\
q^{3} v_{m s-}\end{array}$ & & qvms- & (?) brown, decaying ${ }^{848}$ \\
\hline 18260 & 1966 & & & & $\begin{array}{l}\text { qvn-/ } \\
\text { qvnp- }\end{array}$ & $(?)^{849}$ \\
\hline 18270 & 1967 & & & qvn- & $\begin{array}{l}\text { qvn-* } \\
\text { qvhn }{ }^{3-*}\end{array}$ & to crush, smash, crack \\
\hline 18280 & & $(\mathrm{tq}-+1-)$ & qvns- & $(\mathrm{dq}-+1-)$ & & $\operatorname{itch}(y)^{850}$ \\
\hline 18290 & 1968 & & & & qvnqv- & $\begin{array}{l}\text { to twitch, to move with a jerk, to } \\
\text { shake }\end{array}$ \\
\hline 18300 & 1969 & & & & qvs- & $\begin{array}{l}\text { layer-like thing becomes } \\
\text { detached (e.g. page from book, }\end{array}$ \\
\hline
\end{tabular}

${ }^{845}$ Possibly in KW / Ivarvkmlilas/ (place name said to mean "face out of sight") and KW $/$ [varvk ${ }^{3} \mathrm{utr}^{3} \mathrm{as} / *$ (name of house of one called $\mathrm{KW} / \mathrm{Q}^{3}$ aniqil ${ }^{3} \mathrm{akv} /$ ).

${ }^{846}$ Dog salmon is also known as chum salmon and "chum" is Chinook jargon for "spotted" or "marked." Root is a cross-family stem, cf. South Wakashan (Nuuchahnulth) \{qvah-\} "daubed with red clay" and its derivate /qvahmmis/ "red clay of a certain kind used as paint" in Sapir \& Swadesh (1939:294).

${ }^{847}$ See also the footnote to $\left\{q^{3} v p-, q^{3} p-\right\}$ (root no. 18660).

848 The two KW examples are about dog excrement. The HE/OO examples are HE (Bella Bella) /qvist'u/ HE (Klemtu) /qv'mstu/ OO /qvм³stu/ "brown" and OO /q³ams/ "brown, moss-like." ${ }^{849}$ Possibly in KW /qvнap/ "young sawbill ducks." ${ }^{850}$ Only in OO /qvisa/ "to itch, itchy." 
North Wakashan Comparative Root List (Updated)

\begin{tabular}{|c|c|c|c|c|c|c|}
\hline Root \# & Old \# & $\mathrm{HE}$ & $\mathrm{OO}$ & $\mathrm{HA}$ & KW & Gloss \\
\hline & & & & & & $\begin{array}{l}\text { leaf from stem, skin from flesh), } \\
\text { to peel off }\end{array}$ \\
\hline 18320 & 1972 & & & & qvł- & $\begin{array}{l}\text { to hit, to strike (said of waves); } \\
\text { waves, breakers, ocean swell }\end{array}$ \\
\hline 18330 & 1973 & & $\begin{array}{l}\text { qvlp- } \\
\text { q }^{3} \text { vlp- }\end{array}$ & qvlp- & & dust, powder \\
\hline 18340 & 1974 & qvlx- & qvlx- & qvlx- & & rust-coloured, brownish \\
\hline 18350 & 1975 & & & & qvlx- & $\begin{array}{l}\text { to handle long, loose, pliable } \\
\text { things }\end{array}$ \\
\hline 18360 & 1976 & qvlqv- & qvlqv- & & qvlqv- & to sprain, wrench \\
\hline 18380 & $\begin{array}{l}1979 \\
1980\end{array}$ & $\begin{array}{l}\text { qvyc- } \\
\text { qvys- }\end{array}$ & $\begin{array}{l}\text { qvyc- } \\
\text { qvys- }\end{array}$ & qvys- & & $\begin{array}{l}\text { to squeeze, to tweeze, to hit } \\
\text { against each other (like split ends } \\
\text { of rattle stick); squeaky (voice) }\end{array}$ \\
\hline 18390 & $\begin{array}{l}1977 \\
1978\end{array}$ & $\begin{array}{l}\chi \mathrm{vy}- \\
\chi \mathrm{vys} \bullet-\end{array}$ & ұvys•- & ұvys $\bullet-$ & $\begin{array}{l}\text { qvy- } \\
\text { qvys•- } \\
\text { qvys- }\end{array}$ & $\begin{array}{l}\text { that direction, far, on the far side, } \\
\text { far away }^{852}\end{array}$ \\
\hline 18410 & $\begin{array}{l}1981 \\
1082 \\
1983\end{array}$ & qvył- & qvył- & $\begin{array}{l}\text { qvy£- } \\
\text { qvył- }\end{array}$ & qvył- & $\begin{array}{l}\text { to lose coherence, come apart, } \\
\text { get dismantled; to separate into } \\
\text { components (like juice when } \\
\text { sediment settles); to unravel, to } \\
\text { become detached (like rope) }\end{array}$ \\
\hline 18430 & 1984 & qvyx- & qvyx- & & & to unlock (as with a key) \\
\hline 18440 & $\begin{array}{l}1985 \\
2233\end{array}$ & $\begin{array}{l}\text { qvyqv- } \\
\text { qyq- } \\
\text { qyx- }\end{array}$ & $\begin{array}{l}\text { qvyqv- } \\
\text { qyq- }\end{array}$ & qyq- & & $\begin{array}{l}\text { to wiggle the end of a long thing } \\
\text { (as to free a pole or when }\end{array}$ \\
\hline
\end{tabular}

${ }^{851}$ The form \{qvyc- $\}$ occurs possibly also in $\mathrm{HE} / \mathrm{qvist}^{\prime} \mathrm{H} / \mathrm{OO} / \mathrm{qvi}^{3} \mathrm{stH}_{\mathrm{H}}$ ( an unidentified bird).

$852\{\chi \mathrm{vy}-\}$ only in $\mathrm{HE} / \chi \mathrm{v}^{\prime} \mathrm{iki}{ }^{3} \mathrm{a} /$ "on the far side of the top of sth. bulky (e.g. a cabin)." 853 \{qvy£-\} only in HA /qv'i£a/ "to smash sth. up (e.g. fruit)." 
North Wakashan Comparative Root List (Updated)

\begin{tabular}{|c|c|c|c|c|c|c|}
\hline Root \# & Old \# & $\mathrm{HE}$ & $\mathrm{OO}$ & HA & KW & Gloss \\
\hline & & & & & & $\begin{array}{l}\text { nodding the head or bending it } \\
\text { sideways) }\end{array}$ \\
\hline 18450 & 1988 & qvwnh- & qvwnh- & qwnh- & qwmh- & thumb, big toe \\
\hline 18460 & 1986 & qvwt- & qvwt- & & qwt- & $(?)^{854}$ \\
\hline 18470 & 1987 & qvwt ${ }^{3} h-$ & qvwt ${ }^{3}-$ & $q w t^{3} h-$ & $\begin{array}{l}\text { qwt } t^{3}- \\
\text { qwt- }\end{array}$ & full, filled up ${ }^{855}$ \\
\hline 18480 & $\begin{array}{l}1989 \\
1998\end{array}$ & qvws- & qvws- & qws- & $\begin{array}{l}\text { qws- } \\
\text { qvhs- }\end{array}$ & $\begin{array}{l}\text { (\{qvws-, qws- }\} \text { :) to become } \\
\text { covered with downy material } \\
\text { (like seedhead of dandelion); } \\
\text { (HE/OO/HA also:) to burst into } \\
\text { bloom, to blossom, to flower; } \\
\text { (KW \{qvhs-\}:) to bud, flower, } \\
\text { bloom }\end{array}$ \\
\hline 18500 & 1990 & qvwyq- & qvwyq- & & & to worry ${ }^{856}$ \\
\hline 18510 & 1991 & qvw1- & qvw1- & qw1- & qw1-* & fur, pelt ${ }^{857}$ \\
\hline 18520 & $\begin{array}{l}1992 \\
2240 \\
2241\end{array}$ & $\begin{array}{l}\text { qvw1- } \\
\text { qw1- }\end{array}$ & $\begin{array}{l}\text { qvw1- } \\
\text { qw1- } \\
\text { qws- }\end{array}$ & $\begin{array}{l}\text { qw1- } \\
\text { qw³- } \\
\text { qw³ }^{3}-\end{array}$ & $\begin{array}{l}\text { qwł- } \\
\chi w 1-*\end{array}$ & bent, crooked, warped ${ }^{858}$ \\
\hline
\end{tabular}

${ }^{854}$ In HE/OO \{qvwtyx- $\}$ KW \{qwtyx- $\}$ "ceremonial blanket symbolizing a bride price consisting of material and spiritual property."

${ }^{855} \mathrm{KW}\{$ qwt- $\}$ only in $/$ qut $^{3} \chi \mathrm{u} /<\{$ qwt-+$\chi w\}$ "mouthful."

${ }^{856}$ Stem could be polymorphemic but structure unclear.

${ }^{857}$ In words referring to beaver and muskrat.

${ }^{858} \mathrm{HE} / \mathrm{OO}$ \{qvw1-\} only in HE /qv'ul'ush/ OO /qvulusH/ (name of a supernatural bird). The KW counterpart is /qulus/ (in the Fort Rupert subdialect) and / $\chi$ ulus/* (in the Gwasala subdialect) and means "mythical bird with slanting beak, brother of Thunderbird." The root forms ending in $|s|$ 
North Wakashan Comparative Root List (Updated)

\begin{tabular}{|c|c|c|c|c|c|c|}
\hline Root \# & Old \# & $\mathrm{HE}$ & $\mathrm{OO}$ & HA & KW & Gloss \\
\hline 18530 & 1993 & qvwqv- & qvwqv- & qwqv- & qwqv- & $\begin{array}{l}\text { lump, bulge, hump; (KW also:) } \\
\text { bone }\end{array}$ \\
\hline 18550 & $\begin{array}{l}1995 \\
1996 \\
2346 \\
2348\end{array}$ & & $q v \chi v^{-}$ & $q v \chi^{v-}$ & $\begin{array}{l}q v \chi^{-} \\
q^{3} \chi^{v-} \\
q^{3} \chi^{-}\end{array}$ & $\begin{array}{l}\text { particulate solid matter (e.g. } \\
\text { powder, flour, lime, dust, ashes, } \\
\text { seeds), droplets (of e.g. water); } \\
\text { grey, dusty }\end{array}$ \\
\hline 18560 & & & & qvh- & & to tear off a flat thing \\
\hline 18570 & & & & qvht- & & $(?)^{859}$ \\
\hline 18580 & 1999 & & qvhs- & & & $(?)^{860}$ \\
\hline 18590 & 2000 & qvhq- & $\begin{array}{l}\text { qvik- } \\
\text { qvhq- }\end{array}$ & $\begin{array}{l}\text { qvhk- } \\
\text { quhq- }\end{array}$ & qvihq- & to split apart, to split open ${ }^{861}$ \\
\hline 18600 & 2002 & & & & $\mathrm{qvhk}^{3} \mathrm{v}-*$ & to peel off \\
\hline 18620 & 2005 & qvhqv- & qvhqv- & qvhqv- & & to swing, to sweep \\
\hline 18630 & 2007 & qvh $\chi v-$ & qvh $\chi v-$ & qvh $\chi v-$ & qvh $\chi v-$ & $\begin{array}{l}\text { to display, show, exhibit, bring } \\
\text { out in the open }\end{array}$ \\
\hline 18640 & 2009 & & & & $\mathrm{qvh}^{3} \mathrm{x}-*$ & $(?)^{862}$ \\
\hline 18650 & 2010 & & & & $\mathrm{qvh}^{3} \mathrm{x}-*$ & $(?)^{863}$ \\
\hline
\end{tabular}

are attested only in ОО /qusла/ "crooked, winding in different directions (as a rope, a boat)" НА (Kitamaat dialect) /qusл'a/ "crooked."

${ }^{859}$ In HA /qvad'aułsm/ "bulky in shape and with black spots."

${ }^{860}$ In OO /qvasa/ "to be startled."

${ }^{861} \mathrm{OO}$ \{qvhk-\} only in /qvaka/ the meaning of which is uncertain; consultant believes it is a synonym of OO /qvaqa/ "to split open (e.g. the abdomen of fish or animals)."

${ }^{862} \mathrm{In} \mathrm{KW} / \mathrm{qva}^{3} \mathrm{xa} / *$ [1] "to hurry" (an old word, says Boas).

${ }^{863} \mathrm{In} \mathrm{KW} /$ qva $^{3} \mathrm{xa} / *$ [2] "to straighten up one's body." 
North Wakashan Comparative Root List (Updated)

\begin{tabular}{|c|c|c|c|c|c|c|}
\hline Root \# & Old \# & $\mathrm{HE}$ & $\mathrm{OO}$ & $\mathrm{HA}$ & KW & Gloss \\
\hline 18660 & $\begin{array}{l}2011 \\
2266\end{array}$ & $q^{3} v p-$ & $q^{3} v p-$ & $\begin{array}{l}q^{3} v p- \\
q^{3} p-\end{array}$ & $\begin{array}{l}q^{3} v p- \\
q^{3} p-\end{array}$ & 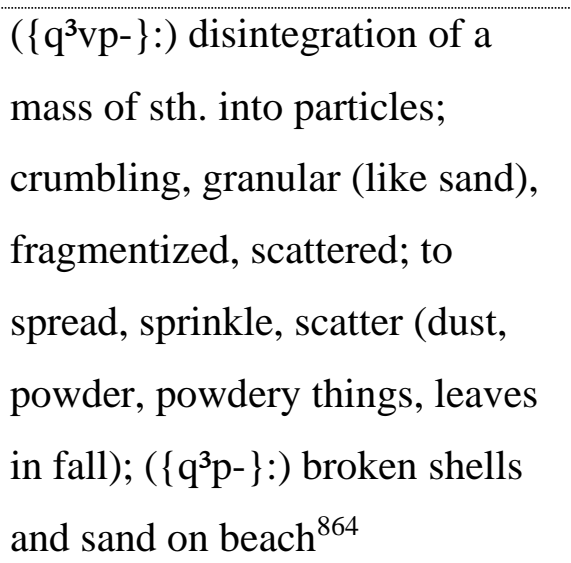 \\
\hline 18670 & $\begin{array}{l}2012 \\
2119 \\
2329\end{array}$ & $\begin{array}{l}q^{3} v w m \infty- \\
q^{3} v w m h-\end{array}$ & $\begin{array}{l}\mathrm{q}^{3} \mathrm{vm}- \\
\mathrm{q}^{3} \mathrm{vwm} \infty- \\
\mathrm{q}^{3} \mathrm{wm} \infty- \\
\mathrm{q}^{3} \mathrm{vwmh-}\end{array}$ & $\begin{array}{l}\mathrm{q}^{3} \mathrm{vm}- \\
\mathrm{q}^{3} \mathrm{wm} \infty-\end{array}$ & $\begin{array}{l}q^{3} w m h- \\
q^{3} w m \infty-\end{array}$ & $\begin{array}{l}\text { to stick out, protrude; notable, } \\
\text { rich, wealthy }{ }^{865}\end{array}$ \\
\hline 18680 & 2014 & $\mathrm{q}^{3} \mathrm{vmt}-$ & $\mathrm{q}^{3} \mathrm{vmt}-$ & & $q^{3} v m t-$ & $\begin{array}{l}\text { to poke into sth., to pierce, to } \\
\text { perforate; (HE/OO also:) to } \\
\text { vaccinate; (KW also:) crabapples } \\
\text { (ripe when pierceable) }\end{array}$ \\
\hline 18690 & 2013 & $q^{3} \mathrm{vms}^{-}$ & $q^{3} v_{m s}-$ & & & fat, rich $^{866}$ \\
\hline 18700 & & & & & $\mathrm{q}^{3} \mathrm{vml}-*$ & (?) thundering \\
\hline
\end{tabular}

${ }^{864}$ Semantically this root seems to be similar to $\{$ qvp- $\}$ (root no. 18240) but with the added notion of scattering. $\{\mathrm{qvp}-\}$ and $\left\{\mathrm{q}^{3} \mathrm{vp}-\right\}$ alternate freely in HA /qvqvpмa/ and $/ \mathrm{q}^{3} \mathrm{vq}^{3} \mathrm{vpma} /$ "to put powder on the face (red ochre etc.)."

8651 . Wealth can refer to cultural property such as privileges, stories, dances, names. $2 .\left\{q^{3} \mathrm{vm}-\right\}$ is probably the root's base form from which the other forms derive by augmentation. 3 . The forms $\left\{q^{3} w m \infty-\right\}$ and $\left\{q^{3} w m h-\right\}$ are also forms of root no. 21601 which refers to howling and wailing.

${ }^{866}$ Probably in $\left\{\mathrm{q}^{3} \mathrm{vm}[\infty] \mathrm{s}-\right\}>\mathrm{HE} / \mathrm{q}^{3} \mathrm{v}^{\prime} \mathrm{MS} / \mathrm{OO} / \mathrm{q}^{3} \mathrm{vmMs} /$ "liver." 
North Wakashan Comparative Root List (Updated)

\begin{tabular}{|c|c|c|c|c|c|c|}
\hline Root \# & Old \# & $\mathrm{HE}$ & $\mathrm{OO}$ & $\mathrm{HA}$ & KW & Gloss \\
\hline 18710 & 2015 & $q^{3} v m x-$ & $q^{3} v m x-$ & & $q^{3} v m x-$ & $\begin{array}{l}\text { land- or rockslide; (KW also:) to } \\
\text { rush down (said of group of } \\
\text { people) }\end{array}$ \\
\hline 18740 & 2018 & $\mathrm{q}^{3} \mathrm{vt}-$ & $q^{3} v t-$ & $q^{3} v t-$ & $\mathrm{q}^{3} \mathrm{vt}-$ & scar, healed wound \\
\hline 18750 & 2019 & $q^{3} \mathrm{vn}-$ & $q^{3} \mathrm{vn}-$ & $q^{3} \mathrm{vn}^{-}$ & $q^{3} \mathrm{vn}-$ & often \\
\hline 18760 & 2020 & $\mathrm{q}^{3} \mathrm{vn}-$ & $\mathrm{q}^{3} \mathrm{vn}-$ & $\mathrm{q}^{3} \mathrm{vn}-$ & $\mathrm{q}^{3} \mathrm{vn}-$ & $\begin{array}{l}\text { (?) to split off, branch off'; node } \\
\text { in network (e.g. in lupine } \\
\text { roots) }\end{array}$ \\
\hline 18770 & $\begin{array}{l}2021 \\
2022\end{array}$ & & $q^{3} v n c-$ & $\begin{array}{l}q^{3} \text { vnc- } \\
q^{3} \text { vns- }\end{array}$ & $q^{3}$ vns- & (?) intertwinement ${ }^{868}$ \\
\hline 18771 & & & & & $\mathrm{q}^{3} \mathrm{vny^{3 } \mathrm { qv }}$ & cottonwood \\
\hline 18780 & 2023 & $q^{3}$ vnk- & $q^{3}$ vnk- & $q^{3}$ vnk- & & fat, stout \\
\hline 18790 & 2024 & $q^{3}$ vnqv- & $q^{3}$ vnqv- & $\mathrm{q}^{3} \mathrm{v} n q \mathrm{v}-$ & & to swallow \\
\hline 18800 & 2025 & & & & $\mathrm{q}^{3} \mathrm{vs}-*$ & to tie with a knot \\
\hline 18810 & 2026 & $q^{3} v^{-}-$ & $\mathrm{q}^{3} \mathrm{vs}-$ & & $q^{3} v^{-}-$ & to drizzle \\
\hline 18820 & 2027 & & & & $\begin{array}{l}\mathrm{q}^{3} \mathrm{vs}-* \\
\mathrm{q}^{3} \mathrm{vhs}-*\end{array}$ & to heal (wound, sore) \\
\hline
\end{tabular}

${ }^{867}$ Possibly in $\mathrm{HE} / \mathrm{q}^{3} \mathrm{VH}^{\prime} \mathrm{M} / \mathrm{OO} / \mathrm{q}^{3} \mathrm{VHM} /$ "instrument with a sharpened edge used to make shakes or take bark off a tree" HA /q $\mathrm{q}^{3} \mathrm{vaH} /$ (a kind of root) $\mathrm{KW} / \mathrm{q}^{3} \mathrm{VH}^{3} \mathrm{i} /$ "lupine roots." 868 The KW examples refer to lupine roots and, in one case, getting drunk on lupine (but unknown if on lupine roots or lupine seeds). The HA examples refer to getting or eating an unidentified kind of root and communicating with the НА/h'ilikла/ dancer. The one OO example of the root form means "friends together." Lupine roots are a network of nodules and perhaps interwovenness is what the various examples have in common semantically. 
North Wakashan Comparative Root List (Updated)

\begin{tabular}{|c|c|c|c|c|c|c|}
\hline Root \# & Old \# & $\mathrm{HE}$ & $\mathrm{OO}$ & HA & KW & Gloss \\
\hline 18830 & & & & $q^{3} v s-$ & & $(?)^{869}$ \\
\hline 18840 & 2028 & & & $q^{3} v f-$ & & to ask for volunteer help \\
\hline 18850 & 2029 & $\mathrm{q}^{3} \mathrm{vl}-$ & $q^{3} v 1-$ & $q^{3} v 1-$ & $q^{3} v 1-$ & to scratch an itch \\
\hline 18860 & 2030 & $\chi v 1-$ & $\chi v 1-$ & 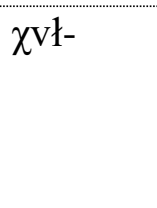 & $\mathrm{q}^{3} \mathrm{vl}-$ & $\begin{array}{l}\text { maternal or paternal uncle; (KW } \\
\text { also:) husband's or wife's brother- } \\
\text { in-law }{ }^{870}\end{array}$ \\
\hline 18880 & 2035 & & $\mathrm{q}^{3} \mathrm{vlp}-$ & $\mathrm{q}^{3}$ vlp- & $\mathrm{q}^{3} \mathrm{vlp}-$ & (?) decay, destruction ${ }^{871}$ \\
\hline 18890 & 2033 & $q^{3} \mathrm{vlm}^{3} \mathrm{~s}-$ & $\begin{array}{l}q^{3} \mathrm{vlm}^{3} \mathrm{c}- \\
\mathrm{q}^{3} \mathrm{vlm} \mathrm{m}^{3} \mathrm{~s}-\end{array}$ & $\mathrm{q}^{3} \mathrm{vlm}^{3} \mathrm{~s}-$ & & rotten, decaying ${ }^{872}$ \\
\hline 18900 & 2036 & $q^{3}$ vlc- & $q^{3}$ vlc- & $q^{3}$ vlc- & $\mathrm{q}^{3} \mathrm{vls}-$ & aging, decaying, rotting \\
\hline 18910 & 2037 & $\begin{array}{l}\text { q3vylh-, } \\
\text { q }^{3} \text { vyly- }\end{array}$ & $\begin{array}{l}\text { q3}^{3} \text { vylh-, } \\
q^{3} \text { vyly- }\end{array}$ & $\begin{array}{l}q^{3} \text { vly- } \\
q^{3} \text { vylh- } \\
q^{3} \text { vyly- }\end{array}$ & $\begin{array}{l}q^{3} \text { vly- } \\
q^{3} \text { vlyh- }\end{array}$ & $\begin{array}{l}\text { acting autonomously, on one's } \\
\text { own accord, by oneself, } \\
\text { unassisted; affecting oneself or } \\
\text { one's own group, to do sth. in } \\
\text { person }^{873}\end{array}$ \\
\hline 18920 & 2038 & $\mathrm{q}^{3} \mathrm{vlqv}-$ & $q^{3}$ vlqv- & $\mathrm{q}^{3}$ vlqv- & $q^{3}$ vlqv- & $\begin{array}{l}\text { ticklish, afraid to touch, tending } \\
\text { to avoid contact; lacking } \\
\text { manliness, tending to chicken out }\end{array}$ \\
\hline
\end{tabular}

${ }^{869}$ Possibly in HA $\left\{\mathrm{q}^{3} \mathrm{vxq}^{3} \mathrm{vywkv}\right\}$ "vegetation."

${ }^{870} \mathrm{KW}$ in $/ \mathrm{q}^{3} \mathrm{vлі}^{3} /$ "uncle (maternal or paternal)" and / $\mathrm{q}^{3} \mathrm{vліs/} \mathrm{"brother-in-law} \mathrm{(of} \mathrm{husband} \mathrm{or}$ wife)."

${ }^{871}$ Only in $\mathrm{OO} / \mathrm{q}^{3} \mathrm{v} \mathrm{J}^{3} \mathrm{Mstu} /$ "rusty colour, colour as of rotten wood; water with moss in it" HA

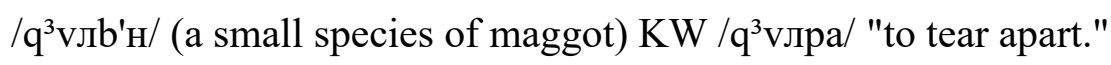

872 In HE $/ \mathrm{q}^{3} \mathrm{vл}^{3 \prime} \mathrm{MS} / \mathrm{OO} / \mathrm{q}^{3} \mathrm{vл}^{3} \mathrm{MS} /$ and $/ \mathrm{q}^{3} \mathrm{vл}^{3} \mathrm{Mc} / \mathrm{HA} / \mathrm{q}^{3} \mathrm{v}^{3} \mathrm{~m}^{3} \mathrm{~s} /$ "having dry rot."

${ }^{873} \mathrm{HE} / \mathrm{OO} / \mathrm{HA}\left\{q^{3} \mathrm{vyly}-\right\}$ is attested only before the suffix $\{-\mathrm{rw}\}$ "together, etc." (suffix no. 4290). 
North Wakashan Comparative Root List (Updated)

\begin{tabular}{|c|c|c|c|c|c|c|}
\hline Root \# & Old \# & $\mathrm{HE}$ & $\mathrm{OO}$ & HA & KW & Gloss \\
\hline 18930 & $\begin{array}{l}2031 \\
2039\end{array}$ & $\mathrm{q}^{3} \mathrm{vlh}-$ & $\mathrm{q}^{3} \mathrm{v} l \mathrm{~h}-$ & $q^{3}$ vlh- & $\begin{array}{l}q^{3} \text { vlh- } \\
q^{3} \text { vylhx- } \\
q^{3} \text { vynht- }\end{array}$ & $\begin{array}{l}\text { giving signs of life, stirring, } \\
\text { twitching; (hence:) alive, to } \\
\text { survive }^{874}\end{array}$ \\
\hline 18940 & $\begin{array}{l}2032 \\
2041 \\
2017\end{array}$ & $\begin{array}{l}\mathrm{q}^{3} \mathrm{vl}^{3}- \\
\mathrm{q}^{3} \mathrm{l}^{3} \mathrm{~h}-\end{array}$ & $\begin{array}{l}\mathrm{q}^{3} \mathrm{vl}^{3}- \\
\mathrm{q}^{3} \mathrm{l}^{3} \mathrm{~h}-\end{array}$ & $\mathrm{q}^{3} \mathrm{vl}^{3}-$ & $\begin{array}{l}\mathrm{q}^{3} \mathrm{vl}^{3-} \\
\mathrm{q}^{3} \mathrm{vm}^{3-*}\end{array}$ & $\begin{array}{l}\text { covered all over, blanketed, } \\
\text { cloaked, concealed, covert } \\
\text { (operation) }^{875}\end{array}$ \\
\hline 18950 & $\begin{array}{l}2034 \\
2040\end{array}$ & $\mathrm{q}^{3} \mathrm{vl}^{3}{ }_{-}$ & $\mathrm{q}^{3} \mathrm{vl}^{3}-$ & $\mathrm{q}^{3} \mathrm{vl}^{3}-$ & $\begin{array}{l}\mathrm{q}^{3} \mathrm{vl}^{3-} \\
\mathrm{q}^{3} \mathrm{vm}^{3-*}\end{array}$ & $\begin{array}{l}\text { 1a. limit, end point, to reach the } \\
\text { end; } 1 \text { b. to bring to an end, to } \\
\text { finish, to complete; } 2 \text {. delimited, } \\
\text { defined, distinct, clear, lucid, } \\
\text { recognizable }{ }^{876}\end{array}$ \\
\hline 18970 & $\begin{array}{l}2042 \\
2053\end{array}$ & & & & $\begin{array}{l}\mathrm{q}^{3} \mathrm{vk}- \\
\mathrm{q}^{3} \mathrm{vkv}-*\end{array}$ & to notch \\
\hline 18980 & 2043 & & & & $\mathrm{q}^{3} \mathrm{vk}-*$ & $(?)^{877}$ \\
\hline 18990 & 2044 & & & & $q^{3} v y-$ & (?) (perhaps onomatopoeic) ${ }^{878}$ \\
\hline 19000 & 2045 & $\mathrm{q}^{3} \mathrm{vyt}-$ & $q^{3} \mathrm{vyt}-$ & $q^{3} v y t-$ & & to hug, to embrace \\
\hline 19001 & & & & & $q^{3} v y t-*$ & to pry, to split off \\
\hline
\end{tabular}

${ }^{874} \mathrm{KW}$ has preserved the original meaning of stirring etc. in $\left\{\mathrm{q}^{3} \mathrm{vylhx}-\right\}$ and $\left.\left\{\mathrm{q}^{3} \mathrm{vynh}\right\}-\right\}$. ${ }^{875}\left\{\mathrm{q}^{3} \mathrm{vl}^{3}-\right\}$ and $\left\{\mathrm{q}^{3} \mathrm{vl}^{3} \mathrm{~h}-\right\}$ occur in e.g. HE $/ \mathrm{q}^{3} \mathrm{v}^{3} \mathrm{k}^{3} \mathrm{H} /$ and $/ \mathrm{q}^{3} \mathrm{v}^{3} \mathrm{ak}^{3} \mathrm{H} / \mathrm{OO} / \mathrm{q}^{3} \mathrm{v}^{3} \mathrm{k}^{3} \mathrm{H} /$ and $/ \mathrm{q}^{3} \mathrm{VI}^{3} \mathrm{ak}^{3} \mathrm{H} / \mathrm{HA} / \mathrm{q}^{3} \mathrm{vJk}^{3} \mathrm{H} /$ "anything covering the body, etc." and in the HE/OO/HA/KW stem $\left\{q^{3} \mathrm{v} l^{3} h f-\right\}$ "to hide sth., to do sth. secretly." For KW this stem is the only example of $\left\{q^{3} \mathrm{vl}^{3}-\right\}$. Some and perhaps all derivates of the form $\left\{\mathrm{q}^{3} \mathrm{vm}^{3}\right\}^{*}$ are from the Koskimo dialect and could not be reelicited anyway.

${ }^{876} \mathrm{KW}\left\{\mathrm{q}^{3} \mathrm{vm}^{3}-\right\}^{*}$ only in $/ \mathrm{q}^{3} \mathrm{vM}^{3} \mathrm{ba} / *$ "at an end" which is from the Koskimo dialect.

${ }^{877} \mathrm{In} \mathrm{KW} / \mathrm{q}^{3} \mathrm{vka} / *$ "to get less."

${ }^{878}$ In KW /q ${ }^{3 i q^{3} v^{2} q^{3} v i / / " c r o s s b i l l " ~(" p e t r e l " ~ a c c o r d i n g ~ t o ~ K o s k i m o ~ s o u r c e) . ~}$ 
North Wakashan Comparative Root List (Updated)

\begin{tabular}{|c|c|c|c|c|c|c|}
\hline Root \# & Old \# & $\mathrm{HE}$ & $\mathrm{OO}$ & $\mathrm{HA}$ & KW & Gloss \\
\hline 19010 & $\begin{array}{l}2046 \\
2047\end{array}$ & $\begin{array}{l}q^{3} v y s- \\
q^{3} v^{3} s-\end{array}$ & $\begin{array}{l}q^{3} v y s- \\
q^{3} v^{3} s-\end{array}$ & $q^{3}$ vys- & $q^{3}$ vys- & $\begin{array}{l}\text { to grip, strangle, squeeze, } \\
\text { wring } \\
879\end{array}$ \\
\hline 19020 & 2048 & & & & $q^{3} v y £-$ & $\begin{array}{l}\text { to be silent, stop speaking, } \\
\text { quieten down }\end{array}$ \\
\hline 19040 & $\begin{array}{l}2049 \\
2050\end{array}$ & $q^{3}$ vył- & $q^{3}$ vył- & $q^{3}$ vyl- & $q^{3} v y 1-$ & $\begin{array}{l}\text { (HE/OO/KW) to break, crumble; } \\
\text { to grind up, crush, shatter, mince; } \\
\text { (HA) disintegrating, rotting } \\
\text { (meat, fruit fish) }\end{array}$ \\
\hline 19070 & 2051 & $q^{3}$ vyqv- & $q^{3}$ vyqv- & & $q^{3} v_{y q v-*}$ & $\begin{array}{l}\text { to twist, warp, loose shape or } \\
\text { structure }\end{array}$ \\
\hline 19080 & 2016 & $q^{3}$ wwmhq- & $q^{3} v w m h q-$ & & & stump ${ }^{880}$ \\
\hline 19090 & 2054 & $q^{3}$ vwt- & $q^{3}$ vwt- & $\mathrm{q}^{3} \mathrm{wt}-$ & $\left(t^{3} q-\right)$ & mushy, jam-like \\
\hline 19100 & 2055 & $q^{3} v w 1-$ & & & & salmon stew, fish mulligan \\
\hline 19110 & 2056 & $q^{3} v w 1-$ & $q^{3} v w 1-$ & & & $\begin{array}{l}\text { to bend or fall forwards (as in } \\
\text { diving, somersaulting) }\end{array}$ \\
\hline 19120 & $\begin{array}{l}2057 \\
2345\end{array}$ & $\begin{array}{l}q^{3} v w q v- \\
q^{3} w q v-\end{array}$ & $\begin{array}{l}q^{3} v w q v- \\
q^{3} w q v-\end{array}$ & $q^{3} w q v-$ & $q^{3} w q v-$ & $\begin{array}{l}\text { stationary, stable, free of flow, } \\
\text { calm (water, weather) }\end{array}$ \\
\hline 19130 & 2058 & $\mathrm{q}^{3} \mathrm{vw} \chi \mathrm{v}-$ & $\mathrm{q}^{3} \mathrm{vw} \chi \mathrm{v}-$ & & $\mathrm{q}^{3} \mathrm{w} \chi \mathrm{V}-$ & to dress \\
\hline 19140 & 2059 & $\begin{array}{l}q^{3} v w q v- \\
q^{3} v w \chi v-\end{array}$ & $\begin{array}{l}q^{3} v q v- \\
q^{3} v \chi v-\end{array}$ & & $\mathrm{q}^{3} \mathrm{vq}-$ & bright light shining, to flash \\
\hline 19150 & & & & $\begin{array}{l}q^{3} v q- \\
q^{3} v h q-\end{array}$ & & to wish for sth. ${ }^{881}$ \\
\hline 19160 & 2062 & & & & $\mathrm{q}^{3} \mathrm{vh}-*$ & $(?)^{882}$ \\
\hline
\end{tabular}

${ }^{879}$ Possibly also in OO /q³ vais/ "my friend!"

${ }^{880}$ Could contain a root $\left\{q^{3} \mathrm{vmq}-\right\}$ or $\left\{q^{3} \mathrm{vwmh}-\right\}$.

${ }^{881}$ In HA / ${ }^{3} \mathrm{vqла/} \mathrm{"to} \mathrm{wish} \mathrm{for} \mathrm{sth.,} \mathrm{etc."} \mathrm{and} \mathrm{HA} \mathrm{/q}{ }^{3} \mathrm{var} \nvdash /$ "what one wishes."

${ }^{882}$ In KW (Koskimo dialect) / $\mathrm{q}^{3} \mathrm{va} / *$ "to live." 
North Wakashan Comparative Root List (Updated)

\begin{tabular}{|c|c|c|c|c|c|c|}
\hline Root \# & Old \# & $\mathrm{HE}$ & $\mathrm{OO}$ & HA & KW & Gloss \\
\hline 19170 & $\begin{array}{l}2064 \\
2065\end{array}$ & $\mathrm{q}^{3} \mathrm{vh}-$ & $\mathrm{q}^{3} \mathrm{vh}-$ & $\mathrm{q}^{3} \mathrm{vh}-$ & $\mathrm{q}^{3} \mathrm{vh}-$ & $\begin{array}{l}\text { standing together, grouped or } \\
\text { bundled together; standing out, } \\
\text { rising }\end{array}$ \\
\hline 19180 & 2066 & & & & $\mathrm{q}^{3}$ vht- & $(?)^{883}$ \\
\hline 19190 & 2068 & $q^{3}$ vht- & $q^{3}$ vht- & $\mathrm{q}^{3} \mathrm{vht}-$ & $\begin{array}{l}q^{3} \text { vht-* } \\
q^{3} \text { vhc- } \\
q^{3} \text { vh }^{3} h c-\end{array}$ & $(?)^{884}$ \\
\hline 19200 & 2067 & $\begin{array}{l}\mathrm{q}^{3} \mathrm{vht}^{3}- \\
\mathrm{q}^{3} \mathrm{vht}^{3} \mathrm{~h}-\end{array}$ & $\begin{array}{l}q^{3} v t^{3}- \\
q^{3} v h t^{3} h-\end{array}$ & $\mathrm{q}^{3} \mathrm{vht} \mathrm{h}^{3} \mathrm{~h}-$ & & crowded $^{855}$ \\
\hline 19210 & 2069 & $q^{3}$ vhs- & $q^{3}$ vhs- & $q^{3}$ vhs- & $q^{3}$ vhs- & $\begin{array}{l}\text { to well up (tears), to weep, to } \\
\text { wail }\end{array}$ \\
\hline 19211 & & & & & $q^{3}$ vhs- & $(?)^{886}$ \\
\hline 19220 & $\begin{array}{l}2070 \\
2072\end{array}$ & $\mathrm{q}^{3} \mathrm{vht-}$ & $\mathrm{q}^{3} \mathrm{vh} 1-$ & $\mathrm{q}^{3} \mathrm{vhl}-$ & $\begin{array}{l}q^{3} v h 1- \\
q^{3} v^{3} l^{3-}\end{array}$ & $\begin{array}{l}\text { to burn to cinders, to destroy by } \\
\operatorname{war}^{887}\end{array}$ \\
\hline 19230 & 2071 & & $q^{3}$ vhlhx- & & $q^{3}$ vhlhx- & dress up for special occasion 888 \\
\hline
\end{tabular}

${ }^{883}$ In KW /q³ vadiqa/ "unhappy, downcast, sad."

${ }^{884}$ Examples refer to slugs and snails and seem to confuse snails, which have a coiled shell, and slugs, which lack it. Boas's glosses mention snails only (1928:268; 1948:364).

${ }^{885}$ A cross-family stem, cf. Proto-Salish *-q ${ }^{3} \mathrm{vac}^{3}-$ "full" in Kuipers (2202:95).

${ }^{886} \mathrm{In} \mathrm{KW} / \mathrm{q}^{3} \mathrm{vaq}^{3} \mathrm{vsH} \chi \varepsilon^{3 /}$ "edges of halibut" $\mathrm{KW} / \mathrm{q}^{3} \mathrm{vaq}^{3} \mathrm{vsaw} \varepsilon^{3 / *}$ "middle piece of salmon" $\mathrm{KW}$ /q ${ }^{3}$ vayus/ (probably:) "lower jaw" KW /q ${ }^{3} \mathrm{vay}^{3} \mathrm{uc}^{3} \mathrm{a} / *$ "soft end of fern $\operatorname{root}$ KW $/ \mathrm{q}^{3} \mathrm{vayal}^{3} \mathrm{ac}^{3} \mathrm{i} / *$ "castoreum." Boas's dictionary lists the first two as derivates of $\left\{q^{3} v h q-\right\}$ "to split etc." They could also be misrecordings. Note the semantic similarity between $\mathrm{KW} / \mathrm{q}^{3} \mathrm{vaq}^{3} \mathrm{vsH} \chi \varepsilon^{3} /$ and the $\left\{q^{3} v h-\right\}$-derivate $\left\{q^{3} v h\left[q^{3} v h\right]-n \chi-\alpha y^{3}\right\}>/ q^{3} v q^{3} v H \chi \varepsilon^{3} /$ "ribs of halibut (standing on edge)." ${ }^{887}$ Is a cross-family stem, cf. Proto-Salish *-q33 vhl-, *-q3vay- "to scorch, (burn to) ashes, black, etc." in Kuipers (2002:95).

${ }^{888} \mathrm{OO}$ only in names of likely KW origin. 
North Wakashan Comparative Root List (Updated)

\begin{tabular}{|c|c|c|c|c|c|c|}
\hline Root \# & Old \# & $\mathrm{HE}$ & $\mathrm{OO}$ & $\mathrm{HA}$ & $\mathrm{KW}$ & Gloss \\
\hline 19240 & 2074 & $\mathrm{q}^{3} \mathrm{vhk}-$ & $\mathrm{q}^{3} \mathrm{vhk}-$ & $q^{3}$ vhk- & & to stand up, to rise \\
\hline 19250 & 2075 & & $q^{3} \mathrm{vhk}-$ & & & $(?)^{889}$ \\
\hline 19260 & $\begin{array}{l}2076 \\
2077 \\
2078\end{array}$ & $q^{3}$ vhqv- & $q^{3}$ vhqv- & $q^{3}$ vhqv- & $q^{3}$ vhqv- & $\begin{array}{l}\text { grey, smoke colour, dogfish } \\
\text { colour; (hence:) (HE/OO/HA) } \\
\text { dogfish; to stick out, rise up, } \\
\text { pierce (like dorsal spines of } \\
\text { dogfish); (KW) 1. to darken (by } \\
\text { smoke, heat), grey (like seagull); } \\
\text { 2. to stretch oneself out with the } \\
\text { arms up }\end{array}$ \\
\hline 19280 & 2079 & $q^{3} v h q-$ & $\begin{array}{l}q^{3} v h q- \\
q^{3} v h \chi_{-}^{-}\end{array}$ & $q^{3} v h q-$ & & to scream, to shout ${ }^{891}$ \\
\hline 19281 & 2080 & & $q^{3} v h q-$ & & $\mathrm{q}^{3} \mathrm{vhq}-*$ & $\begin{array}{l}\text { to split along an axis (like salmon } \\
\text { halves along the backbone prior } \\
\text { to smoking) }\end{array}$ \\
\hline
\end{tabular}

${ }^{889} \mathrm{In} \mathrm{OO} / \mathrm{q}^{3} \mathrm{vaka} /$ "to take salmon out of the trap."

${ }^{890}$ 1. According to https://en.wikipedia.org/wiki/Spiny_dogfish, dogfish are greyish brown at the back but according to http://www.enchantedlearning.com/subjects/sharks/species/Dogfish.shtml the colour is deep grey with some white spots. 2. Also according to https://en.wikipedia.org/wiki/Spiny_dogfish, the dogfish can pierce its captor with its two dorsal spines by arching its back. 3. The association with dogfish survives in KW only in the "stretch out" meaning. 4. Possibly the root occurs with the "pierce" and "stick up" meaning in OO $\left.\left\{\mathrm{q}^{3} \mathrm{vhqv}-{ }^{+} \mathrm{w}^{3} \mathrm{f}\right\}>/ \mathrm{q}^{3} \mathrm{vaq}^{3} \mathrm{vu}\right\} /$ "fence" (which probably refers to a palisade) and in KW $/ \mathrm{q}^{3} \mathrm{vaq}^{3} \mathrm{vu}^{3} \mathrm{Hsm}_{\mathrm{sm}} /$ "barrel." 5. The root is a cross-family stem, cf. Proto-Salish *-q ${ }^{3} \mathrm{va} \chi / \chi \mathrm{v}-$ "to smoke (said of fire), smoke colour" in Kuipers (2002:97).

${ }^{891}\left\{\mathrm{q}^{3} \mathrm{vh} \chi-\right\}$ only in $\mathrm{OO} / \mathrm{q}^{3} \mathrm{vaq}^{3} \mathrm{va} \chi \mathrm{a} /$ [1] "to summon people to a potlatch planning." Possibly this root really refers more generally to a channel's mouth opening wide ("splitting") to let something through, in which case it is related to roots nos. 19281 and 19290. 
North Wakashan Comparative Root List (Updated)

\begin{tabular}{|c|c|c|c|c|c|c|}
\hline Root \# & Old \# & $\mathrm{HE}$ & $\mathrm{OO}$ & HA & KW & Gloss \\
\hline 19290 & $\begin{array}{l}2081 \\
2082 \\
2083\end{array}$ & $\mathrm{q}^{3} \mathrm{vh} \chi-$ & $q^{3} \operatorname{vh} \chi-$ & $\mathrm{q}^{3} \mathrm{vh} \chi-$ & $\mathrm{q}^{3} \mathrm{vh} \chi-$ & $\begin{array}{l}\text { to sprout or fold off a stem (like } \\
\text { growing plant's buds, leaves, } \\
\text { branches); to slope down from a } \\
\text { ridge or stem (like roof's sides, } \\
\text { the down-sweeping branches of a } \\
\text { western hemlock tree); to grow } \\
\text { (like plant), to rise, to expand } \\
\text { (like dough) }\end{array}$ \\
\hline 19300 & 2073 & $\mathrm{q}^{3} \mathrm{vh}^{3} \mathrm{q}_{-}$ & $\begin{array}{l}q^{3} v^{\prime} 1- \\
q^{3} v^{3} t_{-}\end{array}$ & $\mathrm{q}^{3} \mathrm{vh}^{3} \mathrm{l}_{-}$ & $q^{3} v^{3} t^{-}$ & to pick sprouts \\
\hline 19310 & 2084 & & & & $\mathrm{q}^{3} \mathrm{vh}^{3} \mathrm{x}-*$ & to sit up straight, stretch the body \\
\hline 19320 & $\begin{array}{l}2086 \\
2087\end{array}$ & & & & $\chi \mathrm{vmt}-$ & $\begin{array}{l}\text { squirming, writhing, undulatory } \\
\text { motion }^{892}\end{array}$ \\
\hline 19340 & 2088 & $\chi v \mathrm{vms}-$ & $\chi \mathrm{vms}-$ & $\chi \mathrm{vms}-$ & $\chi \vee \mathrm{vms}-$ & venereal disease \\
\hline 19350 & 2089 & $\chi v t-$ & $\chi \mathrm{vt}-$ & & $\begin{array}{l}\chi \mathrm{vt}- \\
\chi \mathrm{vht}-\end{array}$ & $\begin{array}{l}\text { (HE/OO) to cut with a knife; } \\
(\mathrm{KW}) \text { to cut grooves with a knife } \\
\text { on a swelling to make it go } \\
\text { down }^{893}\end{array}$ \\
\hline 19360 & 2090 & $\chi \mathrm{vn}-$ & $\chi \mathrm{vn}-$ & $\chi^{\mathrm{vn}-}$ & $\chi \mathrm{vn}-$ & to shake, shiver, tremble; tense \\
\hline 19370 & 2091 & $\chi \mathrm{vn}-$ & $\chi \mathrm{vn}-$ & & & burnt up (said of fire) \\
\hline 19380 & 2092 & & & & $\chi \mathrm{vnt}-$ & excited, agitated, jumpy, nervous \\
\hline
\end{tabular}

${ }^{892} \mathrm{In} \mathrm{KW}\{[\chi \mathrm{vmt}] \chi \mathrm{vmt}-\mathrm{h}\}>/ \chi \mathrm{vMt}: \chi \mathrm{vMta} /$ "epileptic fits" and words referring to the land otter (= river otter). The link between the two is probably that a land otter can swim by dorsoventral undulation of body and tail (cf. https://en.wikipedia.org/wiki/North_American_river_otter and http://animals.nationalgeographic.com/animals/mammals/american-river-otter/). ${ }^{893}$ HA uses $\{$ tws- $\}$ and $\left\{\mathrm{k}^{3} \mathrm{y}-\right\}$ and KW uses $\left\{\mathrm{t}^{3} \mathrm{ws}-\right\}$ for cutting with a knife in general. 
North Wakashan Comparative Root List (Updated)

\begin{tabular}{|c|c|c|c|c|c|c|}
\hline Root \# & Old \# & $\mathrm{HE}$ & $\mathrm{OO}$ & HA & KW & Gloss \\
\hline 19390 & 2093 & zvnkv- & 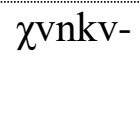 & ұvnkv- & 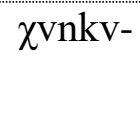 & $\begin{array}{l}\text { child, offspring (of people, } \\
\text { animals) } \\
894\end{array}$ \\
\hline 19400 & 2094 & ұvs- & 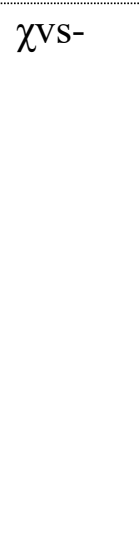 & 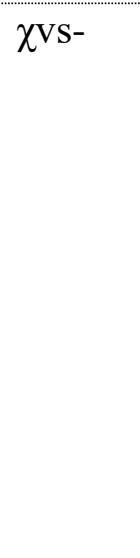 & & $\begin{array}{l}\text { losing shape, cohesion or vigour; } \\
\text { to lose parts (as when plants lose } \\
\text { berries, leaves); to dissolve (like } \\
\text { soap flakes), melt, disintegrate; } \\
\text { (HA also:) to become covered } \\
\text { (e.g. with mud, water), to become } \\
\text { overpowered; to get cleared (of } \\
\text { clutter) }\end{array}$ \\
\hline 19420 & $\begin{array}{l}2094 \\
2101\end{array}$ & $\chi v 1-$ & 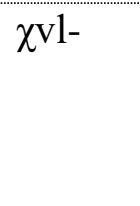 & $\begin{array}{l}\chi \text { vl- } \\
\chi \text { vlc- } \\
\chi \text { vls- }\end{array}$ & $\begin{array}{l}\chi \mathrm{VS}- \\
\chi \mathrm{VZ}-\end{array}$ & $\begin{array}{l}\text { seal blubber; (HA also:) animal } \\
\text { fat }^{895}\end{array}$ \\
\hline 19430 & 2096 & & & & 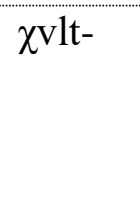 & $\begin{array}{l}\text { to mark a line (as for sawing), to } \\
\text { scratch a mark on sth. (e.g. when } \\
\text { drawing pictures on the sand) }\end{array}$ \\
\hline 19440 & $\begin{array}{l}2097 \\
2098\end{array}$ & $\chi$ vlc- & $\chi$ vlc- & $\chi$ vlc- & $\chi$ vls- & $\begin{array}{l}\text { (?) to turn the head aside; head- } \\
\text { turning, eye-catching, } \\
\text { conspicuous } 896\end{array}$ \\
\hline
\end{tabular}

${ }^{894}$ In all dialects the root occurs also as the free form $\{\chi v n w k v-\}$ in which the augment $|w|$ stems from $|\infty|$. Additionally, in $\mathrm{KW}$ it occurs in a variety of augmented forms whose interpretation is problematic, cf. $\{\chi \mathrm{vnynkv-}\},\{\chi \mathrm{vny} \infty \mathrm{kv}-\},\{\chi \mathrm{vhynkv-}\},\left\{\chi \mathrm{vhn}^{3} \mathrm{wkv}-\right\}$.

$895\{\chi$ vlc- $\}$ or $\{\chi$ vls- $\}$ only in HA $\{\chi$ vlc/s- $£$ ms $\}>/ \chi v^{\prime}$ лs $\lambda$ Ms/ "fat on the belly."

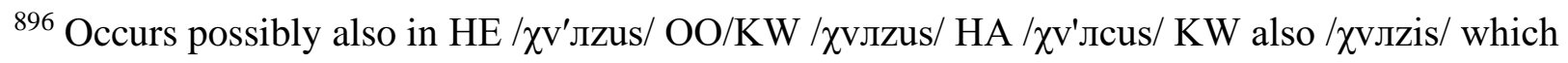
is a fish name consultants say refers to a tommy cod, ratfish or "big-eye." Boas (1947:332) says it is a Hexagrammos superciliaris. On p. 276 of Bella Bella Texts Boas says it means a Hexagrammos octogrammus, which is the masked greenling and a member of a family of brilliantly colourful fishes that also include the non-cod called ling cod (http://greenling.steveclarkhall.com/fish.htm). Bella Bella elder Willie Gladstone said the fish in 
North Wakashan Comparative Root List (Updated)

\begin{tabular}{|c|c|c|c|c|c|c|}
\hline Root \# & Old \# & $\mathrm{HE}$ & $\mathrm{OO}$ & HA & KW & Gloss \\
\hline 19450 & 2099 & & $\chi v 1 k-$ & & $\chi$ vlk- & to mark with a groove \\
\hline 19451 & 2095 & & & & 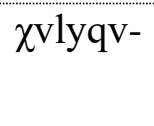 & $\begin{array}{l}\text { to shake the hands (in dancing), } \\
\text { to crumple in the hands }{ }^{897}\end{array}$ \\
\hline 19460 & 2100 & 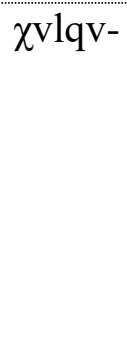 & 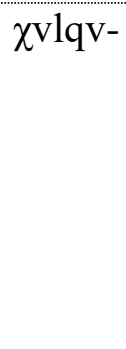 & ¿vlqv- & zvlqv- & $\begin{array}{l}\text { shark, rough like shark skin, to } \\
\text { use sth. rough (like sandpaper, a } \\
\text { file or dogfish skin) in order to } \\
\text { smooth a surface or sharpen an } \\
\text { edge }\end{array}$ \\
\hline 19480 & 2102 & & & & $\chi \mathrm{vk}-$ & crack (as in a rock), groove, ditch \\
\hline 19490 & 2103 & 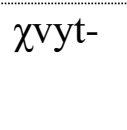 & 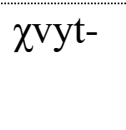 & 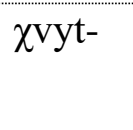 & $\chi v y t-$ & $\begin{array}{l}\text { to stir, to mix by stirring, to mash } \\
\text { with a spoon }\end{array}$ \\
\hline 19500 & 2104 & & & & $\chi v y £-$ & to fart \\
\hline 19510 & 2105 & & & & 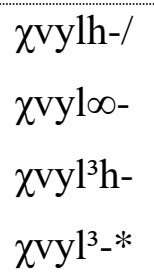 & $(?)^{898}$ \\
\hline
\end{tabular}

question is smaller than a ling cod, which is consistent with it being a greenling. The nickname "big-eye" however points to the ratfish, which is a small shark with a duckbill-shaped snout, a long pointed rat-like tail and large emerald green eyes which are able to reflect light, similar to the eyes of a cat. The North Pacific tommy cod or tomcod (Microgadus proximus) is cod-like and doesn't have conspicuous features like the ratfish or the greenling family.

${ }^{897}$ Could contain a root $\{\chi v 1-\}$ or $\{\chi v 1-\}$, or be an expanded form of $\{\chi v l q v-\}$. Tension could be the shared element of the stem's two senses.

$898\{\chi v y l h-\}$ or $\{\chi v y l \infty 0-\}$ occurs with suffix no. 4220 in the stems $\{\chi v y l h / \infty-w-\}$ and $\{\chi v y l h / \infty-$ $\left.h^{3}-\right\}$ "to spawn." $\left\{\chi v y l^{3} h-\right\}$ is a free form meaning "quartz crystals." Boas's dictionary lists it as $/ \chi \mathrm{vi}^{3} / *<\left\{\chi v y l^{3}-\right\}(1948: 391)$. The semantic connection between spawning and quartz awaits clarification. 
North Wakashan Comparative Root List (Updated)

\begin{tabular}{|c|c|c|c|c|c|c|}
\hline Root \# & Old \# & $\mathrm{HE}$ & $\mathrm{OO}$ & HA & KW & Gloss \\
\hline 19520 & 2106 & & $\chi v y\}-$ & & $\chi \mathrm{v} y \mathrm{x}-$ & $\begin{array}{l}\text { (?) losing shape, cohesion or } \\
\text { vigour }^{899}\end{array}$ \\
\hline 19530 & & & & $\chi v \mathrm{vk}-$ & & $\begin{array}{l}\text { (?) pebbles, loose small hard } \\
\text { things (e.g. potatoes) }\end{array}$ \\
\hline 19540 & 2107 & $\chi \mathrm{vws}-$ & $\chi$ VWS- & & (zs-) & $\begin{array}{l}\text { to boil (as water), to give off } \\
\text { bubbles }\end{array}$ \\
\hline 19580 & 2109 & & & & $\chi$ vht- & lascivious, fond of mating ${ }^{900}$ \\
\hline 19590 & 2110 & & & & $\begin{array}{l}\chi \text { vhnh- } \\
\chi \text { vhnhh- }\end{array}$ & poised for moving or acting 901 \\
\hline 19600 & $\begin{array}{l}2111 \\
2112\end{array}$ & & & $\chi$ vhs- & $\chi^{\text {vhs- }}$ & $\begin{array}{l}\text { (HA) to scrape off inner } \\
\text { cottonwood bark; (KW) to tear } \\
\text { (skin, fabric, a sheet of sth.) }\end{array}$ \\
\hline 19610 & 2113 & $\chi$ vhs- & $\chi$ vhs- & $\chi$ vhs- & $\chi \mathrm{vhs}^{*} *$ & $\begin{array}{l}\text { (HE/OO/HA) maggoty; (KW) } \\
\text { flyblow }^{902}\end{array}$ \\
\hline 19620 & 2114 & & & $\chi v \mathrm{vhf}-$ & $\chi \mathrm{vhf} f-$ & to fillet, to gut, to carve \\
\hline 19630 & 2115 & & & & $\chi v^{2} k^{3} n-*$ & old female salmon \\
\hline 19640 & 2116 & & & & 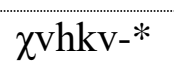 & furrows \\
\hline
\end{tabular}

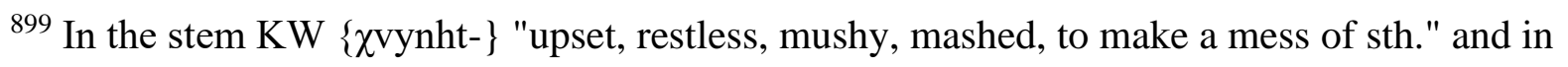
OO $\{\chi v y l h k v\}$ "(sth.) having lost its form again (as e.g. whipped-up berries)."

${ }^{900}$ In words for testicles and the wren. Wrens are associated with sexual activity, cf. p. 137 of Hunting the Wren: Transformation of Bird to Symbol: a Study in Human-Animal Relationships by Elizabeth Atwood Lawrence, 1997, The University of Tennessee Press/Knoxville.

$901\{\chi$ vhnh- $\}$ could be an augmented form of $\{\chi v \mathrm{vn}-\}$ (root no. 19360) with the meaning "tense." It also occurs in the stem $\{\chi$ vhnhł- $\}$ but is here followed by a suffix whose form could be $\{-1\}$ or $\{-$ hł\} while its meaning is unclear.

902 Could be a cross-family stem, cf. proto-Salish *-xv/ $\chi$ vay-a7 "fly, maggot, worm, ant" in Kuipers (2002:122). 
North Wakashan Comparative Root List (Updated)

\begin{tabular}{|c|c|c|c|c|c|c|}
\hline Root \# & Old \# & $\mathrm{HE}$ & $\mathrm{OO}$ & HA & KW & Gloss \\
\hline 19660 & 2117 & & & & $\chi$ vhq- & to scratch ${ }^{903}$ \\
\hline 19670 & 2118 & & & гр- & гр- & $(?)^{904}$ \\
\hline 19680 & & & & Ip- & & bearing the name of $\ldots{ }^{905}$ \\
\hline 19690 & 2120 & Im- & Im- & rm- & Im-* & to lie somewhere 906 \\
\hline 19700 & 2121 & & & & $\begin{array}{l}\text { Imt- } \\
\text { rms-* }\end{array}$ & $\begin{array}{l}\text { to gather up or hold loose things } \\
\text { (e.g. wood, moss, down, fern } \\
\text { fronds) }\end{array}$ \\
\hline 19730 & 2122 & & & & $\operatorname{rm} \chi-$ & $\begin{array}{l}\text { to use one's arms to carry or keep } \\
\text { in place loose things (e.g. } \\
\text { blankets) }\end{array}$ \\
\hline 19740 & 2123 & $\operatorname{Im}^{3} \chi-$ & $\operatorname{Im}^{3} \chi-$ & & $\operatorname{Im}^{3} \chi-$ & left side \\
\hline 19750 & 2124 & & & It- & It- & bent, curved, rounded \\
\hline 19760 & 2125 & In- & In- & In- & $\begin{array}{l}\text { In- } \\
\text { rhnh- }\end{array}$ & female \\
\hline 19770 & 2126 & In- & In- & In- & rn- & to carry a load in one's arms \\
\hline 19780 & 2127 & In- & In- & & rl- & wave \\
\hline
\end{tabular}

${ }^{903}$ Could be an alternant of $\{\mathrm{xvhq}-\}$ (root no. 16730). Is a cross-family stem, cf. *- $\chi \mathrm{vu} / \mathrm{aqv}-$ "to grind, rasp; snore" in Kuipers \{2002:128).

${ }^{904}$ Possibly in KW /rbлuұvstvi3/ "eyeball" and НA /rarabл'ai/ "1. to tickle somebody; 2. the starshaped receptacle which remains after a raspberry or strawberry is picked." What may link these meanings is uncertain. As suggested in Lincoln (n.d.), perhaps the root $\{\mathrm{rp}-\}$ means "inserted, tucked in" and is an alternant of $\{\mathrm{gp}-, \mathrm{kp}-\}$ "to tuck in etc." (root no. 11580).

${ }^{905}$ Used proclitically and with left-hand adjunct terminating $\{\#$ \} $\}$, as in HA /rps I'auləq/ "one called /Г'auləq/."

${ }^{906}$ In HA said of people. 
North Wakashan Comparative Root List (Updated)

\begin{tabular}{|c|c|c|c|c|c|c|}
\hline Root \# & Old \# & $\mathrm{HE}$ & $\mathrm{OO}$ & HA & KW & Gloss \\
\hline 19790 & 2128 & rnys- & Inys- & $\begin{array}{l}\text { Inys- } \\
\text { In }^{3} \text { ys- }\end{array}$ & $\begin{array}{l}\text { rlys- } \\
\text { rl } 1^{3} y s-\end{array}$ & $\begin{array}{l}\text { to screech (like bird), to squeak, } \\
\text { to scream (like siren) }{ }^{907}\end{array}$ \\
\hline 19800 & 2129 & & & & rnf- & $(?)^{908}$ \\
\hline 19810 & 2130 & & & & rnt-* & to beware, to take care $e^{909}$ \\
\hline 19820 & 2131 & & & & rnk- & $\begin{array}{l}\text { thick, viscous (said of a liquid, } \\
\text { fog, mud, oil, paste), pasty, } \\
\text { mushy, to get thick }\end{array}$ \\
\hline 19821 & & & & IS- & & $(?)^{910}$ \\
\hline 19830 & $\begin{array}{l}2132 \\
2133 \\
2134\end{array}$ & qs- & Is- & IS- & $\begin{array}{l}\text { Is- } \\
\text { qs- }\end{array}$ & $\begin{array}{l}\text { round, oval, curving, coiled, } \\
\text { winding; one's eye }\end{array}$ \\
\hline 19840 & 2138 & $\begin{array}{l}\text { Il- } \\
\text { Ilh- }\end{array}$ & $\begin{array}{l}\text { rl- } \\
\text { rlh- }\end{array}$ & rlh- & & to wait for \\
\hline 19850 & $\begin{array}{l}2137 \\
2139\end{array}$ & Il- & Il- & Il- & Il- & $\begin{array}{l}\text { curved shape (like bird's beak), } \\
\text { curving motion, to subtend, to } \\
\text { connect two sides of an angle }\end{array}$ \\
\hline
\end{tabular}

${ }^{907} 1$. The Kitlope consultant for HA used $\left\{\mathrm{In}^{3} \mathrm{ys}-\right\}$ but from the Kitamaat one \{rnys- $\}$ was recorded. KW $\left\{\mathrm{Il}^{3} \mathrm{ys}-\right\}$ is attested only in $\left\{\mathrm{Il}^{3} \mathrm{ys}-\% \mathrm{tw} \alpha \mathrm{y}^{3}\right\}>/ \mathrm{I}^{3} \mathrm{iztv} \varepsilon^{3 /}$ "screaming while going along." 2. Stem could contain a root HE/OO/HA $\left.\left\{\mathrm{IX}^{-}, \mathrm{In}-\right\} \mathrm{KW}\{\mathrm{I}\}-, \mathrm{rl}-\right\}$. Boas reports a term $\mathrm{KW} / \mathrm{I} \nmid \hbar^{3} \mathrm{id} / *$ "to reproach" that perhaps shares a root $\left.\{\mathrm{I}\}-\right\}$ "shrill (piercing) sound or talk" with KW \{rlys- , however, the term could not be re-elicited.

908 Only in $\mathrm{KW} / \mathrm{r} \mathrm{H} \mathrm{a} /$ "to thicken (as oil in cold weather)."

${ }^{909}$ In KW (Koskimo and Newettee dialects)/rnła/* "beware!" and KW /rнłagлił/* "to take care of oneself in the house."

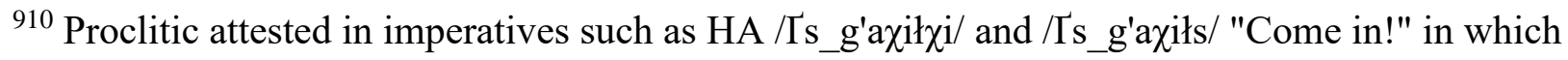
the structural centre is $\{\mathrm{gh} \chi-=\mathrm{y} £\}$ "to come into sth." to which have been joined $\{\# \chi y\}$ (suffix no. 5440) and $\{\# s\}$ (suffix no. 2200). Unknown what the proclitic contributes semantically. 
North Wakashan Comparative Root List (Updated)

\begin{tabular}{|c|c|c|c|c|c|c|}
\hline Root \# & Old \# & $\mathrm{HE}$ & $\mathrm{OO}$ & HA & KW & Gloss \\
\hline & & & & & & $\begin{array}{l}\text { (like muscle), to lie or put } \\
\text { across }^{911}\end{array}$ \\
\hline 19860 & 2140 & rlp- & rlp- & Ilp- & rlp- & $\begin{array}{l}\text { to use hooked fingers or hand for } \\
\text { pulling towards oneself or } \\
\text { hanging on (as when pulling } \\
\text { trigger or holding on to rail or } \\
\text { leash); (in some KW non- } \\
\text { reelicitable examples also:) to } \\
\text { count using finger to bend other } \\
\text { hand's fingers }\end{array}$ \\
\hline 19870 & 2141 & & & & Ils- & to paint, to smear on ${ }^{912}$ \\
\hline 19880 & & & & Ilk- & & $\begin{array}{l}\text { peg or pole placed across sth. } \\
\text { (e.g. a slope) to support or } \\
\text { stabilize it }\end{array}$ \\
\hline 19890 & 2142 & rlxv- & rlxv- & rlxv- & Ilxv- & $\begin{array}{l}\text { hook-shaped, to use the hooked } \\
\text { fingers for pulling, lifting or } \\
\text { counting. }\end{array}$ \\
\hline 19891 & 2143 & rlwhd- & rlwhd- & & & water lily ${ }^{913}$ \\
\hline 19900 & $\begin{array}{l}2144 \\
2145\end{array}$ & rlq- & $\begin{array}{l}\text { rlq- } \\
\text { rl } \chi-\end{array}$ & rlq- & rlq- & $\begin{array}{l}\text { to curve fingers or hand into a } \\
\text { hook or scoop (as for lifting a } \\
\text { container by the handle or } \\
\text { pushing water away with the }\end{array}$ \\
\hline
\end{tabular}

911 Root occurs perhaps also in KW /плаł/* "redbreasted robin." \{rhlh-\} (root no. 20280) and $\left\{\mathrm{Ihl}^{3}-\right\}$ (no. 20290) could be augmented forms of $\{\mathrm{Il}-\}$.

912 This root and $\left\{q^{3} 1 c-, q^{3} l s-\right\}$ may really both refer to rubbing on any mushy or greasy substance with the finger.

913 Only in HE /ז'лw'at/ OO /глwat/ "water lily." 
North Wakashan Comparative Root List (Updated)

\begin{tabular}{|c|c|c|c|c|c|c|}
\hline Root \# & Old \# & $\mathrm{HE}$ & $\mathrm{OO}$ & HA & KW & Gloss \\
\hline & & & & & & $\begin{array}{l}\text { hand), to swim by curving a } \\
\text { fin }^{914}\end{array}$ \\
\hline 19910 & & & & & $\begin{array}{l}\text { Ilq- } \\
\text { rlyq- }\end{array}$ & wild rose \\
\hline 19930 & 2146 & $\mathrm{rl} \chi \mathrm{-}$ & rl $\chi-$ & & $\mathrm{rl} \chi-$ & to twist ${ }^{915}$ \\
\hline 19940 & 2148 & rk- & $\begin{array}{l}\text { rk- } \\
\text { qk- }\end{array}$ & rk- & $\begin{array}{l}\text { rk- } \\
\text { rhk- }\end{array}$ & woman, wife \\
\hline 19950 & 2187 & rhx- & rhx- & IX- & & $(?)^{916}$ \\
\hline 19970 & 2150 & ry- & ry- & $\begin{array}{l}\text { ry- } \\
\text { rhyh- }\end{array}$ & гу- & $\begin{array}{l}\text { long in duration, happening long } \\
\text { ago, late }\end{array}$ \\
\hline 19971 & 2151 & $\begin{array}{l}\text { ry- } \\
\text { ryh- }\end{array}$ & ry- & rh- & ry- & $\begin{array}{l}\text { come!; to come across, } \\
\text { reciprocate, thank }\end{array}$ \\
\hline 19980 & $\begin{array}{l}2152 \\
2177\end{array}$ & $\begin{array}{l}\text { ry- } \\
\text { ry } \\
\text { ry } \\
\text { rh- }\end{array}$ & $\begin{array}{l}\text { ry }{ }^{3}- \\
r y^{3} h-\end{array}$ & $\begin{array}{l}\text { ry- } \\
\text { ry }{ }^{3}- \\
\text { ry }{ }^{3} \mathrm{~h}- \\
\text { rhy }\end{array}$ & $\begin{array}{l}\text { ry }{ }^{3}- \\
\text { ry }{ }^{3} \mathrm{~h}- \\
\text { rhy }\end{array}$ & across, crosswise, sideways ${ }^{917}$ \\
\hline 19990 & 2153 & ryp- & ryp- & ryp- & гур- & birthmark, freckle, mole \\
\hline 20000 & 2154 & & & & ryt- & $(?)^{918}$ \\
\hline 20010 & 2155 & & & & rynht- & to threaten \\
\hline
\end{tabular}

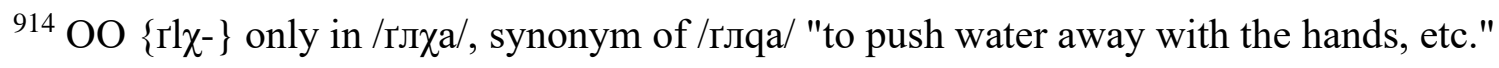
${ }^{915} \mathrm{In} \mathrm{KW} / \mathrm{rl} \chi \cdot \mathrm{h}^{3} \mathrm{id} /$ "to spin threads of bark together" and two HE/OO words referring to handling a bolt or screw.

916 Possibly in $\mathrm{HE} / \mathrm{rałr}^{3} \mathrm{iq}^{3} / \mathrm{OO} / \mathrm{ra}^{3} \mathrm{r}_{\mathrm{H}^{3}} \mathrm{iiq}^{3} / \mathrm{HA} / \mathrm{r} \nmid \mathrm{rHaiq}^{3} /$ "abalone" and related words. ${ }^{917}$ In derivates it may not be diagnosable if $\left\{\mathrm{ry}^{3}-\right\}$ or $\left\{\mathrm{ry}^{3} \mathrm{~h}-\right\}$ is present in which case $\left\{\mathrm{ry}^{3}-\right\}$ is assumed.

918 In KW /rita/ "fresh (said of fish or meat)." 
North Wakashan Comparative Root List (Updated)

\begin{tabular}{|c|c|c|c|c|c|c|}
\hline Root \# & Old \# & $\mathrm{HE}$ & $\mathrm{OO}$ & HA & KW & Gloss \\
\hline 20020 & 2149 & гyn ${ }^{3} y-$ & гyn ${ }^{3} y-$ & & $\begin{array}{l}\text { гуn }{ }^{3}- \\
\text { гуn }{ }^{3} \mathrm{y}-\end{array}$ & $(?)^{919}$ \\
\hline 20030 & 2156 & rys- & rys- & гys- & Гys- & $\begin{array}{l}\text { cow parsnip (Heracleum } \\
\text { lanatum) }\end{array}$ \\
\hline 20040 & $\begin{array}{l}2157 \\
2158 \\
2227\end{array}$ & $\begin{array}{l}\text { qy }^{3} \mathrm{~s}- \\
\text { qys- }\end{array}$ & $\begin{array}{l}\mathrm{qy}^{3} \mathrm{~s}- \\
\text { qys- }\end{array}$ & qys- & $\begin{array}{l}\text { rys- } \\
\text { qys- }\end{array}$ & $\begin{array}{l}\text { (HE/OO/HA and KW \{rys- }\}:) \\
\text { mushy, jelly-like, creamy; to } \\
\text { scrape up anything mushy (food, } \\
\text { paint) with a finger; (KW } \\
\text { \{qys-\}:) smooth, shiny, slippery; } \\
\text { to smooth }\end{array}$ \\
\hline 20050 & 2159 & rys- & rys- & rys- & rys- & to trip up, to trip sb. \\
\hline 20060 & 2160 & ryx- & гух- & гух- & гух- & $\begin{array}{l}\text { to stick on (like a burr, like hair } \\
\text { lice) }\end{array}$ \\
\hline 20070 & 2161 & & & & гуXv- & to hang \\
\hline 20080 & 2161 & $\left(\mathrm{qhm}^{3}-\right)$ & $\left(\mathrm{qhm}^{3}-\right)$ & $\left(\mathrm{qhm}^{3}-\right)$ & гyxv- & deer \\
\hline 20090 & 2162 & qXv- & $\left(q^{3} w p-\right)$ & rXV- & $\begin{array}{l}\text { IXV- } \\
\mathrm{qXV}-*\end{array}$ & to copulate, to cohabit ${ }^{921}$ \\
\hline 20100 & 2163 & rW- & rW- & $\begin{array}{l}\text { IW- } \\
\text { rhwh- }\end{array}$ & Thwh- & $\begin{array}{l}\text { (?) to transpire, to become noted; } \\
\text { to reveal }{ }^{922}\end{array}$ \\
\hline 20110 & $\begin{array}{l}2164 \\
2165\end{array}$ & $\begin{array}{l}\text { rwp- } \\
\text { rwm }{ }^{3} h-\end{array}$ & $\begin{array}{l}\text { rwp- } \\
\text { rwm }{ }^{3} h-\end{array}$ & $\begin{array}{l}\text { гwp- } \\
\text { זWm }\end{array}$ & rwp- & $\begin{array}{l}\text { becoming thin, flat and wide at } \\
\text { one end (like paddle, fish scales); }\end{array}$ \\
\hline
\end{tabular}

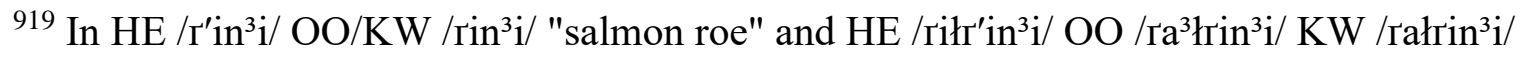
"kidneys."

${ }^{920} \mathrm{HE} / \mathrm{OO}\{$ qys- $\}$ takes the place of $\left\{q^{3}{ }^{3} \mathrm{~s}-\right\}$ when the root undergoes plural and other augmentation.

${ }^{921} \mathrm{KW}\{\mathrm{IXV}-\}$ is attested only in the Koskimo and Newettee dialects.

$922\{\mathrm{rW}-\}$ only in the HE/OO stem $\{\mathrm{rW}-\mathrm{hk}-\}$ "to bestow a name upon sb." and the HA stem \{rW-mH1- $\}$ "aware sth. is near one." KW \{rhwh- $\}$ * perhaps in /rawa/* "head of devilfish." 
North Wakashan Comparative Root List (Updated)

\begin{tabular}{|c|c|c|c|c|c|c|}
\hline Root \# & Old \# & $\mathrm{HE}$ & $\mathrm{OO}$ & HA & KW & Gloss \\
\hline & & & & & & $\begin{array}{l}\text { to flare out at the end, tile- } \\
\text { shaped, plate-like }\end{array}$ \\
\hline 20120 & 2166 & & & & rwt- & $(?)^{923}$ \\
\hline 20130 & 2167 & & & & rwt- & (?) loon 924 \\
\hline 20140 & $\begin{array}{l}2168 \\
2170\end{array}$ & IWs- & IWs- & Iws- & rws- & $\begin{array}{l}\text { protected by a framework of } \\
\text { boards or crossed poles, sheltered }\end{array}$ \\
\hline 20150 & 2169 & & & & rws- & sweaty, steamed up, dewy \\
\hline 20160 & 2171 & & & $\begin{array}{l}\text { rW£- } \\
\text { rWl- }\end{array}$ & זWf- & to claim sth. \\
\hline 20170 & 2172 & & & & $\begin{array}{l}\text { rw1- } \\
\text { rwl }\end{array}$ & $\begin{array}{l}\text { to rake in with fingers, to scrape } \\
\text { up or scoop out with fingers }\end{array}$ \\
\hline 20180 & 2174 & rwł- & Iw1- & Iw1- & rwł- & berry \\
\hline 20190 & 2173 & & & & rwh-* & $(?)^{925}$ \\
\hline 20200 & 2176 & rw $\chi v-$ & 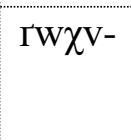 & $\mathrm{rW} \chi \mathrm{V}^{-}$ & rw $\chi^{\prime}-$ & $\begin{array}{l}\text { to scoop up particled solids (e.g. } \\
\text { berries) in the hollow of the hand }\end{array}$ \\
\hline 20210 & & & & & rh- & (onomatopoeic) $^{926}$ \\
\hline 20220 & 2179 & $\mathrm{rh}^{3}-$ & $\mathrm{rh}^{3}-$ & $\begin{array}{l}\text { rh- } \\
\mathrm{rh}^{3}- \\
\mathrm{rhh}^{3}-\end{array}$ & $\begin{array}{l}\mathrm{rh}- \\
\mathrm{rh}^{3}-\end{array}$ & early, early in the morning \\
\hline 20230 & 2180 & rht- & rht- & rht- & rht- & fly, biting of fly \\
\hline 20240 & 2181 & rhnh- & rhnh- & rhnh- & rhnh- & evening, night \\
\hline
\end{tabular}

${ }^{923} \mathrm{In} \mathrm{KW} /$ ruta/ "to punch, strike with fist."

${ }^{924} \mathrm{In} \mathrm{KW} /$ gut $^{3} \mathrm{ga}^{3} \mathrm{z} /$ "cry of loon" KW /rut ${ }^{3}$ ala/* "cry of loon."

925 In two words referring to quartzite rock.

${ }^{926}$ In raven's cry and a word for chicken. 
North Wakashan Comparative Root List (Updated)

\begin{tabular}{|c|c|c|c|c|c|c|}
\hline Root \# & Old \# & $\mathrm{HE}$ & $\mathrm{OO}$ & HA & KW & Gloss \\
\hline 20250 & 2182 & rhzh $\chi-$ & rhzz- & $\begin{array}{l}\text { Ihzq- } \\
\text { Ihzh- }\end{array}$ & rhzq- & $\begin{array}{l}\text { starfish; crosswise, at an angle to } \\
\text { another line, lines forming a } \\
\text { hook-shape }{ }^{927}\end{array}$ \\
\hline 20260 & 2183 & $\mathrm{rh}^{3} \mathrm{xs} \bullet-$ & $\mathrm{Th}^{3} \mathrm{XS} \bullet-$ & & $\begin{array}{l}\text { Ihsx•--, } \\
\text { Ihxs•- }\end{array}$ & $\begin{array}{l}\text { to hook a finger for carrying sth. } \\
\text { (e.g. fish by the gills) or holding } \\
\text { it in position (e.g. blanket thrown } \\
\text { over shoulder) } \\
\text { 928 }\end{array}$ \\
\hline 20270 & 2184 & Th£- & Th£- & Th£- & Th£- & to gaff, to hook, to crochet \\
\hline 20280 & 2186 & & & & rhlh- & $(?)^{929}$ \\
\hline 20290 & $\begin{array}{l}2185 \\
2135\end{array}$ & $\mathrm{rhl}^{3}-$ & $\mathrm{rhl}^{3}-$ & $\mathrm{rhl}^{3}-$ & $\mathrm{rhl}^{3}-$ & to oppose, criticize negatively ${ }^{930}$ \\
\hline 20291 & & rhgv- & rhgv- & & & $(?)^{931}$ \\
\hline 20300 & 2188 & rhxv- & rhxv- & & rhxv- & $\begin{array}{l}\text { to hang down (said of a long } \\
\text { thing), to be hanging on or over } \\
\text { sth., to hang sth. up (e.g. clothes) }\end{array}$ \\
\hline 20310 & 2189 & rhqv- & & & & (?) worm 932 \\
\hline
\end{tabular}

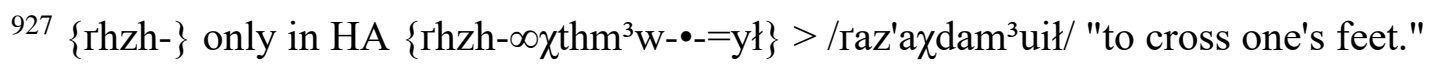
928 Boas (1948:316) mentions the KW root is sometimes $\left\{\mathrm{rhxs}^{\bullet}-\right\}$ instead of $\left\{\mathrm{rhsx}^{\bullet-}\right\}$ but gives no examples. Lincoln (n.d.) provides one example, KW $\{$ Ihsx $\bullet-h\}>/$ rasxa/ and $\{$ Ihxs $\bullet-h\}>$ /raxsa/ "to carry (fish) hooked on fingers."

${ }^{929}$ Possibly in $\{$ rhlh-wp- $\}$ "across, crosswise, to cross over" and \{rhlh-\%wqv-mł $\}>$ KW /raluqvał/ "crooked beak mask (used in Hamatsa dance representing a mythological bird)." Could be an augmented form of $\{$ rl- $\}$ (root no. 19850) 930 Could be an augmented form of $\{$ Il- $\}$ (root no. 19850). ${ }^{931} \mathrm{In} \mathrm{HE} / \mathrm{I}^{\prime} \mathrm{akv} /$ (endearing term; uncertain for whom) and the vocatives HE /ragv'ai/ OO /ragvi/ (with accent on /a/).

932 In HE /I'arvit/ "clam poisoning worm." 
North Wakashan Comparative Root List (Updated)

\begin{tabular}{|c|c|c|c|c|c|c|}
\hline Root \# & Old \# & $\mathrm{HE}$ & $\mathrm{OO}$ & HA & KW & Gloss \\
\hline 20330 & 2191 & $\operatorname{rh} \chi-$ & Ih $\chi-$ & $\operatorname{rh} \chi-$ & $\operatorname{rh} \chi-$ & $\begin{array}{l}\text { to stride, straddle, stand with legs } \\
\text { apart; astride }\end{array}$ \\
\hline 20340 & 2192 & & & & $\operatorname{Ih} \chi_{-}^{-*}$ & $(?)^{933}$ \\
\hline 20360 & 2194 & qp- & qp- & qp- & qp- & $\begin{array}{l}\text { hollow vessel (e.g. canoe, } \\
\text { container) is upside down or } \\
\text { being turned upside down, to } \\
\text { pour out from a vessel }\end{array}$ \\
\hline 20380 & 2197 & & & & qmt- & to notch with knife \\
\hline 20390 & 2198 & & & qm£- & qm£- & to draw in the stomach \\
\hline 20400 & 2199 & qmł- & qmł- & qmł- & & deer \\
\hline 20410 & 2200 & qmk- & qmk- & & qmkv- & $\begin{array}{l}\text { constricting, constricted (like in } \\
\text { trap) }\end{array}$ \\
\hline 20420 & $\begin{array}{l}2201 \\
2374\end{array}$ & qmxv- & qmxv- & qmxv- & $\chi \mathrm{mxv}-$ & $\begin{array}{l}\text { crispy, brittle, crunchy (as } \\
\text { seaweed after curing) }\end{array}$ \\
\hline 20430 & $\begin{array}{l}2202 \\
2268\end{array}$ & & $q^{3} m x v-$ & & qmXv- & $\begin{array}{l}\text { fluffy substance (like down, } \\
\text { cotton) })^{934}\end{array}$ \\
\hline 20440 & 2203 & & & & $\mathrm{qm} \chi-$ & $\begin{array}{l}\text { encircling object slides along the } \\
\text { encircled (as when stripping } \\
\text { berries off a branch by moving } \\
\text { the fingers along the branch } \\
\text { while squeezing it, or when } \\
\text { oarlock slides along oar) }\end{array}$ \\
\hline
\end{tabular}

933 In KW /raqayu/* "long-handled adze." Boas's KW dictionary lists this word as a derivate of $\{\mathrm{rh} \chi-\}$ "to stride, etc." without indicating why.

$934 \mathrm{OO}$ only in $/ \mathrm{q}^{3} \mathrm{Mukv} /<\left\{\mathrm{q}^{3} \mathrm{mxv}-=\mathrm{kv}\right\}$ the meaning of which is not remembered by the consultant except that it had to do with making bits and pieces out of sth. The word could be a KW loan (cf. KW /quukv/ "covered with eagle down") but with /q/ changed to /q3/. 
North Wakashan Comparative Root List (Updated)

\begin{tabular}{|c|c|c|c|c|c|c|}
\hline Root \# & Old \# & $\mathrm{HE}$ & $\mathrm{OO}$ & $\mathrm{HA}$ & KW & Gloss \\
\hline 20441 & & $\mathrm{qdw}^{3} \chi \mathrm{v}-$ & & & & $(?)^{935}$ \\
\hline 20450 & $\begin{array}{l}2204 \\
2205\end{array}$ & qt- & qt- & qt- & qt- & $\begin{array}{l}\text { hinge joint, to jackknife, to } \\
\text { spread or move together legs of } \\
\text { V-shape against resistance; (KW } \\
\text { also:) to make taut or tense (as } \\
\text { when cocking gun or stringing a } \\
\text { bow), to brace, to keep in } \\
\text { position, to counteract, to } \\
\text { contradict }\end{array}$ \\
\hline 20460 & 2206 & $\begin{array}{l}\text { qnc- } \\
\text { qnch- }\end{array}$ & $\begin{array}{l}\text { qnc- } \\
\text { qnch- }\end{array}$ & qnc- & qns- & $\begin{array}{l}\text { lever with sharp edge coming } \\
\text { down (e.g. arm holding a } \\
\text { chopping tool), to adze, to use } \\
\text { mattock; (HA also:) to make taut } \\
\text { or tense (as when stringing a } \\
\text { bow) }{ }^{937}\end{array}$ \\
\hline 20470 & & & & & qnkv- & to frown \\
\hline 20480 & 2207 & & & & qnq-* & to snap (like beak) ${ }^{938}$ \\
\hline 20490 & 2208 & $\mathrm{qn} \chi-$ & $\mathrm{qn} \chi-$ & $\begin{array}{l}\text { qn } \chi- \\
\text { qnq- }\end{array}$ & $\left(q^{3} y^{3} h k-\right)$ & $\begin{array}{l}\text { to straighten the legs of a V- } \\
\text { shape; to kick, to put on shoes; } \\
\text { one's footwear }\end{array}$ \\
\hline
\end{tabular}

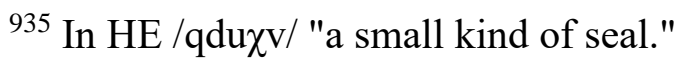

936 Possibly also in KW \{qt-=ws- $\}$ "whirlpool" because whirlpools are caused by conflicting currents. $\{-=w s\}$ would be suffix no. 4400 .

937 \{qnch- $\}$ occurs only in \{qnch-b-hlh\} > HE /q'Hcab'al'a/ OO /qucabala/ "adze" which may have developed under the influence of its synonym $\left\{£^{3}\right.$ hqvh-b-hlh $\}>\mathrm{HE} / £^{3 \prime} \mathrm{aqvab}^{\prime} \mathrm{al} \mathbf{l}^{\prime} \mathrm{a} / \mathrm{OO}$ /£3aqvabala/.

${ }^{938}$ In two words from the $/ \mathrm{DH}^{3} \mathrm{axda}^{3} \chi \mathrm{v} /$ dialect. 
North Wakashan Comparative Root List (Updated)

\begin{tabular}{|c|c|c|c|c|c|c|}
\hline Root \# & Old \# & $\mathrm{HE}$ & $\mathrm{OO}$ & HA & KW & Gloss \\
\hline 20500 & $\begin{array}{l}2209 \\
2210\end{array}$ & $\begin{array}{l}\mathrm{qc}^{3} l 1 c- \\
\mathrm{qc}^{3} \mathrm{yt}- \\
\mathrm{qc}^{3} \mathrm{ws}-\end{array}$ & $\begin{array}{l}\mathrm{qc}^{3} \mathrm{yt}- \\
\mathrm{qc}^{3} \mathrm{ws}-\end{array}$ & $q c^{3} y t-$ & & $(?)^{939}$ \\
\hline 20510 & 2212 & & & $\mathrm{q} f-$ & $q £-$ & to pull in one's stomach ${ }^{940}$ \\
\hline 20520 & & q1- & & & & $(?)^{941}$ \\
\hline 20530 & 2213 & & & & qlt- & to shore up with a post \\
\hline 20550 & 2215 & & & & qlk- & tired \\
\hline 20551 & 2214 & & & & qly3h-* & plover (bird) ${ }^{942}$ \\
\hline 20560 & 2216 & qlkv- & qlkv- & qlkv- & qlkv- & $\begin{array}{l}\text { to lie, to recline (said of animate } \\
\text { beings) } \text { ) }^{943}\end{array}$ \\
\hline 20580 & 2217 & qlxv- & qlxv- & qlxv- & qlxv-* & $\begin{array}{l}\text { to preserve food (especially } \\
\text { berries) by thickening it into a } \\
\text { cake through boiling and/or by } \\
\text { pouring grease over it }\end{array}$ \\
\hline 20590 & $\begin{array}{l}2218 \\
2219\end{array}$ & $\mathrm{ql} \chi-$ & $\mathrm{ql} \chi-$ & $\mathrm{ql} \chi-$ & $\mathrm{ql} \chi-$ & $\begin{array}{l}\text { (?) to leave a trail (e.g. a wake in } \\
\text { the water, offspring, eggs, the }\end{array}$ \\
\hline
\end{tabular}

$939\left\{\mathrm{qc}^{3} \mathrm{ws}-\right\}$ only in HE /qc ${ }^{3} \mathrm{us} / \mathrm{OO} / \mathrm{qc}^{3} \mathrm{us} /$ "rack for drying things (e.g. seaweed, fish slices)."

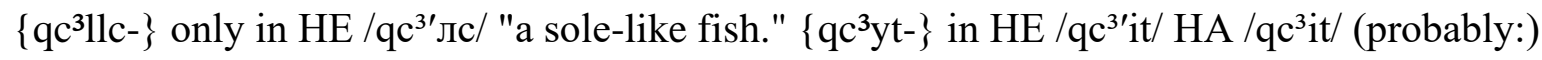
"sole" and HA /q'aqac3ita/ "to fish for /qc 3 it/." The latter word has a synonym HA /q'aqac ${ }^{3} a /$ which could be a backformation.

940 The few derivates include HA/KW $\{\mathrm{q} £-\& y d\}>/ \mathrm{q} 1 . \hbar^{3} \mathrm{id} /$ which the HA consultant said means "to draw in one's stomach" but the KW one "to stop feeling full from eating."

${ }^{941}$ In HE /qł'a/ "to go and pick abalone" (synonyms: /h³ p'a/, /rałr ${ }^{3 \prime i q a /) . ~}$

${ }^{942} \mathrm{In} \mathrm{KW} /$ qлу $^{3} \mathrm{a} / *$ "Aegialitis semipalmata," i.e. semipalmated plover which according to http://findwords.info/term/aegialitis\%20semipalmata is characterized by a ring around the neck that is black in summer but brown or gray in winter.

943 Possibly also in HA /q'aqлkva/ "snake." 
North Wakashan Comparative Root List (Updated)

\begin{tabular}{|c|c|c|c|c|c|c|}
\hline Root \# & Old \# & $\mathrm{HE}$ & $\mathrm{OO}$ & HA & KW & Gloss \\
\hline & $\begin{array}{l}2220 \\
2221\end{array}$ & & & & & $\begin{array}{l}\text { strips left as the scissors move } \\
\text { forward through the sheet), } \\
\text { streaming behind sth. }\end{array}$ \\
\hline 20620 & $\begin{array}{l}2222 \\
2223\end{array}$ & qk- & qk- & qk- & qk- & $\begin{array}{l}\text { tight, jammed in, trapped, fitting } \\
\text { tightly, obstructed, plugged up, } \\
\text { causing an obstruction }{ }^{944}\end{array}$ \\
\hline 20630 & 2224 & qX- & qx- & qX- & qX- & $\begin{array}{l}\text { to bind, fasten, secure with or as } \\
\text { if with a hoop, noose, or ring; to } \\
\text { wear a band }\end{array}$ \\
\hline 20650 & $\begin{array}{l}2226 \\
2248\end{array}$ & qyt- & qyt- & qyt- & qyt- & $\begin{array}{l}\text { to surround, to encircle (like ring } \\
\text { a finger, hat a skull) }\end{array}$ \\
\hline 20660 & 2232 & $\begin{array}{l}\text { qynh-/ } \\
\text { qyx- }\end{array}$ & $\begin{array}{l}\text { qynh-/ } \\
\text { qyx- }\end{array}$ & $\begin{array}{l}\text { qynh-/ } \\
\text { qyx- }\end{array}$ & $\begin{array}{l}\text { qynh-/ } \\
\text { qyx- }\end{array}$ & $(?)^{946}$ \\
\hline 20670 & 2228 & qył- & qył- & qył- & qył- & $\begin{array}{l}\text { to branch off, to head sideways, } \\
\text { to lean to one side; one's head is } \\
\text { turned to one side (as on a } \\
\text { pillow) or moving sideways (as }\end{array}$ \\
\hline
\end{tabular}

${ }^{944} \mathrm{KW}$ only in words referring to bunchberries. The connection with being jammed in and fitting tightly is probably in the springy filaments that are cocked underneath the highly elastic petals of bunchberry flowers. When flipping backward the petals release the springy filaments which snap upward and fling pollen out of containers attached to them (see https://en.wikipedia.org/wiki/Cornus_canadensis). 945 Only in HE/OO/HA/KW \{q[h]yt- $\}>$ HE /q'it/ OO/HA /qait/ /KW /qعt/ "hat, cap" and

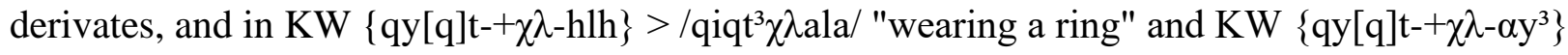
$>/$ qiqt ${ }^{3} \chi \lambda \varepsilon^{3} /$ "ring."

946 In HE/OO/KW \{qynw1-\} HA \{qynhw1-\} "pillow." Stem could contain root no. 20670 if |n| developed from $|1|$. Note that such a development did not happen in e.g. HE $\left.\left.\left\{\mathrm{h}^{3} \mathrm{~h}\right\}-\% \mathrm{w}\right\}\right\}$ $\left\{h^{3} h l w ł\right\}>/ h^{3 \prime}$ al'uł/ "new." 
North Wakashan Comparative Root List (Updated)

\begin{tabular}{|c|c|c|c|c|c|c|}
\hline Root \# & Old \# & $\mathrm{HE}$ & $\mathrm{OO}$ & $\mathrm{HA}$ & KW & Gloss \\
\hline & & & & & & $\begin{array}{l}\text { in beckoning); (KW also:) to stop } \\
\text { on the way }\end{array}$ \\
\hline 20680 & 2229 & & & & qył- & $\begin{array}{l}\text { bog cranberries (probably } \\
\text { Vaccinium oxycoccos) }{ }^{947}\end{array}$ \\
\hline 20690 & 2230 & & & & qyk- & to chew gum ${ }^{948}$ \\
\hline 20700 & 2231 & qyk- & qyk- & & & $(?)^{949}$ \\
\hline 20720 & 2234 & qy $x-$ & qy & qy $\chi-$ & qyx- & $\begin{array}{l}\text { to waste away, to wear out, to } \\
\text { fade (like colour), to become } \\
\text { destroyed; debris left after } \\
\text { destruction }\end{array}$ \\
\hline 20730 & $\begin{array}{l}2235 \\
2236\end{array}$ & qkv- & qkv- & qkv- & qkv- & $\begin{array}{l}\text { 1. (KW) top of structure buckles } \\
\text { and creates overhang; (HA) to } \\
\text { spill over (as when container too } \\
\text { full or boat rocks so that water } \\
\text { washes over its edge); } \\
\text { 2. (KW) house collapses; } \\
\text { 3. (KW and probably HE/OO) to } \\
\text { trap, confine, fit things together } \\
\text { so they cannot loosen themselves }\end{array}$ \\
\hline
\end{tabular}

${ }^{947}$ There is visual similarity between a cranberry blossom sitting on top of a long down-pointing bird beak-like structure and the head of a crane. The similarity has led to cranberries being called "crane berries," see http://cranberry.ca/Farm/facts.html. This root \{qył- $\}$ could therefore really be no. 20670

${ }^{948}$ In KW /qika/ "to chew gum noisily" KW /qik3ala/ "noise made when chewing gum." ${ }^{949}$ In HE /q'ikm'a/ OO /qikma/ "a species of shellfish with a thick shell and attached to the rocks on one side." 
North Wakashan Comparative Root List (Updated)

\begin{tabular}{|c|c|c|c|c|c|c|}
\hline Root \# & Old \# & $\mathrm{HE}$ & $\mathrm{OO}$ & HA & KW & Gloss \\
\hline 20750 & 2237 & $\begin{array}{l}\mathrm{qkv-l} \\
\mathrm{qxv}-\end{array}$ & & & & $(?)^{950}$ \\
\hline 20760 & 2238 & & & qwp- & & $\begin{array}{l}\text { to melt animal fat at the end of a } \\
\text { stick }\end{array}$ \\
\hline 20770 & 2239 & & & & qwt- & $\begin{array}{l}\text { astringency, dry feeling in the } \\
\text { mouth (as caused by tannins in } \\
\text { e.g. chokecherries) }\end{array}$ \\
\hline 20790 & 2242 & & & & $\mathrm{qwl}^{3} \mathbf{3}_{-} *$ & (?) to wish $^{951}$ \\
\hline 20791 & & & & & $q w l^{3} w q v-$ & $\begin{array}{l}\text { to use bird arrow (with kelp knob } \\
\text { as head) }\end{array}$ \\
\hline 20800 & 2243 & qwqv- & qwqv- & qwqv- & qwqv- & $\begin{array}{l}\text { (HE/OO/HA) to drift; (KW) to } \\
\text { roll, to list (ship), to tilt (hollow } \\
\text { vessel); to move in one's sleep, to } \\
\text { worry }\end{array}$ \\
\hline 20810 & 2244 & $\mathrm{qw} \chi \mathrm{v}-$ & $q w \chi v-$ & $q w \chi v-$ & $\mathrm{qw} \chi \mathrm{v}^{-}$ & to scrape (e.g. a hide) \\
\hline 20830 & 2247 & $\mathrm{q} \chi-$ & $\mathrm{q} \chi-$ & $\mathrm{q} \chi-$ & $\mathrm{q} \chi-$ & forked, two-pronged \\
\hline 20840 & 2249 & qhp- & qhp- & qhp- & & spring salmon \\
\hline 20850 & $\begin{array}{l}2195 \\
2250\end{array}$ & qhp- & qhp- & qhp- & $\begin{array}{l}\text { qhbx- } \\
\text { qbx- }\end{array}$ & $\begin{array}{l}\text { (HE/OO) to rise and come } \\
\text { towards one (heat, steam, haze, } \\
\text { smell), to waft, a whiff of sth.; } \\
\text { (HA) to employ smoke; (KW) } \\
\text { heat, heat haze, stuffy air }\end{array}$ \\
\hline
\end{tabular}

${ }^{950}$ In HE /qxvsmkv'il'a/ or /qxvmkv'il'a/ "to win a game by sheer luck."

951 In KW (Koskimo dialect) /qul ${ }^{3} \mathrm{a} / *$ "to wish" and /qul33 $\mathrm{a}^{3} \mathrm{akv} / *$ "(sth.) wished."

952 Boas (1948:340) thought the stem means "knob." It is however possible that the stem's structure is $\left\{\mathrm{qwl}^{3}-\% \mathrm{wqv}-\right\}$, with the root being no. 20790 and meaning "to target, aim or intend" rather than "to wish." The suffix could be no. 4680. 
North Wakashan Comparative Root List (Updated)

\begin{tabular}{|c|c|c|c|c|c|c|}
\hline Root \# & Old \# & $\mathrm{HE}$ & $\mathrm{OO}$ & HA & KW & Gloss \\
\hline 20860 & & qhmh- & & & qhmh- & $(?)^{953}$ \\
\hline 20870 & 2196 & $\mathrm{qhm}^{3}-$ & $\mathrm{qhm}^{3}-$ & $\mathrm{qhm}^{3}-$ & (гyxv-) & deer $^{954}$ \\
\hline 20880 & $\begin{array}{l}2225 \\
2251\end{array}$ & & & qht- & qht- & $\begin{array}{l}\text { to cut crosswise, to slice, to } \\
\text { sever, to cut steaks }\end{array}$ \\
\hline 20890 & & & & & qhs- & $(?)^{955}$ \\
\hline 20900 & $\begin{array}{l}2252 \\
2253\end{array}$ & qhs- & & qht- & qhs- & $\begin{array}{l}\text { to walk, go on foot; (KW also:) } \\
\text { to walk as messenger to invite } \\
\text { people } 956\end{array}$ \\
\hline 20901 & 2254 & qhł- & qh1- & & & $(?)^{957}$ \\
\hline 20910 & $\begin{array}{l}2255 \\
2256\end{array}$ & qh1- & qhł- & & qhł- & bent, encircling 958 \\
\hline 20920 & 2257 & qhk- & qhk- & qhk- & qhk- & to behead, to chop off head \\
\hline 20930 & 2258 & $\mathrm{qhk}^{3} \mathrm{v}-$ & $\mathrm{qhk}^{3} \mathrm{v}-$ & $\mathrm{qhk}^{3} \mathrm{v}-$ & & $\begin{array}{l}\text { to suffer a loss (as in a game or } \\
\text { business) }\end{array}$ \\
\hline 20940 & 2259 & qhwht- & qhwht- & qhwht- & qhwht- & $\begin{array}{l}\text { to hear, to listen, to } \\
\text { communicate }^{959}\end{array}$ \\
\hline
\end{tabular}

953 In HE /q'am'a/ "toy" and possibly KW /qamina/* and /qaminay ${ }^{3} \mathrm{u} / *$ "implement thrown by a $\mathrm{KW} / \mathrm{m}^{3} \mathrm{am}^{3} \mathrm{aq}^{3} \mathrm{a} / . "$

954 In HE /q'am'il'a/ OO /qam³ila/ HA /q'am³ila/ "deer." This root and no. 20860 could be augmented forms of a root $\{\mathrm{qm}-\}$ with diminutive and/or hypocoristic meaning. 955 Possibly in KW /qast/ (address used for male friend of male).

${ }^{956} \mathrm{HE}$ \{qhs- $\}$ only in $\{$ qhs-=hyw $\}>$ /q'ay'ayu/ "stilts" (cf. KW \{qhs-=hyw $\}>$ /qazayu/ "crutch"). The KW free stem $\{\mathrm{qhs}-=\mathrm{y} £\}$ and derivates refer specifically to marriage.

${ }^{957}$ Possibly in the stem \{qhlht-\} "wake of fish streaking by under water, streak in water." 958 Possibly in HE/OO \{qhlhkv-\} "laid beside fire to cook" and KW \{qhlwqv- $\}$ "bent (like horseshoe)."

${ }^{959}$ Could contain a root $\{\mathrm{qhxv}-\},\{\mathrm{qh} \chi \mathrm{v}-\}$ or $\{\mathrm{qhwh}-\}$. 
North Wakashan Comparative Root List (Updated)

\begin{tabular}{|c|c|c|c|c|c|c|}
\hline Root \# & Old \# & $\mathrm{HE}$ & $\mathrm{OO}$ & $\mathrm{HA}$ & KW & Gloss \\
\hline 20950 & 2260 & qhq- & & qhq- & qhq-* & to hang to dry ${ }^{960}$ \\
\hline 20960 & 2262 & & & qhq- & & to saw \\
\hline 20970 & 2263 & & qh $\chi-$ & & & rabbit $^{961}$ \\
\hline 20980 & 2264 & qh $\chi-$ & qh $\chi-$ & $\mathrm{qh} \chi-$ & $\mathrm{qh}^{3} \chi-$ & $\begin{array}{l}\text { (onomatopoeic:) to clear the } \\
\text { throat with a rasping sound }\end{array}$ \\
\hline 20990 & 2265 & $q^{3} p^{3} y-$ & $\mathrm{q}^{3} \mathrm{p}^{3} \mathrm{y}-$ & $\begin{array}{l}q^{3} p^{-} \\
q^{3} p^{3} y-\end{array}$ & $\begin{array}{l}q^{3} p- \\
q^{3} p^{3} y- \\
q^{3} p^{3} h-\end{array}$ & $\begin{array}{l}\text { holding together, un-scattered, } \\
\text { organized; (hence in HA/KW:) } \\
\text { objects or people are gathered } \\
\text { together; (in HE/OO/HA:) to } \\
\text { know things already, good at } \\
\text { learning and not forgetting; (HA } \\
\text { also:) to conform to the standard } \\
\text { or tradition }\end{array}$ \\
\hline
\end{tabular}

${ }^{960} \mathrm{HE}$ only in $/ \mathrm{q}^{\prime} \mathrm{aq}^{3} \mathrm{u} /$ "drying frame." HA only in $/ \mathrm{q}^{\prime} \mathrm{aq}^{3} \mathrm{u} /$ "sliced and smoke-dried meat or fish."

${ }^{961}$ Possibly in OO /qaax/ "rabbit."

962 A HA derivate of $\left\{q^{3} p^{3} y-\right\}, / q^{3} p^{3} i^{1} a l a /$, has probably become contaminated with derivates of $\left\{q^{3} h^{3} p-\right\}$ (root no. 21750) to the effect that instead of $/ q^{3} p^{3} /$ one may also hear (unaccented) $/ \mathrm{q}^{3} \mathrm{ap}^{3} /$. The phenomenon has been observed with both the Kitamaat and Kitlope consultant. Two transcriptions (e.g. HA $/ \mathrm{q}^{3} \mathrm{p}^{3} \mathrm{i}^{\prime} \mathrm{ala} /$ and $/ \mathrm{q}^{3} \mathrm{ap}^{3} \mathrm{i}^{1} \mathrm{ala} /$ ) are given for these cases. The Kitlope consultant is himself aware of the variation and suggests that $\mathrm{HA} / \mathrm{q}^{3} \mathrm{p}^{3} \mathrm{i}^{\prime} \mathrm{ala} / \mathrm{or} / \mathrm{q}^{3} \mathrm{ap} \mathrm{p}^{3} \mathrm{i}^{\prime} \mathrm{ala} /$ is a borrowing from HE. Furthermore, some words that as far as meaning is concerned would seem to be derivates of $\left\{\mathrm{q}^{3} \mathrm{~h}^{3} \mathrm{p}-\right\}$ "on target, on the right track, etc." (root no. 21750), nevertheless have a root allomorph $/ \mathrm{q}^{3} \mathrm{p}-/$. The few cases concerned involve a stem with the form of $\left\{\mathrm{q}^{3} \mathrm{p}\right.$-stw- $\}$, e.g. HA / $\mathrm{q}^{3}$ psduaud/ "to put on the right track." These cases are analyzed as containing a variant $\left\{q^{3} p-\right\}$ of $\left\{q^{3} h p-, q^{3} h^{3} p-\right\}$. 
North Wakashan Comparative Root List (Updated)

\begin{tabular}{|c|c|c|c|c|c|c|}
\hline Root \# & Old \# & $\mathrm{HE}$ & $\mathrm{OO}$ & HA & KW & Gloss \\
\hline 21010 & $\begin{array}{l}2267 \\
2268 \\
2352\end{array}$ & $\mathrm{q}^{3} \mathrm{hmh}-$ & & $\begin{array}{l}\mathrm{q}^{3} \mathrm{~m}- \\
\mathrm{q}^{3} \mathrm{hmh}-\end{array}$ & $\begin{array}{l}\mathrm{q}^{3} \mathrm{~m}- \\
\mathrm{q}^{3} \mathrm{hmh}-* \\
\mathrm{q}^{3} \mathrm{hm}^{3}-*\end{array}$ & $\begin{array}{l}\text { patch for repairing or covering } \\
\text { up; (hence:) } 1 \text {. patch (= stain) on } \\
\text { one's reputation, disgrace; } 2 \text {. to } \\
\text { mend by sewing, to splice (e.g. } \\
\text { netting twine) })^{963}\end{array}$ \\
\hline 21020 & 2269 & $\mathrm{q}^{3} \mathrm{mt}-$ & & & & $(?)^{964}$ \\
\hline 21030 & 2271 & & & $\mathrm{q}^{3} \mathrm{mt}-$ & $\mathrm{q}^{3} \mathrm{mt}-$ & song, singing (for the dancers) \\
\hline 21040 & 2272 & $\mathrm{q}^{3} \mathrm{~ms}-$ & $\mathrm{q}^{3} \mathrm{~ms}-$ & $\mathrm{q}^{3} \mathrm{~ms}-$ & $\mathrm{q}^{3} \mathrm{~ms}-$ & lazy, reluctant \\
\hline 21050 & 2273 & $q^{3} m^{3} s-$ & $q^{3} m^{3} s-$ & $q^{3} m^{3} s-$ & $\mathrm{q}^{3} \mathrm{~ms}-$ & $\begin{array}{l}\text { (KW) salmonberry (Rubus } \\
\text { spectabilis); (HE/OO/HA } \\
\text { possibly:) unripe berry or fruit }{ }^{965}\end{array}$ \\
\hline 21060 & 2274 & & & $\mathrm{q}^{3} \mathrm{ml}-$ & $\mathrm{q}^{3} \mathrm{ml-}$ & $\begin{array}{l}\text { fleshy, meaty (as opposed to thin } \\
\text { and dried out) }\end{array}$ \\
\hline 21070 & 2275 & & & & $q^{3} m k-$ & $\begin{array}{l}\text { barb, barbed (like harpoon), } \\
\text { harpoon point }\end{array}$ \\
\hline 21080 & 2276 & $\mathrm{q}^{3} \mathrm{mkv}-$ & $\mathrm{q}^{3} \mathrm{mkv}-$ & $\mathrm{q}^{3} \mathrm{mkv}-$ & $\mathrm{q}^{3} \mathrm{mkv}-$ & $\begin{array}{l}\text { to bite sth. hard or brittle, to } \\
\text { crunch, crunchy }\end{array}$ \\
\hline
\end{tabular}

$963\left\{q^{3} \mathrm{~m}-\right\}$ refers to excusing or covering up error or embarrassment in the elicited or re-elicited $\mathrm{KW}$ examples in Lincoln (n.d.). Boas however lists many non-reelicitable derivates referring to physical patching and splicing. The only clear HA example of the root is / $\mathrm{q}^{3} \mathrm{am}$ 'agH/ "goldeneye duck" (and note that adult males have a dark head and a circular white patch below the eye).

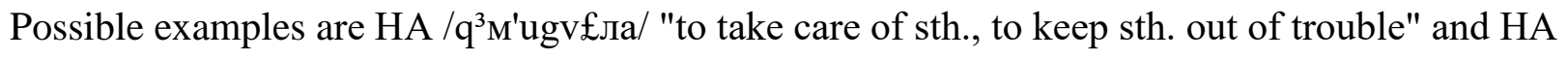
$/ \mathrm{Q}^{3} \mathrm{M}^{3} \mathrm{as} /\left(\right.$ chief's name, meaning unknown). That the root occurs in $\mathrm{HE}$ as $\left\{\mathrm{q}^{3} \mathrm{hmh}-\right\}$ is speculative because its only example is / $\mathrm{Q}^{3 \prime}$ am'akas/, a man's name belonging to the late Edwin Hunt but whose meaning is unknown. Also speculative is the root's occurrence in KW (Koskimo and Newettee dialects) /q ${ }^{3}$ amafla/* "uncle" KW (Koskimo) /q ${ }^{3} \mathrm{am}^{3} \mathrm{a}^{3} \mathrm{al}^{3} \mathrm{a} / *$ (possibly:) "to warn." ${ }^{964} \mathrm{In} \mathrm{HE} / \mathrm{q}^{3 \prime} \mathrm{Md}^{\prime} \mathrm{MS} /$ "small dead hemlock without bark and causing sparks in the fire."

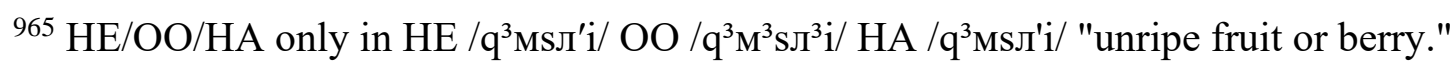


North Wakashan Comparative Root List (Updated)

\begin{tabular}{|c|c|c|c|c|c|c|}
\hline Root \# & Old \# & $\mathrm{HE}$ & $\mathrm{OO}$ & HA & KW & Gloss \\
\hline 21100 & 2277 & & & & $q^{3} m \chi-$ & herring \\
\hline 21110 & $\begin{array}{l}2278 \\
2279\end{array}$ & $\mathrm{q}^{3} \mathrm{t}-$ & $q^{3} t-$ & $\mathrm{q}^{3} \mathrm{t}-$ & $\mathrm{q}^{3 \mathrm{t}-}$ & $\begin{array}{l}\text { patch, to patch; individual or } \\
\text { subgroup differing from main } \\
\text { group }\end{array}$ \\
\hline 21130 & 2280 & $q^{3}$ ht- & $\mathrm{q}^{3} \mathrm{ht}-$ & $\mathrm{q}^{3 \mathrm{t}-}$ & $q^{3} h t-*$ & $(?)^{967}$ \\
\hline 21140 & $\begin{array}{l}2281 \\
2282 \\
2289 \\
2351\end{array}$ & $\begin{array}{l}\mathrm{q}^{3} \mathrm{n}- \\
\mathrm{q}^{3} \mathrm{hnh}-\end{array}$ & $q^{3} n-$ & $\begin{array}{l}q^{3} n- \\
q^{3} h m h-\end{array}$ & $\begin{array}{l}q^{3} n- \\
q^{3} h n y- \\
q^{3} h n^{3}-\end{array}$ & $\begin{array}{l}\text { not coming apart, joined together } \\
\text { (like panels of a patchwork quilt } \\
\text { or cardboard box), fixated, not } \\
\text { running any more (like tree sap), } \\
\text { sticky (like resin) }\end{array}$ \\
\hline 21160 & 2283 & $q^{3} n t-$ & $q^{3} n t-$ & (kt-) & & blast, explosion \\
\hline 21170 & 2284 & $q^{3} \mathrm{nc}^{-}$ & $q^{3} n c-$ & & & to scald (face, hands, etc.) \\
\hline 21180 & $\begin{array}{l}2285 \\
2286 \\
2287\end{array}$ & $\begin{array}{l}q^{3} n c- \\
q^{3} n s-\end{array}$ & $\begin{array}{l}q^{3} n c- \\
q^{3} n s-\end{array}$ & $\begin{array}{l}q^{3} n c- \\
q^{3} n s-\end{array}$ & $q^{3} n s-$ & $\begin{array}{l}\text { not coming apart, joined together } \\
\text { (like the overlapping plates of a } \\
\text { chiton's shell that resemble the } \\
\text { sewn-together panels of a quilt), }\end{array}$ \\
\hline
\end{tabular}

966 Occurs also in KW terms referring to sand fleas, a beach-dwelling species of the Talitridae family of Amphipoda. Their body divides into patch-like segments.

${ }^{967}$ In HE $/ \mathrm{q}^{3} \mathrm{at}^{3} \mathrm{a} / \mathrm{OO} / \mathrm{q}^{3} \mathrm{a} \mathrm{t}^{3} \mathrm{a} / \mathrm{HA} / \mathrm{q}^{3} \mathrm{t}^{3} \mathrm{a} / \mathrm{KW} / \mathrm{q}^{3} \mathrm{at}^{3} \mathrm{a} /{ }^{*}$ "a species of edible sea anemone." Could be root no. 21110 .

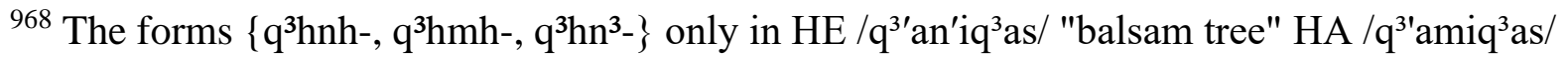
"cottonwood" KW /q $\mathrm{aq}^{3} \mathrm{an}^{3} \mathrm{i}^{3} /$ "black-fruited hawthorn (Crataegus rivularis)." Black cottonwood is a subspecies of balsam poplar. Its buds are sticky with resin that used to be used as an adhesive. The connection between hawthorn and stickiness is that the berries contain a lot of pectin, which is thick and sticky. Possibly $\left\{\mathrm{q}^{3} \mathrm{hnh}-\right\}$ occurs also in $\mathrm{HE} / \mathrm{q}^{3}$ an'isxa/ "to regurgitate (as after eating rancid ooligan oil)" although its suffix(es) are unclear and the only connection with stickiness may be that the oil refuses to go down the digestive tract. 
North Wakashan Comparative Root List (Updated)

\begin{tabular}{|c|c|c|c|c|c|c|}
\hline Root \# & Old \# & $\mathrm{HE}$ & $\mathrm{OO}$ & HA & KW & Gloss \\
\hline & & & & & & $\begin{array}{l}\text { intertwined (like mending twine } \\
\text { and the net or sock being } \\
\text { mended); to patch, mend, darn, } \\
\text { sew } 969\end{array}$ \\
\hline 21190 & 2288 & & & & $q^{3} n s-$ & meat $^{970}$ \\
\hline 21200 & 2290 & & & $q^{3} n £-$ & $q^{3} n £-$ & $\begin{array}{l}\text { (?) to respond to physical impact } \\
\text { (as when drawing in belly and } \\
\text { bending over when hit in } \\
\text { stomach), to renege (on a } \\
\text { decision), to react angrily }\end{array}$ \\
\hline 21210 & 2291 & $q^{3} n ł h-$ & $q^{3} n \nmid h-$ & & & to add sth. to sth. else, to refill \\
\hline 21220 & 2292 & & & $q^{3} n k-$ & & (?) sharp (object, movement) ${ }^{971}$ \\
\hline 21230 & 2293 & $q^{3} n q-$ & $q^{3} n q-$ & & $q^{3} n q-$ & tacky, sticky \\
\hline 21250 & $\begin{array}{l}2294 \\
2295 \\
2296\end{array}$ & $q^{3} s-$ & $\mathrm{q}^{3} \mathrm{~s}-$ & $\begin{array}{l}q^{3} c- \\
q^{3} s-\end{array}$ & $\begin{array}{l}q^{3} s- \\
q^{3} h s-\end{array}$ & $\begin{array}{l}\text { 1. elastic, resilient, rubbery, not } \\
\text { breaking under pressure, chewy } \\
\text { (like meat, fat, tree pitch); (KW } \\
\text { also:) expandable (like one's } \\
\text { chest), taking revenge, do in } \\
\text { return; } \\
\text { 2. pressed together (like spring, } \\
\text { sides of boat, the legs of a hinge } \\
\text { joint such as knee or elbow), to }\end{array}$ \\
\hline
\end{tabular}

969 Possibly also with the sense "not falling off (in autumn)" in HA / $\mathrm{q}^{31} \mathrm{Hc}^{3}$ ialas/ "needles of an evergreen tree."

970 Only in $\mathrm{KW} / \mathrm{q}^{3} \mathrm{Hsq}^{3} \mathrm{as} /$ "to eat meat."

${ }^{971}$ In words whose available glosses are hard to connect, e.g. HA /q ${ }^{31} \mathrm{Hka} /$ "to use an arrow with a sharp bone at the end" and HA /q ${ }^{31} \mathrm{Hxc}^{3} \mathrm{ud} /$ "to snap the eyes at sb." 
North Wakashan Comparative Root List (Updated)

\begin{tabular}{|c|c|c|c|c|c|c|}
\hline Root \# & Old \# & $\mathrm{HE}$ & $\mathrm{OO}$ & HA & KW & Gloss \\
\hline & & & & & & $\begin{array}{l}\text { fit tightly or accurately (like a } \\
\text { shirt a person), close up against, } \\
\text { snug }^{972}\end{array}$ \\
\hline 21300 & 2297 & $\begin{array}{l}q^{3} £^{3} h- \\
q^{3} £^{3}-\end{array}$ & $q^{3} £^{3} h-$ & $q^{3} £^{3} h-$ & $q^{3} £^{3} h-$ & six \\
\hline 21310 & 2298 & $\mathrm{q}^{3} \mathrm{t}-$ & $q^{3} 1-$ & $\mathrm{q}^{3} \mathrm{1}-$ & $q^{31}-$ & $\begin{array}{l}\text { to reach around sth. (e.g. with } \\
\text { one's arms) for lifting or } \\
\text { supporting }\end{array}$ \\
\hline 21320 & 2299 & $\begin{array}{l}\mathrm{q}^{3} 1- \\
\mathrm{q}^{3} \operatorname{lyp}- \\
\mathrm{q}^{3} \operatorname{ll} \chi-\end{array}$ & $\begin{array}{l}\text { q }^{3} l y p- \\
q^{3} \ln \chi-\end{array}$ & $\begin{array}{l}q^{3} l y p- \\
q^{3} \ln \chi-\end{array}$ & $\begin{array}{l}q^{3} \text { nyp- } \\
q^{3} \operatorname{lh} \chi-\end{array}$ & $\begin{array}{l}\left(\left\{\mathrm{q}^{3} l-, \mathrm{q}^{3} l y p-, \mathrm{q}^{3} \mathrm{nyp}-\right\}:\right) \text { to reach } \\
\text { or wrap around sth.; (in } \\
\text { HE/OO/HA developed into:) to } \\
\text { loop around a peg or other point } \\
\text { of support (as suspenders around } \\
\text { one's shoulders or as a padlock's } \\
\text { U-shaped shackle around the ring } \\
\text { it will dangle from), to reach } \\
\text { around (as one's arms around a } \\
\text { pole when climbing); dangling, } \\
\left.\left.\text { suspended; (\{q } \mathrm{q}^{3} \mathrm{l} \chi \chi-\right\}:\right) \text { fence }\end{array}$ \\
\hline
\end{tabular}

$972\left\{\mathrm{q}^{3} \mathrm{c}-\right\}$ is diagnosable only in HA $\left\{\mathrm{q}^{3}[\mathrm{hh}] \mathrm{c}-\right\}>/ \mathrm{q}^{3} \mathrm{aac} /$ "to marry within one's own tribe." Either $\left\{q^{3} c-\right\}$ or $\left\{q^{3} s^{-}\right\}$may occur in HA $/ q^{3} s^{3}{ }^{3} d^{\prime} i /$ "beaver cast, castoreum." A beaver's castor sacs are tightly embedded in surrounding tissue and difficult to cut out, see http://www.youtube.com/watch?feature=player_detailpage\&v=aXFgkwiD_zA.

${ }^{973} \mathrm{HE}\left\{\mathrm{q}^{3} \mathrm{l}-\right\}$ is likely the result of backformation from $\left\{\mathrm{q}^{3} \mathrm{lyp}-\right\}$ because it occurs in just two

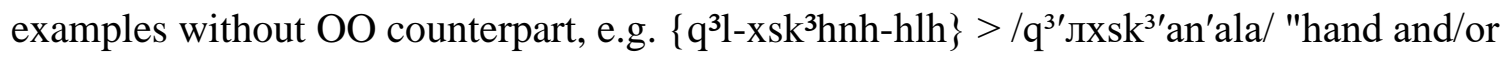
forearm in a sling." The stem $\left\{\mathrm{q}^{3} \mathrm{lh} \chi-\right\}$ "fence" is probably an unanalyzable cross-family stem, cf. Proto-Salish * $\mathrm{q}^{3} \mathrm{l} \chi,{ }^{*} \mathrm{q}^{3} \mathrm{la} \chi$ "round; fence, stockade, corral" mentioned in Kuipers (2002:88). A less likely interpretation is that it contains a root $\left\{q^{3} 1-\right\}$ referring to the slings used to suspend 
North Wakashan Comparative Root List (Updated)

\begin{tabular}{|c|c|c|c|c|c|c|}
\hline Root \# & Old \# & $\mathrm{HE}$ & $\mathrm{OO}$ & HA & $\mathrm{KW}$ & Gloss \\
\hline 21350 & 2300 & $\mathrm{q}^{3} 1^{3-}$ & $\mathrm{q}^{3} 1^{3}-$ & $\begin{array}{l}\mathrm{q}^{3} \mathrm{l}^{-} \\
\mathrm{q}^{3} \mathrm{hlh}- \\
\mathrm{q}^{3} \mathrm{l}^{3-} \\
\mathrm{q}^{3} \mathrm{hl}^{3-}\end{array}$ & & $\begin{array}{l}\text { going a long distance, reaching } \\
\text { far, going deep }\end{array}$ \\
\hline 21360 & $\begin{array}{l}2301 \\
2353\end{array}$ & $q^{3} h t-$ & $\mathrm{q}^{3} \mathrm{ht}-$ & $\begin{array}{l}\mathrm{q}^{3} \mathrm{ht}- \\
\mathrm{q}^{3} \mathrm{~h}^{3 \mathrm{t}}-\end{array}$ & $q^{3}$ ht- & to chisel, to break a copper ${ }^{974}$ \\
\hline 21370 & 2302 & $q^{3} l c-$ & $q^{3} 1 \mathrm{lc}-$ & $q^{3} l c-$ & $\mathrm{q}^{3} 1 \mathrm{~s}-$ & oil, grease, lubrication \\
\hline 21380 & $\begin{array}{l}2303 \\
2304\end{array}$ & $\begin{array}{l}\mathrm{q}^{3} \mathrm{lc}- \\
\mathrm{q}^{3} \mathrm{ls}-\end{array}$ & $\begin{array}{l}\mathrm{q}^{3} \mathrm{lc-} \\
\mathrm{q}^{3} \mathrm{ls}-\end{array}$ & $q^{3} l c-$ & $\mathrm{q}^{3} \mathrm{l}_{\mathrm{s}-}$ & $\begin{array}{l}\text { weighted, held down or } \\
\text { immobilized by weight, anchored }\end{array}$ \\
\hline 21390 & 2305 & & & & $\mathrm{q}^{3} \mathrm{lk}-*$ & to move slowly \\
\hline 21400 & & & & $q^{3} l k-$ & & (?) to braid hair \\
\hline 21410 & 2306 & $q^{3} l x v-$ & $q^{3} 1 x v-$ & $q^{3} l x v-$ & $\begin{array}{l}q^{3} 1 x v- \\
q^{3} l k v-*\end{array}$ & to coil, curl, wriggle; worms ${ }^{975}$ \\
\hline 21420 & 2307 & & & & $\mathrm{q}^{3} 1 \chi-$ & wrinkled, creased \\
\hline 21430 & $\begin{array}{l}2309 \\
2310\end{array}$ & $\mathrm{q}^{3} \mathrm{k}-$ & $\mathrm{q}^{3} \mathrm{k}-$ & $\mathrm{q}^{3} \mathrm{k}-$ & $\mathrm{q}^{3} \mathrm{k}-$ & $\begin{array}{l}\text { to pinch (like crab); to bite with } \\
\text { the teeth, to trap, to clasp, to } \\
\text { close (shell of shellfish) }\end{array}$ \\
\hline 21440 & $\begin{array}{l}2308 \\
2311\end{array}$ & $q^{3} k-$ & $q^{3} k-$ & $q^{3} k-$ & & $\begin{array}{l}\text { piling layer upon layer, to dry } \\
\text { and pound salmon eggs (to make } \\
\text { "Indian cheese") }\end{array}$ \\
\hline
\end{tabular}

one horizontal fence pole from another and that $\{-\mathrm{h} \chi-\}$ is suffix no. 6200 and refers to the suspension.

974 \{ $\left.\mathrm{q}^{3} \mathrm{ht}-\right\}$ occurs possibly also in HA /Q ${ }^{31} \mathrm{at}^{3} \mathrm{i} \mathrm{d} \chi \mathrm{v} /$ "Hartley Bay, people from Hartley Bay." ${ }^{975}\left\{\mathrm{q}^{3} \mathrm{lkv}-\right\}$ only in $\mathrm{KW} / \mathrm{q}^{3} \mathrm{Jgvac}{ }^{3} \chi \mathrm{Sd} \varepsilon^{3} / *$ "rope coiled at bottom of box or basket." 
North Wakashan Comparative Root List (Updated)

\begin{tabular}{|c|c|c|c|c|c|c|}
\hline Root \# & Old \# & $\mathrm{HE}$ & $\mathrm{OO}$ & HA & KW & Gloss \\
\hline 21450 & $\begin{array}{l}2311 \\
2147\end{array}$ & $q^{3} k-$ & & $\mathrm{q}^{3} \mathrm{k}-$ & Ig-* & $(?)^{976}$ \\
\hline 21460 & 2312 & $q^{3} x-$ & $q^{3} x-$ & $q^{3} x-$ & $q^{3} x^{*} *$ & to eat (crunch) raw fish eggs ${ }^{977}$ \\
\hline 21470 & 2313 & $q^{3} y-$ & $q^{3} y-$ & $q^{3} y-$ & $q^{3} y-$ & $\begin{array}{l}\text { high in number, quantity or } \\
\text { degree; much, many }\end{array}$ \\
\hline 21480 & 2324 & $q^{3} y-$ & $q^{3} y-$ & $q^{3} y-$ & & poor, destitute \\
\hline 21490 & & $q^{3} y-$ & $q^{3} y-$ & $\begin{array}{l}q^{3} y- \\
q^{3} h y^{3-}\end{array}$ & $q^{3} h y^{3}-$ & (?) spirit, spiritual ${ }^{978}$ \\
\hline 21500 & 2314 & $q^{3} y^{3}{ }^{3}-$ & & $q^{3} \mathrm{ym}^{3}-$ & & $\begin{array}{l}\text { to troll, to fish with hook and } \\
\text { line }^{979}\end{array}$ \\
\hline 21510 & 2315 & & & & $q^{3} y t-$ & $\begin{array}{l}\text { to fish with rod and baited hook; } \\
\text { (in /Lirviłda }{ }^{3} \chi \mathrm{v} / \text { dialect also:) to } \\
\text { troll }\end{array}$ \\
\hline 21520 & 2316 & & & & $\mathrm{q}^{3} \mathrm{yz}-*$ & cutthroat trout \\
\hline 21530 & $\begin{array}{l}2317 \\
2318\end{array}$ & $q^{3} y s-$ & $\mathrm{q}^{3} \mathrm{ys}-$ & & $q^{3} y s-$ & $\begin{array}{l}\text { pungent, musky, skunky smell } \\
\text { (of e.g. crushed hemlock needles, } \\
\text { underarm, crushed stink currant } \\
\text { leaves) }\end{array}$ \\
\hline
\end{tabular}

${ }^{976}$ In HE / $\mathrm{q}^{3} \mathrm{~g}^{\prime}$ is/ HA /q $\mathrm{q}$ gis/ "transparent to the bottom (water)" and HE $/ \mathrm{q}^{3} \mathrm{k}^{3 \prime} \mathrm{a} /$ "stone on which the stone-throwing game ( $\left.\mathrm{HE} / \mathrm{lk}^{\prime} \mathrm{a} / \mathrm{OO} / \mathrm{KW} / \mathrm{lka} /\right)$ is played." The $\mathrm{KW}$ root occurs only in /rga/* "stone against which players throw in the /lka/ game." This /rga/ could be a misrecording of KW $* / \mathrm{q}^{3} \mathrm{k}^{3} \mathrm{a} /$.

977 Boas (1948:352) gives two examples of this root but they refer to eating raw salmon spawn whereas the HE/OO/HA examples are about eating raw herring eggs. Boas also lists as examples of this root what are really examples of $\left\{q^{3} n q-\right\}$ "tacky, sticky."

${ }^{978}$ In HE/OO/HA $\left\{q^{3} y-\% l h q v-\right\}$ "to drone at ceremony" (with suffix no. 3010) and $\left\{q^{3} h y^{3}-\right.$ \%wqv- (in HA:) "tame the /t'anis/ dancer," (in KW:) "become excited in ceremony." ${ }^{979} \mathrm{HE}$ root is used in Klemtu instead of Bella Bella's $\{\mathrm{dwkv}-\}$. Possibly a Tsimshian borrowing. 
North Wakashan Comparative Root List (Updated)

\begin{tabular}{|c|c|c|c|c|c|c|}
\hline Root \# & Old \# & $\mathrm{HE}$ & $\mathrm{OO}$ & HA & KW & Gloss \\
\hline 21540 & 2320 & $q^{3} y k v-$ & $q^{3} y k v-$ & $q^{3} y k v-$ & & to lie, to deceive, to lure \\
\hline 21541 & & & & & $q^{3} y w d-$ & to talk \\
\hline 21550 & 2321 & & & & $\mathrm{q}^{3} \mathrm{yq}-$ & $(?)^{980}$ \\
\hline 21560 & 2322 & $q^{3} y x-$ & $q^{3} y \chi-$ & & $q^{3} y x-$ & driftwood \\
\hline 21570 & $\begin{array}{l}2319 \\
2323\end{array}$ & $\mathrm{q}^{3} \mathrm{y} \chi \mathrm{h}-$ & $\mathrm{q}^{3} \mathrm{y} \chi \mathrm{h}-$ & $\mathrm{q}^{3} \mathrm{y} \chi \cdot \hbar^{3} \mathrm{~h}-$ & $\mathrm{q}^{3} \mathrm{y} ł-*$ & mosquito $^{981}$ \\
\hline 21571 & & $(\mathrm{qn} \chi-)$ & $\left(\mathrm{qn} \chi^{-}\right)$ & $\begin{array}{l}(q n q-) \\
(q n \chi-)\end{array}$ & $q^{3} y^{3} h k-$ & to kick \\
\hline 21580 & 2325 & $\mathrm{q}^{3} \mathrm{kv}-$ & $\mathrm{q}^{3} \mathrm{kv}-$ & $\mathrm{q}^{3} \mathrm{kv}-$ & $\mathrm{q}^{3} \mathrm{kv}-$ & severed, amputated, cut off \\
\hline 21590 & 2326 & $\begin{array}{l}\mathrm{q}^{3} \mathrm{w}- \\
\mathrm{q}^{3} \mathrm{hw}^{3-}\end{array}$ & $q^{3} w-$ & $\mathrm{q}^{3} \mathrm{w}-$ & $\mathrm{q}^{3} \mathrm{w}-$ & $\begin{array}{l}\text { isolated body of water (e.g. pond, } \\
\text { pool, puddle, lake), standing } \\
\text { water }\end{array}$ \\
\hline 21600 & $\begin{array}{l}2327 \\
2328\end{array}$ & $q^{3} w p-$ & $q^{3} w p-$ & $q^{3} w p-$ & $\mathrm{q}^{3} \mathrm{wp}-$ & $\begin{array}{l}\text { to copulate, insert extended } \\
\text { object (penis, stick) into } \\
\text { receptacle; to wiggle, vibrate, } \\
\text { move up and down and back and } \\
\text { forth }\end{array}$ \\
\hline 21601 & 2330 & $\begin{array}{l}\mathrm{q}^{3} \mathrm{mw}- \\
\mathrm{q}^{3} \mathrm{wmw}-\end{array}$ & $\begin{array}{l}\mathrm{q}^{3} \mathrm{wm} \infty- \\
\mathrm{q}^{3} \mathrm{wmw}-\end{array}$ & $\begin{array}{l}\mathrm{q}^{3} \mathrm{wm} \infty- \\
\mathrm{q}^{3} \mathrm{wmh}-\end{array}$ & $\begin{array}{l}\mathrm{q}^{3} \mathrm{wmh}-* \\
\text { rmwt- }\end{array}$ & $\begin{array}{l}\text { to howl (like wolf, coyote, dog), } \\
\text { to wail (as at funeral) }{ }^{982}\end{array}$ \\
\hline
\end{tabular}

${ }^{980}$ In words referring to (probably ritual) purification and one word glossed as "charm."

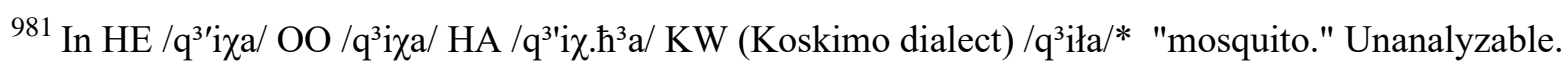
Could be a borrowing.

${ }^{982} \mathrm{KW}\left\{\mathrm{q}^{3} \mathrm{wmh}-\right\}^{*}$ is attested only in / $\mathrm{q}^{3} \mathrm{umala} / *$ "to wail, to sing mourning song for chief in chorus while beating time with stones." Boas (1947:332) mentions KW \{rmwt-h\}>/rMuta/ "wolf howls" as an example of a suffix $\{-$ wt- $\}$ possibly referring to sound. This analysis leaves a problematic root $\{\mathrm{Im}-\}$. 
North Wakashan Comparative Root List (Updated)

\begin{tabular}{|c|c|c|c|c|c|c|}
\hline Root \# & Old \# & $\mathrm{HE}$ & $\mathrm{OO}$ & HA & KW & Gloss \\
\hline 21602 & & & & & $\mathrm{q}^{3} \mathrm{wmh}-$ & $(?)^{983}$ \\
\hline 21610 & 2331 & $\mathrm{q}^{3} \mathrm{wdh}-$ & $\mathrm{q}^{3} \mathrm{wdh}-$ & $\mathrm{q}^{3} \mathrm{wdhq-}$ & $q^{3} w d h q-$ & $(?)^{984}$ \\
\hline 21620 & 2332 & $q^{3} w t-$ & $q^{3} w t-$ & $\mathrm{q}^{3} \mathrm{wt}-$ & $q^{3} w t-$ & $\begin{array}{l}\text { pole or pillar holding sth. up, } \\
\text { open or in place; to use a pole to } \\
\text { brace, shore up, support, poke or } \\
\text { push }^{985}\end{array}$ \\
\hline 21630 & $\begin{array}{l}2333 \\
2334 \\
2335 \\
2336 \\
2338\end{array}$ & $q^{3} w s-$ & $\mathrm{q}^{3} \mathrm{ws}-$ & $q^{3} w s-$ & $q^{3} w s-$ & $\begin{array}{l}\text { (HA/KW) arch (of fish gill), } \\
\text { circle, circular (as a bracelet), to } \\
\text { encircle (as crabs with their } \\
\text { pincers), to circulate, to trade; } \\
\text { (HE/OO) traffic circulation, to } \\
\text { paddle, travel on water, go by } \\
\text { boat }\end{array}$ \\
\hline 21670 & $\begin{array}{l}2339 \\
2340 \\
2341\end{array}$ & $q^{3} w l^{-}-$ & $\mathrm{q}^{3} \mathrm{w} 1-$ & $\left.\mathrm{q}^{3} \mathrm{w}\right\}-$ & $q^{3} w 1-$ & $\begin{array}{l}\text { 1. (HA/KW and possibly in two } \\
\text { OO examples:) ends of long } \\
\text { thing curving towards each other, } \\
\text { crooked, curved, winding, } \\
\text { whirling, to turn around, to } \\
\text { revert, to go back and forth (like } \\
\text { meandering river); }\end{array}$ \\
\hline
\end{tabular}

983 Possibly in $\mathrm{KW} / \mathrm{q}^{3} \mathrm{um}^{3}$ is $/<\left\{\mathrm{q}^{3} \mathrm{wmh}-=\mathrm{ys}\right\}$ "crab."

984 Only in the name of the horned grebe (a bird). Boas (1948:362) says the KW name means "poked in crotch" which means he analyzes it as $\left\{\mathrm{q}^{3} \mathrm{wt}-=\mathrm{hq}\right\}$ with root no. 21620 and suffix no. 6100. Considering the absence of the critical /q/ from the HE/OO term the name more likely has to do with the bird's "distinctive golden-yellow patches of feathers behind its eyes, which resemble short horns. These 'horns' can be erected or lowered, and are only present during the breeding season." (http://www.arkive.org/horned-grebe/podiceps-auritus/) ${ }^{985}$ Possibly also in $\mathrm{KW}\left\{\mathrm{q}^{3} \mathrm{wt}-+\chi \mathrm{wly}\right\}>/ \mathrm{q}^{3} \mathrm{ut}^{3} \chi \mathrm{uli} /$ "little red berry bush growing on rocks." 
North Wakashan Comparative Root List (Updated)

\begin{tabular}{|c|c|c|c|c|c|c|}
\hline Root \# & Old \# & $\mathrm{HE}$ & $\mathrm{OO}$ & $\mathrm{HA}$ & KW & Gloss \\
\hline & & & & & & $\begin{array}{l}\text { 2. (HE/OO/HA/KW) } \\
\text { fermentation, agitation in liquid; } \\
\text { (hence:) to well up, to bubble up, } \\
\text { to fizz, to boil; (HE/OO/HA } \\
\text { also:) to burp, belch, repeat }{ }^{986}\end{array}$ \\
\hline 21680 & 2337 & & & & $q^{3} w y^{3}-$ & $\begin{array}{l}\text { to rub in the hands (leather in } \\
\text { order to soften it, clothes when } \\
\text { laundering) }\end{array}$ \\
\hline 21690 & $\begin{array}{l}2343 \\
2344\end{array}$ & $q^{3} w k v-$ & $\begin{array}{l}q^{3} w k v- \\
q^{3} w x v-\end{array}$ & $q^{3} w k v-$ & $q^{3} w k v-$ & bent, crooked, curved ${ }^{987}$ \\
\hline 21700 & 2342 & $q^{3} w k v-$ & $\mathrm{q}^{3} \mathrm{wkv}-$ & & & bubbling, welling up ${ }^{988}$ \\
\hline 21710 & & & $q^{3} w w-$ & & & $\begin{array}{l}\text { (onomatopoeic: imitation of a } \\
\text { raven cry with the special } \\
\text { significance that someone is } \\
\text { coming by boat) }\end{array}$ \\
\hline 21720 & 2347 & $\mathrm{q}^{3} \mathrm{q}-$ & & & $\mathrm{q}^{3} \mathrm{q}-$ & $(?)^{989}$ \\
\hline 21740 & $\begin{array}{l}524 \\
527 \\
2349 \\
2361\end{array}$ & $\begin{array}{l}q^{3} h- \\
q^{3} h y^{3}-\end{array}$ & $\begin{array}{l}q^{3} h- \\
q^{3} h y^{3}-\end{array}$ & $\begin{array}{l}q^{3} h- \\
q^{3} h y^{3}-\end{array}$ & $\begin{array}{l}\text { nq- } \\
\text { nhq- } \\
q^{3 h-} \\
q^{3 h} y^{3-} \\
q^{3} h^{3-}\end{array}$ & $\begin{array}{l}\text { (HE/OO/HA }\left\{\mathrm{q}^{3} \mathrm{~h}-\right\} \text { and } \mathrm{KW} \\
\{\text { nq- }\} \text { :) free from diversion, } \\
\text { interruption or distraction (hence: } \\
\text { 1. in a straight line, not going } \\
\text { sideways; in the middle, central, } \\
\text { halfway; } 2 \text {. properly ordered, }\end{array}$ \\
\hline
\end{tabular}

986 The OO examples of sense 1 are /q³ ułла/ "crooked" (synonym: /qułла/, /qusла/) and possibly $/ q^{3} u ł a /$ "fish weir."

${ }^{987} \mathrm{KW}$ possibly also in /q⿳3 $\mathrm{q}^{3} \mathrm{usaw}^{3} \mathrm{ani} /$ "Western dock."

988 Only in HE /q'ukv'i/ OO /q ${ }^{3} \mathrm{ukvi} /$ "the bubbling of herrings."

${ }^{989}$ In HE $/ \mathrm{q}^{3} \mathrm{q}^{3} \mathrm{a} /$ "to come loose (said of cedar bark in May-July)" and KW / $\mathrm{q}^{3} \mathrm{q}^{3} \mathrm{H}^{3} \mathrm{i}$ / (probably:) "black hawthorn berries." 
North Wakashan Comparative Root List (Updated)

\begin{tabular}{|c|c|c|c|c|c|c|}
\hline Root \# & Old \# & $\mathrm{HE}$ & $\mathrm{OO}$ & HA & KW & Gloss \\
\hline & & & & & & $\begin{array}{l}\text { correct, complete, to the full } \\
\text { extent, exactly; on course, on } \\
\text { target; } 3 \text {. upright, vertical); } \\
\text { (HE/OO/HA/KW }\left\{\mathrm{q}^{3} \mathrm{~h}-\right\} \text { and the } \\
\text { augmented forms }\left\{\mathrm{q}^{3} h \mathrm{y}^{3}-\right\} \text { and } \\
\left\{\mathrm{q}^{3} \mathrm{~h}^{3}-\right\} \text { also:) to stretch out, } \\
\text { reach out, reach for (hence: } \\
\text { focused on sth. affectively or } \\
\text { cognitively, to love, to worry, to } \\
\text { mind sth., to think, to remember, } \\
\text { to know, to learn, to hit the } \\
\text { target, find what you're looking } \\
\text { for, discover }{ }^{990}\end{array}$ \\
\hline 21750 & 2350 & $\mathrm{q}^{3} \mathrm{hp}-$ & $\mathrm{q}^{3} \mathrm{hp}-$ & $\begin{array}{l}q^{3} p- \\
q^{3} h p- \\
q^{3} h^{3} p-\end{array}$ & $\mathrm{q}^{3} \mathrm{hp}-$ & $\begin{array}{l}\text { to hit a target, be to the point } \\
\text { (words), follow a route; (HA } \\
\text { also:) straight, correct, right, } \\
\text { exact, upright etc. (the senses of } \\
\left.\text { HE/OO/HA }\left\{\mathrm{q}^{3} \mathrm{~h}-\right\}\right)^{991}\end{array}$ \\
\hline
\end{tabular}

990 1. The polysemy of this root resembles that of English "straight," see Webster's Collegiate Dictionary. 2. The augmented forms $\left\{q^{3} h y^{3}-\right\}$ and $\left\{q^{3} h h^{3}-\right\}$ are modifications of $*\left\{q^{3} h h^{3}-\right\} .3$. KW $\{$ nhq- $\}$ occurs in few words and is probably a backformation from the many examples in which a suffix causes augmentation of $\{\mathrm{nq}-\}$ into $\{\mathrm{n}[\mathrm{h}] \mathrm{q}-\}$. 4. KW $\{\mathrm{nq}-\mathrm{h}\}$ means "ten" and likely developed from applying the "full, complete" and "target" sense of \{nq- $\}$ to the fingers of both hands. The HA stem for "ten" is $\left\{\mathrm{q}^{3} \mathrm{hp}-\mathrm{w}-\right\}$ with a root that likely also refers to hitting a target. OO uses $\{$ hyl-w- $\}$ for "ten" and HE $\left\{\mathrm{h}^{3} \mathrm{yk}^{3}-\mathrm{hs}-\right\}$ and $\{$ hyl-w- $\}$.

${ }^{991}$ HA $\left\{q^{3} h p-\right\}$ occurs mostly in $\left\{q^{3} h p-w-\right\}$ "ten." HA $\left\{q^{3} p-\right\}$ occurs only in the stem $\left\{q^{3} p-s t w-\right.$ h\}, e.g. /q $\mathrm{q}^{3}$ psdu'aiła/ "to put back in the same spot" and /q ${ }^{3}$ psduaud/ "to put on the right track." See also the comment on HA $\left\{q^{3} p-, q^{3} p^{3} y-\right\}$ (root no. 20990) 
North Wakashan Comparative Root List (Updated)

\begin{tabular}{|c|c|c|c|c|c|c|}
\hline Root \# & Old \# & $\mathrm{HE}$ & $\mathrm{OO}$ & HA & $\mathrm{KW}$ & Gloss \\
\hline 21780 & 2354 & & $q^{3} \mathrm{hs}-$ & $q^{3} h s-$ & $\mathrm{q}^{3} \mathrm{hs}-*$ & $(?)^{992}$ \\
\hline 21790 & 2355 & $\mathrm{q}^{3} \mathrm{~h}^{3} \mathrm{~s}-$ & $q^{3} h^{3} s-$ & & $q^{3} h s-$ & sea otter \\
\hline 21810 & $\begin{array}{l}2356 \\
2357\end{array}$ & $\begin{array}{l}q^{3} h l h-/ \\
q^{3} h 1-\end{array}$ & $\begin{array}{l}q^{3} h l h-/ \\
q^{3} h ł-\end{array}$ & $\begin{array}{l}\mathrm{q}^{3} \mathrm{l}-/ \\
\mathrm{q}^{3} \mathrm{hlh}-/ \\
\mathrm{q}^{3} \mathrm{~h} 1-\end{array}$ & $q^{3} h 1-$ & $\begin{array}{l}\text { decay, rot, putrefaction, soil, dirt, } \\
\text { bodily waste }\end{array}$ \\
\hline 21820 & 2358 & $\mathrm{q}^{3} \mathrm{~h}$ - & $q^{3} h 1-$ & $\mathrm{q}^{3} \mathrm{~h}$ - & $\mathrm{q}^{3} \mathrm{~h}$ - & $\begin{array}{l}\text { to braid, to plait, interwoven, } \\
\text { winding, writhing (like a worm), } \\
\text { to go zig-zag, unstable (as } \\
\text { quicksand) }{ }^{994}\end{array}$ \\
\hline 21830 & 2360 & & & $q^{3} h x-$ & $q^{3} h x-$ & (?) to stink 995 \\
\hline 21831 & & & & & $q^{3} h y h \chi-$ & (?) surprised, puzzled 996 \\
\hline 21840 & 2362 & $\mathrm{q}^{3} \mathrm{hkv}-$ & $\mathrm{q}^{3} \mathrm{hkv}-$ & $\mathrm{q}^{3} \mathrm{hkv}-$ & $\mathrm{q}^{3} \mathrm{hkv}-$ & $\begin{array}{l}\text { extension of the family, servant, } \\
\text { slave }\end{array}$ \\
\hline
\end{tabular}

${ }^{992} \mathrm{In} \mathrm{OO} / \mathrm{q}^{3}$ aikv/ "overripe berry" and OO /q33ayuqva/ HA /q $\mathrm{q}^{3}$ ayuqva/ KW /q3ayuqva/* "(over)ripe berries."

${ }^{993} \mathrm{HE} / \mathrm{OO} / \mathrm{HA}$ only in $\mathrm{HE} / \mathrm{q}^{3} \mathrm{al}^{\prime} \mathrm{ac}^{3} \mathrm{i} / \mathrm{OO} / \mathrm{q}^{3} \mathrm{alac}^{3} \mathrm{i} / \mathrm{HA}$ (Kitlope) /q $\mathrm{q}^{3} \mathrm{al}^{3} \mathrm{ac}^{3 \mathrm{i}} /$ (Kitamaat) $/ \mathrm{q}^{3} \mathrm{alac}^{3 \mathrm{i}} /$ "chamber pot." What stands in the way of assuming the root form $\left\{\mathrm{q}^{3} \mathrm{~h} 1-\right\}$ for HE/OO/HA is the glottalization of $/ \mathbf{l}^{3} /$ in HA (Kitlope) $/ \mathrm{q}^{3} \mathrm{al}^{3} \mathrm{ac}^{3} \mathrm{i} /$.

${ }^{994}$ Probably also in HE /q'al'лks/ OO /q3aлks/ НА /qªлgs/ "marine pilot."

${ }^{995}$ Possibly in HA / $\mathrm{q}^{3} \mathrm{an}^{3} \mathrm{a} /$ "badger" KW /Q3aniqi1 ${ }^{3} \mathrm{akv} /$ (name of the Transformer in legends) $\mathrm{KW} / \mathrm{q}^{3}$ anixp${ }^{3} \mathrm{ala} / *$ "smell of / $\mathrm{Q}^{3}$ aniqili3akv/." That the root could mean "to stink" is inferable from the Transformer apparently being known for its smell (why else have a term for it) and the fact that a badger has a musk gland on the underside that produces a pungent odor used for defense.

${ }^{996}$ Boas (1948:357) wonders if this stem might derive from a root with the form of $\left\{q^{3} y-\right\}$. If it does it is not clear what would be the suffix. The stem might also contain a root with the form of $\left\{q^{3} y x-\right\}$. 
North Wakashan Comparative Root List (Updated)

\begin{tabular}{|c|c|c|c|c|c|c|}
\hline Root \# & Old \# & $\mathrm{HE}$ & $\mathrm{OO}$ & HA & KW & Gloss \\
\hline 21850 & 2364 & $q^{3} h x v-$ & $q^{3} h x v-$ & $q^{3} h x v-$ & $\mathrm{q}^{3} \mathrm{hxv}-$ & $\begin{array}{l}\text { to emerge from the water (as a } \\
\text { rock or reef when tide goes out), } \\
\text { left high and dry (like beached } \\
\text { boat), shallow water in or on top } \\
\text { of sth. } 997\end{array}$ \\
\hline 21860 & $\begin{array}{l}2363 \\
2365\end{array}$ & $q^{3} h w h-$ & $\mathrm{q}^{3} \mathrm{hwh}-$ & & $\begin{array}{l}q^{3} h w h- \\
q^{3} h w \infty-\end{array}$ & $\begin{array}{l}\text { (?) stinky (like sea otter or rotten } \\
\text { shellfish) })^{998}\end{array}$ \\
\hline 21880 & 2367 & & & & $\begin{array}{l}\chi \text { bls- } \\
\chi \text { hbxv-* } \\
\chi \text { hsbxv-* }\end{array}$ & to bang around, make a racket ${ }^{999}$ \\
\hline 21890 & 2368 & $\begin{array}{l}\chi \mathrm{m}- \\
\chi \mathrm{hmh}-\end{array}$ & $\begin{array}{l}\chi^{\mathrm{m}-} \\
\chi_{\mathrm{hmh}-}\end{array}$ & $\begin{array}{l}\chi \mathrm{m}- \\
\chi \mathrm{hmh}-\end{array}$ & $\begin{array}{l}\chi \mathrm{m}- \\
\chi \mathrm{hmh}-\end{array}$ & $\begin{array}{l}\text { unconnected, alone (hence: } 1 . \\
\text { without influence, lacking in } \\
\text { social power, of low class, } \\
\text { orphan; } 2 \text {. without protecting or } \\
\text { separating layer, without } \\
\text { intermediary, exposed, right next } \\
\text { to sth., to do sth. in person) }\end{array}$ \\
\hline 21900 & $\begin{array}{l}2369 \\
2371\end{array}$ & $\chi \mathrm{ms}-$ & zms- & $\chi \mathrm{ms}-$ & $\chi \mathrm{ms}-$ & $\begin{array}{l}\text { hollow object (e.g. pot, box) is } \\
\text { moved violently or carelessly, } \\
\text { rattling noise from inside a }\end{array}$ \\
\hline
\end{tabular}

${ }^{997}$ Boas (1948:359) gives several non-reelicitable examples referring specifically to fish caught in a stone dam trap.

998 According to http://en.wikipedia.org/wiki/Sea_otter predation of sea otters is not common because "Many predators find the [sea] otter, with their pungent scent glands, distasteful." 999 Boas (1948:385) lists the stem as "xā'bEx̣-" in which "x̣" is likely a typing error for "x̣w" because he mentions a derivate "xā'bEx̣ ${ }^{3} w i d "$. None of Boas's stem's derivates could be reelicited. Boas (1947:328) mentions "xā'sbex̣wiīāla" - "to make noise here and there" which contains an expanded form of the stem but could not be reelicited either. 
North Wakashan Comparative Root List (Updated)

\begin{tabular}{|c|c|c|c|c|c|c|}
\hline Root \# & Old \# & $\mathrm{HE}$ & $\mathrm{OO}$ & HA & KW & Gloss \\
\hline & & & & & & $\begin{array}{l}\text { hollow object, clattering; (HA } \\
\text { also:) to wreck or break into sth. }\end{array}$ \\
\hline 21910 & 2370 & $\chi \mathrm{ms}^{-}$ & $\chi \mathrm{ms}^{-}$ & & 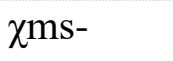 & smoke-dried salmon \\
\hline 21920 & 2372 & $\chi \mathrm{m} £-$ & $\chi \mathrm{m} £-$ & $\chi \mathrm{m} £-$ & & $\begin{array}{l}\text { to dismantle, break down } \\
\text { (objects, relationships) }\end{array}$ \\
\hline 21930 & 2373 & & & & 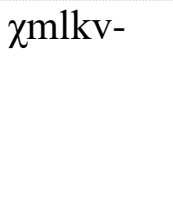 & $\begin{array}{l}\text { to bang around (as when packing } \\
\text { things up, rummaging, scaring } \\
\text { game) }\end{array}$ \\
\hline 21940 & & & & & zmwmw- & backbone \\
\hline 21950 & 2375 & $\chi \mathrm{m}^{3}-$ & $\chi^{m^{3}-}$ & $\chi \mathrm{m}^{3}-$ & $\chi \mathrm{m}^{3}-$ & $\begin{array}{l}\text { to stay overnight, to keep } \\
\text { overnight, to age food }\end{array}$ \\
\hline 21960 & $\begin{array}{l}2376 \\
401\end{array}$ & $\chi^{\mathrm{t}-}$ & $\chi^{t-}$ & $\chi^{t-}$ & $\begin{array}{l}\mathrm{t} \chi- \\
\chi \mathrm{t}-\end{array}$ & carrot (wild or domestic) $)^{1001}$ \\
\hline 21970 & $\begin{array}{l}2377 \\
2427\end{array}$ & $\begin{array}{l}\chi \mathrm{n}- \\
\chi \mathrm{nh}- \\
\chi_{\mathrm{hn}}^{3}-\end{array}$ & 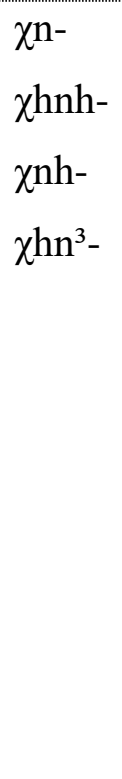 & $\begin{array}{l}\chi \mathrm{n}- \\
\chi \mathrm{nh}- \\
\chi \mathrm{hn}^{3}-\end{array}$ & $\chi \mathrm{hn}^{3}-$ & $\begin{array}{l}\text { 1. ( }\{\chi \mathrm{n}-\} \text { and }\{\chi \mathrm{hnh}-\}:) \text { without } \\
\text { protecting or separating layer, } \\
\text { without intermediary, exposed, } \\
\text { right next to sth., to do sth. in } \\
\text { person (i.e. same as the } 2 \mathrm{nd} \\
\text { series of senses for }\{\chi \mathrm{m}-\text {, } \\
\chi \mathrm{hmh}-\}) \text {; } \\
\text { 2. ( }\{\chi n \mathrm{nh}-\}:) \text { little (in amount), } \\
\text { few (senses probably developed } \\
\text { via "not constituting an obstacle } \\
\text { or separation, negligeable"; KW }\end{array}$ \\
\hline
\end{tabular}

${ }^{1000} \mathrm{In} \mathrm{KW} / \chi \mathrm{MJk}^{3} \mathrm{vala} /$ "noise of moving around" KW / $\chi \mathrm{Mлk}^{3} \mathrm{vga}^{3} \mathrm{k} /$ "sounds of moving (e.g. packing up), making noise to scare game." Stem may contain a root $\{\chi \mathrm{m}-\}$ related to root no. 21900 but form and meaning of suffix unclear.

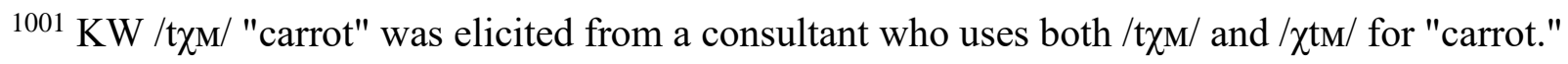


North Wakashan Comparative Root List (Updated)

\begin{tabular}{|c|c|c|c|c|c|c|}
\hline Root \# & Old \# & $\mathrm{HE}$ & $\mathrm{OO}$ & HA & KW & Gloss \\
\hline & & & & & & 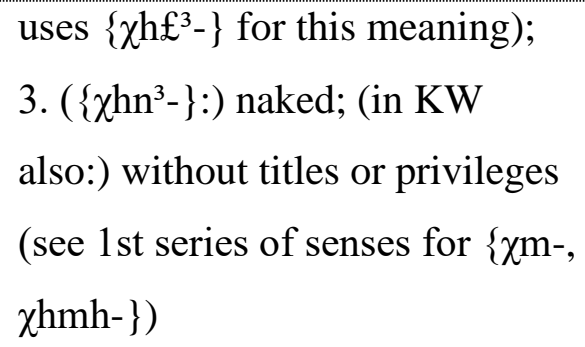 \\
\hline 21980 & 2379 & $\chi$ nt- & $\chi^{n t-}$ & $\chi$ nt- & $\chi^{n t}-$ & to snore \\
\hline 21990 & 2381 & & & & $\chi \mathrm{nf-}$ & too much, very \\
\hline 22000 & 2380 & & & & 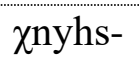 & to marvel, be astonished \\
\hline 22010 & 2382 & $\chi^{\mathrm{s}-}$ & $\chi^{s-}$ & $\chi^{\mathrm{s}-}$ & $\chi^{s-}$ & $\begin{array}{l}\text { cedar material (for canoe, box, } \\
\text { dish, spoon, rope) }\end{array}$ \\
\hline 22020 & 2383 & & & $\chi \mathrm{s}^{-}$ & $\chi \mathrm{s}^{-}$ & $\begin{array}{l}\text { loose things (e.g. grass, roots, } \\
\text { branches, hair) covering sth. (e.g. } \\
\text { for camouflaging) or being } \\
\text { placed somewhere }\end{array}$ \\
\hline 22030 & $\begin{array}{l}2385 \\
2386\end{array}$ & $x^{£-}$ & $x^{£-}$ & $\chi £-$ & $x f-$ & $\begin{array}{l}\text { long object points or pokes out, } \\
\text { to stick up, to poke into, long } \\
\text { thing is set upright; (HA also:) } \\
\text { upstanding, exemplary }\end{array}$ \\
\hline 22040 & 2384 & & & & $\chi £-$ & $\begin{array}{l}\text { to crack by pressure (canoe, } \\
\text { paddle, box), to break a box or a } \\
\text { paddle }\end{array}$ \\
\hline 22050 & 2387 & $\chi 1-$ & $\chi \mathrm{l}^{-}$ & $\chi 1-$ & $\chi 1-$ & $\begin{array}{l}\text { to bail out water; (KW also:) to } \\
\text { scoop up (with strainer, bailer, } \\
\text { dipnet) }\end{array}$ \\
\hline 22060 & 2388 & $\chi l p-$ & $\chi l p-$ & $\chi l p-$ & $\chi l p-$ & $\begin{array}{l}\text { to rake, to scrape with the } \\
\text { fingers; (HA also:) to dig (trench, } \\
\text { grave, clams), to shovel }\end{array}$ \\
\hline
\end{tabular}


North Wakashan Comparative Root List (Updated)

\begin{tabular}{|c|c|c|c|c|c|c|}
\hline Root \# & Old \# & $\mathrm{HE}$ & $\mathrm{OO}$ & HA & KW & Gloss \\
\hline 22070 & & & & 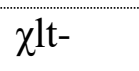 & & $(?)^{1002}$ \\
\hline 22080 & $\begin{array}{l}2389 \\
1514\end{array}$ & & $\chi \mathrm{lc}-$ & & $\chi \mathrm{ls}^{\mathrm{s}}-$ & to claw, to scratch (as in a fight) \\
\hline 22090 & $\begin{array}{l}2390 \\
2431\end{array}$ & $\chi \mathrm{lk}-$ & $\chi \mathrm{lk}-$ & $\chi \mathrm{lk}-$ & $\begin{array}{l}\chi^{\mathrm{lk}-} \\
\chi \mathrm{hk}-\end{array}$ & $\begin{array}{l}\text { hollow-shaped surface (e.g. } \\
\text { bailer), concave, warped, } \\
\text { structure with ribs extending } \\
\text { from a central point or ridge (e.g. } \\
\text { one's rib cage, a four-sided } \\
\text { cottage roof) }\end{array}$ \\
\hline 22100 & 2391 & & & & $\chi \mathrm{lxv}-$ & to cut or scrape with hollow knife \\
\hline 22110 & 2392 & & & & $\chi$ lqv- & bare, bald \\
\hline 22120 & 2393 & & & & $\chi \mathrm{lq}^{3} \mathrm{vh}-$ & (?) hollow ${ }^{1004}$ \\
\hline 22130 & 2394 & & & & $\chi \mathrm{lq}-*$ & $(?)^{1005}$ \\
\hline 22140 & 2395 & & & & $\chi \mathrm{k}^{3}-$ & $\begin{array}{l}\text { to remain in a place or condition } \\
\text { one doesn't belong, to fail to } \\
\text { return, to be stranded }\end{array}$ \\
\hline 22150 & 2378 & & & $\chi^{x-}$ & & (?) to hang the net on a line \\
\hline 22160 & $\begin{array}{l}2396 \\
2397\end{array}$ & $\chi \mathrm{y}^{-}$ & $\chi \mathrm{y}^{-}$ & & $\chi y^{-}-$ & $\begin{array}{l}\text { disconnected, unprotected, bare, } \\
\text { exposed, next to }{ }^{1006}\end{array}$ \\
\hline
\end{tabular}

1002 In НА / Х'лtamił/ (chief's name belonging to the Beaver clan).

1003 \{ $\chi \mathrm{hk}-\}$ only in $\mathrm{KW} / \chi \mathrm{ak}^{3} \mathrm{z \jmath} /$ "backbone of fish."

${ }^{1004} \mathrm{In} \mathrm{KW} / \chi л q^{3} \mathrm{va} /$ "basin, dish, crockery, glassware" (but according to Boas in the Koskimo dialect also "halibut cutting knife").

${ }^{1005}$ In $\mathrm{KW} /$ хлqапаћ ${ }^{3}$ isла/* "to throw blankets to each in turn."

1006 These are also senses of $\left\{\chi \mathrm{m}-, \chi \chi^{\mathrm{hmh}}-\right\}$ and $\left\{\chi \mathrm{n}-, \chi \mathrm{hnh}^{-}\right\}$. 
North Wakashan Comparative Root List (Updated)

\begin{tabular}{|c|c|c|c|c|c|c|}
\hline Root \# & Old \# & $\mathrm{HE}$ & $\mathrm{OO}$ & HA & KW & Gloss \\
\hline 22170 & 2398 & $\chi y-$ & & $\chi y^{3}-$ & $\chi y^{-*}$ & $(\text { probably onomatopoeic) })^{1007}$ \\
\hline 22180 & 2399 & & & & $\chi \mathrm{ym}^{3}-$ & $\begin{array}{l}\text { (?) to wiggle, to drag oneself } \\
\text { forward keeping the body close } \\
\text { to the ground and moving it from } \\
\text { side to side (as when crawling on } \\
\text { hands and knees), to creep sitting } \\
\text { (like child) }\end{array}$ \\
\hline 22190 & 2400 & $\chi y t-$ & $\chi y \mathrm{t}-$ & $\chi y \mathrm{t}-$ & $\chi y \mathrm{t}-$ & $\begin{array}{l}\text { to brush off or shove away (with } \\
\text { back of hand or stick) }\end{array}$ \\
\hline 22200 & 2401 & & & & $\chi y £-*$ & $(?)^{1009}$ \\
\hline 22210 & 2402 & & & & $\chi y £-*$ & $(?)^{1010}$ \\
\hline 22220 & 2403 & $\chi \mathrm{yx}^{-}$ & $\chi y \mathrm{y}-$ & $\chi \mathrm{yx}^{-}$ & $\begin{array}{l}\left(\mathrm{k}^{3} \mathrm{~h} \chi \mathrm{v}^{-}\right) \\
\left(\mathrm{k}^{3} \mathrm{~h} w h \mathrm{v}^{-}\right)\end{array}$ & $\begin{array}{l}\text { to whittle wood, to plane sth., to } \\
\text { shave (wood) }\end{array}$ \\
\hline 22230 & 2404 & zykv- & xykv- & zykv- & xykv- & $\begin{array}{l}\text { to sweep, brush, rake or scrape } \\
\text { away loose things (e.g. dirt, } \\
\text { snow, hair) }\end{array}$ \\
\hline 22240 & 2405 & 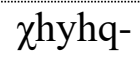 & 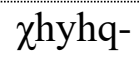 & 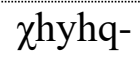 & $\chi \mathrm{yq}-$ & to sit, be in a fixed position ${ }^{1011}$ \\
\hline
\end{tabular}

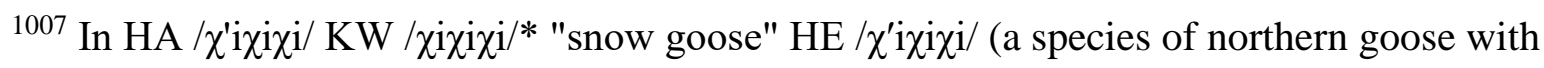
black-tipped wings, probably the snow goose (Chen caerulescens) which exists in two colour plumage morphs, white (snow) or gray/blue (blue), and the white-morph birds are white except for black wing tips).

${ }^{1008}$ In the HE/OO/HA examples associated only with wiping one's bottom.

${ }^{1009} \mathrm{In} \mathrm{KW} / \chi \mathrm{i} £ \mathrm{a} / *$ "to nibble, gnaw."

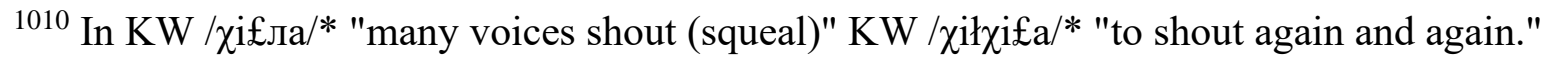
${ }^{1011} \mathrm{HE} / \mathrm{OO} / \mathrm{HA}$ only in the word for barnacles. Barnacles attach themselves permanently to a hard substrate such as rock or a boat's hull and keel. The expanded stem $\{\chi[\mathrm{h}] \mathrm{y}[\mathrm{h}] \mathrm{q}-\}$ occurs in 
North Wakashan Comparative Root List (Updated)

\begin{tabular}{|c|c|c|c|c|c|c|}
\hline Root \# & Old \# & $\mathrm{HE}$ & $\mathrm{OO}$ & $\mathrm{HA}$ & KW & Gloss \\
\hline 22250 & 2406 & & & & $\begin{array}{l}x y q^{-} \\
\chi y \chi^{-} / \\
\chi q^{-} / \\
\chi \chi^{-}\end{array}$ & (?) sore so12 $^{1012}$ \\
\hline 22260 & $\begin{array}{l}2407 \\
2432\end{array}$ & $\chi \mathrm{kv}-$ & $\chi \mathrm{kv}-$ & $\chi \mathrm{kv}-$ & $\chi \mathrm{kv}-$ & $\begin{array}{l}\text { rough to the touch, crusty } \\
\text { outgrowth (e.g. bark of tree, } \\
\text { scab) }\end{array}$ \\
\hline 22270 & 2408 & $\chi \mathrm{w}-$ & $\chi \mathrm{w}-$ & $\chi w-$ & $\chi \mathrm{w}-$ & splitting of wood \\
\hline 22290 & 2409 & $\chi w p-$ & $\chi w p-$ & & & $\begin{array}{l}\text { to scoop together debris or pieces } \\
\text { of wood for the fire }{ }^{1013}\end{array}$ \\
\hline 22300 & 2410 & $\chi w p-$ & $\chi w p-$ & & & $\begin{array}{l}\text { rough to the touch (as opposed to } \\
\text { slippery) }\end{array}$ \\
\hline 22310 & 2411 & & & & $\chi w m h-$ & $\begin{array}{l}\text { disorder, unruliness, } \\
\text { disagreement, quarrelling }{ }^{1014}\end{array}$ \\
\hline 22311 & 2412 & & & & $\chi w m \infty-*$ & rotten $^{1015}$ \\
\hline 22320 & 2413 & $\chi \mathrm{wt}-$ & $\chi w t-$ & $\chi w t-$ & $\chi w t-$ & $\begin{array}{l}\text { to scoop up, ladle out, dip up, } \\
\text { shovel }\end{array}$ \\
\hline 22330 & 2415 & $\chi \mathrm{Wc}^{3}-$ & $\chi \mathrm{wc}^{3}-$ & $\chi w c^{3}-$ & & sledgehammer ${ }^{1016}$ \\
\hline
\end{tabular}

KW too but with the narrowed-down meaning of "sitting directly on" (as opposed to e.g. on a cushion).

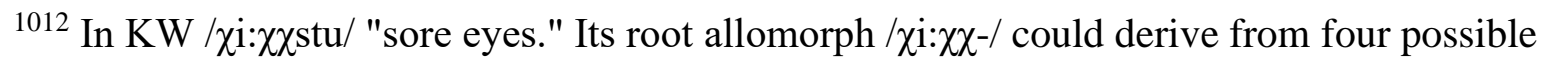
unaugmented roots.

1013 The 1980 root list mistakenly reports a KW derivate */ $\chi$ upa/ of this root.

${ }^{1014}$ In the KW stems $\{\chi w m h t-\}$ "quarrel, fight, battle" and $\{\chi w m h k-\}$ "unruly (hair)." The former may contain a suffix with the form of $\{-1\}$ or $\{-\mathrm{h}\}\}$ and the latter one with the form of $\{-\mathrm{k}\}$ or $\{-\mathrm{hk}\}$.

1015 Possibly in KW / ums/* "rotten."

${ }^{1016}$ Free stem. May contain a root $\{\chi \mathrm{wc}-\}$ or $\{\chi \mathrm{ws}-\}$. 
North Wakashan Comparative Root List (Updated)

\begin{tabular}{|c|c|c|c|c|c|c|}
\hline Root \# & Old \# & $\mathrm{HE}$ & $\mathrm{OO}$ & HA & KW & Gloss \\
\hline 22331 & & & & $\chi \mathrm{WS}-$ & $\chi \mathrm{ws}-*$ & $(?)^{1017}$ \\
\hline 22340 & 2414 & $\chi w s-$ & $\chi w s-$ & $\chi w s-$ & $\chi \mathrm{ws}-$ & to sprinkle, to splash \\
\hline 22350 & 2416 & $\chi w 1-$ & $\chi w 1-$ & & $\chi w 1-$ & a large variety of mussel \\
\hline 22360 & 2417 & & ұw1- & & & (?) to sprinkle $\mathrm{e}^{1018}$ \\
\hline 22370 & 2418 & & & 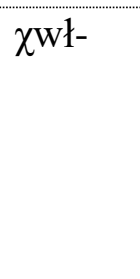 & zw1- & $\begin{array}{l}\text { squirming (like maggots), } \\
\text { writhing, tangled (like strings), } \\
\text { confused (discussion, people), to } \\
\text { shuffle (cards) }\end{array}$ \\
\hline 22380 & 2419 & & 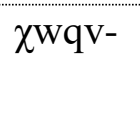 & 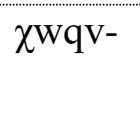 & $\begin{array}{l}\chi \mathrm{wqv}- \\
\chi q \mathrm{q}^{*} *\end{array}$ & skull $^{1019}$ \\
\hline 22381 & & & & & 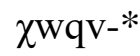 & (?) wearable ornament \\
\hline 22390 & $\begin{array}{l}2420 \\
2421\end{array}$ & & & & $\begin{array}{l}\chi q v^{-} \\
\chi \chi v^{-}\end{array}$ & to gather stones (for cooking) \\
\hline 22400 & 2422 & $\left(\mathrm{k}^{3} \mathrm{nk}-\right)$ & $\left(k^{3} n k-\right)$ & $\left(\mathrm{k}^{3} \mathrm{nk}-\right)$ & $\chi q-$ & to comb \\
\hline 22410 & $\begin{array}{l}2423 \\
2424 \\
2425\end{array}$ & $\begin{array}{l}\chi \mathrm{h}- \\
\chi \mathrm{h}^{3}-\end{array}$ & $\chi \mathrm{h}-$ & $\begin{array}{l}\chi \mathrm{h}- \\
\chi \mathrm{h}^{3}-\end{array}$ & $\chi \mathrm{h}-$ & $\begin{array}{l}\text { outlying; (hence:) outside a main } \\
\text { area, outside or unprotected by } \\
\text { societal rules, escaping } \\
\text { containment, exceeding a } \\
\text { limit }^{1020}\end{array}$ \\
\hline
\end{tabular}

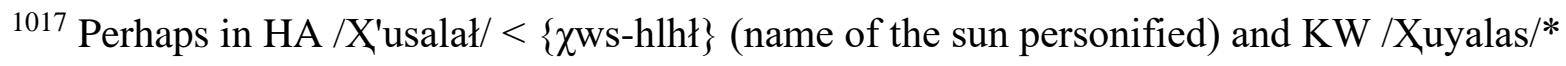
(name of a tribe).

1018 Perhaps in OO / uulalas/ "firemen on top of the building where the potlatch is held." ${ }^{1019} \mathrm{In} \mathrm{KW}$ the root also has an augmented form $\{\chi \mathrm{w}[\mathrm{y}] \mathrm{qv}-\}$. KW $\{\chi q \mathrm{q}-\}^{*}$ occurs in $/ \mathrm{Xqvalu} / *$ (name of probably a person and said to mean "head without body").

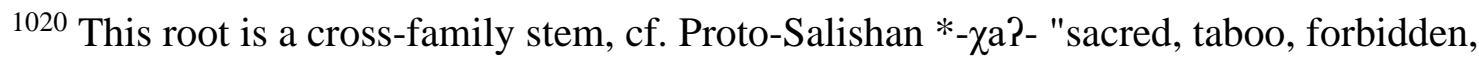
impressive, smart, violent, etc." listed in Kuipers (2002:122). It occurs in the following stems. 1. 
North Wakashan Comparative Root List (Updated)

\begin{tabular}{|c|c|c|c|c|c|c|}
\hline Root \# & Old \# & $\mathrm{HE}$ & $\mathrm{OO}$ & HA & KW & Gloss \\
\hline 22420 & 2426 & & & & $\chi \mathrm{hp}-$ & to grasp in hands or talons \\
\hline 22430 & 2428 & $\chi \mathrm{hs}^{-}$ & $\chi \mathrm{hs}-$ & $\chi \mathrm{hs}^{-}$ & $\chi \mathrm{hs}-$ & $\begin{array}{l}\text { (HE/OO/KW) to rot, go bad, } \\
\text { decompose, deteriorate; to boil } \\
\text { sth. to pieces; (HA) brittle, brittle } \\
\text { things breaking }\end{array}$ \\
\hline 22440 & 2429 & $(\chi \mathrm{nh}-)$ & $\begin{array}{l}\chi \mathrm{h}^{3}- \\
(\chi \mathrm{nh}-)\end{array}$ & $(\chi n h-)$ & $\chi h \AA^{3}-$ & a little 1021 \\
\hline 22450 & 2430 & & & & xh1- & $\begin{array}{l}\text { to raise the voice to mock, } \\
\text { humiliate or frighten away (as in } \\
\text { flushing game), to boo, to jeer at } \\
\text { sb. }\end{array}$ \\
\hline 22470 & & & & $\chi \mathrm{hxv}-$ & & $(?)^{1022}$ \\
\hline
\end{tabular}

In $\{\chi \mathrm{h}-=\mathrm{hp}-\}>\mathrm{HE} / \mathrm{OO} / \mathrm{KW}\left\{\chi \mathrm{h}^{3} \mathrm{p}-\right\}$ HA $\left\{\chi \mathrm{h}^{3} \mathrm{p}-\right\}$ which can refer to cradling on a board but in $\mathrm{HE} / \mathrm{OO} / \mathrm{HA}$ also more generally to being juvenile and not yet having reached the age when one is fully responsible for one's acts although one has stopped being half-wild because still partly belonging to the spirit world. 2 . In the stem $\{\chi \mathrm{h}-=\mathrm{ys}\}$ which occurs in tribal division names associated with the areas of Klemtu and Kitamaat. The root may here refer to being separated from a related larger mainstream population. As far as Kitamaat is concerned, legend has it that it was founded by a chief and his followers fleeing northward from the Rivers Inlet area out of fear of vendetta. 3. Possibly in the stem $\{\chi \mathrm{h}-=\mathrm{ys}\}$ occurring in terms meaning "downstream" in $\mathrm{HE} / \mathrm{OO} / \mathrm{HA}$ as well as "north" in HE/OO but "south" in HA, and in names referring to westerly or northwesterly wind. KW uses $\{\mathrm{gvh}-\}$ for this. 4. In $\mathrm{HE} / \chi^{\prime} \mathrm{ak}^{\prime} \mathrm{a} / \mathrm{OO} / \chi \mathrm{aka} /$ "too much." 5. In $\mathrm{HA} / \mathrm{KW}$ terms referring explicitly to being or going outside containment or bonds. 6. In KW $/ \chi \mathrm{a}^{3} \chi \mathrm{sd} /$ "blueback salmon (i.e. sockeye when living in the ocean)." ${ }^{1021} \mathrm{OO}\left\{\chi \chi^{3} \mathfrak{f}^{3}\right\}$ could be a KW borrowing. 1022 Only in HA / $\chi$ 'axva/ "to flush game or people out (of the bush) by making a noise." 
North Wakashan Comparative Root List (Updated)

\begin{tabular}{|c|c|c|c|c|c|c|}
\hline Root \# & Old \# & $\mathrm{HE}$ & $\mathrm{OO}$ & HA & KW & Gloss \\
\hline 22480 & 2434 & xhwy- & $\begin{array}{l}\chi \text { hwh- } \\
\chi \text { hwy- }\end{array}$ & $\chi \mathrm{hwy}^{3}-$ & $\begin{array}{l}\chi \text { hwh- } \\
\chi \text { hwy- }\end{array}$ & $\begin{array}{l}\text { (?) (at) neck or throat, necklace } \\
\text { (like that of a loon) }^{1023}\end{array}$ \\
\hline 22481 & 2433 & zhwys- & 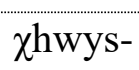 & & & $(?)^{1024}$ \\
\hline 22490 & 2435 & & & & $\begin{array}{l}\chi \chi_{\mathrm{hw} \infty} \infty- \\
\chi \mathrm{hw}^{3}-\end{array}$ & fur seal \\
\hline 22500 & $\begin{array}{l}2436 \\
2437\end{array}$ & $\chi \mathrm{h} \chi-$ & $\begin{array}{l}\chi \mathrm{h} q- \\
\chi \mathrm{h} \chi-\end{array}$ & $\chi \mathrm{h} \chi-$ & $\chi \mathrm{h} \chi-$ & bone \\
\hline 22510 & 2438 & hp- & p- & hhp- & hhp-* & $\begin{array}{l}\text { (?) plant excrescence that is hard } \\
\text { and prickly, thorny or bristly } \\
\text { (like swamp gooseberry berries, } \\
\text { tree cones) }{ }^{1025}\end{array}$ \\
\hline 22520 & 2439 & hp- & hp- & $\begin{array}{l}\text { hp- } \\
\text { hhp- }\end{array}$ & $\begin{array}{l}\text { hp- } \\
\text { hhp- }\end{array}$ & facial and body hair \\
\hline 22530 & $\begin{array}{l}2440 \\
2511\end{array}$ & hp- & hp- & hp- & hp- & $\begin{array}{l}\text { to dip, immerse, dye, hold under } \\
\text { water }\end{array}$ \\
\hline
\end{tabular}

${ }^{1023} 1$. In words referring to the common loon a.k.a. great northern loon (Gavia immer). The bird has a unique cry and seems to be wearing a necklace (cf.

http://www.hww.ca/en/wildlife/birds/loon.html?referrer=https://www.google.ca/). Perhaps therefore this root and the suffix $\{-+\chi h w h\}$ "(at) neck, throat or narrow passage" (suffix no. 5400 ) have a common origin. 2. For HA the form $\left\{\chi h w y^{3}-\right\}$ is assumed because of $/ \hbar^{3} /$ rather than $/ \hbar /$ in the diagnostic derivate $/ \chi$ aw' $^{\prime} \hbar^{3}{ }^{3} \mathrm{u} /$ "3-2 is a loon."

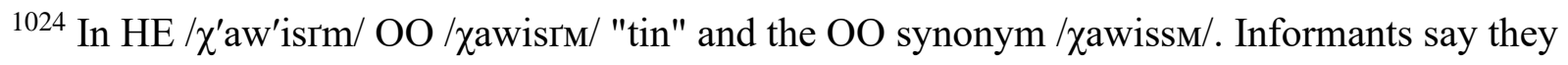
are unaware of any connection with loons (see root no. 22480).

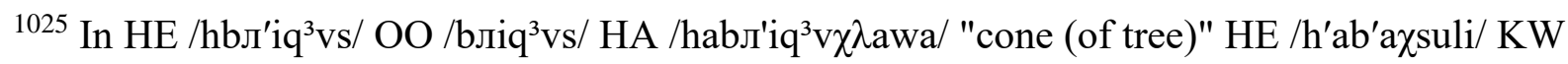
/habaxsuli/* "fruit of swamp gooseberry." The plant is also known as prickly currant and black gooseberry; scientific names Ribes echinatum (Dougl.) and Ribes lacustre (pers.), see p. 72 of The Trees and Shrubs of Western Oregon by Gilbert Benson. Difficult to tell if this root might be identical to that meaning "hair" (root no. 22520). 
North Wakashan Comparative Root List (Updated)

\begin{tabular}{|c|c|c|c|c|c|c|}
\hline Root \# & Old \# & $\mathrm{HE}$ & $\mathrm{OO}$ & HA & KW & Gloss \\
\hline 22540 & 2442 & hm- & $\begin{array}{l}\text { hp- } \\
\text { hm- }\end{array}$ & hm- & $\begin{array}{l}\text { hm- } \\
\text { hmy-* } \\
\text { hm³- }^{3} \\
\text { hhp- }\end{array}$ & $\begin{array}{l}\text { cry of the dancer known as HE } \\
/ \mathrm{h}^{\prime} \mathrm{am}^{\prime} \mathrm{ac}^{3} \mathrm{a} / \mathrm{OO} / \mathrm{KW} / \mathrm{hamac}^{3} \mathrm{a} / \\
\mathrm{HA} / \mathrm{t}^{\prime} \text { anis/ }\end{array}$ \\
\hline 22541 & & hhbh- & hhbh- & & & $\begin{array}{l}\text { (babytalk for "I want a drink of } \\
\text { water!") }\end{array}$ \\
\hline 22550 & 2443 & hm- & hm- & hm- & hm- & to put or hold sth. in the mouth \\
\hline 22560 & 2445 & hmt- & hmt- & hmt- & hmt- & to carry sb. on one's back \\
\hline 22580 & 2446 & $\begin{array}{l}\text { hmc-/ } \\
\text { hms- }\end{array}$ & $\begin{array}{l}\text { hmc-/ } \\
\text { hms- }\end{array}$ & $\begin{array}{l}\text { hmc-/ } \\
\text { hms- }\end{array}$ & $\begin{array}{l}\text { hms- } \\
\text { hm³- }^{3}\end{array}$ & to pick berries ${ }^{1027}$ \\
\hline 22590 & $\begin{array}{l}2444 \\
2447\end{array}$ & $\begin{array}{l}\text { hms- } \\
\text { hm³- }^{3}\end{array}$ & $\begin{array}{l}\text { hms- } \\
\text { hm³ }^{3}\end{array}$ & $\begin{array}{l}\text { hms- } \\
\text { hm³ }^{3}\end{array}$ & 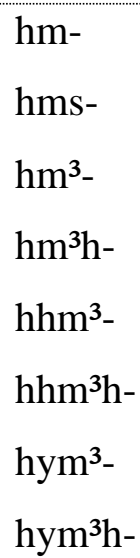 & to eat, food ${ }^{1028}$ \\
\hline
\end{tabular}

${ }^{1026} \mathrm{HA}$ only in $/ \mathrm{hMc}^{3} /<\left\{\mathrm{hm} \# \mathrm{c}^{3}\right\}$ in which $\left\{\# \mathrm{c}^{3}\right\}$ is suffix no. 900 and attested also in e.g. the greeting HA /ya.uc 3 < $\{$ yh-w\#c $\}$ "hello!, hi!."

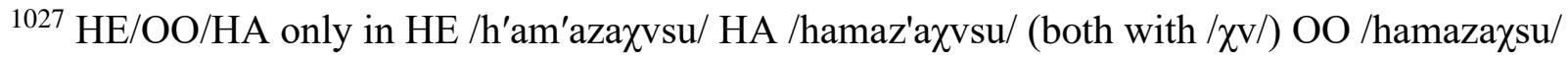
"to feed on berries, to eat berries straight from the plant." That root refers to picking rather than eating berries is inferable only from the many $\mathrm{KW}$ examples.

${ }^{1028} \mathrm{HE} / \mathrm{OO} / \mathrm{HA}\left\{\mathrm{hm}^{3}\right.$ - $\}$ occurs only in the stem $\left\{\mathrm{hm}^{3}\right.$-gylh- $\}$ "to cook" (which stem means "to feed" in KW). In contrast $\{\mathrm{hms}-\}$ meaning "to eat, food" is rare in $\mathrm{KW}$. It can be undecidable if the root form is $\left\{\mathrm{hhm}^{3}-\right\}$ or $\left\{\mathrm{hhm}^{3} \mathrm{~h}-\right\},\left\{\mathrm{hym}^{3}-\right\}$ or $\left\{\mathrm{hym}^{3} \mathrm{~h}-\right\}$ in which case the shorter form is assumed. In some examples $\left\{\mathrm{hym}^{3}-, \mathrm{hym}^{3} \mathrm{~h}-\right\}$ is the plural form of $\left\{\mathrm{hm}^{3}-, \mathrm{hm}^{3} \mathrm{~h}-\right\}$. 
North Wakashan Comparative Root List (Updated)

\begin{tabular}{|c|c|c|c|c|c|c|}
\hline Root \# & Old \# & $\mathrm{HE}$ & $\mathrm{OO}$ & HA & KW & Gloss \\
\hline 22600 & & hms- & hms- & hms- & $\begin{array}{l}\text { hms- } \\
\text { hmz- } \\
\text { hmx' }^{3} \text { - }\end{array}$ & $(?)^{1029}$ \\
\hline 22610 & 2448 & & & & hmkv- & to stoop, hunch the back \\
\hline 22620 & 2449 & & & & $\mathrm{hm} \chi \mathrm{v}-$ & to rush or jump forward \\
\hline 22640 & 2450 & & ht- & ht- & & $\begin{array}{l}\text { (OO) steeply downwards; (HA) } \\
\text { steeply down into a liquid, to } \\
\text { swallow down a liquid }\end{array}$ \\
\hline 22650 & 2451 & $\mathrm{hn}-$ & $\mathrm{hn}-$ & $\mathrm{hn}-$ & hn- & $\begin{array}{l}\text { hollow object (e.g. container, } \\
\text { gun, boat, vehicle) is located or } \\
\text { moves somewhere, container's } \\
\text { content is being manipulated }\end{array}$ \\
\hline 22670 & 2453 & hn£- & hnf- & hn£- & hnf- & $\begin{array}{l}\text { (HE/OO/HA) to shoot with bow } \\
\text { and arrow; (KW) to shoot (with } \\
\text { anything) }\end{array}$ \\
\hline 22671 & 2452 & hny- & hny- & hly- & hny- & $(?)^{1030}$ \\
\hline 22680 & 2455 & & hnkv- & hnkv- & & $\begin{array}{l}\text { to fold on itself, double up, pile } \\
\text { up }\end{array}$ \\
\hline 22690 & 2456 & hnkv- & hnkv- & & hnkv- & to curse, wish ill luck \\
\hline 22700 & 2457 & $\left(\mathrm{k}^{3} \mathrm{hp}-\right)$ & $\left(\mathrm{k}^{3} \mathrm{hp}-\right)$ & $\left(\mathrm{k}^{3} \mathrm{hp}-\right)$ & hnxv- & humpback salmon \\
\hline
\end{tabular}

${ }^{1029}$ In words relating to the dancer known as $\mathrm{HE} / \mathrm{h}^{\prime} \mathrm{am}^{\prime} \mathrm{ac}^{3} \mathrm{a} / \mathrm{OO} / \mathrm{KW} / \mathrm{hamac}^{3} \mathrm{a} / \mathrm{HA} / \mathrm{t}^{\prime}$ anis/. Also in a few words referring to bees or wasps, and a grebe (bird). The connection between the two types of reference is unclear.

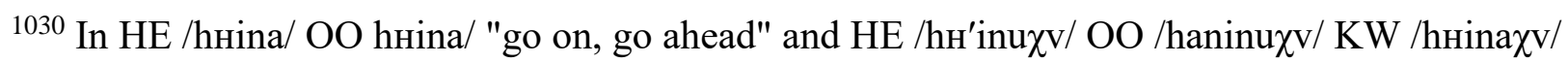
НА һліn ${ }^{3} \mathrm{akv} /$ "to ask for a ride, to ask to go along, to ask for mother or father (said of a child)." 
North Wakashan Comparative Root List (Updated)

\begin{tabular}{|c|c|c|c|c|c|c|}
\hline Root \# & Old \# & $\mathrm{HE}$ & $\mathrm{OO}$ & HA & $\mathrm{KW}$ & Gloss \\
\hline 22710 & 2454 & & & hnqv- & & $(?)^{1031}$ \\
\hline 22720 & 2458 & & & & hnqv- & bent (as a tree by the wind) \\
\hline 22730 & 2459 & hnq- & & hnq- & hnq- & $\begin{array}{l}\text { to fold onto itself (like blanket or } \\
\text { apron so one can carry sth. in it) }\end{array}$ \\
\hline 22740 & 2461 & & hn $\chi-$ & & hnz- & $\begin{array}{l}\text { to look at one's reflection (in } \\
\text { water or mirror) }\end{array}$ \\
\hline 22750 & $\begin{array}{l}2462 \\
2463\end{array}$ & hs- & hs- & hs- & hs- & $(?)^{1032}$ \\
\hline 22760 & 2466 & hł- & hł- & ht- & ht- & $(?)^{1033}$ \\
\hline 22770 & 2467 & hł- & hl- & ht- & hl- & to pause, rest, put to rest \\
\hline 22780 & $\begin{array}{l}2470 \\
2477\end{array}$ & $\begin{array}{l}\text { hl- } \\
\mathrm{hl}^{3}- \\
\mathrm{hyl}^{3-} \\
\mathrm{hyn}^{3-}\end{array}$ & $\begin{array}{l}\text { hl- } \\
\mathrm{hl}^{3}- \\
\mathrm{hyl}^{3}-\end{array}$ & $\begin{array}{l}\text { hl- } \\
\text { hlh- } \\
\text { hl³- }^{3} \\
\text { hyl }^{3-}\end{array}$ & $\begin{array}{l}\text { hl- } \\
\text { hl }^{3}- \\
\text { hyl }^{3}-\end{array}$ & $\begin{array}{l}\text { unstable, unpredictable (hence } \\
\text { e.g. hesitant, wavering, } \\
\text { apprehensive about what's going } \\
\text { to happen next, obtained through } \\
\text { luck, ephemeral, immaterial, } \\
\text { ghostly) }{ }^{1034}\end{array}$ \\
\hline 22790 & 2471 & hls- & hls- & hls- & hls- & $\begin{array}{l}\text { at or close to the critical point, } \\
\text { perilously close to, barely, hardly }\end{array}$ \\
\hline
\end{tabular}

${ }^{1031}$ In HA /h'Hrvist ${ }^{3}$ a/ "fur seal, sea otter."

${ }^{1032} \mathrm{In} \mathrm{HE} / \mathrm{hc}^{3} \chi \mathrm{d}^{\prime} \mathrm{i} / \mathrm{OO} / \mathrm{hc}^{3} \chi \mathrm{di} / \mathrm{KW} / \mathrm{hc}^{3} \chi \mathrm{sd} \varepsilon^{3 /}$ "tail of an animal" HA (Kitlope dialect) /hc $\mathrm{ikv}^{\mathrm{ik}}$ "timberwolf" НА /hsла/ "to charge with the intention of killing (said of any animal)."

${ }^{1033}$ Possibly in (1) \{ht-hq-\} "to dispatch, ship, mail, send goods; to pay what is due (e.g. salary)" and in KW also "to send word"; (2) KW /hłəqa/* "to spread over (e.g. towel over stomach)" KW

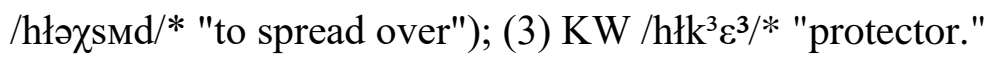

1034 This root's antonym is $\left\{h^{3} 1-\right\}$ (root no. 23600 ). The form $\left\{h y l^{3}-\right\}$ derives from $\{h l-\}$ via $*\left\{\right.$ hhl $\left.^{3}-\right\}$. 
North Wakashan Comparative Root List (Updated)

\begin{tabular}{|c|c|c|c|c|c|c|}
\hline Root \# & Old \# & $\mathrm{HE}$ & $\mathrm{OO}$ & HA & KW & Gloss \\
\hline 22800 & 2472 & & & & hlxv-* & $\begin{array}{l}\text { (?) hesitant, wavering etc. (i.e. } \\
\text { the senses of no. } 22780)^{1035}\end{array}$ \\
\hline 22801 & 2473 & $\mathrm{hl} \chi-$ & $\mathrm{hl} \chi-$ & $\mathrm{hl} \chi-$ & $\left(\mathrm{mh} \chi_{-}\right)$ & killer whale \\
\hline 22810 & $\begin{array}{l}2474 \\
2485\end{array}$ & $\mathrm{hk}^{3} \mathrm{v}-$ & & & $\begin{array}{l}\mathrm{hk}^{3}- \\
\mathrm{hk}^{3} \mathrm{~V}- \\
\mathrm{hhk}^{3} \mathrm{~V}-\end{array}$ & $\begin{array}{l}\text { stuck in a place or condition, } \\
\text { forced to stay, lodged immovably } \\
\text { (e.g. sliver in finger) }\end{array}$ \\
\hline 22820 & $\begin{array}{l}2475 \\
2487\end{array}$ & $\begin{array}{l}\text { hx- } \\
\text { hhx- } \\
\text { hxv- }\end{array}$ & $\begin{array}{l}\text { hx- } \\
\text { hhx- } \\
\text { hxv- }\end{array}$ & $\begin{array}{l}\text { hx- } \\
\text { hhx- }\end{array}$ & $\begin{array}{l}\text { hx- } \\
\text { hhx- }\end{array}$ & $\begin{array}{l}\text { to move the legs of a V-shape } \\
\text { further apart (hence: } 1 \text {. to yawn, } \\
\text { to open the mouth, to open sth. } \\
\text { jaw-like, to play an accordion, to } \\
\text { growl (like dog, wolf); } 2 \text {. to lever } \\
\text { sth. up) })^{1036}\end{array}$ \\
\hline 22821 & 2476 & $\begin{array}{l}\text { hy- } \\
\text { hhyh- } \\
\text { hhy'- }\end{array}$ & hy- & hhyh- & $\begin{array}{l}\text { hy- } \\
\text { hhyh- } \\
\text { hył- }\end{array}$ & to hide, conceal ${ }^{1037}$ \\
\hline
\end{tabular}

${ }^{1035}$ In KW /hлхva/* "to back out (of deal), withdraw (an offer)."

${ }^{1036}$ In the KW examples $\{\mathrm{hhx}-\}$ usually refers more specifically to levering up, the exceptions being KW /haxла/ "yawning" and the proper name KW $\{$ hhx-\%yws $\}>\{$ hhno-yws $\}>/$ Hanəyus/ and /Han ${ }^{3}$ us / "sky keeps on showing openings in clouds on horizon." In the HA examples \{hhx\} refers to levering up with the forearms. $\{\mathrm{hxv}-\}$ occurs diagnosably only in HE/OO $\{\mathrm{hxv}-\mathrm{w}-\}$ "to howl (like dog, wolf, coyote)." In its HA/KW counterpart it is not diagnosable if the stem is $\{\mathrm{hx}-\mathrm{w}-\}$ or $\{\mathrm{hxv}-\mathrm{w}-\}$.

${ }^{1037} \mathrm{HE}\left\{\mathrm{hhy}^{3}-\right\}$ is in most examples augmented to $\left\{[\mathrm{hh}] \mathrm{hhy}^{3}-\right\}$. This contracts to $\left\{\right.$ hhhy $\left.{ }^{3}-\right\}$ whose |hh| will contract further into just $|\mathrm{h}|$ but leave a trace by blocking high tones in suffixes, cf. HE $\left\{[\mathrm{hh}]\right.$ hhy $\left.{ }^{3}-\mathrm{h}-\mathrm{c}^{3} \mathrm{w}-\mathrm{h}\right\}>\left\{\mathrm{hhhy}^{3}-\mathrm{h}-\mathrm{c}^{3} \mathrm{w}-\mathrm{h}\right\}>/ \mathrm{h}^{\prime} \mathrm{ay}^{3} \mathrm{ac}^{3} \mathrm{ua} /$ "person who always hides himself." OO attested only in $\left\{[h h] h y-m^{3} h\right\}>\left\{h h y h-m^{3} h\right\}>/ h a y a m^{3} a /$ "to deny an accusation, hide the truth, keep sth. secret." KW \{hyk- $\}$ only in $\left\{[\right.$ hh]hył-sm-+-h $\}>\{$ hhyht-sm-+-h $\}>/$ hayałsm $^{3}$ a/ "to conceal sth." 
North Wakashan Comparative Root List (Updated)

\begin{tabular}{|c|c|c|c|c|c|c|}
\hline Root \# & Old \# & $\mathrm{HE}$ & $\mathrm{OO}$ & HA & KW & Gloss \\
\hline 22822 & & & & & hymwt- & (?) discontentment ${ }^{1038}$ \\
\hline 22823 & 2477 & & & hynh- & hynh-* & $\begin{array}{l}\text { (?) on the alert, prepared for } \\
\text { things to come }{ }^{1039}\end{array}$ \\
\hline 22824 & 2477 & & & & hynhk- & to do as a last resort ${ }^{1040}$ \\
\hline 22830 & 2478 & hys- & & hys- & & sockeye salmon \\
\hline 22840 & 2479 & hys $\infty \mathrm{kv}-$ & hys $\infty \mathrm{kv}-$ & hys $\propto$ kv- & hys $\propto$ kv- & married $^{1041}$ \\
\hline 22850 & $\begin{array}{l}2480 \\
2483\end{array}$ & $\begin{array}{l}\text { hył- } \\
\text { hyx- } \\
\text { hy³- }\end{array}$ & hyk- & hył- & $\begin{array}{l}\text { hył- } \\
\text { hyx-* }\end{array}$ & $\begin{array}{l}\text { right, proper, on target; lucky, } \\
\text { happy }{ }^{1042}\end{array}$ \\
\hline 22851 & & & & hyłzhqv- & hyłzhqv- & $\begin{array}{l}\text { from the Bella Bella area, } \\
\text { Heiltsuk }^{1043}\end{array}$ \\
\hline 22852 & 2481 & hyly- & hyly- & hyly- & $\begin{array}{l}\text { hyly- } \\
\text { hylo- }\end{array}$ & to hire \\
\hline
\end{tabular}

${ }^{1038}$ In several derivates whose glosses refer to grumbling and to abuse in speaking. Boas (1947:332) believes it contains a suffix $\{-\mathrm{wt}\}$ referring to sound.

${ }^{1039}$ In HA /hin'ala/ "to hear a ghost" KW /has:hina/* "to prepare" and KW /has:hinaqa/* "to overhaul things, to prepare, get oneself ready."

${ }^{1040}$ Could be root no. 22823 with the same extender $|\mathrm{k}|$ found in $\{\mathrm{hlhk}-\}$ (see under no. 11450) and $\{$ hhl'3hk-\} (no. 23142).

${ }^{1041}|\infty|$ between a fricative and another obstruent is phonetic zero in HE/OO but actualized as /a/ in $\mathrm{HA} / \mathrm{KW}$. Stem may be polymorphemic and contain a root $\{$ hy- $\}$ or $\{$ hys- $\}$ but no clues to its meaning or that of the suffix(es). The KW stem $\{$ yhsookv- $\}$ "suet, tallow" presents a similar problem.

${ }^{1042} \mathrm{KW}$ \{hyx-\}* only in /Hixalisлa/* "to set right in the world (name of /Qªniqil3akv/)." ${ }^{1043}$ Dialect borrowing from HE/OO. The HE original means "native, indigenous" and has a structure $\{$ hyył-zhqv $\}$ in which $\{$ hyył- $\}$ could stem from $\{$ hy[h]1- $\},\{[\mathrm{h}]$ hył- $\}$ or $\{$ hy-=ył- $\}$. 
North Wakashan Comparative Root List (Updated)

\begin{tabular}{|c|c|c|c|c|c|c|}
\hline Root \# & Old \# & $\mathrm{HE}$ & $\mathrm{OO}$ & HA & KW & Gloss \\
\hline 22853 & & & & hylw- & & $(?)^{1044}$ \\
\hline 22854 & & & & & hyl ${ }^{3}-$ & $(?)^{1045}$ \\
\hline 22860 & 2482 & hyx- & hyx- & hyx- & hyx- & $\begin{array}{l}\text { (HE/OO/HA) head (of any } \\
\text { animate being); (KW) fish head }\end{array}$ \\
\hline 22880 & 2484 & hyxv- & & hyxv- & hyxv- & (?) to deny, oppose, contest ${ }^{1046}$ \\
\hline 22890 & & & & hkv- & & $(?)^{1047}$ \\
\hline 22900 & 2486 & hxv- & hxv- & & & $(?)^{1048}$ \\
\hline 22920 & & & & $\begin{array}{l}\text { hxv-/ } \\
\text { h } \chi \mathrm{v}-/ \\
\text { hw- }\end{array}$ & & $(?)^{1049}$ \\
\hline 22930 & 2488 & $\begin{array}{l}\text { hw- } \\
\text { hhwh- } \\
\text { hhw3h- }\end{array}$ & $\begin{array}{l}\text { hw- } \\
\text { hhwh- }\end{array}$ & hhwh- & $\begin{array}{l}\text { hw- } \\
\text { hhwh- }\end{array}$ & $\begin{array}{l}\text { arranged crosswise, matted, } \\
\text { interwoven }^{1050}\end{array}$ \\
\hline
\end{tabular}

${ }^{1044}$ In HA /h'ailuxv $\lambda_{H} /<\{[$ hh]hylw-x£n $\}$ "weasel head dance headdress" and derivates.

${ }^{1045}$ In the bound stems $\left\{\mathrm{hyl}^{3}-\mathrm{w}^{3} \mathrm{~S}-\right\},\left\{\mathrm{hyl}^{3}-\mathrm{w}^{3} \mathrm{kV}-\right\}$ and $\left\{\mathrm{hyl}^{3}-\mathrm{w}-\right\}$ which refer to one thing being

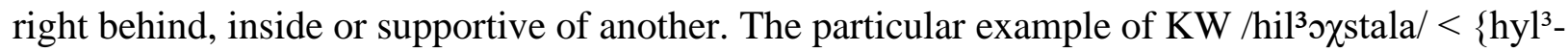
$\mathrm{w}-\mathrm{h} \chi \mathrm{st} \cdot-\mathrm{hlh}\}$ "to hire sb. to speak for one" indicates $\left\{\mathrm{hyl}^{3}-\right\}$ before $\left\{-\mathrm{w},-\mathrm{w}^{3} \mathrm{~s},-\mathrm{w}^{3} \mathrm{kv}\right\}$ could be another variant of $\{$ hylh-, hylø- $\}$ (root no. 22852).

${ }^{1046} \mathrm{HE}$ only in the woman's name /H'ixvaqs/ which is said to mean "denying woman." HA only in /h'ixva/ "to oppose sb.'s right to do sth."

${ }^{1047}$ Possibly in HA /hgvusaw'ai/ "game in which one makes people laugh."

1048 In HE /hxvM³'ait/ OO /hxvM 3 it/ "walrus" OO /hxvM³ai£/ "sea lion."

${ }^{1049} \mathrm{In} \mathrm{HA} / \mathrm{hu}^{3}$ 'ala/ "the kind of sound made by the wings of a certain bird (said to sound like scratching the trees)."

$1050\left\{\mathrm{hhw}^{3} \mathrm{~h}-\right\}$ occurs possibly in HE /h'aw'3'ani/ "brother-in-law"; its root form derives from \{hw\} observable directly in the kinship term's vocative form, /huan'ai/. 
North Wakashan Comparative Root List (Updated)

\begin{tabular}{|c|c|c|c|c|c|c|}
\hline Root \# & Old \# & $\mathrm{HE}$ & $\mathrm{OO}$ & HA & KW & Gloss \\
\hline 22940 & 2489 & & & & hwp ${ }^{3} q-$ & (?) tangled, matted ${ }^{1051}$ \\
\hline 22950 & 2490 & $\begin{array}{l}\text { hwmh- } \\
\text { hwm } \infty-\end{array}$ & $\begin{array}{l}\text { hwmh- } \\
\text { hwm } \infty-\end{array}$ & $\begin{array}{l}\text { hwmh- } \\
\text { hwm } \infty-\end{array}$ & hwmw- & $\begin{array}{l}\text { (HE/OO/HA) to check out, to } \\
\text { obtain information (by watching, } \\
\text { listening and questioning); to ask } \\
\text { permission, to try to make an } \\
\text { arrangement; (KW) to watch, to } \\
\text { observe }\end{array}$ \\
\hline 22960 & 2491 & 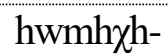 & hwmh $\chi$ h- & & & spider $^{1052}$ \\
\hline 22980 & 2492 & hwt- & hwt- & hwt- & hwt-* & to leave, to abandon; to depart \\
\hline 22990 & $\begin{array}{l}2493 \\
2497\end{array}$ & hws- & hws- & hws- & hws-* & $\begin{array}{l}\text { lumber, shakes or boards split off } \\
\text { a tree }{ }^{1053}\end{array}$ \\
\hline 23000 & 2494 & hws- & hws- & hws- & hws- & $\begin{array}{l}\text { to count, to tally, to calculate; } \\
\text { (HE/OO/HA also:) to keep the } \\
\text { books; (HA also:) to read } \\
\text { books }{ }^{1054}\end{array}$ \\
\hline
\end{tabular}

${ }^{1051}$ Stem's root could be $\{$ hw- $\}$ or $\{$ hwp- $\}$. Occurs in KW /hup ${ }^{3}$ rmala/ "tree stump" and KW /hup ${ }^{3}$ rtuała/ "having one's hair all messed up."

1052 There is no evidence that the name contains suffix no. 6200, $\{-\mathrm{h} \chi-\}$ "down from higher level, off." The meaning of the root would also be unclear.

${ }^{1053} \mathrm{KW}$ possibly in /huzac ${ }^{3} \mathrm{i} / *$ [1] "isolation shed for widows, menstruating women or sick people." One informant has $\mathrm{KW} / \mathrm{xuzac}^{3 \mathrm{i}}$ / "rest shed" which seems to derive from KW \{ $\mathrm{xws}-$ \} "rest, breather; to take a rest" (root no. 14240) but is possibly a restructuring of $/ \mathrm{huzac}^{3} \mathrm{i} / *$ based on folk etymology.

${ }^{1054}$ Could be root no. 22990 if recording a tally was done by putting a stick or chip in a receptacle for every object tallied. KW has a word suggesting this practice, $\left\{\right.$ hws $\left.-=\mathrm{hc}^{3} \mathrm{y}\right\}>$ /huzaci3i/ [2] "receptacle to count into." 
North Wakashan Comparative Root List (Updated)

\begin{tabular}{|c|c|c|c|c|c|c|}
\hline Root \# & Old \# & $\mathrm{HE}$ & $\mathrm{OO}$ & HA & KW & Gloss \\
\hline 23020 & 2499 & hwł- & & & hw1- & $(?)^{1055}$ \\
\hline 23030 & & & & hwy ${ }^{3}-$ & & $(?)^{1056}$ \\
\hline 23031 & & hwkv- & hwkv- & $\begin{array}{l}\text { hwkv- } \\
\text { hwxv- }\end{array}$ & $h w^{3} x v-$ & $\begin{array}{l}\text { arranged crosswise, matted, } \\
\text { interwoven }^{1057}\end{array}$ \\
\hline 23040 & 2500 & & & & hwkv- & $(?)^{1058}$ \\
\hline 23050 & $\begin{array}{l}2503 \\
2504\end{array}$ & hqv- & hqv- & hqv- & 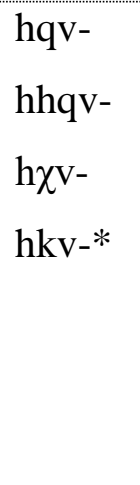 & $\begin{array}{l}\text { 1. to press down on, to sit on } \\
\text { eggs, to lie flat, to lie face down; } \\
\text { 2. to bend down (hence: to stoop, } \\
\text { to bend or fall forward; to hunch } \\
\text { one's back, a hump, a mountain); } \\
\text { 3. (HA also:) baby faces back or } \\
\text { chest of carrying adult }{ }^{1059}\end{array}$ \\
\hline 23070 & 2505 & & & & hqv- & $(?)^{1060}$ \\
\hline
\end{tabular}

${ }^{1055}$ In $\{$ hwlh $\}$ HE "small (in size)" KW "few, little (in amount), a bit." Uncertain if analyzable as $\{$ hwt- $=\mathrm{h}\}$, with a suffix carrying diminutive meaning.

1056 Possibly in HA /h'uy3ala/ "to respect sb."

${ }^{1057} \mathrm{KW}$ only in $/ \mathrm{hu}^{3} \mathrm{Xvl}^{3} \mathrm{a} /$ "log jam."

${ }^{1058}$ In KW \{[hwkv]hwkv- $\}>$ /huxv:hukv/ (name of a mythological man-eating bird) and derivates.

${ }^{1059}$ Boas (1948:93) lists a root $\{\mathrm{hkv}-\}^{*}$ and three derivates referring to stooping and people or trees leaning over. They are however not mentioned in Lincoln (n.d.) and probably typing errors. $\mathrm{KW} / \mathrm{h} \chi \mathrm{va} /[1]$ "to fall (bend) forward" is the only certain example of $\{\mathrm{h} \chi \mathrm{v}-\}$ as variant of $\{\mathrm{hqv}-$ \} .

${ }^{1060}$ In $\{$ hrvm- $\}$ "to join or stay with others, to join the dead" and derivates. Root could be no. 23050 . 
North Wakashan Comparative Root List (Updated)

\begin{tabular}{|c|c|c|c|c|c|c|}
\hline Root \# & Old \# & $\mathrm{HE}$ & $\mathrm{OO}$ & HA & KW & Gloss \\
\hline 23080 & $\begin{array}{l}2506 \\
2508\end{array}$ & $\mathrm{~h} \chi \mathrm{v}-$ & $\mathrm{h} \chi \mathrm{v}-$ & $\mathrm{h} \chi \mathrm{v}-$ & $\mathrm{h} \chi \mathrm{V}-$ & $\begin{array}{l}\text { to climb (tree, pole, steep rock, } \\
\text { rope) }\end{array}$ \\
\hline 23090 & $\begin{array}{l}2507 \\
2620\end{array}$ & $\begin{array}{l}\mathrm{h} \chi \mathrm{v}- \\
\mathrm{h}^{3} \chi \mathrm{v}-\end{array}$ & $\mathrm{h}^{3} \chi_{\mathrm{V}-}$ & $\mathrm{h}^{3} \chi^{\mathrm{V}-}$ & $\mathrm{h} \chi \mathrm{V}-$ & $\begin{array}{l}\text { thrush (Hylocichla ustulata } \\
\text { according to Boas }(1928: 179)^{1062}\end{array}$ \\
\hline 23100 & 2509 & & & & hq- & to swell (said of body parts) \\
\hline 23110 & 2512 & hht- & hht- & & hht- & (?) cross, uncooperative $\mathrm{e}^{1063}$ \\
\hline 23120 & 2514 & hht- & hht- & hht- & hht- & black cod \\
\hline 23130 & 2515 & & & & $\begin{array}{l}\text { hhny-*/ } \\
\text { hhwy-* }\end{array}$ & $(?)^{1064}$ \\
\hline 23131 & 2516 & & & & hhnhk- & to request permission \\
\hline 23140 & $\begin{array}{l}2517 \\
2518 \\
2519 \\
2520 \\
2521 \\
2522\end{array}$ & hhs- & hhs- & hhs- & hhs- & $\begin{array}{l}\text { breath, whiff, air, spirit, soul, } \\
\text { ghost, vital force in animate } \\
\text { beings, blood; to contain, emit or } \\
\text { inhale air, vital force, etc.; air- } \\
\text { filled, spongy, fluffy, airborne } \\
\text { (like smell or sound); to cure (by } \\
\text { blowing) }\end{array}$ \\
\hline
\end{tabular}

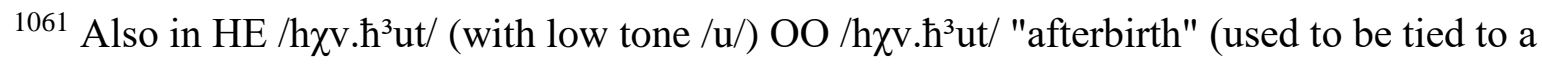
young tree to climb up as the tree grows).

${ }^{1062} \mathrm{KW}$ only in $/ \mathrm{hu}^{3} \mathrm{ala} /[2]$ "song of the thrush" and $/ \mathrm{H} \chi \mathrm{vam}^{3}$ is/ (name of the extinct WakemanSound tribe; according to legend they were named after the thrush).

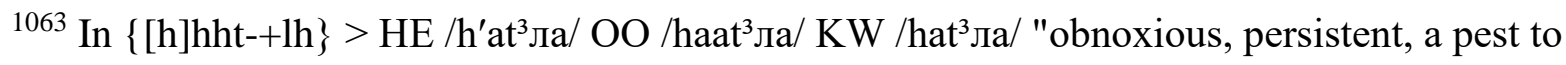
others" and a word listed by Boas $(1928: 95,199)$ but not reelicited, sc OO $\{$ hht-\&yd $\}>$ /hat.h3it/* "to disobey."

${ }^{1064}$ Possibly in KW /h:hani/* and its Newettee synonym /h:hawi/* which are spelled "hahā'ne" and "hahā'we" in Boas (1948:84) and glossed as Clarengula hyemalis. This must be Clangula hyemalis or long-tailed duck, formerly known as the oldsquaw duck. 
North Wakashan Comparative Root List (Updated)

\begin{tabular}{|c|c|c|c|c|c|c|}
\hline Root \# & Old \# & $\mathrm{HE}$ & $\mathrm{OO}$ & HA & KW & Gloss \\
\hline 23141 & & hhs- & hhs- & hhs- & $\mathrm{h}^{3} \mathrm{hs}^{-}$ & $(?)^{1065}$ \\
\hline 23142 & 2510 & hhl'3hk- & hhl'3hk- & $\begin{array}{l}\text { hhl'h- } \\
\text { hhl'3hk- }\end{array}$ & 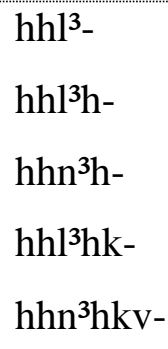 & to proceed with haste \\
\hline 23160 & & & & hhxv- & & $\begin{array}{l}\text { to gang up on sb., to go after sth. } \\
\text { as a group }\end{array}$ \\
\hline 23170 & 2523 & & & & hhqv-* & roasted salmon ${ }^{1066}$ \\
\hline 23180 & & hhqv- & & & hhqv- & $(?)^{1067}$ \\
\hline 23190 & $\begin{array}{l}2525 \\
2604\end{array}$ & $h^{3} b-$ & $\begin{array}{l}h^{3} b- \\
h^{3} g v-\end{array}$ & $h^{3} b-$ & $h^{3} b-$ & mother ${ }^{1068}$ \\
\hline 23200 & 2526 & $h^{3} p-$ & $h^{3} p-$ & & $h^{3} p-$ & abalone $^{1069}$ \\
\hline 23210 & $\begin{array}{l}2527 \\
2628\end{array}$ & $h^{3} \mathrm{hp}-$ & & & $\mathrm{h}^{3} \mathrm{p}-*$ & (?) all, in full ${ }^{1070}$ \\
\hline
\end{tabular}

${ }^{1065}$ Possibly in HE /h'ay ${ }^{3} \mathrm{asu} / \mathrm{OO} / \mathrm{hay}^{3} \mathrm{asu} / \mathrm{HA} / \mathrm{h}^{\prime} \mathrm{ay}{ }^{3} \mathrm{asu} / \mathrm{KW} / \mathrm{h}^{3} \mathrm{l}^{3} \mathrm{a}^{3} \mathrm{su} /$ "one's hand." ${ }^{1066} \mathrm{In} \mathrm{KW} / \mathrm{h} \chi:$ haqv/* "to eat fresh roasted salmon" and KW /ha :haqvamas/* "to eat whole." ${ }^{1067}$ Possibly in $\mathrm{HE} / \mathrm{h}^{\prime}$ arvuy ${ }^{3} \mathrm{u} /$ (name of a monster) $\mathrm{KW} /$ harvala/ "to get revenge, to lie in wait (for game or people)."

$1068\left\{\mathrm{~h}^{3} \mathrm{gv}-\right\}$ only in OO $/ \mathrm{h}^{3}$ gvani/ "grandmother."

${ }^{1069} \mathrm{In} \mathrm{HE} / \mathrm{h}^{3} \mathrm{p}^{\prime} \mathrm{a} / \mathrm{OO} / \mathrm{h}^{3} \mathrm{pa} / \mathrm{KW} / \mathrm{h}^{3} \mathrm{ah}^{3} \mathrm{ap}{ }^{3} \mathrm{a} /$ "to go after abalone."

${ }^{1070}$ Perhaps in KW $\left\{\mathrm{h}^{3} \mathrm{p}-\% \mathrm{yqv}-\mathrm{h}\right\}>/ \mathrm{h}^{3}$ biqva/* "gale, storm" and HE $\left\{\left[\mathrm{h}^{3} \mathrm{~h}\right] \mathrm{h}^{3} \mathrm{hp}-\mathrm{w}-\mathrm{d}\right\}>\left\{\mathrm{h}^{3} \mathrm{~h}^{3} \mathrm{p}-\right.$ $\mathrm{w}-\mathrm{d}\}>/ \mathrm{h}^{3}$ ap'ut/ "to walk or run as fast as one can, to use all one's strength in doing sth., to run an engine at full speed." For HE the form $\left\{h^{3} h p-\right\}$ rather than $\left\{h^{3} p-\right\}$ is assumed because $/ h^{3} a p^{\prime} u t /$ has a synonym $\left\{\left[h^{3} h\right] h^{3} h g-w-d\right\}>\left\{h^{3} h^{3} g-w-d\right\}>/ h^{3} a g^{\prime} u t /$. 
North Wakashan Comparative Root List (Updated)

\begin{tabular}{|c|c|c|c|c|c|c|}
\hline Root \# & Old \# & $\mathrm{HE}$ & $\mathrm{OO}$ & HA & KW & Gloss \\
\hline 23230 & 2529 & $\begin{array}{l}\mathrm{h}^{3} \mathrm{~m}- \\
\mathrm{w}^{3} \mathrm{~W}-\end{array}$ & $\begin{array}{l}h^{3} m- \\
h^{3} h m^{3} h- \\
w^{3} w-\end{array}$ & $\begin{array}{l}\mathrm{h}^{3} \mathrm{~m}- \\
\mathrm{h}^{3} \mathrm{hmh}- \\
\mathrm{h}^{3} \mathrm{hm}^{3} \mathrm{~h}-\end{array}$ & $\begin{array}{l}h^{3} \mathrm{~m}- \\
\mathrm{h}^{3} \mathrm{~W}-\end{array}$ & $\begin{array}{l}\text { screened off, constricted, pinched } \\
\text { off (hence: } 1 . \text { sealed, blocked, } \\
\text { watertight, solid; } 2 \text {. tapered, } \\
\text { beginning or ending in a point, } \\
\text { centering around or spreading out } \\
\text { from a point, first in series) })^{1071}\end{array}$ \\
\hline 23240 & 2530 & $\mathrm{~h}^{3} \mathrm{mhk}-$ & $\mathrm{h}^{3} \mathrm{mhk}-$ & $\begin{array}{l}\mathrm{h}^{3} \mathrm{mhk}- \\
\mathrm{h}^{3} \mathrm{hmhk}-\end{array}$ & $\mathrm{h}^{3} \mathrm{mhk}-$ & to defecate ${ }^{1072}$ \\
\hline 23250 & 2531 & $\begin{array}{l}\mathrm{h}^{3} \mathrm{hm} \alpha \mathrm{y}^{3}- \\
\mathrm{h}^{3} \mathrm{hm}^{3} \mathrm{~h}-\end{array}$ & $\begin{array}{l}\mathrm{h}^{3} \mathrm{hm} \alpha \mathrm{y}^{3}- \\
\mathrm{h}^{3} \mathrm{hm}^{3} \mathrm{~h}-\end{array}$ & $\begin{array}{l}\mathrm{h}^{3} \mathrm{~m} \alpha \mathrm{y}^{3}- \\
\mathrm{h}^{3} \mathrm{mhys}- \\
\mathrm{h}^{3} \mathrm{hm}^{3} \mathrm{~h}-\end{array}$ & $h^{3} m \alpha y^{3}-$ & small $^{1073}$ \\
\hline 23260 & 2532 & & & & $\mathrm{~h}^{3} \mathrm{~m}-$ & yes $^{1074}$ \\
\hline 23270 & 2534 & $h^{3} m^{3} t-$ & $h^{3} m^{3} t-$ & $h^{3} m^{3} t-$ & $\begin{array}{l}h^{3} m t- \\
h^{3} m^{3} t-\end{array}$ & (?) bulbous, spherical ${ }^{1075}$ \\
\hline 23280 & 2535 & $\mathrm{~h}^{3} \mathrm{~ms}-$ & $\mathrm{h}^{3} \mathrm{~ms}-$ & $\mathrm{h}^{3} \mathrm{~ms}-$ & & $\begin{array}{l}\text { thick (box, snow, layer), dense } \\
\text { (fog, brush) }\end{array}$ \\
\hline 23290 & 2536 & & & & $\mathrm{~h}^{3} \mathrm{~ms}-$ & $\begin{array}{l}\text { supernatural, dangerous, taboo, to } \\
\text { be respected }\end{array}$ \\
\hline
\end{tabular}

${ }^{1071} \mathrm{KW}\left\{\mathrm{h}^{3} \mathrm{~W}-\right\}$ only in $\left\{\mathrm{h}^{3} \mathrm{w}-\%\right.$ lhqv- $\}$ "silence falls"; HE/OO has $\left\{\mathrm{h}^{3} \mathrm{~m}-\%\right.$ lhqv- $\}$ instead.

$\mathrm{HE} / \mathrm{OO}\left\{\mathrm{w}^{3} \mathrm{w}-\right\}$ substitutes for $\left\{\mathrm{h}^{3} \mathrm{~m}-\right\}$ before $\left\{-\mathrm{p}^{3} \mathrm{n}-\right\}$ "times" or its many derivatives, see suffix no. $220 \mathrm{ff}$. In HA the sense "first" is associated mostly (but not exclusively) with the augmented form $\left\{h^{3} h m^{3} h-\right\}$.

1072 Stem may contain a suffix $\{$-hk- $\}$ with privative meaning attached to $\left\{h^{3} \mathrm{~m}-\right\}$ (root no. 23230).

${ }^{1073} \mathrm{HA}\left\{\mathrm{h}^{3} \mathrm{~m} \alpha \mathrm{y}^{3}-\right\}$ and $\left\{\mathrm{h}^{3} \mathrm{mhys}-\right\}$ have plural forms $\left\{\mathrm{h}^{3} \mathrm{ym} \alpha \mathrm{y}^{3}-\right\}$ and $\left\{\mathrm{h}^{3} \mathrm{ymhys}-\right\}$ that can mean "equally small," cf. HA $\left\{\mathrm{h}^{3} \mathrm{ymhys-tw}\right\}>/ \mathrm{h}^{3} \mathrm{im}$ 'aisdu/ "as small as." On the use of a plural form to signify equal degree of sth. see also the case of HA $\left\{\mathrm{h}^{3} \mathrm{ywh}-\right\}$ mentioned under root no. 17820. 1074 Occurs also as $\{\# \& m$, etc. $\}$ (suffix no. 470).

${ }^{1075}$ In terms for sea urchin or sea eggs; in KW also in terms referring to a boil or carbuncle. 
North Wakashan Comparative Root List (Updated)

\begin{tabular}{|c|c|c|c|c|c|c|}
\hline Root \# & Old \# & $\mathrm{HE}$ & $\mathrm{OO}$ & HA & KW & Gloss \\
\hline 23310 & & & & $\mathrm{~h}^{3} \mathrm{ml}-$ & $\mathrm{h}^{3} \mathrm{ml}-$ & $\begin{array}{l}\text { concession, exception; strange, } \\
\text { different, novel }{ }^{1076}\end{array}$ \\
\hline 23320 & $\begin{array}{l}2537 \\
2615\end{array}$ & $\begin{array}{l}\mathrm{h}^{3} \mathrm{ml-}- \\
\mathrm{h}^{3} \mathrm{~m}^{3} \mathrm{l}- \\
\mathrm{h}^{3} \mathrm{w} 1-\end{array}$ & $\begin{array}{l}\mathrm{h}^{3} \mathrm{ml}- \\
\mathrm{h}^{3} \mathrm{wl}-\end{array}$ & $\begin{array}{l}\mathrm{h}^{3} \mathrm{ml}- \\
\mathrm{h}^{3} \mathrm{w}{ }^{-} \\
\mathrm{h}^{3} \mathrm{~h} 1-\end{array}$ & $\begin{array}{l}\mathrm{h}^{3} \mathrm{ml}- \\
\mathrm{h}^{3} \mathrm{~h} 1-\end{array}$ & $\begin{array}{l}\text { playful, for entertainment, to play } \\
\text { games; (HA/KW also:) to } \\
\text { dance }^{1077}\end{array}$ \\
\hline 23321 & & & & & $\mathrm{~h}^{3} \mathrm{mlyxv-}$ & staying at home \\
\hline 23322 & 2538 & $h^{3} m y^{3} h \chi-$ & $h^{3} m y^{3} h \chi-$ & $\mathrm{h}^{3} \mathrm{my}{ }^{3} \mathrm{~h} \chi-$ & $\mathrm{h}^{3} \mathrm{myh} \chi-$ & $\begin{array}{l}\text { to pray, praise, honour, pay } \\
\text { tribute }\end{array}$ \\
\hline 23330 & $\begin{array}{l}2539 \\
2540\end{array}$ & $\begin{array}{l}h^{3} m q v- \\
h^{3} m \chi-\end{array}$ & $\begin{array}{l}\mathrm{h}^{3} \mathrm{mqv}- \\
\mathrm{h}^{3} \mathrm{~m} \chi-\end{array}$ & $\begin{array}{l}h^{3} m q v- \\
h^{3} m \chi-\end{array}$ & $h^{3} m \chi-$ & $\begin{array}{l}\text { blocked off, choked off, screened } \\
\text { off, sealed, watertight, halting }{ }^{1078}\end{array}$ \\
\hline 23350 & 2533 & $\mathrm{~h}^{3} \mathrm{hm}^{3} \mathrm{~h}^{3} \mathrm{t}$ & $\begin{array}{l}\mathrm{h}^{3} \mathrm{~m}^{3}- \\
\mathrm{h}^{3} \mathrm{~m}^{3} \mathrm{~h} 1- \\
\mathrm{h}^{3} \mathrm{hm}^{3} \mathrm{~h} 1-\end{array}$ & $\mathrm{h}^{3} \mathrm{hm}{ }^{3} \mathrm{~h} 1-$ & $\begin{array}{l}\mathrm{h}^{3} \mathrm{~m}^{3} \mathrm{~h} \nmid- \\
\mathrm{h}^{3} \mathrm{hm} \mathrm{m}^{3} \mathrm{~h} 1-\end{array}$ & $\begin{array}{l}\left(\mathrm{HE} / \mathrm{HA} / \mathrm{KW} \text { and } \mathrm{OO}\left\{\mathrm{h}^{3} \mathrm{~m}^{3}-\right\}:\right) \\
\text { to become aware of sth. strange, } \\
\text { unusual, or supernatural; (OO } \\
\left.\left.\left\{\mathrm{h}^{3} \mathrm{hm}^{3} \mathrm{~h}\right\}-\right\}:\right) \text { to hear }\end{array}$ \\
\hline 23360 & & & & & $\mathrm{~h}^{3} \mathrm{hm}^{3} \mathcal{L}_{-}$ & strange, unusual, different ${ }^{1079}$ \\
\hline 23370 & 2541 & $\mathrm{~h}^{3} \mathrm{dmkV}-$ & $\mathrm{h}^{3} \mathrm{dmkV}-$ & $\mathrm{h}^{3} \mathrm{hdmkV}-$ & $\mathrm{h}^{3} \mathrm{dmkv}-$ & sandhill crane \\
\hline 23380 & 2543 & & $\mathrm{~h}^{3} \mathrm{t}-$ & & $\mathrm{h}^{3} \mathrm{t}-$ & $(?)^{1080}$ \\
\hline
\end{tabular}

${ }^{1076}$ HA only in $/ \mathrm{h}^{31} \mathrm{MłM} /$ "even though, in spite of".

${ }^{1077} \mathrm{KW}\left\{\mathrm{h}^{3} \mathrm{~h} \nmid-\right\}$ occurs only in $\left\{\mathrm{h}^{3} \mathrm{~h} \nmid-\% \mathrm{hxv}-\right\}$ "bone game, (s)lahel game."

$1078\left\{\mathrm{~h}^{3} \mathrm{mqv}-\right\}$ only in HE/OO/HA $\left\{\mathrm{h}^{3} \mathrm{mqv}-\mathrm{h}\right\}$ "to stutter" and OO $/ \mathrm{h}^{3} \mathrm{Mr} \mathrm{rm} /$ "material for making watertight."

${ }^{1079}$ Lincoln (n.d.) indicates accent on $/ \mathrm{M}^{3} /$ in derivates, e.g. $\mathrm{KW} / \mathrm{h}^{31} \mathrm{M}^{3} £ \mathrm{a} /$ "strange, different, etc." It means the stem must be $\left\{h^{3}[h] m^{3} £-\right\}$ which may derive from $*\left\{h^{3} m £-\right\}$, a possible alternant of $\left\{h^{3} \mathrm{~m} t-\right\}$ (root no. 23320).

${ }^{1080} \mathrm{In} \mathrm{KW} / \mathrm{h}^{3} \mathrm{t}^{3} \mathrm{Hxva} /$ "to persist, to keep on doing sth." and $\mathrm{OO} / \mathrm{h}^{3} \mathrm{dis} /$ "still." 
North Wakashan Comparative Root List (Updated)

\begin{tabular}{|c|c|c|c|c|c|c|}
\hline Root \# & Old \# & $\mathrm{HE}$ & $\mathrm{OO}$ & $\mathrm{HA}$ & KW & Gloss \\
\hline 23390 & 2544 & $h^{3} n-$ & & $\begin{array}{l}h^{3} \mathrm{n}- \\
\mathrm{h}^{3} \mathrm{hnh} 1-\end{array}$ & & $\begin{array}{l}\text { to pay salary, to pay what is due, } \\
\text { to do sth. in return for sth. (e.g. } \\
\text { thank for gift or feast) }\end{array}$ \\
\hline 23400 & 2545 & & $h^{3} n-$ & $h^{3} n-$ & & rockslide, landslide \\
\hline 23410 & 2546 & $h^{3} n-$ & $h^{3} n-$ & $h^{3} n-$ & $h^{3} n-$ & $\begin{array}{l}\text { fire, to look after the fire (starting } \\
\text { it, fueling it) }\end{array}$ \\
\hline 23430 & 2549 & $h^{3} n-$ & $h^{3} n-$ & & $\begin{array}{l}\mathrm{h}^{3} \mathrm{l}- \\
\mathrm{h}^{3} \mathrm{n}-\end{array}$ & (?) spruce pitch, resin, glue $e^{1083}$ \\
\hline 23431 & 2550 & $h^{3} n-$ & $h^{3} n-$ & $h^{3} n-$ & $h^{3} n-$ & $(?)^{1084}$ \\
\hline 23440 & 2551 & $\mathrm{~h}^{3} \mathrm{ynh}-$ & $\mathrm{h}^{3} \mathrm{ynh}-$ & $h^{3} y n h-$ & $\begin{array}{l}\mathrm{h}^{3} \mathrm{n}- \\
\mathrm{hn}-\end{array}$ & $(?)^{1085}$ \\
\hline
\end{tabular}

$1081\left\{h^{3} h n h 1-\right\}$ only in HA / $h^{3}$ an'ałzaqv/ "to thank sb. for sth. (feast, gift)."

1082 Perhaps in HE/OO $\left\{\mathrm{h}^{3} \mathrm{n}^{3} \mathrm{yq}-\right\}$ HA $\left\{\mathrm{h}^{3} \mathrm{nyq}-\right\} \mathrm{KW}\left\{\mathrm{h}^{3} \mathrm{ny}^{3} \mathrm{q}-\right\}$ "firewood, fuel" $\mathrm{KW}\left\{\mathrm{h}^{3} \mathrm{n}^{3} \mathrm{q}-\right\}$ "to light fire (by putting coals in)" $\mathrm{HE}\left\{\mathrm{h}^{3} \mathrm{n}-\mathrm{x}^{\prime} \mathrm{yt}\right\} \mathrm{OO}\left\{\mathrm{h}^{3} \mathrm{n}-\mathrm{xyt}\right\} \mathrm{HA}\left\{\mathrm{h}^{3} \mathrm{n}-\mathrm{xyt}\right\}$ "pitchwood used to transfer and start a fire." Boas (1948:08) lists several more words to do with making or moving fire and pointing to a root $\left\{\mathrm{h}^{3} \mathrm{n}-\right\}$ but they could not be reelicited, e.g. $\mathrm{KW} / \mathrm{h}^{3} \mathrm{H}^{3} \mathrm{a} / *$ "to put fire on rock."

1083 Possibly in $\mathrm{HE} / \mathrm{h}^{3} \mathrm{H}^{\prime} \mathrm{iw}^{3} \mathrm{as} / \mathrm{OO} / \mathrm{h}^{3} \mathrm{Hiw}^{3} \mathrm{as} / \mathrm{KW} / \mathrm{h}^{3}{ } \mathrm{Niw}^{3} \mathrm{as} /$ (in the Koskimo dialect $/ \mathrm{h}^{3} \mathrm{Hiw}^{3} \mathrm{as} /$ ) "spruce tree." It may contain a stem HE/OO $\left\{\mathrm{h}^{3} \mathrm{n}-\% \mathrm{yxv}-\right\} \mathrm{KW}\left\{\mathrm{h}^{3} \mathrm{l}-\% \mathrm{yxv}-\right\}$ in which $\{-\% \mathrm{yxv}-\}$ is suffix no. 3980 . Note there is also $\mathrm{HE} / \mathrm{OO} / \mathrm{KW}\left\{\mathrm{h}^{3} \mathrm{l}[\mathrm{y}] \mathrm{xv}-\right\}$ "to hunt with a canoe, etc." discussed in the comment on $\left\{\mathrm{h}^{3} 1 \mathrm{xv}-\right\}$ (root no. 23642).

${ }^{1084}$ In $\left\{h^{3} n-y s\right\}$ "maternal or paternal aunt." Could also be an $|y|$-augmented root $\left\{h^{3} n s-\right\}$ but $\left\{h^{3} n-\right\}$ is more likely because $\{-y s\}$ (suffix no. 2160) also occurs in another kinship term. ${ }^{1085} \mathrm{In} \mathrm{KW} / \mathrm{h}^{3} \mathrm{a}^{3} \mathrm{H} /$ and $/ \mathrm{ha}^{3} \mathrm{H} / \mathrm{HE} / \mathrm{h}^{3} \mathrm{a}^{3}$ in'ix/ OO $/ \mathrm{h}^{3} \mathrm{a}^{3}$ inix/ HA $/ \mathrm{h}^{3} \mathrm{ah}^{3}$ ainik/ "eyebrow" and derivates. 
North Wakashan Comparative Root List (Updated)

\begin{tabular}{|c|c|c|c|c|c|c|}
\hline Root \# & Old \# & $\mathrm{HE}$ & $\mathrm{OO}$ & HA & KW & Gloss \\
\hline 23441 & 2591 & & & & $\begin{array}{l}h^{3} n- \\
h^{3} y n h-\end{array}$ & (?) to quarrel ${ }^{1086}$ \\
\hline 23450 & 2552 & & $h^{3} n p-$ & & & heartburn \\
\hline 23460 & $\begin{array}{l}2553 \\
2554\end{array}$ & $h^{3} n t-$ & $\mathrm{h}^{3} \mathrm{nt}-$ & $h^{3} n t-$ & $h^{3} n t-$ & $\begin{array}{l}\text { chewy, crunchy (like herring } \\
\text { eggs); to use front teeth to strip } \\
\text { off whatever covers a layer (e.g. } \\
\text { herring eggs); (HE/HA also:) to } \\
\text { gnaw (like beaver, rodents) }\end{array}$ \\
\hline 23470 & $\begin{array}{l}2555 \\
2556\end{array}$ & $\mathrm{~h}^{3} \mathrm{nc}-$ & $\begin{array}{l}h^{3} n c- \\
h^{3} n c \cdot-\end{array}$ & $h^{3} \mathrm{nc}-$ & $\mathrm{h}^{3} \mathrm{~ns}-$ & $\begin{array}{l}\text { (HE/OO/HA) to move little by } \\
\text { little, to shove, to move } \\
\text { sideways, to slide; (KW) to } \\
\text { snuggle up, to relax amorously, } \\
\text { to lie with lover or spouse }\end{array}$ \\
\hline 23490 & $\begin{array}{l}2557 \\
2558\end{array}$ & & & & $h^{3} n y^{3}-$ & $\begin{array}{l}\text { 1. lacking specifics, pointless, } \\
\text { without direction, misdirected, } \\
\text { foolish; } \\
\text { 2. to leave food uneaten, fail to } \\
\text { finish meal }\end{array}$ \\
\hline 23500 & $\begin{array}{l}2559 \\
2560\end{array}$ & $\mathrm{~h}^{3} \mathrm{hgvh}-$ & h³hgvh- & $h^{3}$ ngvh- & $\mathrm{h}^{3} \mathrm{ngvh}-$ & $\begin{array}{l}\text { identity, social rank, being } \\
\text { somebody; "who?" }\end{array}$ \\
\hline 23510 & 2561 & $h^{3} n x v-$ & $h^{3} n x v-$ & & $\begin{array}{l}h^{3} n x v- \\
h^{3} n k-\end{array}$ & $\begin{array}{l}\text { (HE/OO) bruise, bruised; (KW) } \\
\text { dark or bushy eyebrows }\end{array}$ \\
\hline 23511 & 2547 & $\begin{array}{l}\mathrm{h}^{3} \mathrm{n}^{3} \mathrm{wkV}- \\
\mathrm{h}^{3} \mathrm{hnh}{ }^{-} \\
\mathrm{h}^{3} \mathrm{nh} \nmid \mathrm{xn}-\end{array}$ & $\begin{array}{l}\mathrm{h}^{3} \mathrm{nwkv-} \\
\mathrm{h}^{3} \mathrm{hnh} \nmid- \\
\mathrm{h}^{3} \mathrm{nh} \nmid \mathrm{kn}-\end{array}$ & $\mathrm{h}^{3} \mathrm{nh}$ xkn- & $\begin{array}{l}\mathrm{h}^{3} n^{3} \mathrm{wkv-} \\
\mathrm{h}^{3} \mathrm{hnhh}-\end{array}$ & $\begin{array}{l}\text { (?) to keep trying, proceed little } \\
\text { by little }{ }^{1087}\end{array}$ \\
\hline
\end{tabular}

$1086\left\{h^{3} y n h-\right\}$ possibly in KW / $h^{3}$ inuxva/ "to make threatening gestures." $\left\{h^{3} n-\right\}$ in KW $\left\{\left[h^{3} y\right] h^{3} n\right\}>/ h^{3} i \hbar^{3} \mathrm{H} /$ "verbal fight (quarrel) between husband and wife" and derivates. ${ }^{1087}$ Unanalyzable stems with initial $\left|h^{3} n\right|,\left|h^{3} n^{3}\right|$ or $\left|h^{3} h n\right|$ and vague semantic similarity. HE $\left\{h^{3} n^{3} w k v-\right\}$ OO $\left\{h^{3} n w k v-\right\}$ and KW $\left\{h^{3} n^{3} w k v-\right\} *$ from the Smith Inlet dialect means "to keep 
North Wakashan Comparative Root List (Updated)

\begin{tabular}{|c|c|c|c|c|c|c|}
\hline Root \# & Old \# & $\mathrm{HE}$ & $\mathrm{OO}$ & HA & KW & Gloss \\
\hline 23520 & $\begin{array}{l}2562 \\
2563\end{array}$ & $\begin{array}{l}h^{3} n q v- \\
h^{3} n^{3} q v \\
h^{3} n w-\end{array}$ & $\mathrm{h}^{3} \mathrm{nqv}-$ & $\begin{array}{l}h^{3} n q v- \\
h^{3} n w-\end{array}$ & $\begin{array}{l}h^{3} n q v- \\
h^{3} n w \infty- \\
h^{3} n w h-\end{array}$ & $\begin{array}{l}\text { cloud, fog, overcast; sky } \\
\text { obscured by a layer }{ }^{1088}\end{array}$ \\
\hline 23530 & 2564 & $\mathrm{~h}^{3} \mathrm{nq}-$ & $\mathrm{h}^{3} \mathrm{nq}-$ & $\mathrm{h}^{3} \mathrm{nq}-$ & $h^{3} n q-$ & $\begin{array}{l}\text { to strain, to squeeze through slit } \\
\text { or pipe, to force through or let } \\
\text { out a bit at a time (e.g. sound), to } \\
\text { babble (like baby), to moan }\end{array}$ \\
\hline 23550 & $\begin{array}{l}2465 \\
2567 \\
2569 \\
2634\end{array}$ & $\begin{array}{l}\mathrm{s}- \\
\mathrm{h}^{3} \mathrm{~s}- \\
\mathrm{h}^{3} \mathrm{~s}^{\bullet-} \\
\mathrm{h}^{3} \mathrm{hs}-\end{array}$ & $\begin{array}{l}\mathrm{h}^{3} \mathrm{~s}- \\
\mathrm{h}^{3} \mathrm{~s} \bullet- \\
\mathrm{h}^{3} \mathrm{~h} s-\end{array}$ & $\begin{array}{l}\mathrm{h}^{3} \mathrm{~s}- \\
\mathrm{h}^{3} \mathrm{~s}^{\bullet-} \\
\mathrm{h}^{3} \mathrm{hs}-\end{array}$ & $\begin{array}{l}\text { hs-* } \\
h^{3}{ }^{3}-\end{array}$ & $\begin{array}{l}\text { (?) otherworldly, supernatural, } \\
\text { dangerous, to be respected; to be } \\
\text { kept away from, to ward off, to } \\
\text { shoo (vermin, evil, dog) }{ }^{1089}\end{array}$ \\
\hline 23560 & $\begin{array}{l}2211 \\
2464\end{array}$ & $\mathrm{~h}^{3} \mathrm{~s}^{\bullet-}$ & $\mathrm{h}^{3} \mathrm{~s}^{\bullet-}$ & $\mathrm{h}^{3} \mathrm{~s}^{\bullet-}$ & $\begin{array}{l}\text { hs- } \\
\text { qs- } \\
h^{3} p^{\bullet-}\end{array}$ & $\begin{array}{l}\left(\mathrm{HE} / \mathrm{OO} / \mathrm{HA}\left\{\mathrm{h}^{3} \mathrm{~s}^{\bullet}-\right\} \mathrm{KW}\{\mathrm{hs}-\right. \\
\text { qs- }\}:) \text { to belong to the other one } \\
\text { (i.e. neither the speaker nor the } \\
\text { addressee); (HE/OO/HA }\left\{\mathrm{h}^{3} \mathrm{~s}^{\bullet}\right\} \\
\left.\mathrm{KW}\left\{\mathrm{h}^{3} \mathrm{ps} \bullet\right\}:\right) \text { the other or } \\
\text { opposite side, one side (as } \\
\text { opposed to both) }\end{array}$ \\
\hline
\end{tabular}

trying, persevere." $\left\{\mathrm{h}^{3} \mathrm{hnh}\right.$ - $\}$ occurs in HE $/ \mathrm{h}^{3}$ an'ałла/ OО $/ \mathrm{h}^{3}$ anałла/ "to coax sb. into doing sth." and $\mathrm{KW} / \mathrm{h}^{3}$ analas/ "funnel." The stems HE $\left\{\mathrm{h}^{3} \mathrm{nh} \nmid \mathrm{xn}-\right\}$ OO $\left\{\mathrm{h}^{3} \mathrm{nh} \nmid \mathrm{kn}-\right\} \mathrm{HA}\left\{\mathrm{h}^{3} \mathrm{nh} \mathrm{xkn}-\right\}$ mean "to gasp for breath."

${ }^{1088} \mathrm{HE} / \mathrm{HA}\left\{\mathrm{h}^{3} \mathrm{nw}-\right\} \mathrm{KW}\left\{\mathrm{h}^{3} \mathrm{nw} \infty / \mathrm{h}-\right\}$ may have developed from $\left\{\mathrm{h}^{3} \mathrm{n} \chi \mathrm{v}-\right\}$ as suggested by Boas (1948:10; 1947:323).

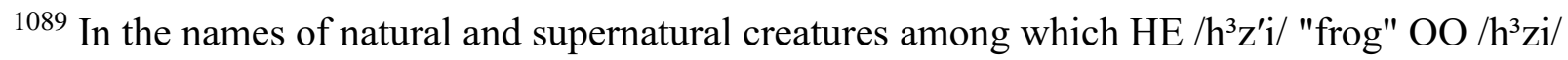
"child-snatching ogre with the basket" $\mathrm{HA} / \mathrm{h}^{3} \mathrm{ziq}^{3} /$ "worm, caterpillar or any creature that moves by squirming; ghost" $\mathrm{KW} / \mathrm{h}^{3} \mathrm{su} /$ (a kind of rock cod) $\mathrm{KW} / \mathrm{h}^{3} \mathrm{sam}^{3} \mathrm{a} / *$ (a long red crab of medium size). The feature $|\bullet|$ in $\left\{\mathrm{h}^{3} \mathrm{~s}^{\bullet-}\right\}$ and $\left\{\mathrm{h}^{3} \mathrm{~h} \mathrm{~s}^{\bullet-}\right\}$ is posited to explain why a following suffix no. 3210 takes the form of $\{-\mathrm{k}-\}$ rather than $\{-\&-\}$. 
North Wakashan Comparative Root List (Updated)

\begin{tabular}{|c|c|c|c|c|c|c|}
\hline Root \# & Old \# & $\mathrm{HE}$ & $\mathrm{OO}$ & HA & KW & Gloss \\
\hline 23570 & & & $\mathrm{~h}^{3} £ \mathrm{bw}-$ & & $h^{3} \lambda b w-$ & seven $^{1090}$ \\
\hline 23580 & $\begin{array}{l}2570 \\
2572\end{array}$ & & & $h^{3} \AA_{-}$ & $\mathrm{h}^{3} \mathcal{f}-$ & $\begin{array}{l}\text { to snap, break off (like rope, tree } \\
\text { branch); (HA also:) severed, } \\
\text { apart, separated (from company, } \\
\text { structure), dispersed, lost (in } \\
\text { space or time); (KW also:) } \\
\text { dentalia }^{1091}\end{array}$ \\
\hline 23590 & $\begin{array}{l}2571 \\
2638\end{array}$ & & & & $\begin{array}{l}\mathrm{h}^{3} £^{3} \mathrm{k}- \\
\mathrm{h}^{3} \mathrm{~h} £^{3} \mathrm{k}-*\end{array}$ & filled up, swollen ${ }^{1092}$ \\
\hline 23600 & $\begin{array}{l}2573 \\
2575 \\
2576\end{array}$ & $h^{3} l_{-}$ & $h^{3} 1-$ & $h^{3} l_{-}$ & $h^{3} 1-$ & $\begin{array}{l}\text { secure, stable, lingering, lasting, } \\
\text { fast, tight, settled (like } \\
\text { agreement), motionless, rigid, } \\
\text { dead }^{1093}\end{array}$ \\
\hline 23620 & 2577 & & & $\begin{array}{l}\mathrm{h}^{3} \mathrm{lz}- \\
\mathrm{h}^{3} \mathrm{lc}-/ \\
\mathrm{h}^{3} 1 \mathrm{~s}-\end{array}$ & $\begin{array}{l}\mathrm{h}^{3} \mathrm{lz}- \\
\mathrm{h}^{3} \mathrm{l} \mathrm{s}-\end{array}$ & $\begin{array}{l}\text { flesh, meat, fleshy; (KW also:) } \\
\text { body fat, fat (as opposed to lean), } \\
\text { fleshy }\end{array}$ \\
\hline
\end{tabular}

1090 Sapir reports a South Wakashan root \{7a£-\} "two" (1939:247) and a suffix \{-p.- $\}$ "to go across" (1939:325). The HE and HA words for "seven" consist of $\left\{\mathrm{mh}^{3} 1-\right\}$ "two" plus HE \{hw $^{3}$ s $\}$ HA $\{$-hws $\}$ "across" (in this case to the fingers of the other hand). The HE/HA suffix derives from suffix no. 4220 which has many forms including $\{-\mathrm{w}\}$ and shades of meaning including "across." It is therefore at least conceivable that root no. 23570 contains the two mentioned South Wakashan morphemes plus native $\{-\mathrm{w}\}$ "across."

${ }^{1091}$ All examples but one of the dentalia meaning involve a stem $\left\{\mathrm{h}^{3} \mathfrak{f}-\mathrm{lh}-\right\}$ (with suffix no. 2960). Uncertain which scenario could link dentalia and breaking off or being dispersed. Perhaps it is that some types of dentalia were used as money, see https://www.nativewomenscollective.org/regaliastoriesdentalia.html.

1092 Two of Boas's examples (1948:20) were re-elicited but with a root $\left\{h^{3} £^{3} k-\right\}$, not $\left\{h^{3} h £^{3} k-\right\}$. 1093 Antonym of $\{$ hl-, etc. $\}$ (root no. 22780). 
North Wakashan Comparative Root List (Updated)

\begin{tabular}{|c|c|c|c|c|c|c|}
\hline Root \# & Old \# & $\mathrm{HE}$ & $\mathrm{OO}$ & HA & KW & Gloss \\
\hline 23630 & 2578 & $\begin{array}{l}h^{3} l c- \\
h^{3} l^{3} h s-\end{array}$ & $\begin{array}{l}h^{3} l c- \\
h^{3} l^{3} h s-\end{array}$ & $\begin{array}{l}h^{3} l c- \\
h^{3} l: h^{3} h s-\end{array}$ & $\mathrm{h}^{3} \mathrm{ls}-$ & sea cucumber ${ }^{1094}$ \\
\hline 23640 & 2579 & $\mathrm{~h}^{3} \mathrm{lk}-$ & & & $\mathrm{h}^{3} \mathrm{lk}-$ & $\begin{array}{l}\text { to shuck clams, remove meat } \\
\text { from shellfish }{ }^{1095}\end{array}$ \\
\hline 23641 & 2580 & $\mathrm{~h}^{3} \mathrm{lkv}-$ & $\mathrm{h}^{3} \mathrm{lkv}-$ & (blk-) & $\begin{array}{l}\mathrm{h}^{3} \mathrm{lkv}- \\
\mathrm{h}^{3} 1 \mathrm{xv}-\end{array}$ & $\begin{array}{l}\text { blood, to lose blood, to bleed; } \\
\text { (OO also:) precious }{ }^{1096}\end{array}$ \\
\hline 23642 & 2574 & $\mathrm{~h}^{3} 1 \mathrm{xv}-$ & $\mathrm{h}^{3} 1 \mathrm{xv}-$ & $\mathrm{h}^{3} \mathrm{lxv}-$ & $\mathrm{h}^{3} 1 \mathrm{xv}-$ & $\begin{array}{l}\text { to hunt with a canoe, to hunt at } \\
\text { sea }{ }^{1097}\end{array}$ \\
\hline 23643 & & & & $\mathrm{~h}^{3}$ lqhi- & & soaked $^{1098}$ \\
\hline 23650 & 2583 & $h^{3} l \chi-$ & $h^{3} l \chi-$ & $h^{3} l \chi-$ & $\left(\mathrm{k}^{3} \mathrm{y} \chi^{-}\right)$ & to kill, murder, beat up \\
\hline 23660 & 2584 & $h^{3} l \chi_{-}^{-}$ & $h^{3} l \chi-$ & $h^{3} l \chi_{-}^{-}$ & & to chew \\
\hline
\end{tabular}

${ }^{1094}$ Informants aren't sure if the examples are about sea cucumber or sea slugs. Boas says on $\mathrm{p}$. 13 of his KW dictionary and on p. 180 of Bella Bella Texts that the root refers to the sea slug or Holothuria. What he means is probably the sea cucumber because Holothuria is a genus of softbodied armless invertebrates resembling a cucumber and some species of Holothuria are harvested and sold as sea cucumber. Sea slugs do not necessarily resemble a cucumber and may not be edible.

${ }^{1095} \mathrm{HE}$ only in the place name $/ \mathrm{H}^{3} \pi \mathrm{XdM}^{3} \mathrm{i}^{\prime} \mathrm{as} /$ "site of shucking clams."

1096 Root occurs also in a few OO endearing terms for infants.

${ }^{1097}$ In KW $\left\{\mathrm{h}^{3} 1 \mathrm{xv}-=\mathrm{zW}-\alpha \mathrm{y}^{3}\right\}>/ \mathrm{h}^{3} л u z v \varepsilon^{3} /$ which according to Boas (1947:212) means "sea hunter on flat." To this gloss he adds "(i.e. in sky, Orion)." Root probably also occurs in the stem $\left\{h^{3} 1[y] x v-\right\}$ of terms referring to $(\mathrm{HE})$ hunting for sea mammals, $(\mathrm{OO})$ hunting in general, HA (Kitlope dialect) hunting using a canoe but (KW) getting ready (e.g. to leave). Boas (n.d.:13) lists many examples of KW \{h3lyxv-\} referring to sea hunting but hardly any could be re-elicited. 1098 Only two examples, НА / ${ }^{3}$ лqаһ. $\hbar^{3 \prime}$ Н/ "soaked (said of one's entire body)" НА / ${ }^{3}$ лqałs'is/ "soaked (said of one's feet)," both with word-final accent. 
North Wakashan Comparative Root List (Updated)

\begin{tabular}{|c|c|c|c|c|c|c|}
\hline Root \# & Old \# & $\mathrm{HE}$ & $\mathrm{OO}$ & HA & $\mathrm{KW}$ & Gloss \\
\hline 23661 & & & & & $\mathrm{~h}^{3} \mathrm{lhq}-$ & $(?)^{1099}$ \\
\hline 23670 & 2585 & & & $\mathrm{~h}^{3} \mathrm{ky}-$ & $h^{3} \mathrm{k}-$ & $\begin{array}{l}\text { to bend forward with the head } \\
\text { pointing down and the rear end } \\
\text { up, to jump on the surface of } \\
\text { water (said of whales, dolphins, } \\
\text { fish) }\end{array}$ \\
\hline 23680 & 2586 & & & & $\mathrm{~h}^{3} \mathrm{k}-*$ & firm $^{1100}$ \\
\hline 23690 & $\begin{array}{l}2588 \\
2602 \\
2642 \\
2643\end{array}$ & $\begin{array}{l}h^{3} y- \\
h^{3} h y h-\end{array}$ & $\begin{array}{l}h^{3} y- \\
h^{3} h y h-\end{array}$ & $\begin{array}{l}h^{3} y- \\
h^{3} h y h-\end{array}$ & $\begin{array}{l}h^{3} y- \\
h^{3} h y h-\end{array}$ & $\begin{array}{l}\text { 1. to bend, fold, double (sth. or } \\
\text { oneself, e.g. over an edge or log } \\
\text { or when squatting), to go in a } \\
\text { loop (as when rounding a point of } \\
\text { land), to teeter (as a long thing } \\
\text { with only its middle on top of a } \\
\text { rock), to seesaw, to pitch (like a } \\
\text { boat); } \\
2 . \text { (in several non-reelicitable } \\
\text { Boas-Hunt examples:) to send } \\
\text { payment (for shamanic services } \\
\text { rendered) }\end{array}$ \\
\hline
\end{tabular}

${ }^{1099} \mathrm{In} \mathrm{KW} \mathrm{/h^{3 } л а q / ~ " a l m o s t " ~ a n d ~ / h ³ л а q а / ~ " a l m o s t ~ t h e r e ~ ( е . g . ~ a b o u t ~ t o ~ g i v e ~ b i r t h ) , ~ t o ~ h a v e ~ a ~}$ narrow escape."

${ }^{1100} \mathrm{In} \mathrm{KW} / \mathrm{h}^{3} \mathrm{k} / *$ "firmly."

1101 1. Possibly also with the sense of "to come around" in the exclamation HA $/ \mathrm{h}^{3}$ ayuh'ali/ that invites people to enjoy themselves at the celebration. 2. Unaugmented $\left\{h^{3} y-\right\}$ occurs only in $\mathrm{HE} / \mathrm{OO} / \mathrm{HA} / \mathrm{KW}\left\{\mathrm{h}^{3} \mathrm{y}-\mathrm{hs}-\right\}$ "to urinate (said of a female)" and the mostly KW stem $\left\{\mathrm{h}^{3} \mathrm{y}-\mathrm{wh}-\right\}$ "to round a point (before reaching the village)" in which latter $\{-w,-w h-\}$ is likely suffix no. 4220 with the sense of "completely." Instead of $\left\{h^{3} y-w h-\right\}$ HE/OO usually have $\left\{h^{3} h y h-w-\right\}$ and $\left\{h^{3}\right.$ hyh-hwh- $\}$. 
North Wakashan Comparative Root List (Updated)

\begin{tabular}{|c|c|c|c|c|c|c|}
\hline Root \# & Old \# & $\mathrm{HE}$ & $\mathrm{OO}$ & $\mathrm{HA}$ & KW & Gloss \\
\hline 23700 & 2589 & $\mathrm{~h}^{3} \mathrm{yp}-$ & $\mathrm{h}^{3} \mathrm{yp}-$ & $\mathrm{h}^{3} \mathrm{yp}-$ & $h^{3} y p-$ & $\begin{array}{l}\text { to pinch, to seize with two } \\
\text { fingers }\end{array}$ \\
\hline 23721 & 2593 & & & & $\begin{array}{l}h^{3} y s- \\
h^{3} y^{3} s-\end{array}$ & abalone shells \\
\hline 23730 & 2592 & & & & $h^{3} y s h y^{3} w-$ & (?) sympathy, pity, mercy ${ }^{1102}$ \\
\hline 23731 & 2596 & $\left(w^{3} w k v-\right)$ & $\left(w^{3} w k v-\right)$ & $h^{3} y g h-$ & & to store away, to save $\mathrm{e}^{1103}$ \\
\hline 23790 & $\begin{array}{l}2597 \\
2599\end{array}$ & $\begin{array}{l}h^{3} y k- \\
h^{3} y^{3} x-\end{array}$ & $\begin{array}{l}h^{3} y k- \\
h^{3} y^{3} x-\end{array}$ & $\mathrm{h}^{3} \mathrm{yk}-$ & $\mathrm{h}^{3} \mathrm{yk}-$ & $\begin{array}{l}\text { good, nice, fine, well; smooth, } \\
\text { level, free of obstruction, } \\
\text { uninterrupted }^{1104}\end{array}$ \\
\hline 23800 & 2598 & $h^{3} y^{3}-$ & $\mathrm{h}^{3} \mathrm{yk}^{3}-$ & $\mathrm{h}^{3} \mathrm{yk}^{3}-$ & $\begin{array}{l}\mathrm{h}^{3} \mathrm{yk}^{3}- \\
\mathrm{h}^{3} \mathrm{y}^{3} \mathrm{f}_{-} *\end{array}$ & $\begin{array}{l}\text { up, high up, above, on the upper } \\
\text { side; heaven, supreme (in space } \\
\text { or rank) }{ }^{1105}\end{array}$ \\
\hline 23810 & 2600 & & & & $h^{3} y k v-$ & to clean up, clear up \\
\hline 23820 & 2601 & & & $h^{3} y x v-$ & $h^{3} y x v-$ & $\begin{array}{l}\text { (?) to anticipate (eagerly in HA, } \\
\text { with hope or fear in } \mathrm{KW} \text { ) }\end{array}$ \\
\hline 23830 & 2603 & $h^{3} y q v-$ & $h^{3} y q v-$ & $\mathrm{h}^{3} \mathrm{yqv}-$ & $h^{3} y q v-$ & to dodge, to get out of the way \\
\hline 23850 & 2605 & & & & $\mathrm{~h}^{3} \mathrm{xV}-$ & red cedar bark \\
\hline
\end{tabular}

${ }^{1102} \mathrm{In} \mathrm{KW} / \mathrm{h}^{3} \mathrm{isay}^{3} \mathrm{u} /$ "to sympathize" and derivates.

${ }^{1103} \mathrm{HA}$ in $\left\{\mathrm{h}^{3} \mathrm{ygh}-\right\}>/ \mathrm{h}^{3} \mathrm{ig}$ 'a/ "to store away" and derivates including e.g. $\left\{\mathrm{h}^{3} \mathrm{ygh}-\mathrm{yl}-\mathrm{h}\right\}>$ $/ \mathrm{h}^{3}$ ig'aiła/ "to put sth. away indoors."

${ }^{1104}$ Both $\left\{h^{3} y k-\right\}$ and $\left\{h^{3} y^{3} x-\right\}$ occur as free forms. In both HE and $O O\left\{h^{3} y^{3} x-\right\}$ must be used instead of $\left\{\mathrm{h}^{3} \mathrm{yk}-\right\}$ before some suffixes of the kind that can only follow a complete word stem. Whereas in $\mathrm{OO}$ the two stems alternate freely before deictics with grammatical function, in HE $\left\{h^{3} y k-\right\}$ is used before some of them but $\left\{h^{3} y^{3} x-\right\}$ before others. $\left\{h^{3} y^{3} x-\right\}$ may have the structure $\left\{h^{3} \mathrm{yk}^{-}\right\}$.

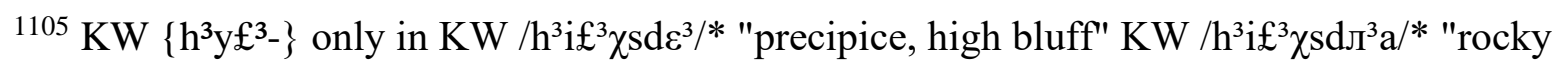
precipice." 
North Wakashan Comparative Root List (Updated)

\begin{tabular}{|c|c|c|c|c|c|c|}
\hline Root \# & Old \# & $\mathrm{HE}$ & $\mathrm{OO}$ & HA & KW & Gloss \\
\hline 23860 & $\begin{array}{l}2607 \\
2645 \\
2617\end{array}$ & $\mathrm{~h}^{3} \mathrm{w}-$ & $\mathrm{h}^{3} \mathrm{~W}-$ & $\begin{array}{l}h^{3} w- \\
h^{3} h w h-\end{array}$ & $\begin{array}{l}h^{3} h w h- \\
h^{3} w y h-\end{array}$ & $\begin{array}{l}\text { solidified, stopped, inert, } \\
\text { resisting change or action (hence } \\
\text { not leaving a place, settled, } \\
\text { reluctant to get going, operating } \\
\text { slowly); (HA }\left\{\mathrm{h}^{3} \mathrm{w}-\right\} \text { also:) to } \\
\text { leave sth. somewhere for a } \\
\text { while }^{1106}\end{array}$ \\
\hline 23861 & 2613 & & & $\begin{array}{l}\mathrm{h}^{3} \mathrm{wS} \cdot- \\
\mathrm{h}^{3} \mathrm{wsl-}\end{array}$ & $\begin{array}{l}h^{3} w- \\
h^{3} w s h-\end{array}$ & $\begin{array}{l}\text { (HA) lying somewhere; (KW) by } \\
\text { itself, apart }{ }^{1107}\end{array}$ \\
\hline 23870 & 2608 & $\mathrm{~h}^{3} \mathrm{w}-$ & & $\mathrm{h}^{3} \mathrm{w}-$ & & $(?)^{1108}$ \\
\hline 23890 & 2609 & $h^{3} w p-$ & $\mathrm{h}^{3} \mathrm{wp}-$ & $\mathrm{h}^{3} \mathrm{wp}-$ & & to use a wood chisel ${ }^{1109}$ \\
\hline 23900 & & (wwl-) & (wwl-) & $\mathrm{h}^{3} \mathrm{wp}-$ & & mound, heap \\
\hline 23910 & 2610 & & $h^{3} w p-$ & & $\mathrm{h}^{3} \mathrm{wp}-$ & to whisper \\
\hline 23920 & 2611 & $\mathrm{~h}^{3} \mathrm{wmh}-$ & & & $\mathrm{h}^{3} \mathrm{wm} \infty-$ & $(?)^{1110}$ \\
\hline 23930 & 2612 & $\mathrm{~h}^{3} \mathrm{wm}^{3}-$ & $h^{3} w^{3}-$ & $h^{3} w^{3}-$ & $\begin{array}{l}\mathrm{h}^{3} \mathrm{wmh}- \\
\mathrm{h}^{3} \mathrm{wm}^{3-}\end{array}$ & pain eases or stops \\
\hline 23940 & & & & & $h^{3} w d h x-$ & suddenly \\
\hline
\end{tabular}

1106 Probably also in HA $/ \mathrm{h}^{3} \mathrm{aw}^{\prime} \mathrm{am}^{3} \mathrm{ru} / \mathrm{and} / \mathrm{h}^{3} \mathrm{aw}^{\prime} \mathrm{aum}{ }^{3} \mathrm{ru} /$ "to amalgamate."

${ }^{1107} \mathrm{HA}\left\{\mathrm{h}^{3} \mathrm{ws}^{\bullet-}\right\}$ only in $/ \mathrm{h}^{3} \mathrm{us}$ 'ala£ла/ "lying on a raised surface (said of any person, animal, object)"

${ }^{1108}$ Perhaps in $\left\{\mathrm{h}^{3} \mathrm{w}-\% \mathrm{hkv}-\right\}$ (with assumed suffix no. 6060) "to keep sth. in one's mouth (e.g. candy, chewing tobacco)."

1109 The only HA example, /h'upa/ "to use a wood chisel," is from a Kitamaat informant but not recognized by the Kitlope one.

${ }^{1110}$ Possibly in the HE proper name $/ \mathrm{H}^{3} \mathrm{um}$ 'a£a/ and in $\mathrm{KW} / \mathrm{h}^{3} \mathrm{a}^{3} \mathrm{ums} /<\left\{\left[\mathrm{h}^{3}\right] \mathrm{h}^{3} \mathrm{wm} \infty-\mathrm{s}\right\}$ "mortal, not magic or supernatural, ordinary." 
North Wakashan Comparative Root List (Updated)

\begin{tabular}{|c|c|c|c|c|c|c|}
\hline Root \# & Old \# & $\mathrm{HE}$ & $\mathrm{OO}$ & $\mathrm{HA}$ & KW & Gloss \\
\hline 23950 & & & & $\mathrm{~h}^{3} \mathrm{w} 1-$ & $\begin{array}{l}h^{3} w 1- \\
h^{3} w l-\end{array}$ & evident, discernable, noticeable \\
\hline 23970 & 2616 & & & & $\begin{array}{l}h^{3} \mathrm{w}^{3} \mathrm{~S}^{-} \\
\mathrm{h}^{3} \mathrm{w}^{3} 1^{3-}\end{array}$ & wolf $^{1111}$ \\
\hline 23980 & 2606 & $\mathrm{~h}^{3} \mathrm{wkv}-$ & $\mathrm{h}^{3} \mathrm{wkV}-$ & $\mathrm{h}^{3} \mathrm{wkV}-$ & & pity, mercy, compassion \\
\hline 24000 & & $\mathrm{~h}^{3} \mathrm{w} \chi \mathrm{v}-$ & & & & $(?)^{1112}$ \\
\hline 24010 & 2622 & $\mathrm{~h}^{3} \mathrm{q}-$ & $\mathrm{h}^{3} \mathrm{q}-$ & $\mathrm{h}^{3} \mathrm{q}-$ & $\mathrm{h}^{3} \mathrm{q}-$ & $\begin{array}{l}\text { wide, wide open, to gape (said of } \\
\text { hole or mouth) }\end{array}$ \\
\hline 24030 & 2625 & $h^{3} h-$ & $\mathrm{h}^{3} \mathrm{~h}-$ & & & to add grease to one's cooking \\
\hline 24050 & 2626 & $\mathrm{~h}^{3} \mathrm{~h}-$ & $\mathrm{h}^{3} \mathrm{~h}-$ & $\mathrm{h}^{3} \mathrm{~h}-$ & $h^{3} h-$ & $\begin{array}{l}\text { ah! (in exclamations and forms of } \\
\text { phatic communion) }\end{array}$ \\
\hline 24060 & 2627 & $\mathrm{~h}^{3} \mathrm{hp}-$ & $\mathrm{h}^{3} \mathrm{hp}-$ & $\mathrm{h}^{3} \mathrm{hp}-$ & $\mathrm{h}^{3} \mathrm{hp}-$ & maggoty \\
\hline 24070 & 2629 & $\mathrm{~h}^{3} \mathrm{hmws-}$ & $\mathrm{h}^{3} \mathrm{hmws}-$ & $\mathrm{h}^{3} \mathrm{hmw1-}$ & $\mathrm{h}^{3} \mathrm{hmws}-$ & $\begin{array}{l}\text { 1. (HE/OO/KW) to decorate (e.g. } \\
\text { Christmas tree); } \\
\text { 2. (HE/HA/KW) to practise a } \\
\text { craft, do skilled work }{ }^{1113}\end{array}$ \\
\hline 24071 & 2629 & $\begin{array}{l}h^{3} h m w y^{3}- \\
h^{3} w m w y^{3}-\end{array}$ & $h^{3} h m w y^{3}-$ & $h^{3} h m w y^{3}-$ & & $\begin{array}{l}\text { (HE/HA) surplus, extra, excess } \\
\text { (that one gives away for free); } \\
\text { (OO) to decorate }\end{array}$ \\
\hline
\end{tabular}

${ }^{1111}$ In the stems $\left\{\mathrm{h}^{3} \mathrm{w}^{3} \mathrm{~s}-\mathrm{yk}-\right\}$ and $\left\{\mathrm{h}^{3} \mathrm{wl}^{3}-\mathrm{yk}-\right\}$ both meaning "wolf."

1112 Perhaps in $\mathrm{HE} / \mathrm{h}^{3 \prime} \mathrm{u} \chi \mathrm{va} /$ "empty clam shell; an unidentified small kind of clam" HE $/ h^{3 \prime}$ u $\chi$ vліs/ "empty clam shells on the beach." In both examples the root, if it is $\left\{\mathrm{h}^{3} \mathrm{w} \chi \mathrm{v}-\right\}$, must be augmented to $\left\{\mathrm{h}^{3} \mathrm{w}[\infty] \chi \mathrm{V}-\right\}>\left\{\mathrm{h}^{3} \mathrm{ww} \chi \mathrm{V}-\right\}$ because only low tones can occur in suffix allomorphs. 1113 Only HA example is $/ \mathrm{h}^{31}$ amul ${ }^{3}$ ini $\chi \mathrm{v} /$ "carver" (term said to be obsolete and now replaced by HA $/ \chi v^{\prime} a £^{3}$ ini $\left.\chi v /\right)$.

${ }^{1114} \mathrm{HE}\left\{\mathrm{h}^{3} \mathrm{wmw} \mathrm{y}^{3}-\right\}$ occurs in examples from elder Eli Wallace; other HE informants used $\left\{h^{3} h m w y^{3}-\right\}$. The OO gloss may be wrong. 
North Wakashan Comparative Root List (Updated)

\begin{tabular}{|c|c|c|c|c|c|c|}
\hline Root \# & Old \# & HE & $\mathrm{OO}$ & HA & $\mathrm{KW}$ & Gloss \\
\hline 24080 & 2631 & $\mathrm{~h}^{3} \mathrm{ht}-$ & $\mathrm{h}^{3} \mathrm{ht}-$ & $\mathrm{h}^{3} \mathrm{ht}-$ & $\begin{array}{l}\mathrm{h}^{3} \mathrm{t}- \\
\mathrm{h}^{3} \mathrm{ht}-\end{array}$ & $\begin{array}{l}\text { sinew, tendon, muscle, tenseness; } \\
\text { to strain (muscles, vocal cords, at } \\
\text { stool, in child birth) })^{1115}\end{array}$ \\
\hline 24090 & 2633 & (nqv-) & (nqv-) & $\mathrm{h}^{3} \mathrm{hs}-$ & $\left(n \chi v^{-}\right)$ & $\begin{array}{l}\text { soapberries (probably Canada } \\
\text { buffaloberry - Shepherdia } \\
\text { canadensis) }^{1116}\end{array}$ \\
\hline 24100 & 2635 & $\mathrm{~h}^{3} \mathrm{hs}-$ & $\mathrm{h}^{3} \mathrm{hs}-$ & & & $\begin{array}{l}\text { to use the longline to catch } \\
\text { halibut }\end{array}$ \\
\hline 24110 & 2636 & $\begin{array}{l}h^{3} h f- \\
h^{3} h £ \bullet-\end{array}$ & $\begin{array}{l}h^{3} h £- \\
h^{3} h £ \bullet-\end{array}$ & $\begin{array}{l}h^{3} h £- \\
h^{3} h £ \bullet-\end{array}$ & $\begin{array}{l}h^{3} h \lambda- \\
h^{3} h £- \\
h^{3} h £ y- \\
h^{3} h £ h- \\
h^{3} h £ \cdot-\end{array}$ & $\begin{array}{l}\text { away from open space (hence } \\
\text { away from the centre of the } \\
\text { house and towards the wall, away } \\
\text { from the beach and towards the } \\
\text { land, away from the sea and } \\
\text { towards the mainland, towards } \\
\text { the interior, inland, shorewards, } \\
\text { behind the house) }{ }^{1117}\end{array}$ \\
\hline 24111 & & & & & $\mathrm{~h}^{3} \mathrm{~h} \AA-$ & wolf $^{1118}$ \\
\hline 24120 & 2637 & & & & $\mathrm{~h}^{3} \mathrm{~h} £-$ & else, or \\
\hline
\end{tabular}

$1115 \mathrm{KW}\left\{\mathrm{h}^{3} \mathrm{t}-\mathrm{\}}\right.$ only in $/ \mathrm{h}^{3} \mathrm{dabiz} \varepsilon^{3 /}$ "tendon behind knee". Informant may have been in error as this is likely KW / $\mathrm{h}^{3}$ adabize ${ }^{3 / *}$ glossed by Boas as "calf of leg" (1948:17) and "sinew at heel" (1947:313).

1116 Likely a Tsimshian loan.

${ }^{1117} \mathrm{HA}\left\{\mathrm{h}^{3} \mathrm{~h} £-\right\}$ is attested only before the suffix $\{-\mathrm{tw}\}$.

${ }^{1118}$ A root form $\left\{h^{3} h £-\right\}$ is extractable from KW $/ h^{3} a £^{3} a l a /<\left\{h^{3} h £-+h l h\right\}$ "wolf sound" and perhaps KW $/ \mathrm{h}^{3} \mathrm{a} £ \mathrm{Lul}^{3}$ inu $\chi \mathrm{v} / *<\left\{\mathrm{h}^{3} \mathrm{~h} £-\right.$ w1-+ynw $\left.\chi \mathrm{v}\right\}$ "wolf." This form could be a backformation from a large number of unanalyzable and possibly borrowed free stems meaning "wolf" and consisting of $/ \mathrm{h}^{3} \lambda /, / \mathrm{h}^{3} \mathrm{a} \lambda /, / \mathrm{h}^{3} £ /, / \mathrm{h}^{3} \mathrm{a} £ /$ followed by $/ \mathrm{H}^{3} \mathrm{M} /, / \mathrm{an}^{3} \mathrm{M} /, / \mathrm{J}^{3} \mathrm{M} /$ or $/ \mathrm{al}{ }^{3} \mathrm{M} /$. 
North Wakashan Comparative Root List (Updated)

\begin{tabular}{|c|c|c|c|c|c|c|}
\hline Root \# & Old \# & $\mathrm{HE}$ & $\mathrm{OO}$ & HA & KW & Gloss \\
\hline 24130 & 2638 & $\mathrm{~h}^{3} \mathrm{~h} £^{3}-$ & $h^{3} h £^{3}-$ & $h^{3} h £^{3}-$ & $\mathrm{h}^{3} \mathrm{~h} £^{3}-$ & $\begin{array}{l}\text { up in the woods, inland, in the } \\
\text { interior; to build a canoe }\end{array}$ \\
\hline 24140 & 2639 & $\mathrm{~h}^{3} \mathrm{~h} 1-$ & $\mathrm{h}^{3} \mathrm{~h} 1-$ & $\mathrm{h}^{3} \mathrm{~h} 1-$ & $\mathrm{h}^{3} \mathrm{~h} 1-$ & $\begin{array}{l}\text { adjoining in space, time or } \\
\text { ranking (hence } 1 . \text { next, second, } \\
\text { following after, recent, new, } \\
\text { again, recently, lately; } 2 \text {. in } \\
\text { support of, subordinate to, } \\
\text { assisting } 1119\end{array}$ \\
\hline 24150 & 2640 & & & & $\mathrm{~h}^{3} \mathrm{hly}-$ & to search, go here and there \\
\hline 24160 & 2641 & $\mathrm{~h}^{3} \mathrm{hg}-$ & $\mathrm{h}^{3} \mathrm{hg}-$ & $\mathrm{h}^{3} \mathrm{hg}-$ & $\begin{array}{l}\left(\mathrm{w}^{3} \mathrm{yl}^{3}-\right. \\
\left.\mathrm{w}^{3} \mathrm{yl}^{3} \mathrm{~h}-\right)\end{array}$ & all, everything, everybody \\
\hline 24170 & & $\mathrm{~h}^{3} \mathrm{hxhdy-}$ & $\mathrm{h}^{3} \mathrm{~h} x h d y-$ & & & $\begin{array}{l}\text { fortified island at the mouth of an } \\
\text { inlet or river }\end{array}$ \\
\hline 24180 & 2644 & $h^{3} h x v-$ & $\mathrm{h}^{3} \mathrm{hxv-}$ & $h^{3} h x v-$ & $h^{3} h x v-$ & to foam, to skim off foam \\
\hline 24181 & & & & & $\mathrm{~h}^{3} \mathrm{hwhqv-}$ & summer seat, sundeck ${ }^{1120}$ \\
\hline 24190 & & & & $\mathrm{~h}^{3} \mathrm{hqv-}$ & & $(?)^{1121}$ \\
\hline 24210 & 2648 & $\mathrm{~h}^{3} \mathrm{hq}-$ & $\mathrm{h}^{3} \mathrm{hq}-$ & $\mathrm{h}^{3} \mathrm{hq}-$ & $\mathrm{h}^{3} \mathrm{hq}-$ & $\begin{array}{l}\text { to arrive, appear on the scene, } \\
\text { show up, loom up, become }\end{array}$ \\
\hline
\end{tabular}

${ }^{1119}$ Likely also the root of HA $\left\{\mathrm{h}^{3} \mathrm{~h} h \mathrm{hqv}-\right\}$ "weasel dance." This dance is the Kitlope equivalent of the OO $/ \Lambda \mathrm{u}^{3}$ лаха/ dance. The latter name derives from the HE/OO/HA stem $\left\{\lambda \mathrm{w}^{3} l_{-}\right\}$"again" related to $\mathrm{KW}\left\{\lambda \mathrm{W}^{3}-\right\}$ "and, and also, along with, together with, accompanied by."

${ }^{1120}$ Stem's structure could be $\left\{\mathrm{h}^{3} \mathrm{hxv}-\% \mathrm{hqv}-\right\}$ with suffix no. 4020 but unknown meaning of the root. The root could also be $\left\{\mathrm{h}^{3} \mathrm{hwh}-\right\}$ (no. 23860) with the meaning "to linger, to lounge" and suffix no. 4020.

${ }^{1121}$ Possibly in HA $\left\{\mathrm{h}^{3} \mathrm{hq}^{3} \mathrm{vn}\right\}$ "sand dune." 
North Wakashan Comparative Root List (Updated)

\begin{tabular}{|c|c|c|c|c|c|c|}
\hline Root \# & Old \# & $\mathrm{HE}$ & $\mathrm{OO}$ & HA & KW & Gloss \\
\hline & & & & & & $\begin{array}{l}\text { visible, open up, unfurl, } \\
\text { reveal }^{1122}\end{array}$ \\
\hline
\end{tabular}

${ }^{1122}$ Only two certain KW examples. Boas's dictionary doesn't seem to be aware of this root at all. 


\section{Index of Root Forms}

Alphabetic Order:

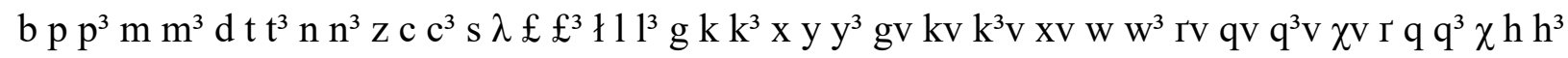

\begin{tabular}{|c|c|c|c|c|}
\hline & $\mathbf{b}$ & bwł- $\quad 200,210$ & pnqv- & 410 \\
\hline & & bwxv- 220 & $\mathrm{pn} \chi \mathrm{V}-$ & 420 \\
\hline bns- & 10 & bwqv- 230 & pnq- & 410,430 \\
\hline$b^{3}-$ & 20,30 & bqv- 240 & $\mathrm{pn}^{3} 1_{-}$ & 400 \\
\hline bs- & 40 & $\mathrm{~b} \chi-\quad 250$ & ps- & 440 \\
\hline bł-* & 450 & bh- $\quad 260$ & $\mathrm{p} £-$ & 450 \\
\hline bl- & 50 & bhs- $\quad 270$ & pł- & 450 \\
\hline blk- & 60 & bh£- $\quad 280$ & $\mathrm{płc}^{3}-$ & 3200 \\
\hline blxv- & 70 & bhł- * 280 & pl- & 460 \\
\hline blq-* & 80 & bhkv- $\quad 160,290,300$ & plk- & 470 \\
\hline $\mathrm{bl} \chi^{-*}$ & 90 & 310,311 & plq- & 80,480 \\
\hline $\mathrm{bx}-*$ & 140 & bhxv- $\quad 320,330,340$ & pk- & 500 \\
\hline by- & 100,260 & bhqv- $\quad 240,350$ & px- & 510 \\
\hline bys- & 110 & bh $\chi \mathrm{v}-\quad 360$ & pys- & 520,521 \\
\hline by1- & 120 & & py£- & 530 \\
\hline byk- & 130 & $\mathbf{p}$ & pyk- & 540 \\
\hline byx- & 140 & & pyqv- & $-\quad 550$ \\
\hline bkv- & 150 & p- $\quad 22510$ & py $\chi \mathrm{v}-$ & 550 \\
\hline bkv- & 160 & pt- $\quad 370$ & pyq-* & 540 \\
\hline bw- & 170 & pn- $\quad 380$ & $\mathrm{py}^{3} \mathrm{~s}-$ & 520,521 \\
\hline bwt- & 180 & pnc- 390 & $p^{3} k_{-}$ & 740 \\
\hline bws- & 190 & pns- $\quad 390$ & pkv- & 150 \\
\hline bw£- & 200 & pn£- $\quad 400$ & & \\
\hline
\end{tabular}


North Wakashan Comparative Root List (Updated)

\begin{tabular}{|c|c|c|}
\hline pkv- 580 & $\mathrm{p}^{31-} \quad 870$ & $\mathrm{~m}$ \\
\hline pxv- $\quad 590$ & $\mathrm{p}^{3} 1-\quad 880,890$ & \\
\hline pw- 600 & $\mathrm{p}^{3} \mathrm{lc}-\quad 900$ & mmm- 1190 \\
\hline pws- $\quad 610,611$ & $\mathrm{p}^{3} \mathrm{lxv}-\quad 890$ & mmk- 1200 \\
\hline pwf- $\quad 620$ & $\mathrm{p}^{3} \mathrm{lqv}-\quad 910$ & mt- $\quad 1210,1211$ \\
\hline pwxv- $\quad 630,640$ & $\mathrm{p}^{3} 1 \chi_{\mathrm{V}-} \quad 890$ & mn- 1212 \\
\hline pwqv- 650 & $\mathrm{p}^{3} \mathrm{lq}-\quad 910$ & mnz- $\quad 1220$ \\
\hline pwqv- 660 & $\mathrm{p}^{3} 1 \chi_{-} \quad 920$ & mnt- 1230 \\
\hline pw $\chi v-\quad 660,670$ & $\mathrm{p}^{3} \mathrm{k}-\quad 940$ & mnk- $\quad 1240$ \\
\hline pqv- 240 & $\mathrm{p}^{3} \mathrm{x}-\quad 940$ & mnxv- 1250 \\
\hline $\mathrm{p} \chi \mathrm{V}-\quad 680$ & $p^{3} y s-960$ & mnr-* 1260 \\
\hline pq- $\quad 690$ & $p^{3} y x v-\quad 970$ & mnq- $\quad 1270$ \\
\hline $\mathrm{p} \chi-\quad 700,710$ & $\mathrm{p}^{3} \mathrm{yq}^{3} \mathrm{v}-* \quad 980$ & mc- 1280 \\
\hline ph- $\quad 720$ & $\mathrm{p}^{3} \mathrm{yq}-\quad 990$ & ms- $\quad 1280,1290,1300$ \\
\hline phs- $\quad 440$ & $\mathrm{p}^{3} \mathrm{kv}-\quad 580,1000$ & mł- $\quad 1320,1330$ \\
\hline ph£- $\quad 740$ & $\mathrm{p}^{3} \mathrm{xv}-\quad 590$ & $m \nvdash \bullet-\quad 1320$ \\
\hline phł- $\quad 740,750,760$ & $\mathrm{p}^{3} \mathrm{w}-1010$ & młh- $\quad 1320$ \\
\hline phxv- $\quad 590,770$ & $\mathrm{p}^{3} \mathrm{ws}-\quad 1020$ & ml- $\quad 1340,1410$ \\
\hline phqv- 780 & $\mathrm{p}^{3} \mathrm{w}-1 \quad 1030,1040$ & mlc- 1360 \\
\hline phq- $\quad 690$ & $\mathrm{p}^{3} \mathrm{wkv}-\quad 1050$ & mls- $\quad 1360$ \\
\hline ph $\chi-\quad 710,790$ & $\mathrm{p}^{3} \mathrm{wqv}-\quad 1060$ & mlk- $\quad 1370,1380$ \\
\hline $\mathrm{ph}^{3} \mathrm{k}-\quad 740$ & $\mathrm{p}^{3} \mathrm{qv}-\quad 1070$ & mlx- 1380 \\
\hline & $\mathrm{p}^{3} \mathrm{q}-\quad 1080$ & mlxv- 1250 \\
\hline $\mathbf{p}^{3}$ & $\mathrm{p}^{3} \mathrm{~h}-\quad 1090,1100$ & mlqv- $\quad 1400$ \\
\hline & $\mathrm{p}^{3} \mathrm{hs}-\quad 440,830$ & $\mathrm{ml} \chi \mathrm{v}-\quad 1400$ \\
\hline $\mathrm{p}^{3} \mathrm{dk}-\quad 800$ & $\mathrm{p}^{3} \mathrm{~h} £-\quad 1130$ & mlq- $\quad 1400,1401$ \\
\hline $\mathrm{p}^{3 \mathrm{t}-} \quad 810$ & $\left.\mathrm{p}^{3} \mathrm{~h}\right\}-\quad 1140$ & $\mathrm{ml}^{3-} \quad 1410$ \\
\hline $\mathrm{p}^{3} \mathrm{tk}-\quad 800$ & $\mathrm{p}^{3} \mathrm{hx}-\quad 1150$ & mk- $\quad 1200,1420$ \\
\hline $\mathrm{p}^{3} \mathrm{nq}-\quad 820$ & $\mathrm{p}^{3} \mathrm{hq} \mathrm{q}^{3} \mathrm{v}-1170$ & mx- 1440 \\
\hline $\mathrm{p}^{3} \mathrm{~s}-\quad 440,830,850$ & $\mathrm{p}^{3} \mathrm{hq}^{3-} \quad 1170$ & mxs- $\quad 1300$ \\
\hline $\mathrm{p}^{3} £-\quad 860$ & & my- $\quad 260,1450$ \\
\hline
\end{tabular}


North Wakashan Comparative Root List (Updated)

\begin{tabular}{|c|c|c|}
\hline myt- $\quad 1460,1470$ & $\mathrm{~m} \chi \mathrm{V}-\mathrm{s}$ & $\operatorname{mh} \chi \mathrm{v}-\quad 1810,2100$ \\
\hline mys- $\quad 1480,1490$ & mq- 1830 & mhq- $\quad 1890,2120$ \\
\hline myg- $\quad 1510$ & $\mathrm{~m} \chi-\quad 1840,1850$ & $\operatorname{mh} \chi-\quad 2130,2140$ \\
\hline mygh- $\quad 1510$ & mh- $\quad 260,1860$ & $\mathrm{mh}^{3}{ }^{3-} \quad 2150$ \\
\hline myk- 1520 & mhp-* 1890 & $\mathrm{mh}^{3} \mathrm{yl}^{-*} \quad 2021$ \\
\hline myk $^{3}-\quad 1520$ & mhdh- 1920 & \\
\hline myx- $\quad 140,1530,1531$, & mht- 1210 & $\mathbf{m}^{3}$ \\
\hline 1540 & mhnys- 1930 & \\
\hline mykv- 1550 & mhnh- $\quad 2200$ & $m^{3} n-\quad 2160$ \\
\hline myqv- 1560 & mhn'hys- 1930 & $\mathrm{~m}^{3} \mathrm{nc}-\quad 2170,2180$ \\
\hline my $\chi_{\mathrm{v}-*} \quad 1570$ & mhnn $^{3} y s-\quad 1930$ & $\mathrm{~m}^{3} \mathrm{~ns}-\quad 2170,2180$ \\
\hline myq-* 1580 & mhz- 1940 & $m^{3} n y-\quad 2160$ \\
\hline myx- 1590 & mhzh- 1940 & $m^{3} n q-\quad 2190$ \\
\hline mkv- $\quad 1600,1610$ & mhs- $\quad 1940,1950,1960$ & $\mathrm{~m}^{3} \mathrm{nh}-\quad 2200$ \\
\hline mxv- $\quad 1610,1620,1630$ & mhs $\infty-1940$ & $m^{3} n^{3} c-\quad 2170$ \\
\hline mw- $\quad 1640,1641,1650$ & mh£- 1970 & $\mathrm{~m}^{3} \mathrm{c}-\quad 2210$ \\
\hline mwdh-* 1920 & mhł- $\quad 1320,1330,1971$ & $\mathrm{~m}^{3} \mathrm{~s}-\quad 1300,2210$ \\
\hline mwt- 1660 & $1980,2000,2020$ & $\mathrm{~m}^{3}$ stq- $\quad 2220$ \\
\hline mwc- 1670 & $\mathrm{mhl}^{3}-\quad 1410$ & $\mathrm{~m}^{3} \mathrm{~s} \chi^{-*} \quad 2220$ \\
\hline mws- $\quad 1670,1680$ & mhk- 2010 & $\mathrm{~m}^{3} \chi \mathrm{stq}-\quad 2220$ \\
\hline mwl- $\quad 1690,1700,1710$ & mhx- $\quad 1440,2020$ & $\mathrm{~m}^{3} 1_{-} * \quad 2410$ \\
\hline $\mathrm{mwl}^{3-} \quad 1710$ & mhył- 2021 & $\mathrm{~m}^{3} \mathrm{l}-\quad 2230$ \\
\hline $\mathrm{mwl}^{3} \mathrm{~W}-\quad 1710$ & mhyh-* 2030 & $\mathrm{~m}^{3} \mathrm{lqv}-\quad 2240$ \\
\hline mwl'sh- 1710 & mhyht- 2030 & $\mathrm{~m}^{3} \mathrm{lq}-\quad 2250$ \\
\hline mwkv- $\quad 1720,1730$ & mhyh $^{3} x^{-} \quad 2030$ & $m^{3} y-\quad 1450$ \\
\hline mwxv- $\quad 1730,1740$ & mhy ${ }^{3-} \quad 1450$ & $\mathrm{~m}^{3} \mathrm{ys}-\quad 1480$ \\
\hline mwqv- $\quad 1750,1760$, & mhkv- $\quad 2040,2050$ & $\mathrm{~m}^{3} \mathrm{y} £-\quad 2270$ \\
\hline 1770,1780 & mhxv- $\quad 1620,2050$ & $\mathrm{~m}^{3} \mathrm{yl}-\quad 2270$ \\
\hline mw $\chi^{\mathrm{v}-} \quad 1790$ & mhw- 2060 & $\mathrm{~m}^{3} \mathrm{yk}-\quad 2300$ \\
\hline mrv-* 1791 & $\mathrm{mhw}^{3} \mathrm{y}-\quad 1640$ & $m^{3} y^{3} \mathcal{E}-\quad 2270$ \\
\hline mqv- 1800 & mhqv- $\quad 2070,2090$ & $\mathrm{~m}^{3} \mathrm{kv}-\quad 2310$ \\
\hline
\end{tabular}




\section{North Wakashan Comparative Root List (Updated)}

\begin{tabular}{|c|c|c|}
\hline $\mathrm{m}^{3} \mathrm{xV}-1620$ & d & $\mathrm{dy}^{3}-2780$ \\
\hline $\mathrm{m}^{3} \mathrm{~W}-\quad 2320$ & & dkv- $\quad 160,2790$ \\
\hline $\mathrm{m}^{3} \mathrm{wt}-\quad 2330$ & dm- $\quad 2450,2460$ & dxv- $\quad 2800$ \\
\hline $\mathrm{m}^{3} \mathrm{wc}-\quad 2340$ & dmt- 2470 & dw- $\quad 2810,2820$ \\
\hline $\mathrm{m}^{3} \mathrm{w} £-\quad 2350$ & dmkv- 2480 & dwt- 2830 \\
\hline $\left.\mathrm{m}^{3} \mathrm{w}\right\}-\quad 2350$ & dmxv- * 2490 & dws- $\quad 2840$ \\
\hline $\mathrm{m}^{3} \mathrm{wkv}-* \quad 2360$ & dmqv- 2500 & dw£- $\quad 2850$ \\
\hline $\mathrm{m}^{3} \mathrm{wqv}-\quad 1760,2370$ & $\mathrm{dm} \chi_{-} \quad 2510,2520$ & dw1- 2860 \\
\hline $\mathrm{m}^{3} \mathrm{w}^{3} \mathrm{qv}-\quad 2380$ & dn- 2530 & dwlw- 2870 \\
\hline $\mathrm{m}^{3} \mathrm{q}-\quad 2390$ & dnt- $\quad 2550$ & dwkv- 2880 \\
\hline $\mathrm{m}^{3} \mathrm{~h}-\quad 2400$ & dns- 2560 & dwxv- 2900 \\
\hline $\mathrm{m}^{3} \mathrm{hny}-\quad 2200$ & dnkv- * 2570 & dwqv- $\quad 2500,2910$ \\
\hline $\mathrm{m}^{3} \mathrm{hnh}-\quad 2200$ & dnxv- 2580 & dq- 2920 \\
\hline $\mathrm{m}^{3} \mathrm{hs}-\quad 1960,2401$ & $\operatorname{dn} \chi_{-} \quad 2590,2600$ & $\mathrm{~d} \chi-\quad 2930$ \\
\hline $\mathrm{m}^{3} \mathrm{hsk}-\quad 2401$ & $\mathrm{dn}^{3-*} \quad 2610$ & dh- $\quad 2780,2940$ \\
\hline $\left.\mathrm{m}^{3} \mathrm{~h}\right\}-\quad 2410,2420$ & $\mathrm{dn}^{3} \mathrm{~h}-\quad 2610$ & dhp- $\quad 2950,2960$ \\
\hline $\mathrm{m}^{3} \mathrm{hy}-\quad 2401$ & dt- $\quad 2620$ & dhc- 2780 \\
\hline mhysk- 2401 & dls- 2630 & dhs- 2970 \\
\hline$m^{3} h y^{3} h £-\quad 2401$ & dlk- 2640 & dht- 2980 \\
\hline mhyh- 2160 & dlx- $\quad 2650$ & dhy- 2690 \\
\hline $\mathrm{m}^{3} \mathrm{hkv}-\quad 2310,2430$ & dlhyd- 2981 & dhyd- 2981 \\
\hline $\mathrm{m}^{3} \mathrm{hxv}-\quad 2440$ & dk- $\quad 2670$ & dhyh- 2690 \\
\hline $\mathrm{m}^{3} \mathrm{~h} w h-\quad 2320$ & $d x-\quad 2680$ & dhy ${ }^{3}-\quad 2940$ \\
\hline $\mathrm{m}^{3} \mathrm{hw}^{3}-\quad 2320$ & dy- $\quad 2690$ & dhk-* 2982 \\
\hline $\mathrm{m}^{3} \mathrm{hqv}-\quad 2070$ & dyk- 2700 & dhxv- 2990 \\
\hline $\mathrm{m}^{3} \mathrm{~h} \chi \mathrm{v}-\quad 2100$ & dyk- 2710 & dhq- $\quad 3000,3010$ \\
\hline mhhs- 2401 & dyx- 2720 & $\mathrm{dh}^{31} \mathrm{k}-3020$ \\
\hline \multirow[t]{4}{*}{$m^{3}$ hhł- $\quad 2401$} & dykv- 2730 & \\
\hline & dyxv- $\quad 2740$ & $\mathbf{t}$ \\
\hline & dyqv- $\quad 2750,2760$ & \\
\hline & dy $x-\quad 2770$ & tp- 3030 \\
\hline
\end{tabular}


North Wakashan Comparative Root List (Updated)

\begin{tabular}{|c|c|c|}
\hline tm- $\quad 3040$ & tyq- 3330 & $\mathrm{t}^{3} \mathrm{mxv}-\quad 3090$ \\
\hline tms- 3050 & tyx- 3350 & $\mathrm{t}^{3} \mathrm{mq}-\quad 3590$ \\
\hline tmsx •- $\quad 3050$ & ty $^{3} \mathrm{sx}-\quad 3210$ & $\mathrm{t}^{3} \mathrm{mhkv-} \quad 3570$ \\
\hline tmt- 3060 & ty $^{3} \mathrm{sx} \bullet-\quad 3210$ & $\mathrm{t}^{3} \mathrm{~m}^{3} \mathrm{~s}-\quad 3540$ \\
\hline $\operatorname{tmx}-\quad 3070$ & ty $^{3} x^{x-} \quad 3210$ & $t^{3} n-\quad 3140$ \\
\hline tmkv- $\quad 3080$ & ty $^{3} x^{\bullet}-\quad 3210$ & $\mathrm{t}^{3} \mathrm{nc}-\quad 3600$ \\
\hline tmxv- $\quad 3090$ & tkv- $\quad 3360$ & $t^{3} n s-\quad 3600,3610$ \\
\hline tmqv- $\quad 3100$ & txv- 2800 & $t^{3} n x-\quad 3620$ \\
\hline $\operatorname{tm} \chi v^{-} \quad 3110$ & tw- 3400 & $\mathrm{t}^{3} \mathrm{nqv}^{-*} \quad 3630$ \\
\hline tmq- $\quad 3120,3130$ & twp- 3410 & $\mathrm{t}^{3} \mathrm{n} \chi-\quad 3650$ \\
\hline $\operatorname{tm} \chi-\quad 3130$ & tws- $\quad 3420$ & $\mathrm{t}^{3} \mathrm{~s}-\quad 3660,3670$ \\
\hline tn- $\quad 3140,3150$ & twl-* 3430 & $t^{3} 1-\quad 3680$ \\
\hline tns- $\quad 3150$ & twqv- $\quad 3440$ & $\mathrm{t}^{3} \mathrm{lc}-\quad 3690$ \\
\hline tnk- 3160 & tw $\chi v-\quad 3441$ & $\mathrm{t}^{3} 1 \mathrm{~s}-\quad 3690,3700$ \\
\hline $\operatorname{tnx}-\quad 3170$ & tqv- 3450 & $t^{3} 1 x v-\quad 3710$ \\
\hline $\operatorname{tn} \chi-\quad 3180$ & tq- $\quad 2920,3450$ & $\mathrm{t}^{3} \mathrm{q}^{3-}-3720$ \\
\hline ts- 3190 & $t \chi-\quad 700,2920,21960$ & $\mathrm{t}^{3} \mathrm{k}-\quad 3280,3730$ \\
\hline ttto- $\quad 3200$ & th- 3470 & $\mathrm{t}^{3} \mathrm{x}-\quad 3280,3740,3750$ \\
\hline $\mathrm{ttc}^{3}-\quad 3200$ & thm 3 hqv- 3100 & 3760 \\
\hline $\mathrm{ttx} \cdot-\quad 3210$ & thw $^{3-} \quad 3400$ & $\mathrm{t}^{3} \mathrm{y}-\quad 3770,3780$ \\
\hline tlp- 3220 & thqv- $3270,3500,4120$ & $\mathrm{t}^{3} \mathrm{yp}-\quad 3800$ \\
\hline tlkv- $\quad 3230,3240,3250$ & th $\chi \mathrm{v}-\quad 3110$ & $\mathrm{t}^{3} \mathrm{ys}-\quad 3810$ \\
\hline tlqv- $\quad 3260,3270$ & thq- 3010 & $\mathrm{t}^{3} \mathrm{y} \mathbf{l}_{-} \quad 3820$ \\
\hline tk- $\quad 3280,3281$ & & $t^{3} y k-\quad 3830,3840$ \\
\hline tx- $\quad 510,2680,3281$ & $\mathbf{t}^{3}$ & $t^{3} y x-\quad 3840,3850,3860$ \\
\hline tys- $\quad 521,3210$ & & $\mathrm{t}^{3} \mathrm{ykv}-\quad 3870$ \\
\hline tył- 3290 & $\mathrm{t}^{3} \mathrm{p}-\quad 3520$ & $t^{3} y x v-\quad 3880$ \\
\hline tyłx•- 3210 & $\mathrm{t}^{3} \mathrm{~m}-\quad 3540$ & $t^{3} y q v-\quad 3890,3900$ \\
\hline tyk- 3280 & $\mathrm{t}^{3} \mathrm{~ms}-\quad 3550$ & $\mathrm{t}^{3} \mathrm{yq}-\quad 3910$ \\
\hline tyx- 3310 & $t^{3} \mathrm{ml}-\quad 3560$ & $\mathrm{t}^{3} \mathrm{y} \chi-\quad 3920$ \\
\hline tykv- 3320 & $\mathrm{t}^{3} \mathrm{mkv}-\quad 3570,3580$ & $\mathrm{t}^{3} \mathrm{y}^{3} 1-\quad 3930$ \\
\hline
\end{tabular}




\section{North Wakashan Comparative Root List (Updated)}

\begin{tabular}{|c|c|c|}
\hline$t^{3} y^{3} k-\quad 3940$ & $\mathbf{n}$ & nwgvh- $\quad 4421$ \\
\hline$t^{3} y^{3} k v-\quad 3940$ & & nwkv- $\quad 4500,4510$ \\
\hline $\mathrm{t}^{3} \mathrm{kV}-\quad 2790,3950$ & np- $\quad 4160$ & nw $\chi \mathrm{v}^{-} \quad 4520$ \\
\hline $\mathrm{t}^{3} \mathrm{w}-\quad 3960$ & nm- 2460 & nqv- 4530 \\
\hline $\mathrm{t}^{3} \mathrm{wp}-\quad 3410$ & nms- $\quad 4180$ & $\mathrm{n} \chi \mathrm{v}-\quad 4530,11410$ \\
\hline $\mathrm{t}^{3} \mathrm{WS}-\quad 3420,3970$ & nt- $\quad 4190$ & nq- $\quad 4540,4550,21740$ \\
\hline $\mathrm{t}^{3} \mathrm{w} 1-\quad 2860$ & nn- $\quad 4210$ & $\mathrm{n} \chi-\quad 4550,4560$ \\
\hline $\mathrm{t}^{3} \mathrm{wxV}-\quad 3980,3990$ & nnxw- 4211 & nh- $\quad 4580,4590,4600$ \\
\hline $\mathrm{t}^{3} \mathrm{wqv}-\quad 4000,4010$ & nnkv- $\quad 4220,4230$ & 11450 \\
\hline $\mathrm{t}^{3} \mathrm{w} \chi \mathrm{v}-* \quad 4010,4020$ & ns-* 4240 & nhp- $\quad 4160,4610$ \\
\hline $\mathrm{t}^{3} \mathrm{qv}-\quad 4030$ & nt- $\quad 4250,4260$ & nhn- $\quad 4611$ \\
\hline $\mathrm{t}^{3} \mathrm{q}-\quad 2920,4040$ & nl- $\quad 4260$ & nhs- $\quad 4240,4640$ \\
\hline $\mathrm{t}^{3} \chi^{-} \quad 2920$ & nk- 4290 & nh- $\quad 4470$ \\
\hline $\mathrm{t}^{3} \mathrm{~h}-\quad 4060,4070$ & $n x-\quad 4300$ & nhk- $\quad 4650$ \\
\hline $\mathrm{t}^{3} \mathrm{hm}^{3-} \quad 3540$ & ny- $\quad 4310$ & nhx- $\quad 4660$ \\
\hline $\mathrm{t}^{3} \mathrm{hm}^{3} \mathrm{hkv}-\quad 3570$ & nynwyh- 4420 & nhxv- $\quad 4670,4680$ \\
\hline$t^{3} h n y^{3-} \quad 3140$ & nys- $\quad 4320,4330,4800$ & nhwh- 4420 \\
\hline $\mathrm{t}^{3} \mathrm{hnh}-\quad 3140$ & nył- 4350 & nh $\chi \mathrm{v}-\quad 4670$ \\
\hline $\mathrm{t}^{3} \mathrm{hs}-\quad 3660,3670$ & nyk- 4360 & nhq- $\quad 4550,4690,4700$, \\
\hline $\mathrm{t}^{3} \mathrm{hl}-\quad 4071$ & nykv- $\quad 4370$ & 21740 \\
\hline $\mathrm{t}^{3} \mathrm{hk}-\quad 4080$ & nyqv- 1560 & \\
\hline$t^{3} h x-\quad 4080$ & ny $\chi-4380$ & $\mathbf{n}^{3}$ \\
\hline$t^{3} h y^{3}-\quad 3780$ & ngv- 4390 & \\
\hline $\mathrm{t}^{3} \mathrm{hkv}-\quad 4100$ & nkv- $\quad 4370,4400$ & $\mathrm{n}^{3} \mathrm{~m}-\quad 2160,2460$ \\
\hline $\mathrm{t}^{3} \mathrm{hqv}-\quad 4110,4120$ & nw- $\quad 4420,4421$ & $\mathrm{n}^{3} \mathrm{mh}-\quad 2200$ \\
\hline$t^{3} h q-\quad 4130$ & nwt- 4430 & $\mathrm{n}^{3} \mathrm{mhy} \mathrm{y}^{3} \mathrm{~h}-\quad 2200$ \\
\hline $\mathrm{t}^{3} \mathrm{~h} \chi-\quad 4130$ & nwn $\infty-\quad 4440$ & $n^{3} n-\quad 4710$ \\
\hline $\mathrm{t}^{3} \mathrm{~h}^{3} \mathrm{~s}-\quad 3670$ & nws- $\quad 4320,4450$ & $\mathrm{n}^{3} \mathrm{nk}-\quad 4720,4730$ \\
\hline$t^{3} h^{3} \chi-\quad 4150$ & nwł•- $\quad 4460$ & $\mathrm{n}^{3} \mathrm{nq}-\quad 820$ \\
\hline & nwyh- $\quad 4420$ & $\mathrm{n}^{3} \AA_{-}-4740$ \\
\hline & nw1- 4470 & $\mathrm{n}^{3} 1-* \quad 4741$ \\
\hline
\end{tabular}


North Wakashan Comparative Root List (Updated)

\begin{tabular}{|c|c|c|}
\hline $\mathrm{n}^{3} 1 \mathrm{~s}-* \quad 4742$ & $\mathrm{n}^{3} \mathrm{hkv}-\quad 5060$ & zyk- $\quad 5370,5380$ \\
\hline$n^{3} 1 \chi-\quad 4750$ & $\mathrm{n}^{3} \mathrm{hxv}-\quad 4860,5070$ & zyx- $\quad 5400$ \\
\hline$n^{3} l h-\quad 4760$ & $\mathrm{n}^{3} \mathrm{hk}^{3} \mathrm{v}-* \quad 5070$ & zykv- $\quad 5410,5420$ \\
\hline $\mathrm{n}^{3} \mathrm{k}-\quad 4720,4770,4810$ & $\mathrm{n}^{3}$ hqv- $\quad 5090,5100$ & zyqv- 5420 \\
\hline$n^{3} y-\quad 4780,4790$ & $\mathrm{n}^{3} \mathrm{~h} \chi-\quad 5110$ & zyq- $\quad 5430$ \\
\hline$n^{3}$ ys- $\quad 4800$ & & zyq- $\quad 5440$ \\
\hline $\mathrm{n}^{3} \mathrm{yk}-\quad 4790$ & $\mathbf{z}$ & zXv- $\quad 5450$ \\
\hline$n^{3} y 1-\quad 4810$ & & zw- 5460 \\
\hline$n^{3} y k-\quad 4810$ & zblx- $\quad 5120$ & zwp- $\quad 5480$ \\
\hline $\mathrm{n}^{3} \mathrm{ykv}-\quad 4370,4810,4840$ & zp- $\quad 5480$ & zwmyg- 5481 \\
\hline$n^{3} \mathrm{xv}-\quad 4860$ & $\mathrm{zm}-\quad 5130,5140$ & zwmyk- 5481 \\
\hline$n^{3} x v^{\bullet-} \quad 4870$ & zmxv- 5150 & zwt-* 5490 \\
\hline $\mathrm{n}^{3} \mathrm{xvh}-\quad 4870$ & zt- $\quad 5170$ & zwnh- 5491 \\
\hline $\mathrm{n}^{3} \mathrm{~W}-\quad 4880,4890$ & zn- $\quad 5180$ & zws- $\quad 5500$ \\
\hline $\mathrm{n}^{3} \mathrm{w} 1-\quad 4900$ & znk- 5190 & zwxv- $\quad 5510,5520$ \\
\hline $\mathrm{n}^{3}$ wlh- $\quad 4910$ & znq- $\quad 5200,5210$ & zwqv- $\quad 5540,5550$ \\
\hline $\mathrm{n}^{3}$ wyqv- $\quad 4940$ & $\mathrm{zn}^{3-} \quad 5211$ & zqv- $\quad 5560,5570,5580$ \\
\hline $\mathrm{n}^{3} \mathrm{WXv}-\quad 4920,4930$ & zc- 5212 & $z \chi-\quad 5590$ \\
\hline $\mathrm{n}^{3} \mathrm{wqv}_{-} * \quad 4940$ & zs- $\quad 5212,5220$ & zh-* 12090 \\
\hline$n^{3} w^{3} s-\quad 4890$ & zł- $\quad 5230$ & zhmy- 5130 \\
\hline $\mathrm{n}^{3} \mathrm{qv}-\quad 4950$ & zlk- $\quad 5240$ & $\mathrm{zhm}^{3-} \quad 5140$ \\
\hline $\mathrm{n}^{3} \chi \mathrm{vh}-\quad 4970$ & zlx- 5250 & zhc- 5600 \\
\hline $\mathrm{n}^{3} \mathrm{~h}-\quad 4590,4980,4990$ & zlxv- $\quad 5260$ & zhs- $\quad 5600,5610$ \\
\hline 5000,5040 & $\mathrm{zl} \chi-\quad 5270$ & zhkv- $\quad 5620$ \\
\hline $\mathrm{n}^{3} \mathrm{hs}-\quad 5010$ & zk- $\quad 5280,5300,5370$ & zhxv- $\quad 5620,5630,5640$ \\
\hline $\mathrm{n}^{3} \mathrm{~h}^{3}-\quad 5020$ & zx- 5310 & zhwhdly- 5641 \\
\hline $\mathrm{n}^{3} \mathrm{~h} 1-\quad 5030$ & zy- $\quad 5320$ & zhqv- 5650 \\
\hline $\mathrm{n}^{3} \mathrm{hlh}-\quad 5040$ & zyp- $\quad 5321$ & $\mathrm{zhq}^{3} \mathrm{v}-\quad 5660$ \\
\hline $\mathrm{n}^{3} \mathrm{hl} \infty \mathrm{-}-\quad 5050$ & zyt- $\quad 5330,5340$ & zhq- $\quad 5670$ \\
\hline $\mathrm{n}^{3} \mathrm{hk}-\quad 5050$ & zys-* 5350 & $\mathrm{zh}^{3} \mathrm{n} \chi-\quad 5671$ \\
\hline $\mathrm{n}^{3} \mathrm{hk}^{3-} \quad 5050$ & zył- 5360 & $\mathrm{zh}^{31} 1_{-} \quad 5680$ \\
\hline
\end{tabular}


North Wakashan Comparative Root List (Updated)

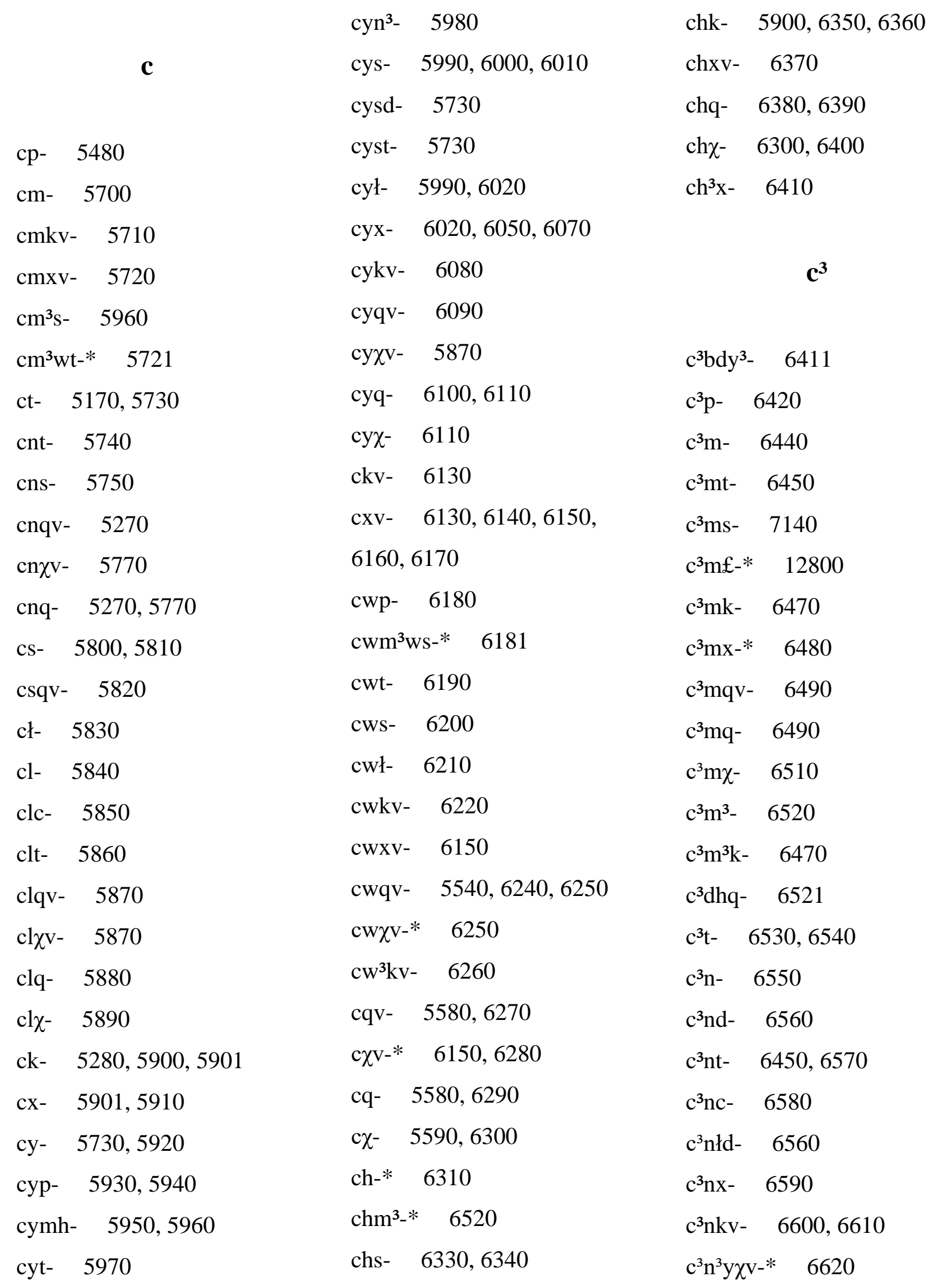




\section{North Wakashan Comparative Root List (Updated)}

\begin{tabular}{|c|c|c|}
\hline$c^{3} n^{3} y x^{*}$ & $c^{3} \mathrm{yk}-\quad 6920,6930,6940$ & $c^{3}$ hp- $\quad 6420$ \\
\hline$c^{3} s^{-} \quad 5820,6540,6630$ & 6950 & $\mathrm{c}^{3} \mathrm{hm}^{3}-\quad 6440,6520$ \\
\hline $\mathrm{c}^{3}$ sk- $\quad 5820$ & $c^{3} \mathrm{yk}^{3} \mathrm{~h} 1-\quad 6960$ & $c^{3}$ ht- $\quad 6540$ \\
\hline $\mathrm{c}^{3} \mathrm{skv}_{-} * \quad 5820$ & $c^{3} y_{x-} \quad 6970,6980,6990$ & $\mathrm{c}^{3} \mathrm{hs}-\quad 7350,7370,7380$ \\
\hline $\mathrm{c}^{3} \mathrm{sqv}-\quad 5820,6640$ & 7000 & $\mathrm{c}^{3} \mathrm{hk}-\quad 4080,6770,7400$ \\
\hline$c^{3} 1-\quad 6540,6560,6650$ & $c^{3}$ ykv- $\quad 6920,7020$ & 7430 \\
\hline$c^{3} 1 d-\quad 6540$ & $c^{3} y x v-\quad 7040$ & $c^{3} \mathrm{hx}-\quad 6790,6810,7440$ \\
\hline$c^{3} x^{\bullet}-\quad 6560$ & $c^{3}$ yqv- 7030 & $7450,7460,7470$ \\
\hline$c^{3} 1-\quad 6650,6660$ & $c^{3} y \chi v-\quad 7040$ & $c^{3}$ hyq- 7471 \\
\hline$c^{3} 1 d-\quad 6560$ & $\mathrm{c}^{3} \mathrm{yq}-\quad 6100,7030$ & $c^{3} h^{3}-\quad 7480$ \\
\hline$c^{3} 1 t-\quad 6670$ & $c^{3} y x-\quad 7070$ & $c^{3} \mathrm{hkv}-\quad 7490$ \\
\hline$c^{3} 1 s-\quad 6680$ & $c^{3} y^{3} p-\quad 6840$ & $c^{3} h x v-\quad 6370$ \\
\hline$c^{3} 1 k-\quad 6690$ & $c^{3} y^{3} s-\quad 6890,13260$ & $c^{3} h w \infty-\quad 7500$ \\
\hline$c^{3} 1 x-\quad 5250,6690$ & $\mathrm{c}^{3} \mathrm{kv}-\quad 7080,7090,7100$ & $c^{3} \mathrm{hw}^{3-} \quad 7130$ \\
\hline$c^{3} 1 \mathrm{kv}-\quad 6600,6610,6710$ & $c^{3} x v-\quad 7110$ & $c^{3} \mathrm{hqv}-\quad 7510,7520,7530$ \\
\hline$c^{3} l q v-\quad 6720$ & $c^{3} W^{-} \quad 7130,7140$ & $c^{3} h \chi v-7530$ \\
\hline$c^{3} 1 \chi v-\quad 6730$ & $\mathrm{c}^{3} \mathrm{wp}-\quad 5480$ & $\mathrm{c}^{3} \mathrm{hr}-\quad 7540$ \\
\hline$c^{3} l q-\quad 6740,6750$ & $c^{3} w t-\quad 7160$ & $\mathrm{c}^{3} \mathrm{hq}-\quad 7260,7550,7551$ \\
\hline$c^{3} 1 \chi_{-} \quad 6760$ & $c^{3}$ wnh- $\quad 7170$ & $c^{3} \mathrm{~h} \chi-\quad 6510$ \\
\hline$c^{3} \mathrm{k}-\quad 6770$ & $\mathrm{c}^{3} \mathrm{ws}-\quad 7140,7180$ & $c^{3} h^{3} q-\quad 7560$ \\
\hline$c^{3} x-\quad 6540,6790,6800$ & $c^{3} w 1-\quad 7190$ & $\mathrm{c}^{3} \mathrm{~h}^{3} \chi-\quad 7560$ \\
\hline 6810 & $c^{3} \mathrm{wkv}-\quad 7200$ & \\
\hline$c^{3} \mathrm{y}-\quad 5920,6830$ & $c^{3} \mathrm{WXv}-\quad 7210$ & $\mathbf{s}$ \\
\hline$c^{3}$ yp- $\quad 6840$ & $\mathrm{c}^{3} \mathrm{wws}-\quad 7140$ & \\
\hline$c^{3}$ ymhws- $\quad 6850$ & $\mathrm{c}^{3} \mathrm{wqv}-\quad 7220,7230$ & s- $\quad 23550$ \\
\hline$c^{3}$ yt- $\quad 6540$ & $\mathrm{c}^{3} \mathrm{w} \chi \mathrm{V}^{-} \quad 7230$ & sbh- $\quad 7570$ \\
\hline$c^{3}$ ynh- $\quad 6870$ & $\mathrm{c}^{3} \mathrm{~W}^{3} \mathrm{~s}-\quad 7140$ & sp- $\quad 7570$ \\
\hline$c^{3} y s-\quad 6880,6890$ & $c^{3} q^{-}-6270,7240,7250$ & $\mathrm{sp}^{3}-\quad 7580$ \\
\hline$c^{3} \mathrm{yl}_{-} * \quad 5990$ & $c^{3} q^{-} \quad 7260$ & smp- $\quad 7590$ \\
\hline$c^{3} y \nmid \chi v-\quad 6910$ & $7270,7280,7290$ & sms- $\quad 7600$ \\
\hline$c^{3} y_{1} w^{3}-\quad 6910$ & $\mathrm{c}^{3} \mathrm{~h}-\quad 7300,7310$ & smk- $\quad 7610$ \\
\hline
\end{tabular}


North Wakashan Comparative Root List (Updated)

\begin{tabular}{|c|c|c|}
\hline st- $\quad 7620$ & sy $\chi v-7970$ & shwh- 8050 \\
\hline sn- $\quad 7630,7640,7650$ & syq- 7980 & shqv- $\quad 8120,8430$ \\
\hline snt- 7660 & sy $^{3} x^{-} \quad 7990$ & shq $^{3} v-\quad 8120$ \\
\hline sny ${ }^{3}-\quad 7670$ & sy $^{3} \mathrm{kv}-\quad 8000$ & $\operatorname{sh} \chi v-\quad 8410,8440$ \\
\hline snkv- $\quad 7680$ & skv- $\quad 8010,8020,8391$ & shq- $\quad 8450,8460$ \\
\hline snq- $\quad 7690$ & sw- $\quad 8040,8050,8060$ & $\mathrm{sh}^{3} \mathrm{x}-\quad 8370$ \\
\hline ss- 7700 & swp- $\quad 6180,8070$ & $\mathrm{sh}^{3} \mathrm{kv}-\quad 8390$ \\
\hline sss- $\quad 5730$ & sw1- 8080 & $\operatorname{sh}^{3} \chi v-\quad 8470$ \\
\hline st- $\quad 7710,7720$ & swkv- 8090 & \\
\hline słbxv- 7730 & swqv- 8110 & $\lambda$ \\
\hline sł- $\quad 7740$ & sqv- 8120 & \\
\hline sl- $\quad 7740,7750$ & $\mathrm{sq}^{3} \mathrm{v}-\quad 8120$ & $\lambda \mathrm{m}-\quad 8480$ \\
\hline slp- $\quad 7760$ & $\mathrm{~s} \chi \mathrm{v}-* \quad 8120$ & $\lambda \mathrm{ms}-\quad 8490$ \\
\hline slt- $\quad 7780,7790$ & sq- $\quad 8130$ & $\lambda \mathrm{mxv}-\quad 8500$ \\
\hline slth- 7791 & $\mathrm{~s} \chi-\quad 8150,8160$ & $\lambda \mathrm{n}-\quad 8510,8520$ \\
\hline sls- $\quad 7800$ & sh- $\quad 8170$ & $\lambda$ ns- $\quad 8530$ \\
\hline sly³- 7670 & shp- 8200 & $\lambda n £-\quad 8540$ \\
\hline slqv- 7810 & sht- 8210 & $\lambda n-* \quad 8550$ \\
\hline $\mathrm{sl} \chi \mathrm{v}^{-} \quad 7820$ & 8240 & $\lambda \mathrm{nqv}-* 8560$ \\
\hline slq- $\quad 7810$ & shs- $\quad 8240,8250$ & $\lambda \mathrm{k}-\quad 8580$ \\
\hline sk- $\quad 7840$ & sht- $\quad 8260,8270$ & $\lambda x-\quad 8590$ \\
\hline $\mathrm{sk}^{3} \mathrm{~s}^{\bullet-} \quad 12970$ & shlh- $\quad 7750,8290$ & $\lambda y-\quad 8600,8601$ \\
\hline $\mathrm{sk}^{3} \mathrm{~h}-\quad 7850$ & $\mathrm{shl}^{3}-\quad 8300$ & $\lambda y s-\quad 8610$ \\
\hline sx-* 7860 & shk- $\quad 8310,8320,8340$ & $\lambda y k v-\quad 8620$ \\
\hline sy- $\quad 7880$ & 8360 & $\lambda y q-\quad 8630$ \\
\hline syt- 7890 & shx- $\quad 8360,8370$ & $\lambda \mathrm{y}^{3} \mathrm{kv}-\quad 8620$ \\
\hline synh- $\quad 7900$ & shyht- 8290 & $\lambda \mathrm{kv}-\quad 8640$ \\
\hline sys- 7910 & shy³_- 7880 & $\lambda w-\quad 8650,8670$ \\
\hline sył- $\quad 7920,7930$ & shkv- $\quad 8380,8390,8391$ & $\lambda w s-\quad 8670$ \\
\hline syx- $\quad 7940,7950$ & shxv- 8400 & $\lambda$ wgv- $\quad 8680$ \\
\hline sykv- 7960 & shxv- 8410 & $\lambda$ wgvm- 8690 \\
\hline
\end{tabular}


North Wakashan Comparative Root List (Updated)

\begin{tabular}{|c|c|c|}
\hline$\lambda w k v-\quad 8680$ & Enkv- 8960 & Eh- 9330,9340 \\
\hline$\lambda q v-\quad 8700,8710$ & Enqv- $\quad 8970,8980$ & £hp- $\quad 8830,9040,9350$ \\
\hline$\lambda \mathrm{q}^{* *} \quad 8710$ & £nq-* 8990 & £hmw- 8500 \\
\hline$\lambda \chi-\quad 8730,8740$ & £s- 9000 & £ht- 9360 \\
\hline$\lambda \mathrm{h}-\quad 8750$ & £ł- 9010 & fhs- $\quad 9380,9400$ \\
\hline$\lambda$ hmh- $\quad 8500$ & $£ 1-\quad 9020$ & Ehk- 9050,9410 \\
\hline$\lambda \mathrm{hmhw}^{3}{ }_{-} \quad 8500$ & flp-* 9040 & Ehx- $\quad 9420,9430$ \\
\hline$\lambda \mathrm{hp}-\quad 8760$ & $£ \mathrm{k}-\quad 8580,8590,9050$ & Ehqv- 9440,9450 , \\
\hline$\lambda$ ht- 8761 & 9060 & 10910 \\
\hline$\lambda$ hk- 8770 & $£ x^{-} \quad 8590,9080$ & £hq- $\quad 9300,9470$ \\
\hline 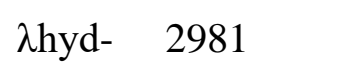 & Exs- 9090 & £h $\chi-\quad 9480,9490$ \\
\hline$\lambda \mathrm{hkv-} \quad 8620,8640$ & $£ x^{\bullet-} \quad 8590$ & $\mathrm{fh}^{3} \mathrm{~s}-\quad 9000,9400$ \\
\hline$\lambda \mathrm{h} \chi \mathrm{v}-\quad 8800,8810$ & £ys- 9100 & \\
\hline$\lambda \mathrm{h}^{3} \mathrm{p}-\quad 8820$ & £ył- $\quad 9110,9120,9130$ & $\mathfrak{f}^{\mathbf{3}}$ \\
\hline & £yx- $\quad 9110,9120$ & \\
\hline$£$ & £ykv- 9150 & $\mathfrak{£}^{3} p-\quad 9510$ \\
\hline & fyqv- $\quad 9160,9170$ & $£^{3} \mathrm{~ms}-\quad 9530$ \\
\hline fp- $\quad 8830$ & £yq- $\quad 9190,9200$ & $£^{3} \mathrm{~m} 1-\quad 9550$ \\
\hline$£ \mathrm{~m}-\quad 8840$ & $£ y x-\quad 9200$ & $£^{3} \mathrm{mkv}-\quad 9560,9570$ \\
\hline £ms-* 8850 & £y $\mathrm{y}^{3} \mathrm{~s}-\quad 9100$ & $£^{3} \mathrm{~m} \chi \mathrm{v}^{-} \quad 9571$ \\
\hline £mt- 8860 & £kv- 8640 & $£^{3} \mathrm{mq}-\quad 9580,9590$ \\
\hline £mk- 8870 & £wp- 9230 & $£^{3} \mathrm{mh}-\quad 9600$ \\
\hline £mkv- 8880 & £wmh- 9240 & $£^{3}$ mhkv-* 9601 \\
\hline$£$ mxv- $\quad 8500$ & £wmo- 9240 & $\mathfrak{£}^{3} \mathrm{mh} \chi \mathrm{v}-\quad 9602$ \\
\hline$£ \mathrm{~m} \chi \mathrm{v}^{-} * \quad 9571$ & £wl-* 9250 & $\mathfrak{f}^{3} \mathrm{~m}^{3} \mathrm{~s}-\quad 9530,9603$ \\
\hline £mq- $\quad 8900,8910$ & $£ w y^{3}-\quad 9260$ & $\mathfrak{f}^{3} \mathrm{t}-\quad 9610$ \\
\hline ft- $\quad 8920,8930$ & $£ w \chi v-\quad 9270$ & $£^{3}$ ns- $\quad 9620$ \\
\hline £n-* 8940 & £qv- $\quad 8700,9280,10910$ & $£^{3}$ nk- $\quad 9640,9650$ \\
\hline £ns-* 8950 & $£ \chi v^{-} \quad 9290$ & $\mathfrak{£}^{3} n y^{3}-* \quad 9660$ \\
\hline £nk- 8550 & £q- $\quad 9300,9310,9320$ & $£^{3} n q-\quad 9670,9680$ \\
\hline £ny ${ }^{3}-\quad 8950$ & $£ \chi-\quad 8730$ & $\mathfrak{£}^{3} n^{3} q-\quad 9681$ \\
\hline
\end{tabular}


North Wakashan Comparative Root List (Updated)

\begin{tabular}{|c|c|c|}
\hline $\mathfrak{£}^{3} \mathrm{~s}-\quad 9690$ & $\mathfrak{f}^{3}$ ws- $\quad 10080$ & $£^{3}$ hyh- $\quad 9820$ \\
\hline $\mathfrak{E}^{3} 1-\quad 9710,9720$ & $\mathfrak{f}^{3}$ wsnhk- $\quad 10090$ & $£^{3}$ hyhqv-* \\
\hline $\mathfrak{f}^{3} 1-\quad 9730,9740$ & $\mathfrak{E}^{3}$ wskv- 10100 & $\mathfrak{f}^{3} \mathrm{hy}^{3}-\quad 9820,10390$ \\
\hline$£^{3} 1^{3} \chi-\quad 9750$ & $\left.\mathfrak{E}^{3} w\right\}_{-} \quad 10110,10120$ & 10400 \\
\hline $\mathfrak{£}^{3} \mathrm{k}-\quad 9760,9780,9790$ & $\mathfrak{f}^{3} \mathrm{wk}^{3} \mathrm{v}-10130$ & $£^{3} \mathrm{hkv}-$ \\
\hline $\mathfrak{E}^{3} \mathrm{x}-\quad 9800$ & $\mathfrak{f}^{3} \mathrm{wk}^{3} \mathrm{vp}-10060$ & $\mathfrak{f}^{3} \mathrm{hxv}-\quad 10020,10420$ \\
\hline $\mathfrak{f}^{3} \mathrm{y}-\quad 9810,9820$ & $\mathfrak{£}^{3} \mathrm{wk}^{3} \mathrm{vm}-\quad 10060$ & $\mathfrak{f}^{3} \mathrm{hwq}^{3} \mathrm{v}-\quad 10421$ \\
\hline$£^{3} y p-\quad 9830$ & $\mathfrak{£}^{3} \mathrm{wqv}-\quad 10140,10150$ & $£^{3}$ hqv- 10430 \\
\hline$£^{3} \mathrm{yn}^{3} \mathrm{~h}-\quad 9840,9850$ & $\mathfrak{£}^{3} \mathrm{w} \chi \mathrm{V}^{-} \quad 10151,10160$ & $\mathfrak{£}^{3} \mathrm{~h} \chi \mathrm{v}-\quad 10440$ \\
\hline $\mathfrak{f}^{3} \mathrm{yc}-\quad 9860$ & $\mathfrak{E}^{3} w \chi v £^{3} w \chi v-10161$ & $£^{3} \mathrm{hq}-\quad 10450,10460$ \\
\hline$£^{3}$ ys- $\quad 9860,9880,9890$ & $\mathfrak{£}^{3} \mathrm{w} \chi \mathrm{v}^{3} \chi \mathrm{V}^{*} * \quad 10161$ & $\mathfrak{f}^{3} \mathrm{~h} \chi-\quad 10210,10230$ \\
\hline$£^{3}$ yst- $\quad 9891$ & $\mathfrak{E}^{3} \mathrm{~W}^{3} \mathrm{xV} \bullet-\quad 10170$ & 10480 \\
\hline$£^{3} y £-\quad 9900$ & $£^{3} \mathrm{qv}^{-} \quad 10180,10910$ & $\mathfrak{f}^{3} \mathrm{~h}^{3} \mathrm{WXV}-\quad 10481$ \\
\hline$£^{3} y x-9910$ & $£^{3} \chi \mathrm{V}^{-} \quad 10190$ & $\mathfrak{f}^{3} \mathrm{~h}^{3} \mathrm{hnys}^{*} * \quad 10301$ \\
\hline$£^{3} \mathrm{yx}^{\bullet-} \quad 8590$ & $\mathfrak{f}^{3} \mathrm{q}^{-} \quad 10200$ & $\mathfrak{f}^{3} h^{3} h £^{3} y-\quad 10482$ \\
\hline$£^{3} y k v-\quad 9920,9960$ & $\mathfrak{E}^{3} \chi^{-} \quad 10210,10220$ & \\
\hline$£^{3} y x v-9960$ & 10230 & $\mathbf{1}$ \\
\hline$£^{3}$ ywhx-* 9961 & $£^{3 h}-\quad 10240,10250$ & \\
\hline $\mathfrak{f}^{3}$ yq- $\quad 9970,9980$ & $\mathfrak{f}^{3}$ hbht- $\quad 10280$ & tp- 10490 \\
\hline$£^{3} y \chi-\quad 10000$ & $£^{3}$ hp-* 10290 & $\mathrm{~lm}-\quad 10500,10510$ \\
\hline$£^{3} y^{3}-9820$ & $£^{3}$ ht- $\quad 9610,10300$ & łmp- $\quad 10530$ \\
\hline $\mathfrak{E}^{3} y^{3} \chi-\quad 10000$ & $\mathfrak{£}^{3}$ hnys-* 10301 & łms- $\quad 10540$ \\
\hline $\mathfrak{f}^{3} \mathrm{kv}-\quad 10010$ & $\mathfrak{f}^{3} \mathrm{hs}-\quad 10310,10320$ & łmqv- 10550 \\
\hline $\mathfrak{f}^{3} \mathrm{XV}-\quad 10020$ & 10350 & łmq- $\quad 10560$ \\
\hline $\mathfrak{f}^{3} \mathrm{wp}-\quad 10030$ & $£^{3} h$ hh- $\quad 10350$ & $\operatorname{lm} \chi-\quad 10570$ \\
\hline $\mathfrak{f}^{3}$ wp•- $\quad 10040$ & $\mathfrak{f}^{3} \mathrm{hs} \bullet-10350$ & tt- 10580 \\
\hline$£^{3} w p^{3} k-10060$ & $£^{3} h \nmid-\quad 10360$ & łn- $\quad 10590,10600$ \\
\hline $\mathfrak{f}^{3} \mathrm{wp}^{3} \mathrm{kv}-\quad 10060$ & $£^{3} \mathrm{hk}-\quad 9760,10361$ & lnp- $\quad 10610$ \\
\hline $\mathfrak{f}^{3} \mathrm{wm}^{3-} \quad 10030$ & $\mathfrak{f}^{3} \mathrm{hx}-\quad 10370$ & Int- $\quad 10620,10630$ \\
\hline $\mathfrak{f}^{3} \mathrm{wt}-\quad 10070$ & $£^{3}$ hykm- 10371 & lns- $\quad 11011$ \\
\hline $\mathfrak{£}^{3} \mathrm{wc}-\quad 10071$ & $£^{3}$ hyqv- 10372 & lnxv- $\quad 10650$ \\
\hline
\end{tabular}


North Wakashan Comparative Root List (Updated)

\begin{tabular}{|c|c|c|}
\hline łnq- $\quad 8980,10660$ & thp- $\quad 10980$ & lyk-* 11190 \\
\hline $\ln \chi-\quad 10670$ & $\mathrm{thm}^{3}-10500$ & lyx- $\quad 11170,11200$ \\
\hline łs-* 10680 & tht- $\quad 10980,11010$ & 11210 \\
\hline łl- $\quad 10600,10690$ & tht $^{3}-\quad 11010$ & lyxv- 11220 \\
\hline $11^{3}-\quad 10690$ & łhnc- 11011 & lyqv- $\quad 11230,11231$ \\
\hline tx-* 10700 & ths- 11012 & lyq- $\quad 11240,11250$ \\
\hline łxs- 9090 & tht- 11013 & lyx- 11260 \\
\hline ły- 10710 & thl ${ }^{3}-10690$ & lkv- 11270 \\
\hline łym $^{3}-* \quad 10720$ & thk- 11020 & lxv- 11270 \\
\hline lyt- 10730 & thx- $\quad 11030$ & $1 w-\quad 11300,11310$, \\
\hline łync- 11011 & thxv- 11050 & 11320 \\
\hline łyc- 10740 & łhws- 11012 & lwp- 11330 \\
\hline łys- $\quad 10740,10760$ & thwhs- 11012 & $\operatorname{lwm}^{3}-\quad 11340$ \\
\hline łyk- 11020 & thqv- 10910 & lwt- 11350 \\
\hline łyx- $\quad 9110,9120,10770$ & thq- 10920,11060 & lws- 11360 \\
\hline łyxv- 10780 & th $\chi-\quad 11070,11080$ & lwt- 11370 \\
\hline łyq- $\quad 10770$ & & lwqv- $\quad 11380,11390$ \\
\hline łyx- 10770 & $\mathbf{l}$ & lw $\chi v-\quad 11400$ \\
\hline łkv- $\quad 10800,10810$ & & $1 w^{3} \chi v-\quad 11410$ \\
\hline 10820 & th $^{3} \mathrm{hq}^{-} \quad 11060$ & lqv- 11420 \\
\hline łxv- 10830,10840 & $1 p-* \quad 4160$ & $1 \chi \mathrm{v}-\quad 11410$ \\
\hline łwp- 10530 & lmxv- 11090 & lrw- 11421 \\
\hline łwm $\infty-* \quad 10850$ & $\operatorname{lm} \chi v^{-} \quad 11100$ & $1 \chi-\quad 11430,11440$ \\
\hline łwt- $\quad 10870$ & $\operatorname{lm} \chi-\quad 11110$ & lh- 11450 \\
\hline łwxv- 10880 & ls- $\quad 11120$ & lhxv- 11460 \\
\hline łwqv- $\quad 10890,10900$ & lk- $\quad 11130,11140$ & $\mathrm{lhq}^{3} \mathrm{vt}-\quad 11461$ \\
\hline $\mathrm{lw}^{3} \mathrm{t}-\quad 10870$ & ly- 11450 & $\operatorname{lh} \chi \mathrm{v}-\quad 11470$ \\
\hline łqv- 10910 & lyp- 11150 & lh $\chi-\quad 11490$ \\
\hline łq- $\quad 10920,10930$ & lyt- 11160 & \\
\hline $1 \chi-\quad 10940$ & lynoo- 11170 & \\
\hline th- 10970 & lys- 11180 & \\
\hline
\end{tabular}


North Wakashan Comparative Root List (Updated)

\begin{tabular}{|c|c|c|}
\hline $\mathbf{1}^{3}$ & gnw ${ }^{3} \mathrm{~h}-\quad 11710$ & gwnœ- 11710 \\
\hline & gn $\chi \mathrm{V}-\quad 11720,11730$ & gws- 11980 \\
\hline $1^{3} \mathrm{p}-\quad 1410$ & $\mathrm{gn}^{3} \mathrm{c}-\quad 11680$ & gwł- 11990 \\
\hline $1^{3} y x-\quad 11500$ & gł- $\quad 11740$ & $\mathrm{gwl}^{3-} \quad 11991$ \\
\hline $1^{3} \chi \mathrm{vw}-\quad 11510$ & gl- $\quad 11750,11760$ & gwys- 11980 \\
\hline $1^{3} \mathrm{q}-\quad 11520$ & glt- 11780 & gwy3hlh- 11891 \\
\hline lih $^{3} \quad 11530$ & glx- $\quad 11790$ & 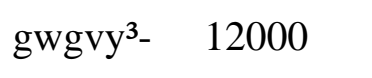 \\
\hline $1^{3 h}$ - $\quad 11540$ & glxv-* 11800 & gwgvy $^{3} \mathrm{w}-\quad 12000$ \\
\hline 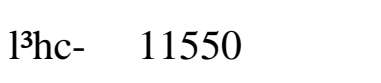 & $\mathrm{glw}^{3-} \quad 11800$ & gwkv- 12010 \\
\hline l3hqv- 11560 & gl $\chi v-11730$ & gwXv-* 12010 \\
\hline $1^{3} \mathrm{~h} \chi \mathrm{v}-\quad 2100,11560$ & gl $\chi-\quad 11730$ & $\mathrm{gw}^{3-} \quad 12030$ \\
\hline & glh- $\quad 11820$ & $\mathrm{gw}^{3} f-\quad 12040$ \\
\hline g & $\mathrm{gl}^{3} \chi-\quad 11730$ & $\mathrm{~g} \chi \mathrm{v}-\quad 11911,12050$ \\
\hline & gy- $\quad 11830,11850$ & $\mathrm{~g} \chi-\quad 12070$ \\
\hline g- $\quad 11570$ & gyc- $\quad 11870$ & gh- 11830,12080, \\
\hline gp- 11580 & gys- $\quad 11850,11860$, & 12090 \\
\hline gm- $\quad 11590$ & 11870 & 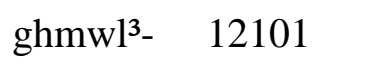 \\
\hline gmt- $\quad 11600,11610$ & gy£- $\quad 11880$ & ghmh- 12100 \\
\hline gmxv- 11620 & gyg- 11890 & ghd- 12110 \\
\hline $\operatorname{gd} \chi v-\quad 11630$ & gyk- $\quad 11890$ & ghlh- 12091 \\
\hline gt- $\quad 11640,11650$ & gyx- 11850 & ghlø- 12091 \\
\hline gn- $\quad 11660,11670$ & gyw3hlh- $\quad 11891,11900$ & $\mathrm{ghl}^{3-} \quad 11991,12130$ \\
\hline 11690 & gyy $^{3}-\quad 11850$ & ghy- 12092 \\
\hline gnc- 11680 & gy $\chi v-\quad 11910,11911$ & ghyh- 12092 \\
\hline gnch- 11690 & gyq- $\quad 11920$ & ghy ${ }^{3}-11850$ \\
\hline gnc•- 11690 & gy $\chi-11930$ & ghwyq- 12140 \\
\hline gns- 11690 & $\mathrm{gy}^{3}-\quad 11830,11850$ & ghwhq- 12140 \\
\hline gnt- 11670 & gy $^{3} \mathrm{~s}-\quad 11850$ & $\operatorname{gh} \chi-\quad 12093$ \\
\hline gnłh- 11660 & gw- $\quad 11931$ & gh $\chi^{\bullet-} \quad 12093$ \\
\hline gnw $^{3-} \quad 11710$ & $\begin{array}{l}\text { gwmh- } 11940 \\
\text { gwt- } 11950\end{array}$ & ghhlh- \\
\hline
\end{tabular}




\section{North Wakashan Comparative Root List (Updated)}

\begin{tabular}{|c|c|c|}
\hline $\mathbf{k}$ & kyxv- 12430 & $\operatorname{kh} \chi-\quad 12570$ \\
\hline & ky $\chi-\quad 12440$ & \\
\hline kp- $\quad 11580,12150$ & kxv- $\quad 12450$ & $\mathbf{k}^{3}$ \\
\hline km-* 12151 & kwp- 12460 & \\
\hline kmt- $\quad 12160$ & kwt- 12470 & $\mathrm{k}^{3} \mathrm{p}-\quad 12740$ \\
\hline kms-* 12170 & kws- $\quad 12480,12490$ & $\mathrm{k}^{3} \mathrm{~m}-\quad 12750$ \\
\hline kmk-* 12151 & kwt- $\quad 12500$ & $\mathrm{k}^{3} \mathrm{mt}-\quad 12760,12770$ \\
\hline kmx-* 12151 & kwkv- 12510 & $\mathrm{k}^{3} \mathrm{~ms}-\quad 12780,12790$ \\
\hline $\mathrm{kmy}^{3}-\quad 12170$ & kwxv- 12520 & $\mathrm{k}^{3} \mathrm{~m} £-\quad 12800$ \\
\hline kmq- $\quad 12190,12200$ & kwqv- 12460 & $\mathrm{k}^{3} \mathrm{my}^{3-} \quad 12760$ \\
\hline kdh- $\quad 12220$ & $\mathrm{kw} \chi \mathrm{v}-\quad 12530,12540$ & $\mathrm{k}^{3} \mathrm{mxv}-\quad 12810$ \\
\hline kt- $\quad 12230$ & $\mathrm{kw}^{3} \mathrm{kv}-\quad 12520$ & $\mathrm{k}^{3} \mathrm{mq}-\quad 12820$ \\
\hline kn- $\quad 12240$ & $\mathrm{k} \chi \mathrm{V}-\quad 12550$ & $\mathrm{k}^{3} \mathrm{~m} \chi-\quad 12830$ \\
\hline knc- $\quad 12250$ & kq- $\quad 12560$ & $\mathrm{k}^{3} \mathrm{mhq}-\quad 12820$ \\
\hline kns-* 12250 & $\mathrm{k} \chi-\quad 12570$ & $\mathrm{k}^{3} \mathrm{mh} \chi-^{*} \quad 12820$ \\
\hline knk- $\quad 12260$ & kh- 12580,12590 , & $\mathrm{k}^{3 \mathrm{t}-} \quad 12840,12860$ \\
\hline knx- $\quad 12290$ & 12591 & $\mathrm{k}^{3} \mathrm{n}-\quad 12880,12890$ \\
\hline knq- $\quad 12310$ & khp- $\quad 12600$ & $\mathrm{k}^{3} \mathrm{nt}-* \quad 12900$ \\
\hline $\mathrm{kn}^{3}-\quad 12240$ & kht- 12610,12620 , & $\mathrm{k}^{3} \mathrm{nl}-* \quad 12900$ \\
\hline ks- $\quad 12330$ & 12630 & $k^{3} n k-\quad 12930$ \\
\hline $\mathrm{ksp}^{3-} \quad 12340$ & khnh- 12240 & $\mathrm{k}^{3}$ nyl-* $\quad 12900$ \\
\hline $\mathrm{kf-} \quad 12350,12360$ & $\mathrm{khn}^{3}-12240$ & $\mathrm{k}^{3} \mathrm{nkv-} \quad 12940$ \\
\hline k1- $\quad 12370$ & $\mathrm{khc}^{3} \mathrm{~s}-\quad 12640$ & $\mathrm{k}^{3} \mathrm{nxv}-* \quad 12900,12940$ \\
\hline klx- $\quad 11790,12380$ & khs- $\quad 12620,12650$, & $\mathrm{k}^{3}$ nwt- $\quad 12900$ \\
\hline klxv- 12390 & 12660 & $\mathrm{k}^{3} \mathrm{nq}-\quad 12960$ \\
\hline $\mathrm{kl} \chi \mathbf{-}^{*} \quad 12400$ & khk- $\quad 6360$ & $\mathrm{k}^{3} \mathrm{nh}-\quad 12890$ \\
\hline klhkv- 12410 & khx- 12690 & $\mathrm{k}^{3} \mathrm{~s}-\quad 12970$ \\
\hline kyc- $\quad 11870$ & khy $^{3}-\quad 12700$ & $\mathrm{k}^{3} \mathrm{~s}^{\bullet}-\quad 12970$ \\
\hline kys- $\quad 11870,12330$ & khwt- 12710 & $\mathrm{k}^{3 \mathfrak{f}-}-12990,12991$ \\
\hline kyf- $\quad 12420$ & khwht- 12710 & $\mathrm{k}^{31-}-\quad 13000,13010$ \\
\hline kykhł- 12370 & khq- $\quad 12560$ & $\mathrm{k}^{3} 1-\quad 13010,13020$ \\
\hline
\end{tabular}


North Wakashan Comparative Root List (Updated)

\begin{tabular}{|c|c|c|}
\hline $\mathrm{k}^{3} \mathrm{lp}-\quad 13030$ & $\mathrm{k}^{3} \mathrm{y} \chi \mathrm{v}-\quad 13350$ & $\mathrm{k}^{3} \mathrm{hmh}-\quad 12750$ \\
\hline $\mathrm{k}^{3} \mathrm{lt}-\quad 13040$ & $\mathrm{k}^{3} \mathrm{yq}-13360$ & $\mathrm{k}^{3} \mathrm{hmhs}-\quad 12790$ \\
\hline $\mathrm{k}^{3} \mathrm{ls}-\quad 13050,13060$ & $\mathrm{k}^{3} \mathrm{y} \chi^{-} \quad 12440,13370$ & $\mathrm{k}^{3} \mathrm{ht}-\quad 12840,13630$ \\
\hline $\mathrm{k}^{3} \mathrm{lk}-\quad 13070$ & $\mathrm{k}^{3} \mathrm{yhw}-\quad 13341$ & 13640 \\
\hline $\mathrm{k}^{3} \mathrm{~lx}-\quad 13080$ & $\mathrm{k}^{3}$ yhws- $\quad 13260$ & $\mathrm{k}^{3} \mathrm{hs}-\quad 13650,13660$ \\
\hline $\mathrm{k}^{3} 1 \mathrm{kv}-\quad 13090$ & $\mathrm{k}^{3} \mathrm{y}^{3}-\quad 13371$ & $\mathrm{k}^{3} \mathrm{~h} £-* \quad 13670$ \\
\hline $\mathrm{k}^{3} \mathrm{lqv}-\quad 13100$ & $k^{3} y^{3} s-13260$ & $\mathrm{k}^{3} \mathrm{~h} h-\quad 13680,13690$ \\
\hline $\mathrm{k}^{3}$ lq- $\quad 13110$ & $\mathrm{k}^{3} \mathrm{y}^{3} \mathrm{~s}^{\bullet-} \quad 13260$ & 13700 \\
\hline $\mathrm{k}^{3} 1 \chi-\quad 13120,13130$ & $\mathrm{k}^{3} \mathrm{xv}-\quad 13380$ & $\mathrm{k}^{3} \mathrm{hk}-\quad 13710$ \\
\hline 13140 & $\mathrm{k}^{3} \mathrm{~W}-\quad 13390,13400$ & $\mathrm{k}^{3} \mathrm{hyw}-13341$ \\
\hline $\mathrm{k}^{3} \mathrm{lhm}-\quad 13021$ & $\mathrm{k}^{3} \mathrm{wp}-\quad 13410$ & $\mathrm{k}^{3}$ hyws $\quad 13260$ \\
\hline $\mathrm{k}^{3} 1^{3} \mathrm{x}-\quad 13080$ & $\mathrm{k}^{3} \mathrm{wm}^{3-} \quad 15900$ & $k^{3}$ hyh- $\quad 13200,13210$ \\
\hline $\mathrm{k}^{3} \mathrm{k}-\quad 13150,13160$ & $\mathrm{k}^{3} \mathrm{wt}-\quad 13420,13430$ & $\mathrm{k}^{3} \mathrm{hy}^{3}-\quad 3780,13200$ \\
\hline 13170 & 13440,13450 & $\mathrm{k}^{3} \mathrm{hy}^{3} \mathrm{~h} \chi \mathrm{v}-* \quad 13730$ \\
\hline $\mathrm{k}^{3} \mathrm{x}-\quad 13180,13190$ & $\mathrm{k}^{3} \mathrm{wc}-\quad 13470$ & $\mathrm{k}^{3} \mathrm{hwh}-\quad 13341$ \\
\hline $\mathrm{k}^{3} \mathrm{y}-\quad 13200,13210$ & $\mathrm{k}^{3} \mathrm{ws}-\quad 13460,13470$ & $\mathrm{k}^{3} \mathrm{~h} w h \chi \mathrm{V}-\quad 13720$ \\
\hline $\mathrm{k}^{3} \mathrm{yp}-\quad 6970$ & $\mathrm{k}^{3} \mathrm{w} £-\quad 13480$ & $\mathrm{k}^{3} \mathrm{~h} \chi \mathrm{v}-\quad 13720$ \\
\hline$k^{3} y_{m h}-\quad 12750$ & $\left.\mathrm{k}^{3} \mathrm{w}\right\}-\quad 13490,13500$ & $\mathrm{k}^{3} \mathrm{hq}-\quad 12560,13750$ \\
\hline $\mathrm{k}^{3} \mathrm{yt}-\quad 13230,13240$ & $\mathrm{k}^{3} \mathrm{wW}-\quad 13341$ & $\mathrm{k}^{3} \mathrm{~h} \chi-\quad 12570$ \\
\hline 13250 & $\mathrm{k}^{3} \mathrm{wkv}-\quad 13510$ & $\mathrm{k}^{3} \mathrm{~h}^{3} \mathrm{~s}-\quad 13660$ \\
\hline$k^{3} y^{3}-\quad 13260,13290$ & $\mathrm{k}^{3} \mathrm{Wxv}-\quad 13520$ & $\mathrm{k}^{3} \mathrm{~h}^{3} k_{-} \quad 13760$ \\
\hline $\mathrm{k}^{3} \mathrm{y} £-\quad 13300$ & $\mathrm{k}^{3} \mathrm{Wws}-13260$ & $\mathrm{k}^{3} \mathrm{~h}^{3} \mathrm{x}-\quad 13770,13780$ \\
\hline $\mathrm{k}^{3} \mathrm{y} 1-\quad 13310,13320$ & $\mathrm{k}^{3} \mathrm{wqv}-\quad 13530$ & $\mathrm{k}^{3} \mathrm{~h}^{3} \mathrm{qv}-\quad 13550$ \\
\hline 13330 & $\mathrm{k}^{3} \mathrm{w} \chi \mathrm{v}-\quad 13540$ & $\mathrm{k}^{3} \mathrm{~h}^{3} \mathrm{qw}-13790$ \\
\hline $\mathrm{k}^{3} \mathrm{yl}^{3-}-13320$ & $\mathrm{k}^{3} \mathrm{~W}^{3} \mathrm{~s}-\quad 13470$ & \\
\hline $\mathrm{k}^{3} \mathrm{y} \mathbf{l}^{3} \mathrm{~h}-\quad 13320$ & $\mathrm{k}^{3} \mathrm{qv}-\quad 13550$ & $\mathbf{x}$ \\
\hline$k^{3}$ ygh- 13340 & $\mathrm{k}^{3} \mathrm{q}^{-} \quad 13560$ & \\
\hline$k^{3} y k-13340$ & $\mathrm{k}^{3} \chi-\quad 13570$ & xmt- $\quad 13810$ \\
\hline$k^{3} y^{3}-13200$ & $\mathrm{k}^{3} \mathrm{~h}-\quad 13580,13590$ & xms- $\quad 13820,13830$ \\
\hline $\mathrm{k}^{3} \mathrm{yw}-13341$ & 13600 & 13840 \\
\hline$k^{3}$ yws- 13260 & $\mathrm{k}^{3} \mathrm{hp}-\quad 13610,13620$ & $\mathrm{xm}^{3}-\quad 13841$ \\
\hline
\end{tabular}




\section{North Wakashan Comparative Root List (Updated)}

\begin{tabular}{|c|c|c|}
\hline xt- 13850 & xws- $\quad 14220,14230$ & yl $\chi-\quad 14480$ \\
\hline xnt- $\quad 13860,13880$ & 14240,14250 & yk- 14490,14500 \\
\hline 13890 & xw£-* 14260 & $y x-14510,14520$ \\
\hline 13890 & xw1- 14270 & yxh- 14510 \\
\hline $\mathrm{xnk}^{3-*} 13900$ & xwkv- $\quad 14280,14281$ & $y x^{\bullet-} \quad 14510$ \\
\hline xnqv- 13910 & xwqv-* 14290 & yy- 14380 \\
\hline xnq- 13920 & xwq-* 14290 & $y k v-\quad 14530,14550$ \\
\hline$x_{z X}-\quad 13940,13950$ & xqv- 14300 & yxv- $\quad 14550,14560$ \\
\hline xs- $\quad 13950,13970$ & $\mathrm{xhm}^{3}{ }_{-} * \quad 14310$ & 14570 \\
\hline$x £-\quad 13980$ & xht- 14320 & yw- $\quad 14580,14581$ \\
\hline xł- 13990 & $\mathrm{xhc}^{3-} \quad 13950$ & ywd- 14590 \\
\hline 14000,14020 & xhs- 13950 & ywt- 14600 \\
\hline 14030,14040 & xht- 14130 & ywnwqv- 4940 \\
\hline xlt- 14050 & xhlh- $\quad 14340$ & yws- 14610 \\
\hline xls-* 14060 & xhk- 14350 & yw£-* 14620 \\
\hline xlkv- 14070 & xhxv-* 14360 & ywt- 14600 \\
\hline xlq- $\quad 14080$ & $\mathrm{xhw}^{3}-\quad 14370$ & ywlh- 14630 \\
\hline $\mathrm{xk}^{3}-14090$ & & ywlœ-* 14630 \\
\hline xyt- 14100 & $\mathbf{y}$ & ywkv- 14640 \\
\hline xyc- 14110 & & yqv- $\quad 14660,14661$ \\
\hline $\mathrm{xyc}^{3} \mathrm{hx}-\quad 14111$ & $y-\quad 14380$ & $y \chi v-\quad 14670$ \\
\hline 14110 & yp- 14390 & yq- $\quad 14661,14680$ \\
\hline xył- 14120 & yn- 14400 & 14690 \\
\hline$x y^{3}-\quad 14130$ & ynt-* 14410 & yh- $\quad 14380,14700$ \\
\hline xyk-* 14140 & ynk- $\quad 4360,4720$ & 14701 \\
\hline 14110,14150 & $\mathrm{ynk}^{3}-\quad 4720$ & yhp- 14710 \\
\hline 14110 & ys- $\quad 14420,14421$ & yhmh- 14702 \\
\hline xkv-* 14160 & $y £-\quad 14430$ & yht- 14720,14730 , \\
\hline xwp- $\quad 14170,14180$ & yls- 14440 & 14740 \\
\hline$x_{w m}^{3} s-\quad 14190$ & yl $\chi v-* \quad 14460$ & 14750 \\
\hline xwt-* 14200 & ylq-* 14480 & yhs $\infty$ kv- 14760 \\
\hline
\end{tabular}


North Wakashan Comparative Root List (Updated)

\begin{tabular}{|c|c|c|}
\hline yh£- 14780 & $\mathrm{y}^{3} \mathrm{ys}-15040$ & gvlt- 15310 \\
\hline yh1- 14790,14800 , & $\mathrm{y}^{3} \mathrm{yxh}-\quad 15050$ & gvls- 15301 \\
\hline 14810 & $\mathrm{y}^{3} \mathrm{yx}^{\bullet}-\quad 15050$ & gvlkv- $\quad 15330,15340$ \\
\hline $\mathrm{yhHk}^{3}-* \quad 14820$ & $\mathrm{y}^{3} \mathrm{yq}-15060$ & gvx- 15250 \\
\hline yhx- 14510 & $\mathrm{y}^{3} \mathrm{y} \chi-\quad 15070$ & gvy- $\quad 11931,15350$ \\
\hline yhxk $^{3}-\quad 14820$ & $\mathrm{y}^{3} \mathrm{wmh}-15080$ & gvyt- 15360 \\
\hline yhx- 14830 & $\mathrm{y}^{3} \mathrm{wk}-* \quad 14940$ & gvyn $^{3}$ p- $\quad 15260$ \\
\hline yhxv- 14570 & $\mathrm{y}^{3} \mathrm{wgv}-\quad 15100$ & gvyc-* 15370 \\
\hline yhwhs- 14840 & $\mathrm{y}^{3} \mathrm{wgvh}-\quad 15100$ & gvys*- 15370 \\
\hline $\mathrm{yhw}^{3} \mathrm{yx}-14850$ & $y^{3} \chi^{v-} \quad 15110$ & gvył- 15380 \\
\hline yhqv- $\quad 14860,14870$, & $y^{3} \mathrm{~h}-\quad 14380$ & gvwmh- 11940 \\
\hline 14880,14890 & $\mathrm{y}^{3} \mathrm{hp}-\quad 14941$ & gvwt- 15390 \\
\hline yh $\chi v^{-} * * 14900$ & $\mathrm{y}^{3} \mathrm{hs}-* \quad 15130$ & gvws- 11980 \\
\hline yhrhł- 14910 & $\mathrm{y}^{3} \mathrm{~h} £-\quad 14780$ & gvw1- 11990 \\
\hline yhq- $\quad 14690,14920$ & $\mathrm{y}^{3} \mathrm{~h}$ lhq- $\quad 15150$ & gvwgvy3- 12000 \\
\hline yh $\chi-\quad 14690,14930$ & $\mathrm{y}^{3} \mathrm{hk}-\quad 15160$ & gvwkv- 12010 \\
\hline & $y^{3} h x v-\quad 15160$ & gvq- $\quad 15400$ \\
\hline $\mathbf{y}^{\mathbf{3}}$ & $\mathrm{y}^{3} \mathrm{hr}-\mathrm{e} \quad 15170$ & gvh- $\quad 15410,15420$ \\
\hline & & 15470 \\
\hline $\mathrm{y}^{3} \mathrm{mt}-\quad 14940$ & gv & gvhnh- 15430 \\
\hline$y^{3} \mathrm{mk}-\quad 14941$ & & gvhn $^{3}-\quad 15240,15430$ \\
\hline$y^{3} m x-\quad 14941$ & gvd- $\quad 15200,15201$ & gvhs- $\quad 15280,15460$, \\
\hline $\mathrm{y}^{3} \mathrm{mqv}-* \quad 14950$ & gvt- $\quad 15210,15230$ & 115470 \\
\hline $\mathrm{y}^{3} \mathrm{mq}^{*} * 14960$ & gvn- $\quad 15240,15250$ & gvhsh- 15470 \\
\hline$y^{3}$ nk- $\quad 4720$ & gvnp- $\quad 15260$ & gvhs•- 15470 \\
\hline$y^{3} 1-\quad 14970$ & gvnyp- 15260 & gvhk- 15420 \\
\hline $\mathrm{y}^{3} \mid x-\quad 14980$ & gvn $\chi^{*} * 15270$ & gvhx- 15420 \\
\hline $\mathrm{y}^{3} \mathrm{lkv}-* \quad 14990$ & gvs- $\quad 15280$ & gvhkv- 15490 \\
\hline$y^{3} x-\quad 15000$ & gv£- 15290 & gvhxv- 15500 \\
\hline$y^{3} y-\quad 4780,15010,15020$ & gvł- $\quad 15300$ & gvhq $^{31-} \quad 15510$ \\
\hline $\mathrm{y}^{3} \mathrm{yt}-15030$ & gvl- $\quad 15301$ & \\
\hline
\end{tabular}


North Wakashan Comparative Root List (Updated)

\begin{tabular}{|c|c|c|c|}
\hline & kv & kvyq- 15760 & $\mathrm{k}^{3} \mathrm{vml}-\quad 15950,15980$ \\
\hline & & kvyx- 15770 & $\mathrm{k}^{3} \mathrm{vml}^{3-} \quad 15950$ \\
\hline kvm- & 15520 & kvxv- 12450 & $\mathrm{k}^{3}$ vmws- $\quad 15940$ \\
\hline kvmt- & 15530 & kvwp- 12460 & $\mathrm{k}^{3} \mathrm{vm}^{3-} \quad 15900$ \\
\hline kvmc- & 15540 & kvwmyt- $\quad 15771$ & $\mathrm{k}^{3} \mathrm{vt}-\quad 15990,16000$ \\
\hline kvmt- & 15550 & kvwt- 12470 & 16010 \\
\hline kvmx- & 15550 & kvw£- 15780 & $\mathrm{k}^{3} \mathrm{vn}-\quad 16020$ \\
\hline kvdkv- & 15560 & kvwł- 15781 & $\mathrm{k}^{3} \mathrm{vnc}-\quad 16030$ \\
\hline kvt- & 15210,15570 & kvwkv- 12510 & $\mathrm{k}^{3} \mathrm{vnl}-\quad 16040$ \\
\hline kvn- & 15580 & kvwxv- 12520 & $\mathrm{k}^{3} \mathrm{vnxv}-\quad 16050$ \\
\hline kvnc- & 15590 & $\mathrm{kv} \chi \mathrm{v}-\quad 12550$ & $\mathrm{k}^{3} \mathrm{vnq}-\quad 16060$ \\
\hline kvns- & 15590 & kvq- $\quad 15800$ & $\mathrm{k}^{3} \mathrm{vc}-\quad 16070$ \\
\hline kvnxv- & 15600 & kv $\chi-\quad 12440$ & $\mathrm{k}^{3} \mathrm{vs}-\quad 15650,16070$ \\
\hline kvnq- & 15610 & kvhdkv- 15560 & 16080, 16090, 16091, \\
\hline $\mathrm{kvn}^{3} \mathrm{q}-$ & 15610 & $\mathrm{kvhn}^{3-} \quad 15580$ & 16100 \\
\hline $\operatorname{kvn}^{3} \chi-$ & 15610 & kvhs- 15810 & $\mathrm{k}^{3} \mathrm{vl}-\quad 16110,16120$ \\
\hline kvs- & 15630,15650 & kvh£- $\quad 15820$ & $\mathrm{k}^{3} \mathrm{vlp}-\quad 16150,16160$ \\
\hline kvsx- & 15630 & kvhx- 15830 & $\mathrm{k}^{3}$ vlt- $\quad 16170$ \\
\hline kvsxh- & 15630 & kvhkv- 15840 & $\mathrm{k}^{3}$ vls- $\quad 16180$ \\
\hline $\operatorname{kvsx} \bullet-$ & 15630 & kvhxv- 15850 & $\mathrm{k}^{3} \mathrm{v} l \mathrm{xv}-\quad 16190$ \\
\hline $\mathrm{kvf-*}$ & 15660 & kvhq- 15800 & $\mathrm{k}^{3} \mathrm{v} l \chi \mathrm{v}-\quad 16190$ \\
\hline $\mathrm{kv£m-}$ & 15660 & $\mathrm{kvh}^{3} \mathrm{c}-\quad 15860$ & $\mathrm{k}^{3} \mathrm{vlq}-\quad 16200$ \\
\hline $\mathrm{kvf} \bullet-$ & 15660 & $\mathrm{kvh}^{3} \mathrm{~s}-\quad 15860$ & $\mathrm{k}^{3} \mathrm{vl} \chi-\quad 16210$ \\
\hline kvł- & 15670 & & $\mathrm{k}^{3} \mathrm{vl}^{3} \mathrm{k}-\quad 16211$ \\
\hline kvl- & 15580,15680 & $\mathbf{k}^{\mathbf{3}} \mathbf{v}$ & $\mathrm{k}^{3} \mathrm{vl}^{3} \mathrm{xv}-\quad 16190$ \\
\hline kvlxv- & 15690 & & $\mathrm{k}^{3} \mathrm{vl}^{3} \chi \mathrm{v}-16190$ \\
\hline kvk- & 15700,15710 & $\mathrm{k}^{3} \mathrm{vp}-\quad 15870,15890$ & $\mathrm{k}^{3} \mathrm{vk}-\quad 13170$ \\
\hline kvyt- & 15720 & $\mathrm{k}^{3} \mathrm{vm}-\quad 15900,15920$ & $\mathrm{k}^{3} \mathrm{VX}-\quad 16220$ \\
\hline kvys- & 15730 & $\mathrm{k}^{3} \mathrm{vmt}-\quad 15930$ & $\mathrm{k}^{3} \mathrm{vy}-16090$ \\
\hline kvyx- & 15590 & $\mathrm{k}^{3} \mathrm{vms}-\quad 15940,15950$ & $\mathrm{k}^{3} \mathrm{vyt}-16230$ \\
\hline kvykv- & 15750 & $\mathrm{k}^{3} \mathrm{vm} \mathcal{}-\quad 15960,15970$ & $\mathrm{k}^{3}$ vys- $\quad 16240$ \\
\hline
\end{tabular}




\section{North Wakashan Comparative Root List (Updated)}

\begin{tabular}{|c|c|c|}
\hline $\mathrm{k}^{3}$ vył- $\quad 16250$ & $\mathrm{k}^{3} \mathrm{vh}^{3} \mathrm{qv}-\quad 13550,16450$ & xvwp- $\quad 14170,14180$ \\
\hline $\mathrm{k}^{3} \mathrm{vykv-} \quad 16260$ & $\mathrm{k}^{3} \mathrm{vh}^{3} \mathrm{q}-\quad 16440$ & xvwmyt- 15771 \\
\hline $\mathrm{k}^{3} \mathrm{vyxv-} \quad 16270$ & & xvwt- 12470 \\
\hline $\mathrm{k}^{3} \mathrm{vy} \chi \mathrm{v}-\quad 16280$ & $\mathbf{X V}$ & xvws- $\quad 14230,14240$ \\
\hline $\mathrm{k}^{3} \mathrm{vyq}-\quad 16290,16300$ & & xVwł- $\quad 14270$ \\
\hline $\mathrm{k}^{3} \mathrm{vy} \chi-$ & xvp- 16460 & xvwkv- 14280 \\
\hline $\mathrm{k}^{3} \mathrm{VXV}-\quad 13380$ & xvm- 16461 & xvh- 16660 \\
\hline $\mathrm{k}^{3} \mathrm{VW}-\quad 13390,13400$ & xvmt- $\quad 15310,16470$ & $\mathrm{xvhm}^{3}-\quad 16461$ \\
\hline $\mathrm{k}^{3} \mathrm{vwp}-\quad 13410$ & xvmc- 16480 & xvht- 16670 \\
\hline $\mathrm{k}^{3} \mathrm{Vwt}-\quad 13450$ & xvms- 16480 & xvhs- $\quad 16520,16680$ \\
\hline $\mathrm{k}^{3} \mathrm{VWS}-$ & xvmqv- 16490 & xvhł- 16690 \\
\hline $\mathrm{k}^{3}$ Vw1- $\quad 13490$ & xvmq- 16490 & xvhkv- $\quad 16700,16710$ \\
\hline $\mathrm{k}^{3} \mathrm{VwkV}-\quad 13510$ & xvt- 16500 & 16720 \\
\hline $\mathrm{k}^{3} \mathrm{VWXV}-\quad 13520$ & $x t^{3} q-\quad 16501$ & xvhq- $\quad 16730$ \\
\hline $\mathrm{k}^{3} \mathrm{vq}^{-} \quad 16320$ & xvn-* 16502 & $\mathrm{xvh}^{3} \mathrm{~s}-$ \\
\hline $\mathrm{k}^{3} \mathrm{v} \chi-\quad 16290,16330$ & xvn£-* 16503 & \\
\hline $\mathrm{k}^{3} \mathrm{vh}-\quad 16340,16350$ & xvnys- 16510 & $\mathbf{w}$ \\
\hline $\mathrm{k}^{3} \mathrm{vhp}-$ & xvs- $\quad 16520,16540$ & \\
\hline $\mathrm{k}^{3} \mathrm{vhm}^{3}-\quad 15900$ & xvlp- & w- $\quad 17820$ \\
\hline 13640,15990 & 15310 & wp- 16740 \\
\hline 16380 & 16570 & wn- $\quad 16760,16800$ \\
\hline $\mathrm{k}^{3}$ vhs- $\quad 16100,16400$ & 16580 & wnmt- 16790 \\
\hline 16220 & 16590 & wnc- 16810 \\
\hline $\mathrm{k}^{3} \mathrm{vhk}-$ & 16600,16610 & 16810 \\
\hline $\mathrm{k}^{3} \mathrm{vhx}-$ & 16620 & wnł- $\quad 16820,16830$ \\
\hline $\mathrm{k}^{3}$ vhxs- 16100 & xvykv- 16580 & wnk- 16760 \\
\hline$k^{3}$ vhywkv- 16410 & xvyqv- 16580 & wnx- $\quad 16760,16820$ \\
\hline $\mathrm{k}^{3} \mathrm{vhy}^{3}{ }^{3}-\quad 16420$ & 16630,16640 & wny- 16780 \\
\hline $\mathrm{k}^{3} \mathrm{vhxv-} \quad 15850,16430$ & 16650 & wnyqv- 4940 \\
\hline $\mathrm{k}^{3}$ vhwh-* 16431 & $x y^{3} s-$ & wnqv-* \\
\hline $\mathrm{k}^{3} \mathrm{vhq}-\quad 16440$ & $\mathrm{xVw}-*$ & wn $\chi v-\quad 4940,16860$ \\
\hline
\end{tabular}




\section{North Wakashan Comparative Root List (Updated)}

\begin{tabular}{|c|c|c|}
\hline wnq- 16870 & wy£- $\quad 17100,17110$ & wh1- $\quad 17390,17400$, \\
\hline wnq $^{3-} \quad 16871$ & wył- 17120 & 17410 \\
\hline wnhqv- 4940 & wyl $^{3}-\quad 17130$ & whłqy- \\
\hline$w^{3} h 1-\quad 16872$ & wyk- 17131,17140 & whłqys-* 17380 \\
\hline wc- $\quad 16880,16881$ & 17140 & 16960 \\
\hline ws- $\quad 16880,16881$, & wykv- 15750 & whk- $\quad 17420,17430$ \\
\hline $16890,16900,17190$ & wyqv- 17160 & 17440 \\
\hline wsdys- 16920 & 17180 & why- 17041 \\
\hline $\mathrm{w} £-\quad 16930,16940$, & $\mathrm{wy}^{3} \chi-\quad 17181$ & whylyq- 17121 \\
\hline 16941 & ww- $\quad 17041,17220$ & whkv- 17450 \\
\hline w1- $\quad 16941,16950$, & wws- 17190 & whxv- $\quad 17460,17470$ \\
\hline 17400 & Ww£- 17200 & whqv- $\quad 17480,17490$ \\
\hline wl- 16960 & wwł- 17210 & wh $\chi v-17500$ \\
\hline wlt-* 16970 & wwkv- 17430 & 17480 \\
\hline wlk- 16980 & www- 17220 & 17510 \\
\hline wlx- 16980 & wwqv- 17230 & 17820 \\
\hline wly- 16780 & $\mathrm{ww}^{3} \mathrm{kV}-\quad 17430$ & 17181,17520 \\
\hline wlyqv- 16990 & $w^{3} v^{-} \quad 17810$ & \\
\hline wly ${ }^{3} \chi v_{-} * \quad 16990$ & wq- $\quad 17250,17260$, & $\mathbf{w}^{3}$ \\
\hline wlxv- 17000 & 17270,17480 & \\
\hline wl $\chi v-17010$ & $\mathrm{w} \chi-\quad 17290,17300$ & $\mathrm{~W}^{3}-\quad 17820$ \\
\hline wlq- $\quad 16870,17020$, & 17310 & $w^{3} p-\quad 17540$ \\
\hline 17030 & wh- $\quad 17041,17330$ & 17550 \\
\hline wl $\chi^{-*} \quad 17030$ & wht- $\quad 17340,17350$ & $w^{3} n-\quad 17560,17870$ \\
\hline wk- 17040 & whnm- 16790 & 17880 \\
\hline wy- $\quad 17041,17050$ & $w n^{3}-\quad 16800$ & $\mathrm{w}^{3} \mathrm{nk}-\quad 17570$ \\
\hline 17060 & whz-* 17380 & $\mathrm{w}^{3} \mathrm{nxv}^{-*} \quad 17580,17590$ \\
\hline wyp-* 17070 & 17360, 17370, & $\mathrm{w}^{3} \mathrm{nh}-\quad 16872$ \\
\hline wynh- 17080 & 17380 & $w^{3} n^{3} h 1-\quad 16872$ \\
\hline wyn $\infty-\quad 17080$ & 16930 & $w^{3} s-\quad 17600,17610$ \\
\hline wys- 17090 & & $\mathrm{w}^{3} 1-\quad 17620,17630$ \\
\hline
\end{tabular}


North Wakashan Comparative Root List (Updated)

\begin{tabular}{|c|c|c|}
\hline w3lwp-* 17631 & $\mathrm{w}^{3} \mathrm{w}^{3} \mathrm{zh}-\quad 17740$ & \\
\hline$w^{3} x^{3}-17640$ & $\mathrm{w}^{3} \mathrm{qv}-17810$ & IV \\
\hline $\mathrm{w}^{3} \mathrm{xs}-\quad 17600$ & $\mathrm{w}^{3} \mathrm{qvh}-\quad 17810$ & \\
\hline$w^{3} y-\quad 17650$ & $w^{3} q^{3} v-\quad 17810$ & rvms- 17960 \\
\hline w $^{3}$ yp- $\quad 17660$ & $\mathrm{w}^{3} \mathrm{q}^{3} \mathrm{vh}-\quad 17810$ & rvn- $\quad 17970,17980$ \\
\hline$w^{3}$ ynh- $\quad 17560$ & $\mathrm{w}^{3} \mathrm{~h}-\quad 17820,17840$ & 17990,18020 \\
\hline $\mathrm{w}^{3} \mathrm{yn} \infty-\quad 17560$ & $\mathrm{w}^{3} \mathrm{hp}-\quad 17850$ & rvnt- 18000 \\
\hline$w^{3}$ ys- $\quad 17650$ & $\mathrm{w}^{3} \mathrm{hmwt}-2330$ & rvnt $^{3}-18000$ \\
\hline $\mathrm{w}^{3} \mathrm{ys} \bullet-17650$ & $\mathrm{w}^{3} \mathrm{hm}^{3-} \quad 2320,17850$ & rvnc- $\quad 17960,18010$ \\
\hline $\left.\mathrm{w}^{3} \mathrm{y}\right\}-\quad 17670$ & $w^{3} \mathrm{hm}^{3} \mathrm{p}-\quad 17850$ & rVns- 18010 \\
\hline $\mathrm{w}^{3} \mathrm{yl}^{3-}-17680$ & $w^{3} h n w f-\quad 17881$ & rvl- $\quad 18020$ \\
\hline$w^{3} \mathrm{yl}^{3} \mathrm{~h}-\quad 17680$ & $\mathrm{w}^{3}$ hnwl-* $\quad 17881$ & rvlx- 18030 \\
\hline$w^{3} y_{k}-\quad 17690$ & $\mathrm{w}^{3} \mathrm{hnh}-\quad 17560,17870$ & rvy- $\quad 18050,18060$ \\
\hline $\mathrm{w}^{3} \mathrm{yx}-\quad 17140,17700$ & 17880 & rvyc- 18070 \\
\hline $\mathrm{w}^{3} \mathrm{yx}^{\bullet-} \quad 17700$ & $\mathrm{~W}^{3} \mathrm{hn} \infty-\quad 17560,17870$ & rvys- $\quad 18070,18080$ \\
\hline $\mathrm{w}^{3} \mathrm{yw} £-\quad 17711$ & $\mathrm{w}^{3} \mathrm{hn}^{3}-\quad 17560$ & rvyk- 18090 \\
\hline $\mathrm{w}^{3} \mathrm{yq}-\quad 17710$ & $\mathrm{w}^{3} \mathrm{hs}-17890$ & rvy -18100 \\
\hline$w^{3} y^{3} \chi v-\quad 17920$ & $w^{3} h £-\quad 17891$ & rvy $^{3}-\quad 18110$ \\
\hline $\mathrm{w}^{3} \mathrm{w}-\quad 17041,17720$ & $\mathrm{w}^{3} \mathrm{~h}$ - $\quad 17900$ & IVW- 17720 \\
\hline 23230 & $\mathrm{w}^{3} \mathrm{hl}^{3-}-16960$ & rvwł- $\quad 18120,18130$ \\
\hline $\mathrm{w}^{3} \mathrm{wt}-\quad 17730$ & $w^{3} h_{x s-} \quad 17840$ & IVwlh- 18120 \\
\hline $\mathrm{w}^{3} \mathrm{wnw}-\quad 17590$ & $\mathrm{w}^{3} \mathrm{hxs} \bullet-17840$ & IVwy $^{3-} \quad 18110$ \\
\hline $\mathrm{w}^{3} \mathrm{Wnw}^{3-} \quad 17590$ & wºwh- $^{3} \quad 17720$ & IVw $\chi \mathrm{V}-18140$ \\
\hline $\mathrm{w}^{3} \mathrm{WZ}-\quad 17740$ & $w^{3} \mathrm{hw}^{3-} \quad 17820$ & $\mathrm{rVW}^{3}-18110$ \\
\hline $\mathrm{w}^{3}$ wzh- $\quad 17740$ & $\mathrm{w}^{3} \mathrm{~h} \chi \mathrm{V}-\quad 17920$ & rvqv- 18150 \\
\hline wºlh- $^{3} \quad 17750$ & $w^{3}$ hq- $\quad 17930,17940$ & rVxV- 12050 \\
\hline $\mathrm{w}^{3} \mathrm{wgv}-\quad 17760$ & $\mathrm{w}^{3} \mathrm{~h}^{3} \mathrm{xS}^{\bullet-} \quad 17840$ & гV $\chi \mathrm{V}-12570$ \\
\hline $\mathrm{w}^{3} \mathrm{wkv}-\quad 17761$ & $\mathrm{w}^{3} \mathrm{~h}^{3} \mathrm{q}^{3} \mathrm{v}-\quad 17810$ & rvh- $\quad 17960,18160$, \\
\hline $\mathrm{W}^{3} \mathrm{WW}-\quad 17770$ & $\mathrm{w}^{3} \mathrm{~h}^{3} \mathrm{q}^{3} \mathrm{vh}-\quad 17810$ & 18170 \\
\hline $\mathrm{w}^{3} \mathrm{wqv}-\quad 17780,17790$ & $\mathrm{w}^{3} \mathrm{~h}^{3} \chi^{\mathrm{v}}-17920$ & rvht- 18180 \\
\hline $\mathrm{w}^{3} \mathrm{whw}^{3}{ }_{-} \quad 17820$ & $\mathrm{w}^{3} \mathrm{~h}^{3} \chi_{-} \quad 17920$ & rvhn $^{3}-17980$ \\
\hline
\end{tabular}


North Wakashan Comparative Root List (Updated)

\begin{tabular}{|c|c|c|}
\hline Ivhs- 15470 & qvyc- 18380 & $\mathbf{q}^{3} \mathbf{v}$ \\
\hline rvhsh- 15470 & qvys- $\quad 18380,18390$ & \\
\hline rvhs• 15470 & qvys•- 18390 & $q^{3} \mathrm{vp}-18660$ \\
\hline rvh£- 18190 & qvy£- 18410 & $\mathrm{q}^{3} \mathrm{vm}-\quad 15920,18670$ \\
\hline rvhł- 18200 & qvyl- $\quad 18410$ & $\mathrm{q}^{3} \mathrm{vmt}-\quad 18680$ \\
\hline rvhlh- 18160 & qvyx- 18430 & $\mathrm{q}^{3} \mathrm{vms}-\quad 18250,18690$ \\
\hline rvhlhs- 18210 & qvyqv- 18440 & $\mathrm{q}^{3} \mathrm{vml}-* \quad 18700$ \\
\hline rvhk- 18220 & qvwt- 18460 & $\mathrm{q}^{3} \mathrm{vmx}-\quad 18710$ \\
\hline rvhy³- 18060 & qvwt $^{3-} \quad 18470$ & $\mathrm{q}^{3} \mathrm{vm}^{3}-* \quad 18940,18950$ \\
\hline rvhkv- 12570 & qvwt $t^{3 h}-18470$ & $\mathrm{q}^{3} \mathrm{vt}-18740$ \\
\hline rvhqv- 12570 & qvwnh- 18450 & $q^{3} \mathrm{vn}-\quad 18750,18760$ \\
\hline $\operatorname{rvh} \chi \mathrm{v}-\quad 12570$ & qvws- 18480 & $q^{3}$ vnc- $\quad 18770$ \\
\hline 18230 & qvwł- $\quad 18510,18520$ & $\mathrm{q}^{3}$ vns- $\quad 18770$ \\
\hline & qvwyq- 18500 & $\mathrm{q}^{3} \mathrm{vny}{ }^{3} \mathrm{qv}-\quad 18771$ \\
\hline $\mathbf{q v}$ & qvwqv- 18530 & $q^{3}$ vnk- $\quad 18780$ \\
\hline & qvqv- 18150 & $q^{3}$ vnqv- 18790 \\
\hline qvp- $\quad 18240$ & $\mathrm{qv} \chi \mathrm{v}-\quad 18550$ & $q^{3}$ vs- $\quad 15650,18800$, \\
\hline qvmc- 18250 & qv $\chi-\quad 18550$ & $18810,18820,18830$ \\
\hline qvms- 18250 & qvh- $\quad 18560$ & $q^{3} v £-\quad 18840$ \\
\hline qvn- $\quad 18260,18270$ & qvhp- 15870 & $q^{3}$ v1- $\quad 18850$ \\
\hline qvnp- 18260 & qvht- 18570 & $q^{3} \mathrm{vl}-\quad 18750,18860$ \\
\hline qvns- 18280 & qvhn $^{3-*} \quad 18270$ & $q^{3}$ vlp- $\quad 18330,18880$ \\
\hline qvnqv- 18290 & qvhs- $\quad 18480,18580$ & $\mathrm{q}^{3} \mathrm{vlm}^{3} \mathrm{c}-\quad 18890$ \\
\hline qvs- 18300 & qvhk- 18590 & $\mathrm{q}^{3} \mathrm{vlm}^{3} \mathrm{~s}-\quad 18890$ \\
\hline qv£- 15660 & qvhk $^{3} \mathrm{v}_{-} * \quad 18600$ & $q^{3}$ vlc- 18900 \\
\hline qv£m- 15660 & qvhqv- $\quad 15850,18150$ & $q^{3}$ vls- $\quad 18900$ \\
\hline qv1- $\quad 18320$ & 18620 & $q^{3}$ vly- 18910 \\
\hline qvlp- 18330 & qvh $\chi v^{-} \quad 18630$ & $q^{3}$ vlyh- 18910 \\
\hline qvlx- $\quad 18340,18350$ & qvhq- 18590 & $q^{3}$ vlqv- $\quad 18920$ \\
\hline qvlqv- 18360 & $\mathrm{qvh}^{3} \mathrm{x}^{-*} \quad 18640,18650$ & $q^{3}$ vlh- 18930 \\
\hline vy- $\quad 18390$ & & $\mathrm{q}^{3} \mathrm{vl}^{3-}-18940,18950$ \\
\hline
\end{tabular}




\section{North Wakashan Comparative Root List (Updated)}

\begin{tabular}{|c|c|c|}
\hline$q^{3}$ vk- $\quad 18970,18980$ & $q^{3}$ vhs- $\quad 18820,19210$ & 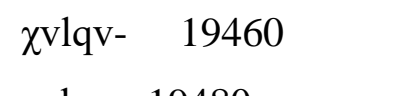 \\
\hline$q^{3} v^{3}-18990$ & 19211 & 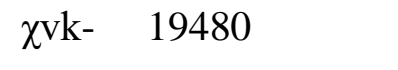 \\
\hline$q^{3}$ vyt- $\quad 19000,19001$ & $q^{3}$ vhł- $\quad 19220,19300$ & $\chi v \mathrm{vy}-18390$ \\
\hline q $^{3}$ vynht- $\quad 18930$ & $q^{3}$ vhlhx- 19230 & $\chi v$ vyt- 19490 \\
\hline$q^{3}$ vys- 19010 & $\mathrm{q}^{3} \mathrm{vhl}^{3-} \quad 19220$ & 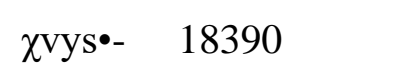 \\
\hline$q^{3} \mathrm{vyf}-19020$ & $q^{3}$ vhk- $\quad 19240,19250$ & 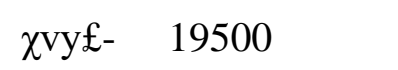 \\
\hline$q^{3}$ vył- 19040 & $q^{3}$ vhqv- 19260 & zvyl- 19520 \\
\hline$q^{3}$ vyly- 18910 & $q^{3}$ vhq- $\quad 19150,19280$ & zvylh- 19510 \\
\hline$q^{3}$ vylh- 18910 & 19281 & $\chi$ vyloo- 19510 \\
\hline 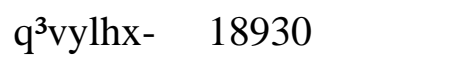 & $\mathrm{q}^{3} \mathrm{vh} \chi-\quad 19280,19290$ & $\chi \mathrm{vyl}^{3}-* \quad 19510$ \\
\hline$q^{3}$ vyqv- 19070 & $q^{3} \mathrm{vh}^{31-} \quad 19300$ & $\chi$ vyl $^{3 h}-19510$ \\
\hline$q^{3} \mathrm{vy}^{3} \mathrm{~s}-19010$ & $\mathrm{q}^{3} \mathrm{vh}^{3} \mathrm{x}-* \quad 19310$ & $\chi v y x-19520$ \\
\hline $\mathrm{q}^{3} \mathrm{vkv}^{-*} \quad 18970$ & $q^{3} \mathrm{vh}^{3} \mathrm{hc}-19190$ & 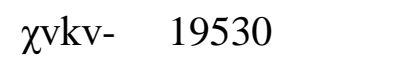 \\
\hline $\mathrm{q}^{3} \mathrm{vw}-\quad 17720$ & & $\chi$ vws- 19540 \\
\hline $\mathrm{q}^{3} \mathrm{vwmh}-\quad 18670$ & $\chi \mathbf{v}$ & $\chi$ vht- $\quad 19350,19580$ \\
\hline$q^{3}$ vwmhq- 19080 & & $\chi$ vhnh- 19590 \\
\hline$q^{3}$ vwm $\infty-\quad 18670$ & 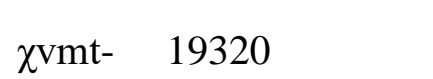 & $\chi$ vhnhł- 19590 \\
\hline$q^{3}$ vwt- 19090 & $\chi$ vms- $^{-} 19340$ & $\chi$ vhs- $\quad 19600,19610$ \\
\hline$q^{3}$ vwł- $\quad 19100,19110$ & $\chi v \mathrm{vt}-19350$ & $\chi \operatorname{vhf-} 19620$ \\
\hline $\mathrm{q}^{3} \mathrm{vwqv-} \quad 13530,19120$ & $\chi^{\mathrm{vn}-} \quad 19360,19370$ & $\chi \mathrm{vhk}^{3} \mathrm{n}-* \quad 19630$ \\
\hline 19140 & $\chi$ vnt- 19380 & 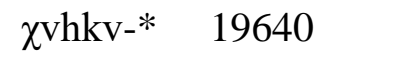 \\
\hline $\mathrm{q}^{3} \mathrm{vw} \chi \mathrm{V}^{-} \quad 19130,19140$ & $\chi v \mathrm{vnkv-} \quad 19390$ & $\chi$ vhq- 19660 \\
\hline$q^{3}$ vqv- $\quad 13550,19140$ & $\chi v \mathrm{VZ}-\quad 19420$ & \\
\hline$q^{3} v \chi^{v-} \quad 19140$ & $\chi \mathrm{vs}-\quad 19400,19420$ & I \\
\hline$q^{3} \mathrm{vq}^{-} \quad 19140,19150$ & $\chi v 1-\quad 18860$ & \\
\hline $\mathrm{q}^{3} \mathrm{vh}-\quad 19160,19170$ & $\chi v 1-\quad 19420$ & I- 14661 \\
\hline$q^{3}$ vht- $\quad 19180,19190$ & 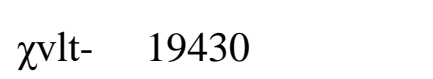 & гр- $\quad 19670,19680$ \\
\hline $\mathrm{q}^{3} \mathrm{vht}^{3}-\quad 19200$ & 19420,19440 & rm- 19690 \\
\hline$q^{3} v^{3} t^{3 h}-19200$ & $\chi$ vls- $\quad 19420,19440$ & rmt- 19700 \\
\hline vhc- 19190 & 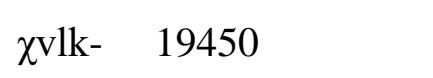 & rms-* 19700 \\
\hline & $\chi$ vlyqv- 19451 & rmwt- 21601 \\
\hline
\end{tabular}




\section{North Wakashan Comparative Root List (Updated)}

\begin{tabular}{|c|c|c|}
\hline $\operatorname{rm} \chi-\quad 19730$ & rynh1- 20010 & rhnh- 19760,20240 \\
\hline $\mathrm{rm}^{3} \chi-\quad 19740$ & ryn ${ }^{3}-\quad 20020$ & 20250 \\
\hline It- 19750 & ryn ${ }^{3} y-\quad 20020$ & 20250 \\
\hline In- $\quad 19760,19770$, & rys- $\quad 20030,20040$, & rhzh- 20250 \\
\hline 19780 & 20050 & rhzh $\chi-\quad 20250$ \\
\hline Inf- 19800 & ryx- 20060 & rhc- 2780 \\
\hline Int-* 19810 & ryxv- $\quad 20070,20080$ & rhsx•- 20260 \\
\hline rnk- 19820 & ryh- 19971 & rh£- $\quad 20270$ \\
\hline rnys- 19790 & ry³- 19980 & rhlh- 20280 \\
\hline In $^{3} y s-\quad 19790$ & ry ${ }^{3} \mathrm{~h}-\quad 19980$ & rhl $^{3}-\quad 20290$ \\
\hline Is- $\quad 19821,19830$ & Ixv- 20090 & rhk- 19940 \\
\hline rl- $\quad 18020,19780$ & IW- $\quad 17720,20100$ & rhx- 19950 \\
\hline 19840,19850 & rwp- 20110 & rhxs•- 20260 \\
\hline rlp- 19860 & rwm $^{3} \mathrm{~h}-\quad 20110$ & rhyh- 19970 \\
\hline rls- 19870 & rwt- $\quad 20120,20130$ & rhy³ -19980 \\
\hline rlk- 19880 & rws- $\quad 20140,20150$ & rhgv- 20291 \\
\hline rlys- 19790 & IW£- $\quad 20160$ & Ihxv- 20300 \\
\hline rlyq- 19910 & rwł- 18120,18130 , & rhwh- 20100 \\
\hline Ilxv- 19880 & 20160, 20170, 20180, & rhqv- 20310 \\
\hline rlwhd- 19891 & 20190 & rhq- $\quad 12570$ \\
\hline rlq- $\quad 19900,19910$ & rwlh- 18120 & rh $\chi-\quad 12570,20330$ \\
\hline rl $\chi-\quad 19900,19930$ & rwlo- 18120 & 20340 \\
\hline rlh- 19840 & rwl ${ }^{3}-\quad 20170$ & $\mathrm{rhh}^{3}-\quad 20220$ \\
\hline rl $l^{3}$ ys- 19790 & rwy ${ }^{3} h-\quad 18110$ & $\mathrm{rh}^{3}-\quad 2780,20220$ \\
\hline rg-* 21450 & rwqv- $\quad 13530,18150$ & $\mathrm{rh}^{3} \mathrm{xs} \bullet-\quad 20260$ \\
\hline rk- 19940 & IW $\chi v-\quad 18140,20200$ & $\mathrm{rh}^{3} \mathrm{q}-\quad 2780$ \\
\hline rx- 19950 & rqv- 18150 & $\mathrm{rh}^{3} \mathrm{~h}-\quad 2780$ \\
\hline гу- 19970,19971, & rq- $\quad 18150$ & \\
\hline 19980 & rh- $\quad 2780,19971,20210$ & $\mathbf{q}$ \\
\hline rур- 19990 & 20220 & \\
\hline ryt- 20000 & rht- $\quad 2780,20230$ & q- $\quad 14661$ \\
\hline
\end{tabular}


North Wakashan Comparative Root List (Updated)

\begin{tabular}{|c|c|c|}
\hline qbx- $\quad 20850$ & qyt- 20650 & $\mathrm{qhm}^{3}-\quad 20870$ \\
\hline qp- 20360 & qynh- 20660 & qht- 20880 \\
\hline qmt- 20380 & qys- $\quad 20040$ & qhs- $\quad 20890,20900$ \\
\hline qm£- 20390 & qył- $\quad 20670,20680$ & qhł- 20900,20901 , \\
\hline qmł- 20400 & qyk- $\quad 20690,20700$ & 20910 \\
\hline qmk- 20410 & qyx- 20660 & qhk- 20920 \\
\hline qmkv- 20410 & qyq- $\quad 18440$ & $\mathrm{qhk}^{3} \mathrm{v}-\quad 20930$ \\
\hline qmxv- $\quad 20420,20430$ & qy $\chi_{-} \quad 18440,20720$ & qhwht- 20940 \\
\hline $\mathrm{qm} \chi-\quad 20440$ & qy $^{3} \mathrm{~s}-\quad 20040$ & qhq- 18150,20950 \\
\hline $\mathrm{qdw}^{3} \chi^{\mathrm{v}-} \quad 20441$ & qkv- $\quad 20730,20750$ & 20960 \\
\hline qt- 20450 & qXv- $\quad 20090,20750$ & qh $\chi_{-} \quad 20970,20980$ \\
\hline qnc- $\quad 20460$ & qwp- 20760 & $\mathrm{qh}^{3} \chi_{-} \quad 20980$ \\
\hline qnch- 20460 & qwmh- 18450 & \\
\hline qns- $\quad 20460$ & qwt- $\quad 18460,18470$, & $\mathbf{q}^{3}$ \\
\hline qnkv- 20470 & 20770 & \\
\hline qnq- $\quad 20480,20490$ & $\mathrm{qwt}^{3}-\quad 18470$ & $q^{3} p^{-} \quad 18660,20990$ \\
\hline qn $\chi-\quad 20490$ & qwt $^{3} h-\quad 18470$ & 21750 \\
\hline $\mathrm{qc}^{3} 1 \mathrm{lc}-\quad 20500$ & qwnh- 18450 & $q^{3} p^{3} y-\quad 20990$ \\
\hline $\mathrm{qc}^{3} \mathrm{yt}-\quad 20500$ & qws- $\quad 18480,18520$ & $q^{3} p^{3} h-\quad 20990$ \\
\hline $\mathrm{qc}^{3} \mathrm{ws}-\quad 20500$ & qw1- $\quad 18510,18520$ & $q^{3} m-\quad 21010$ \\
\hline qs- $\quad 19830,23560$ & $\mathrm{qwl}^{3-*} \quad 20790$ & $\mathrm{q}^{3} \mathrm{mt}-\quad 21020,21030$ \\
\hline$q £-\quad 20510$ & $\mathrm{qwl}^{3} \mathrm{wqv}-\quad 20791$ & $\mathrm{q}^{3} \mathrm{~ms}-\quad 21040,21050$ \\
\hline q1- 20520 & qwqv- $\quad 18530,20800$ & $\mathrm{q}^{3} \mathrm{ml}-\quad 21060$ \\
\hline qlt- 20530 & qw $\chi v^{-}-20810$ & $q^{3} \mathrm{mk}-\quad 21070$ \\
\hline qlk- $\quad 20550$ & $\mathrm{qw}^{3} \mathrm{~s}-\quad 18520$ & $q^{3} \mathrm{mkv}-\quad 21080$ \\
\hline qly3h-* 20551 & $\mathrm{qw}^{3} \mathrm{k}-\quad 18520$ & $q^{3} m x v-\quad 20430$ \\
\hline qlkv- 20560 & qq- $\quad 18150$ & $q^{3} m w-\quad 21601$ \\
\hline qlxv- 20580 & $\mathrm{q} \chi-\quad 20830$ & $q^{3} m \chi-$ \\
\hline $\mathrm{q} l \chi-\quad 20590$ & qhbx- 20850 & $\mathrm{q}^{3} \mathrm{~m}^{3} \mathrm{~s}-\quad 21050$ \\
\hline qk- $\quad 19940,20620$ & qhp- $\quad 20840,20850$ & $q^{3 t}-\quad 21110,21130$ \\
\hline qx- 20630 & qhmh- 20860 & $q^{3} n-\quad 21140$ \\
\hline
\end{tabular}


North Wakashan Comparative Root List (Updated)

\begin{tabular}{|c|c|c|}
\hline$q^{3}$ nt- $\quad 21160$ & $q^{3} y t-\quad 21510$ & $q^{3} \chi-\quad 18550$ \\
\hline$q^{3}$ nc- $\quad 21170,21180$ & $q^{3} y z-* \quad 21520$ & $q^{3} \mathrm{~h}-\quad 21740$ \\
\hline ns- $\quad 21180,21190$ & $q^{3} y s-\quad 21530$ & $q^{3} \mathrm{hp}-\quad 21750$ \\
\hline$n £-\quad 21200$ & $q^{3} y ł h-* \quad 21570$ & $q^{3} \mathrm{hmh}-\quad 21010,21140$ \\
\hline$q^{3}$ nyp- $\quad 21320$ & $q^{3} y k v-\quad 21540$ & $\mathrm{q}^{3} \mathrm{hm}^{3-*} \quad 21010$ \\
\hline$q^{3}$ nłh- $\quad 21210$ & $q^{3} y w d-\quad 21541$ & $q^{3}$ ht- $\quad 21130,21360$ \\
\hline nk- $\quad 21220$ & $q^{3} y q-\quad 21550$ & $q^{3}$ hny- $\quad 21140$ \\
\hline nq- $\quad 21230$ & $\mathrm{q}^{3} \mathrm{y} x-\quad 21560$ & $q^{3}$ hnh- 21140 \\
\hline c- 21250 & $\mathrm{q}^{3} \mathrm{y} \chi \mathrm{h}-\quad 21570$ & $\mathrm{q}^{3} \mathrm{hn}^{3}-\quad 21140$ \\
\hline $\mathrm{q}^{3} \mathrm{~s}-\quad 21250$ & $q^{3} y x \cdot \hbar^{3} h-\quad 21570$ & $q^{3}$ hc- $\quad 2780$ \\
\hline$q^{3 \mathfrak{f}^{3}-} \quad 21300$ & $q^{3} y^{3} h k-\quad 21571$ & $q^{3} \mathrm{hs}-\quad 21250,21780$ \\
\hline $\mathrm{q}^{3} £^{3} \mathrm{~h}-, \quad 21300$ & $\mathrm{q}^{3} \mathrm{kv}-\quad 21580$ & 21790 \\
\hline$q^{31-} \quad 21310$ & $\mathrm{q}^{3} \mathrm{w}-\quad 21590$ & $q^{3} h 1-\quad 21810,21820$ \\
\hline$q^{3} 1-\quad 21320,21810$ & $q^{3} w p-\quad 21600$ & $q^{3}$ hlh- $\quad 21350,21810$ \\
\hline$q^{3} 1 y p-\quad 21320,21350$ & $q^{3} w m w-\quad 21601$ & $\mathrm{q}^{3} \mathrm{hl}^{3-}-\quad 21350,21740$ \\
\hline$q^{3} 1 t-\quad 21360$ & $q^{3} w m h-\quad 21601,21602$ & $q^{3} h x-\quad 21830$ \\
\hline lc- $\quad 21370,21380$ & $\mathrm{q}^{3} \mathrm{wm} \infty-\quad 21601$ & $q^{3} h y h \chi-\quad 21831$ \\
\hline ls- $\quad 21370,21380$ & $q^{3} w d h-\quad 21610$ & $q^{3} h^{3}{ }^{3}-21490,21740$ \\
\hline$q^{3} 1 \mathrm{k}-\quad 21390,21400$ & $q^{3} w d h q-\quad 21610$ & $\mathrm{q}^{3} \mathrm{hkv}-\quad 21840$ \\
\hline $\mathrm{q}^{3} \mathrm{lkv}-* \quad 21410$ & $q^{3} w t-\quad 19090,21620$ & $q^{3} h x v-21850$ \\
\hline$q^{3} \mid x v-\quad 21410$ & $q^{3} w s-\quad 21630$ & $\mathrm{q}^{3} \mathrm{hwh}-\quad 21860$ \\
\hline $1 \chi_{-} \quad 21420$ & $q^{3} w 1-\quad 21670$ & $\mathrm{q}^{3} \mathrm{~h} w \infty-21860$ \\
\hline $\mathrm{q}^{3} \operatorname{lh} \chi-\quad 21320$ & $q^{3} w \nmid \bullet-\quad 21670$ & $q^{3} h^{3} w_{-} \quad 21590$ \\
\hline $1^{3}-21350$ & $q^{3} w y^{3}-\quad 21680$ & $q^{3} h^{3} p-\quad 21750$ \\
\hline$q^{3} 3^{3} h-\quad 18940$ & $q^{3} w k v-\quad 21690,21700$ & $q^{3} h^{3} t-\quad 21360$ \\
\hline k- $\quad 21430,21440$ & $q^{3} w x v-\quad 21690$ & $q^{3} h^{3} s-\quad 21790$ \\
\hline 450 & $q^{3} w w-\quad 21710$ & \\
\hline 21460 & $q^{3} w q v-19120$ & $\chi$ \\
\hline$y-\quad 21470,21480$ & $\mathrm{q}^{3} \mathrm{w} \chi \mathrm{v}-19130$ & \\
\hline & $q^{3} \chi v^{-} \quad 18550$ & $\chi^{-} \quad 14661$ \\
\hline $\mathrm{Im}^{3-} \quad 21500$ & $q^{3} q-\quad 21720$ & $\chi$ bls- $\quad 21880$ \\
\hline
\end{tabular}


North Wakashan Comparative Root List (Updated)

\begin{tabular}{|c|c|c|}
\hline$\chi \mathrm{m}-\quad 21890$ & $\chi y £-* \quad 22210$ & $\chi$ hk- 22090 \\
\hline$\chi \mathrm{ms}-\quad 21900,21910$ & $\chi y x-\quad 22220$ & xhyhq- $\quad 22240$ \\
\hline$\chi \mathrm{m} £-\quad 21920$ & xykv- 22230 & $\chi$ hxv- $\quad 22470$ \\
\hline$\chi$ mlkv- 21930 & $\chi y q-\quad 22240,22250$ & 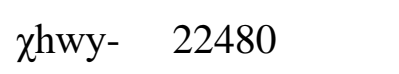 \\
\hline$\chi m x v-\quad 20420$ & $\chi \mathrm{y} \chi-\quad 22250$ & $\chi$ hwys- 22481 \\
\hline 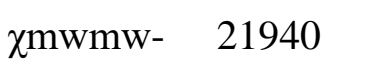 & $\chi \mathrm{y}^{3-}-22170$ & $\chi \mathrm{hwy}^{3}-\quad 22480$ \\
\hline$\chi \mathrm{m}^{3}-\quad 21950$ & $\chi \mathrm{kv}-\quad 22260$ & $\chi$ hwh- 22480 \\
\hline$\chi^{\mathrm{t}-} \quad 21960$ & $\chi w-\quad 22270$ & $\chi$ hwoo- 22490 \\
\hline$\chi^{n-} \quad 21970$ & $\chi w p-\quad 22290,22300$ & $\chi \mathrm{hw}^{3}-\quad 22490$ \\
\hline xnt- 21980 & $\chi w m h-\quad 22310$ & $\chi$ hq- $\quad 22500$ \\
\hline$\chi \mathrm{n} £-\quad 21990$ & $\chi w m \infty-* \quad 22311$ & 22500 \\
\hline ұnyhs- 22000 & $\chi w t-\quad 22320$ & $\chi^{h^{3}-} \quad 22410$ \\
\hline ఒnh- $\quad 21970$ & $\chi \mathrm{wc}^{3}-\quad 22330$ & \\
\hline$\chi s^{-} \quad 22010,22020$ & $\chi w s-\quad 22331,22340$ & h \\
\hline$\chi £-\quad 22030,22040$ & $\chi w 1-\quad 18520,22350$ & \\
\hline$\chi 1-\quad 22050$ & 22360,22370 & h- $\quad 14380,14661$ \\
\hline$\chi \mathrm{lp}-\quad 22060$ & $\chi w q v-\quad 22380,22381$ & hp- $\quad 22510,22520$ \\
\hline zlt- $\quad 22070$ & $\chi q v-\quad 22380,22390$ & 22530,22540 \\
\hline$\chi \mathrm{lc}-\quad 22080$ & $\chi \chi \chi^{V}-\quad 22390$ & hm- $\quad 1190,22540$ \\
\hline$\chi 1 \mathrm{ls}-\quad 22080$ & $\chi q-\quad 22250,22400$ & 22550,22590 \\
\hline 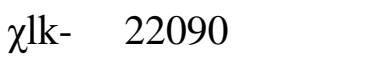 & $x x^{-} \quad 22250$ & hmm- 1190 \\
\hline$\chi 1 \mathrm{xv}-\quad 22100$ & $\chi \mathrm{h}-\quad 22410$ & hmt- 22560 \\
\hline$\chi$ lqv- $\quad 22110$ & $\chi \mathrm{hbxv}-* \quad 21880$ & hmz- 22600 \\
\hline$\chi \mathrm{lq}^{3} \mathrm{vh}-\quad 22120$ & $\chi$ hp- $\quad 22420$ & hmc- 22580 \\
\hline$\chi \mathrm{lq}-* \quad 22130$ & $\chi \mathrm{hmh}-\quad 21890$ & hms- $\quad 22580,22590$, \\
\hline$\chi \mathrm{k}^{3}-\quad 22140$ & $\chi$ hnh- $\quad 21970$ & 22600 \\
\hline$\chi x-\quad 22150$ & $\chi \mathrm{hn}^{3}-\quad 21970$ & hmk- $\quad 1200$ \\
\hline$x y-\quad 22160,22170$ & $\chi$ hs- $\quad 22430$ & hmy-* 22540 \\
\hline$\chi \mathrm{ym}^{3}-\quad 22180$ & xhsbxv-* 21880 & hmkv- 22610 \\
\hline xyt- 22190 & $\chi \mathrm{h}^{3} £^{3} \quad 22440$ & hm $\chi \mathrm{v}-\quad 22620$ \\
\hline ұy£-* 22200 & xht- 22450 & \\
\hline
\end{tabular}


North Wakashan Comparative Root List (Updated)

\begin{tabular}{|c|c|c|}
\hline $\mathrm{hm}^{3-} \quad 22540,22580$ & hy- $\quad 14380,22821$ & hws- $\quad 22990,23000$ \\
\hline 22590 & hymwt- 22822 & hwł- 23020 \\
\hline $\mathrm{hm}^{3} \mathrm{xs}-\quad 22600$ & hym $^{3-} \quad 22590$ & hwy ${ }^{3-} \quad 23030$ \\
\hline $\mathrm{hm}^{3} \mathrm{~W}-\quad 1190$ & hym³ ${ }^{3}$ - $\quad 22590$ & hwkv- 23031,23040 \\
\hline $\mathrm{hm}^{3} \mathrm{~h}-\quad 22590$ & hynh- 22823 & hwxv- 23031 \\
\hline ht- 22640 & hynhk- 22824 & hww-* 17220 \\
\hline hn- $\quad 4210,22650,23440$ & hyn $^{3-} \quad 22780$ & hwqv- 17230 \\
\hline hnf- $\quad 22670$ & hys- 22830 & hw $\chi v-17290$ \\
\hline hny- 22671 & hys $œ k v-\quad 22840$ & $\mathrm{hw}^{3} \mathrm{xv}-\quad 23031$ \\
\hline hnkv- $\quad 22680,22690$ & hył- 22821,22850 & hqv- $\quad 23050,23070$ \\
\hline hnxv- 22700 & hyłzhqv- 22851 & $\mathrm{~h} \chi \mathrm{v}-\quad 22920,23050$, \\
\hline hnqv- 22710,22720 & hyly- 22852 & 23080,23090 \\
\hline hnq- $\quad 4550,22730$ & hylw- 22853 & hq- 23100 \\
\hline hn $\chi_{-} \quad 22740$ & hylh- 22852 & hh- $\quad 11450$ \\
\hline hnh- $\quad 4550$ & hylœ- 22852 & hhbh- 22541 \\
\hline $\mathrm{hc}^{3-} \quad 6540$ & hyl $^{3}{ }_{-} \quad 22780,22854$ & hhp- $\quad 22510,22520$ \\
\hline $\mathrm{hc}^{3 \mathrm{t}-} \quad 6540$ & hyx- $\quad 22850,22860$ & 22540 \\
\hline $\mathrm{hc}^{3} \mathrm{~s}-\quad 6540$ & hyxv- 22880 & $\mathrm{hhm}^{3-} \quad 22590$ \\
\hline hs- $\quad 22750,23550$, & hy $^{3} \mathrm{x}-\quad 22850$ & $\mathrm{hhm}^{3} \mathrm{~h}-\quad 22590$ \\
\hline 23560 & hkv- $\quad 22890,23050$ & hhd- 2780 \\
\hline ht- $\quad 22760,22770$ & $\mathrm{hk}^{3} \mathrm{v}-\quad 22810$ & hht- 23110,23120 \\
\hline hl- $\quad 22780$ & hxv- 22820,22900 & hhny-* 23130 \\
\hline hls- $\quad 22790$ & 22920 & hhnhk- 23131 \\
\hline hly- $\quad 22671$ & hw- 17220,22920 , & hhn ${ }^{3}$ h- $\quad 23142$ \\
\hline hlxv-* 22800 & 22930 & hhnhkv- 23142 \\
\hline hll- $\quad 22801$ & hwpª- 22940 & hhs- $\quad 23140,23141$ \\
\hline hlh- $\quad 11450,22780$ & hwmw- 22950 & hhl $^{3}-\quad 23142$ \\
\hline hlhk- 11450 & hwmh- 22950 & hhl'h- 23142 \\
\hline $\mathrm{hl}^{3} \mathrm{~s}_{-} \quad 22780$ & hwmos- $\quad 1190,22950$ & hhl'3hk- 23142 \\
\hline $\mathrm{hk}^{3-} \quad 22810$ & hwmh $\chi$ h- 22960 & hhx- 22820 \\
\hline hx- 22820 & hwt- 22980 & 17041 \\
\hline
\end{tabular}


North Wakashan Comparative Root List (Updated)

\begin{tabular}{|c|c|c|}
\hline hhyh- 22821 & $\mathrm{~h}^{3} \mathrm{~m}^{3} \mathrm{k}-\quad 23320$ & $\mathrm{~h}^{3} \mathrm{c}-\quad 2780$ \\
\hline hhy $^{3-} \quad 22821$ & $\mathrm{~h}^{3} \mathrm{~m}^{3} \mathrm{hl}-\quad 23350$ & $\mathrm{~h}^{3} \mathrm{~s}-\quad 6540,23550$ \\
\hline $\mathrm{hhk}^{3} \mathrm{v}-\quad 22810$ & $\mathrm{~h}^{3} \mathrm{~d}-\quad 2780$ & $\mathrm{~h}^{3} \mathrm{~s}^{\bullet}-\quad 23550,23560$ \\
\hline hhxv- 23160 & $\mathrm{~h}^{3} \mathrm{dmkv}-\quad 23370$ & $\mathrm{~h}^{3} \lambda \mathrm{bw}-\quad 23570$ \\
\hline hhw- 17041 & $\mathrm{~h}^{3} \mathrm{t}-\quad 23380,24080$ & $\mathrm{~h}^{3 £}-\quad 23580$ \\
\hline hhwy-* 23130 & $\mathrm{~h}^{3} \mathrm{n}-\quad 23390,23400$ & $\mathrm{~h}^{3} £ b w-\quad 23570$ \\
\hline hhwh- 22930 & $23410,23430,23431$ & $\mathrm{~h}^{3 \mathfrak{f}^{3} \mathrm{k}-} \quad 23590$ \\
\hline hhw³ h- 22930 & 23440,23441 & $\mathrm{~h}^{3} 1-\quad 23430,23600$ \\
\hline hhqv- 23050,23170 , & $\mathrm{h}^{3} \mathrm{np}-\quad 23450$ & $\mathrm{~h}^{3} \mathrm{lz}-\quad 23620$ \\
\hline 23180 & $\mathrm{~h}^{3} \mathrm{nt}-\quad 23460$ & $\mathrm{~h}^{3} 1 \mathrm{c}-\quad 23620,23630$ \\
\hline & $\mathrm{h}^{3} \mathrm{nc}-\quad 23470$ & $\mathrm{~h}^{3} 1 \mathrm{~s}-\quad 23620,23630$ \\
\hline $\mathbf{h}^{3}$ & $h^{3} n c \cdot-\quad 23470$ & $\mathrm{~h}^{3} \mathrm{lk}-\quad 23640$ \\
\hline & $h^{3}$ ns- $\quad 23470$ & $\mathrm{~h}^{3} \mathrm{lkv}-\quad 23641$ \\
\hline $\mathrm{h}^{3-} \quad 14661$ & $\mathrm{~h}^{3} \mathrm{nk}-\quad 23510$ & $\mathrm{~h}^{3} \mathrm{lxv}-\quad 23641,23642$ \\
\hline $\mathrm{h}^{3} \mathrm{~b}-\quad 23190$ & $h^{3} n y^{3}-\quad 23490$ & $\mathrm{~h}^{3} l q v-\quad 2100$ \\
\hline $\mathrm{h}^{3} \mathrm{p}-\quad 23200,23210$ & $h^{3}$ ngvh- $\quad 23500$ & $h^{3}$ lqhł- $\quad 23643$ \\
\hline $\mathrm{h}^{3} \mathrm{ps}^{\bullet-} \quad 23560$ & $h^{3} n x v-\quad 23510$ & $\mathrm{~h}^{3} 1 \chi_{-} \quad 23650,23660$ \\
\hline $\mathrm{h}^{3} \mathrm{~m}-\quad 23230,23260$ & $\mathrm{~h}^{3} \mathrm{nw}-\quad 23520$ & $\mathrm{~h}^{3}$ lhq- $\quad 23661$ \\
\hline 23350 & $\mathrm{~h}^{3} \mathrm{nwkv-} \quad 23511$ & $\mathrm{~h}^{3} 1: \mathrm{h}^{3} \mathrm{hs}-\quad 23630$ \\
\hline $\mathrm{h}^{3} \mathrm{mt}-\quad 23270$ & $h^{3}$ nwh- $\quad 23520$ & $\mathrm{~h}^{3} \mathrm{l}^{3 \mathrm{~h} s-} \quad 23630$ \\
\hline $\mathrm{h}^{3} \mathrm{~ms}-\quad 23280,23290$ & $h^{3} n w \infty-\quad 23520$ & $\mathrm{~h}^{3} \mathrm{k}-\quad 23670,23680$ \\
\hline $\mathrm{h}^{3} \mathrm{ml}-\quad 23310,23320$ & $\mathrm{~h}^{3} \mathrm{nh} ł \mathrm{kn}-\quad 23511$ & $\mathrm{~h}^{3} \mathrm{ky}-\quad 23670$ \\
\hline $\mathrm{h}^{3}$ mlyxv- $\quad 23321$ & $h^{3} n h ł x n-\quad 23511$ & $\mathrm{~h}^{3} \mathrm{y}-\quad 4780,14380$ \\
\hline $\mathrm{h}^{3} \mathrm{myh} \chi-\quad 23322$ & $\mathrm{~h}^{3} \mathrm{n}^{3} \mathrm{w}-\quad 4890$ & $15010,15020,23690$ \\
\hline$h^{3} m y^{3} h x-\quad 23322$ & $\mathrm{~h}^{3} \mathrm{n}^{3} \mathrm{wkv}-\quad 23511$ & $\mathrm{~h}^{3} \mathrm{yp}-\quad 23700$ \\
\hline $\mathrm{h}^{3} \mathrm{mqv}-\quad 23330$ & $\mathrm{~h}^{3}$ nqv- $\quad 23520$ & $\mathrm{~h}^{3} \mathrm{yt}-15030$ \\
\hline $\mathrm{h}^{3} \mathrm{~m} \chi-\quad 23330$ & $\mathrm{~h}^{3} \mathrm{nq}-\quad 820,4550,23530$ & $\mathrm{~h}^{3} \mathrm{ynh}-\quad 23440,23441$ \\
\hline $\mathrm{h}^{3} \mathrm{mhk}-\quad 23240$ & $h^{3}$ nhxvh- $\quad 4970$ & $h^{3} y n h x v h-\quad 4970$ \\
\hline $\mathrm{h}^{3}$ mhys- $\quad 23250$ & $h^{3} n h \chi v-\quad 4970$ & $h^{3} y s-\quad 13260,15040$ \\
\hline $\mathrm{h}^{3} \mathrm{may}^{3}-\quad 23250$ & $\mathrm{~h}^{3} \mathrm{nh} \chi \mathrm{vh}-\quad 4970$ & 23721 \\
\hline $\mathrm{h}^{3} \mathrm{~m}^{3} \mathrm{t}-\quad 23270$ & $h^{3} n^{3} q v-\quad 23520$ & $\mathrm{~h}^{3} \mathrm{yshy}{ }^{3} \mathrm{w}-\quad 23730$ \\
\hline
\end{tabular}


North Wakashan Comparative Root List (Updated)

\begin{tabular}{|c|c|c|}
\hline $\mathrm{h}^{3} \mathrm{y}^{3}{ }^{3} * * \quad 23800$ & $\mathrm{~h}^{3} \mathrm{WZW}-\quad 17740$ & $\mathrm{~h}^{3} \mathrm{hmhh}-\quad 23240$ \\
\hline$h^{3}$ ygh- $\quad 23731$ & $\mathrm{~h}^{3} \mathrm{wzh}-\quad 17740$ & $\mathrm{~h}^{3} \mathrm{hmay}^{3}-\quad 23250$ \\
\hline$h^{3} y k-\quad 23790$ & $\mathrm{~h}^{3} \mathrm{Wc} \bullet-\quad 17740$ & $\mathrm{~h}^{3} \mathrm{hm}^{3} \mathcal{3}_{-} \quad 23360$ \\
\hline $\mathrm{h}^{3} \mathrm{yk}^{3}-\quad 23800$ & $\mathrm{~h}^{3} \mathrm{Wsl}-\quad 23861$ & $\mathrm{~h}^{3} \mathrm{hm}^{3} \mathrm{~h}-\quad 23230,23250$ \\
\hline$h^{3} y x h-15050$ & $\mathrm{~h}^{3} \mathrm{wsh}-\quad 23861$ & $\mathrm{~h}^{3} \mathrm{hm}^{3} \mathrm{~h} \mathrm{l}-\quad 23350$ \\
\hline $\mathrm{h}^{3} \mathrm{yx}^{\bullet}-\quad 15050$ & $\mathrm{~h}^{3} \mathrm{Ws}^{\bullet-} \quad 23861$ & $\mathrm{~h}^{3} \mathrm{hm}^{3} \mathrm{~h}^{3} \mathrm{k}-\quad 23350$ \\
\hline $\mathrm{h}^{3} \mathrm{ykv}-\quad 23810$ & $\mathrm{~h}^{3} \mathrm{w} 1-\quad 4900,23320$ & $\mathrm{~h}^{3} \mathrm{hd}-\quad 2780$ \\
\hline$h^{3} y x v-\quad 23820$ & 23950 & $\mathrm{~h}^{3}$ hdhc- $\quad 2780$ \\
\hline $\mathrm{h}^{3} \mathrm{ywh}-\quad 17820$ & $\mathrm{~h}^{3} \mathrm{wl}-\quad 23950$ & $\mathrm{~h}^{3} \mathrm{ht}-\quad 24080$ \\
\hline $\mathrm{h}^{3} \mathrm{yqv}-\quad 23830$ & $\mathrm{~h}^{3}$ wlh- $\quad 17750$ & $\mathrm{~h}^{3} \mathrm{hnhl-} \quad 23511,23390$ \\
\hline $\mathrm{h}^{3} \mathrm{yq}-\quad 15060$ & $\mathrm{~h}^{3}$ wyq- $\quad 17710$ & $\mathrm{~h}^{3} \mathrm{hc}-\quad 2780$ \\
\hline$h^{3} y \chi-\quad 15070$ & $\mathrm{~h}^{3} \mathrm{wyh}-\quad 23860$ & $\mathrm{~h}^{3} \mathrm{~h} s-\quad 6540,23550$ \\
\hline$h^{3} y^{3} s-\quad 13260,23721$ & $\mathrm{~h}^{3} \mathrm{wgv}-\quad 17760$ & $24090,24100,23141$ \\
\hline$h^{3} y^{3} s^{\bullet-} \quad 17840$ & $\mathrm{~h}^{3} \mathrm{wkv}-\quad 23980$ & $\mathrm{~h}^{3} \mathrm{hs} \bullet-\quad 23550$ \\
\hline$h^{3} y^{3} x-\quad 23790$ & $\mathrm{~h}^{3} \mathrm{wW}-\quad 17041$ & $\mathrm{~h}^{3} \mathrm{~h} \lambda-\quad 24110$ \\
\hline $\mathrm{h}^{3} \mathrm{y}^{3} \mathrm{qv}-17710$ & $\mathrm{~h}^{3} \mathrm{wqv}-\quad 2370,17790$ & $\mathrm{~h}^{3} \mathrm{~h} f-\quad 24110,24111$, \\
\hline $\mathrm{h}^{3} \mathrm{y}^{3} \chi \mathrm{v}-17710$ & $\mathrm{~h}^{3} \mathrm{w} \chi \mathrm{V}-\quad 24000$ & 24120 \\
\hline $\mathrm{h}^{3} \mathrm{gv}-\quad 23190$ & $\mathrm{~h}^{3} \mathrm{wh}-\quad 17041$ & $h^{3} h £ y-\quad 24110$ \\
\hline $\mathrm{h}^{3} \mathrm{xv}-\quad 23850$ & $\mathrm{~h}^{3} \mathrm{~W}^{3} \mathrm{Z}-\quad 17740$ & $\mathrm{~h}^{3} \mathrm{~h} £ \mathrm{~h}-\quad 24110$ \\
\hline $\mathrm{h}^{3} \mathrm{~W}-\quad 17770,17820$ & $\mathrm{~h}^{3} \mathrm{~W}^{3} \mathrm{~s}-\quad 23970$ & $\mathrm{~h}^{3} \mathrm{~h} £ \cdot-\quad 24110$ \\
\hline $23230,23860,23861$, & $\mathrm{h}^{3} \mathrm{w}^{3} \mathrm{l}^{3-} \quad 23970$ & $h^{3} h £^{3}-\quad 24130$ \\
\hline 23870 & $\mathrm{~h}^{3} \chi \mathrm{V}^{-} \quad 4970,23090$ & $\mathrm{~h}^{3} \mathrm{~h} £^{3} \mathrm{k}-* \quad 23590$ \\
\hline $\mathrm{h}^{3} \mathrm{wp}-\quad 23890,23900$ & $\mathrm{~h}^{3} \chi \mathrm{vh}-\quad 4970$ & $\mathrm{~h}^{3} \mathrm{~h} 1-\quad 23320,24140$ \\
\hline 23910 & $\mathrm{~h}^{3} \mathrm{q}-\quad 24010$ & $\mathrm{~h}^{3} \mathrm{hly}-\quad 24150$ \\
\hline $\mathrm{h}^{3} \mathrm{wmwy}^{3-} \quad 24071$ & $\mathrm{~h}^{3} \chi_{-}-2920,4970$ & $\mathrm{~h}^{3} \mathrm{hg}-\quad 24160$ \\
\hline $\mathrm{h}^{3} \mathrm{wmh}-\quad 23920,23930$ & $\mathrm{~h}^{3} \mathrm{~h}-\quad 24030,24050$ & $\mathrm{~h}^{3} \mathrm{hxhdy}-\quad 24170$ \\
\hline$h^{3} w m \infty-\quad 23920$ & $\mathrm{~h}^{3} \mathrm{hp}-\quad 23210,24060$ & $h^{3}$ hyh- $\quad 14380,23690$ \\
\hline $\mathrm{h}^{3} \mathrm{wm}^{3-} \quad 23930$ & $\mathrm{~h}^{3} \mathrm{hm} w s-\quad 24070$ & $\mathrm{~h}^{3} \mathrm{hgvh}-\quad 23500$ \\
\hline$h^{3} w d h x-\quad 23940$ & $\mathrm{~h}^{3} \mathrm{hmww}-\quad 24070$ & $h^{3} h x v-\quad 24180$ \\
\hline $\mathrm{h}^{3} \mathrm{wt}-\quad 17730$ & $\mathrm{~h}^{3} \mathrm{hmw} \mathrm{y}^{3-} \quad 24071$ & $\mathrm{~h}^{3} \mathrm{hwh}-\quad 17720,17820$ \\
\hline $\mathrm{h}^{3} \mathrm{WZ}-\quad 17740$ & $\mathrm{~h}^{3} \mathrm{hmh}-\quad 23230$ & 23860 \\
\hline
\end{tabular}


North Wakashan Comparative Root List (Updated)

$\mathrm{h}^{3}$ hwhqv- 24181

$\mathrm{h}^{3} \mathrm{hw}^{3-} \quad 17720$

h ${ }^{3}$ hqv- 24190

$\mathrm{h}^{3} \mathrm{~h} \chi \mathrm{v}-\quad 17790$

$\mathrm{h}^{3} \mathrm{hr}-15170$

$\mathrm{h}^{3} \mathrm{hq}^{-} \quad 24210$ 


\section{References}

Boas, Franz. 1928. Bella Bella Texts. Columbia University Press, New York.

Boas, Franz. 1947. Kwakiutl Grammar with a Glossary of the Suffixes. Op. posth. edited by Helene Yampolsky with the collaboration of Zellig S. Harris. Transactions of the American Philosophical Society, New Series Volume 37, Part 3, pp. 201-377.

Boas, Franz. (n.d.) Kwakiutl Dictionary. Unpublished typescript edited by Helene Boas Yampolsky. In 1948 added to the Boas Collection of the American Philosophical Society.

Fortescue, Michael D. 2007. Comparative Wakashan Dictionary. Lincom studies in native American linguistics, Volume 57. Munich: Lincom Europa.

Galloway, Brent D. 2009. Dictionary of Upriver Halkomelem. University of California Publications in Linguistics, Volume 141.

Kuipers, Aert H. 1967. The Squamish Language. Mouton \& Co., The Hague, The Netherlands.

Kuipers, Aert H. 2002. Salish Etymological Dictionary. University of Montana Occasional Papers in Linguistics No. 16, Missoula, MT 59812

Lincoln, Neville J. (n.d.) Kwakwala Dictionary. Unpublished typescript. 


\section{North Wakashan Comparative Root List (Updated)}

Lincoln, Neville J. and John C. Rath. 1980. North Wakashan Comparative Root List. National Museum of Man, Mercury Series, Canadian Ethnology Service Paper No. 68.

Nater, Hank. (n.d.) The Nuxalk Language (lexicon, names, narratives, grammar). Unpublished typescript.

Sapir, Edward and Morris Swadesh. 1939. William Dwight Whitney Linguistic Series, Linguistic Society of America, University of Pennsylvania, Philadelphia.

Swadesh, Morris. 1953. Salish-Wakashan Lexical Comparisons Noted by Boas. IJAL Volume 19. 